\title{
PREDICTING TOXIC GAS CONCENTRATIONS RESULTING FROM ENCLOSURE FIRES USING THE LOCAL EQUIVALENCE RATIO CONCEPT LINKED TO FIRE FIELD MODELS
}

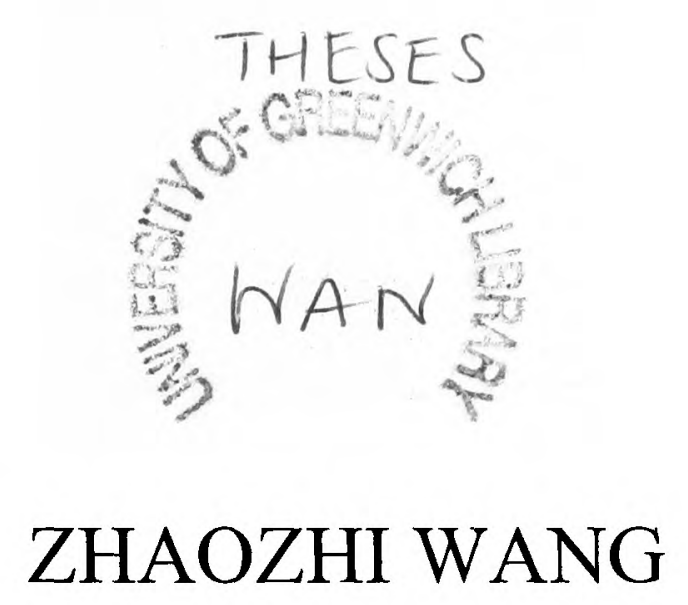

A thesis submitted in partial fulfilment of the requirements of the University of Greenwich for the degree of Doctor of Philosophy

March 2007

School of Computing and Mathematical Science

The University of Greenwich

30 Park Row, Greenwich, London SE10 9LS 


\section{ACKNOWLEDGEMENTS}

I am deeply indebted to my supervisors, Prof. Ed Galea and Dr. Fuchen Jia, for their great interesting, enthusiasm and guidance in my research. They provided me with many helpful comments and suggestions during my studies and on the writing of research papers and this thesis. I would like to thank my internal examiner, Dr. Mayur $\mathrm{K}$. Patel, for the useful guidance in improving the final form of the thesis.

Part of the fire experiments for validating the toxicity models developed in this work carried out at the fire laboratory at the Rockwool International, Denmark. The author would like to thank Mr. A. Jakobsen and Mr. K. Ejlersen and other staff at that fire laboratory for carrying out the tests and the Rockwool International for financial support.

I grateful acknowledge the financial support of FSEG of the University of Greenwich through its research bursary programme. I also wish to thank Dr. J. Ewer, Dr. A. Grandison, Dr. S. Gwynne, Mr. Y. Wang and all my friends for their advice and help in my research.

Finally, I would like to thank my parents for their encouragement and my wife, Ms Ruixia Deng for her understanding and support during many years of the study. 


\section{ABSTRACT}

Fire field modelling is based on the techniques of computational fluid dynamics (CFD), which provide detailed variable solutions throughout the computational domain quickly, repeatedly and cheaply compared with fire experiments. A main component of fire effluent are toxic gases and one of the main toxic gases responsible for a significant number of fire fatalities is Carbon Monoxide (CO). Current engineering fire field models either ignore the prediction of toxic gas generation or require the use of detailed chemistry to predict toxic gas generation. These detailed chemistry models require considerable data which is not generally available for most common building materials. The main objective of the study presented in this thesis is to develop practical and reasonable engineering fire field models to simulate the production and movement of toxic gases in enclosure fires. The developed toxicity models should be capable of working within the framework of the current popular combustion models in fire safety engineering. In addition, the developed models should not rely too heavily on hard to obtain experimental data.

The central idea behind the newly developed toxicity model is the use of the Local Equivalence Ratio (LER). The species yields as functions of the Global Equivalence Ratio (GER) and temperature are input parameters of this model. Correlations for most building materials are available from small-scale fire experiments. Similar approaches to this method are also developed using the $\mathrm{CO} / \mathrm{CO}_{2}$ and $\mathrm{H}_{2} / \mathrm{H}_{2} \mathrm{O}$ mole ratios. The LER methodology is further refined by an approach which divides the computational domain for the calculation of toxic gases into two parts, a control region in which the toxic gases are dependent on the LER and temperature, and a transport region in which the toxic gas concentrations are dependent on the mixing of hot gases with fresh air.

The toxicity model is then extended to two-fuel cases. In the two-fuel model, the LER is a function of the two mixture fractions, which are used to represent the mixture of the two different fuels, oxygen and combustion products. This model is useful in simulating residential fires, in which wood lining of sidewalls or ceilings is the second fuel. 
Finally, the transportation of $\mathrm{HCl}$ within fire compartments is considered. $\mathrm{HCl}$ generated from the combustion of materials containing chlorine is both a potent sensory irritant and a strong pulmonary irritant. $\mathrm{As} \mathrm{HCl}$ is transported in the gaseous flows produced by fire, a considerable amount of $\mathrm{HCl}$ may be deposited on enclosure walls and thus removed from the general transport flow. It is therefore necessary to develop models which take into account the amount of $\mathrm{HCl}$ deposited on the walls and removed from the flow. A mathematical model is developed to simulate the exchange of $\mathrm{HCl}$ between gas boundary and wall surfaces and the reaction of $\mathrm{HCl}$ with walls.

All the toxicity models developed in this study can be integrated into the practical volumetric heat source approach and the Eddy Break-up (EBU) combustion model typically used in practical engineering analysis. Finally, the integrated fire models developed in this study are validated using a range of fire tests. 


\section{CONTENTS}

\section{CHAPTER 1}

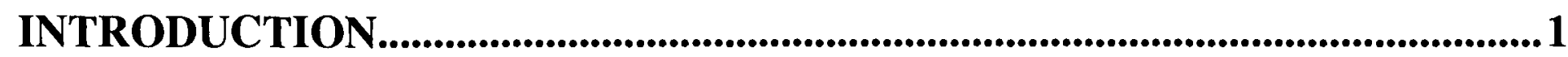

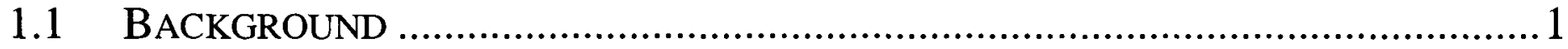

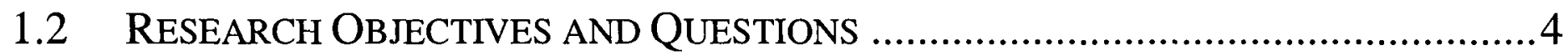

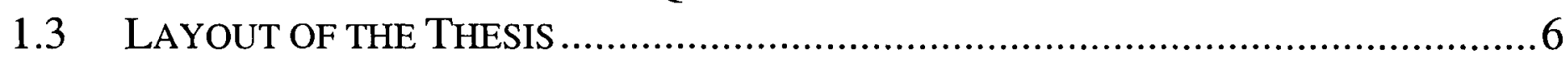

\section{CHAPTER 2}

BACKGROUND AND LITERATURE REVIEW ............................................8

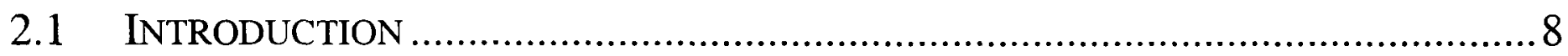

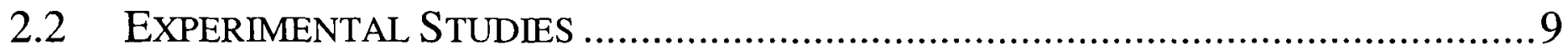

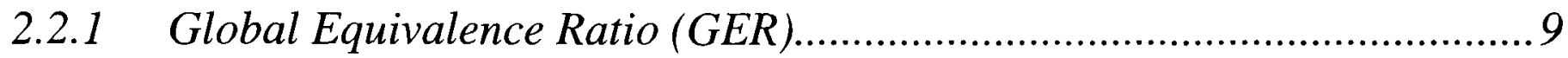

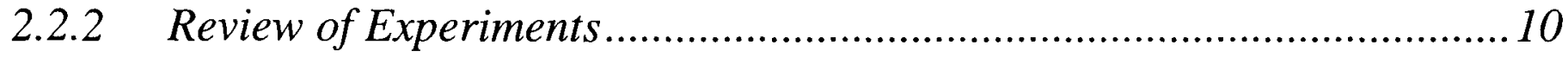

2.2.2.1 Earlier Small-scale experiments .................................................... 10

2.2.2.2 The Purser Furnace experiments .................................................. 11

2.2.2.3 Reduced-room experiments ...................................................... 13

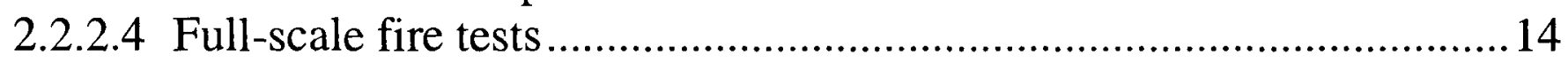

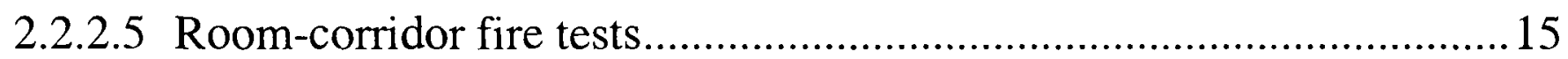

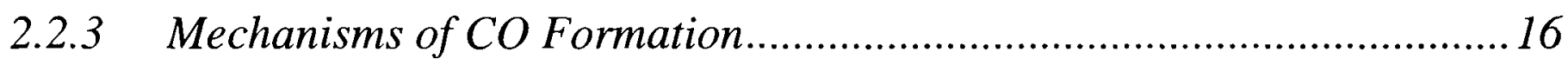

2.2.4 Engineering CO Yield Correlations ..................................................... 18

2.2.4.1 Correlations for polymers ......................................................... 18

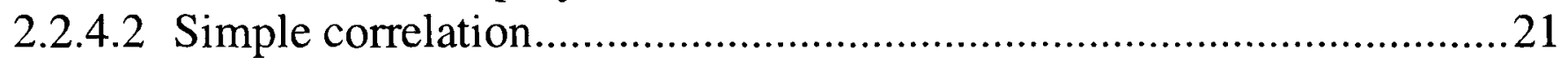

2.2.4.3 Correlations for hexane and methane ............................................22

2.2.4.4 Correlations for plastics...............................................................22

2.3 ABSORPTION OF HCL BY WALLS OF ENCLOSURES .....................................2 23

2.4 SMOKE PROPERTIES ............................................................................ 25

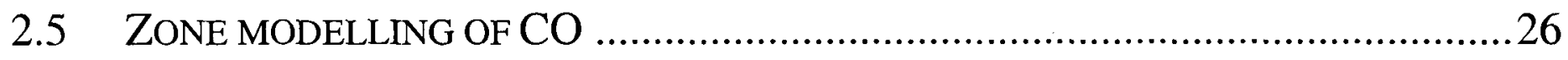

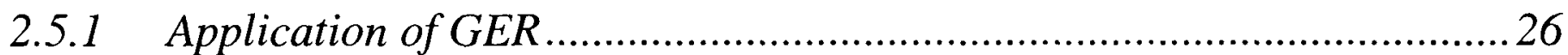

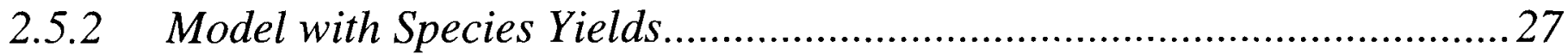

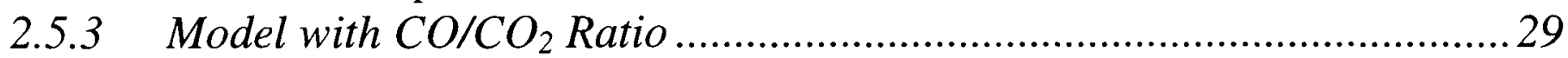

2.5.4 Limits of Applying GER in Zoon Modelling ....................................... 30

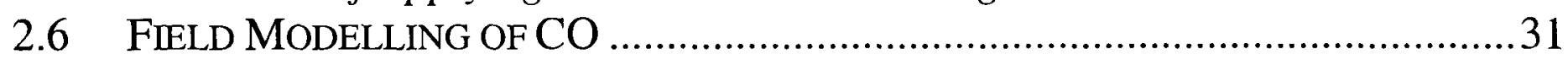

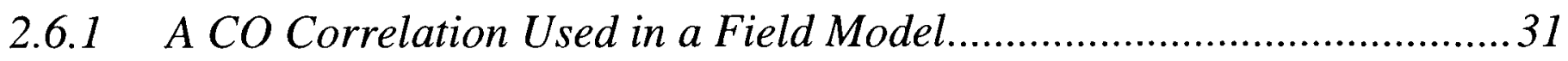

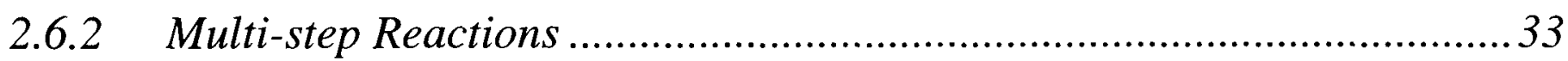

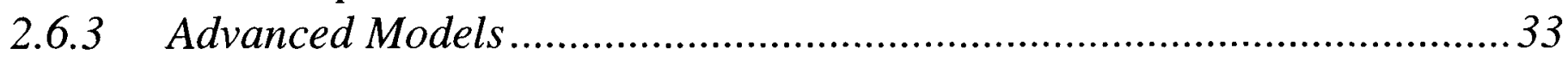

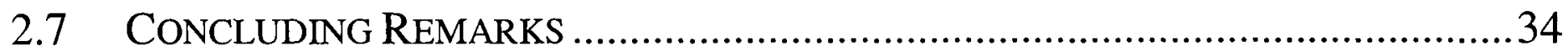

\section{CHAPTER 3}

MATHEMATICAL MODELS FOR ENCLOSURE FIRES.............................36

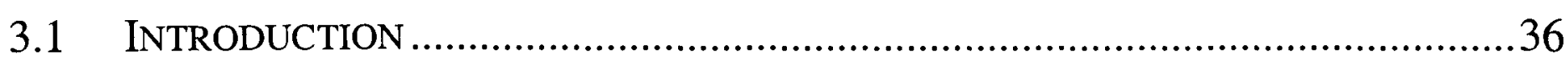

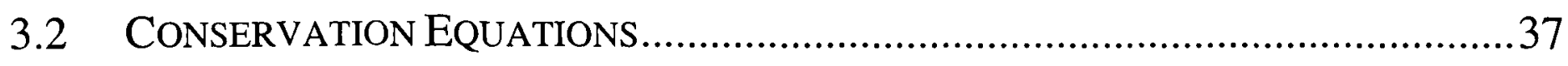

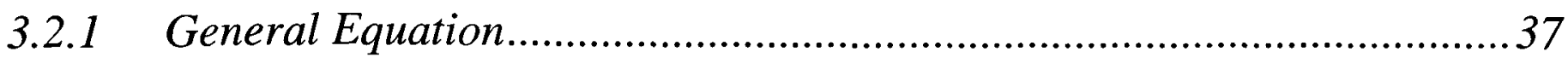

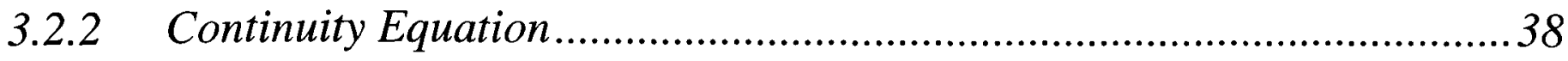

3.2.3 Momentum Equation .................................................................. 38 


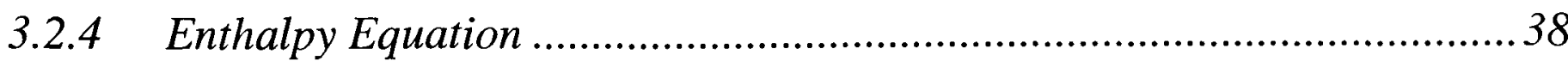

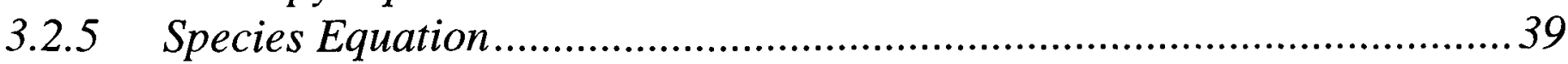

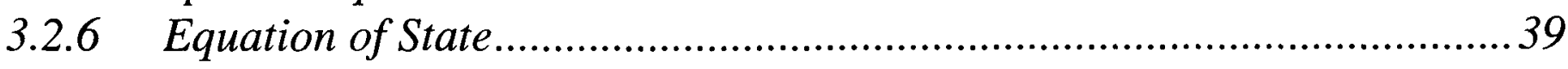

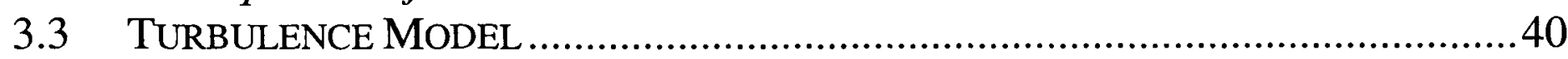

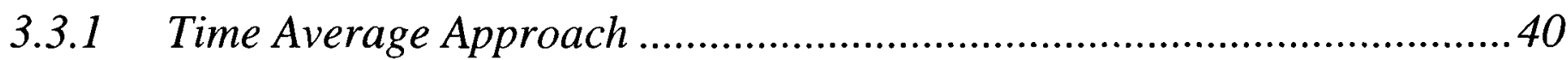

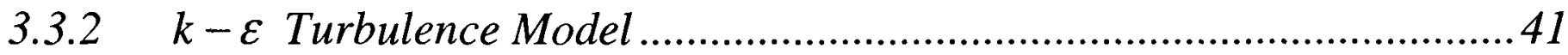

3.3.3 Large Eddy simulation model ........................................................... 43

3.4 CoMBUSTION MODELS .............................................................................43

3.4.1 Volumetric heat source approach ....................................................... 44

3.4.2 Conserved scalar approach .............................................................. 45

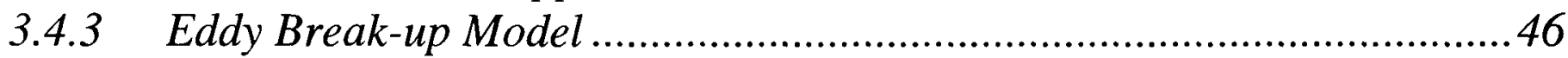

3.4.3.1 Eddy Break-up model .....................................................................46

3.4.3.2 Two-fuel Eddy Break-up Model.........................................................4 4

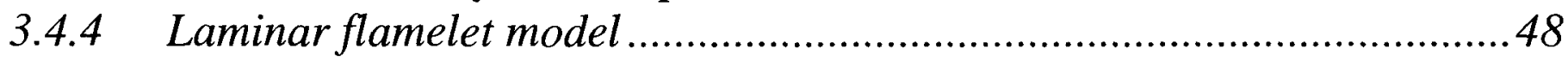

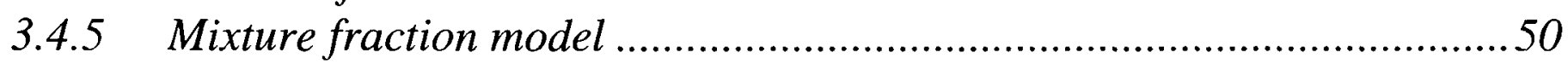

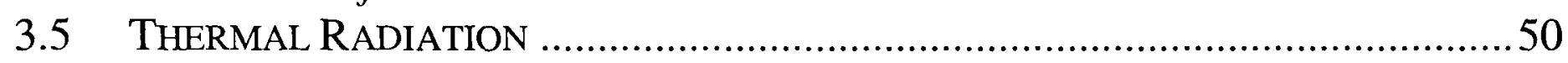

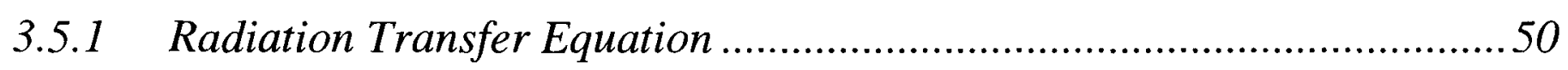

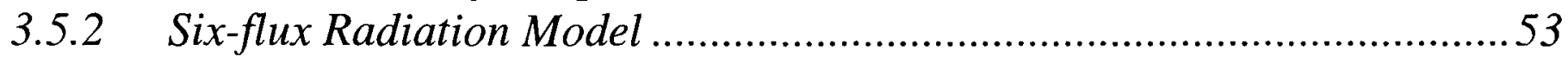

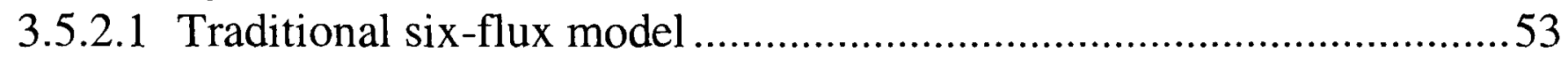

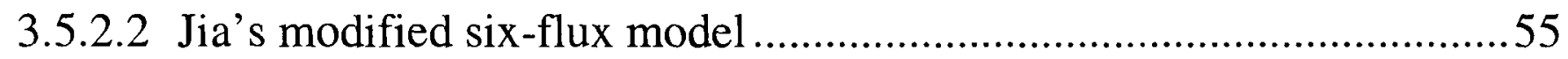

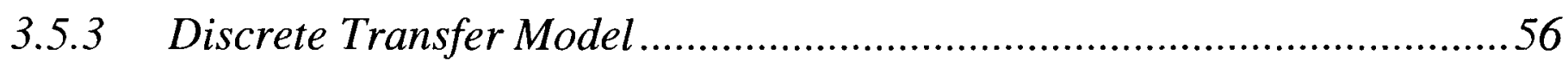

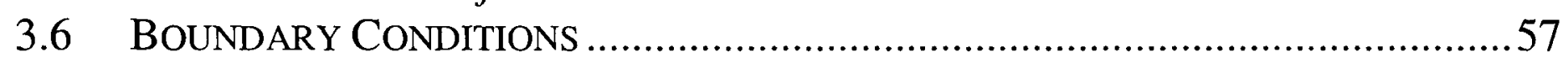

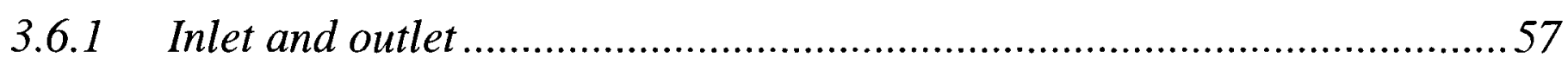

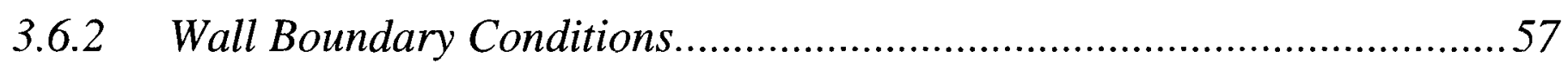

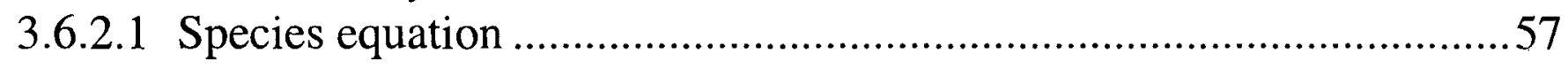

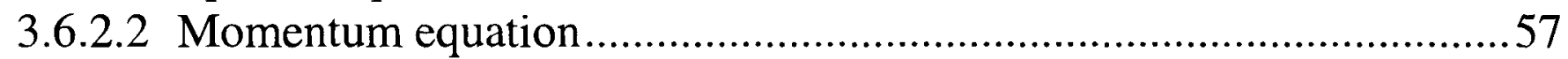

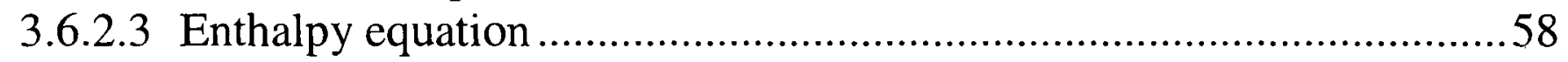

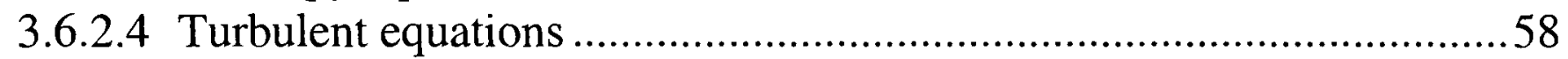

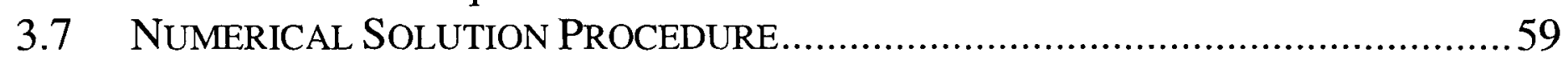

3.7.1 Discrete form of Conservation Equations............................................59

3.7.2 Solution Method .............................................................................60

3.8 SimUlating ONE OF THE CIB W 14 Round RobIN TEST CASES ...................61

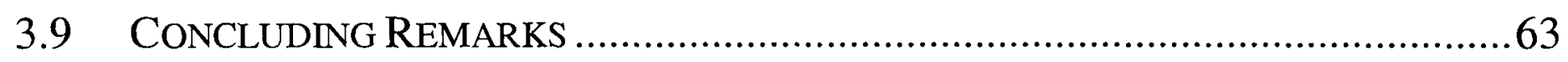

\section{CHAPTER 4}

TOXICITY MODELS USING LOCAL EQUIVALENCE RATIO ...................64

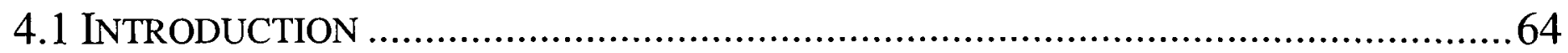

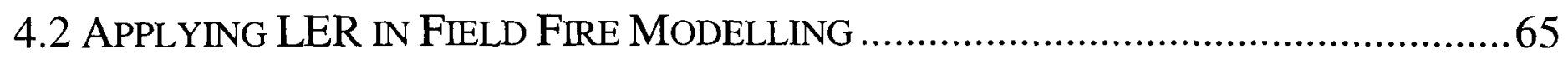

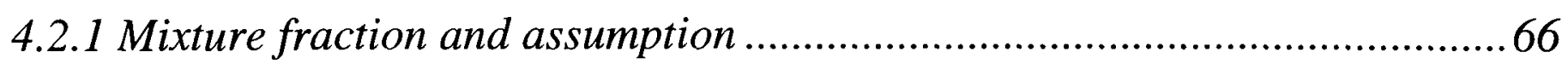

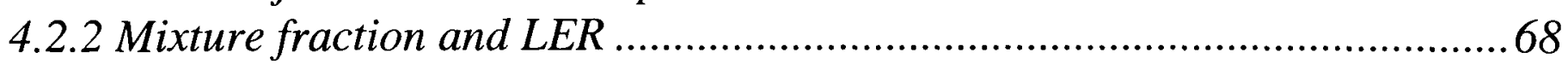

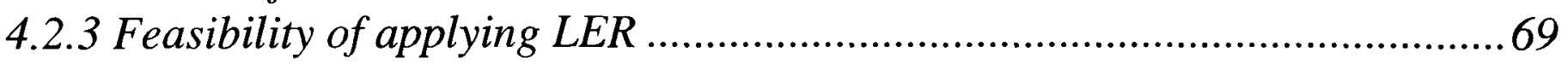

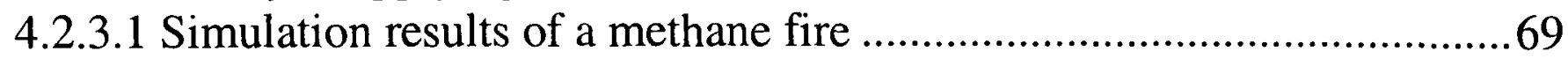

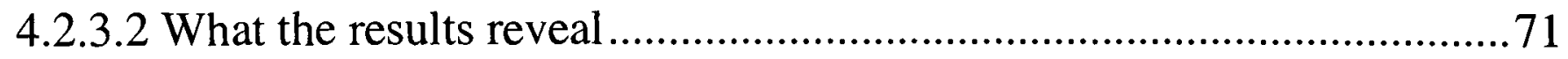

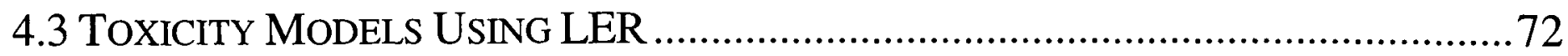

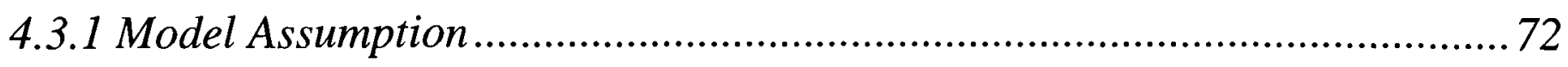

4.3.2 Model based on species yields-Model I ................................................ 73

4.3.3 Model based on $\mathrm{CO} / \mathrm{CO}_{2}$ ratio-Model $\mathrm{II}$................................................76 76

4.3.4 Model based on ratios of $\mathrm{CO} / \mathrm{CO}_{2}$ and $\mathrm{H}_{2} / \mathrm{H}_{2} \mathrm{O}-$ Model $\mathrm{III}$..................... 78 


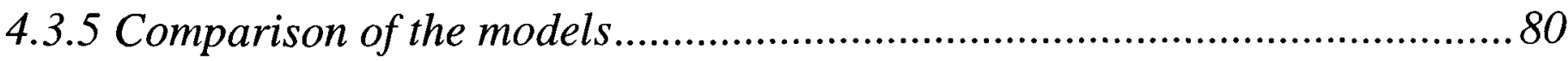

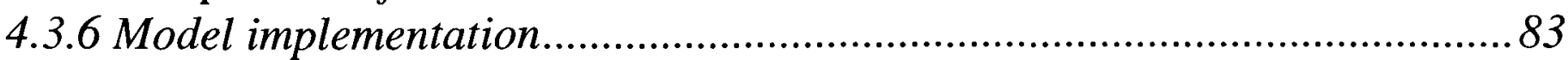

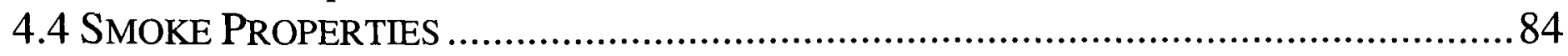

4.4.1. Calculation of smoke properties ....................................................... 85

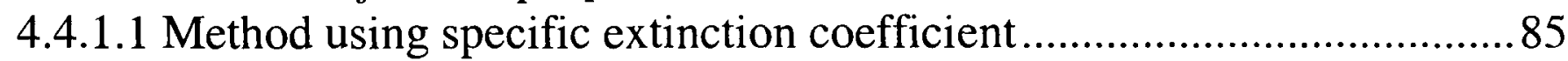

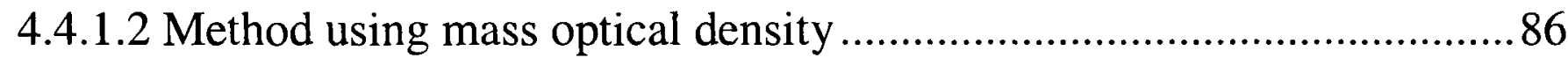

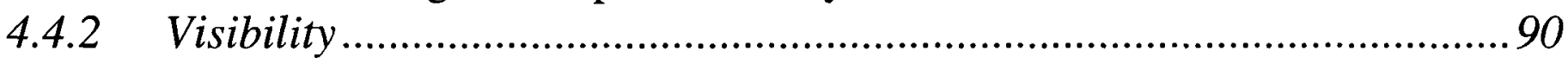

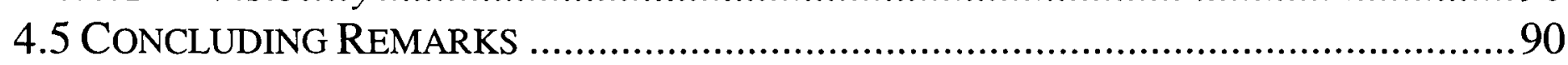

\section{CHAPTER 5}

SIMULATIONS OF ENCLOSURES FIRES...............................................92

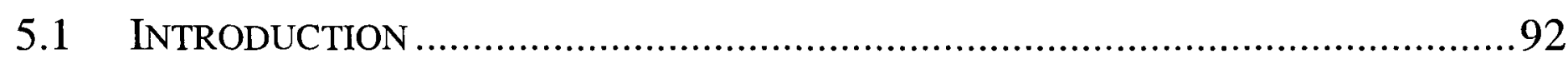

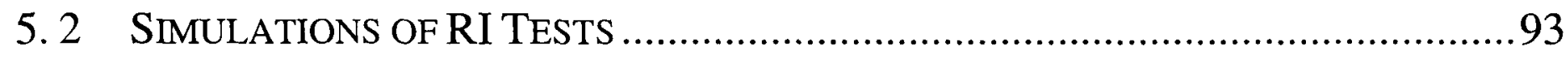

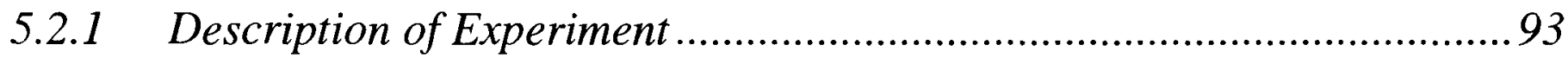

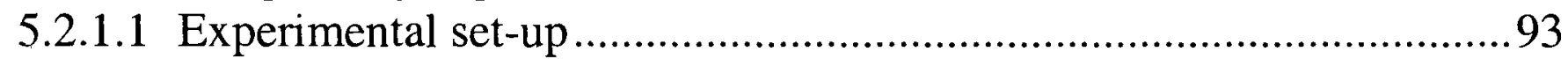

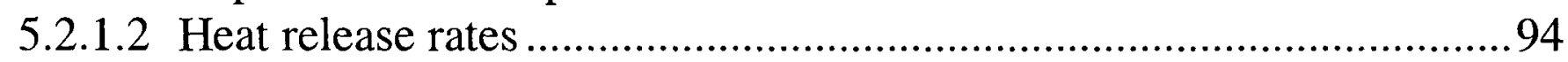

5.2.1.3 Burning processes of the tests ................................................ 96

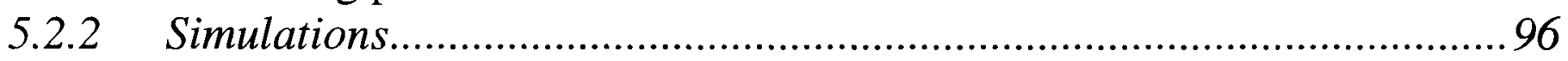

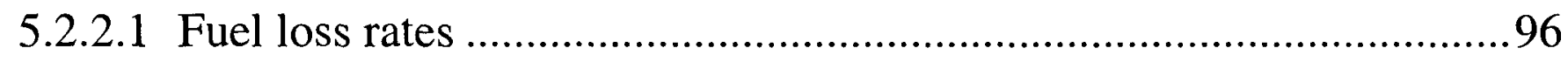

5.2.2.2 Modelling of wood cribs and meshes ........................................... 97

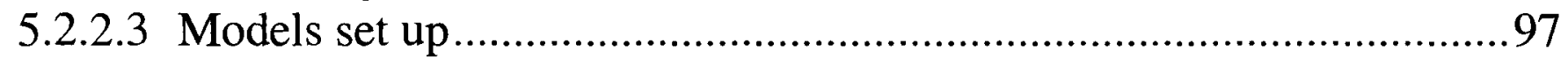

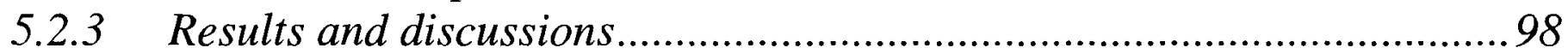

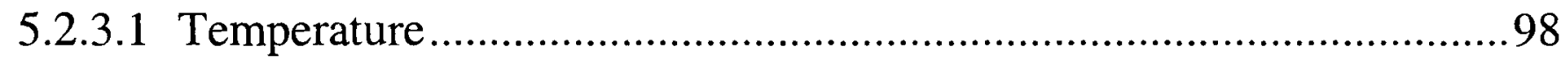

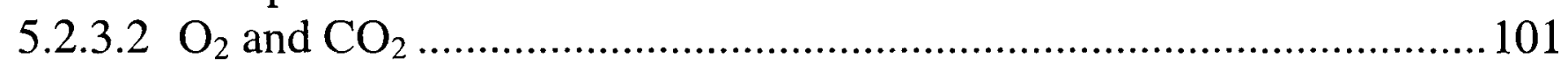

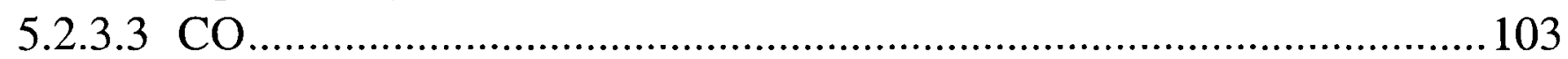

5.3 SIMULATION OF ONE OF THE TOXFIRE TESTS ......................................... 104

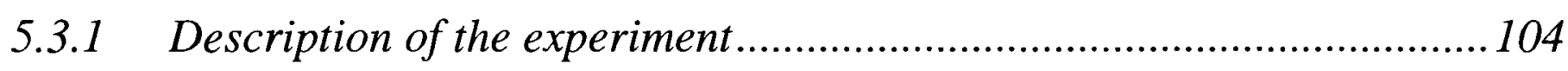

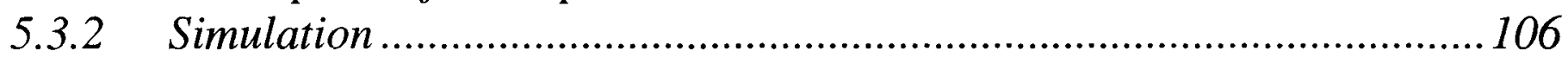

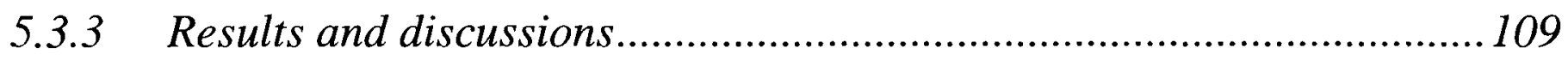

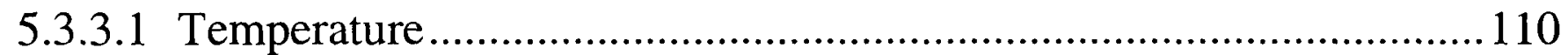

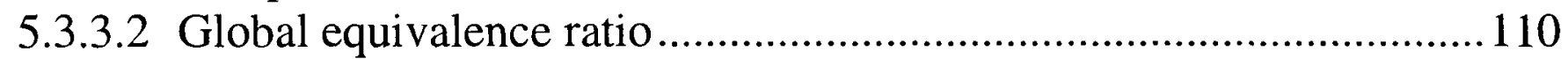

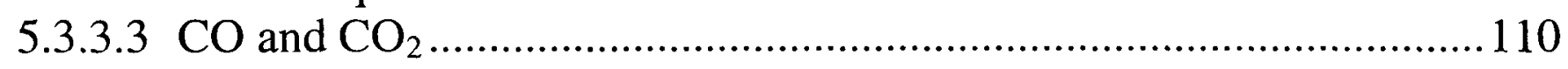

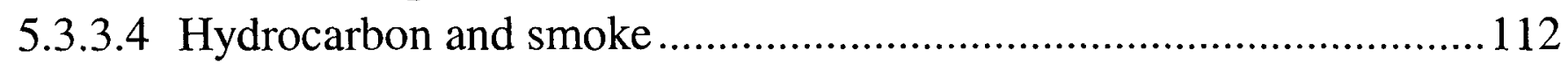

5.4 SIMULATION OF W14-B3 FIRE TEST .................................................. 112

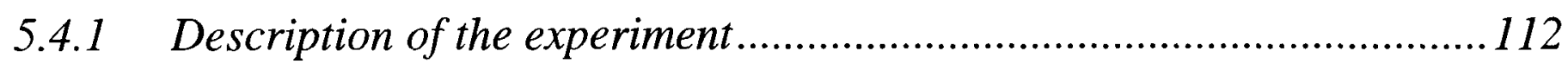

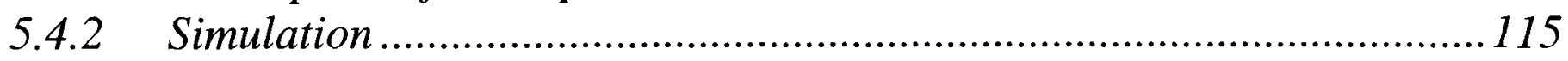

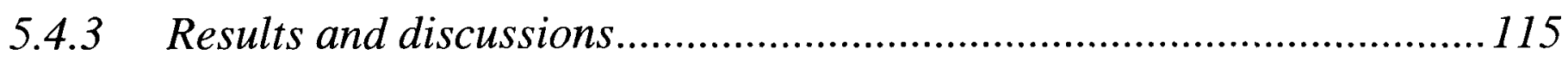

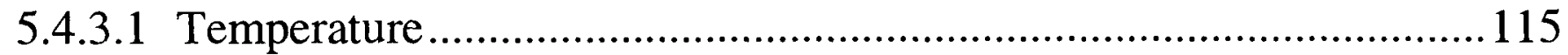

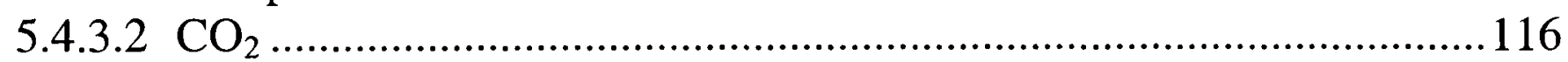

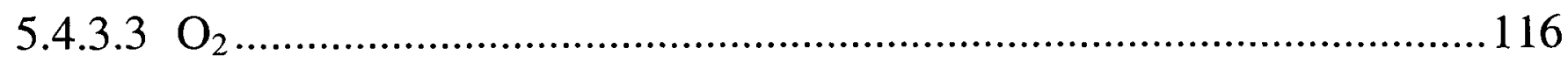

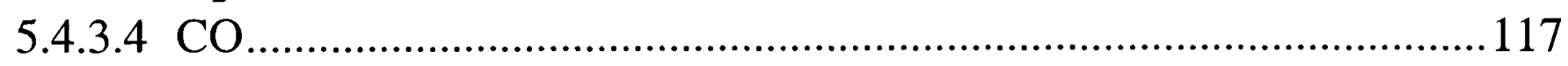

5. 5 Simulation of THE Borealis CABLE FIRE TEST .................................... 120

5.5.1 Description of the experiment ....................................................... 120

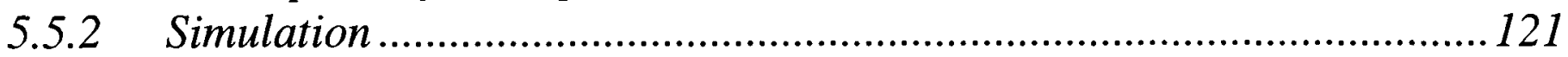

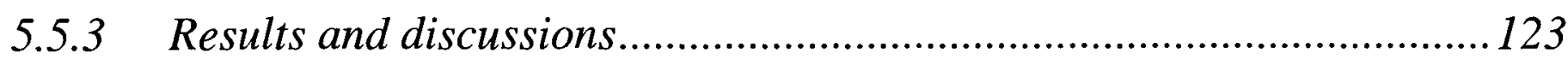

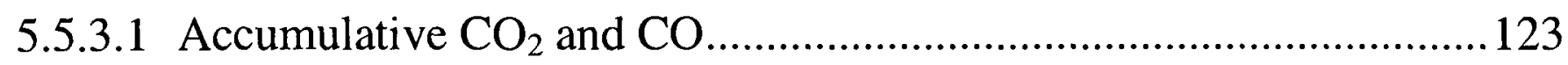

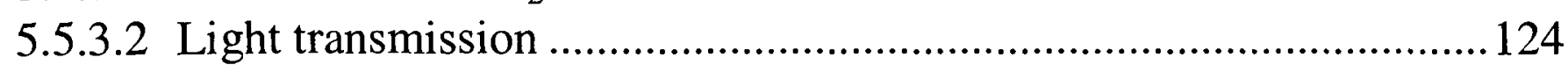

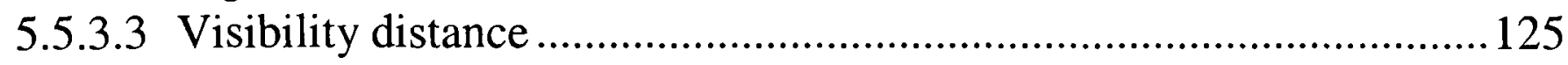


5.6 SIMULATION OF A COMPARTMENT-HALLWAY FIRE TEST ….........................126

5.6.1 Description of the experiment ......................................................... 126

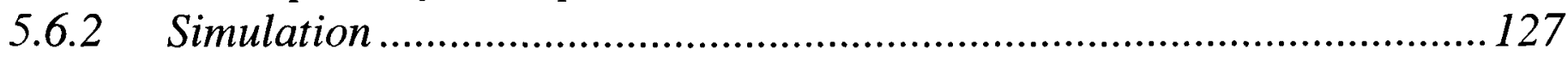

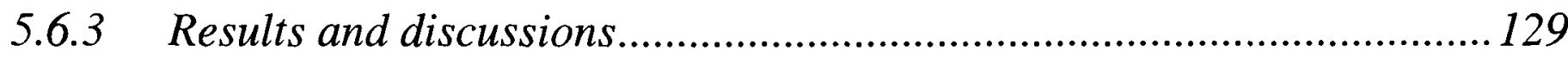

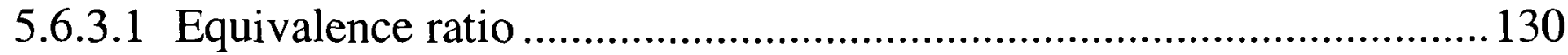

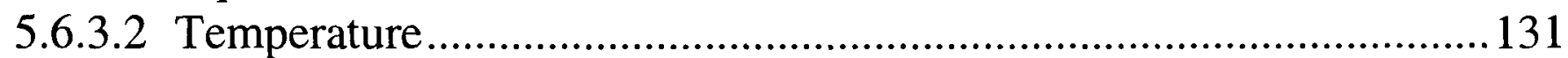

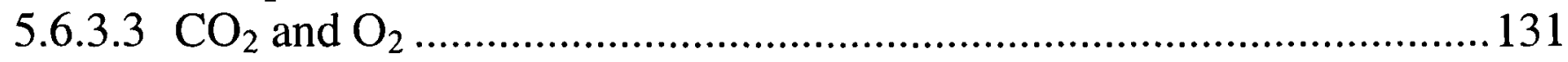

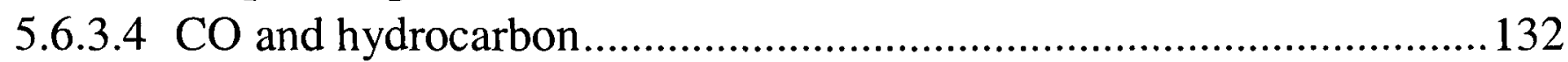

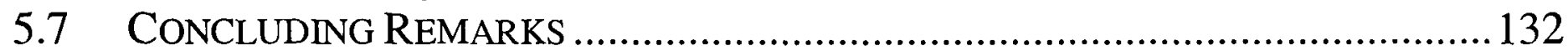

\section{CHAPTER 6}

APPROACH TO TOXIC GASES AT REMOTE LOCATIONS ......................135

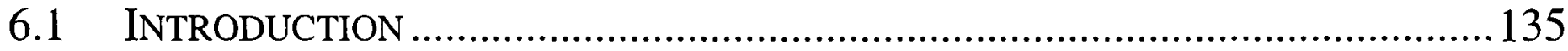

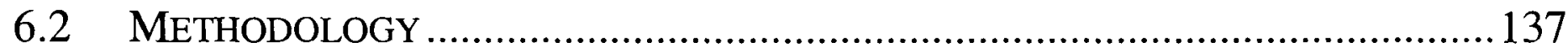

6.2.1 Division of the computational domain ...............................................137

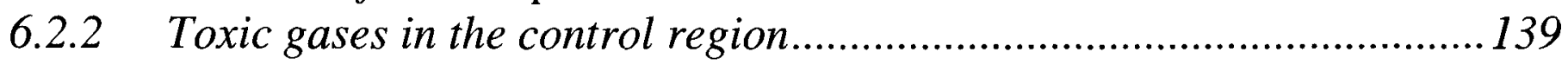

6.2.3 Toxic gases in the transport region ................................................ 140

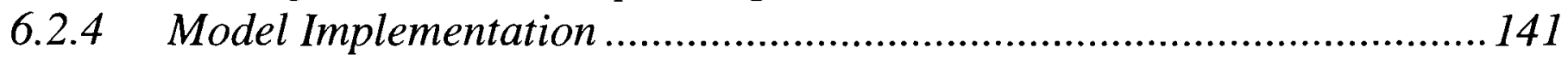

6.2.5 Applicable Conditions................................................................... 141

6.3 Simulations OF ONE OF THE COMPARTMENT-HALl WAy HEXANE FIRES ..... 142

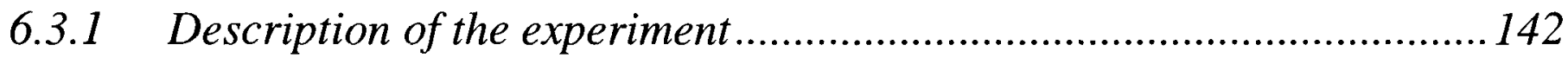

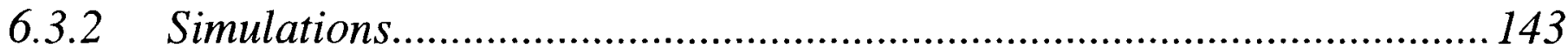

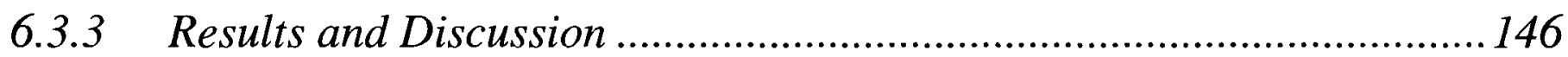

6.3.3.1 Transient compartment temperature ............................................. 146

6.3.3.2 Predicted control region during steady state.................................. 147

6.3.3.3 Temperatures and species during steady state .............................. 148

6.3.3.4 Comparison with Model I........................................................... 150

6.3.3.5 Sensitivity of species on critical equivalence ratio..........................150

6.4 SIMULATIONS OF A CORRIDOR CABLE FIRE ............................................ 151

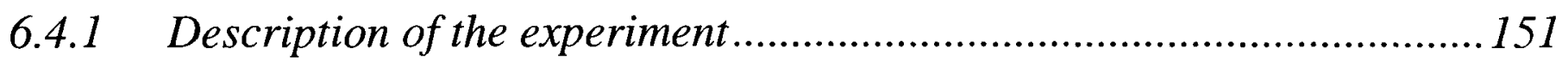

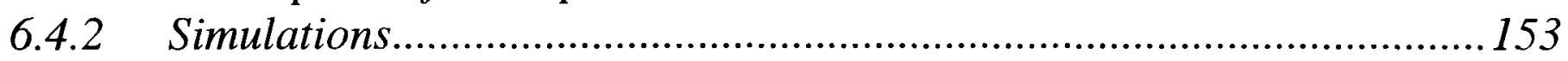

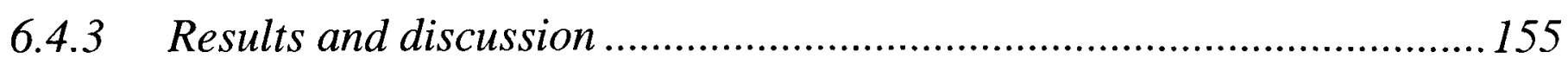

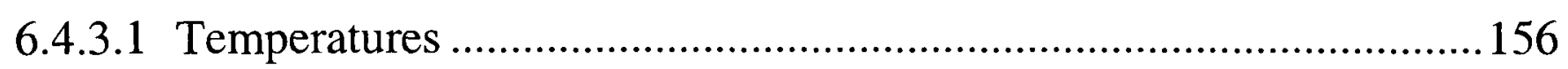

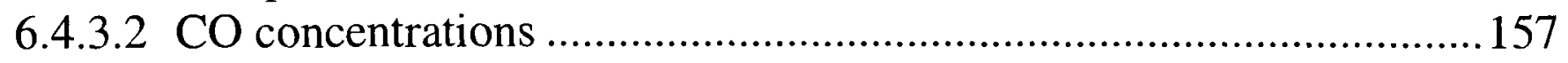

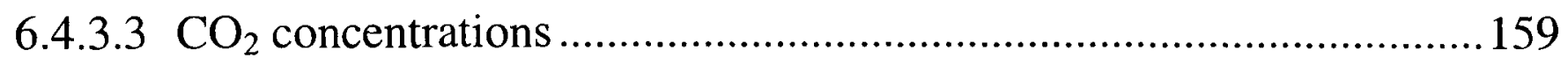

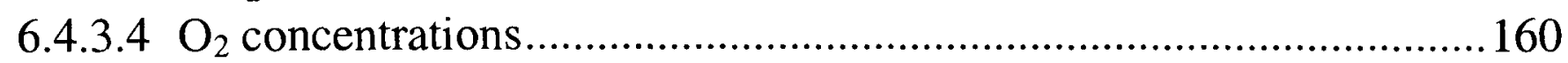

6.4.3.5 Predicted control region at time of peak burning rate ..................... 160

6.4.3.6 Comparison with Model I.............................................................. 161

6.4.3.7 Sensitivity of species on combustion efficiency.............................162

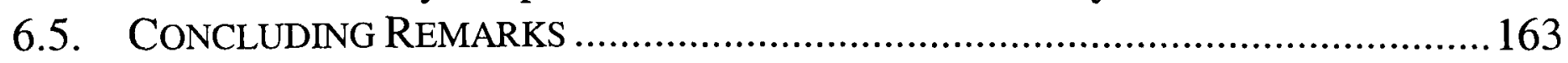

\section{CHAPTER 7}

TWO-FUEL TOXICITY MODEL AND APPLICATION.......................................165

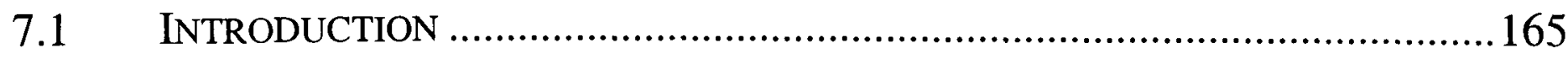

7.2 LOCAL EQUIVALENCE RATIO FOR TWO-FUEL FIRES ...............................166

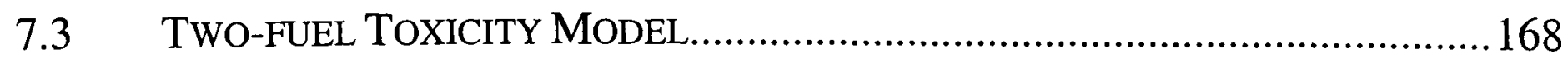

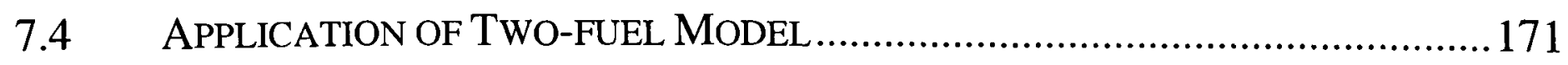


7.4.1 Description of fire experiments................................................ 171

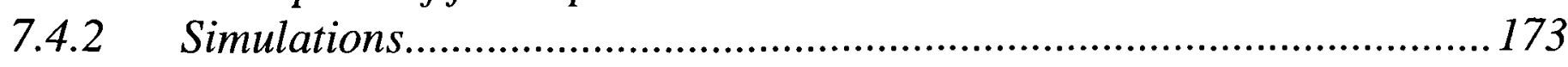

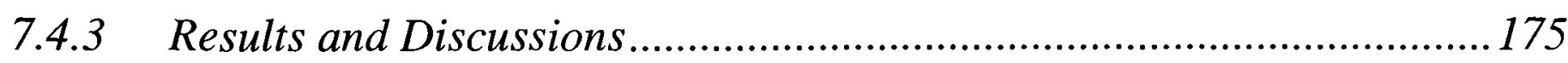

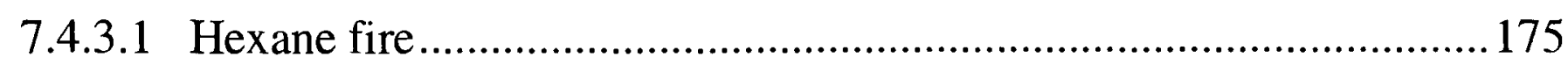

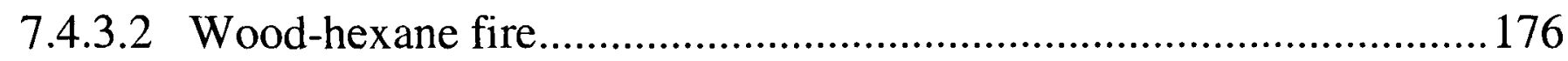

7.4.3.3 Effect of wood on combustion products ........................................179

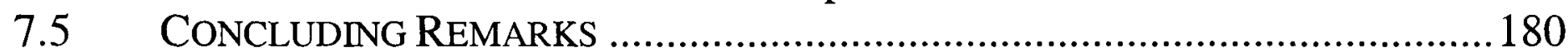

\section{CHAPTER 8}

DEPOSITION OF HCL ON SOLID SURFACES................................................182

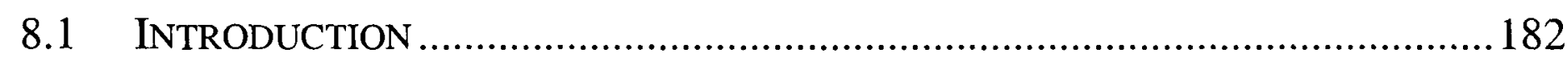

8.2 THE ORIGINAL MODEL ………………................................................... 183

8.2.1 Movement of HCL to Boundary Layer.................................................183

8.2.2 Equilibrium between Boundary Layer and Wall Surfaces...................... 184

8.2.3 Diffusion and Reaction with Wall....................................................... 185

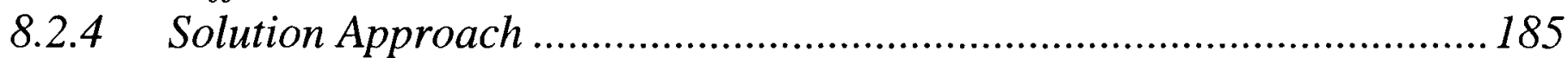

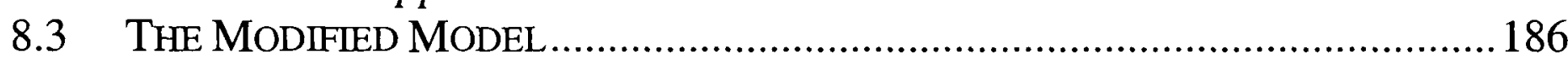

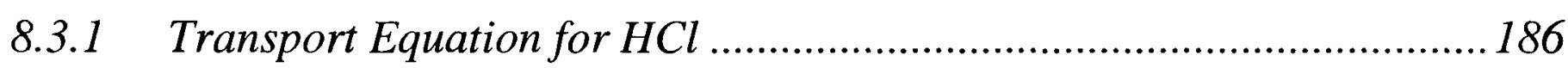

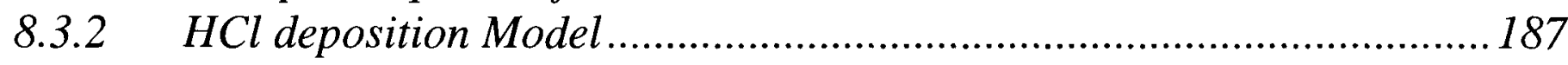

8.3.2.1 On the movement of $\mathrm{HCl}$ to boundary layer .................................... 187

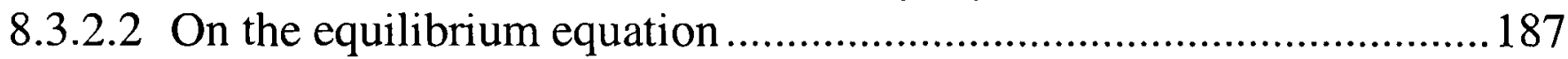

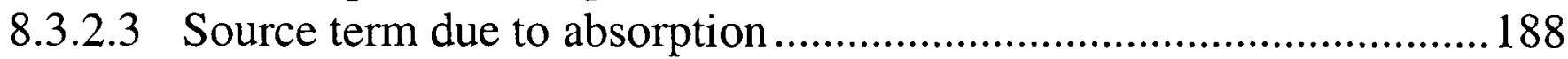

8.3.3 Implementation of the $\mathrm{HCl}$ decay model ..............................................191

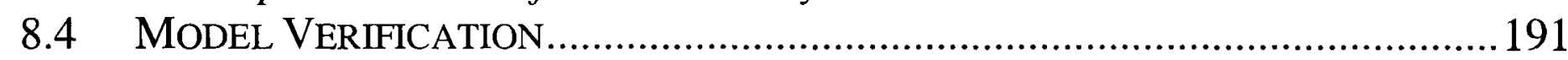

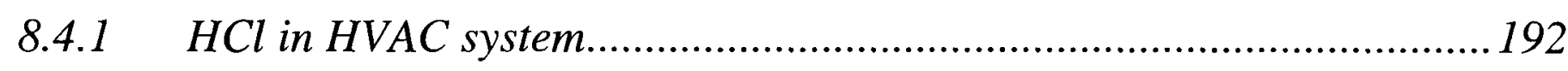

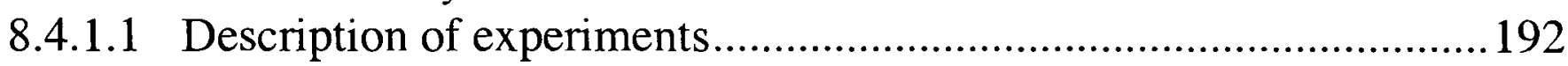

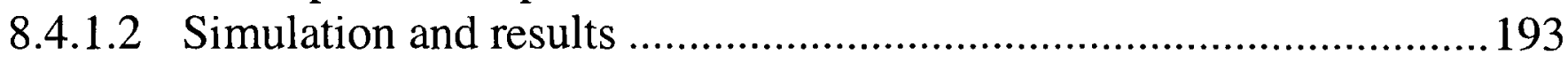

8.4.2 Small-scale chamber PVC fires ......................................................196

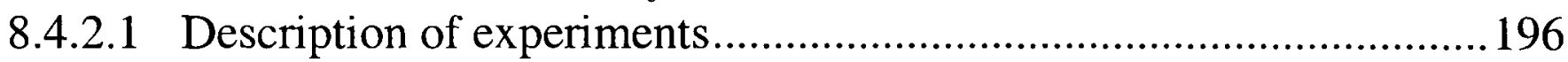

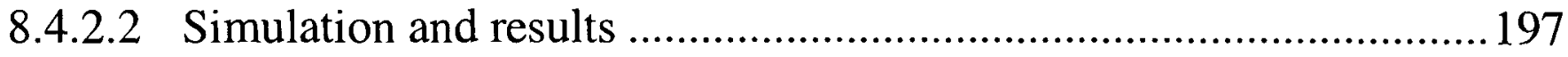

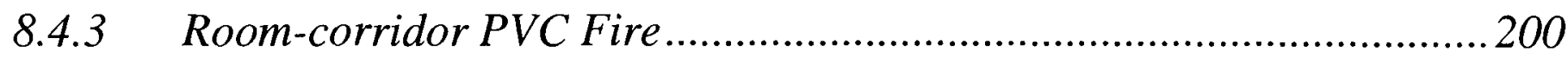

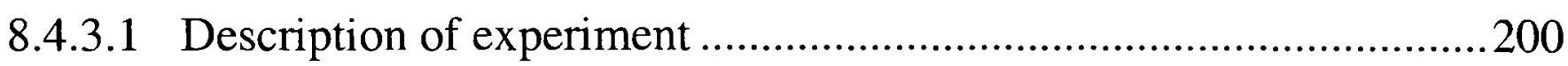

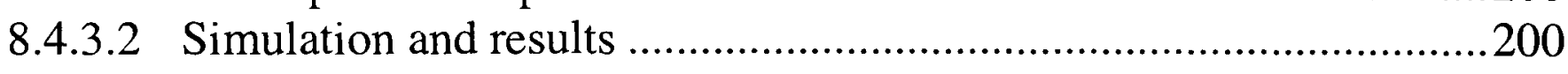

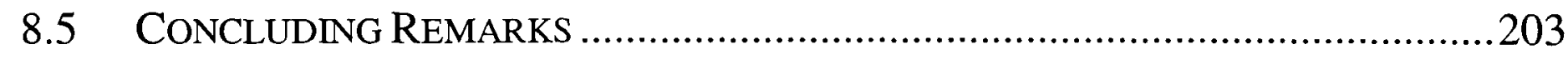

\section{CHAPTER 9}

CONCLUSIONS .............................................................................................................205

\section{CHAPTER 10}

FURTHER WORK .................................................................................................213

10.1 EFFECT OF HIGH TEMPERATURES ON CO.....................................................213

10.2 EFFECT OF PYROL YSIS OF SOLID FUEL ON CO ………..................................214

10.3 CRITERION FOR DETERMINING THE CONTROL REGION …….............................214

10.4 TOXICITY MODELS FOR HCN AND SOOT .....................................................215

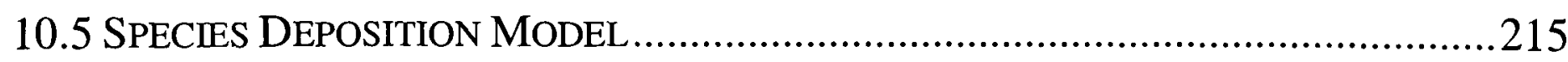




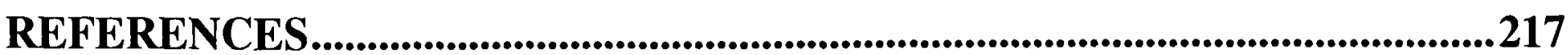

APPENDIX A

SIMULATING ONE OF THE CIB W14 ROUND ROBIN TEST CASES USING

THE SMARTFIRE FIRE FIELD MODEL ..................................................................2234

APPENDIX B

PUBLICATIONS

256 


\section{LIST OF FIGURES}

Figure 2.2.1: Schematic of the Purser furnace (reproduced from [BS03]). 12

Figure 2.2.2: Ratio of the yield at well ventilation condition to the yields with various equivalence ratios for (a) $\mathrm{CO}_{2}$ and (b) $\mathrm{CO}$ (reproduced from [Te95])... 19

Figure 2.2.3: Yields of $\mathrm{CO}$ as function of equivalence ratio from Purser Furnace NHMH cable experiments.

Figure 3.8.1: Depiction of fire compartment geometry showing location of fire source and the location of the three thermocouple trees.

Figure 3.8.2: Predicted (symbols) and measured (solid lines) temperatures at the top and bottom thermal couples along the thermal couple tree $B B^{\prime}$.

Figure 4.2.1: Predicted temperature contours at the vertical plane passing through the thermocouple trees.

Figure 4.2.2: Predicted LERs on (a) the central plane and (b) its parallel plane.........70

Figure 4.2.3: Flow field in the central plane through the doorway.............................71

Figure 4.3.1: Predicted $\mathrm{CO}_{2}$ mass fractions as function of the LER........................ 81

Figure 4.3.2: Predicted $\mathrm{O}_{2}$ mass fractions as function of the LER........................82

Figure 4.3.3: Predicted CO mass fractions as function of the LER ..........................82

Figure 5.2.1: Schematic of RI fire experimental set up..........................................94

Figure 5.2.2: Heat release rates (KW) of RI fire tests. ...........................................95

Figure 5.2.3: Fire test in Rockwool International: (a) ignition of wood cribs; (b) early stage of the fire; (c) side view of the fire plume in the doorway; (d) normal view of the fire.

Figure 5.2.4: (a) Mole ratio of $\mathrm{CO}$ to $\mathrm{CO}_{2}$ in the duct; (b) the combustion efficiencies used in the simulation.........................................................................96

Figure 5.2.5: Predicted temperature $(\mathrm{K})$ contours in the central plane through the doorway at 6 minutes.

Figure 5.2.6: Measured and predicted temperatures at (a) thermocouple tree A; (b) thermocouple tree B; (c) thermocouple tree C; (d) thermocouple tree D.

Figure 5.2.7: Measured and predicted temperatures at thermocouple tree $\mathrm{D}$ as function of height at 6 minutes.

Figure 5.2.8: Measured and predicted $\mathrm{O}_{2}$ volume fractions (a) at the doorway; (b) inside the room.

Figure 5.2.9: Measured and predicted $\mathrm{CO}_{2}$ volume fractions (a) at the doorway; (b) inside the room.

Figure 5.2.10: Predicted mass fractions of unburned fuel inside the room (a) and at the doorway (b) by model II and Model III.

Figure 5.2.11: Measured and predicted CO volume fractions (a) at the doorway; and

(b) inside the room.

Figure 5.3.1: (a) Side view of the room and the probe positions in the opening and in the exhaust duct to the calorimeter system and (b) top view of the fire room.

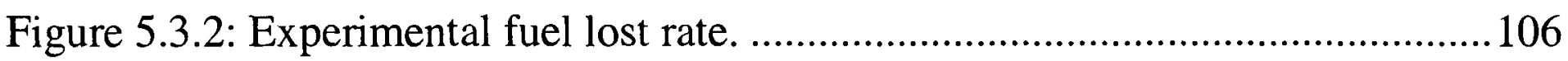

Figure 5.3.3: Total heat releases rate. .......................................................... 106

Figure 5.3.4: Yields of combustion products for polypropylene. Note: the units are 10 $\mathrm{kg} / \mathrm{kg}$ for $\mathrm{CO}_{2}, 1 \mathrm{~kg} / \mathrm{kg}$ for $\mathrm{CO}, 5 \mathrm{~kg} / \mathrm{kg}$ for $\mathrm{HC}$ and $1 \mathrm{~kg} / \mathrm{kg}$ for SOOT. 
Figure 5.3.5: The computational mesh in the compartment opening (each cell measures $0.1 \mathrm{~m} \times 0.11 \mathrm{~m}$ ) and the gas-sampling probe being placed from the top left corner to the middle of other side of the opening.

Figure 5.3.6: Predicted (open) and measured (solid) temperatures as functions of time.

Figure 5.3.7: Predicted (open) and measured (solid) GER as a function of time......111

Figure 5.3.8: Predicted (open) (at positions from $\mathrm{A}$ to $\mathrm{H}$ and their average) and measured (solid) $\mathrm{CO}$ as functions of time.

Figure 5.3.9: Predicted (open) and measured (solid) $\mathrm{CO}_{2}$ volume fraction (\%) as a function of time.

Figure 5.3.10: Predicted (open) and measured (solid) hydrocarbon volume fractions $(\mathrm{ppm})$ in the opening as functions of time.

Figure 5.3.11: Predicted (open) and measured (solid) soot rates $\left(\mathrm{m}^{2} / \mathrm{s}\right)$ in the opening as functions of time.

Figure 5.4.1: The geometry of W14-b3 test and the locations of the instrumentation.

Figure 5.4.2: Fuel loss rates of the two fires.

Figure 5.4.3: (a) The effective heat of combustion (data from Figure 8 in [HK98]); (b) the heat release rates of the two fires.

Figure 5.4.4: Predicted (open) and measured (solid) temperatures as functions of time.

Figure 5.4.5: Predicted (open) and measured (solid) concentrations of $\mathrm{CO}_{2}$ as

functions of time. .................................................................. 116

Figure 5.4.6: Predicted (open) and measured (open) concentrations of $\mathrm{O}_{2}$ as functions of time.

Figure 5.4.7: Predicted (open) and measured (solid) concentrations of $\mathrm{CO}$ as functions of time.

Figure 5.4.8: Predicted $\mathrm{CO}$ contours in the central plane through the opening and the gas collector at 28 minutes. Note: the black square depicts the gas collector and the measured $\mathrm{CO}$ volume fraction is $3.86 \%$...... 118

Figure 5.4.9: Predicted CO contours in the plane through the gas collector and thermocouple tree $\mathrm{Tb}$ at 28 minutes. Note: the black square depicts the gas collector and the measured $\mathrm{CO}$ volume fraction is $3.86 \%$.

Figure 5.4.10: Predicted CO contours in opening window at 28 minutes................. 119

Figure 5.5.1: Schematic of cable fire tests set up [Bo01] .....................................120

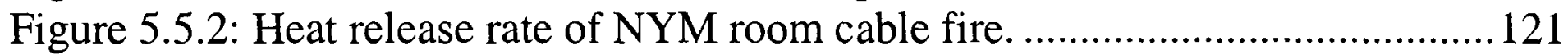

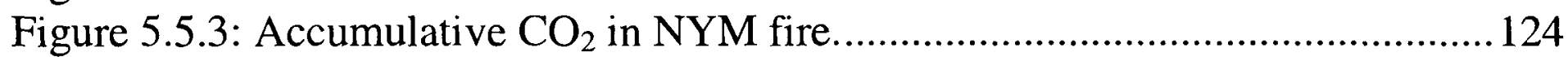

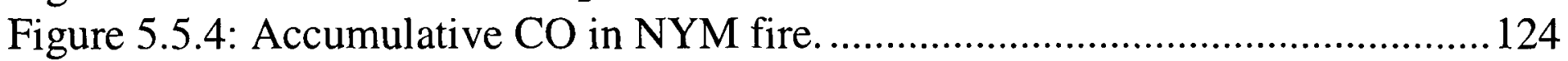

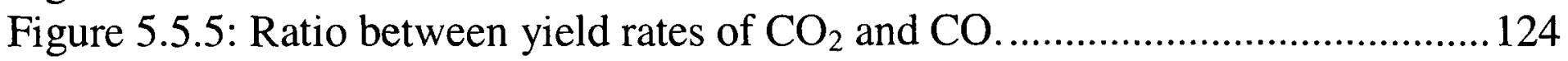

Figure 5.5.6: Measured and predicted light transmission as function of time for NYM cable fire.

Figure 5.5.7: Predicted light extinction coefficient as function of time for NYM cable fire.

Figure 5.5.8: Smoke viewed through the doorway at (a): 1 minute; (b) 2 minutes and

(c) 3 minutes.

Figure 5.6.1: The compartment-hallway fire facility (reproduced from [LE94])..... 127

Figure 5.6.2: Yield of CO for hexane fire........................................................ 129

Figure 5.6.3: Predicted LERs contours at the window at (a) 40 seconds and (b) 60 seconds. 
Figure 5.6.4: Predicted LERs contours at the central plane through the window at 60 seconds.

Figure 5.6.5 : Axial temperatures in the upper-layer of the hallway. The normalizing value is $1300 \mathrm{~K}$

Figure 5.6.6: Axial profile of $\mathrm{CO}_{2}$ and $\mathrm{O}_{2}$ in the upper-layer of the hallway. The normalizing values are $20 \%\left(\mathrm{CO}_{2}\right)$ and $10.5 \%\left(\mathrm{O}_{2}\right)$.

Figure 5.6.7: Axial profile of the $\mathrm{CO}$ and unburned hydrocarbon (UHC) the upperlayer of the hallway. The normalizing values are $2.56 \%(\mathrm{CO})$ and $4.36 \%$ (UHC).

Figure 6.1.1: Control volume used for calculating the CVER by Lattimer et al...... 136

Figure 6.3.1: (a) Fire test facility and (b) sample locations ([LV97]).

Figure 6.3.2: (a) Fuel loss rates and (b) air mass flow rates into fire compartment. 143

Figure 6.3.3: Set up of the compartment-hallway hexane fire by SMARTFIRE (ceiling and floor are removed to allow visualizations of the inside compartment and hallway).

Figure 6.3.4: (a) Measured compartment equivalence ratios and (b) correlations between the yields of CO and CVER and GER.

Figure 6.3.5: Measured and predicted temperatures at Location $a$ inside the compartment.

Figure 6.3.6: Predicted distributions of (a) local equivalence ratios and the control region (shadow); (b) temperatures; (c) velocities; and (d) Oxygen in the vertical plane passing through the window.

Figure 6.4.1: Top views of test geometry and measurement locations.

Figure 6.4.2: Measured (a) cable burning rate and (b) total heat release rate of the NHMH cable fire.

Figure 6.4.3: Yield of $\mathrm{CO}$ (a) and combustion efficiency as function of equivalence ratio (b) for NHMH cable.

Figure 6.4.4: Set up of the room-corridor cable tray fire by SMARTFIRE (ceilings are removed to allow visualization of the inside corridor and room). ....... 155

Figure 6.4.5: Predicted $\mathrm{CO}(\%)$ contours at the horizontal plane $0.1 \mathrm{~m}$ below the ceiling at 13 minutes.

Figure 6.4.6: Measured and predicted temperatures at (a) Location 5; (b) Location 9;

(c) Location 12 and (d) Location 15.

Figure 6.4.7: Measured and predicted CO concentrations at (a) Location 5; (b) Location 9; (c) Location 12 and (d) Location 15.

Figure 6.4.8: Measured and predicted $\mathrm{CO}_{2}$ concentrations at (a) Location 5; (b) Location 9; (c) Location 12 and (d) Location 15.

Figure 6.4.9: Measured and predicted $\mathrm{O}_{2}$ concentrations at (a) Location 5; (b) Location 9; (c) Location 12 and (d) Location 15

Figure 6.4.10: Predicted control region in the horizontal plane $0.15 \mathrm{~m}$ below the ceiling at 13 minutes.

Figure 7.4.1: Schematic of hexane-wood fires: (a) Side view of the facility used in the test (reproduce from [LV98]) and (b) plan view of the facility showing the wood in the upper layer of the compartment and the gas sampling positions.

Figure 7.4.2: Fuel loss rates of the liquid $n$-hexane and the wood (data from [LV98]).

Figure 7.4.3: Vertical plane through the middle of the fire sources depicting the computational mesh.

Figure 7.4.4: Measured and predicted temperatures for the 'Hexane' fire. 
Figure 7.4.5: Measured and predicted normalised species concentrations in the 'Hexane' fire. Symbols and normalised values: square-CO (15\%),

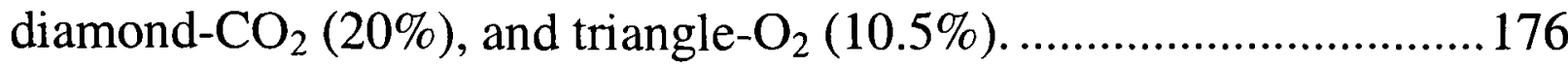

Figure 7.4.6: Predicted temperatures at the five positions for gas sampling in the 'Wood-hexane' fire.

Figure 7.4.7: Measured and predicted averages of temperatures in the 'Wood-hexane' fire.

Figure 7.4.8: Measured and predicted $\mathrm{CO}$ volume fractions at (a) the rear of the compartment; (b) the front of the compartment and at locations in the hallway with (c) $0.36 \mathrm{~m}$, (d) $1.83 \mathrm{~m}$ and (e) $3.2 \mathrm{~m}$ to the compartment. 177

Figure 7.4.9: The measured and predicted normalised species concentrations of the 'Wood-hexane' fire (Symbols and normalised values see Figure 7.4.5).

Figure 8.2. 1: Mechanisms of $\mathrm{HCl}$ deposition on wall.

Figure 8.4.1: Schematic layout of HVAC experimental system (dots are positions for the measurements of $\mathrm{HCl}$ ).

Figure 8.4.2: Measured and predicted $\mathrm{HCl}$ concentrations of Case 1 at (a) 2 minutes;

(b) 10 minutes and (c) 28 minutes using the modified model (present) and earlier model (old).

Figure 8.4.3: Measured and predicted $\mathrm{HCl}$ concentrations of Case 2 at (a) 2 minutes;

(b) 10 minutes and (c) 28 minutes.

Figure 8.4.4: Schematic of small-scale chamber PVC fire tests.

Figure 8.4.5: Predicted deposition rates of $\mathrm{HCl}$ on PMMA wall surfaces and concrete species surfaces (a) Case 1; (b) Case 2 and (c) Case 3.

Figure 8.4.6: Plan view of Schematic of PVC room-corridor fire.

Figure 8.4.7: Burning rate of PVC.

Figure 8.4.8: Measured and predicted $\mathrm{HCl}$ concentrations at (a) doorway and locations in the corridor with distances of (b) $1.24 \mathrm{~m}$, (c) $6.15 \mathrm{~m}$, (d) $11.02 \mathrm{~m}$ and (e) $15.91 \mathrm{~m}$.

Figure 8.4.9: Predicted $\mathrm{HCl}$ concentrations at $6.15 \mathrm{~m}$ from the closed end of the corridor produced by the current model (present) and the earlier model (old). 


\section{LIST OF TABLES}

Table 2.2.1: yields of products and heat of combustion for well-ventilated fires (data from [Te95])..................................................................... 19

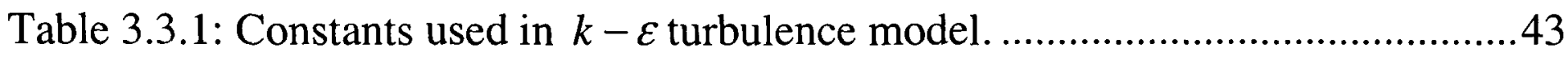

Table 5.1.1: Fire model specification summary.................................................... 93

Table 5.2.1: Parameters of correlation (5.2.2) for wood.........................................98

Table 5.2.2: Comparison of measured and predicted averages (over the time period said in the table) of species volume fraction (\%) inside the room and at the doorway.

Table 5.3.1: Parameters of correlation (5.3.2) for polypropene................................. 108

Table 5.3.2: Carbon recovery in the opening and the duct (data from [LBM96]) .... 110

Table 5.5.1: Parameters of correlation (5.5.1) for PVC.......................................... 121

Table 5.5.2: Predicted visibility distances within the test room. ............................. 126

Table 6.3.1: Parameters for the yields of combustion products of $n$-Hexane............ 145

Table 6.3.2: Measured and predicted temperatures and species concentrations........ 149

Table 6.3.3: Experimental and predicted species concentrations at Location $m$ generated using Model I and the present model.

Table 6.3.4: Sensitivity of remote species concentrations on the critical equivalence ratio at Location $m$ for the compartment-hallway fire. .......................151

Table 6.4.1: Parameters for the yields of combustion products of NHMH cable...... 154

Table 6.4.2: Measured and predicted peak species concentrations.

Table 6.4.3: Predicted peak species concentrations at Location 5 and 15 using Model I and the present modified model.

Table 6.4.4: Sensitivity of remote peak species concentrations on combustion efficiency at Location 15 for the U-shaped corridor.

Table 7.4.1: Effect of wood on the averaged productions of combustion products within the fire compartment and the model predictions.

Table 7.4.2: $\mathrm{O}_{2}$ concentration at the end of the hallway and the $\mathrm{CO}$ reduction percentage from the fire compartment level to that at the end of the hallway.

Table 8.4.1: Model parameters for various wall materials [GH91, JF00].

Table 8.4.2: Absorption percentages of chloride by PMMA walls and concrete specimens. 


\section{Chapter 1}

\section{INTRODUCTION}

\subsection{Background}

Uncontrolled fire generates heat and toxic gases. It threatens people's lives and damage properties. Unwanted enclosure fires are particularly lethal to people due to the nature of the gaseous environment within the enclosures; it blocks the exits from the fire building, exposing residents inside to unbearable high temperature, heat fluxes and toxic gases thus reducing the visibility for escaping due to dense smoke.

The threat of fire is everywhere around us, for example at home, at work, within a pub, train, shopping centres and underground stations, etc. Unwanted fires have not only damaged properties but also endangered people's lives. Throughout the world, 6575,000 people die in fires annually [Br97, Ji99]. It is realized that most of the fire death are caused by the inhalation of smoke and toxic gases [HA82, HH89]. A building fire at Luoyang, the capital city of central Chinese province of Henan, killed more than 300 people [Co00]. Because there was no fire alarm and the exits were blocked, the emergency services delayed and people could not escape before smoke filled the corridors. 'Most people in the building had died from smoke inhalation' [Co00]. In UK, around 700 people die in fires each year [WG00]. The most common cause of fire deaths is overcoming by smoke and toxic gases. In 1998, 668 lives lost and 18,170 injured. Of those killed, $27 \%$ died from burning, $46 \%$ died from the effect of gas and smoke while $20 \%$ died from the combined effect of burning and smoke [WG00].

Carbon monoxide $(\mathrm{CO})$ is the main toxic gas generated in enclosure fires. It is produced from both smouldering and flaming combustions. The hygienic limit of $\mathrm{CO}$ for a short duration of exposure $(15 \mathrm{~min})$ in working condition is $0.01 \%(\mathrm{~V} / \mathrm{V})$ [LBM96]. The incapacitation exposure limit recommended by Purser for CO exposure is $0.067 \%$ over 15 minutes [Pu95]. In practical terms, a $70 \mathrm{~kg}$ man at rest exposed to a $\mathrm{CO}$ concentration of $1 \%$ will lose consciousness in 9 minutes. This exposure duration 
is reduced to approximately 3 minutes if the activity level of the man is equivalent to that of light work [Pu95]. Enclosure fires usually generate several percent of carbon monoxide, which is several times higher than the fatal level discussed above. Carbon monoxide concentration in an adjacent hallway of the fire room in tests by Lattimer et al [LV98] is as high as $2 \%$ high. This explains why many fire deaths occurred in the room remote from the original room of fire [LV98, Co00].

Fires with materials containing halogens produce halogen acids such as $\mathrm{HF}, \mathrm{HCl}$ and $\mathrm{HBr}$. Polyvinylchloride (PVC) is widely used in cables as insulation and jacket materials. $\mathrm{HCl}$ from the decomposition of PVC is both a potent sensory irritant and a strong pulmonary irritant [Ha96]. Acrolein is an organic irritant, which is extremely irritating to the eyes and the upper respiratory tract. However, the $\mathrm{HCl}$ concentrations decay quickly in the process of transport within fire enclosures because $\mathrm{HCl}$ is easily absorbed by the wall surfaces [MP79, GH89].

Fire development within a compartment or a building is extremely complex. Chemical and physical changes of the combustible materials and the surrounding conditions are involved in fire phenomena. Therefore, the numerical simulations of enclosure fires involve knowledge of many different disciplines of science and technology. With progress in the understanding of physical and chemical principles involved in fire, a number of mathematical models have been developed and reported for simulating compartment fires. There are essentially two types of fire models: Zone Models [Wa95] and Field Models [St95]. Zone models divide the fire compartment into several zones deemed relatively uniform in temperatures and gas compositions. In each zone, variables are uniform and can vary with time. Mass and energy in each zone are described with ordinary differential equations. Numerical methods are than used to solve these equations. Zone models involve numerous empirical relationship and many constants derived from experiments. However, the representations of the relationship more often than not depend on the type of enclosure. The assumption of uniform variables in each zone may be invalid for complex fire scenarios [Ga89]. The shortcomings of zone models are evident when applied to complex enclosure fire scenarios. 
Field models involve the use of Computational Fluid Dynamics (CFD) tool. Compared with zone modelling, field models solve a set of conservation equations at a more fundamental level where the fluid flow is governed by these three-dimensional, partial differential equations. In general, field models consist of the following equations:

(1) continuity equation,

(2) three momentum equations that govern the conservation of momentum per unit mass in each of the three space dimensions,

(3) energy conservation equation,

(4) turbulence model and

(5) other scalar variables such as mass fractions and mixture fraction.

General-purpose CFD packages solve these governing equations numerically at discrete points in space and time.

CFD modelling appears more versatile and uses a minimum of empiricism. It becomes an attractive approach to simulate enclosure fires fast due to the rapid progress of computer technology. Field models can provide quantitative solutions to the physical variables throughout the computational grid. Compared with real fire tests, field modelling can also provide more detailed information than can be easily and cost effectively be gathered from experiments. On the other hand, numerical simulations can be repeated many times with changes of the environmental conditions to understand how parameters affect the results. Fire field models have been widely used to investigate the development of hazardous conditions within fire enclosures such as high rise building [GB96], underground [SW92, CH98], tunnel [WB00], aircraft cabin [GM91, JP06a-b], and fire-sprinkler interactions [HG88]. Descriptions of other applications of fire field models are detailed in [Bi94, Co95, St95, No01, Mc05].

Using the field modelling approach [Ga89] to fire simulation, the fire source can be treated as a simple time dependent prescribed source of heat and smoke [KM94, Na00, WJ01a] or using relatively simple one-step reaction mechanisms for gaseous or liquid fuels such as methane [LM97, HH99, MK99, XH01]. Furthermore, advances have been made in predicting the spread of flames over solid surfaces [Ka92, Op95, JG97, JG99a, JG99b, JG99c, YH96, JP06a-b]. However, the weakest component of fire field 
model is its ability to predict the generation and spread of toxic fire products, in particular CO. The introduction of flamelet-based combustion models offer the additional prospect of incorporating more detailed chemistry, for example in relation to $\mathrm{CO}$ in room fire prediction [LM97, HM99, Hy00, WH00]. One key limitation of the flamelet model, as a general engineering tool, is its heavy reliance on data for detailed chemical kinetics. For the most part, this is available for only a few gaseous fuels and notably not for common building materials.

\subsection{Research Objectives and Questions}

In the development of fire field models today, there is lack of practical approaches to predicting the production of smoke and toxic gases. As protection of lives from building fires is a key objective of fire safety research, it is desirable for fire models to be capable of predicting the spread of toxic gases within fire enclosures. Hence, the current study concentrates on the development of toxicity models within a fire field modelling framework to simulate the production and movement of smoke and toxic gases from and within fire enclosures.

The main aim of the present study is to extend the fire field model generally used, i.e. the volumetric heat source model and the Eddy-Break-up approach, to include the capability of predicting the production of smoke and toxic gases within fire environments. The goals set to be achieved in the development of the toxicity model in this study are:

- To identify the input data, necessary to represent the behaviour of the toxic gases, which are

(1) measurable,

(2) can be obtained from small-scale experiments and/or

(3) can be found in literature, for most furniture and building materials.

- That the model is able to be integrated within the framework of CFD fire modelling.

- That the simulation overheads incurred for the toxicity model should be minimal compared to typical CFD runtime requirements. 
In order to achieve theses objectives, in this study, an approach to make use of yield data of toxic species collected from small-scale tests to develop toxicity models. From small-scale hood tests, it has been found that the generation of many combustion products, such as $\mathrm{CO}, \mathrm{CO}_{2}$, can be correlated with the Global Equivalence Ratio (GER) [Be86, Pi95]. The yield data based on GER are available for a wide range of building materials. However, the direct use of GER correlations to large scale enclosure fires is problematic [Ba95, GR95, Pi95, BG98, PF04, Pi04, WV04a-b, LV05], in particular when relatively simple zone modelling technology is applied.

As the model development is set within the framework of CFD fire modelling, a fundamental question arises when these correlations are employed, i.e., Can the correlations derived from small-scale experiments be applied to predict the generation and transport of toxic gases within fire enclosures, and if so, how can they be used?

In addition to the development of toxicity models within a CFD fire modelling framework, the author will also attempt to answer some other questions associated with the fundamental question stated above. The sub-questions are:

- Why does the GER approach fail in certain circumstances to predict correctly the levels of toxic gases within full-scale fire enclosures? How can a field model address this problem?

- How are species concentrations calculated using the concept of local equivalence ratio (LER)? In what region is the approach valid?

- How is the region, in which the changes of species concentrations are only due to mixing of hot gases with fresh air, determined? Furthermore, how are the species concentrations calculated in this region?

- Can smoke properties, such as optical density or visibility distance, be calculated using the LERs via simple relationships?

- How are the species concentrations in two-fuel fires calculated using correlations from the experiments with a single burning fuel? 
As one of the combustion products from materials containing chlorine, namely $\mathrm{HCl}$ has a tendency to be absorbed by solid obstacles and walls in the course of transportation within enclosures, in this study, efforts will be directed to model the decay of $\mathrm{HCl}$ in fire enclosures due to absorption.

In this study, substantial effort has been directed towards developing relatively simple and practical engineering models to simulate the production and movement of smoke and toxic gases within fire enclosures. Attention has been focused on all the problems and issues presented above.

\subsection{Layout of the Thesis}

Chapter 2 presents a in depth background study for the generation of toxic gases in experiments and mathematical models. The mechanisms of $\mathrm{CO}$ generation are summarised and correlations discussed for a wide range of experimental studies. Mathematical models for the prediction of $\mathrm{CO}$ are reviewed and their application limitations discussed. The experimental and modelling studies on the decay of $\mathrm{HCl}$ due to the absorption by solid surfaces are also reviewed in this chapter.

Chapter 3 details the equations and numerical modelling techniques used by the CFD approach to solve fire modelling problems. The conservation equations for continuity, momentum, enthalpy and species are presented. Sub-models involved in fire simulations such as turbulence, radiation, and combustion models are discussed. Finally, as an application of the models, a simulation to a complex enclosure fire scenario is performed and the simulation results are compared with the experimental data.

Three mathematical models for the prediction of toxic gases based on the concept of LER are developed and presented in Chapter 4. These models require correlations between the yields of toxic gases or $\mathrm{CO} / \mathrm{CO}_{2}$ ratios and the equivalence ratios. $\mathrm{A}$ simple approach for the calculation of smoke properties, such as the light extinction coefficient, is also developed and presented in this chapter. 
The toxic gas models developed in Chapter 4 are validated in Chaptr range of full-scale fire tests and reduced compartment-corridor firt sampling locations are within or close to the combustion region. The different enclosure sizes, opening types and fuels. One of the tests is the author's assistance and others are taken from the publications $c$ carried out by the Fire Safety Engineering Group (FSEG) at th Greenwich. Predictions of temperatures and combustion products are corresponding experimental data.

A methodology is proposed in Chapter 6 to divide the computational parts, a control region and a transport region, for the calculations of species concentrations in the control region are calculated with the $\mathrm{m}$ in Chapter 4 while they are only for the mixing of the combustion pr air in the transport region. This methodology is tested with two room-1

A two-fuel toxic gas model is developed in Chapter 7 to study the linings, on walls and/or ceiling, on the toxic gas level of enclost chapter, the model is used to simulate hexane fire scenarios with ar. liners on the ceiling as a second fuel.

A deposition model for the exchange of $\mathrm{HCl}$ between gas/wal developed in Chapter 8. This model is validated using experiments or $\mathrm{HCl}$ within a HVAC (heating, ventilation and air conditioning), enclosure and a small-scale chamber.

Finally, a summary of conclusions and recommendations for $f$ presented in Chapter 9 and Chapter 10 respectively. 


\section{Chapter 2}

\section{BACKGROUND AND LITERATURE REVIEW}

\section{$2.1 \quad$ Introduction}

Fire hazard includes two parts, the generation and the spread of heat and toxic gases. The productions of species as functions of the fuel loss rate are generally the input parameters in combustion models. The lack of understanding of physics and chemistry of fire, especially for the toxic gases, hinders the development of mathematical modelling. Therefore, correct experimental correlations need to be chosen from a large number of publications and used in sub-combustion modelling [Ga89].

Combustion is associated with two major processes. In the first process, the gasified materials are converted mostly to products of incomplete combustion, such as carbon monoxide, smoke, hydrocarbons and other products. In the second oxidation process, the gasified material and the incomplete products further oxide mostly to carbon dioxide and water [Te96].

Combustion products in compartment fires are determined by various conditions, the geometry, ventilation, fluid dynamics, thermal environment, chemistry and the mode of burning [GR95, KQ00]. The mode of burning and ventilation are two of the key conditions for generation of toxic species. The fires can be classified into three categories, smouldering, over ventilated and under ventilated.

In this chapter, the experiments for the combustion yields and the mechanisms of $\mathrm{CO}$ formation are briefly reviewed. Correlations between combustion products and the Global Equivalence Ratio (GER) from literatures are presented here and used later on in this thesis. The advancement of zone and filed fire modelling for the predictions of $\mathrm{CO}$ within fire enclosures are also reviewed. 


\subsection{Experimental Studies}

\subsubsection{Global Equivalence Ratio (GER)}

Enclosure fire is concerned with the burning rate of fuel, the entraining rate of air and the structure of the plume. The concept of global equivalence ratio (GER), which reflects the relationship between fuel and air, describes the vitiated burning condition within the fire enclosure.

Before describing the concept of the equivalence ratio, the concept of stoichiometry is first introduced here. A chemical reaction is referred to by complete combustion if all the products are in their most stable state. For example, combustion of methane $\left(\mathrm{CH}_{4}\right)$ with only $\mathrm{CO}_{2}$ and $\mathrm{H}_{2} \mathrm{O}$ as products is

$$
\mathrm{CH}_{4}+2 \mathrm{O}_{2}=\mathrm{CO}_{2}+2 \mathrm{H}_{2} \mathrm{O}
$$

Stoichiometry means that the proportion of fuel to oxygen (air) is exact for all of the reactants completely convert to products. The mass ratio of fuel to air at stoichiometric state is called stoichiometric ratio, and noted as $r$.

The concept of GER can be used to express the ventilation situation of enclosure fires in zone fire modelling. There are two main definitions for the equivalence ratio in the review paper by Pitts [Pi95]. One is the plume equivalence ratio $\left(\phi_{p}\right)$, the other one is upper layer global equivalence ratio $\left(\phi_{g}\right)$. 'The plume equivalence ratio will be defined as the fuel mass-flow rate divided by the air mass-entrainment rate into the plume below the layer normalized by the stoichiometric ratio for the fuel'. 'The global equivalence ratio $\left(\phi_{g}\right)$ will refer to the ratio of the mass of the gas in the upper layer derived from the fuel divided by that introduced from air normalized by the stoichiometric ratio' [Pi95]. For a fuel lean mixture (or over ventilated conditions), the global equivalence ratio $\phi_{g}$ is less than one; for stoichiometric mixture $\phi_{g}$ equal to one, and for fuel rich mixture (under ventilation conditions) $\phi_{g}$ is greater than one. The plume equivalence ratio $\phi_{p}$ and the global equivalence ratio $\phi_{g}$ are identical if no air or fuel enters the upper layer except by the way of the plume and the fire is in steady state. 


\subsubsection{Review of Experiments}

In order to study the generation of major species in compartment fires, a large number of experiments using various apparatus have been undertaken by researchers. These experiments include small-scale hood-type experiments, reduced-scale room fires and full-scale enclosure fires.

\subsubsection{Earlier Small-scale experiments}

Some of early experiments to study $\mathrm{CO}$ generation were performed in a small-scale two-layer environment that is called hood experiment. These experiments were used to develop models by treating room fires as two layers- the hot upper layer and the cold lower layer. The fires in these experiments were allowed to burn long enough to reach a steady state for temperatures and compositions in the upper layer.

Beyler [Be86a, Be86b] studied two-layer fires via hood experiments with Wood, PMMA, polyethylene and a range of gas fuels. A homogeneous nature of the upper layer gases has been seen by sampling the gases at a variety of locations within the hood. Beyler's study showed that the species yields correlated with the plume equivalence ratio very well. A little $\mathrm{CO}$ production was observed if the plume equivalence ratio is below 0.5 . The $\mathrm{CO}$ production increased with the increase of the plume equivalence ratio (in his experiments the GERs were less than 2). Although the levels of $\mathrm{CO}$ varied with fuels, the correlations were qualitatively similar.

Toner et al [TZ87], Zukoski et al [ZT89] and Morehart et al [MZ90] performed similar hood-type experiments and came to similar results concluded by Beyler. Toner et al concluded that the species concentrations were well correlated with the upper layer equivalence ratio, which was equal to the plume equivalence ratio in their experiments. He has also found that the species concentrations were not sensitive to temperatures in the range of $480-870 \mathrm{~K}$. As air was injected into the upper layer, Zukoski and Morehart found that species concentrations changed with the upper layer equivalence ratio, which was different from the plume equivalence ratio, in a welldefined correlation. 
Tewarson used a flammability apparatus, which satisfies the hood conditions for low $\phi$ values and allows the air enter the hot upper layer directly, to study the yields of combustion products [Te95, Te96]. Studies showed that the concentrations of incomplete combustion products depend on the chemical structures of the materials. For the same GER value, $\mathrm{CO}$ concentrations are higher for materials with oxygen atoms in the structure. In Tewarson's experiments, combustion was found in the nonflaming mode if the GER is above 3.5. Generalized correlations to calculate the heat of combustion and yields of products at various equivalence ratios for a wide range of materials have been derived by Tewarson.

Mulholland Et al [MJ91] have studied the yields of CO and smoke with different oxygen concentration using a modified cone calorimeter. They concluded that the $\mathrm{CO}$ yield for a given fuel in a free ambient condition is mainly controlled by the flame temperature and increases by at least a factor of two as the oxygen concentration is decreased from $21 \%$ to $14 \%$. However, the smoke yield is less sensitive than the yield of $\mathrm{CO}$ to the $\mathrm{O}_{2}$ concentrations. The fuels in their study were methane, propane, PMMA, ABS, polyethylene, and Douglas fir. In Mulholland's tests, the flame extinction occurred when the $\mathrm{O}_{2}$ concentration is less than $14 \%$. Mulholland has not concluded if this minimal $\mathrm{O}_{2}$ concentration is valid or not in real full-scale enclosure fires, in which high temperature may keep the flame on in more vitiated air conditions.

\subsubsection{The Purser Furnace experiments}

Difficulties may arise to gather useful data from small-scale experiments to mimic real full-scale fires since the combustion conditions in the former fire scenarios are sometimes quite different from the latter in the available oxygen concentrations and temperatures, which control the generation of $\mathrm{CO}$ [Ba95]. As been summarised by Gottuk [Go92], the hood type experiments differ from actual compartment fires in several ways. First, actual fire would contain most of the radiation, resulting in higher wall and upper layer temperatures than in the hood. Secondly, in real compartment fires, the air is limited by the ventilation and air in the lower layer can be convectively heated by hot compartment surfaces prior to fire plume entrainment. Thirdly, the dynamic flame structures enhance mixing of the upper layer in actual compartment 
fire and extend the flame zone beyond the plume. Lastly, rather than the steady-state burning conditions, actual fires are usually quite transient in nature.

However, these problems may be solved in part by the development of the Purser Furnace (see Figure 2.2.1) [BS03]. The tube furnace, with a heating zone is equipped with an adjustable electric heating system capable of controlling temperature. The inlet end of the furnace tube allows the primary air blow into the tube. The test materials are transported to the combustion zone through a drivable boat. The species concentrations are measured in a cubic chamber, which is adjacent to the furnace tuber. An air supply tube passes through the wall of the chamber and ends approximately $70 \mathrm{~mm}$ above the end of the quartz furnace tube. The British Standard [BS03] provides the definitions and formulas for the calculation of toxic product yields against equivalence ratio.

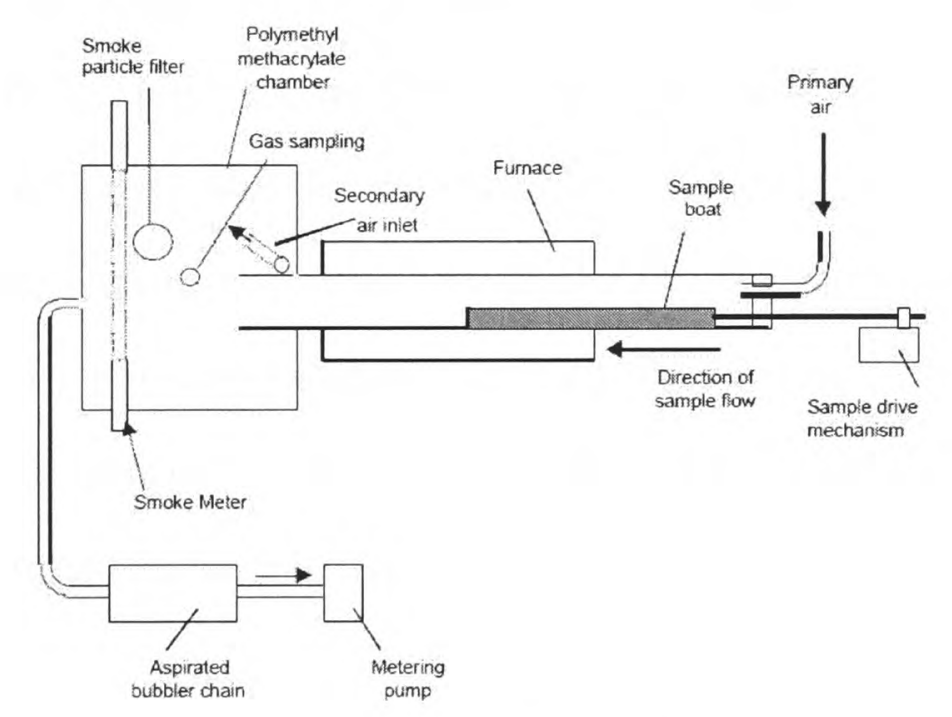

Figure 2.2.1: Schematic of the Purser furnace (reproduced from [BS03]).

The fire in small-scale experiment is usually non-flaming if the GER is very high. However, the Purser furnace is able to replicate the condition of combustion with high temperature and low oxygen concentrations (under ventilated), which often appears in full-scale enclosure fires. 'In the Purser furnace the rate of burning is fixed and the heat flux is allowed to vary' [HQ02]. 'This modified tube furnace method is capable of exposing materials and their gas phase products to a wide range of decomposition conditions, and of producing toxic products with similar yields to those found in large scale fires of different types and stages' [PF94]. A two-dimension correlation between 
the species yields and the GERs and temperatures are available, based on experiments with the Purser furnace [PF94, HP99, HQ02, HP04, HL05].

\subsubsection{Reduced-room experiments}

An experimental system especially designed to study the correlation between gas productions and the GER has been developed at VPISU [Go92]. The dimensions of the reduced enclosure are $1.2 \mathrm{~m}$ wide, $1.5 \mathrm{~m}$ long and $1.2 \mathrm{~m}$ high. Air enters the enclosure only by a low inlet and the upper layer gas flows out through a high window. Thus, the plume equivalence ratio can be easily calculated by the directly measured burning rate and air entrainment rate. Four fuels (hexane, PMMA, spruce and polyurethance) were used to study the correlation between $\mathrm{CO}$ generation and the GER. The results of the reduced room tests showed qualitatively similar curves as been observed from hood experiments. However, quantitatively different products yields have been found. Higher $\mathrm{CO}$ production was observed in hood experiments compared with that in reduced room fires when the GER is approximately 0.5 . The $\mathrm{CO}$ production can be neglected if the global equivalence ratio is less than 1 for reduced enclosure fires. More $\mathrm{CO}$ was produced in the reduced enclosure fires than that in hood experiments for large values of equivalence ratios. The higher upper layer temperatures in the reduced room fire tests caused these differences in the CO yields between the two different fire scenarios.

A systematic investigation of the behaviours of enclosure fires concerned with the formation of $\mathrm{CO}$ for natural gas fires was reported in [BJ94] and cited in [Pi94a]. The reduced room for these fires has dimensions of $0.98 \mathrm{~m}$ wide, $1.46 \mathrm{~m}$ long and $0.98 \mathrm{~m}$ high. The heat release rate varied from 10 to $670 \mathrm{~kW}$. For fires with high values of GERs, the CO concentrations in the front were much higher than that in the rear of the compartment.

In a recent work by Wieczorek et al [WV99], pool fires in a reduced room with different door widths were conducted to study the formation of CO. It was found that the concentrations of combustion products correlated well with the global equivalence ratios and followed the same trends as data from other hood experiments and reduced room fires. However, the door width also had slight effects on the levels of $\mathrm{CO}_{2}$ and 
CO. Wieczorek et al [WV04a, WV04b] found that the concentrations of combustion products at the exit of the fire enclosure can well be correlated to a non-dimensional heat release rate, which provides an indication of the burning conditions at the compartment exit.

By placing wood on the ceiling of the reduced room and burning methane, it was found that the $\mathrm{CO}$ concentrations are four times higher than that in the same methane fire without the wood as the second fuel [PJ94].

\subsubsection{Full-scale fire tests}

The earliest experimental study on the correlation between the combustion products and the reciprocal equivalence ratio in full-scale fire scenarios was conducted by Tewarson [Te84]. The shapes of the correlation for wood are similar to that from the hood experiments. Unified fire property data correlation between the normalized yields to the reciprocal equivalence, based on Tewarson's experimental data, has been presented by $\mathrm{Yu}[\mathrm{Yu} 93]$.

Investigations with full-scale enclosure natural gas fires were reported in [PB95]. Compared with an early study of reduced room fires [BJ94], the upper-layer temperatures and $\mathrm{CO}$ concentrations in the full-scale room fires were significantly higher than that observed in reduced room tests. $\mathrm{CO}$ concentrations of $6 \%$ were found in the upper layer of full-scale and fuel rich fires. These $\mathrm{CO}$ levels are two times higher than that in reduced room fires or three times higher than that in hood experiments while the three fire scenarios had approximately the same value of GERs [PB95].

A series of tests in the ISO 9705 room [LBM96] and large storage [LBM96b] on the generation of toxic gases were conducted at the department of Fire Technology at SP Swedish National Testing and Research Institute. The materials burned are PP (polypropene), nylon 66, TMTM (tetrametylthruiuram monosulfide), CNBA (4cholor-3-nitrobenzoic acid) and CB (chlorobenzene). Experiments showed that it is possible to achieve oxygen-depleted conditions on a large-scale fire enclosure 
involving these materials. Yields for species measured at the opening depended on the ventilation conditions. Temperature was another factor affecting the formation of $\mathrm{CO}$.

In 1997, CIB W14 Subgroup 2 undertook a Round Robin Testing exercise designed to investigate the accuracy of computer based fire models and their users in simulating a range of test fire scenarios [HK98]. Together with the local temperatures, the species concentrations including $\mathrm{CO}$ have been measured in these tests. This exercise included a range of zone and field models and a range of modelers, from fire safety engineers to the actual software developers.

In 1999 a series of large-scale cable fire tests were undertaken in the Borealis Antwerp fire research laboratory [Bo01, RS02, RS04]. The room for cable fire tests was with dimensions of $3 \times 3 \times 3 \mathrm{~m}$. The combustion products passing through the exit of the test room were measured.

Among these full-scale fire experiments, one case from [PB95] is used to analyse the feasibility of applying local equivalence ratio to predict the species concentrations within the fire enclosures. Cases from [LBM96, HK98, RS02] are also used to validate the model developed in Chapter 5.

A full-scale wood fire experiment which is designed to validate the toxicity models in this study has been conducted at Rockwool International with the author's assistance. Details of the experiment are described in Chapter 5.2.

\subsubsection{Room-corridor fire tests}

The above studies on $\mathrm{CO}$ formation in enclosure fires have focused on its generation and spread within the fire room. However, the toxic gases at locations far away from the fire source are sometimes essential for the evacuation in building fires. A series of tests were conducted by Lattimer et al [LE94, LV97, LV98, LV05] to study the movements of combustion products from a burning room into its adjacent corridor. The fuels in the tests were hexane or hexane and wood. The fire scenarios were varied by changing the size of fire within the compartment, the soffit heights at either end of the corridor and the window-style exhaust vent area, which connects the compartment 
and the corridor. These experiments allowed the air to enter the fire compartment only through a duct. The GERs of the compartment fires could be estimated precisely. However, the local equivalence ratio (LER) within the corridor varied with space. These experiments are excellent for model validations. One test selected from [LE94] is used to validate the one-fuel toxicity model developed in Chapter 5. One test from [LV98] is used to validate the two-fuel toxicity model developed in Chapter 7. One test from [LV97] is used to validate the methodology for calculating CO concentrations at remote locations in Chapter 6.

A series of large-scale room-corridor fire tests were conducted to study the generation of $\mathrm{CO}$ in vitiated conditions and its movement in the corridor [FR94, PR99, Pu03]. Wood cribs were the primary fuel in these tests. In two of the four tests, PMMA or PVC was the secondary fuel. The corridor was $12 \mathrm{~m}$ long. In these tests, the measured $\mathrm{CO}$ concentrations at the open end of the corridor were as high as $2 \%$ and $\mathrm{O}_{2}$ concentrations there were higher than $10 \%$. These tests showed that the flame extinction caused by air vitiation is an important reason for the high $\mathrm{CO}$ concentrations away from the fire source.

A series of full large-scale room-corridor cable fires were conducted at the department of Fire Technology at SP Swedish National Testing and research Institute [Pe06]. The corridor was $44.6 \mathrm{~m}$ long. As been observed in [FR94], high level of $\mathrm{CO}$ and $\mathrm{O}_{2}$ concentrations at remote locations from the fire origin were also measured. One case from [Pe06] is used to validate the model in Chapter 6.

\subsubsection{Mechanisms of CO Formation}

Understanding the generation of combustion products involves a detailed knowledge of the fire chemistry [Si95]. The complicated chemical mechanisms of toxic generation are not intended to discuss here. We focus on the effect of ventilation and combustion modes on the generation of $\mathrm{CO}$ in enclosure fires.

It has been found in hood-type experiments that one mechanism responsible for the formation of $\mathrm{CO}$ is the quenching [Pi95, Pi97] of a fire plume upon entering an upper 
layer of rich combustion products. As reviewed from hood-type experiments, the toxic gases produced by this process are well correlated with the GER.

Hood-type experiments and reduced-room fires show that the yields of products are dependent on the upper layer temperature. In real full-scale fires, the high temperature drives the reaction on even the oxygen concentration is low, resulting in significant concentrations of carbon monoxide and other products of incomplete combustion. Pitts [Pi94b] and Gottuk et al [GRB95] studied this effect of temperature on carbon monoxide formation in compartment fires using detailed kinetic chemistry. Higher temperature accelerates the conversion rates of both $\mathrm{CO}$ to $\mathrm{CO}_{2}$ and hydrocarbon to CO. For most situations, upper layer temperature below $800 \mathrm{k}$ indicate chemical uncreative layer. When the upper layer is about $900 \mathrm{~K}$ or higher, $\mathrm{CO}$ is completely converted to $\mathrm{CO}_{2}$ for over ventilated and slightly under ventilated conditions. However, the hydrocarbon oxidation is much faster than $\mathrm{CO}$ oxidation. Therefore, the net $\mathrm{CO}$ production is high at fuel rich and high temperature locations.

The presence of wood in an oxygen-depleted upper layer of a fire enclosure can cause a significantly higher CO level. When wood pyrolysis in the upper layer with high temperature and low level of oxygen, the oxygen contained in the fuel forms $\mathrm{CO}$ directly. Because there is limited oxygen in the wood, the CO cannot fully convert to $\mathrm{CO}_{2}$ in the process of pyrolysis. This is an important mechanism for $\mathrm{CO}$ formation in enclosure fires.

The mechanisms for $\mathrm{CO}$ formation in enclosure fires are summarized by Pitts [Pi95, $\mathrm{Pi} 97]$ via investigating a wide range of literatures. They are:

$M 1$ quenching of a turbulent fire plume upon entering a rich upper layer

M2 mixing of oxygen directly into a rich high-temperature upper layer with subsequent reaction

M3 Pyrolysis of wood in high-temperature and vitiated environments

M4 Approach to full-equilibrium combustion productions in a rich, hightemperature upper layer.

Combustion mode is also a key factor to the generation of toxic gases. Polymer fires usually start from non-flaming or smouldering combustion [Oh95]. Smouldering fires 
produce high yields of irritant smoke and CO compared with flaming fires [Oh95, $\mathrm{Pu} 00$, Ts94]. The $\mathrm{CO} / \mathrm{CO}_{2}$ mole ratios in combustion of wood are 0.9 and 0.0035 for pyrolysis and flaming modes respectively [Ts94].

\subsubsection{Engineering $\mathrm{CO}$ Yield Correlations}

\subsubsection{Correlations for polymers}

Species yield is usually defined as

$$
y_{i}=\frac{m_{i}}{m_{f}} \quad(\mathrm{~kg} / \mathrm{kg})
$$

where $m_{i}$ is the mass of species $i$ and $m_{f}$ is the mass of fuel. Note that the mass $m_{f}$ refers to the total fuel entering the gas mixture, not the net reacted fuel. Yields of combustion products in a range of different diffusion flames are reported by Tewarson [Te95]. For well-ventilated enclosure fires, small levels of toxic gases are generated. The yields of fire products for various materials at this condition, denoted by $y_{i, \infty}$, are given in [Te95 (Table 3-3.11)]. The subscript $\infty$ here represents infinite amount of air. Table 2.2.1 presents the yields for a few polymer materials, some of which will be used in the present study.

For under ventilated fires, a generalized relationship, to calculate the yields of products at various equivalence ratios, is given by Tewarson [Te95] and discussed in [MT94]. The generalized relationship between the yields of combustion products $y_{i}(\phi)$ and the global equivalence ratio is expressed by the following formula

$$
y_{i}(\phi)=y_{i, \infty}\left[1+\frac{\alpha}{\exp (\phi / \beta)^{-\zeta}}\right]
$$

where $\alpha, \beta$ and $\xi$ are the correlation coefficients and depend on the characteristic of the chemical structures of the polymers.

Yield of carbon dioxide and consumption of oxygen for non-halogenated polymers are given in equation (2.2.4) and (2.2.5) respectively 


$$
\begin{aligned}
& y_{\mathrm{CO} 2}=y_{\mathrm{CO} 2, \infty}\left[1-\frac{1.0}{\exp (\phi / 2.15)^{-1.2}}\right] \\
& y_{\mathrm{O} 2}=y_{\mathrm{O} 2, \infty}\left[1-\frac{0.97}{\exp (\phi / 2.14)^{-1.2}}\right]
\end{aligned}
$$

Table 2.2.1: yields of products and heat of combustion for well-ventilated fires (data from [Te95]).

\begin{tabular}{|l|l|l|l|l|l|}
\hline Material & $\boldsymbol{\Delta H}_{\mathbf{T}}(\mathbf{k J} / \mathbf{g})$ & $\mathbf{y}_{\mathbf{C O} 2}(\mathbf{g} / \mathbf{g})$ & $\mathbf{y}_{\mathbf{C O}}(\mathbf{g} / \mathbf{g})$ & $\mathbf{y}_{\mathbf{C H}}(\mathbf{g} / \mathbf{g})$ & $\mathbf{y}_{\mathbf{S}}(\mathbf{g} / \mathbf{g})$ \\
\hline PS & 39.2 & 2.33 & 0.060 & 0.014 & 0.164 \\
\hline PP & 43.4 & 2.79 & 0.024 & 0.006 & 0.059 \\
\hline PE & 43.6 & 2.76 & 0.024 & 0.007 & 0.060 \\
\hline PMMA & 25.2 & 2.12 & 0.010 & 0.001 & 0.022 \\
\hline Wood (pine) & 17.9 & 1.33 & 0.005 & 0.001 & - \\
\hline Nylon & 30.8 & 2.06 & 0.038 & 0.016 & 0.075 \\
\hline
\end{tabular}

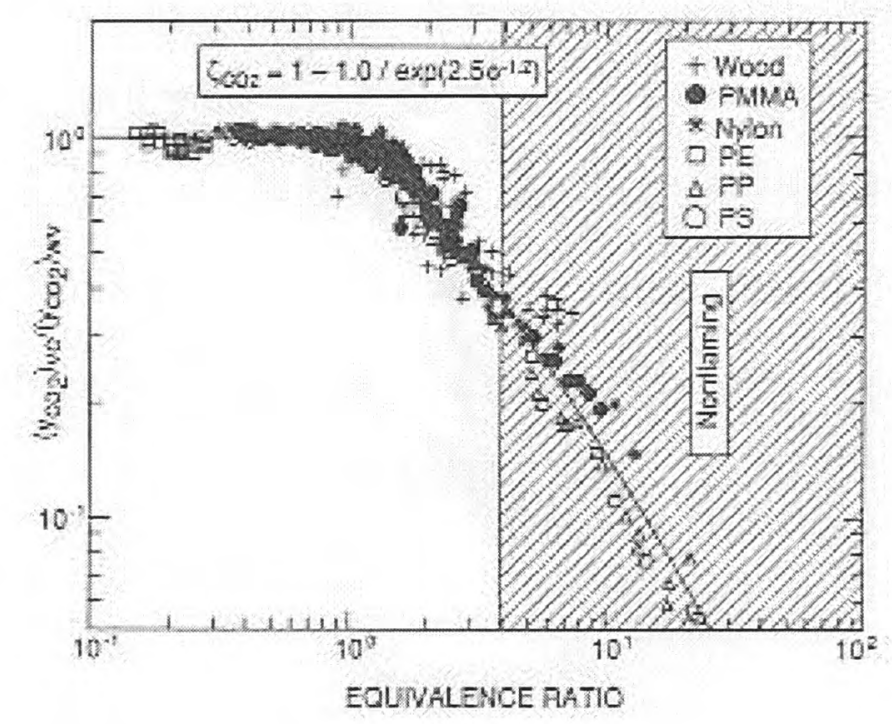

(a)

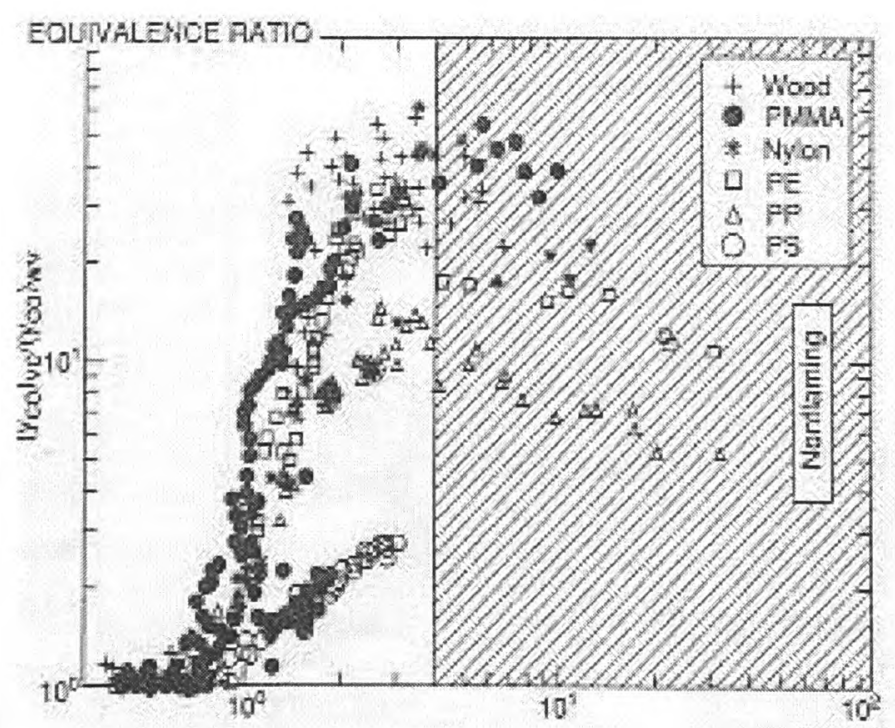

(b)

Figure 2.2.2: Ratio of the yield at well ventilation condition to the yields with various equivalence ratios for (a) $\mathrm{CO}_{2}$ and (b) $\mathrm{CO}$ (reproduced from [Te95]). 
From equation (2.2.4) and (2.2.5), it is seen that the ratio $y_{\mathrm{CO} 2} / y_{\mathrm{CO} 2, \infty}$ (also see Figure 2.2.2(a)) and $y_{O 2} / y_{O 2, \infty}$ are fuel independent. However, the ratios for soot, hydrocarbon and $\mathrm{CO}$ (al so see Figure 2.2.2(b)) significantly depend on the fuel. The parameters of equation (2.2.3) for the yields of soot, hydrocarbon and $\mathrm{CO}$ for the fuels listed in Table 2.2.1 can be found in [Te95].

Tewarson [Te96] indicated that the relationship for the yield of $\mathrm{CO}$ is suitable to enclosure fires under the following conditions. 'The upper layer temperature is less than $700 \mathrm{~K}$; the upper layer is lean and is at a high temperature $(>900 \mathrm{~K})$, or air enters the hot upper layer (>900K) directly or through the plume'.

Purser furnace experiments can produce correlations between yields of products, the GER and the temperature for polymers. Correlations for materials such as low-density polyethylene, wood and PVC can be found in [PF94, HP99]. The correlations between the yields of combustion products and the equivalence ratio for various cables have been previously studied using the Purser Furnace at the University of Bolton [HL05]. These correlations can be expressed with functions like Equation (2.2.3) that was used by Tewarson. As an example, Figure 2.2.3 depicts the experimental yields of $\mathrm{CO}$ from $\mathrm{NHMH}$ cable at $800^{\circ} \mathrm{C}$. In this figure, the approximation is given by Equation (2.2.6)

$$
y_{C O}(\phi)=0.009 \times\left[1+\frac{33.5}{\exp (\phi / 0.9)^{-5.0}}\right]
$$

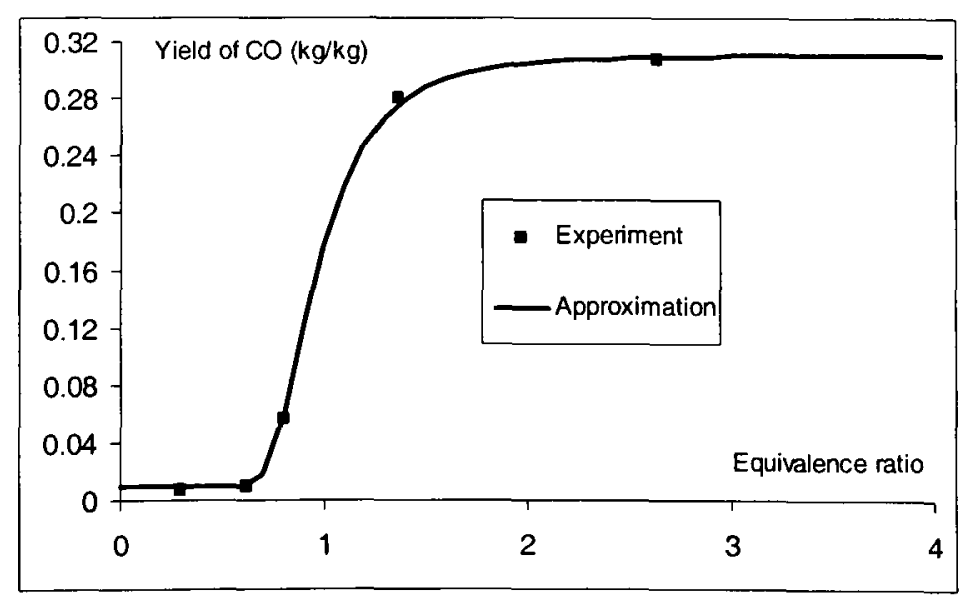

Figure 2.2.3: Yields of $\mathrm{CO}$ as function of equivalence ratio from Purser Furnace NHMH cable experiments. 


\subsubsection{Simple correlation}

Several correlations used as guidelines for protection engineering are presented in [GR95]. The following equation presents a 'zero order' correlation between $\mathrm{CO}$ yield and the GER. The normalized yield, $f_{i}$, is defined as

$$
f_{i}=\frac{y_{i}}{K_{i}}
$$

where $K_{i}$ is the maximum theoretical yield of specie $i$. For example, $K_{C O}$ can be calculated by assuming that all carbon in the fuel is converted to $\mathrm{CO}$ (no $\mathrm{CO}_{2}$ is generated). The normalized yields of $\mathrm{CO}_{2}, \mathrm{O}_{2}$ and $\mathrm{H}_{2} \mathrm{O}$ are well correlated to the GER [GR95]

$$
\begin{array}{ll}
f_{\mathrm{CO} 2}=1 & \text { for } \phi<1 \\
f_{\mathrm{CO} 2}=B_{\mathrm{CO} 2} / \phi & \text { for } \phi>1 \\
f_{O 2}=1 & \text { for } \phi<1 \\
f_{O 2}=B_{O 2} / \phi & \text { for } \phi>1 \\
f_{H 2 O}=1 & \text { for } \phi<1 \\
f_{H 2 O}=B_{H 2 O} / \phi & \text { for } \phi>1
\end{array}
$$

where the $B_{\mathrm{CO} 2}, \quad B_{\mathrm{O} 2}$ and $B_{\mathrm{H} 2 \mathrm{O}}$ are the yields coefficients. Gottul and Roby [GR95] found that a good correlation between the $\mathrm{CO}$ yield to the equivalence ratio rather than that with the normalized $\mathrm{CO}$ yield could be obtained for a number of different fuels. Based on Beyler's hood tests [Be86a], a simple CO yield formula for methanol, ethanol, isopropanol, propane and acetone are given in [KQ00]

$$
\begin{array}{ll}
y_{C O}=0 & \text { for } \phi<0.5 \\
y_{C O}=0.3 \phi-0.15 & \text { for } 0.5<\phi<1.2
\end{array}
$$




$$
y_{C O}=0.21 \quad \text { for } \phi>1.2
$$

A formula for the CO yield is similar to (2.2.14), as suggested in [GR95].

\subsubsection{Correlations for hexane and methane}

As reviewed in section 2.2.3, the yield of $\mathrm{CO}$ depends on not only the global equivalence ratio, but also the temperature of the gas mixture. The effect of temperature on $\mathrm{CO}$ production for hexane fires is best represented by the following two equations

$$
\begin{aligned}
& y_{\mathrm{CO}}=(0.19 / 180) * \tan ^{-1}(10(\phi-0.8))+0.095 \text { for } T<800 K \\
& y_{\mathrm{CO}}=(0.22 / 180) * \tan ^{-1}(10(\phi-1.25))+0.11 \quad \text { for } T>900 K
\end{aligned}
$$

Equations (2.2.15) and (2.2.16) are derived from hood and enclosure hexane experimental data respectively [GR95]. They can be used to predict the minimum of $\mathrm{CO}$ production in compartment fires for other fuels. An alternative form of equation (2.2.16) is given in [LV97]

$$
y_{C O}=\frac{0.22}{1+\left(\frac{\phi}{1.2}\right)^{-6}}
$$

The CO yield of methane fire is approximately the average of yields given by formulas (2.2.15) and (2.2.16) [GR95]. However, this correlation is only suitable for methane fires with the temperature below 900K. From room fire tests [BJ94, PB95] and kinetic modelling [Pi94b, GRB95], it is known that higher temperature cause high $\mathrm{CO}$ concentration in rich methane fires.

\subsubsection{Correlations for plastics}

Generalized correlation between the yields of combustion products, corrosion and the equivalence ratio for the halogenated polymers has been provided by Tewarson et al 
[TC94]. The polymers are four fluoro- and five chloropolymers and a polyethylenepolyvinylchloride cable. The yield of $\mathrm{CO}_{2}$ decreases and the yields of $\mathrm{CO}$, hydrocarbons and smoke increase for halogen contained polymers with the increase of the GERs. Compared with the non-halogenated polymers, the combustion of halogenated polymers is very inefficient and strongly effected by a slight reduction of the flow rate of air. The yield of $\mathrm{CO}$ reaches a maximum when the equivalence ratio reaches 0.6. This is quite different from the non-halogenated polymers. The yield of $\mathrm{CO}$ for non-halogenated polymers just shows slight increase until the equivalence ratio is greater than one.

The correlations between the yields and the equivalence ratio for halogenated polymers have the form of equation (2.2.3) too, i.e.

$$
y_{i}(\phi)=y_{i, \infty}\left[1+\frac{\alpha}{\exp (\phi / \beta)^{-\zeta}}\right]
$$

where i stands for $\mathrm{CO}_{2}, \mathrm{CO}$, hydrocarbon and soot, $\alpha, \beta$ and $\xi$ are the correlation coefficients [TC94]. The consumption of oxygen for halogenated polymer fire is expressed with

$$
y_{\mathrm{O} 2}=y_{\mathrm{O} 2, \infty}\left[1-\frac{0.30}{\exp (\Phi / 0.53)^{-11}}\right]
$$

Masaik et al [MC01] studied the influence of variable temperatures and the flow of air on the yields of the toxic gases during burning of plastic. The plastic materials are polystyrene foam, polyvinyl chloride floor covering, polyamide carpet and flexible polyurethane foam. Yields of the combustion products are obtained at $500^{\circ} \mathrm{C}$ and $800^{\circ} \mathrm{C}$ with the equivalence ratio from 0.5 to 1.5 . The detailed correlation are depicted with figures in [MC01] and not represented here.

\subsection{Absorption of $\mathbf{H C l}$ by Walls of Enclosures}

The non-thermal fire damages are usually caused by smoke, toxics and corrosive products from the fires. This kind of damages depends on the chemical properties of the products and the deposition of products on the walls, ceiling, building furnishings, 
equipment, etc., and the environmental conditions. Most commercial and industrial occupancies are susceptible to non-thermal fire damages. Examples of typical commercial and industrial occupancies are telephone central offices, computer rooms, power plant control rooms, etc. On 8 May 1988, a disruptive fire occurred in power and communication cables of a central telephone exchange in Hinsdale, IL [AF00]. The total loss was about 60 million dollars.

Fires involving chlorine element produce hydrogen chloride $(\mathrm{HCl}) . \mathrm{HCl}$ is a sensory and pulmonary irritant [Ha96]. It is dangerous to humans remaining within fire enclosures [Pu02]. Polyvinylchloride (PVC) is one of the most widely used materials in electrical equipment and in wires and cables as insulation and jacket materials. The one product given off by PVC fire, which is not given off by natural materials, is $\mathrm{HCl}$. The $\mathrm{HCl}$ yields seem to be largely independent of the equivalence ratio [LBM96, LBM96b, MC01, AM03].

Unlike other toxic gases produced within enclosure fires, such as $\mathrm{CO}$, the concentration of $\mathrm{HCl}$ in the fire effluent decreases rapidly due to the absorption by wall surfaces. In order to better understand the deposition properties of $\mathrm{HCl}$ within enclosure fires, a series of fire tests involving a PVC floor covering within a small $(2.8 \mathrm{~m}$ (wide) $\times 2.8 \mathrm{~m}($ deep $) \times 2.4 \mathrm{~m}($ high $))$ unventilated room were performed in 1979 [Mp79]. These trials demonstrated that the ratio of $\mathrm{HCl}$ deposited on walls to the total of $\mathrm{HCl}$ generated strongly depended on the nature of the wall surface materials and the compartment humidity. It was found that wall coatings consisting of matt and stain paints resulted in approximately $50 \%$ of the original chloride ions in PVC being deposited on the walls within 20 minutes. High gloss paint and plastic film lead to high concentrations of $\mathrm{HCl}$ in the room atmosphere while unpainted asbestos cement could absorb large quantities of $\mathrm{HCl}$. The absorption capability of wall surfaces increases with increased atmospheric humidity.

More recently, a series of full-scale and large-scale fire tests with materials containing chloride [LBM96, LBM96b] reported that less than $60 \%$ of the theoretical yield of $\mathrm{HCl}$ was measured at enclosure openings. The time to reach peak $\mathrm{HCl}$ concentration 
at the enclosure opening was also found to occur much later than the peak in $\mathrm{CO}$ concentration. In some cases, this delay was more than 10 minutes.

A series experimental involving the transport of $\mathrm{HCl}$ within enclosures were conducted for developing a $\mathrm{HCl}$ decay model involving the absorption by wall surfaces [GH89, GH90, GH91a, GH91b, GH92]. A wide range of wall materials were studied, such i.e. PMMA, painted gypsum, ceiling tile, cement block etc. The experiments were performed with varied geometries, small boxes, compartment, buildings including room and corridor and a heating, ventilation and air conditioning (HVAC) system. Model parameters for different materials have been derived from the experiments in [GH89, GH92].

Climent-Llorca et al [CV98] have studied the absorption and diffusion of chloride with concrete in small-scale PVC resin fires. The test chamber had dimensions of $40 \mathrm{~cm}$ wide, $40 \mathrm{~cm}$ high and $80 \mathrm{~cm}$ long and was constructed of $14 \mathrm{~mm}$ thick PMMA sheet. Concrete specimens with different exposed area were located in the test chamber in order to study the chloride contamination of concrete by interaction with PVC combustion gases. Within 30 minutes, up to $54 \% \mathrm{HCl}$ from the pyrolysis of PVC within the chamber has been absorbed by the concrete target.

The transport of smoke including $\mathrm{HCl}$ has been reviewed by Butler et al [BM04], in which the absorption of $\mathrm{HCl}$ by smoke particles were also discussed.

Several scenarios with the transport of $\mathrm{HCl}$ from the previous reviewed publications [GH91a, GH92, CV98] are used to validate the $\mathrm{HCl}$ deposition model developed in Chapter 8.

\subsection{Smoke Properties}

Smoke is one of the basic phenomenon in enclosure fires. The amount and character of the smoke generated is strongly affected by the combustion environment, combustion modes and the materials. The light extinction coefficient and smoke optical density are in connection with the visibility of objectives and are mostly 
concerned in studying enclosure fires. Optical dense smoke affects the ability to find the way to escape and the run or walk speed.

In the mathematical modelling of smoke movement in fires, conservation equations are usually used to represent the soot formation and oxidation. Magnussen et al. [MH77, MH78] proposed a soot model for turbulent diffusion flame based on the eddy dissipation concept. Soot is formed in two stages - radical nuclei formation and soot particle formation, which are represented by two partial differential equations. Novozhilov et al [NM96] proposed a soot model in which a one-step global chemical reaction is considered and a parameter is used to represent the mole ratio of soot to the total carbon in the reaction. However, how to get the empirical value of the parameter was not discussed in the article. Models to predict the smoke optical density have been developed by using $\mathrm{CO}_{2}$ concentration, or $\mathrm{O}_{2}$ concentration or temperatures in [IP99, Na00]. Compared with experimental data, the predicted smoke optical density based on the $\mathrm{O}_{2}$ concentrations showed good agreement with the measured data while the predictions based on the temperatures overestimated the smoke optical density considerably.

\subsection{Zone modelling of $\mathrm{CO}$}

\subsubsection{Application of GER}

An algorithm frame considering the mechanisms of $\mathrm{CO}$ formation in enclosure fires (See Section 2.2.3) has been presented by Pitts [Pi97]. In this algorithm frame, each factor for the generation of $\mathrm{CO}$ has been accounted and incorporated into a GER description. Developing toxicity model along this line is interesting. In this section, how the concept of GER is applied in zone modelling is specially reviewed.

Although the generality of experimentally derived yield data are not well established, they provide the best way to estimate species concentrations [KQ00]. Because the concept of global equivalence ratio (GER) is developed for two-layer zone fire modelling, the basic idea about predictions of carbon monoxide in enclosure fires using zone model is briefly introduced here. For any chemical models, the composition of fuel must be known. The SFPE handbook [SFPE95] provides compositions of a wide range of fuels. For the estimate of the GER, the fuel loss rate 
(or fuel flow rate) must be given as an input. This can be calculated using the plume equation (Chapter 4 in [KQ00]). In zone modelling, the airflow rate into the upper layer of the compartment can be obtained, approximately, by calculating the air rate through the opening [JT92].

Once the GER is obtained, the species yields can be calculated using the correlations that are reviewed in the Section 2.2. Cooper [Co94] developed a global equivalence ratio model (GGERM) to predict the upper layer mass fractions of fuel, $\mathrm{O}_{2}, \mathrm{CO}$ and other products. The GGERM model utilizes the correlations between the yields of combustion products and the global equivalence ratios based on hood experiments. Yamada et al [YT94] proposed a similar zone model for the prediction of CO. Tsuchiya [Ts94] developed a chemical sub-model in zone modelling for fuel containing $\mathrm{C}, \mathrm{H}$ and $\mathrm{O}$ elements. In his model, $\mathrm{CO}, \mathrm{CO}_{2}, \mathrm{H}_{2} \mathrm{O}$, and the unburned gasified fuel are considered as the combustion products. In this model, the $\mathrm{CO} / \mathrm{CO}_{2}$ ratio against GER is applied. The representations of the applications of GER in zone modelling are described in forms with species yields and $\mathrm{CO} / \mathrm{CO}_{2}$ ratio in the following sections respectively.

\subsubsection{Model with Species Yields}

In this section, the calculation of species concentrations in the upper layer are represented with the simple correlations in [GR95]. The yields of the combustion products can be expressed with their concentrations in the upper layer by

$$
y_{i}(\phi)=\frac{C_{i_{\text {wet }}}\left(\dot{m}_{f}+\dot{m}_{a}\right) M_{i}}{\dot{m}_{f} M_{\text {mix }}}
$$

where $C_{i_{\text {wet }}}$ is the wet mole fraction of species $i, \dot{m}_{a}$ is the rate of airflow into the upper layer, $\dot{m}_{f}$ is the fuel loss rate, $M_{i}$ is the molecular weight of species i and $M_{m i x}$ is the molecular weight of the air mixture. The consumption of oxygen (or air) is expressed with [GR95]

$$
y_{O 2}(\phi)=\frac{0.21 \dot{m}_{a} \frac{M_{O 2}}{M_{a}}-C_{O 2_{\text {wet }}}\left(\dot{m}_{f}+\dot{m}_{a}\right) \frac{M_{O 2}}{M_{m i x}}}{\dot{m}_{f}}
$$


From (2.5.1) and (2.5.2), the mole fractions of combustion products and oxygen can be expressed with yields by equation (2.5.3) and (2.5.4)

$$
\begin{aligned}
C_{i_{\text {wet }}}= & \frac{y_{i}(\phi) \dot{m}_{f} M_{m i x}}{\left(\dot{m}_{f}+\dot{m}_{a}\right) M_{i}} \\
C_{O 2_{\text {wet }}} & =\frac{0.21 \dot{m}_{a} \frac{M_{m i x}}{M_{a}}-y_{O 2}(\phi) \dot{m}_{f} \frac{M_{m i x}}{M_{O 2}}}{\dot{m}_{f}+\dot{m}_{a}}
\end{aligned}
$$

Note the correlation between the mole fraction $C_{i}$ and the mass fraction $Y_{i}$ is given by

$$
Y_{i}=C_{i} \frac{M_{i}}{M_{m i x}}
$$

The mass fractions of species can be expressed as

$$
\begin{aligned}
& Y_{i_{\text {wet }}}=\frac{y_{i}(\phi) \dot{m}_{f}}{\left(\dot{m}_{f}+\dot{m}_{a}\right)} \\
& Y_{O 2_{\text {wet }}}=\frac{0.21 \dot{m}_{a} \frac{M_{O 2}}{M_{a}}-y_{O 2}(\phi) \dot{m}_{f}}{\dot{m}_{f}+\dot{m}_{a}}
\end{aligned}
$$

The correlation between the wet concentration and the dry concentration is expressed by [GR95]

$$
C_{i_{\text {wet }}}=\left(1-C_{H_{2} O_{\text {wet }}}\right) C_{i_{d r y}}
$$

The yield of water is seldom given because it is difficult to obtain reliable measurements. For simplifying the calculation, it is assumed that the mole ratio, $\alpha$, of $\mathrm{H}_{2} \mathrm{O}$ to $\mathrm{CO}_{2}$ at any equivalence ratio is equal to the calculated ratio at stoichiometric conditions. With this assumption, equation (2.5.8) is simplified, using the $\mathrm{CO}_{2}$ concentration [GR95] 


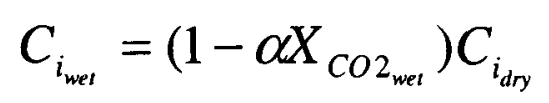

\subsubsection{Model with $\mathrm{CO} / \mathrm{CO}_{2}$ Ratio}

Equations (2.5.6-2.5.9) give the average species concentrations in the upper layer of a fire enclosure using the yields of combustion products. A chemical model developed by Tsuchiya provides an approach to calculate the species concentrations using the mole ratio of $\mathrm{CO}$ to $\mathrm{CO}_{2}$ [Ts94, Ts95]. In Tsuchiya's model, the combustion products, $\mathrm{CO}, \mathrm{CO}_{2}, \mathrm{H}_{2} \mathrm{O}$, and the unburned gasified fuel are involved in the chemical reaction

$$
\begin{aligned}
(\alpha \mathrm{C}+\beta \mathrm{H}+\gamma \mathrm{O}) & +(1-p)[(1-q / 2) \alpha+\beta / 4-\gamma / 2] \mathrm{O}_{2} \\
& =(1-p)\left[\alpha(1-q) \mathrm{CO}_{2}+\alpha q \mathrm{CO}\right. \\
& \left.+\beta / 2 \mathrm{H}_{2} \mathrm{O}\right]+p(\alpha \mathrm{C}+\beta \mathrm{H}+\gamma \mathrm{O})
\end{aligned}
$$

where $(\alpha \mathrm{C}+\beta \mathrm{H}+\gamma \mathrm{O})$ is the gasified fuel, $p$ is the unburned fuel carbon fraction, $q$ is the carbon fraction converted to $\mathrm{CO}$ in the burned fuel. The values of parameters $\alpha, \beta$ and $\gamma$ represent the fuel compositions.

The rates of $\mathrm{CO}_{2}, \mathrm{CO}$, and $\mathrm{H}_{2} \mathrm{O}$ generation are then calculated by [Ts95]

$$
\begin{aligned}
& \dot{m}_{\mathrm{CO}_{2}}=(1-p)(1-q) \alpha M_{\mathrm{CO}_{2}} \dot{m}_{f} / M_{f} \\
& \dot{m}_{\mathrm{CO}}=(1-p) q \alpha M_{\mathrm{CO}} \dot{m}_{f} / M_{f} \\
& \dot{m}_{\mathrm{H}_{2} \mathrm{O}}=\frac{1}{2}(1-p) \beta \alpha M_{\mathrm{H}_{2} \mathrm{O}} \dot{m}_{f} / M_{f}
\end{aligned}
$$

where $\dot{m}_{f}$ is fuel loss rate. The $\mathrm{O}_{2}$ remaining after combustion is

$$
\begin{aligned}
& \dot{m}_{\mathrm{O}_{2}}=(\alpha+\beta / 4-\gamma / 2) M_{\mathrm{O} 2} \dot{m}_{f} /\left(M_{f} \phi\right) \\
& -(1-p)[(1-q / 2) \alpha+\beta / 4-\gamma / 2] M_{O 2} \dot{m}_{f} / M_{f}, \quad \dot{m}_{\mathrm{O} 2} \geq 0
\end{aligned}
$$

Parameters $p$ and $q$ are empirically functions of equivalence ratio $\phi$. In general, the value of $p$ is determined by the following assumptions, $p=0$ if $\phi<1$ and at $\dot{m}_{\mathrm{O} 2}=0$ if $\phi \geq 1$. The value of $q$ is derived from the $\mathrm{CO} / \mathrm{CO}_{2}$ mole ratio. An empirical relationship for burning wood cribs is [Ts95] 


$$
q=0.0875 \phi /(1+0.0875 \phi)
$$

By divided by the total generation rate, the mass fraction of species $i$ at steady burning state is expressed as

$$
Y_{i}=\frac{\dot{m}_{i}}{\sum_{j} \dot{m}_{j}}
$$

where $\mathrm{j}$ covers all the species such that the denominator of equation (2.5.16) represents the rate of mass including both fuel and air.

Note that the numerator of equation (2.5.6) is the generation rate of species $i$. Therefore, both equation (2.5.6) and (2.5.16) calculate the average of species concentrations utilizing the species generation rate and total rate of fuel loss and air entrainment. From this point of view, the two approaches are essentially consistent. Detailed $\mathrm{CO} / \mathrm{CO}_{2}$ ratios at different combustion conditions for various combustion materials are reviewed by Tsuchiya [Ts94].

\subsubsection{Limits of Applying GER in Zoon Modelling}

The GER concept based on the 'hood' experiments is only applicable to enclosure fires with the following conditions [Pi95]

C1 Fires for which the upper layer temperature is less than $700 \mathrm{~K}$,

C2 Fires for which the upper layer is lean and very hot $(>900 \mathrm{~K})$,

C3 Fires for which the only route for oxygen (air) to enter the upper layer is through the fire plume and the upper layer is very hot $(>900 \mathrm{~K})$.

The GER concept based on the 'hood' experiments is not applicable to enclosure fires with the following conditions 
C4 $\phi>0.5$ and intermediate temperature $(700-900 \mathrm{~K})$ in the upper layer,

C5 Fires with temperatures greater than $900 \mathrm{~K}$ for which oxygen (air) enters a rich upper layer directly.

Through the Purser Tube Furnace experiments, correlations between species yields and the equivalence ratio and temperature of gas mixture are available. Therefore, using proper experimental apparatus the inapplicable conditions of GER based on 'Hood' type experiments, $C 4$ and $C 5$, can be reduced to

$C 5^{\prime}$ Fires in which oxygen (air) enters a rich upper layer directly.

The concept of GER is proposed for zone fire modelling for the prediction of CO within fire enclosures. It is still impossible to solve problem $C 5^{\prime}$ in the two layer zone models for fire with complicated geometry.

More discussions on the limits of GER in fire simulations can be found in [Ba95, Pi95, GRB95, PB95, Pi01, WV04, LV05].

\subsection{Field Modelling of $\mathrm{CO}$}

\subsubsection{A CO Correlation Used in a Field Model}

A few papers are concerned with the correlation of $\mathrm{CO}$ generation in field fire modelling. Luo et al [LB94, LB96a, LB96b] applied the correlation between $\mathrm{CO}, \mathrm{CO}_{2}$ and $\mathrm{O}_{2}$ in the simulations of enclosure fires. The fuels in their studies are propane and polyurethane respectively. The chemical reaction for propane fire is

$$
\mathrm{C}_{3} \mathrm{H}_{8}+(2+2 a+b) \mathrm{O}_{2}=a \mathrm{CO}_{2}+b \mathrm{CO}+4 \mathrm{H}_{2} \mathrm{O}
$$

where parameters $a$ and $b$ are determined by

$$
\frac{y_{C O}}{y_{C O 2}}=\frac{28 a}{44 b}
$$

and 


$$
\frac{60 y_{O 2}}{23}=\frac{44 b}{28 a}
$$

Here $y$ refers to the mass fraction. Correlation given by equation (2.6.2) and (2.6.3) is an approximation from many experimental data in [WF82] and is very rough for various fuels.

Equations (2.6.2) and (2.6.3) imply the following equation

$$
\frac{y_{C O}}{y_{C O 2}}=\frac{23}{60 y_{O 2}}
$$

Note that the $\mathrm{O}_{2}$ mass fractions may be approximately zero in the fuel rich region. This means the right hand side of equation (2.6.4) tends to infinite if the LER is greater than 1.0. Thus, the mass ratio of $\mathrm{CO}$ to $\mathrm{CO}_{2}$ is extremely high in very fuel rich region according to equation (2.6.4). Infinite $\mathrm{CO} / \mathrm{CO} 2$ ratio is unrealistic for the GERs between 1.0 and 3.0 [Pi95, Ts94, Te95]. Therefore, the above correlations, using the predicted $\mathrm{O}_{2}$ mass fraction, are unreliable to determine the parameters $a$ and $b$ in chemical equation (2.6.1) in locations where the LERs are greater than 1.0. The above correlation for the calculation of parameters $a$ and $b$ can be used to represent locations away from the fire source, where the local equivalence ratios (LER) are usually less than 1.0 .

Luo et al tried to connect the mass fractions of $\mathrm{CO}_{2}, \mathrm{CO}$ and $\mathrm{O}_{2}$ at a location via the two parameters $a$ and $b$. Given the fuel to air ratio is determined at a given location, the coefficient $(2+2 a+b)$ in equation $(2.6 .1)$ is known. Therefore, only one extra equation including $a$ and $b$ is sufficient to give the values of $a$ and $b$. In other words, only one of the two parameters $a$ and $b$ is independent.

As reviewed in Section 2.2, experimental correlations between the yields of combustion products and the GERs are available for various materials. A suitable parameter is needed in field modelling to calculate the concentrations of toxic gases using these correlations. The LER is obviously a logic option to replace the GER. Mathematical models with the LER are developed to predict generation and spread of combustion products within fire enclosures later in this thesis. 


\subsubsection{Multi-step Reactions}

Simplified chemical reaction mechanisms possibly be used to predict $\mathrm{CO}$ in enclosure fires. This method may be applicable for some gas-fuel fires that the toxic gases may be seriously temperature dependent [Pi94b, PB95, GRB95] and the multi-step chemical reaction rates are available from literatures [HD81,WD81, JL88].

Bilger [Bi94] has ever proposed an idea of using Eddy Break-up (EBU) model based on 4-step reduced mechanisms for the prediction of CO. The multi-step EBU model, in which $\mathrm{CO}$ is one of the intermediate products, was sufficient to account for the effect of inefficient oxygen, slow fuel evaporation and slow reaction rate on the extinction processes [BK99, BM00, HC97], produced good flame base height and other flame characteristics of cylindrical tube methane flames [KC99]. However, as an engineering method for the prediction of $\mathrm{CO}$ in fire enclosures, the multi-step EBU model, for fuel rich scenarios, is in question by Brink [Br04] according to his experience in this area [BK99, BM00].

Hyde et al [HM99] simulated axi-symmetric flame using a two-step EBU model, in which the reaction rates of the two steps are controlled by the mixing rate but with different coefficient for each step. The predicted CO level was modest to Hyde's special fire scenarios.

\subsubsection{Advanced Models}

Wen [WH00] et al has simulated under ventilation-controlled propane fires using the flamelet model [Pe84] and the constrained equilibrium model [KK91]. The predicted $\mathrm{CO}$ distributions are different in scenarios with different combinations of the combustion models and radiation approaches. Only one of three scenarios produced a good approximation to the measured $\mathrm{CO}$ concentrations. As the $\mathrm{CO}$ concentrations were only measured at a fixed location for the steady fires, no confidence conclusions have been drawn from these simulations using these advanced combustion models. 
Hyde [HM99, Hy00] has investigated different numerical approaches to the CO levels in vitiated compartment ethylene fires. Numerical studies showed that chemical equilibrium is a poor model for describing the $\mathrm{CO}$ in either a flame or an upper layer. This is because the time for equilibrium to reach is prohibitively long, compared the residence time of the gas in the compartment. He indicated that the $\mathrm{CO}$ level in vitiated compartment fires is solely determined by quenching the flame plume in the upper layer. In the combustion region, the $\mathrm{CO}$ profiles predicted by the laminar flamelet are more representative of the experimental data along the centre line of jet fires than the two-step EBU model. Numerical results to a hypothetical compartment fire demonstrated that the two-step EBU failed to predict high enough levels of CO that has been usually observed from full-scale fires from literatures, while the laminar flamelet approach seems preferable.

A disadvantage of the flamelet model is that the data are available only for some gas/liquid fuels, not commonly used building materials that are concerned by fire engineers.

\subsection{Concluding Remarks}

The fire modelling approach for prediction of combustion products is limited by reliable species yields. The species yields depend on the combustible materials and the combustion environment. Ventilation is an important factor on the generation of toxic gases in enclosure fires. The concept of GER describes the mixing state of fuel and air for two-layer room fire modelling. Correlation between the production of the toxic gases and the GER are available from small-scale experiments. In this chapter, experiments for species yields are reviewed and the mechanisms for $\mathrm{CO}$ generation are analyzed. Correlations between the yields of toxic gases and the GERs from small-scale experiments are presented and application conditions are discussed.

The main points from the review in this chapter are summarised below:

1) The yields of combustion products correlated well with the GER from hoodtype experiments. 
2) Correlations between the generation of $\mathrm{CO}$ and GER derived from hood-type experiments sometimes fail to the enclosure fires, in which there is continuing combustion in the upper layer due to the high temperatures.

3) The Purser Furnace is capable of mimicking the local combustion conditions including the equivalence ratio and the temperature.

4) The GER can represent the uniform mixture of fuel, air and combustion products in the hood-type experiments, while this single value cannot reflect the spatial varies of the mixture in the full-scale fire scenarios.

5) The zone modelling is not capable of predicting the $\mathrm{CO}$ concentrations for fire scenarios with complicated fire geometries.

6) The multi-step reaction approach may be in question for fuel rich fire scenarios.

7) The flamelet model is a preferable approach for prediction $\mathrm{CO}$ in enclosure fires; however, model data are only available for a few gas and liquid fuels.

8) Unlike $\mathrm{CO}$ and $\mathrm{CO}_{2}, \mathrm{HCl}$ can be absorbed by wall surfaces in the course of transportation within fire enclosures.

Points 6) and 7) indicate that the advanced combustion models are not suitable as an engineering tool for the predictions of $\mathrm{CO}$ in fire enclosures at present due to either unfitting the vitiated fire or the lack of data for most of the building materials. From points 1) to 3 ), reliable correlations between the species yields and equivalence ratio with different temperature ranges could be derived from properly small-scale experiments; however, points 4) and 5) means these invaluable experimental results could not be used in fire simulations in zone modelling as been proposed.

Could these correlations derived from small-scale experiments be applied in fire field modelling for the prediction of combustion products including $\mathrm{CO}$ ? 


\section{Chapter 3}

\section{MATHEMATICAL MODELS FOR ENCLOSURE FIRES}

\subsection{Introduction}

The field approach is an attractive route to investigate the behaviour of enclosure fires. Field modelling is based on Computational Fluid Dynamics (CFD). Conduction, Convection, Radiation and Combustion are the key components of a CFD fire model. The transport phenomena are core to the fire field model and consider the manner in which mass, momentum, and energy are transported and conserved throughout the flow domain. Modelling chemical reactions is necessary for the prediction of combustion products and smoke which impose a threat to people trapped in enclosures that are on fire. In field models, sub-models are required to account for some of distinctive fire phenomena, such as turbulence, chemical reaction rates, visibility, etc.

Field models are more versatile and involve a minimum of empiricism, as opposed to zone models [St95]. By dividing the domain of interest into a large number of control volumes, acceptably accurate solutions of the equations can be obtained numerically. Field models have been used to investigate the development of hazardous conditions within fire enclosures [Ku86, Ga89, LW93, Bi94, Co95, Co95b, Tu95, St95, Ji99, Hy00, No01, Mc05, JP06a-b].

The objective of this chapter is to describe the basic methods and models used in field based fire simulations. The general conservation equations are presented in Section 3.2. Section 3.3 describes the turbulence model used in fire simulations. Sub-models for combustion and radiation are reviewed in Section 3.4 and Section 3.5 respectively. The boundary conditions are summarised in Section 3.6. The numerical methods and techniques for the solutions of the governing equations are described in Section 3.7. As an example, in Section 3.8, the simulation results to a complex enclosure fire using SMARTFIRE are briefly summarised. Finally, conclusions are drawn in section 3.9. 


\subsection{Conservation Equations}

\subsubsection{General Equation}

The mathematical statements of the transport processes involved in fires are called conservation equations, which are based on the three conservation laws

- Conservation of mass,

- Conservation of momentum,

- Conservation of energy.

The general conservation equation takes the following form with Cartesian tensor notation [Co95]

$$
\begin{array}{r}
\frac{\partial(\rho \varphi)}{\partial t}+\frac{\partial}{\partial x_{j}}\left(\rho u_{j} \varphi\right)=\frac{\partial}{\partial \mathrm{x}_{j}}\left(\Gamma_{\varphi} \frac{\partial}{\partial x_{j}} \varphi\right)+S_{\varphi} \\
\text { Transient }+ \text { Convection }=\text { Diffusion }+ \text { Source }
\end{array}
$$

where $\varphi$ represents the dependent variable to be solved, $\rho$ is the density of the fluid and $u_{j}$ is velocity of the fluid in the $j^{\text {th }}(x, y$, or $z)$ direction. $\Gamma_{\varphi}$ is diffusion coefficient for the variable $\varphi . S_{\varphi}$ is the additional source term of variable $\varphi$.

The first term in equation (3.2.1) is the transient term, at which $\varphi$ is accumulative per unit volume. The second term in equation (3.2.1) is the convection term, which represents the accumulation of $\varphi$ per unit volume due to the divergence in its convective flux field. The third term in equation (3.2.1) is the diffusion term, which represents the accumulation of $\varphi$ per unit volume due to the divergence in its diffusive flux field. The last term of equation (3.2.1) is the source term, which includes all the additional sources of $\varphi$, for example, the change of heat via radiation is a source term of energy conservation equation.

In the following section, the conservation equations of mass, momentum, energy and species [Co95] are briefly described. Similar presentations can be found in [Tu95]. 


\subsubsection{Continuity Equation}

The overall mass continuity within a control volume is described with the continuity equation,

$$
\frac{\partial \rho}{\partial t}+\frac{\partial}{\partial x_{j}}\left(\rho u_{j}\right)=0
$$

This equation states that the change rate of the density in the control volume is balanced by the net rate of flow across the control volume boundaries.

\subsubsection{Momentum Equation}

Equations describing the conservation of momentum are in the form of equation (3.2.3)

$$
\frac{\partial}{\partial t}\left(\rho u_{i}\right)+\frac{\partial}{\partial x_{j}}\left(\rho u_{j} u_{i}\right)=-\frac{\partial p}{\partial x_{i}}+\frac{\partial \tau_{i j}}{\partial x_{j}}+g_{i}
$$

where $g_{\mathrm{i}}$ is the body force term per unit volume in the $i^{\text {th }}$ direction. $P$ is the pressure. $\tau_{i, j}$ is the stress tensor, which is defined as

$$
\tau_{i j}=\mu\left(\frac{\partial u_{i}}{\partial x_{j}}+\frac{\partial u_{j}}{\partial x_{i}}\right)-\frac{2}{3} \mu \frac{\partial u_{k}}{\partial x_{k}} \delta_{i j}
$$

where $\mu$ denotes the dynamic viscosity of the fluid and $\delta_{i j}=1$ for $i=j$ and 0 for $i \neq j$. The left-hand side of equation (3.2.3) represent the local rate of change of momentum for a volume of element travelling. The first term on the right-hand side is the pressure force. The second term in this side is the viscous forces (given by equation 3.2.4) and the third term is the body force.

\subsubsection{Enthalpy Equation}

The conservation of energy is given by the following equation

$$
\frac{\partial}{\partial t}(\rho h)+\frac{\partial}{\partial x_{j}}\left(\rho u_{j} h\right)=\frac{\partial p}{\partial t}+\frac{\partial}{\partial x_{j}}\left[\frac{k}{c_{p}} \frac{\partial h}{\partial x_{j}}-\dot{q}_{j}^{R}\right]
$$

where $h$ is the mixture static enthalpy expressed as 


$$
h=c_{p} T+\sum_{\alpha} Y_{a} H_{\alpha}
$$

here $c_{p}$ is the specific of the mixture, $Y_{\alpha}$ and $H_{\alpha}$ are the mass fraction and the heat of reaction of the species $\alpha$ respectively. The thermal diffusivity can be simply expressed by the viscosity and the Prandtl number $\sigma$ as

$$
k=c_{p} \frac{\mu}{\sigma}
$$

The first term on the left-hand side of equation (3.2.5) is the change rate of the energy per unit volume. The second term is the net heat flux by convection. The first term on the right-hand side is the pressure work. The last term includes two parts: the transport of energy due to conduction and radiation.

\subsubsection{Species Equation}

For a species $\alpha$, the conservation equation is given as

$$
\frac{\partial}{\partial t}\left(\rho Y_{\alpha}\right)+\frac{\partial}{\partial x_{j}}\left(\rho u_{j} Y_{\alpha}\right)=\frac{\partial}{\partial x_{j}}\left(\rho D \frac{\partial Y_{a}}{\partial x_{j}}\right)+S_{\alpha}
$$

where $Y$ is the mass fraction of species $\alpha, D$ is the diffusion coefficient, and $S_{\alpha}$ is the chemical source term of species $\alpha$ due to the reactions. Equation (3.2.8) is a statement that the change rate of species $\alpha$ is balanced by the net flow into the control volume due to the convection and diffusion and the generation or destruction of this species.

\subsubsection{Equation of State}

In order to close the four equations of conservation, an additional equation is needed. This equation is known as the state equation. For ideal gases the state equation is expressed as

$$
P=\rho R T
$$

where $\mathrm{R}$ is a constant, which is specific to the mixture of gases. 


\subsection{Turbulence Model}

\subsubsection{Time Average Approach}

Previously in Section 3.2 the equations for conservations of mass, momentum, enthalpy and species have been described. With proper time step sizes and meshes, numerical solutions to these equations can be obtained by Direct Numerical Simulation (DNS). For most building fires, in which turbulence is significant, DNS requires extremely small time-step-size and fine meshes. This leads a prohibitively long computational time that makes DNS unfeasible in fire applications.

Without representing turbulence effects, solving these equations is pointless, if accurate predictions are sought. On the other hand, the instantaneous values of the flow variables are not so important for the engineering problems. Therefore, considering the random pattern of fluctuations in turbulent flow, statistical rather than instantaneous treatment is used to develop the conservation equations. This is achieved by averaging the fluctuations of the flow variables.

A mean value $\bar{\varphi}$ of variable $\varphi$ is defined as

$$
\bar{\varphi}=\frac{1}{2 \Delta t} \int_{t-\Delta t}^{+\Delta t} \varphi(t) \mathrm{d} t
$$

Equation (3.3.1) is the simple time (or Reynolds) average to variable $\varphi$. For compressible flows, the Faver average is density-weighted averaging and defined by

$$
\widetilde{\varphi}=\frac{\overline{\rho \varphi}}{\bar{\rho}}
$$

and the instantaneous value of $\phi$ is expressed in the form of (3.3.3)

$$
\varphi=\widetilde{\varphi}+\varphi^{\prime \prime}
$$

with $\overline{\rho \varphi}=0$, where $\varphi^{\prime \prime}$ is the fluctuation and the superposed bar indicates the mean value.

With Favre averaging, the equation for continuity (3.2.2), momentum equation (3.2.3), enthalpy equation (3.2.5) and species equation (3.2.8) can be represented as 


$$
\begin{aligned}
& \frac{\partial \bar{\rho}}{\partial t}+\frac{\partial}{\partial x_{j}}\left(\bar{\rho} \widetilde{u}_{j}\right)=0 \\
& \frac{\partial}{\partial t}\left(\bar{\rho}_{u_{i}}\right)+\frac{\partial}{\partial x_{j}}\left(\overline{\rho u_{j}} \tilde{u}_{i}\right)=-\frac{\partial \bar{p}}{\partial x_{i}}+\frac{\partial}{\partial x_{j}}\left(\bar{\tau}_{i j}-\bar{\rho} \overline{u_{i}^{\prime \prime} u_{j}^{\prime \prime}}\right)+\bar{g}_{i} \\
& \frac{\partial}{\partial t}(\bar{\rho} \tilde{h})+\frac{\partial}{\partial x_{j}}\left(\bar{\rho}_{j} \tilde{h}\right)=\frac{\partial \bar{p}}{\partial t}+\frac{\partial}{\partial x_{j}}\left[\frac{k}{c_{p}} \frac{\partial \tilde{h}}{\partial x_{j}}-\bar{\rho} \overline{u_{j}^{\prime \prime} h^{\prime \prime}}\right]-\frac{\partial \dot{q}_{j}^{R}}{\partial x_{j}} \\
& \frac{\partial}{\partial t}\left(\bar{\rho} \widetilde{Y}_{\alpha}\right)+\frac{\partial}{\partial x_{j}}\left(\bar{\rho} \tilde{u}_{j} \widetilde{Y}_{\alpha}\right)=\frac{\partial}{\partial x_{j}}\left(\bar{\rho} D \frac{\partial \tilde{Y}_{\alpha}}{\partial x_{j}}-\bar{\rho} \overline{u_{j}^{\prime \prime} Y_{\alpha}^{\prime \prime}}\right)+\bar{S}_{\alpha}
\end{aligned}
$$

The effect of Favre averaging on the general equation (3.2.1) is

$$
\frac{\partial(\bar{\rho} \tilde{\varphi})}{\partial t}+\frac{\partial}{\partial x_{j}}\left(\widehat{\rho}_{j} \widetilde{\varphi}\right)=\frac{\partial}{\partial \mathrm{x}_{\mathrm{j}}}\left(\Gamma_{\varphi} \frac{\partial \widetilde{\varphi}}{\partial x_{j}}-\bar{\rho} \overline{u_{j}^{\prime \prime} \varphi^{\prime \prime}}\right)+S_{\varphi}
$$

As seen in equations (3.3.4), the continuity equation, with the averaged variables, has the same form of equation (3.2.2) with the instantaneous variables. However, the other conservation equations (3.3.5-3.3.8) contain additional turbulent terms on their right-hand sides. These are the Reynolds stress $\left(\rho \overline{u_{i} u_{j}^{\prime \prime}}\right)$ and the Reynolds fluxes $\left(\rho \overline{u_{i}} \varphi^{\prime \prime}\right)$. These terms need to be calculated via some form of turbulence model. A review on field modelling of turbulent flows has been given by Kumar in [Ku83]. In the next section, the widely used $k-\varepsilon$ model in fire simulations is described.

\subsection{2 $k-\varepsilon$ Turbulence Model}

The $k-\varepsilon$ turbulence model is based on the eddy viscosity hypothesis [Bo77, LS74]. Eddy viscosity hypothesis is that the Reynolds stresses can be related to the mean velocity gradients in a manner analogous to the relationship between the stress and strain tensors in laminar Newtonian flow. This assumption gives

$$
-\overline{u_{i}^{\prime \prime} u_{j}^{\prime \prime}}=\mu_{t}\left(\frac{\partial \tilde{u}_{i}}{\partial x_{j}}+\frac{\partial \widetilde{u}_{j}}{\partial x_{i}}\right)-\frac{2}{3} \delta_{i j}\left(k+\mu_{t} \frac{\partial \tilde{u}_{k}}{\partial x_{k}}\right)
$$


Here $\mu_{t}$ is a local eddy or turbulent kinetic viscosity and is calculated by

$$
\mu_{t}=C_{\mu} \frac{k^{2}}{\varepsilon} \bar{\rho}
$$

$C_{\mu}$ in equation (3.3.10) is an empirical constant. $k$ is the turbulent kinetic energy and $\epsilon$ is the turbulent dissipation rate.

Analogous to the eddy viscosity hypothesis, we have the eddy diffusivity hypothesis, that the Reynolds fluxes of a scalar are linearly related to the mean scalar gradient.

$$
\overline{u_{i}^{\prime \prime} \varphi^{\prime \prime}}=-\Gamma_{t} \frac{\partial \tilde{\varphi}}{\partial x_{i}}
$$

Where $\Gamma_{t}$ is the approximate eddy or turbulent diffusivity for scalar $\phi$ and is expressed as

$$
\Gamma_{t}=\frac{\mu_{t}}{\sigma_{t}}
$$

where $\sigma_{\mathrm{T}}$ is the turbulent Prandtl number.

The transport equations for the turbulent kinetic energy $k$ and the turbulent dissipation rate $\epsilon$ are given by the following equations

$$
\begin{aligned}
\frac{\partial}{\partial t}(\bar{\rho} k)+\tilde{u}_{i} \frac{\partial}{\partial x_{i}}(\bar{\rho} k) & =\frac{\partial}{\partial x_{i}}\left[\left(\frac{\mu_{t}}{\sigma_{k}}+\mu_{t}\right) \frac{\partial k}{\partial x_{i}}\right]+\mu_{t}\left(\frac{\partial \tilde{u}_{i}}{\partial x_{j}}+\frac{\partial \tilde{u}_{j}}{\partial x_{i}}\right) \frac{\partial \tilde{u}_{j}}{\partial x_{i}} \\
- & \beta g \frac{\mu_{t}}{\sigma_{k}} \frac{\partial \widetilde{T}}{\partial x_{i}}-\bar{\rho} \varepsilon \\
\frac{\partial}{\partial t}(\bar{\rho} \varepsilon)+\tilde{u}_{i} \frac{\partial}{\partial x_{i}}(\bar{\rho} \varepsilon) & =\frac{\partial}{\partial x_{i}}\left[\left(\frac{\mu_{t}}{\sigma_{\varepsilon}}+\mu\right) \frac{\partial \varepsilon}{\partial x_{i}}\right] \\
& +C_{1} \frac{\varepsilon}{k}\left(\mathrm{G}_{\mathrm{K}}+\mathrm{G}_{\mathrm{B}}\right)\left(1+C_{3} \mathrm{R}_{\mathrm{f}}\right)-C_{2} \bar{\rho} \frac{\varepsilon^{2}}{k}
\end{aligned}
$$

where $C_{1}, C_{2}, C_{3}$ and $\sigma_{\varepsilon}$ are further empirical constants and the shear and buoyancy source term are 


$$
\mathrm{G}_{\mathrm{K}}=\mu_{t}\left(\frac{\partial \tilde{u}_{i}}{\partial x_{j}}+\frac{\partial \tilde{u}_{j}}{\partial x_{i}}\right) \frac{\partial \tilde{u}_{j}}{\partial x_{i}}
$$

and

$$
\mathrm{G}_{\mathrm{B}}=-\beta g \frac{\mu_{t}}{\sigma_{t}} \frac{\partial \tilde{T}}{\partial x_{j}}
$$

$\mathrm{R}_{\mathrm{f}}$ is the flux Richardson number $=-\mathrm{G}_{\mathrm{B}} / \mathrm{G}_{\mathrm{K}}$ and $\beta$ is expressed with

$$
\beta=-\frac{1}{\bar{\rho}}\left(\frac{\partial \bar{\rho}}{\partial \widetilde{T}}\right)_{p}
$$

The constants of the $k-\varepsilon$ turbulence model used in SMARTFIRE are represented in Table 3.3.1 [EJ02].

Table 3.3.1: Constants used in $k-\varepsilon$ turbulence model.

\begin{tabular}{|c|c|c|c|c|c|}
\hline$C_{\mu}$ & $\sigma_{k}$ & $\sigma_{\varepsilon}$ & $C_{1}$ & $C_{2}$ & $C_{3}$ \\
\hline 0.09 & 1.0 & 1.3 & 1.44 & 1.92 & 1.0 \\
\hline
\end{tabular}

\subsubsection{Large Eddy simulation model}

Time averaging is not necessary if the problem is allowed to be solved with smallest time step size and finest meshes. This approach is called Direct Numerical Simulation (DNS). Large Eddy Simulation (LES) [Sm63, Gr03] is attempt to maintain the accuracy of DNS but without the mesh size required to solve the finest eddies.

\subsection{Combustion Models}

In the process of combustion, species are formed and destroyed together with the release of energy. All these occur at the molecular level. In order to avoid this complexity, the heat release rate and species generation rates are usually estimated at each control volume of the computational domain. The majority of practical fire field modelling applications [Ga89] have been concerned with the spread of heat and smoke in complex structures and so the combustion process has either been ignored or greatly simplified. 
The volumetric heat source approach treats the fire as a source of heat. A volumetric space in which energy or heat is assumed to be release from combustion processes given before a fire simulation starts.

A mixture fraction is often used to represent the mixture of fuel, air and combustion products. The mass fractions of combustion products are calculated using the mixture fraction and a pre-assumed type of probability density function (pdf). The pdf can be determined by the mean value of the mixture fraction and its variance which is governed by a partial differential equation.

By adding a fuel transport equation, the combustion products are predicted using the mixture fraction in the Eddy Break-Up (EBU) model [MH77], with the assumption of single, one-step and infinitely fast chemical reactions.

Knowing the derivation of oxygen mass fraction against the mixture fraction, the released heat, due to combustion, is calculated using the mixture fraction and its gradient in the mixture fraction model.

For the prediction of the incomplete combustion products, such as $\mathrm{CO}$, the effect of finite rate chemical kinetics should also be considered. One of the most promising approaches that is able to adequately represent the effect of finite rate kinetics is the laminar flamelet model [Li83, Pe84]. This model assumes that the combustion occurs within thin laminar flamelets that are embedded within the turbulent flow field. However, the required input data for flamelet models are only known for a few gaseous fuels, such as methane and propane [Li83, Pe84, WH00]

\subsubsection{Volumetric heat source approach}

The simplest form of field fire combustion models is the volumetric heat source approach (or heat release rate model). In this model, the fire is treated as a simple volumetric source of heat and is patched into the computational domain. The heat source is added to the source term in the energy conservation equation. Because this heat source approach does not reflect the fire structure correctly, it is usually used to 
simulate the flow trends far from the combustion region. When the heat source approach is used, the flame length should be small compared to the size of the enclosure. The volumetric heat source approach does not include the species transport equations and cannot predict the species concentrations within the computational domain.

\subsubsection{Conserved scalar approach}

Most of enclosure fires consist of non-premixed diffusion flames. The convenient method to deal with this kind of combustion is by using the conserved scalars approach. Bilger [Bi76] concluded that the instantaneous state of mixture could be described with the mixture fraction $\xi$, i.e.

$$
\varphi=\varphi(\xi)
$$

where $\varphi$ stands for the physical properties of the mixture of gas, such as species concentration and temperature. One-step irreversible combustion reaction can be represented by

$$
F+r O \rightarrow(1+r) P
$$

where $r$ is the stoichiometric oxidant $(O)$ to fuel $(F)$ mass ratio. A mixture fraction is defined as

$$
\xi=\frac{\beta-\beta_{2}}{\beta_{1}-\beta_{2}}
$$

where

$$
\beta=Y_{f}-Y_{o} / r
$$

where $Y_{f}, Y_{o}$ are the mass fractions of fuel and oxidant respectively and subscripts 1 and 2 refer to the fuel and oxidant stream. The source term of the mixture fraction is 0 if all species diffusivities are equal [Bi80]. For the case of infinitely fast reaction, the fuel and oxidant cannot co-exist in the same region, i.e.

$$
Y_{f} \cdot Y_{o}=0
$$


At the stoichiometric state, both of the fuel and oxidant disappear, i.e. $Y_{f}=0$ and $Y_{o}=0$. From equation (3.4.4), stoichiometric state means the value of $\beta$ is zero. In fires, the mass fraction of fuel in the fuel stream and the mass fraction of air in air stream are normally 1.0 . Therefore, the stoichiometric mixture fraction, $\xi_{s}$ is

$$
\xi_{s}=\frac{-\beta_{2}}{\beta_{1}-\beta_{2}}=\frac{1}{r+1}
$$

In fuel rich $\left(\xi>\xi_{s}\right)$ and fuel lean $\left(\xi<\xi_{s}\right)$ regions, the mass fractions of fuel and oxidant can be obtained from equation (3.4.3), (3.4.4) and (3.4.5) and expressed with the following equations respectively

$$
\begin{array}{ll}
Y_{o}=0, Y_{f}=\left(\beta_{1}-\beta_{2}\right) \xi+\beta_{2} & \text { if } \xi>\xi_{s} \\
Y_{f}=0, Y_{o}=-r\left(\left(\beta_{1}-\beta_{2}\right) \xi+\beta_{2}\right) & \text { if } \xi<\xi_{s}
\end{array}
$$

The mean mass fractions are obtained as following

$$
\widetilde{Y}=\int_{0}^{1} Y(\xi) P(\xi) d \xi
$$

where $P(\xi)$ a pre-assumed probability density function (pdf) of the mixture fraction $\xi$. A wide range of pdfs are discussed by Tuovinen [Tu95].

\subsubsection{Eddy Break-up Model}

\subsubsection{Eddy Break-up model}

In non-premixed turbulent fires, the fuel and oxidant are in separate eddies. The combustion process is usually faster than the eddy mixing process. By considering the local turbulence level, Spalding [Sp71] suggested that the rate of the reaction is controlled by the rate at which the larger unburned eddies are successfully broken into smaller ones until the sizes of the small eddies allow the chemical reaction to occur. 
Therefore, the combustion rate can be determined by the rate of intermixing on a molecular scale of fuel and oxygen eddies.

The most commonly used form of the eddy break-up model is that proposed by Magnussen and Hjertager [MH77], in which the fuel-burning rate is given by

$$
R_{f}=A \rho \frac{\varepsilon}{\kappa} \min \left\{Y_{\mathrm{f}}, \frac{Y_{o}}{r}, B \frac{Y_{p}}{1+r}\right\}
$$

where the model constants $A$ and $B$ usually take the values of 4.0 and 1.0 respectively, $\rho$ is the density, $k$ and $\varepsilon$ are the turbulent kinetic energy and the turbulent dissipation rate respectively, $r$ is the stoichiometric mass ratio of oxidant (air) to fuel. The mass fractions of oxidant $Y_{o}$ and product $Y_{p}$ are given by

$$
Y_{o}=1-Y_{f}-r\left(\xi-Y_{f}\right)
$$

and

$$
Y_{p}=1-Y_{f}-Y_{o}
$$

where $\xi$ is the mixture fraction. Here, the mass fraction $Y$ is time-averaged.

The EBU coefficient $A$ of 4.0 is an empirical value. In fact, a wide range of values of $A$ has been used in various applications. Brizuela and Bilger [BB96] tried to evaluate its correct value from theoretical basis and recommended an approximation of its theoretical value derived using two presumed probability functions.

\subsubsection{Two-fuel Eddy Break-up Model}

The uncontrolled enclosure fires usually include more than one fuel. Two-fuel or twooxidant eddy break-up combustion models have been presented in the CFD software CFX-4.2 [CFX97]. The two-fuel model is introduced here. There are two mixture fractions $\xi_{1}$ and $\xi_{2}$ corresponding to the two different fuels. The source terms of the fuels, the mass fractions of the fuels and oxygen are given by 


$$
\begin{aligned}
& R_{f \alpha}=A \rho \frac{\varepsilon}{\kappa} \min \left\{Y_{f \alpha} \frac{Y_{o} Y_{f \alpha}}{r_{\alpha} Y_{f \alpha}+r_{\beta} Y_{f \beta}}, B \frac{Y_{p \alpha}}{1+r_{\alpha}}\right\} \\
& Y_{p \alpha}=\left(1+r_{\alpha}\right)\left(\xi_{\alpha}-Y_{f \alpha}\right) \\
& Y_{o}=1-Y_{f 1}-Y_{f 2}-Y_{p 1}-Y_{p 2}
\end{aligned}
$$

where $\alpha$ can be 1 or 2 representing one of the two fuels while $\beta$ refers to the other fuel and is 2 or 1. From equation (3.4.13), the burning rates of the two fuels are controlled by the mass fractions of fuel, oxidant and products. For the case of fuel rich, (3.4.13) implies that two fuels share the limited oxidant according to how much oxidant is needed to completely consume the fuels. That is to say, $Y_{o} r_{\alpha} Y_{f \alpha} /\left(r_{\alpha} Y_{f \alpha}+r_{\beta} Y_{f \beta}\right)$ of the oxidant is assigned to the fuel $\alpha$ and $Y_{o} r_{\beta} Y_{f \beta} /\left(r_{\alpha} Y_{f \alpha}+r_{\beta} Y_{f \beta}\right)$ of the oxidant is assigned to the fuel $\beta$. This mode of sharing oxidant only considers the chemical composition of the two fuels and ignores their ability to consume the oxidant. No applications of the present two-fuel eddy break-up model have been found in simulations of enclosure fires. In the present study, this model is used to model the heat released from fires with wood and hexane as fuels in Chapter 7.

\subsubsection{Laminar flamelet model}

The fast chemistry assumption is successful in the prediction of temperature and the main combustion products $\left(\mathrm{CO}_{2}, \mathrm{O}_{2}, \mathrm{H}_{2} \mathrm{O}\right)$ in the Eddy Break-up model. However, the main toxic gas, carbon monoxide, is not available without considering the finite kinetics effects. The often-used approach with finite rate chemistry is the laminar flamelet model.

In the laminar flamelet approach, 'where the diffusion flame is considered as a statistical ensemble of thin laminar flames which are called flamelet' [No01]. The turbulent flame can be treated as amount of microscopic elements, which have the structure of the laminar diffusion flame. Therefore, the instantaneous state variables 
are expressed with the mixture fraction $\xi$. Detailed description of the flamelet concept for non-premixed flame can be found in literatures [Li83, Pe84, Pe86].

The mass fractions and temperature are governed by [Pe84]

$$
\rho \frac{\partial Y_{i}}{\partial t}-\frac{\rho}{2} \chi \frac{\partial^{2} Y_{i}}{\partial \xi^{2}}=\dot{\omega}_{i}
$$

where $i$ stand for the species and temperature and $\chi$ is the scalar dissipation rate [Bi80]

$$
\chi=2 D \nabla \xi \nabla \xi
$$

and $D$ is the common diffusion coefficient. $\dot{\omega}_{i}$ is the source term of species $i$ or the temperature. In the mixing layer of a laminar flame, the accumulation effect is of little importance compared with the diffusion effect. Therefore, the equation (3.4.16) is simplified to be

$$
-\rho \chi \frac{d^{2} Y_{i}}{2 d \xi^{2}}=\dot{\omega}
$$

Equation (3.4.18) shows that the chemical calculation is decoupled from the turbulent flow field calculation. The state relationships for all variables such as the species concentration are unique functions of the mixture fraction $\xi$. At each strain rate, a table of the properties versus the mixture fraction is calculated and stored in a look-up table. In order to obtain this relationship library, detailed chemical kinetic mechanisms for the combustion are needed. Another method to evaluate the relationships is by experimental measurements of laminar diffusion flames. As seen in equation (3.4.9), the mean values of the variables are then obtained through a preassumed probability density function.

As mentioned previously, the correlation between the mixture fraction and the species concentration are calculated before the calculation of the flow field. These kind of correlations are stored in a look-up table and used for the calculations of various variables by flamelet models in fire simulations. 


\subsubsection{Mixture fraction model}

In the Eddy Break-up model, two variables for the mixture fraction and the fuel mass fraction are solved. In the flamelet model, the mean value of the mixture fraction equation is still solved and the mixture fraction variance is other variable. The probability density function, which is used to calculate the mean properties of other variables, is obtained by using the mean value of the mixture fraction and its variance. From the point of view of extra scalars, both of the EBU and the laminar flamelet combustion model require similar computations.

Floyd, et al [FB01] developed a mixture fraction model, in which only one extra scalar is required to calculate the heat released due to combustion. In this model, the consumption rate of oxygen is a function of the gradient of mixture fraction and the derivation of oxygen mass fraction against the mixture fraction. With the assumption that the combustion is one-step, complete and infinite fast, the derivation of oxygen mass fraction against the mixture fraction is zero in the fuel rich region and a constant in the fuel lean region. Therefore, the consumption rate of oxygen is only a function of the mixture fraction at any control volume. Compared with the EBU model, the mixture fraction model reduces computational cost in terms of solving one less extra partial differential equation. However, the gradient of the mixture fraction is involved in the mixture fraction model. Therefore, the mesh sensitivity of the mixture fraction model should carefully considered when used for applications.

\subsection{Thermal Radiation}

\subsubsection{Radiation Transfer Equation}

Thermal radiation is one of the important heat transfer modes in enclosure fires. Due to the radiative heat losses, the flame temperature is less than the adiabatic flame temperature. It is known that radiation from flame to fuel surfaces becomes the dominant mode of heat transfer for characteristic flame lengths greater than $0.2 \mathrm{~m}$ [TL95]. Therefore, radiation from the hot layer is partially responsible for the ignition of combustible materials and flame spread. It also exposes a severe threat to people 
remaining in the burning enclosures. A Mathematical model for the calculation of radiation is needed for simulating enclosure fires.

The equation of radiation transfer is expressed by [CR93, Ji99]

$$
\begin{aligned}
\frac{d}{d l} I(\Omega, r) & =-(a+s) I(\Omega, r)+a I_{b}(r) \\
& +\frac{s}{4 \pi} \int_{2^{\prime}=4 \pi} I\left(\Omega^{\prime}, r\right) \Phi\left(\Omega^{\prime} \rightarrow \Omega\right) d \Omega^{\prime}
\end{aligned}
$$

where $\Omega$ is a specified direction; $l$ represent the path length along direction $\Omega ; \mathrm{r}$ is the position of a point; $I(\Omega, r)$ stands for the radiation intensity along $\Omega$ at position $r ; a$ and $s$ are the absorption and the scattering coefficients of the medium respectively; $I_{b}(r)$ is the black radiation intensity; and $\Phi\left(\Omega^{\prime} \rightarrow \Omega\right)$ is the scattering phase function. The above equation is the basis of numerical methods for the solution of radiation heat transfer.

In many engineering applications, scattering may be ignored since 'gas molecules and small soot particles scatter negligibly, as long as the wave length of the principal radiation is much greater than their diameter' [Le98]. Only in the coal combustion and the dense oil spray in diesel engines or the near burner region of furnaces scatter is important [Sa86]. When scatter is neglected in fire simulation, equation (3.5.1) is then simplified as

$$
\frac{d}{d l} I(\Omega, r)=-a I(\Omega, r)+a I_{b}(r)
$$

The absorption of radiation of a gas mixture depends on the wavelength of the radiation beam. The calculation involving the entire wavelength is complicated and time consuming. The spectral dependence of absorption is simplified by introducing the concept of a grey body for which the absorption is independent of wavelength. In most of fires, both gas and soot contribute the radiative emission and absorption. The calculation of the absorption coefficient is then

$$
\alpha=\alpha_{g}+\alpha_{s}
$$


Reasonable estimates of absorption coefficients due to gas phase and soot are given by $[\mathrm{NoO1}]$

$$
\begin{aligned}
& \alpha_{g}=0.28 \exp (-T / 1135) \\
& \alpha_{s}=1264 f_{v} T
\end{aligned}
$$

where $f_{v}$ is the soot volume fraction.

The temperature dependent absorption coefficient used in SMARTFIRE is specified in equation (3.5.6)

$$
\begin{array}{ll}
\alpha=0.01, & \text { if } T<323 \mathrm{~K} \\
\alpha=0.01+c_{1}(T-323) / 323, & \text { if } 323 \leq T \leq 700 \\
\alpha=0.01+377 c_{1}+c_{2}(T-700) / 700 & \text { if } T>700
\end{array}
$$

where $T$ is the gas temperature and $c_{1}$ and $c_{2}$ are constants, which depend on the combustible materials. Values of the parameters for various fuels can be found in the manual of SMARTFIRE [GK99].

It is impossible to obtain the analytical solution of equation (3.5.2) except some extremely simple cases. Mathematical methods to the solution of equation (3.5.2) are usually the zone method, Monte Carlo method and discrete method. As discussed by Jia [Ji99], both zone method and Monte Carlo method are practical only for relatively coarse meshes. In fire field modelling, "very-fine" meshes are often used for the complicated cases. A suitable method is necessary for incorporating the radiation transfer into field fire modelling. The six-flux model [HM88], one of the discrete methods, is frequently used in fire modelling [KB97, JG97, JG99a, JG99b and WJ01a]. 'The use of the six-flux model is more preferable than the discrete transfer, as it exhibits most interesting features concerning accuracy and CPU time' [KB97].

The discrete transfer radiation method was proposed by Lockwood and Shah [LS81]. In this method, the radiation transfer equation is solved along a number of prescribed rays. This method avoids the disadvantage in discretisation only along the coordinate directions caused by the six-flux model. It also improves accuracy by increasing the 
number of rays. Another advantage of this method is that it can be applicable to arbitrary geometrical configurations. However, with the increase of the number of rays, the associated increases in computational overheads are also significant.

\subsubsection{Six-flux Radiation Model}

As discussed in the previous section, it is impossible to obtain the analytical solution of equation (3.5.2) except for some extremely simple cases. In this section, the sixflux radiation model [HM88, Ji99] is described.

\subsubsection{Traditional six-flux model}

The six radiation fluxes in the positive and negative directions of the coordinate $x, y$ and $z$ are denoted by $F_{x}^{+}, F_{x}^{-}, F_{y}^{+}, F_{y}^{-}, F_{z}^{+}$and $F_{z}^{-}$respectively. From equation (3.5.2), we have

$$
\begin{aligned}
& \frac{d F_{\alpha}^{+}}{d \alpha}=-a F_{\alpha}^{+}+a E_{b} \\
& \frac{d F_{\alpha}^{-}}{d \alpha}=a F_{\alpha}^{-}-a E_{b}
\end{aligned}
$$

where $\alpha$ represents the coordinate directions $x, y$ and $z ; E_{b}$ is the black-body emissive power

$$
E_{b}=\sigma T^{4}
$$

and $\sigma$ is the Stefan-Boltzmann constant $\left(\sigma=5.6678 \mathrm{E}-8 \mathrm{w} / \mathrm{m}^{2} / \mathrm{K}^{4}\right)$.

At the wall surface, the radiation fluxes have the following relation

$$
F_{n}^{+}=\varepsilon_{w} E_{b}+\left(1-\varepsilon_{w}\right) F_{n}^{-}
$$

where $\varepsilon_{w}$ the wall emissivity and $n$ is the outward going unit normal direction to the wall surface. At open boundaries, there is no reflection involved in the calculation; the radiation flux going into the calculation domain is then 


$$
F_{i n}=\varepsilon_{g} \sigma T^{4}
$$

where $\varepsilon_{g}$ is the emissivity of gas mixture and $T$ is the corresponding temperature.

Let $R_{x}=\left(F_{x}^{+}+F_{x}^{-}\right) / 2, R_{y}=\left(F_{y}^{+}+F_{y}^{-}\right) / 2, R_{z}=\left(F_{z}^{+}+F_{z}^{-}\right) / 2$. The following second-order ordinary differential equations can be derived from (3.5.7) and (3.5.8) [KB97]

$$
\frac{d}{d \alpha}\left(\frac{1}{a} \frac{d R_{\alpha}}{d \alpha}\right)=a R_{\alpha}-a E_{b}
$$

At the wall boundary,

$$
\frac{d}{d n} R_{n}=-\frac{a \varepsilon_{w}\left(E_{b}-R_{n}\right)}{2-\varepsilon_{w}}
$$

where $n$ is the outward going unit normal direction to the wall surface.

A source term to the enthalpy equation (3.3.6) due to radiation is expressed as

$$
S_{r a d}=2 a\left(R_{x}+R_{y}+R_{z}-3 E_{b}\right)
$$

A method to implement the six-flux radiation model was provided in [KB97]. Note that the heat flux equation (3.5.12) obeys the general form of CFD governing equation

$$
\frac{\partial \rho R_{\alpha}}{\partial t}+\nabla \cdot\left(\rho \vec{U} R_{\alpha}-\Gamma_{R \alpha} \nabla R_{\alpha}\right)=S_{R \alpha}
$$

where

$$
\Gamma_{R \alpha}=\frac{1}{a}
$$

and

$$
S_{R \alpha}=-a\left(R_{\alpha}-E_{b}\right)
$$


Therefore, the source term of energy equation due to radiation is obtained by introducing three solved variables.

\subsubsection{Jia's modified six-flux model}

The conventional six-flux model transforms the heat transfer equation from one-order problem (3.5.7)-(3.5.8) to a two-order problem (3.5.15). By testing examples with analytical solution, Jia has found that 'the conventional six-flux model suffers two kinds of error - the uncertainty due to the dependence of the boundary conditions at wall on the limit value of the absorption coefficient of the media and the unrealistic transformation from one-way parabolic problem to a two-way elliptic problem'[Ji99].

A modified form of the conventional six-flux model has been developed by Jia [Ji99]. Instead of using the radiation fluxes $R_{x}, R_{y}$ and $R_{z}$, Jia utilizes the original six fluxes $F_{x}^{+}, F_{x}^{-}, F_{y}^{+}, F_{y}^{-}, F_{z}^{+}$and $F_{z}^{-}$in his model. From equation (3.5.7) and (3.5.8), the radiation flux after going through a cell along a coordinate direction is given by the following recurrence equation [Ji99],

$$
F_{n+1}=F_{n} e^{-a \delta \alpha}+\left(1-e^{-a \delta \alpha}\right) E_{b}
$$

where $F_{n}$ and $F_{n+1}$ are respectively the radiation fluxes entering and leaving the cell and $\delta \alpha$ is the length of the cell along $\alpha$ direction. The boundary condition at the wall is given by (3.5.10).

The source term of enthalpy at a cell contributed by radiation is

$$
S_{\text {rad }}=a\left(F_{x}^{+}+F_{x}^{-}+F_{y}^{+}+F_{y}^{-}+F_{z}^{+}+F_{z}^{-}-6 E_{b}\right) V
$$

where $V$ is the volume of the cell.

The advantage of Jia's modified method is avoiding the possible error caused by process of transferring a first-order problem to a second-order problem. However, this modification increases the number of equations to be solved. 


\subsubsection{Discrete Transfer Model}

The six-flux model is intended for applications where heat loss from the enclosure fires. However, this model sometimes is insufficient for applications where the accuracy of the heat flux is crucial [Ji99, JP06a-b]. When modelling the flame spread over the burnable materials, the six-flux model sometimes fails to predict the properly ignition time due to the angles between the surface and the six fluxes. The discrete transfer model provides the increased angular resolution required for situations such as flame spread.

The discrete transfer method was proposed by Lockwood and Shah [Ls81]. The radiation is solved along a number of prescribed ray directions that discretise the $4 \pi$ solid angle. Each cell through which the ray passes is treated as isothermal, i.e., the temperature and properties are uniform in the cell. Thus, the radiation intensity along the ray is calculated by the following recurrence equation that is the solution to equation (3.5.2) within the cell [Ji99]

$$
I_{n+1}=I_{n} e^{-a \delta l}+I_{b}\left(1-e^{-a \delta l}\right)
$$

where $I_{\mathrm{n}}$ and $I_{\mathrm{n}+1}$ are respectively the radiation intensities of entering and leaving the cell along the ray and $\delta l$ is the ray segment within the cell.

Assuming a grey wall surface, the wall boundary is calculated by

$$
I=\frac{\varepsilon_{w} I_{B}}{\pi}+\left(1-\varepsilon_{w}\right) I^{-}
$$

where $I^{-}$is the incident radiation on wall.

The source term of enthalpy at a cell due to the radiation with discrete transfer model is represented by

$$
S_{\text {rad }}=\sum_{\text {rays }}\left(I_{n+1}-I_{n}\right) \vec{\Omega} \cdot \Delta \vec{A} \Delta \Omega
$$

where $\vec{\Omega}$ is a ray direction and $\Delta A$ is the normal face area of a cell volume. 


\subsection{Boundary Conditions}

\subsubsection{Inlet and outlet}

At an inlet, the velocity into the domain is specified. It is also necessary to give the values of other fluid properties such as species concentrations. At an outlet, which is typically used in fire modelling for allowing mass to vent in and out of a domain, the value of pressure is prescribed.

\subsubsection{Wall Boundary Conditions}

\subsubsection{Species equation}

Walls are used to confine the flow within an enclosure. No mass fluxes passes through a wall. Therefore, for species $\alpha$

$$
\frac{\partial Y_{\alpha}}{\partial n}=0
$$

However, if the wall can absorb a species, such as HCL, an additional deposition model to describe this is needed.

\subsubsection{Momentum equation}

At a control volume containing node $P$ with a wall surface, a source term due to the shear force, $F \mathrm{~s}$, is added to the momentum equation. The calculation of the shear force depends on that this node lie in the laminar layer or in the turbulent layer. The following relation is used to determine where this node lies.

$$
y^{+}=\frac{\sqrt{\rho \tau_{w}}}{\mu} \Delta y_{p}
$$

Where

$$
\tau_{w}=\mu \frac{u_{p}}{\Delta y_{p}}
$$


and $\Delta y_{p}$ is the distance to the wall from the node $P$.

If $y^{+}(3.6 .2)$ is less than 11.63 then the near wall flow is laminar [Gr03]. In this case, the shear force is calculate by

$$
F_{s}=-\mu \frac{u_{p}}{\Delta y_{p}} A_{\text {wall }}
$$

where $A_{\text {wall }}$ is the surface area. Otherwise, the near flow is turbulent and the shear force is

$$
F_{s}=-\frac{\rho C_{\mu}^{3 / 4} k_{p}^{1 / 2} u_{p} A_{\text {wall }}}{u^{+}}
$$

where

$$
u^{+}=\frac{1}{\kappa} \ln \left(E y^{+}\right)
$$

here $\kappa$ and $E$ are model constants.

\subsubsection{Enthalpy equation}

For a wall surface with temperature, $T_{\mathrm{w}}$, the convective heat flux into the wall is

$$
q_{s}=h_{c}\left(T_{p}-T_{w}\right)
$$

where $h_{\mathrm{c}}$ is the heat transfer coefficient [Gr03] and $T_{p}$ is the temperature at cell $P$. More efficient calculations of $T_{\mathrm{w}}$ has been described by Jia [Ji99].

\subsubsection{Turbulent equations}

No special modifications are necessary for the $k$ equation. The dissipation rate in the near wall cell is calculate from the value of $k$ by

$$
\varepsilon=\frac{C_{\mu}^{3 / 4} k^{3 / 2}}{\kappa \Delta y_{p}}
$$




\subsection{Numerical Solution Procedure}

The governing equations for turbulent reacting flows described in Section 3.3 consist of a set of three dimensional, time dependent, non-linear partial differential equations. In general, their exact solutions cannot be obtained by analytical methods. The method to solve these equations is briefly introduced in this section.

\subsubsection{Discrete form of Conservation Equations}

The basic principle of CFD is to transfer the continuous differential governing equations to simple discrete algebraic equations that can be solved with relative ease. First, the calculation domain is divided into a number of control volumes (or cells) such that there is one control volume surrounding each grid point. The general differential equation (3.2.1) is integrated about time and space over each control volume as

$$
\begin{gathered}
\int_{C V}\left(\int^{+\Delta t} \frac{\partial(\rho \varphi)}{\partial t} d t\right) d V+\int^{+\Delta t}\left(\int_{A} \vec{n} \cdot(\rho \varphi \vec{u}) d A\right) d t \\
=\int^{+\Delta t}\left(\int_{A} \vec{n} \cdot(\Gamma \nabla \varphi) d A\right) d t+\int^{+\Delta t} \int_{C V} S_{\varphi} d V d t
\end{gathered}
$$

The result is the discrete equations that contain the value of $\varphi$ for a group of grid points. Details of the discrete method to each conservation equation can be found in [Gr03, Pa80]. The general algebraic equations at a control volume is mathematically expressed as

$$
\left\{\frac{(\rho \Delta V)_{P}}{\Delta t}+\sum_{\text {All faces }} a_{A}\right\} \varphi_{\mathrm{P}}=\sum_{\text {All adjacentnodes }} a_{A} \varphi_{A}+\frac{\left(\rho \varphi_{P}\right)_{P}^{\text {old }} \Delta V}{\Delta t}+S_{\varphi, P} \Delta V
$$

In equation (3.7.2), $\varphi$ is the appropriate variable. $a_{A}$ is commonly known as the coefficients, which contains contributions from the convection and diffusion terms. The source term $S_{\varphi, P}$ is linearized [Pa80, Sp81] as seen in equation (3.7.3)

$$
S_{\phi, P}=S_{C}+S_{P} \phi_{P}
$$




\subsubsection{Solution Method}

The discrete algebraic equations (3.7.2-3) can be written as

$$
a_{\mathrm{P}} \phi_{\mathrm{P}}=\sum_{\text {All adjacentnodes }} a_{A} \phi_{A}+b
$$

where

$$
\begin{aligned}
& a_{P}=\left\{\frac{(\rho \Delta V)_{P}}{\Delta t}+\sum_{\text {All faces }} a_{A}-S_{P} \Delta V\right\} \\
& b=\frac{\left(\rho \phi_{P}\right)_{P}^{\text {old }} \Delta V}{\Delta t}+S_{C} \Delta V
\end{aligned}
$$

Equations (3.7.4 - 3.7.6) are non-linear and coupled. Therefore, a sequential procedure is adopted for calculating fluid flow. A set of nominally linear algebraic equations for the particular variable for every control volume of the fluid domain is obtained by updating the new values of the other variables into the corresponding discrete equation (3.7.4).

A problem is that there is not a special equation for the pressure variable. The pressure gradient forms a part of the source term for the momentum equations. It causes the difficulty in the calculation of the velocity field. However, the pressure field generated from the momentum equation by using appropriate pressure field values must satisfy the continuity equation. Thus, a mechanism of iterative pressure correction and velocity correction is established aimed at reducing the mass residual source term of the continuity equation to zero. A well-known method implementing this mechanism is called SIMPLE (Semi-Implicit Method for Pressure-Linked Equations) algorithm. The SIMPLE algorithm is simply described here while a detailed description can be found in [PS72]:

Step 1: Guess the pressure field $\left(\mathrm{p}^{*}\right)$.

Step 2: Solve the momentum equations to obtain $\mathrm{u}^{*}, \mathrm{v}^{*}$, and $\mathrm{w}^{*}$. 
Step 3: Solve the pressure-correction equation ( $\left.p^{\prime}\right)$ which is derived from the continuity equation.

Step 4: Apply pressure corrections to velocities and pressure. e.g. $\left(u=u^{*}+u^{\prime}\right)$

Step 5: solve equations for other variables, e.g. enthalpy.

Step 6: Repeat Steps (2) to (5) until convergence.

\subsection{Simulating One of the CIB W14 Round Robin Test Cases}

In this section, the main simulation findings to one of the CIB W14 Round Robin tests [HK98] using SMARTFIRE [GK99] by the author [WJ00, WJ01a] are used to demonstrate the salient features of the field modelling approach to enclosure fires. In the following, the simulation results compared with the experimental data are briefly represented. Details can be found in Appendix A or the publications [WJ00, WJ01a].

The test scenario is with a compartment measured $14.4 \mathrm{~m} \times 7.2 \mathrm{~m}$ in plan and $3.53 \mathrm{~m}$ in height and contained a door of dimensions $2.97 \mathrm{~m} \times 2.13 \mathrm{~m}$ (See Figure 3.8.1). It was intended to represent an industrial building in which the fire source was concentrated in a single location. The wood fire was located on the floor in the centre of the room. Gas temperatures were measured using three vertical rakes of thermocouples identified as location $\mathrm{A}, \mathrm{B}$ and $\mathrm{C}$ in Figure 3.8.1, which were in the rear, the centre and the front corner of the room respectively.

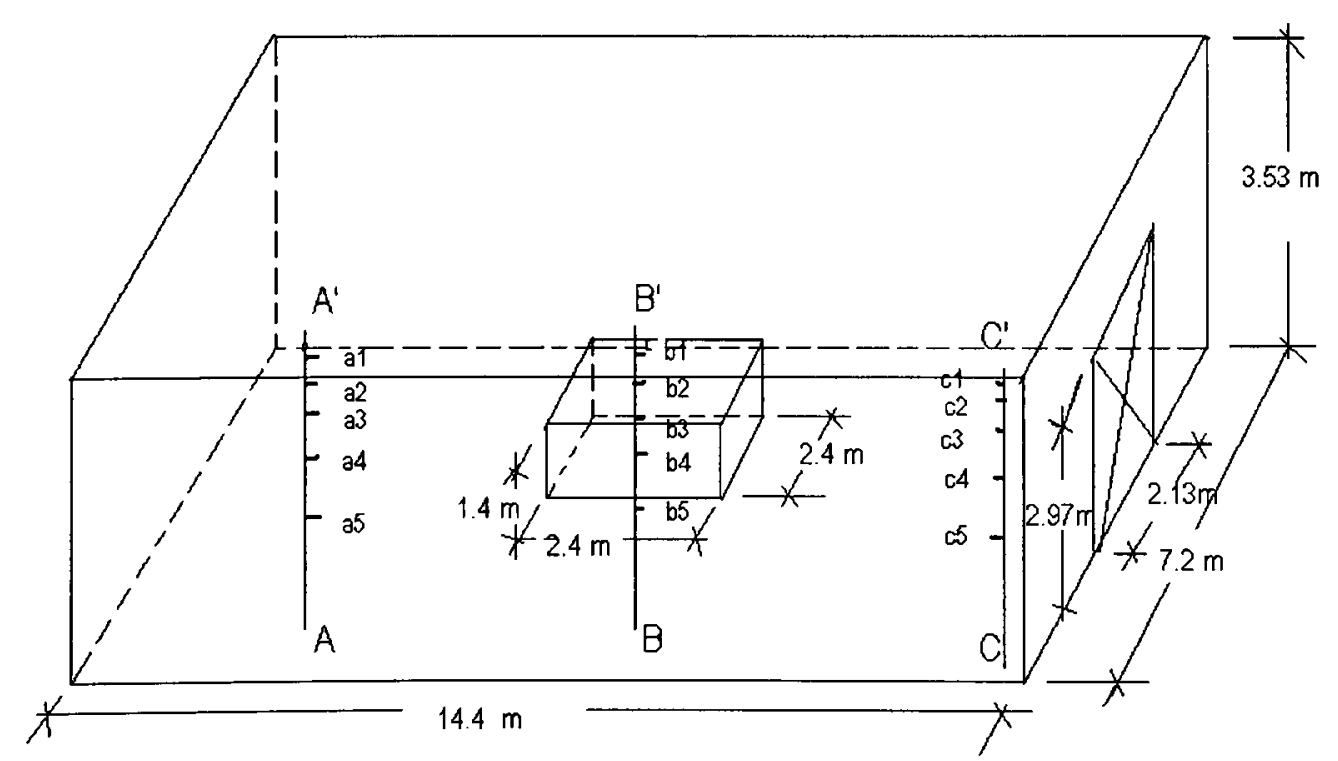

Figure 3.8.1: Depiction of fire compartment geometry showing location of fire source and the location of the three thermocouple trees.

The simulation was performed with the following features

- the volumetric heat approach - the heat release rate model; 
- the $k-\varepsilon$ turbulence model;

- six-flux radiation model;

- meshes of 41,325 (i.e. $57 \times 25 \times 29$ );

- Time step size of 5 seconds;

- using a $400 \mathrm{MHz}$ Pentium III PC with $256 \mathrm{MB}$ of memory

The simulation was run for an equivalent of 60 minutes of real time and required approximately 174 hours of computational time. The simulation was able to reproduce the qualitative features of the experimental data. As an example, the measured and predicted temperatures at the top and bottom thermal couples along the thermal couple tree $B B^{\prime}$ are depicted in Figure 3.8.2. As can be seen from this figure, the measured temperatures reach a maximum value of some $1214^{\circ} \mathrm{C}$ after approximately 40 minutes and then show a gradual decline for the next 20 minutes. The predicted temperatures appear to follow this trend throughout the first 60 minutes. In particular, there is also a great level of stratification along this vertical line predicted as been observed from the experimental data. The peak in the numerical predictions is $1360^{\circ} \mathrm{C}$ (an error of $12 \%$ ) and occurs after 30 minutes. The average predicted temperature after 30 minutes along the vertical line is approximately $1310^{\circ} \mathrm{C}$ while the measured average temperature is $1116^{\circ} \mathrm{C}$. Between 25 minutes and 50 minutes, the predicted average temperature along the vertical line differ from the measured values by between 6 and $17 \%$.

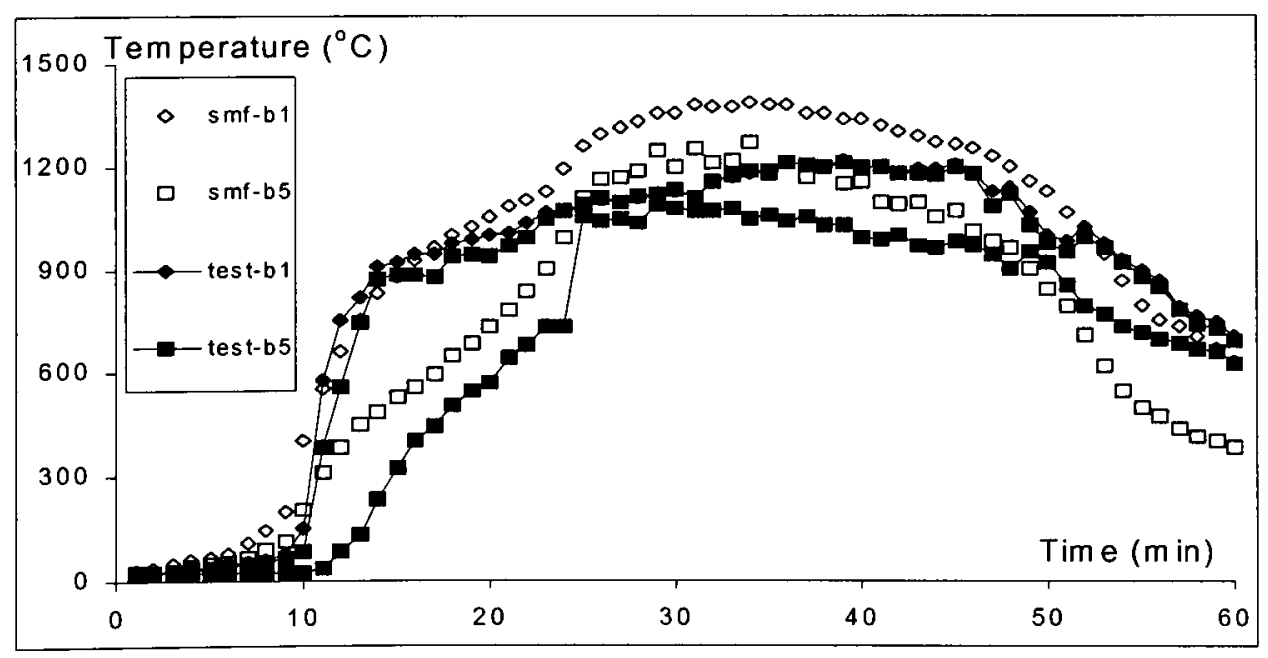

Figure 3.8.2: Predicted (symbols) and measured (solid lines) temperatures at the top and bottom thermal couples along the thermal couple tree $B B^{\prime}$.

The volumetric heat source approach used in this simulation is shown to be capable of generating a reasonable representation of the gas temperature distribution in a moderately large compartment subjected to a large non-spreading fire. 


\subsection{Concluding Remarks}

The framework of field fire modelling including the governing equations, turbulent models, combustion models, radiation models, boundary conditions and the numerical solution methods is discussed in this chapter. By introducing the $k-\varepsilon$ approach to model turbulence, the field fire modelling is a promising approach to simulate enclosure fires due to its repeatability and low cost compared with conducting real fire experiments.

Simple combustion models, such as volumetric heat approach (heat source model) and the practical eddy break-up model could produce reasonable temperature predictions away from the fire source. The simulation to one of Round Robin W14 fires using the simple volumetric heat source model further demonstrates this point. However, these models do not account for the production and movement of smoke and toxic gases. As mentioned in the introduction in Chapter 1, toxic gases are generated in almost all fires and are responsible for most of fire casualties.

An alternative approach, which not only does not completely lose the chemistry of the combustion of interest like the over-simplified one-step global reaction but also consider the useable data for the toxicity in enclosure fires, is required. In the remainder of this thesis, combining with the practical volumetric heat source approach or EBU combustion model, the concept of local equivalence ratio (LER) is applied in fire field modelling to predict hazard products in enclosure fires. The absorption of HCL by solid walls in fire is also studied. 


\section{Chapter 4}

\section{TOXICITY MODELS USING LOCAL EQUIVALENCE RATIO}

\subsection{Introduction}

The understanding of $\mathrm{CO}$ formation and the correlation between $\mathrm{CO}$ generation and equivalence ratio comes mostly from small-scale fire tests, such as hood experiments. The Global Equivalence Ratio (GER) in this type of experiments usually refers to the plume GER because the cold air enters the hot upper layer only through the fire plume. The correlation between CO generation and the GER for various fuels from smallscale tests have been reviewed in Section 2.2. It was expected that hood experiments produce similar burning environments to those in the burning region of real enclosure fires. If this was the case, then the correlation could be used for the predictions of $\mathrm{CO}$ concentration in the hot upper layer of enclosures in zone modelling as described previously, in Chapter 2.

From reduced and full-scale room fire tests [Go92, BJ94, PB95], it was found that the correlation between the $\mathrm{CO}$ concentration and the GER depends on the scale of enclosures. The measured $\mathrm{CO}$ concentrations in the upper layer are quite different for reduced and full-scale fire scenarios in which the GERs are similar. Therefore, the application of the correlation from small-scale experiments is limited by the complexity of fire enclosures.

An example from Pitts et al's tests [PB95] is introduced here to demonstrate that the GER concept cannot be directly used in simulating enclosure fires in zoon modelling. This test is also used to analyse the feasibility of applying CO correlation based on small-scale tests in field modelling and is presented in Chapter 4.2. The fire room is $2.44 \mathrm{~m} \times 2.44 \mathrm{~m} \times 3.67 \mathrm{~m}$ with a $0.76 \mathrm{~m} \times 2.03 \mathrm{~m}$ door. A $35-\mathrm{cm}$-diameter burner located centrally in the enclosure with the burner lip at $38 \mathrm{~cm}$ above the floor. The heat release rate of the methane fire considered was $1250 \mathrm{Kw}$. The fuel rate was $0.0232 \mathrm{~kg} / \mathrm{s}$ and the rate of air entering into the room was $0.395 \mathrm{~kg} / \mathrm{s}$. The GER of this test is close to 1.0. It was later reported [Pi01] that a coding error was found in calculating the airflow rate, which resulted in 'an overestimate of the air mass flow by a factor of 
$\sim 2.8$ ' (equivalent to reducing the previously calculated GERs by a factor of $\sim 2.8$ ). Therefore, the real airflow rate should have been $1.106 \mathrm{~kg} / \mathrm{s}$ and the resulting GER should be 0.365 . From the hood tests, the upper layer $\mathrm{CO}$ concentration is negligible for a GER of less than 0.4 [Pi95]. The upper layer experimental measurements were found to be $\sim 1 \%$ (rear of the room) and $\sim 0.2 \%$ (front of the room).

This case clearly demonstrates that although the GERs are similar for the hood experiments and the full-scale fire test, there are significant differences in the $\mathrm{CO}$ concentrations between the two fire scenarios. As reviewed in Chapter 2, experimental correlations between the yields of combustion products and the GERs are available for various materials. Suitable parameters are required in the field modelling approach to calculate the concentrations of toxic gases using these correlations. The Local Equivalence Ratio (LER) concept is a practical option to get a better local representation of toxic species.

In the following sections, mathematical models with the LER are developed to predict generation and spread of combustion products within fire enclosures. An assumption for the concept of mixture fraction and the relationship between the mixture fraction and the local equivalence ratio (LER) are given first. Secondly, the feasibility of applying the concept of LER in field fire modelling is discussed. Thirdly, mathematical models using the concept of LER are developed. One model uses the correlation between the species yields and the equivalence ratio and two models use the $\mathrm{CO} / \mathrm{CO}_{2}$ mole ratio. The correlations about yields of products from small-scale experiments are used as model input parameters. Comparison between the predictions of these models is presented. Finally, simple methods for calculating the smoke optical density based on the LER are developed. The specific light extinction coefficient or the mass optical density is used as input parameters in these present smoke properties approaches.

\subsection{Applying LER in Field Fire Modelling}

Most enclosure fires are in nature non-premixed combustion. In non-premixed combustion, a fuel element mass fraction can be defined to represent the mass fraction of all elements originating from the fuel source and an oxidant element mass fraction 
to represent the mass fraction of oxidant originating from the oxidant source. The mixture fraction, a conserved scalar, is defined from the two elements mass fractions to express the mixture of fuel, oxidant and combustion products.

In this study, the conservation equation for mixture fraction is used to calculate the combustion products and the mixture fraction is use to obtain the LER. In the following sections, the concept of mixture fraction is presented together with the assumption used. This is followed by the derivation of the correlation between the mixture fraction and the LER. The fire scenario in [PB95], described in Section 4.1, was used to demonstrate LER concept.

\subsubsection{Mixture fraction and assumption}

In analogy to the mass fraction of a species, the mass fraction of an element can be defined as the mass of the element divided by the total mass in a control volume. The mass fraction of element $i, Z_{i}$ can be expressed in terms of the mass fractions of all species

$$
Z_{i}=\sum_{j=1}^{s} \mu_{i j} Y_{j}
$$

where $s$ denotes the number of species involved in combustion and $\mu_{i j}$ denotes the mass proportion of element $i$ in the species $j$ [WM99] and $Y$ is the mass-fraction of the species $j$.

For non-premixed fires, there are usually two streams: the fuel and the oxygen (or air). A mixture fraction based on the element $i$ can be defined as

$$
\xi=\frac{Z_{i}-Z_{i 2}}{Z_{i 1}-Z_{i 2}}
$$

where $Z_{i 1}$ and $Z_{i 2}$ are the mass fraction of element $i$ in the streams of fuel and air respectively. As mixing proceeds, $\xi$ takes values between 0 and 1 . The governing equation for element mass fraction $Z_{i}$ is expressed as follows: 


$$
\frac{\partial\left(\bar{\rho} \tilde{Z}_{i}\right)}{\partial t}+\frac{\partial}{\partial x_{i}}\left(\bar{\rho} \tilde{u}_{i} \tilde{Z}_{i}\right)=\frac{\partial}{\partial \mathrm{x}_{\mathrm{i}}}\left\{\left(\Gamma_{i}+\Gamma_{t}\right) \frac{\partial \tilde{Z}_{i}}{\partial x_{i}}\right\}
$$

where $\Gamma_{i}$ and $\Gamma_{t}$ are the diffusion and turbulent exchange coefficient for species $i$, respectively. For the present study, the diffusion coefficients and the turbulent exchange coefficient are assumed to be the same for all species, thus replacing, $\left(\Gamma_{i}+\Gamma_{t}\right)$ by $\Gamma$. The implications of this assumption are discussed below.

The assumption of equal diffusion coefficients creates a single variable, $\xi$ containing the information about all of the elements through (4.2.2). This approach is widely used to analyze laminar and turbulent diffusion flames [Pe86]. This approximation for the diffusivity is reasonable for some fire scenarios [Bi76, SF90]. Pope et al [PS99] studied the variation of the equivalence ratio and the element ratio of premixed laminar flames. It was reported that the atomic $\mathrm{C} / \mathrm{O}$ ratio varies by up to $25 \%$ from the inlet value and atomic $\mathrm{C} / \mathrm{H}$ ratio varies by up to $10 \%$. It shows that sometimes this assumption may appear too restrictive for laminar fire. For high Reynolds numbers it is believed that differential diffusion effect is confined to the high-wave-number end of the spectrum, with consequently small contributions to instantaneous values and to means and other lower moments' [Bi89]. Under the assumption that all the species diffuse equally fast (which is justified in most turbulent flows of practical interest at moderate to high Reynolds numbers [Bi80]), the source term for the mixture fraction disappears from the governing equation [No01]. Enclosure fires are usually in the regime of high turbulence, thus the assumption of equal diffusivity for all species should be satisfied.

Thus, all mixture fractions defined by (4.2.2) are equal. From equation (4.2.1) and (4.2.2), the mixture fraction $\xi$ dependents linearly on species mass fractions. Using (4.2.2) and (4.2.3), the conservation equation for the mixture fraction $\xi$ can be derived

$$
\frac{\partial(\bar{\rho} \tilde{\xi})}{\partial t}+\frac{\partial}{\partial x_{i}}\left(\bar{\rho} \tilde{u}_{i} \tilde{\xi}\right)=\frac{\partial}{\partial \mathrm{x}_{\mathrm{i}}}\left\{(\Gamma) \frac{\partial \tilde{\xi}}{\partial x_{i}}\right\}
$$




\subsubsection{Mixture fraction and LER}

The LER, $\phi$, in a control volume is defined as the ratio of the mass, which originates from the fuel source, to the mass, which originates from the air stream, divided by the stoichiometric fuel to air ratio [Gu95]. The LER is obtained from the mixture fraction.

Following analysis taken in [Pe84 and Tu95], we take element $i$ is the carbon element and ignoring the mass fraction of carbon in the air is, the mixture fraction (4.2.2) becomes

$$
\xi=\frac{Z_{c}}{Z_{c 1}}
$$

The mass of carbon in a control volume is then expressed by

$$
m_{c}=\rho \times v \times Z_{c}
$$

where $\rho$ and $v$ are the density and volume of the control volume. Under the assumption of equal diffusivity for all elements, the ratios among elements (which are from fuel source) do not change in the processes of chemical reaction and transport. The carbon mass (4.2.6) corresponds to the following amount of fuel mass

$$
m_{\text {fuel }}=m_{c} / Z_{c 1}=\rho \times v \times Z_{c} / Z_{c 1}
$$

The LER, using equations (4.2.5) and (4.2.7) is given by

$$
\phi=\frac{m_{\text {fuel }} / m_{\text {air }}}{r_{s t}}=\frac{\left(\rho v Z_{c} / Z_{c 1}\right) /\left(\rho v-\rho v Z_{c} / Z_{c 1}\right)}{r_{s t}}=\frac{\xi /(1-\xi)}{r_{s t}}
$$

where $r_{s t}$ is the stoichiometric fuel-air mass ratio. The LER, $\phi$, is then expressed in terms of the mixture fraction, $\xi$, and the stoichiometric mixture fraction values, $\xi_{s t}$,

$$
\phi=\frac{\xi /(1-\xi)}{\xi_{s t} /\left(1-\xi_{s t}\right)}=\frac{\xi\left(1-\xi_{s t}\right)}{\xi_{s t}(1-\xi)}
$$




\subsubsection{Feasibility of applying LER}

Recalling the fire experiment described in Section 4.1, a question is raised as to why high levels $\mathrm{CO}$ concentration were found in the upper layer of full-scale enclosure experiments for low values of GER $(<0.5)$ ? The field model results obtained with SMARTFIRE using the LER approach can be used to explain the reasons why this discrepancy can exist.

\subsubsection{Simulation results of a methane fire}

The methane fire in [PB95] is simulated to demonstrate the feasibility of using LER to predict the species concentrations. In the simulation, the EBU combustion model, six-flux radiation model are applied. Due to the symmetry of this fire scenario, only half of the geometry is simulated with a mesh number of 34336. A simulation with more fine meshes has produced similar results. The predicted temperature contours in a vertical plane passing the thermocouple trees inside the test room are depicted in Figure 4.2.1. The measured temperature in the front of the room is about $950^{\circ} \mathrm{C}$ $(1220 \mathrm{~K})$, which is under predicted by $100^{\circ} \mathrm{C}$. The measured temperature in the rear of the room is about $850^{\circ} \mathrm{C}(1120 \mathrm{k})$, which is under predicted by $150^{\circ} \mathrm{C}$. The error of predictions is less than $17.6 \%$.

The predicted airflow rate entering the room and the GER are $0.91 \mathrm{~kg} / \mathrm{s}$ and 0.43 , which are close to experimental results, $1.106 \mathrm{Kg} / \mathrm{s}$ and 0.365 respectively. The simulation produced reasonable temperatures and GER compared with the experimental results.

The predicted LER in the central plane and its parallel plane $0.6 \mathrm{~m}$ away are depicted in Figure 4.2.2(a)-(b). At the first glance, the fire plume slightly leans to the doorway (see Figure 4.2.2(a)). This phenomenon is caused by the high burner lip (38 $\mathrm{cm}$ above the floor) in the fire test. As seen in Figure 4.2.2, the LERs in the upper layer of the fire room are not uniform. The rear of the room has a deep layer with the LER over 1.0. However, only a very thin layer in the front of the room has high LER. According to the small-scale methane experiments, the $\mathrm{CO}$ concentration is about $1 \%$ if the GER 
is 1 [Pi95]. From the distribution of LER, the CO concentrations in the rear of the room would reach or over $1 \%$ and nearly $0 \% \mathrm{CO}$ in the front of the room would be expected. The estimated CO concentrations based on LER are close to the measured values in the front and rear of the room.

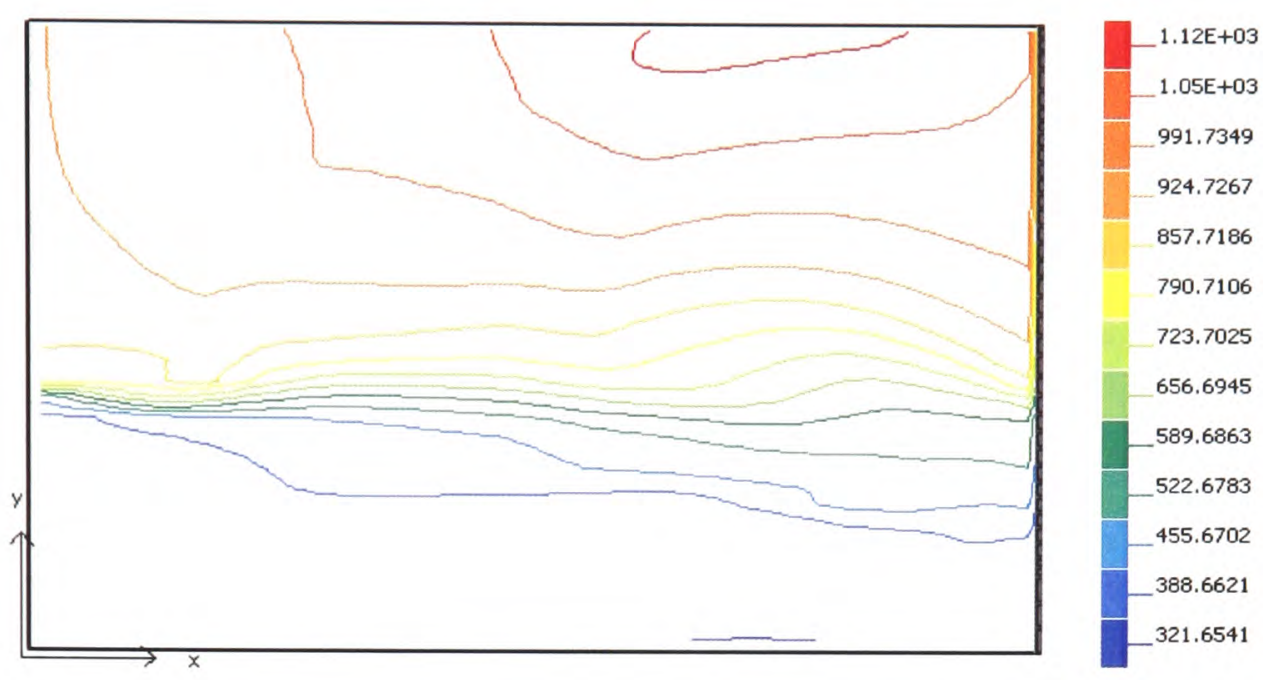

Figure 4.2.1: Predicted temperature contours at the vertical plane passing through the thermocouple trees.

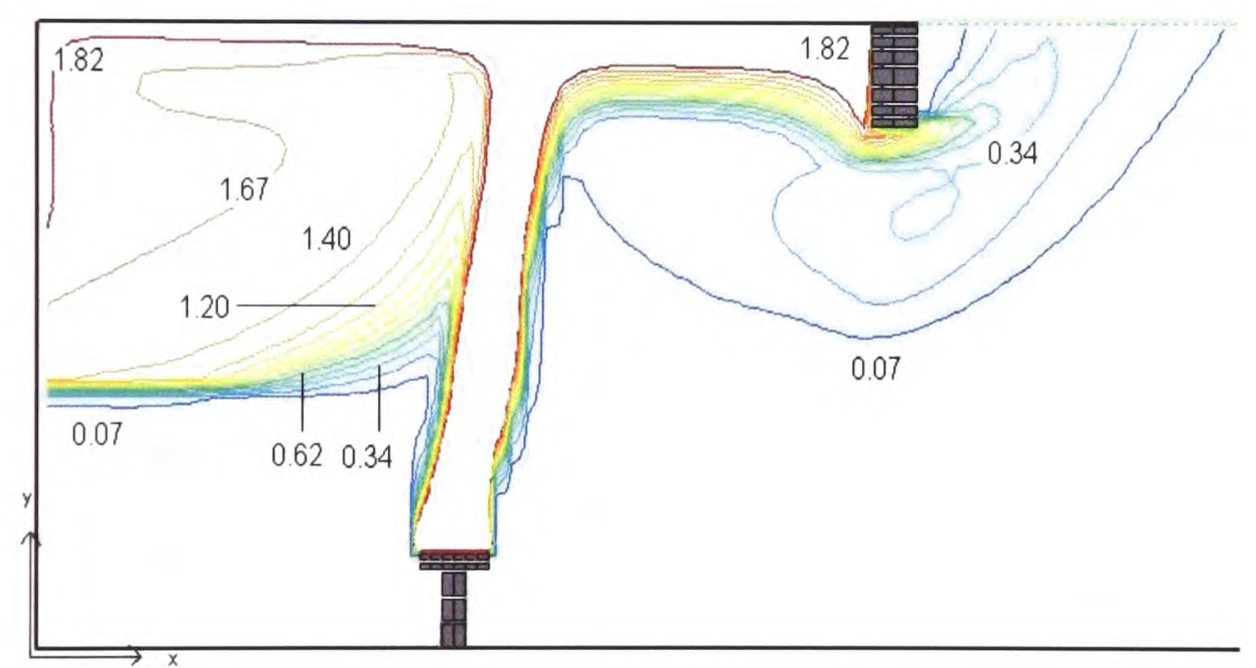

(a)

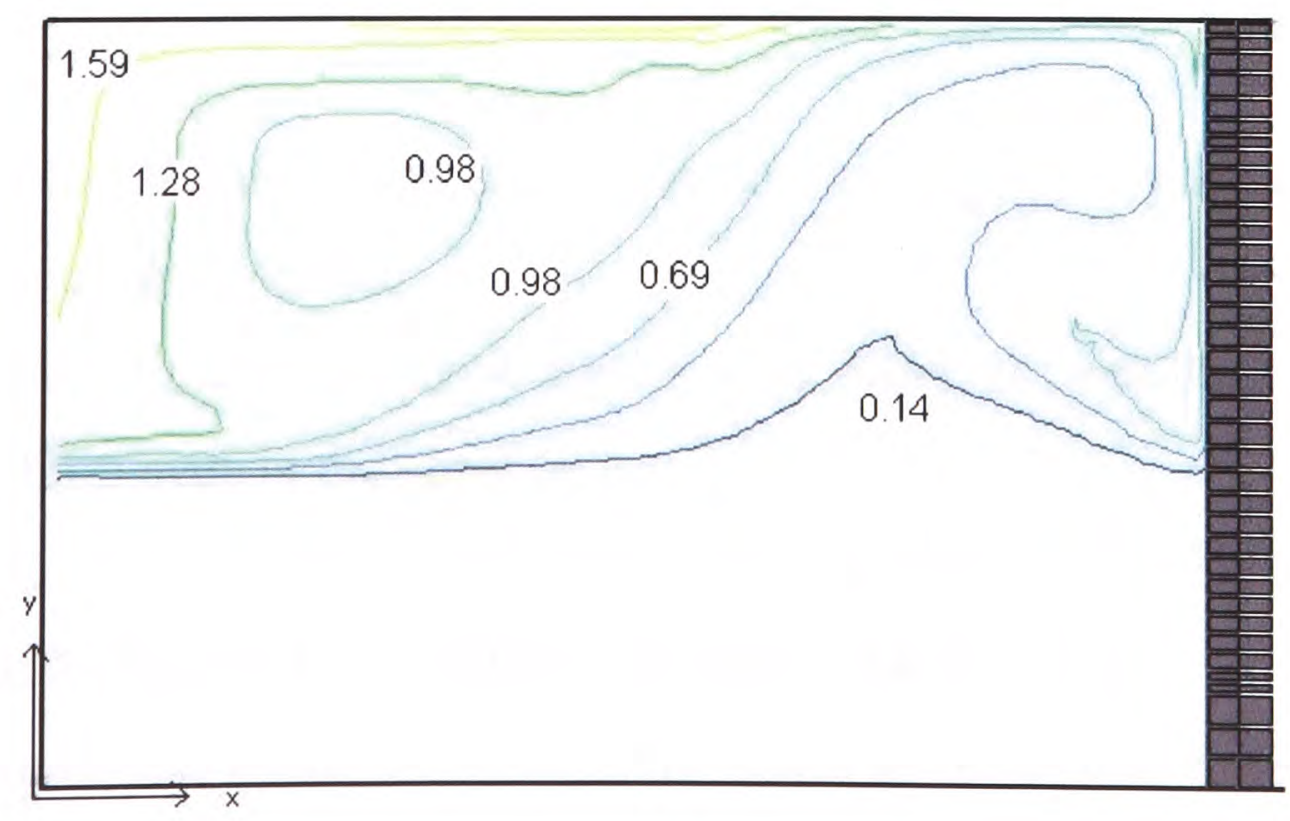

(b)

Figure 4.2.2: Predicted LERs on (a) the central plane and (b) its parallel plane. 
The distribution of predicted velocities in the central plane pass through the doorway is shown in Figure 4.2.3. As seen in this figure, the predicted neutral plane in the doorway is $1.0 \mathrm{~m}$ above the floor. It is clearly seen that some of air entering the doorway exits without well mixing with the upper layer gases. Therefore, the upper layer GER cannot be simply calculated using the air rate entering the doorway. The fraction of air going out of the room without mixing with the fuel rich gases is essential to get accurate estimate of the upper layer equivalence ratio but is not easy to be estimated in zone modelling. In field modelling, we do not need to calculate this fraction (while it is easy to do) because field modelling provides the LERs in the whole computation domain.

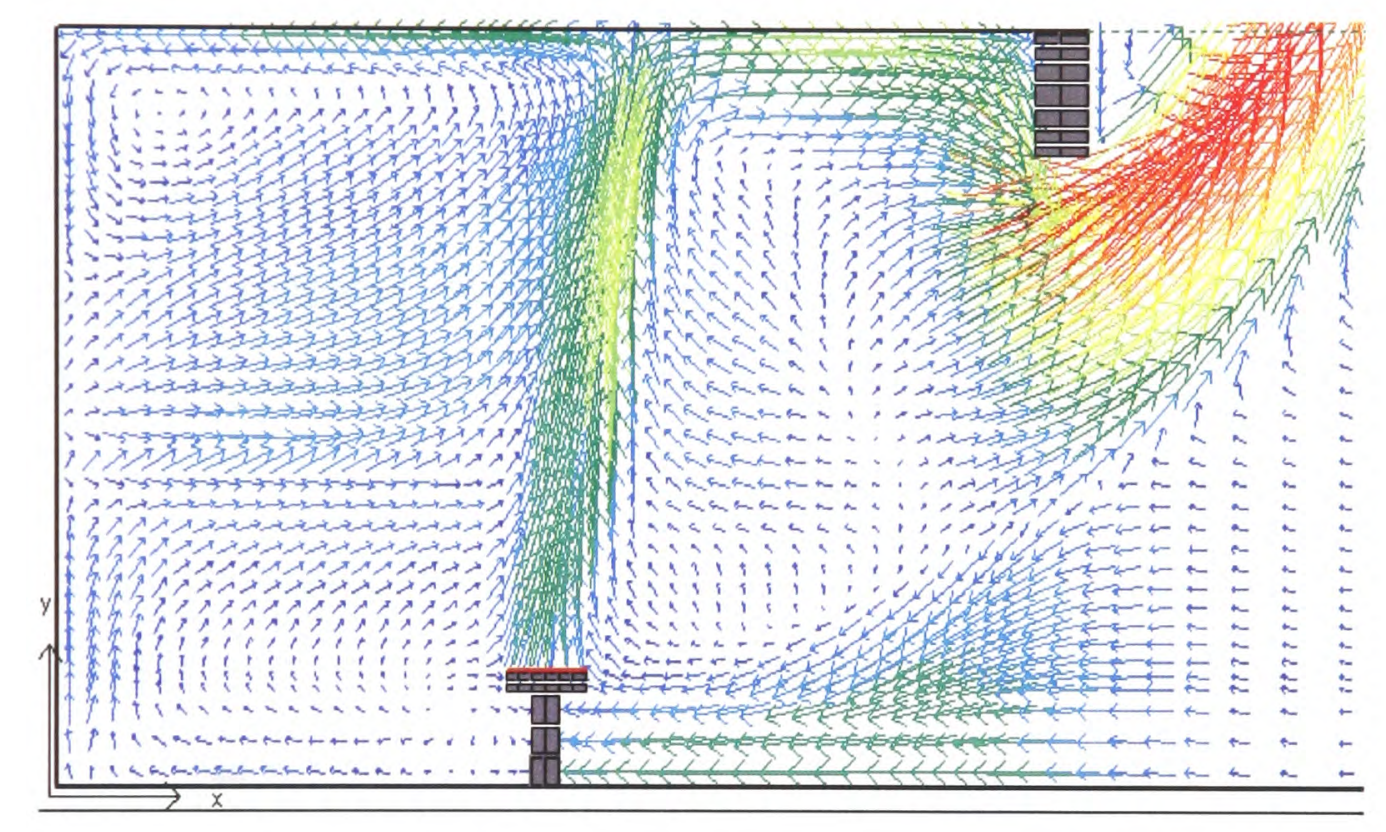

Figure 4.2.3: Flow field in the central plane through the doorway.

\subsubsection{What the results reveal}

From the results of the above simulation to the full-scale methane fire, some important findings are obtained

- The GER of the fire room is significantly different from the LERs, which is not uniform even in the upper layer.

- The CO concentrations calculated with the GER are much lower than the measured $\mathrm{CO}$ levels especially in the rear of the fire room.

- It is possible to make use of the LER values to obtain better predictions of the $\mathrm{CO}$ concentrations at different locations. 
These findings are encouraging to the use of the concept of LER in field fire modelling for predicting toxic gases.

\subsection{Toxicity Models Using LER}

Three models have been developed in this study using the concept of LER. The first model uses the species yields while others use the ratio of $\mathrm{CO}$ to $\mathrm{CO}_{2}$ from smallscale experiments.

\subsubsection{Model Assumption}

The feasibility of use of the equivalence data collected from small scale fire tests to predict $\mathrm{CO}$ generation within enclosure fires has been experimentally examined by a number of researchers recently. Wieczorek et al [WV04a, WV04b] found that a well mixed uniform upper layer, for which a single-point local measurement represent the upper layer composition, could be provided by specially designed test apparatus; in compartments with prototypical building features, a well-mixed upper layer is not necessary achieved and spatially variations in the species levels are expected within the layer. They indicated, by reviewing some published results, that measurement locations are critical in obtaining representative data. It has been found in their experimental study that the concentrations of combustion products at the exit of the fire enclosure can well be correlated to a non-dimensional heat release rate, which provides an indication of the burning conditions at the compartment exit.

Fardell et al [FP04] have compared the yields of combustion products from the Purser Tube Furnace experiments and full scale fire tests. In their tests, the equivalence ratio and temperature within the Purser Tube Furnace were similar to the values at the sampling location of the full scale tests. They conclude that the local CO concentration within full-scale enclosure can be simulated with equivalence ratio data collected from bench scale experiments.

A concept of control volume equivalence ratio (CVER) has been proposed by Lattimer et al in the study to compartment-hallway fires [LV05]. A control volume is 
defined as the fire compartment and a space extended into the hallway up to the location where the species need to be determined. For remote locations, the control volume needs to extend as far as to the flame tips in the adjacent hallway. The CVER is calculated using the fuel loss rate and the rate of air entering the defined control volume. It was concluded that species concentration are determined by the CVER which is location dependent [LV05].

The common point in these experimental studies is that local species concentrations within a fire enclosure can be correlated with the local burning conditions (local equivalence ratio or non-dimensional heat release rate or CVER and temperatures). Therefore, based on the experimental findings, one assumption used for the toxicity model development is presented below.

\section{Assumption 4.3.1}

In this study, it is assumed that each control volume in the combustion region is similar to a miniature combustion apparatus. The combustion conditions within a control volume and the associated LER and temperature is assumed approximately equivalent to that of a small-scale fire experiment with the same value of GER and upper layer temperature.

With this assumption, the correlation between yields of combustion products and GER obtained from experiments with small-scale combustion apparatus is then used in this study to model the production of combustion products by replacing the GER in the correlation by the LER at a control volume.

\subsubsection{Model based on species yields-Model I}

In this model, it is assumed that only the fuel, $\mathrm{CO}_{2}, \mathrm{CO}$, hydrocarbon and soot contain the carbon element in the gas mixture. Therefore, the mixture fraction (4.2.5) based on the conservation of carbon can be expressed by

$$
\xi=\frac{\left(\mu_{f, c} Y_{f}+\frac{12}{44} Y_{C O 2}+\frac{12}{28} Y_{C O}+Y_{S O O T}+\mu_{H C, c} Y_{H C}\right)-0}{\mu_{f, c}-0}
$$


where $Y$ represents the mass fraction and $\mu_{i, j}$ represents the mass fraction of element $j$ in species $i . \mu_{f, c}$ here is $Z_{c 1}$ in equation (4.2.5), which refers to the fraction of carbon in the fuel.

An equivalent of the equation (4.3.1) is

$$
\rho V\left(\xi-Y_{f}\right) \mu_{f, c}=\rho V\left(\frac{12}{44} Y_{C O 2}+\frac{12}{28} Y_{C O}+Y_{S O O T}+\mu_{H C, C} Y_{H C}\right)
$$

where $\rho$ is the density and $V$ is the volume of a control volume. Equation (4.3.2) implies that the mass of carbon element in each control volume is conserved in the process of the chemical reactions and species transport.

In the correlation expression between the yields of species and the equivalence ratio derived from small-scale experimental data, $y_{f}(\phi, T), y_{\mathrm{CO} 2}(\phi, T), y_{C O}(\phi, T)$, $y_{\text {soot }}(\phi, T), y_{h c}(\phi, T)$ represent the yields of fuel, $\mathrm{CO}_{2}, \mathrm{CO}$, soot and hydrocarbon per kilogram fuel with the equivalence ratio $\phi$ and the temperature $T$. Then the conservation of the carbon element is expressed as a function of the yields of species by

$$
\begin{aligned}
\mu_{f, c} & =\mu_{f, c} y_{f}(\phi, T)+\frac{12}{44} y_{C O 2}(\phi, T)+\frac{12}{28} y_{C O}(\phi, T) \\
& +y_{S O O T}(\phi, T)+\mu_{H C, c} y_{H C}(\phi, T)
\end{aligned}
$$

If the mass from the fuel source is $\rho V \xi \mathrm{kg}$, the conservation equation of carbon element is obtained from equation (4.3.3) by multiplying $\rho V \xi$

$$
\begin{aligned}
\rho V \xi\left(1-y_{f}(\phi, T)\right) \mu_{f, c} & =\rho V \xi\left(\frac{12}{44} y_{C O 2}(\phi, T)+\frac{12}{28} y_{C O}(\phi, T)\right. \\
& \left.+y_{S O O T}(\phi, T)+\mu_{H C, c} y_{H C}(\phi, T)\right)
\end{aligned}
$$

By comparing equation (4.3.2) and equation (4.3.4), mass fractions of combustion products can be expressed with the mixture fraction and the LER $\phi$ by the following equations 


$$
\begin{aligned}
& Y_{C O 2}=y_{C O 2}(\phi, T) \xi \\
& Y_{C O}=y_{C O}(\phi, T) \xi \\
& Y_{S O O T}=y_{S O O T}(\phi, T) \xi \\
& Y_{H C}=y_{H C}(\phi, T) \xi
\end{aligned}
$$

Similarly, the mixture fraction (4.3.1) can also be expressed with the oxygen element as

$$
\xi=\frac{\left(\frac{32}{44} Y_{C O 2}+\frac{16}{28} Y_{C O}+\frac{16}{18} Y_{H 2 O}+\mu_{f, o} Y_{f}+Y_{O 2}\right)-\mu_{a i r, o}}{\mu_{f, o}-\mu_{a i r, o}}
$$

Corresponding to equation (4.3.3) and (4.3.4), the following equations are obtained according to the balance of oxygen element

$$
\begin{aligned}
\rho V\left(\xi \mu_{f, o}+(1-\xi) \mu_{a i r, o}\right) & =\rho V\left(\frac{32}{44} Y_{C O 2}+\frac{16}{28} Y_{C O}+\frac{16}{18} Y_{H 2 O}\right. \\
& \left.+\mu_{f, o} Y_{f}+Y_{O 2}\right) \\
\rho V \xi\left(\mu_{f, o}+y_{O}(\phi, T)\right) & =\rho V \xi\left(\frac{32}{44} y_{C O 2}(\phi, T)+\frac{16}{28} y_{C O}(\phi, T)\right. \\
+ & \left.\frac{16}{18} y_{H 2 O}(\phi, T)+\mu_{f, o} y_{f}(\phi, T)\right)
\end{aligned}
$$

Comparing equation (4.3.10) and (4.3.11), the mass fraction of oxygen can be derived from the mixture fraction

$$
Y_{O 2}=\mu_{a i r, o}(1-\xi)-y_{O}(\phi, T) \xi
$$

where $y_{o}(\phi)$ is consumption of oxygen per kilogram fuel in the correlation based on small-scale experiments. 
As the mixture fraction $\xi$ is related to the LER $\phi$ by correlation (4.2.9), the mass fractions of the combustion products $(4.3 .5-4.3 .8,4.3 .12)$ are functions of the LER and temperature.

\subsubsection{Model based on $\mathrm{CO} / \mathrm{CO}_{2}$ ratio-Model II}

For some fuels, instead of the species yields, the experimental correlation between the $\mathrm{CO} / \mathrm{CO}_{2}$ mole ratio and the GER is available [Ts94]. Therefore, an alternative approach to toxic gases based on the $\mathrm{CO} / \mathrm{CO}_{2}$ ratio is required. Tsuchiya [Ts95] has developed a sub-model for estimating the average upper layer $\mathrm{CO}$ concentration. In addition to the chemical balance equation, the empirical $\mathrm{CO} / \mathrm{CO}_{2}$ mole ratio is used to calculate the species yields. In a steady fire, the ratio of fuel loss rate to the air entrainment rate is can be approximated as the ratio of the mass from the fire source to the mass from the air stream in the upper layer. Therefore, instead of the mass in the upper layer, attributed to the fire source and the air stream, Tsuchiya [Ts95] uses the fuel loss rate together with the air entrainment rate.

In Tsuchiya's model, the chemical reaction is expressed with (2.5.10), i.e.

$$
\begin{aligned}
(\alpha \mathrm{C}+\beta \mathrm{H}+\gamma \mathrm{O}) & +(1-p)[(1-q / 2) \alpha+\beta / 4-\gamma / 2] \mathrm{O}_{2} \\
& =(1-p)\left[\alpha(1-q) \mathrm{CO}_{2}+\alpha q \mathrm{CO}\right. \\
& \left.+\beta / 2 \mathrm{H}_{2} \mathrm{O}\right]+p(\alpha \mathrm{C}+\beta \mathrm{H}+\gamma \mathrm{O})
\end{aligned}
$$

where $(\alpha \mathrm{C}+\beta \mathrm{H}+\gamma \mathrm{O})$ is the gasified fuel, $p$ is the unburned fuel carbon fraction, $q$ is the carbon fraction converted to $\mathrm{CO}$ in the burned fuel. The values of parameters $\alpha, \beta$ and $\gamma$ represent the composition of the fuel. The unburned fuel, $p(\alpha \mathrm{C}+\beta \mathrm{H}+\gamma \mathrm{O})$, includes incomplete combustion products, such as hydrocarbon and soot.

As seen in section 4.2, in a control volume of the computational domain $\rho \times v \times \xi$ can be considered as the mass that correspond to the fuel from the fire source and $\rho \times v \times(1-\xi)$ as the mass from the air stream. By replacing the fuel loss rate with $\rho \times v \times \xi$, the sub-model in [Ts95] can be applied in field fire models for the prediction of $\mathrm{CO}$ concentration. Analogous to (2.5.11-14), the mass of combustion products in a control volume are calculated as follows. The mass of the unburned fuel is 


$$
m_{f}=p \times \rho v \xi
$$

The masses of $\mathrm{CO}_{2}, \mathrm{CO}$, and $\mathrm{H}_{2} \mathrm{O}$ are

$$
\begin{aligned}
& m_{\mathrm{CO} 2}=(1-p)(1-q) \alpha M_{\mathrm{CO} 2} \rho v \xi / M_{f} \\
& m_{\mathrm{CO}}=(1-p) q \alpha M_{\mathrm{CO}} \rho v \xi / M_{f} \\
& m_{\mathrm{H} 2 \mathrm{O}}=\frac{1}{2}(1-p) \beta \alpha M_{\mathrm{H} 2 \mathrm{O}} \rho v \xi / M_{f}
\end{aligned}
$$

The $\mathrm{O}_{2}$ remaining after combustion is

$$
\begin{gathered}
m_{\mathrm{O} 2}=(1-\xi) \rho v \mu_{\text {air }, O}-(1-p)[(1-q / 2) \alpha+\beta / 4-\gamma / 2] \\
\times M_{\mathrm{O} 2} \rho v \xi / M_{f}, \\
m_{\mathrm{O} 2} \geq 0
\end{gathered}
$$

The mass of $\mathrm{N}_{2}$ is expressed by

$$
m_{N 2}=\rho v(1-\xi)\left(1-\mu_{a i r, O 2}\right)
$$

The mass fraction of species $i$ is expressed using the sub-component mass fractions from (4.3.14) to (4.3.19) by

$$
Y_{i}=\frac{m_{i}}{m_{f}+m_{C O 2}+m_{C O}+m_{O 2}+m_{N 2}+m_{H 2 O}}
$$

where $i$ represents fuel, $\mathrm{CO}_{2}, \mathrm{CO}, \mathrm{O}_{2}, \mathrm{~N}_{2}$, and $\mathrm{H}_{2} \mathrm{O}$.

There are two unknown parameters $p$ and $q$ in this model. The parameter $q$ in the model is calculated from the $\mathrm{CO} / \mathrm{CO}_{2}$ mole ratio [Ts95] by

$$
q=\frac{r_{\mathrm{CO} / \mathrm{CO} 2}}{1+r_{\mathrm{CO} / \mathrm{CO} 2}}
$$

where the $\mathrm{CO} / \mathrm{CO}_{2}$ mole ratio $r_{\mathrm{CO} / \mathrm{CO} 2}$ is a function of the LER and temperature. Just like the species yield used in the model in section 4.3 .2 , the correlation between 
$r_{\mathrm{CO} / \mathrm{CO} 2}$ and the LER and temperature is derived from small-scale experiments with the concept of the GER. It is not easy to estimate the factor $p$ of the unburned fuel in the chemical equation (4.3.13). However, here it is assumed that the fuel and oxygen could not co-exist [Ts95], thus yielding the exprssion

$$
p \times m_{O 2}=0
$$

Since there is incomplete combustion product $(\mathrm{CO})$ in chemical reaction equation (4.3.13), the mass of oxygen in a control volume may not be zero when the LER is slightly greater than one. Set the mass of $\mathrm{O}_{2}$ to be 0 , than equation (4.3.18) becomes

$$
(1-\xi) \rho v \mu_{\text {air }, O}-(1-p)((1-q / 2) \alpha+\beta / 4-\gamma / 2) M_{O 2} / M_{f} \rho v \xi=0
$$

The solution of equation (4.3.23) is

$$
\bar{p}=1-\frac{(1-\xi) \mu_{a i r, O}}{((1-q / 2) \alpha+\beta / 4-\gamma / 2) \times M_{o 2} / M_{f} \times \xi}
$$

From equation (4.3.21), parameter $q$ is a function of the mole ratio of $\mathrm{CO}$ to $\mathrm{CO}_{2}$. As a result, $q$ is also a function of the LER and temperature. When the mass of oxygen is positive, the left hand of equation (4.3.23) is greater than zero. At the condition with positive $\mathrm{O}_{2}$ in a control volume, $\bar{p}$ given by (4.3.24) is negative. Therefore, the parameter $p$ is expressed as

$$
p=\max \{0, \bar{p}\}
$$

\subsubsection{Model based on ratios of $\mathrm{CO} / \mathrm{CO}_{2}$ and $\mathrm{H}_{2} / \mathrm{H}_{2} \mathrm{O}-$ Model III}

There is little $\mathrm{H}_{2}$ in the turbulent fires when the equivalence ratio is less than one. However, the mole fraction of $\mathrm{H}_{2}$ increases rapidly as the equivalence ratio increases beyond one. When the LER is over 2, the mole fraction of $\mathrm{H}_{2}$ is sometimes as high as $\mathrm{CO}$ in the hot upper layer in the hood test (See Figure 10 and 11 in [Pi95]). In the following equations, the intermediate products are simply represented with $\mathrm{H}_{2}$, and 
CO. The mole ratio of $\mathrm{H}_{2}$ to $\mathrm{H}_{2} \mathrm{O}$ is assumed to be similar to that of $\mathrm{CO} / \mathrm{CO}_{2}$ mole ratio $q$ in this study. The chemical equation (4.3.13) is extended to

$$
\begin{aligned}
(\alpha \mathrm{C}+\beta \mathrm{H}+\not \mathrm{O})+ & (1-p)[(1-q / 2) \alpha+\beta(1-q) / 4-\gamma / 2] \mathrm{O}_{2} \\
& =(1-p)\left[\alpha(1-q) \mathrm{CO}_{2}+\alpha q \mathrm{CO}+\beta / 2 q \mathrm{H}_{2}\right. \\
& \left.+\beta / 2(1-q) \mathrm{H}_{2} \mathrm{O}\right]+p(\alpha \mathrm{C}+\beta \mathrm{H}+\gamma \mathrm{O})
\end{aligned}
$$

Similar to the analysis in section 4.3.3, we have that

$$
\begin{aligned}
m_{f}= & p \times \rho v \xi \\
m_{\mathrm{CO} 2}= & (1-p)(1-q) \alpha M_{\mathrm{CO} 2} \rho v \xi / M_{f} \\
m_{\mathrm{CO}}= & (1-p) q \alpha M_{\mathrm{CO}} \rho v \xi / M_{f} \\
m_{\mathrm{H} 2 \mathrm{O}}= & \frac{1}{2}(1-p)(1-q) \beta \alpha M_{\mathrm{H} 2 \mathrm{O}} \rho v \xi / M_{f} \\
m_{\mathrm{H} 2}= & \frac{1}{2}(1-p) q \beta \alpha M_{\mathrm{H} 2} \rho v \xi / M_{f} \\
m_{\mathrm{O} 2}= & \rho v(1-\xi) \mu_{a i r, O}-(1-p)[(1-q / 2) \alpha+(1-q) \beta / 4-\gamma / 2] \\
& \times M_{\mathrm{O} 2} \rho v \xi / M_{f}, \quad m_{\mathrm{O} 2} \geq 0
\end{aligned}
$$

The mass of $\mathrm{N}_{2}$ within the control volume is

$$
m_{N 2}=\rho v(1-\xi) \times\left(1-\mu_{\text {air }, O}\right)
$$

The mass fraction of species $i$ is expressed using the sub-component mass fractions from (4.3.27) to (4.3.33) by

$$
Y_{i}=\frac{m_{i}}{m_{f}+m_{C O 2}+m_{C O}+m_{O 2}+m_{N 2}+m_{H 2 O}+m_{H 2}}
$$

where $i$ represents fuel, $\mathrm{CO}_{2}, \mathrm{CO}, \mathrm{O}_{2}, \mathrm{~N}_{2}, \mathrm{H}_{2} \mathrm{O}$ and $\mathrm{H}_{2}$. The parameter $q$ for the $\mathrm{CO} / \mathrm{CO}_{2}$ and $\mathrm{H}_{2} / \mathrm{H}_{2} \mathrm{O}$ ratio is 


$$
q=\frac{r_{\mathrm{CO} / \mathrm{CO} 2}}{1+r_{\mathrm{CO} / \mathrm{CO} 2}}
$$

The value of the parameter $\mathrm{p}$ is expressed as

$$
p=\max \{0, \bar{p}\}
$$

where $\bar{p}$ is the solution of equation (4.3.37)

$$
(1-\xi) \mu_{a i r, o}-(1-p)((1-q / 2) \alpha+\beta(1-q) / 4-\gamma / 2) M_{o 2} \xi / M_{f}=0
$$

\subsubsection{Comparison of the models}

Model I in Section 4.3.2 is based on the species yield of each species involved in combustion. These species yields, as functions of the equivalence ratio, are obtained from small-scale experiments. In this model, the mass fraction of each species is derived from the experimentally obtained species yield via the conservation of the carbon element in a control volume. Since the GER in the small-scale experiments are usually less than 3.5 for flaming fires [Te95], the correlations may not be reliable for extremely high values of LER in full-scale enclosure fires. The accuracy of the model depends on the precision of the empirical yield of the species. The use of these experimental correlations is described in detail in the next section.

Model II and III are based on the balance of the chemical reactions with the assumption that all of the carbon element convert to $\mathrm{CO}_{2}, \mathrm{CO}$ or remain in the unburned fuel. $\mathrm{H}_{2}$ is generated in model III while the $\mathrm{H}_{2} / \mathrm{H}_{2} \mathrm{O}$ mole ratio is similar to the $\mathrm{CO} / \mathrm{CO}_{2}$ ratio.

To compare the models developed in this chapter, pinewood is selected as fuel. The yields of $\mathrm{CO}, \mathrm{CO}_{2}$, and the consumption of $\mathrm{O}_{2}$ for pinewood are given in [Te95]

$$
y_{\mathrm{CO} 2}(\phi)=1.33\left[1-\frac{1.0}{\exp (\phi / 2.15)^{-1.2}}\right]
$$




$$
\begin{aligned}
& y_{\mathrm{CO}}(\phi)=0.005\left[1+\frac{44.0}{\exp (\phi / 1.30)^{-3.5}}\right] \\
& y_{\mathrm{O} 2}(\phi)=1.00\left[1-\frac{0.97}{\exp (\phi / 2.14)^{-1.2}}\right]
\end{aligned}
$$

It is noted that these correlation are only functions of the equivalence ratio. The $\mathrm{CO} / \mathrm{CO}_{2}$ mole ratio required by model II and III can be derived from the yields of $\mathrm{CO}$ and $\mathrm{CO}_{2}$, given by

$$
r_{C O / C O 2}=\frac{y_{C O}(\phi) / M_{C O}}{y_{C O 2}(\phi) / M_{C O 2}}
$$

The range of the equivalence ratios is from 0 to 4 in the following comparison. The predicted mass fractions of $\mathrm{CO}_{2}, \mathrm{O}_{2}$ and $\mathrm{CO}$ as functions of the LERs are depicted in Figures 4.3.1-4.3.3. The theoretical maximum of $\mathrm{CO}$ and $\mathrm{CO}_{2}$ mass fractions and the minimum of $\mathrm{O}_{2}$ mass fractions are given in these figures. The theoretical maximum of $\mathrm{CO}_{2}$ refers to the production of $\mathrm{CO}_{2}$ when all of the $\mathrm{C}$ elements (fuel lean case) or all of the $\mathrm{O}$ elements (fuel rich case) convert to $\mathrm{CO}_{2}$. The theoretical maximum of $\mathrm{CO}$ means that only $\mathrm{CO}$ contains $\mathrm{C}$ element in the products. The theoretical minimum of $\mathrm{O}_{2}$ means that the combustion is complete. As seen in Figure 4.3.1-4.3.2, the three toxic gas models generally agree on the predictions of $\mathrm{CO}_{2}$ and $\mathrm{O}_{2}$ mass fractions. In the fuel lean region, predictions from Model II and III are closer to the theoretical maximum values since it is assumed that the fuel and oxygen cannot co-exist in one control volume. The predicted $\mathrm{O}_{2}$ mass fraction by all the three models decreases to 0 as the LER increase to 1.5. Predictions of $\mathrm{O}_{2}$ from Model II and III are closer to the theoretical minimum.

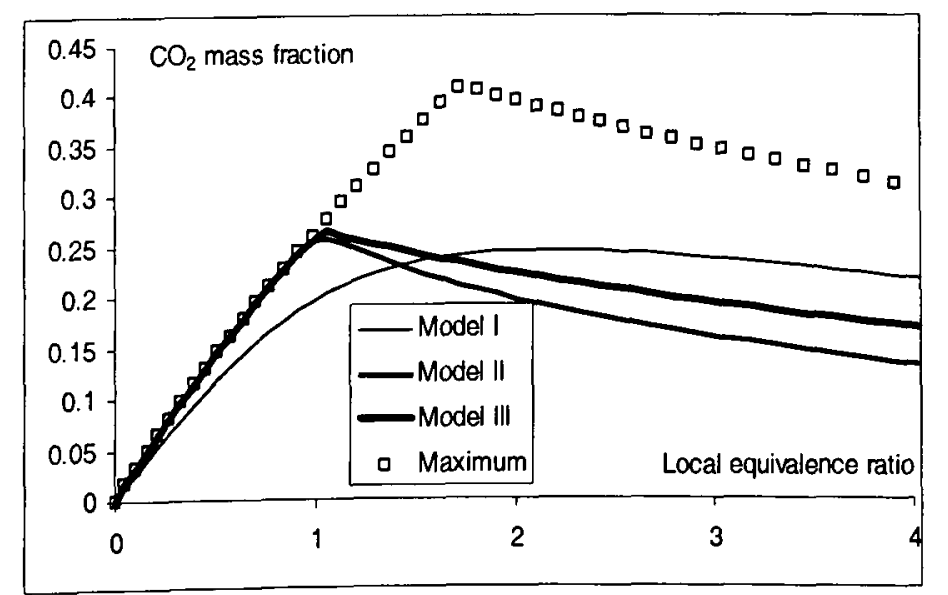

Figure 4.3.1: Predicted $\mathrm{CO}_{2}$ mass fractions as function of the LER. 


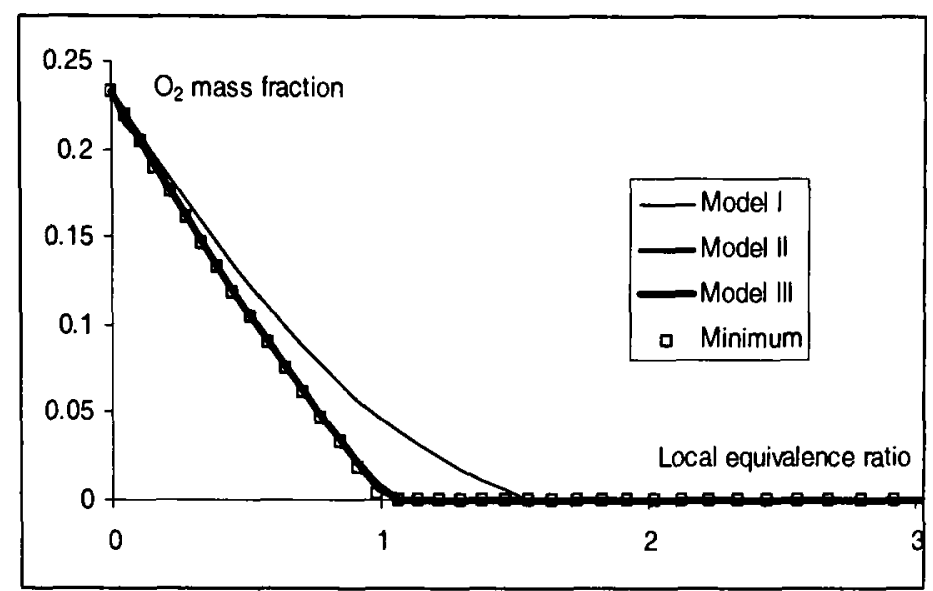

Figure 4.3.2: Predicted $\mathrm{O}_{2}$ mass fractions as function of the LER.

The predicted trends of $\mathrm{CO}$ mass fraction from three models are depicted in Figure 4.3.3. In the fuel lean region, three models produce similar CO mass fractions. When the equivalence ratio is above 2.5 , the differences between the predictions from the three models become significant.

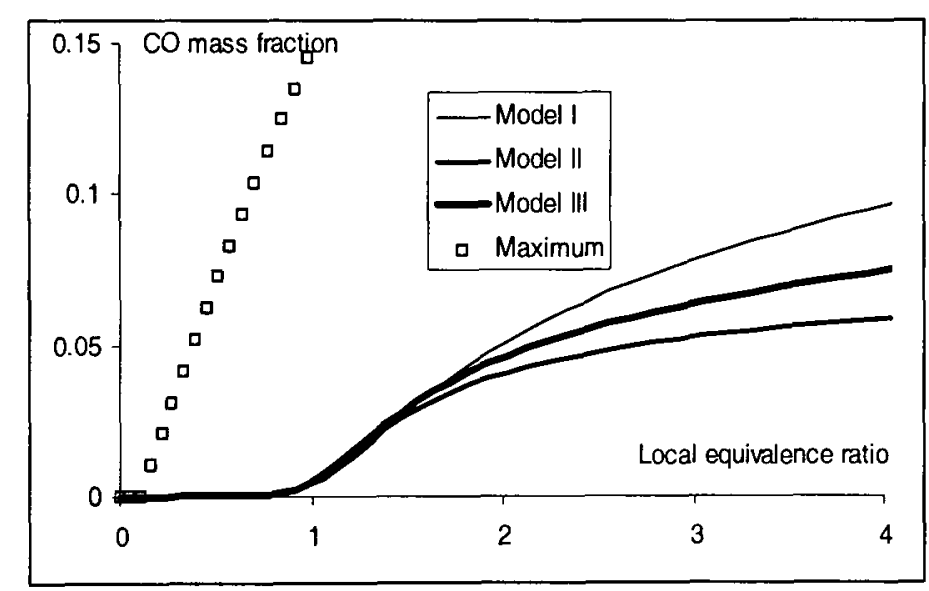

Figure 4.3.3: Predicted CO mass fractions as function of the LER.

The features of the three toxic gas models are described as follows.

(1) Model I uses the species yields while Model II uses the $\mathrm{CO} / \mathrm{CO}_{2}$ mole ratio and Model III uses the $\mathrm{CO} / \mathrm{CO}_{2}$ ratio together with the $\mathrm{H}_{2} / \mathrm{H}_{2} \mathrm{O}$ ratio. The empirical correlation used in the derivation of Model I, II and III can be obtained from turbulent fire tests based on small-scale apparatus with the concept of the GER. If the fire is "steady-state" in nature, the LER in the hotter upper layer is nearly uniform. Therefore, the GER within the smallscale test is actually close to the LER. Thus, the correlation between the species yields and the GER derived from small-scale tests are representative correlations between the species yields and the LER. By assuming each 
control volume in field models as a miniature combustion apparatus, the combustion condition within a control volume with a value of the LER is assumed close to that in the small-scale fire experiment with the same value of GER. The correlation between the yields of combustion products and the GER obtained from tests on small-scale combustion apparatus is then used in this study to model the production of combustion products. The GER in the correlation is replaced by the LER at a control volume in the present developed models.

(2) Models produce similar $\mathrm{CO}_{2}$ and $\mathrm{O}_{2}$ predictions and the predictions from Model II and III are closer to the theoretical maximum values in fuel lean region. Models produce similar CO predictions in the fuel lean region and the region where the LER is less than 2.0.

(3) Apart from predicting $\mathrm{CO}, \mathrm{CO}_{2}$ and $\mathrm{O}_{2}$, Model I can also predict the mass fractions of other products such as hydrocarbon and soot while Model III is able to predict the mass fractions of $\mathrm{H}_{2}$ and the unburned fuel.

\subsubsection{Model implementation}

As toxicity models developed in this chapter are not coupled with the released heat due to combustion, these models could be incorporated with the volumetric heat source approach and the EBU combustion model for the calculation of combustion products.

The correlations between the yields of combustion products and the GERs are derived from the small-scale experiments and are only reliable within a limited range of GERs. One shortcoming of the $\mathrm{CO}$ yield, equation (4.3.39), is that it overestimates the $\mathrm{CO}$ yields when GERs become too large. Usually, with the increase of GER, the yield of $\mathrm{CO}$ increases. Equation (4.3.39) gives high $\mathrm{CO}$ yield even when GERs become so large that no combustion takes place. In reality, when the equivalence ratio is beyond a critical point, the yield of $\mathrm{CO}$ decreases too. The maximal equivalence ratio for $\mathrm{CO}$ generation is 3.5 for flammable combustion, for most unhalogenated materials, while 
it is approximately 2.0 for halogenated polymers according to Tewarson's work [Te95].

To rectify this shortcoming, an inflexion point of the curve representing the relationship between the yields of $\mathrm{CO}$ and equivalence ratios, $\phi_{\text {inflexion }}$, is introduced to represent the critical value of the equivalence ratio beyond which the $\mathrm{CO}$ yields decrease. When the equivalence ratio $\phi$ is greater than $\phi_{\text {inflexion }}$, the yield of $\mathrm{CO}, y_{C O}(\phi)$, is calculated using the yields of $\mathrm{CO}_{2}$ (at equivalence ratio $\phi$ ) and the yield ratio of $\mathrm{CO}$ to $\mathrm{CO}_{2}$ at the inflexion point $\phi_{\text {inflexion }}$. Therefore, the correlation for $\mathrm{CO}$ is expressed as

$$
y_{C O}(\phi)= \begin{cases}y_{C O}(\phi) & \phi<\phi_{\text {inflexion }} \\ y_{C O 2}(\phi) \cdot \frac{y_{C O}\left(\phi_{\text {inflexion }}\right)}{y_{C O 2}\left(\phi_{\text {inflexion }}\right)} & \phi \geq \phi_{\text {inflexion }}\end{cases}
$$

In the calculation of the toxic gas concentrations using Model II and Model III, the $\mathrm{CO}$ to $\mathrm{CO}_{2}$ mole ratio at various LERs is given by

$$
r_{C O / C O 2}(\phi)= \begin{cases}\frac{y_{C O}(\phi) / M_{C O}}{y_{C O 2}(\phi) / M_{C O 2}} & \phi<\phi_{\text {inflexion }} \\ \frac{y_{C O}\left(\phi_{\text {inflexion }}\right) / M_{C O}}{y_{C O 2}\left(\phi_{\text {inflexion }}\right) / M_{C O 2}} & \phi \geq \phi_{\text {inflexion }}\end{cases}
$$

where $M$ represents the molecular weight of $\mathrm{CO}$ or $\mathrm{CO}_{2}$.

\subsection{Smoke Properties}

Mathematical models to predict the smoke optical density have been developed by using $\mathrm{CO}_{2}$ concentration, or $\mathrm{O}_{2}$ concentration or temperatures in [IP99, Na00]. In this study, instead of using extra conserved scalars in CFD modelling, smoke properties are calculated using the mixture fraction and the specific extinction coefficient or mass optical density of the fuel. The visibility distance is calculated from the predicted light extinction coefficient. 


\subsubsection{Calculation of smoke properties}

\subsubsection{Method using specific extinction coefficient}

In section 4.3.2, the mass fraction of soot are calculated using the toxic gas modelModel I

$$
Y_{\text {soot }}=y_{\text {soot }}(\phi, T) \xi
$$

The soot concentration $\left(\mathrm{kg} / \mathrm{m}^{3}\right)$ is then expressed by

$$
C_{\text {soot }}=\rho Y_{\text {soot }}
$$

The extinction coefficient, $K$, can be expressed as the product of an extinction coefficient per unit mass, $K_{\mathrm{m}}$, and the mass concentration of smoke aerosol [Mu95],

$$
K=K_{m} \cdot C_{\text {smoke }}
$$

$K_{m}$ is also known as the specific extinction coefficient. Assume that the soot concentration is equal to the smoke aerosol concentration, than the extinction coefficient can be expressed, using the LER and temperature, as

$$
K=K_{m} \cdot \rho Y_{\text {soot }}=K_{m} \rho y_{\text {soot }}(\phi, T) \xi
$$

Here the specific extinction coefficient, $K_{m}$, depends on the size distribution and optical properties of the smoke. The value of $K_{m}$ is $7.6 \mathrm{~m}^{2} / \mathrm{g}$ for smoke produced during flaming combustion of wood and plastic or $4.4 \mathrm{~m}^{2} / \mathrm{g}$ for smoke produced during the pyrolysis of these materials. As mentioned in [Mu95], these specific extinction coefficients are obtained by researchers through small-scale experiments. Dobbin [Do94] has summarized specific extinction coefficients for various fuels.

'The physical basis for light extinction measurements is Bouguer's law, which relates the intensity, $I_{0}$ of the incident monochromatic light of wavelength $\lambda$ and the 
intensity of light, $I_{\lambda}$ transmitted through path length L, of the smoke' [Mu95]. The correlation between the ratio of light intensity $I_{\lambda}$ to $I_{0}$ through the path length $L$ and the light extinction coefficient $K$ is

$$
\frac{I_{\lambda}}{I_{\lambda}^{0}}=e^{-K L}
$$

Substitute (4.4.4) into (4.4.5), the light transmission is expressed as a function of the mixture fraction and the LER by

$$
\frac{I_{\lambda}}{I_{\lambda}^{0}}=e^{-K_{m} \rho y_{s o o t}(\phi, T) \xi}
$$

Another form of equation (4.4.5) is

$$
\frac{I_{\lambda}}{I_{\lambda}^{0}}=10^{-D L}
$$

where the quantity $\mathrm{D}$ is the optical density per meter. The correlation between the light extinction coefficient $K$ and the smoke optical density $D$ is then

$$
D=K / 2.3
$$

Therefore, the optical density can be calculated using the local equivalence ratio

$$
D=K_{m} \rho y_{\text {soot }}(\phi) \xi / 2.3
$$

\subsubsection{Method using mass optical density}

The mass optical density is defined as [Mu95]

$$
D_{m}=\frac{D V}{\Delta M}
$$

where $V$ is the volume of the chamber in the small-scale smoke experiments and $\Delta M$ is the loss of fuel mass. Corresponding to the small-scale experiments, $V$ is the volume of a cell and $\Delta M$ is the mass that originates from the fuel source in field 
modelling. The volumetric mass $\Delta M / V$, which originates from the fuel source, can be expressed using the mixture fraction in field modelling by

$$
\frac{\Delta M}{V}=\rho \xi
$$

where $\rho$ is the density of the gas mixture and $\xi$ is the mixture fraction. By substituting (4.4.11) into (4.4.10), the optical density can be expressed in terms of the mixture fraction as

$$
D=D_{m} \frac{\Delta M}{V}=D_{m} \rho \xi
$$

Using the correlation (4.4.8), the light extinction coefficient is related to the optical density by

$$
K=2.3 D_{m} \rho \xi
$$

The mass optical density can be measured from the small-scale experiments for various combustion materials. Mulholland [Mu95] has summarized the optical density of wood and plastics fires under flaming and shouldering combustion conditions. In [IP99], Ingason et al has provided some mass optical density data about vehicle fires.

At locations distant from the fire source, the unburned fuel can be supposed to be 0 and ignored. In these locations, the amount of mass that originates from the fuel source is approximately proportional to the $\mathrm{CO}_{2}$ mass or to the amount of oxygen consumed. The mass from the fuel source at locations far from the fire source can be approximately expressed with the $\mathrm{CO}_{2}$ mass fraction as

$$
\rho \xi V=\rho Y_{\mathrm{CO} 2} V / r_{\mathrm{CO} 2}
$$

where $r_{\mathrm{CO} 2}$ is the stoichiometric yield of $\mathrm{CO}_{2}$ as one unit mass of fuel is consumed.

The mass originated from the air source is $\rho V(1-\xi)$. The consumed oxygen, $m_{\mathrm{O} 2}$, can be expressed with the $\mathrm{O}_{2}$ mass fraction as 


$$
m_{O 2}=(\rho V(1-\xi)) Y_{O 2, \infty}-\rho V Y_{O 2}
$$

where $Y_{O 2, \infty}$ refers to the oxygen mass fraction in the ambient air. Let the stociometric $\mathrm{O}_{2}$ to fuel ratio be $r_{O 2}(\mathrm{~kg} / \mathrm{kg})$, the consumed oxygen can also approximated to

$$
m_{O 2}=\rho V \xi \cdot r_{O 2}
$$

Eliminating $m_{\mathrm{O} 2}$ in (4.4.15) and (4.4.16), the amount of mass from the fuel source can be expressed as a function of $\mathrm{O}_{2}$ mass fraction by

$$
\rho \xi V=\rho V \frac{Y_{O 2, \infty}-Y_{O 2}}{r_{O 2}+Y_{O 2, \infty}}
$$

From (4.4.12), (4.4.14) and (4.4.17), the optical density can be related to the $\mathrm{CO}_{2}$ mass fraction by

$$
D=D_{m} \rho \xi=D_{m} \rho Y_{\mathrm{CO} 2} / r_{\mathrm{CO} 2}
$$

and with the $\mathrm{O}_{2}$ mass fraction as

$$
D=D_{m} \rho \xi=D_{m} \rho \frac{Y_{O 2, \infty}-Y_{O 2}}{r_{O 2}+Y_{O 2, \infty}}
$$

In fire simulations, equation (4.4.12) is the first choice for the calculation of the optical density if the mixture fraction is available. Equation (4.4.18) and (4.4.19) use $\mathrm{CO}_{2}$ or $\mathrm{O}_{2}$ mass fraction to derive an approximation of the mass from the fuel source. For simulations involving volumetric heat and $\mathrm{CO}_{2}$ sources, expression (4.4.18) can be used to derive the smoke optical density. Isaksson et al [IP99] reported a similar relationship to equation (4.4.19), given below

$$
D / l=D_{m} \rho\left(Y_{O 2, \infty}-Y_{O 2}\right) / r_{O 2}
$$


In equation (4.4.20), $D /$ is the optical density. If the stochiometric $\mathrm{O}_{2}$ to fuel ratio is large compared to the ambient $\mathrm{O}_{2}$ mass fraction, equation (4.4.19) can be simplified to give equation (4.4.20).

If the volumetric heat source model is applied to fire simulations where there are no combustion products available, the smoke optical density can be estimated using the temperature distribution [IP99].

The basic idea is to estimate the amount of mass that originates from the fuel source using the change in temperature at each cell. At a cell, let $M$ be the mass that originates from the fuel source. The heat released from the fuel is

$$
Q=M \cdot \Delta H
$$

where $\Delta H$ is the heat of combustion. Supposing that all the heat released from combustion is used to raise the temperature of the gas, then we have

$$
Q=\rho V C_{p}\left(T-T_{\infty}\right)
$$

where $C_{p}$ is the specific heat of gas mixture and $T_{\infty}$ is the ambient temperature. From (4.4.21) and (4.4.22), the mass originating from the fuel source is given by

$$
M=\rho V C_{p}\left(T-T_{\infty}\right) / \Delta H
$$

From (4.4.12) and (4.4.23), the optical density, based on gas temperature is evaluated by

$$
D=D_{m} \rho C_{p}\left(T-T_{\infty}\right) / \Delta H
$$

Note that the assumption about the correlation between the temperature changes and the mass from fire source is not true for cases in which there are heat losses through the wall. This limits the application of the method based on the local temperatures. 


\subsubsection{Visibility}

The reduction of visibility in fire environment affects people in (1) finding their way to a safe place and (2) impacts on their travel speed. It also makes the rescue by fire fighters more difficult.

In this section, visibility is derived from the calculated light extinction coefficient. The following expression is used to calculate the visibility distance $S$ reported in [Mu95],

$$
K \cdot S=C
$$

where constant $C$ is

$$
C= \begin{cases}8 & \text { Light }- \text { emitting sign } \\ 3 & \text { Light }- \text { reflecting sign }\end{cases}
$$

Combining this with the light extinction approaches of (4.4.4) and (4.4.12), the visibility distance can be evaluated using

$$
S=\frac{C}{K_{m} \rho y_{\text {soot }}(\phi, T) \xi}
$$

or

$$
S=\frac{C}{2.3 D_{m} \rho \xi}
$$

\subsection{Concluding Remarks}

In this chapter, the limitation of applying the concept of GER in zone modelling is indicated. The mixture fraction that represents the mixture of the fuel, oxidant and combustion products is described. The correlation between the LER and the mixture fraction is derived. 
Three models for toxic gases are developed in this study. The first one uses the correlation between the species yields and the local equivalence ratio and temperature. The second model uses $\mathrm{CO} / \mathrm{CO}_{2}$ mole ratio. The third model uses both $\mathrm{CO} / \mathrm{CO}_{2}$ and $\mathrm{H}_{2} / \mathrm{H}_{2} \mathrm{O}$ ratios. All of the three models produce similar predictions of the combustion products in the region where the LER is not large. The assumption that the mole ratio of $\mathrm{H}_{2}$ to $\mathrm{H}_{2} \mathrm{O}$ is similar to that of $\mathrm{CO} / \mathrm{CO}_{2}$ may be too rough. A more realistic correlation between $\mathrm{H}_{2}$ and $\mathrm{H}_{2} \mathrm{O}$ should be considered in the further study.

Simple methods for the calculation of smoke properties (optical density, light extinction coefficient and visibility distance) are developed in this chapter. In these approaches, smoke properties are calculated using the local LERs or mixture fractions or mass fractions of $\mathrm{CO}_{2}\left(\right.$ or $\left.\mathrm{O}_{2}\right)$.

The models developed in this chapter can be used with the practical volumetric heat source approach or the EBU combustion model. For cases involving the volumetric heat source approach, an extra variable is used to represent the mixture of combustion gases.

The toxicity model Model I have been applied to fire scenarios simulations and reported in [WJ01b, WJ06] and some of the approaches relating to light extinction coefficients in this chapter have been applied for fires on ships [GG03, GL03, GL04]. The models developed in this chapter will be validated in the next chapter by simulating a wide range of fire scenarios. 


\section{Chapter 5}

\section{SIMULATIONS OF ENCLOSURES FIRES}

\subsection{Introduction}

To validate the toxicity models developed in Chapter 4 , four full-scale fire tests, RI (Rockwool International) fire, PP3 fire [LBM96], W14-b3 fire [HK98], Borealis cable fire [Bo01, RS02] and one reduced compartment-hallway (RCH) [LE94] fire are simulated in this chapter. Simulations are performed using SMARTFIRE or CFX (Version 4.2) modelling software. In the simulations, the released heat from combustion is modelled with the volumetric heat source approach or the eddy breakup combustion model while the combustion products and light extinction coefficients are calculated from the models developed in Chapter 4.

In the simulations in this chapter, the inflexion point $\phi_{\text {inf lexion }}$ in equation (4.3.42) takes 5.0 except 2.0 for cable fire.

Toxicity Model I, II and III, developed in Chapter 4, produce similar CO concentrations in the region far away from the fire source. The RI fire is simulated using all three models while the other fires are simulated using model I only. The approach to calculate smoke properties based on the local equivalence ratio (or mixture fraction) is used to predict the light transmission of Borealis's cable fire. Part of the simulation results in this chapter can be found in [WJ01b, WJ02, WJ06, WJ06c].

The summary of the tests and simulations is shown in table 5.1.1. 
Table 5.1.1: Fire model specification summary.

\begin{tabular}{|l|l|l|l|l|l|}
\hline Tests & RI tests & $\begin{array}{l}\text { PP3 } \\
{[\text { LBM96] }}\end{array}$ & W14-b3 [HK98] & $\begin{array}{l}\text { Borealis fire } \\
{[\text { Bo01, RS04] }}\end{array}$ & $\begin{array}{l}\text { RCH } \\
\text { tests[LE94] }\end{array}$ \\
\hline Fire Fuel & $\begin{array}{l}\text { Pine wood crib } \\
\mathrm{CH}_{1.7} \mathrm{O}_{0.83}\end{array}$ & $\begin{array}{l}\text { Polypropylene } \\
{\left[\mathrm{C}_{3} \mathrm{H}_{6}\right]_{n}}\end{array}$ & $\begin{array}{l}\text { Pine wood crib } \\
\mathrm{CH}_{1.7} \mathrm{O}_{0.83}\end{array}$ & $\begin{array}{l}\text { PVCcable } \\
\mathrm{CH}_{1.5} \mathrm{Cl}_{0.5}\end{array}$ & $\begin{array}{l}\text { Hexane } \\
{\left[\mathrm{C}_{6} \mathrm{H}_{14}\right]_{n}}\end{array}$ \\
\hline $\begin{array}{l}\text { Geometry (length, } \\
\text { height, width) }\end{array}$ & $3.6 \mathrm{~m}, 2.4 \mathrm{~m}, 2.4 \mathrm{~m}$ & $3.6 \mathrm{~m}, 2.4 \mathrm{~m}, 2.4 \mathrm{~m}$ & $7.4 \mathrm{~m}, 3.6 \mathrm{~m}, 7.2 \mathrm{~m}$ & $3.0 \mathrm{~m}, 3.0 \mathrm{~m}, 3.0 \mathrm{~m}$ & $\begin{array}{l}4.88 \mathrm{~m}, 1.67 \mathrm{~m}, \\
1.22 \mathrm{~m}\end{array}$ \\
\hline $\begin{array}{l}\text { Mesh in } \\
\text { simulation }\end{array}$ & $45^{*} 23^{*} 11=11385$ & $40^{*} 20^{*} 12=9600$ & $35 * 27 * 15=14175$ & $\begin{array}{l}26^{*} 22^{*} 19 \\
=10868\end{array}$ & $\begin{array}{l}84^{*} 26^{*} 17 \\
=37128\end{array}$ \\
\hline $\begin{array}{l}\text { Symmetry taken } \\
\text { into consideration }\end{array}$ & Yes & Yes & No & No & Yes \\
\hline Species Yields & $\begin{array}{l}\text { Equation }(2.2 .3) \\
\text { for wood }\end{array}$ & $\begin{array}{l}\text { Equation }(2.2 .3) \\
\text { for } \\
\text { plypropylene }\end{array}$ & $\begin{array}{l}\text { Equation }(2.2 .3) \\
\text { for pine wood }\end{array}$ & $\begin{array}{l}\text { Equation }(2.2 .3) \\
\text { for cable }\end{array}$ & $\begin{array}{l}\text { Equation } \\
(2.2 .8-13,15- \\
16) \text { for hexane }\end{array}$ \\
\hline $\begin{array}{l}\text { Model or method } \\
\text { in Chapter 4 }\end{array}$ & $\begin{array}{l}\text { Model I, Model II } \\
\text { and Mode1 III }\end{array}$ & Model I & Model I & $\begin{array}{l}\text { Model I and } \\
\text { method for } \\
\text { smoke }\end{array}$ & Model I \\
\hline $\begin{array}{l}\text { Heat released due } \\
\text { to combustion }\end{array}$ & EBU model & $\begin{array}{l}\text { Volumetric heat } \\
\text { source approach }\end{array}$ & $\begin{array}{l}\text { Volumetric heat } \\
\text { source approach }\end{array}$ & EBU model & EBU model \\
\hline Radiation model & $\begin{array}{l}\text { Six-flux radiation } \\
\text { model }\end{array}$ & $\begin{array}{l}\text { Six-flux } \\
\text { radiation model }\end{array}$ & $\begin{array}{l}\text { Six-flux radiation } \\
\text { model }\end{array}$ & $\begin{array}{l}\text { Six-flux } \\
\text { radiation model }\end{array}$ & $\begin{array}{l}\text { Six-flux } \\
\text { radiation model }\end{array}$ \\
\hline $\begin{array}{l}\text { Type of computer } \\
\text { and Software used }\end{array}$ & $\begin{array}{l}\text { PC } \\
\text { SMARTFIRE }\end{array}$ & $\begin{array}{l}\text { Deck } \\
\text { CFX-4.2 }\end{array}$ & $\begin{array}{l}\text { Deck } \\
\text { CFX-4.2 }\end{array}$ & $\begin{array}{l}\text { PC } \\
\text { SMARTFIRE }\end{array}$ & $\begin{array}{l}\text { PC } \\
\text { SMARTFIRE }\end{array}$ \\
\hline $\begin{array}{l}\text { Simulation time } \\
\text { of real fire }\end{array}$ & 18 minutes & 20 minutes & 45 minutes & 35 minutes & 1 minute \\
\hline $\begin{array}{l}\text { Running time of } \\
\text { simulation }\end{array}$ & 60 hours & 42.3 hours & 480 hours & 48 hours & 108 hours \\
\hline
\end{tabular}

\section{2 Simulations of RI Tests}

\subsubsection{Description of Experiment}

\subsubsection{Experimental set-up}

The experimental facility at Rockwool International was used to conduct corner fire tests in the ISO 9705 test room. The facility was modified to conduct two fire tests with wood cribs as fuels (see Figure 5.2.1). The dimensions of the compartment was $3.6 \times 2.4 \times 2.4 \mathrm{~m}^{3}$. The walls were made of concrete and $0.15 \mathrm{~m}$ thick. A $0.6 \times 1.2 \mathrm{~m}^{2}$ door to the room was centrally located on one of the short walls. In order to protect the structure of the test room, the ceiling and the walls were covered with $10 \mathrm{~cm}$ thick Rockwool. A hood that connected to an exhaust duct collected the combustion gases from the fire compartment.

Pinewood with a density of $503 \mathrm{~kg} / \mathrm{m}^{3}$ was used as a fuel source in the tests. The wood cribs measured $1.05 \mathrm{~m} \times 1.05 \mathrm{~m} \times 0.5 \mathrm{~m}$ high and were located in the centre of the floor and $1.0 \mathrm{~m}$ from the rear wall. Half of the volume of the crib was filled with $50 \mathrm{~mm} \times 50 \mathrm{~mm}$ battens. Gas temperatures were measured using four vertical rakes of thermocouples identified as locations A, B, C and D. Tree A (7 thermocouples 
separated by $0.3 \mathrm{~m}$ ) was located in the central plane of the room and $0.55 \mathrm{~m}$ from the rear wall. Tree B (three thermocouples separated by $0.3 \mathrm{~m}$ ) was at the centre of the room. Tree $\mathrm{C}$ (seven thermocouples separated by $0.3 \mathrm{~m}$ ) was in the right front corner and $0.25 \mathrm{~m}$ to the walls ( $0.15 \mathrm{~m}$ to the covered wool). The top thermocouple in each tree was placed $0.2 \mathrm{~m}$ below the ceiling $(0.1 \mathrm{~m}$ to the covered wool). Thermocouple tree $\mathrm{D}$ was positioned in the central line of the doorway (11 thermocouples separated by $0.1 \mathrm{~m}$, the top one is $0.1 \mathrm{~m}$ to the soffit of the opening). The fire test was repeated twice. The volume fractions of $\mathrm{CO}, \mathrm{CO}_{2}$ and $\mathrm{O}_{2}$ were measured by two gas collectors inside the room or in the doorway in the two tests. The gas collector inside the room was on the central plane of the room, $0.55 \mathrm{~m}$ to the rear wall and $0.2 \mathrm{~m}$ below the ceiling. The gas collector in the doorway was a $20 \mathrm{~cm}$ long pipe with 20 small holes and was located in the opening. The position of this gas collector was $0.15 \mathrm{~m}$ below the soffit of the opening. Note that the thermocouple tree D and this gas collector were in the same vertical plane at the outside surface of the front wall. A hood outside the room was used to collect the combustion gases.

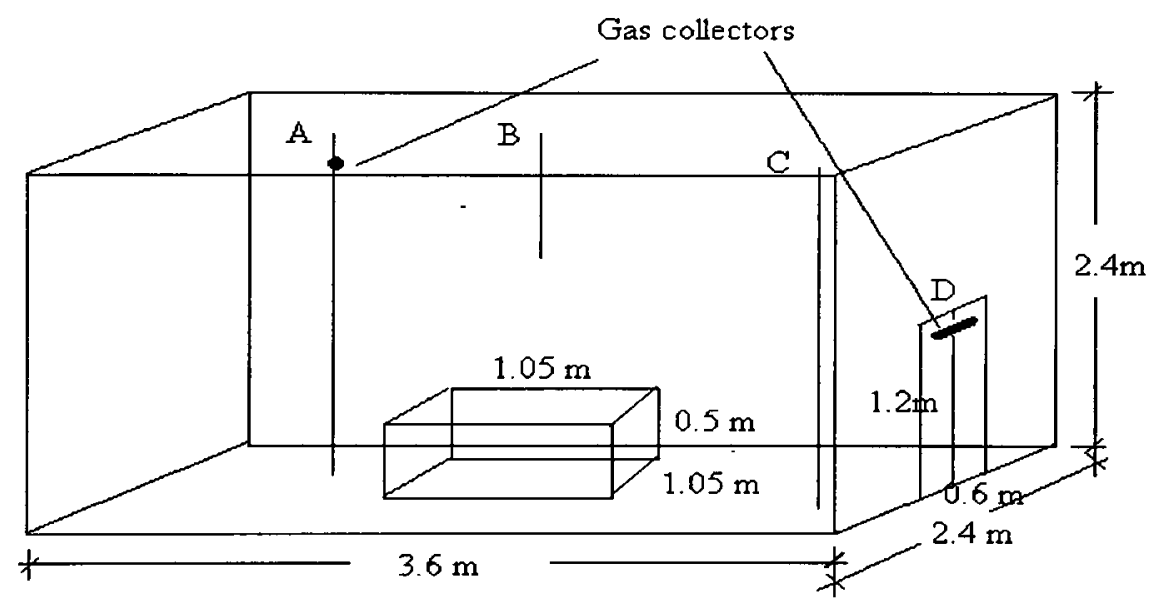

Figure 5.2.1: Schematic of RI fire experimental set up.

\subsubsection{Heat release rates}

In one test, the volume fractions of combustion species inside the room were measured and in the other one values in the doorway were measured. The wood cribs were allowed to burn for 15 minutes after ignition and then the fire was extinguished.

The volume fractions of $\mathrm{CO}, \mathrm{CO}_{2}, \mathrm{O}_{2}$, the gas temperature, light intensity and fluid velocity in the duct were measured. The heat release rates were calculated based on the measurements in the duct. As seen in Figure 5.2.2, the measured heat release rates $(\mathrm{KW})$ appear to be similar in the two tests. 


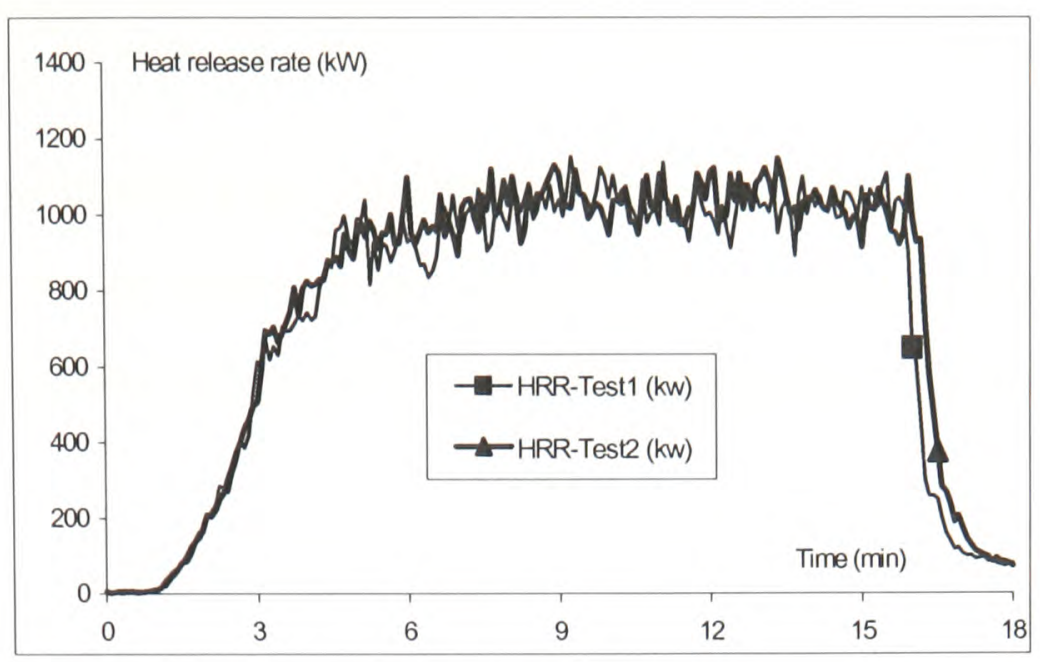

Figure 5.2.2: Heat release rates (KW) of RI fire tests.

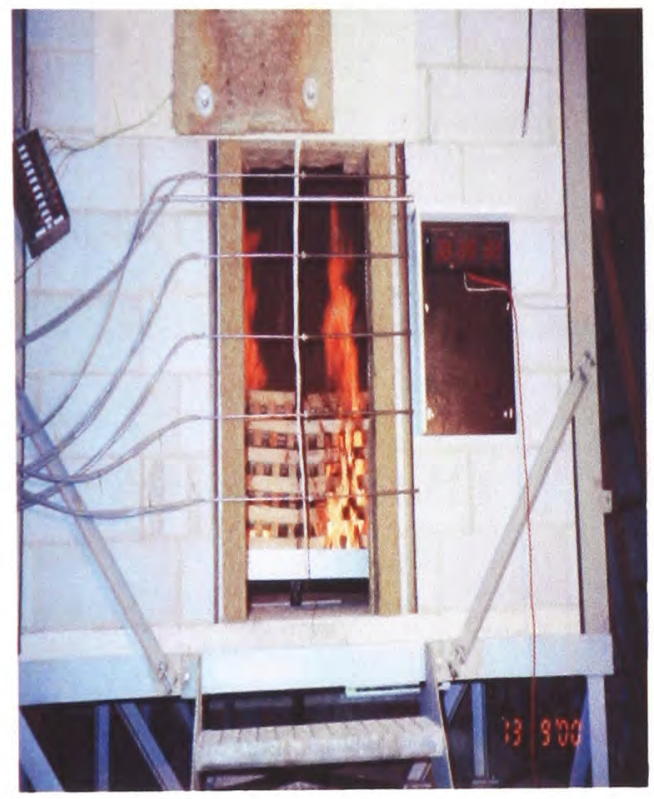

(a)

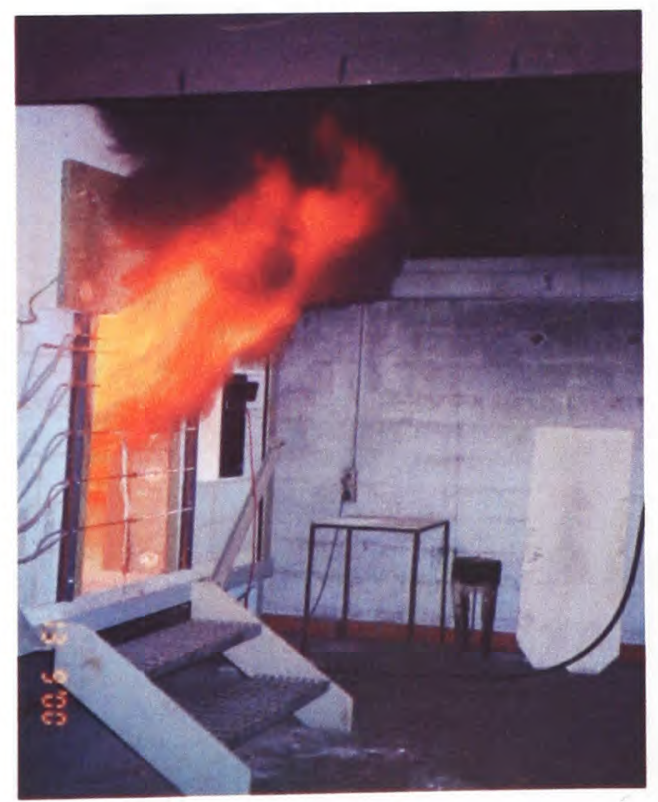

(c)

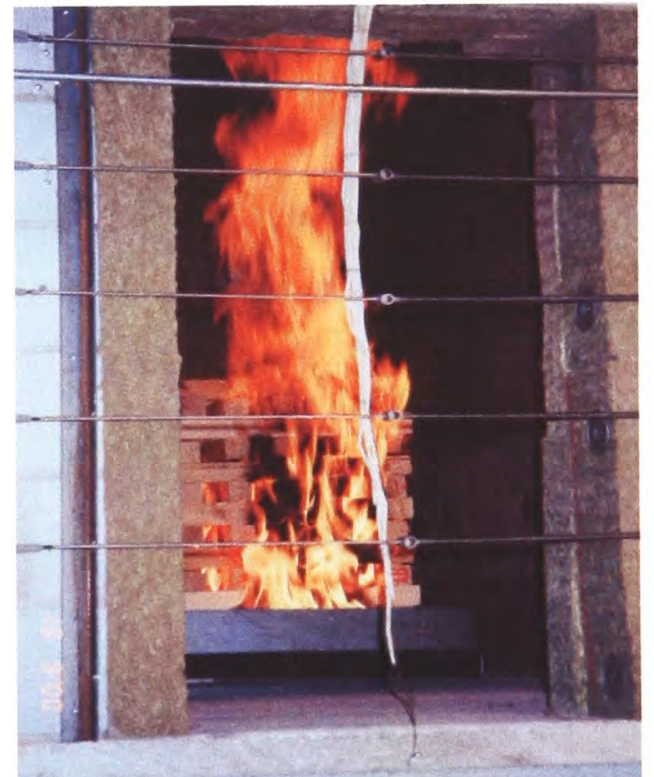

(b)

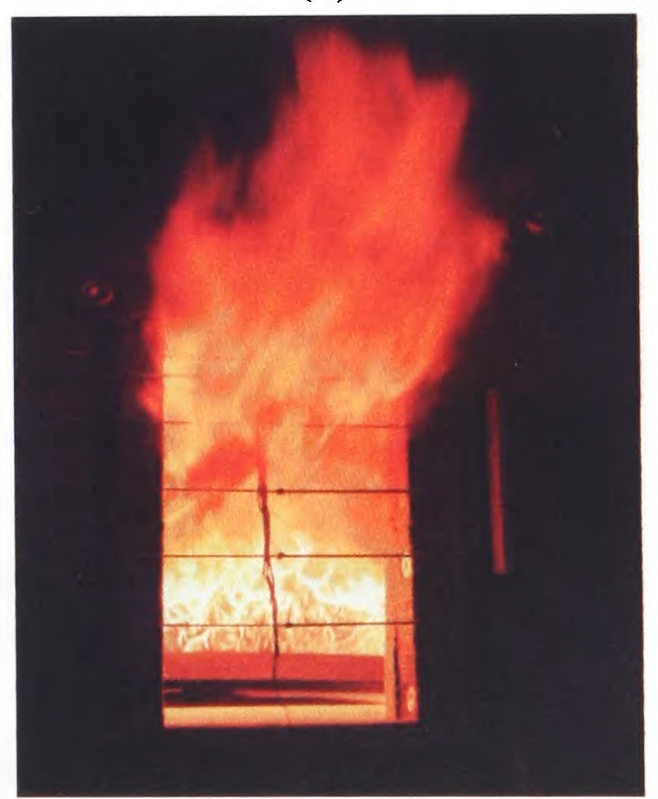

(d)

Figure 5.2.3: Fire test in Rockwool International: (a) ignition of wood cribs; (b) early stage of the fire; (c) side view of the fire plume in the doorway; (d) normal view of the fire. 


\subsubsection{Burning processes of the tests}

The burning process is briefly seen in Figure 5.2.3. The wood cribs were ignited using alcohol. Pictures 4.2.3(c-d) were taken around 7 minutes after the wood cribs were ignited. In the test, the flame protruded through the opening door and thick smoke was generated.

\subsubsection{Simulations}

\subsubsection{Fuel loss rates}

As seen in figure 5.2.2, the heat release rates of the two repeated fire tests were very similar and so the averaged heat release rates are used in the simulation. The fuel loss rate of the fire is calculated by equation (5.2.1)

$$
\dot{m}=\dot{Q} /(\chi \cdot \Delta H)
$$

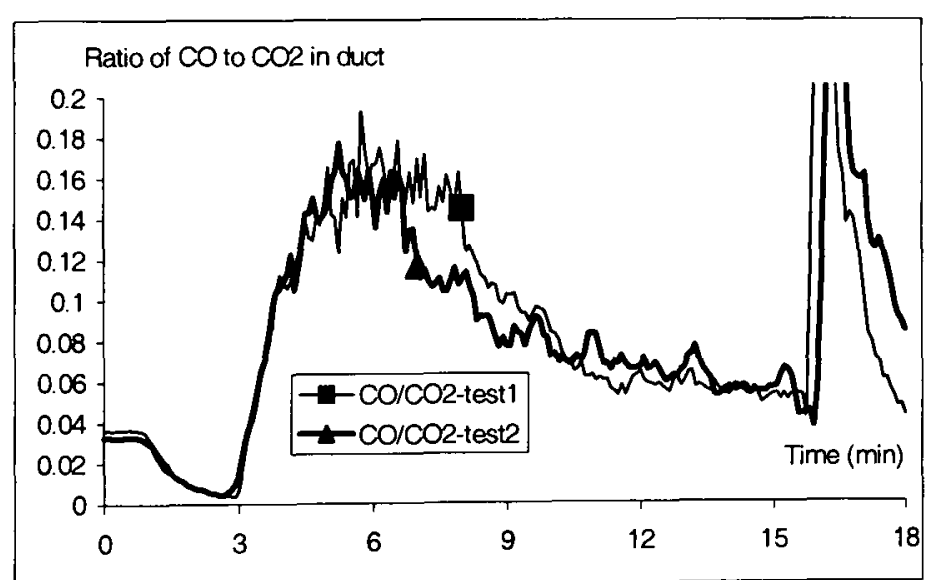

(a)

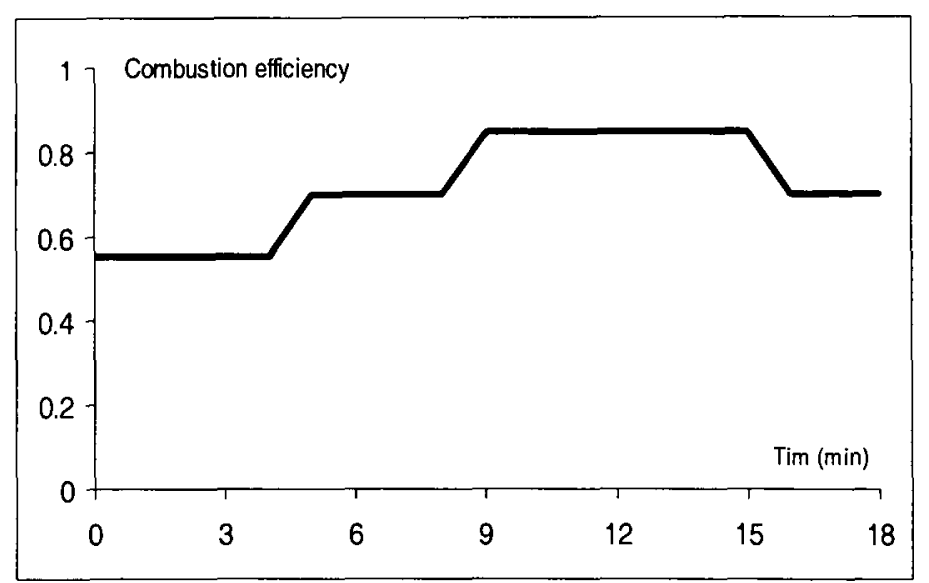

(b)

Figure 5.2.4: (a) Mole ratio of $\mathrm{CO}$ to $\mathrm{CO}_{2}$ in the duct; (b) the combustion efficiencies used in the simulation.

The heat of combustion $\Delta H$ is $17.8 \mathrm{MJ} / \mathrm{kg}$ for burning wood [Te95, HK98]. The combustion efficiency of wood is usually set as 0.7 . Varied combustion efficiency (time dependent) from 0.4 to 1.0 was found in a large enclosure fire with an opening window [HK98]. According to the measured $\mathrm{CO} / \mathrm{CO}_{2}$ ratio in the duct (see Figure 5.2.4(a)), the combustion efficiency appears to increase after 5 minutes. In the initial combustion stage, the combustion efficiency may be low because of the low 
temperatures. The simulations are performed with varied combustion efficiencies from 0.55 to 0.85 (Figure 5.2.4(b)).

\subsubsection{Modelling of wood cribs and meshes}

Ignoring the effect of wood cribs and simply treating the fuel as a heat source, a fire with constant heat release rate of $1000 \mathrm{KW}$, which is approximately the peak vale of the heat release rate in the real test, is simulated using the volumetric heat source approach. It is found that the predicted fire plume leans away from the doorway to the rear wall. The low part of thermocouple tree A (see Figure 5.2.1 for the position of thermocouple tree A) is then in the predicted fire plume. The predicted temperature there is higher than that under the ceiling by $250 \mathrm{k}$. This phenomenon is quite different from the measured trend in the tests. In a simulation with treating wood cribs as solid obstacles, wood cribs have prevented the plume from moving to the rear wall. The predicted upper layer temperature along the thermocouple tree $\mathrm{A}$ is higher than that in the lower layer as observed in the experiments. Therefore, the assumption of wood cribs being modelled as solid obstacles in the simulation is a "good" approximation.

The mesh used in the simulations consisted of 11385 (i.e. $45 \times 23 \times 11$ ) computational cells for half of the compartment. Symmetric boundary conditions are applied to the central plane, which pass through the fire source. Free surface boundary conditions were automatically applied on all open surfaces of the extended region. All air entrained into the solution field entrained air at ambient conditions. A $600 \mathrm{MHz}$ Pentium PC with $256 \mathrm{MB}$ of memory was used to carry out the simulations.

\subsubsection{Models set up}

The EBU model is adopted here to simulate the released heat due to combustion. Toxicity Models I, II and III in section 4.3 are used in this simulation to calculate the combustion products using the predicted LERs. Yields of species of wood burning are given by (2.2.3), which is repeated here by 


$$
y_{i}(\phi)=y_{i, \infty}\left[1+\frac{\alpha}{\exp (\phi / \beta)^{-\zeta}}\right]
$$

where all the parameters are given in Table 5.2.1 [Te95].

Table 5.2.1: Parameters of correlation (5.2.2) for wood.

\begin{tabular}{|l|l|l|l|l|}
\hline & $y_{i, \infty}(\mathrm{kg} / \mathbf{k g})$ & $\alpha$ & $\beta$ & $\zeta$ \\
\hline $\mathbf{C O}$ & 0.005 & 44.0 & 1.3 & -3.5 \\
\hline $\mathbf{C O}_{2}$ & 1.33 & -1.0 & 2.15 & -1.2 \\
\hline $\mathbf{O}_{2}$ & 1.00 & -0.97 & 2.14 & -1.2 \\
\hline
\end{tabular}

In the calculation of the toxic gas concentrations using Model II and Model III, the $\mathrm{CO}$ to $\mathrm{CO}_{2}$ mole ratio at various LERs is given by (4.3.43) with the following expression

$$
r_{C O / C O 2}(\phi)= \begin{cases}\frac{y_{C O}(\phi) / M_{C O}}{y_{C O 2}(\phi) / M_{C O 2}} & \phi<\phi_{\text {inflexion }} \\ \frac{y_{C O}\left(\phi_{\text {inflexion }}\right) / M_{C O}}{y_{C O 2}\left(\phi_{\text {inflexion }}\right) / M_{C O 2}} & \phi \geq \phi_{\text {inflexion }}\end{cases}
$$

where $M$ represents the molecular weight of $\mathrm{CO}$ or $\mathrm{CO}_{2}$.

\subsubsection{Results and discussions}

\subsubsection{Temperature}

During the experiments, the flame was observed protruding through the opening door in the tests (see Figure 5.2.3 (c) and (d)). The simulation reproduced this phenomenon reasonably accurately. The predicted temperature contours in the central plane at 6 minutes are depicted in Figure 5.2.5. The maximum of predicted temperatures outside of the room reaches $750^{\circ} \mathrm{C}$, whereas the predicted temperatures around the fire source are about $1000^{\circ} \mathrm{C}$.

The temperatures as functions of time at different thermocouple trees are depicted in Figures 5.2.6(a)-(d). As can be seen in Figure 5.2.6, the measured temperatures at location $\mathrm{A}, \mathrm{B}$ and $\mathrm{C}$ show a rapid increase over the first 4 minutes followed by a very 
gradual increase from 4 minutes to 15 minutes. The sharp drop in temperature at around 15 minutes is due to the injection of water spray, used to put the fire out at the end of the experiment. Stratification within the back of the compartment is seen with the lower region being considerably cooler while the upper region being hotter (Figure 5.2.6(a)). This kind of stratification in the front corner is not so clear after 5 minutes (Figure 5.2.6(c)).

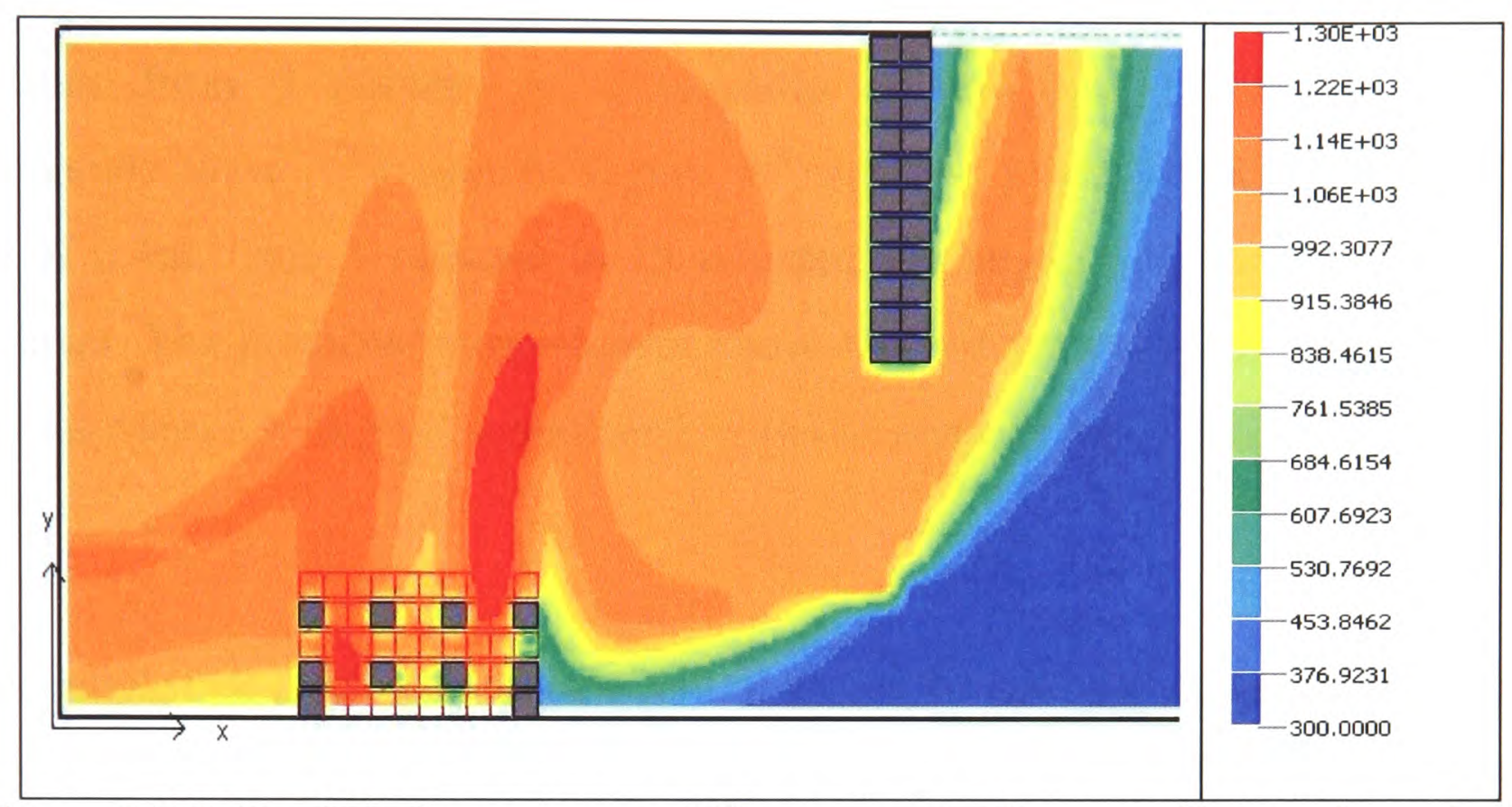

Figure 5.2.5: Predicted temperature $(\mathrm{K})$ contours in the central plane through the doorway at 6 minutes.

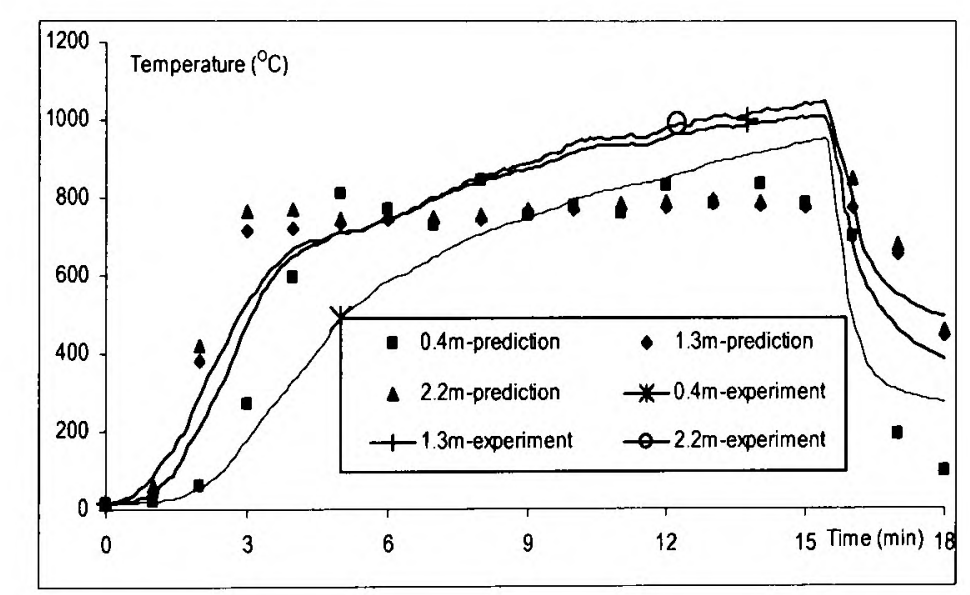

(a)

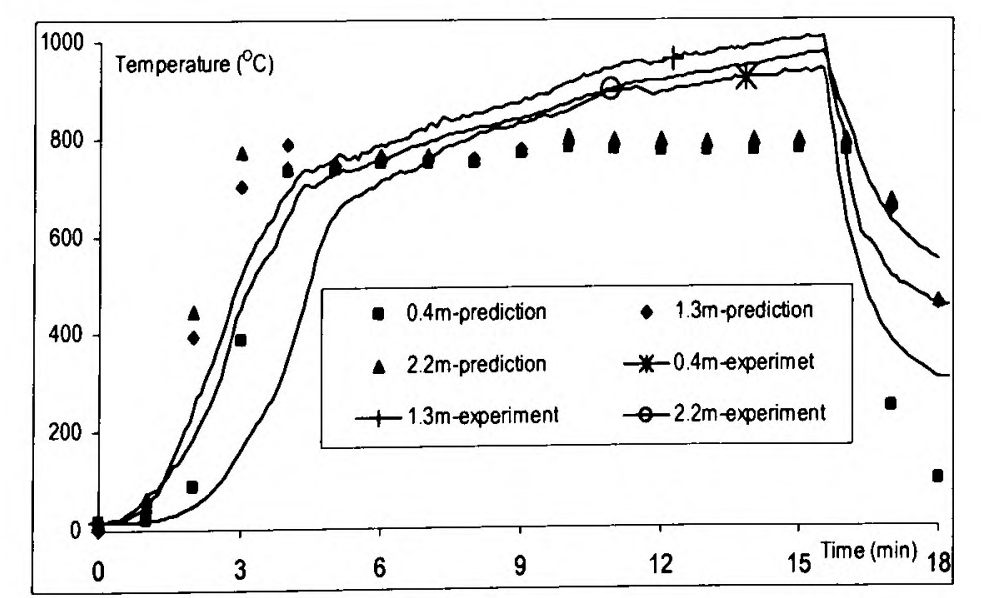

(c)

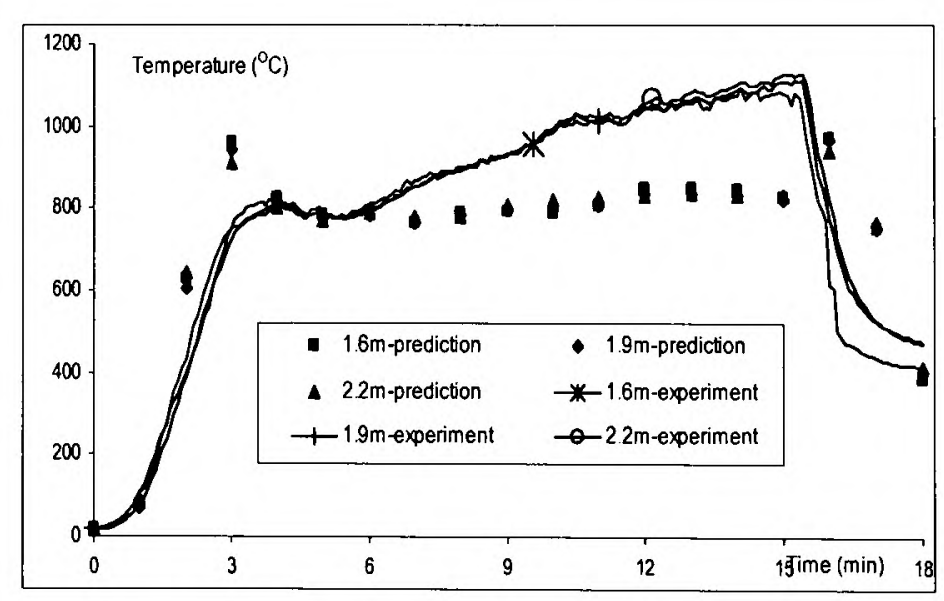

(b)

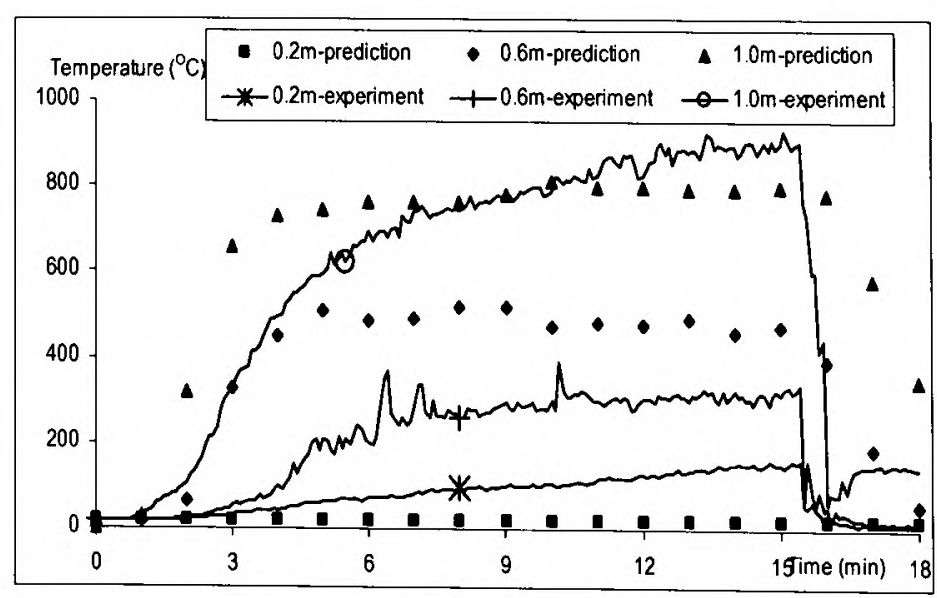

(d)

Figure 5.2.6: Measured and predicted temperatures at (a) thermocouple tree $A$; (b) thermocouple tree B; (c) thermocouple tree C; (d) thermocouple tree D. 
The predicted temperatures appear to follow the measured trends throughout the first 5 minutes (the fire growing stage). From 5 minutes to 15 minutes, the predicted temperatures appear to be near constant. This is due to the quasi-steady heat release rates used in the simulation (see Figure 5.2.2). At $2.2 \mathrm{~m}$ height, the maximum of prediction error between the average of the measurements and the average of predictions from 5 minutes to 15 minutes is less than $15.6 \%$ for the three thermocouple trees. As seen in Figure 5.2.6(a), the predicted temperatures at the height of $0.4 \mathrm{~m}$, from 5 minutes to 15 minutes, are slightly higher than that in the upper layer. The predicted temperatures above the fire source and in the front corner of the test room are in good agreements with the measurements (see Figure 5.2.6(b)(c)).

Thermocouple tree D is located in the central line of the doorway and on the same plane with the outside surface of the front wall. The predicted temperatures at a height of $1.0 \mathrm{~m}$ are qualitatively good (See Figure 5.2.6(d)). From 5 minutes to 15 minutes, the average temperature of $794.4^{\circ} \mathrm{C}$ is under-predicted by $14.1{ }^{\circ} \mathrm{C}$ (i.e. $1.8 \%$ ). However, the average temperature at a height of $0.6 \mathrm{~m}$ is over-predicted by approximately $80 \%$ in the simulation. Note that this position is in the transient region between the hot upper layer and the cold lower layer (Figure 5.2.5). This difference is probably caused by under prediction of the mixing between the lower layer and the upper layer and by the use of small absorption coefficient for ambient air. In addition, temperatures were measured by bare thermocouples in the tests. At lower positions in the cool fresh air steam, they are more likely to record higher temperatures by absorbing radiative energy from the fire, the hot upper layer and hot walls and the ceiling. It is very difficult to produce good predictions within this rapidly changing region. At the height of $0.2 \mathrm{~m}$, the predicted temperatures are closer to the ambient temperature of $16.5^{\circ} \mathrm{C}$ while the average of measured temperature is $107.1^{\circ} \mathrm{C}$.

The predicted and measured doorway temperatures at 6 minutes are depicted in Figure 5.2.7. The lower layer temperatures in the doorway are under predicted in the simulation. The predicted cold layer is about $0.2 \mathrm{~m}$ lower than the measurement. As 
explained before, this disparity may be caused by the inadequate model treatment to the lower layer radiation absorption.

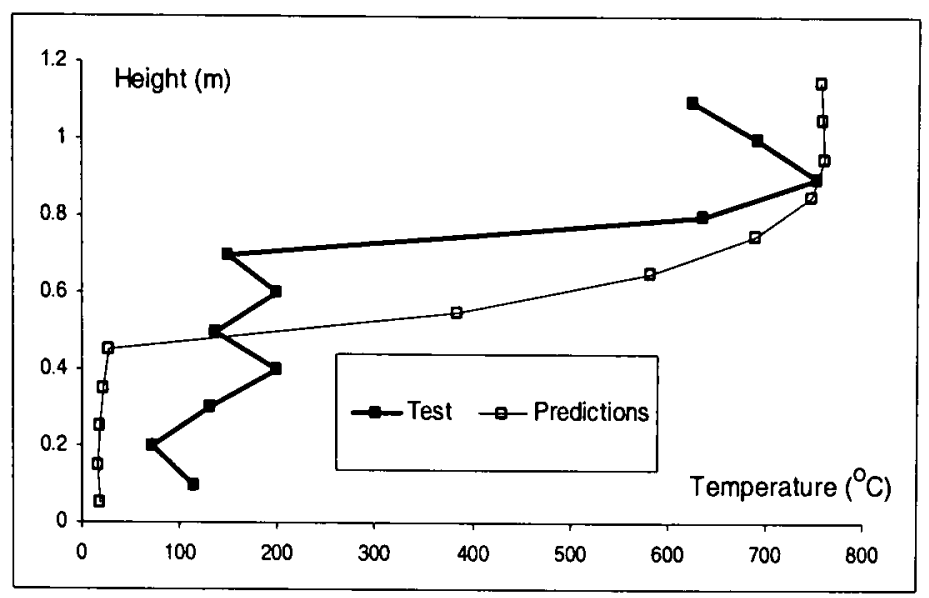

Figure 5.2.7: Measured and predicted temperatures at thermocouple tree D as function of height at 6 minutes.

\subsubsection{2 $\mathrm{O}_{2}$ and $\mathrm{CO}_{2}$}

As seen in Figure 5.2.8, the measured $\mathrm{O}_{2}$ volume fractions in the doorway and inside the room decrease smoothly in the first minute followed by a rapid decrease from 1 minute to 3.5 minutes. The measured $\mathrm{O}_{2}$ volume fractions are close to $0 \%$ from 5 minutes to 15 minutes. The trends of measured $\mathrm{CO}_{2}$ volume fractions in the doorway and inside the room (see Figure 5.2.9) are completely inversion of those for $\mathrm{O}_{2}$ volume fractions.

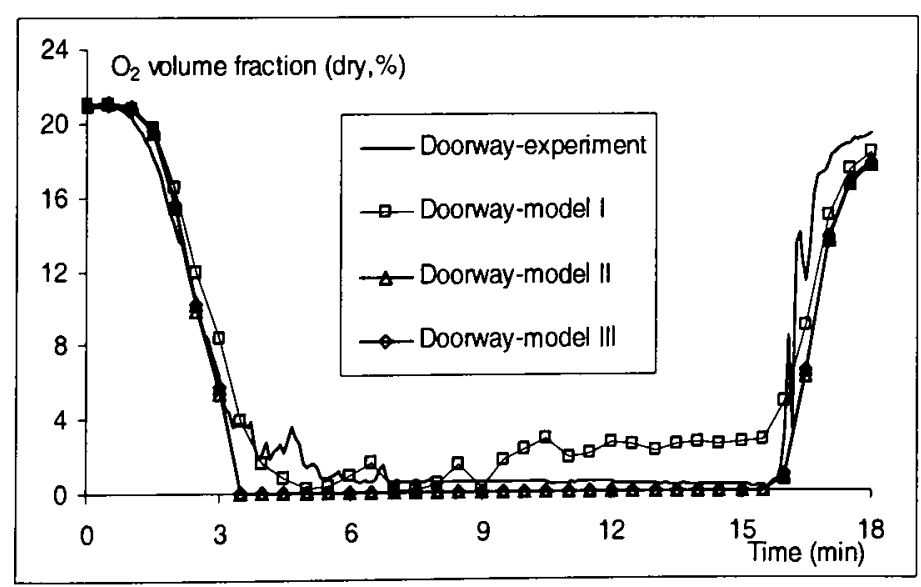

(a)

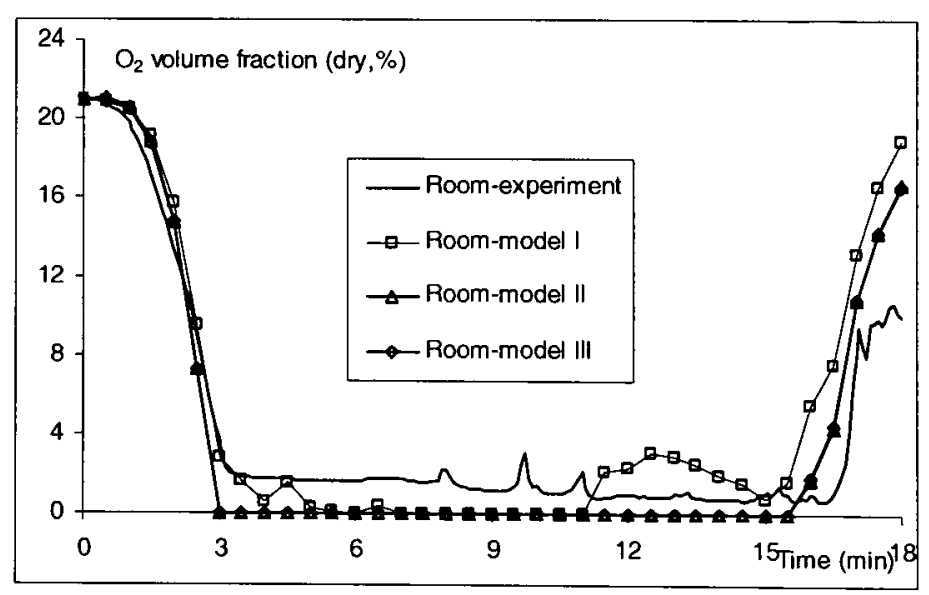

(b)

Figure 5.2.8: Measured and predicted $\mathrm{O}_{2}$ volume fractions (a) at the doorway; (b) inside the room.

The predicted concentrations of $\mathrm{CO}_{2}$ and $\mathrm{O}_{2}$ by the three models follow the measured trends. The $\mathrm{CO}_{2}$ concentrations inside the room predicted by model II are somewhat lower than those by other models (see Figure 5.2.9(b)). Model II and III have 
accounted for the unburned fuels. The unburned fuel mass fractions inside the room and at the doorway, predicted by model II and III, are depicted in Figure 5.2.10. From 6 minutes to 10 minutes, the fuel mass fraction predicted by model II and III inside the room is approximately 0.1 (see Figure 5.2.10 (a)) and 0.06 (see Figure 5.2.10(b)) in the doorway, respectively. The high mass fraction of unburned fuel predicted by the models may be the reason for the low predictions of $\mathrm{CO}_{2}$ inside the room in this period. Compared with Model III, Model II produced lower $\mathrm{CO}_{2}$ and higher fuel concentrations.

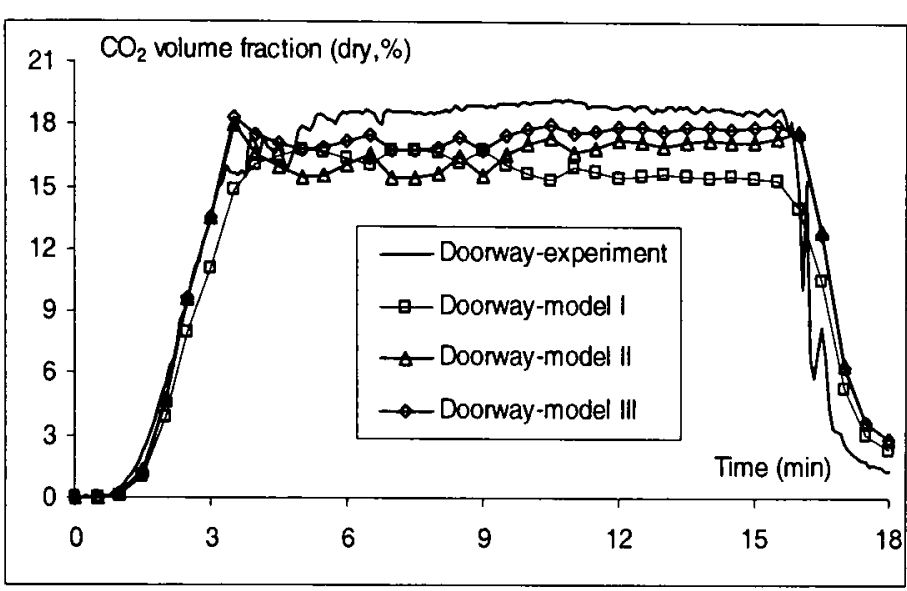

(a)

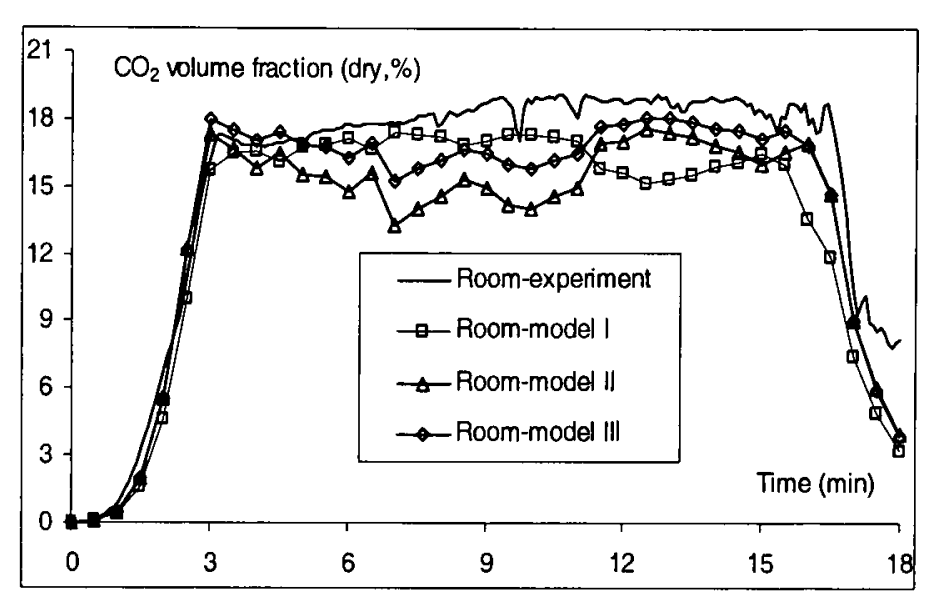

(b)

Figure 5.2.9: Measured and predicted $\mathrm{CO}_{2}$ volume fractions (a) at the doorway; (b) inside the room

Table 5.2.2: Comparison of measured and predicted averages (over the time period said in the table) of species volume fraction $(\%)$ inside the room and at the doorway.

\begin{tabular}{|l|l|l|l|l|l|l|}
\hline Location & Species & $\begin{array}{l}\text { Time } \\
\text { period } \\
(\mathbf{m i n})\end{array}$ & Exp. (\%) & $\begin{array}{l}\text { Model } \\
\mathbf{I}(\%)\end{array}$ & $\begin{array}{l}\text { Model } \\
\text { II (\%) }\end{array}$ & $\begin{array}{l}\text { Model III } \\
(\%)\end{array}$ \\
\hline \multirow{5}{*}{ Doorway } & $\mathrm{O}_{2}$ & $5-15$ & 0.579 & 1.61 & 0.0 & 0.0 \\
\cline { 2 - 7 } & $\mathrm{CO}_{2}$ & $5-15$ & 18.77 & 16.0 & 16.6 & 17.5 \\
\cline { 2 - 7 } & $\mathrm{CO}$ & $4-9$ & 3.68 & 2.82 & 2.69 & 2.89 \\
\cline { 2 - 7 } & $10-15$ & 1.24 & 1.45 & 1.59 & 1.65 \\
\hline \multirow{4}{*}{ Room } & $\mathrm{O}_{2}$ & $5-15$ & 1.30 & 0.86 & 0.0 & 0.0 \\
\cline { 2 - 7 } & $\mathrm{CO}_{2}$ & $5-15$ & 18.44 & 16.6 & 15.6 & 16.8 \\
\cline { 2 - 7 } & $\mathrm{CO}$ & $4-9$ & 4.75 & 3.82 & 3.33 & 3.67 \\
\cline { 3 - 7 } & & $10-15$ & 1.85 & 2.43 & 2.30 & 2.48 \\
\hline
\end{tabular}

The measured and predicted averages of $\mathrm{O}_{2}$ and $\mathrm{CO}_{2}$ volume fractions from 5 to 15 minutes are compared in Table 5.2.2. The averages of experimentally measured $\mathrm{O}_{2}$ volume fractions inside the room and at the doorway are below $1.3 \%$ while the averages of the simulation predictions are below 1.61 (Model I) and 0.0\% (Model II 
and III). The average of experimentally measured $\mathrm{CO}_{2}$ volume fraction in the doorway is $18.77 \%$ compared with the simulation predicted average value of $16.0 \%$ (Model I), producing an error of $14.8 \%$. The simulation prediction errors for the averaged $\mathrm{CO}_{2}$ volume fractions inside the room and at the doorway are less than $15.4 \%$ for all three models.

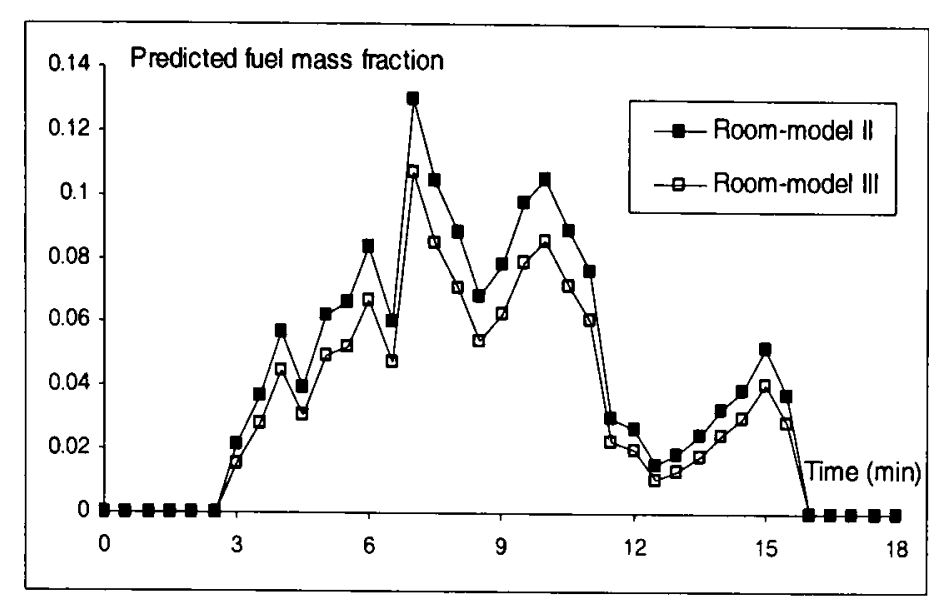

(a)

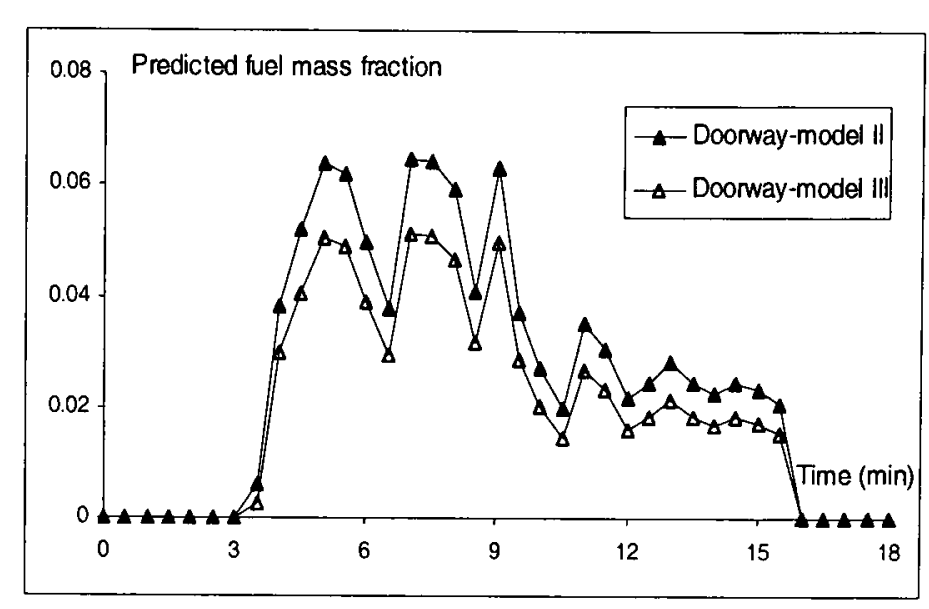

(b)

Figure 5.2.10: Predicted mass fractions of unburned fuel inside the room (a) and at the doorway (b) by model II and Model III.

\subsubsection{CO}

The $\mathrm{CO}$ volume fractions in the doorway and inside the room as functions of time are depicted in Figure 5.2.11(a)-(b). The measured $\mathrm{CO}$ volume fractions at the doorway are near $0 \%$ in the first 3 minutes. After 3 minutes, the $\mathrm{CO}$ volume fraction increases rapidly. It reaches $5.3 \%$ at approximately 8 minutes, and then rapidly drops between 9-10 minutes. A period of quasi-steady state is observed between 10-15 minutes. The measured $\mathrm{CO}$ volume fractions inside the room are close to $0 \%$ in the first 3 minutes. After 3 minutes, the $\mathrm{CO}$ volume fraction rapidly increases and reaches a value of $6.76 \%$ before 5 minutes, and then decreases to $0.5 \%$ at 8 minutes. The $\mathrm{CO}$ volume fractions fluctuate between $0.5 \%$ and $3 \%$ between $8-15$ minutes.

The predicted trends of doorway $\mathrm{CO}$ volume fractions by the three models are close to the measured values. The rapid drop of CO concentration, between 9-11 minutes, observed in the test results are similarly predicted by the LER based models. The measured peak CO volume fraction at 7.75 minutes of $5.29 \%$ is under predicted by $1.88 \%$ (relative error of $35.5 \%$ ) at 7.5 minutes in the simulation using Model I. Inside 
the room, the measured $\mathrm{CO}$ volume fractions are fluctuated between $0.5 \%$ and $3 \%$ from 8-15 minutes. The peak $\mathrm{CO}$ volume fraction $6.79 \%$ at 5 minutes is underpredicted by $0.74 \%$ (i.e. a relative error of $10.9 \%$ ) at 7 minutes by model I.

Since $\mathrm{H}_{2}$ is introduced as an intermediate product in model III, the unburned fuel mass fraction predicted by model III is lower than that by model II (see Figure 5.2.10). As a result, the predicted $\mathrm{CO}_{2}$ and $\mathrm{CO}$ concentrations predicted by model III are slightly higher than those predicted by model II (see Figure 5.2.9 and 5.2.11). However, the difference is not significant.

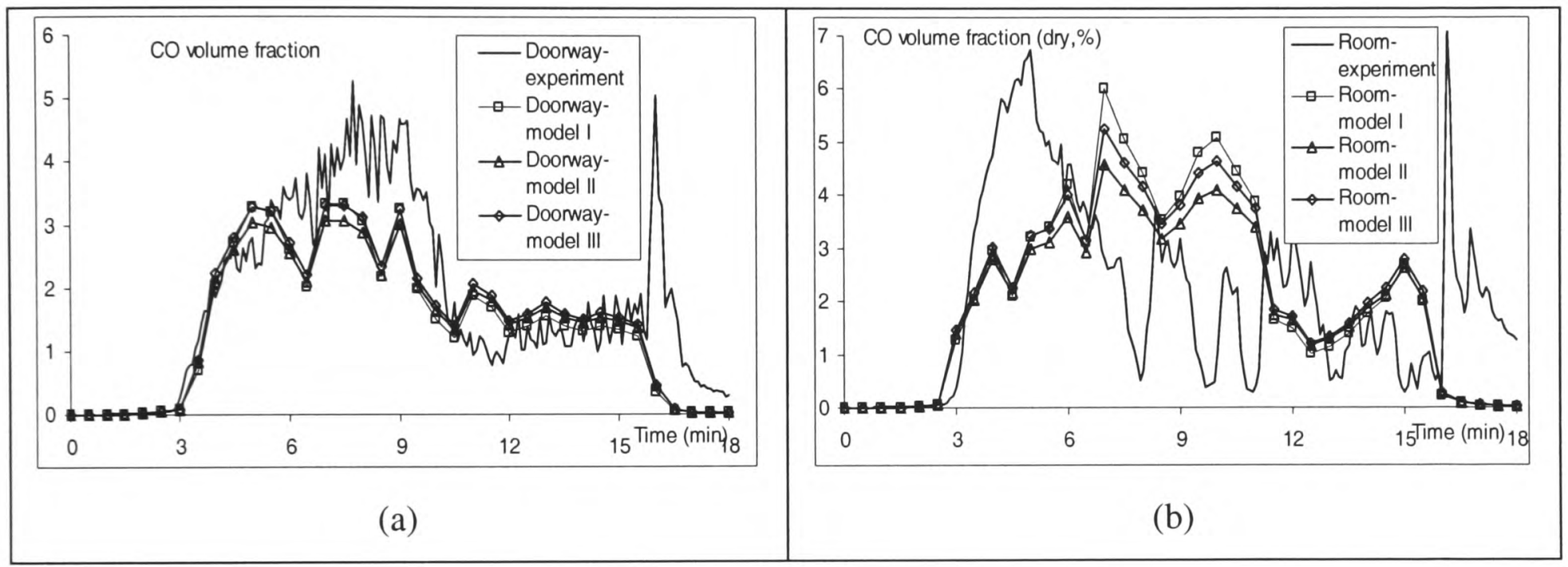

Figure 5.2.11: Measured and predicted $\mathrm{CO}$ volume fractions (a) at the doorway; and (b) inside the room.

The measured and predicted averages of $\mathrm{CO}$ volume fractions over the period of 4-9 minutes and 10-15 minutes are compared in Table 5.2.2. Model I, II and III predicted similar average $\mathrm{CO}$ concentrations.

As Models I, II and III predict similar results for the $\mathrm{CO}$ concentration except for significant high LER (See Chapter 4.3), in what follows, only Model I is used.

\subsection{Simulation of One of the TOXFIRE Tests}

\subsubsection{Description of the experiment}

A series of full-scale fire experiments have been reported in [LBM96]. The test selected here, 'PP3', is one of the polypropene $\left(\left[\mathrm{C}_{3} \mathrm{H}_{6}\right]_{n}\right)$ tests in the series. The test 
was conducted inside a lightweight concrete room with dimensions $(3.6 \mathrm{~m} \times 2.4 \mathrm{~m} \times$ $2.4 \mathrm{~m}$ ) and is shown in Figure 5.3.1. The ceiling and the upper walls were covered with $5 \mathrm{~cm}$ thick high-density mineral wool to protect the structure of the compartment. An opening of $0.8 \mathrm{~m} \times 0.89 \mathrm{~m}$ is centrally located on one of the short walls. The top of the opening was $2.0 \mathrm{~m}$ above the floor in the room. The fuel was placed in a pan, which had a rim of height $0.3 \mathrm{~m}$.

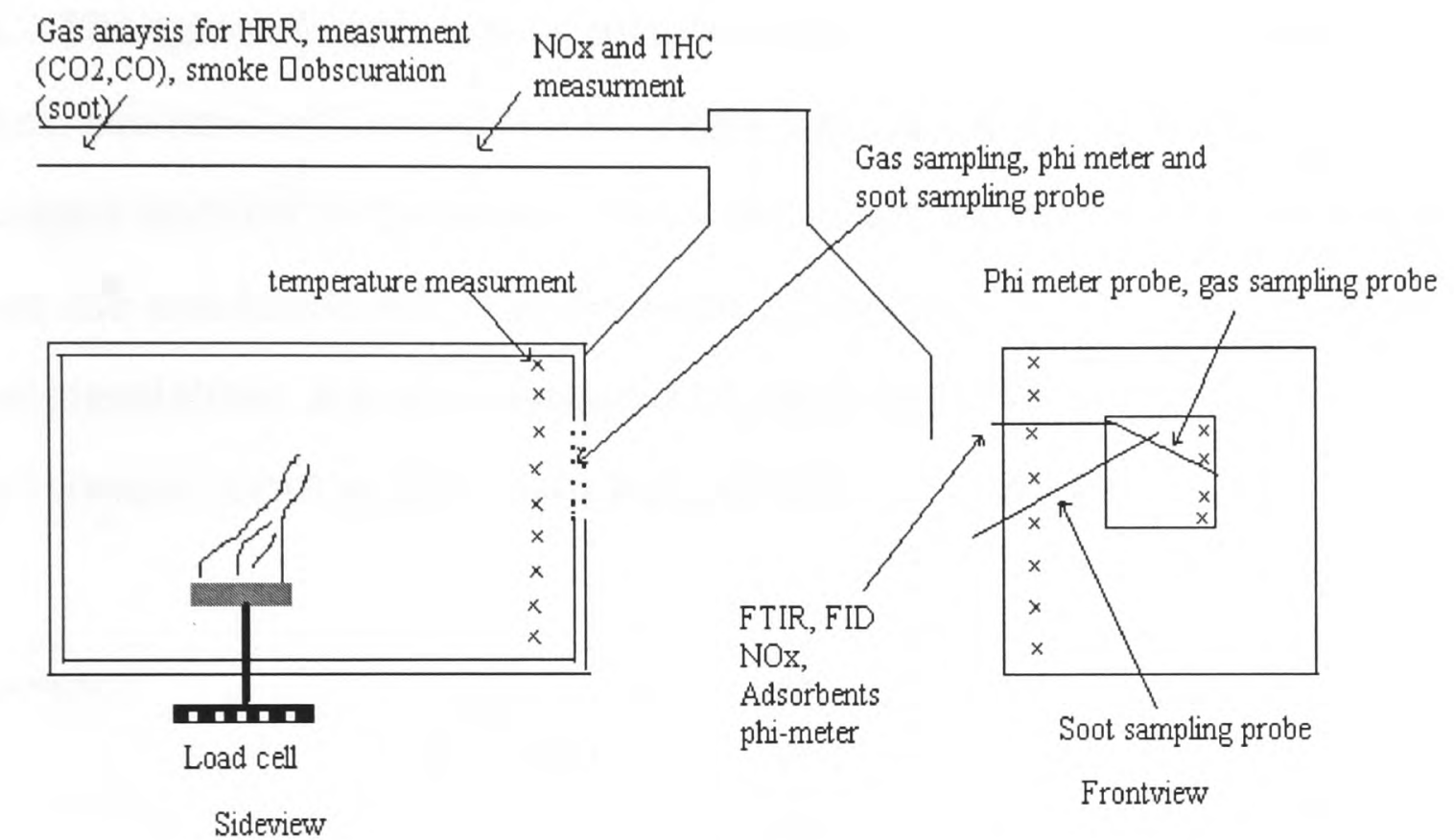

(a)

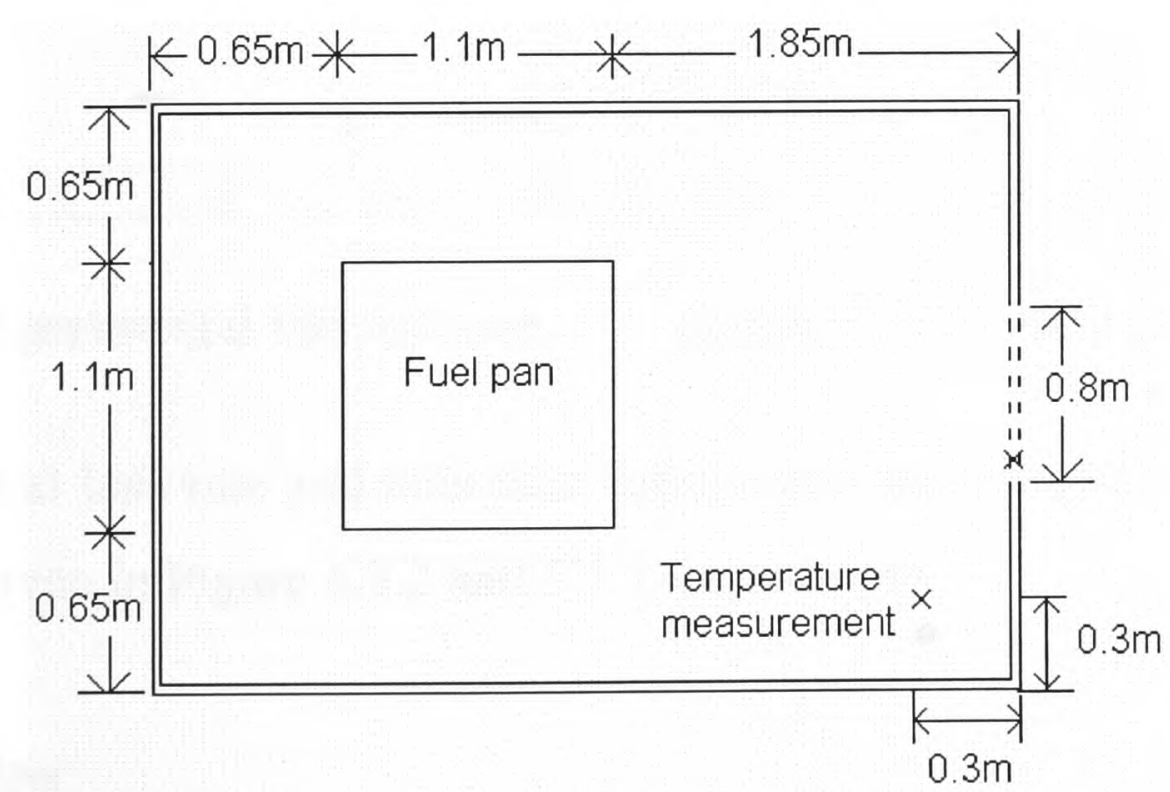

(b)

Figure 5.3.1: (a) Side view of the room and the probe positions in the opening and in the exhaust duct to the calorimeter system and (b) top view of the fire room.

A thermocouple tree, with 15 thermocouples, was mounted inside the room and was located $0.3 \mathrm{~m}$ from the two walls in the front left corner (see figure 5.3.1(b)). A second vertical thermocouple tree was placed in the opening, $0.1 \mathrm{~m}$ from the right hand edge of the opening. The combustion products from the room were collected by 
a hood connected to an exhaust duct. The HRR was determined using the production rates of $\mathrm{CO}_{2}, \mathrm{CO}$, soot and THC (total amount of unburned hydrocarbons) in the duct. A gas-sampling probe and a phi meter probe were placed diagonally from the top left corner to the middle of the other side of the opening. The suction end was in the top corner. The phi meter was used to measure the GER of the fire room.

In test PP3, $60 \mathrm{~kg}$ polypropene was placed in a $1.2 \mathrm{~m}^{2}$ pan. The ambient temperature was $19^{\circ} \mathrm{C}$. The ignition time was 1 minute and 25 second, and the total test time was 38 minutes. Because of the high heat release rate, some of the insulation in the ceiling was dislodged and fell to the floor. From the measured fuel loss rate in [LBM96], the time when the insulation fell was between 22 minutes and 25 minutes, therefore the numerical simulations are not continued beyond 22 minutes as the original conditions of the test compartment might have changed after this timeline.

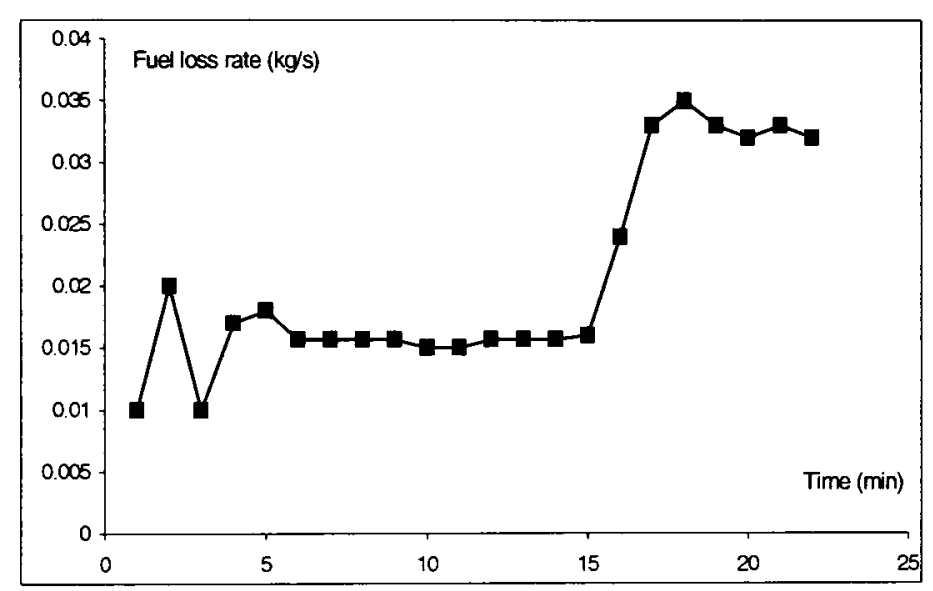

Figure 5.3.2: Experimental fuel lost rate.

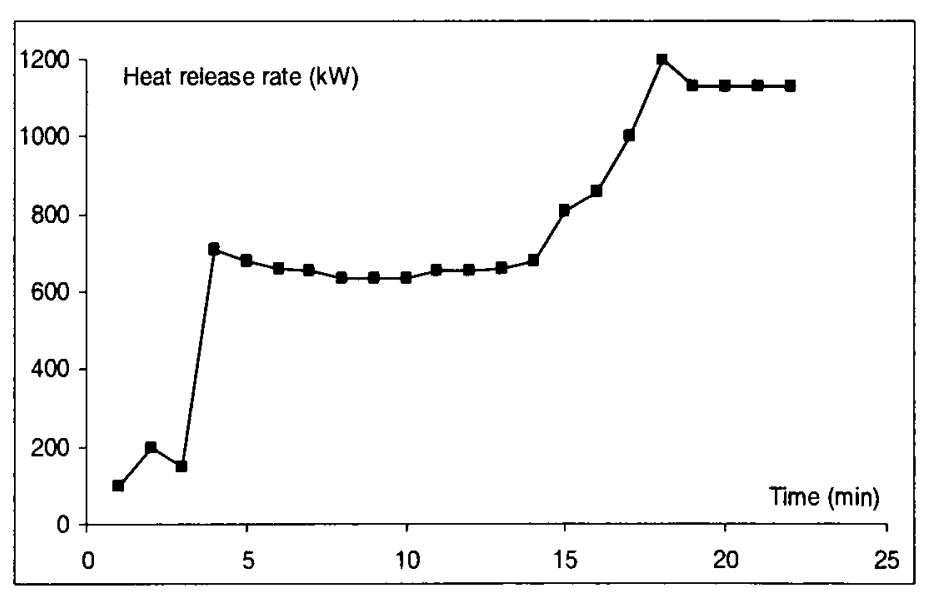

Figure 5.3.3: Total heat releases rate.

The measured fuel loss rate and total heat release rate according to the measurement in the duct are given in Figure 5.3.2 and 5.3.3 respectively.

\subsubsection{Simulation}

In the experiment, the total heat release rate (see Figure 5.3.3) was based on measurements made in the hood exhaust duct. However, since some fuel is transported outside of the compartment, which does not participate in the fire dynamics within the compartment, the heat released within the enclosure will be less than that measured in the exhaust duct. Therefore, the overall heat release rate measured (Figure 5.3.3) needs to be corrected. For the simulation that follow, a 
correction value of 0.7 is used as the compartment GER of this fire test reached as high as 1.4 .

In the experiment, only the upper parts of the walls are covered by insulation. In the model, the walls are assumed to be adiabatic. Therefore, it is necessary to adjust the heat release rate of the fire to compensate for the heat loss through the lower part of the walls. The heat loss rate by conduction through the walls is approximated by the following expression:

$$
\dot{q}=k \cdot \frac{\Delta T}{\Delta L} \quad\left(\mathrm{~W} / \mathrm{m}^{2}\right)
$$

where $k$ is the wall conductivity $(1.4 \mathrm{~W} / \mathrm{mK}$ for concrete), $\Delta T$ is the temperature differences between wall and the ambient (from 300 to $800^{\circ} \mathrm{C}$ ), $\Delta L$ is the thickness of the wall. According to equation (5.3.1), approximately $10 \%$ of the total heat released by the fire is lost through the wall. The heat release rate used in the simulation is therefore further adjusted to take the heat loss through the walls into account. The combined effect uses only $60 \%$ of the experimentally measured heat release. The simple volumetric heat source model is adopted here to simulate this released heat due to combustion.

The yields of species of polypropene burning are given by $(2.2 .3)$, which is repeated here by

$$
y_{i}(\phi)=y_{i, \infty}\left[1+\frac{\alpha}{\exp (\phi / \beta)^{-\zeta}}\right]
$$

where all the parameters are given in Table 5.3.1 [Te95].

The yields of products based on equations (5.3.2) are depicted in Figure 5.3.4. With the increase of GER, the generation of $\mathrm{CO}_{2}$ decreases while the generation of other species increase. These changes are small if GER is less than 0.8. The effect of GER on the generation of soot is minimal. However, the generation of $\mathrm{CO}, \mathrm{CO}_{2}$ and hydrocarbon are significantly affected by the GER. The yield of hydrocarbon is higher than $1 \mathrm{~kg} / \mathrm{kg}$ if the equivalence ratio is over 3.2. The $\mathrm{CO}$ yield is a monotonously increasing function with the equivalence ratio. These are unrealistic since the law of mass conservation is breached. Therefore, these correlations, 
particularly for hydrocarbon, are only valid to a limited range of LERs. However, as far as engineering applications of these correlations are concerned, we are primarily interested in locations distant from the fire source where LERs are not extremely high and so this is not considered to be a serious limitation with the approach.

Table 5.3.1: Parameters of correlation (5.3.2) for polypropene.

\begin{tabular}{|c|c|c|c|c|}
\hline & $y_{i, \infty}(\mathbf{k g} / \mathbf{k g})$ & $\alpha$ & $\beta$ & $\zeta$ \\
\hline $\mathbf{C O}$ & 0.024 & 10.0 & 1.39 & -1.8 \\
\hline $\mathbf{C O}_{\mathbf{2}}$ & 2.79 & -1.0 & 2.15 & -1.2 \\
\hline $\mathbf{O}_{\mathbf{2}}$ & 3.04 & -0.97 & 2.14 & -1.2 \\
\hline Hydrocarbon & 0.006 & 1800 & 1.58 & -3.5 \\
\hline Soot & 0.059 & 1.6 & 4.61 & -0.6 \\
\hline
\end{tabular}

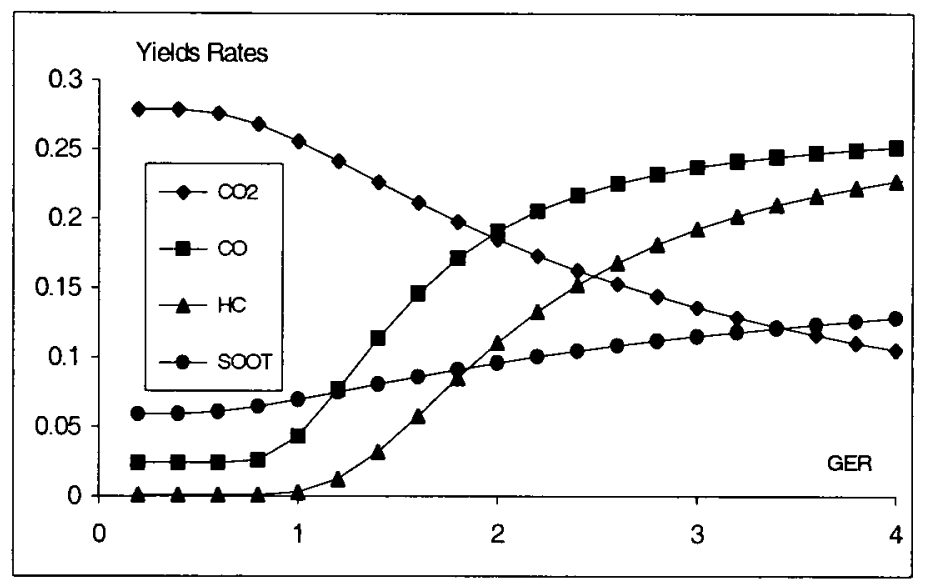

Figure 5.3.4: Yields of combustion products for polypropylene. Note: the units are 10 $\mathrm{kg} / \mathrm{kg}$ for $\mathrm{CO}_{2}, 1 \mathrm{~kg} / \mathrm{kg}$ for $\mathrm{CO}, 5 \mathrm{~kg} / \mathrm{kg}$ for $\mathrm{HC}$ and $1 \mathrm{~kg} / \mathrm{kg}$ for SOOT.

Only half of the enclosure was simulated and the central plane across the fire and the window was specified as a symmetric plane boundary condition. The mesh consisted of 10560 (i.e. $44 \times 20 \times 12$ ) computational cells. The volume fractions of $\mathrm{CO}, \mathrm{CO}_{2}$ and hydrocarbon were predicted at positions where the sampling probe was placed. The mesh in the opening and the eight positions $(\mathrm{A}, \mathrm{B} \ldots \mathrm{H})$ where the gas-sampling probe covered is shown in Figure 5.3.5. Soot was sampled in the opening using a probe at the position close to cell $\mathrm{D}$. Therefore, the predicted soot rate is the product of the predicted soot mass fraction at position $\mathrm{D}$ and the mass flow rate through the opening. A factor $\left(10000 \mathrm{~m}^{2} / \mathrm{kg}\right.$, see [LBM96]) was used for the conversion from light obscuration $\left(\mathrm{m}^{2} / \mathrm{s}\right)$ to soot production rate $(\mathrm{kg} / \mathrm{s})$. This factor is also used to convert the predicted soot production rate to the light obscuration $\left(\mathrm{m}^{2} / \mathrm{s}\right)$. 


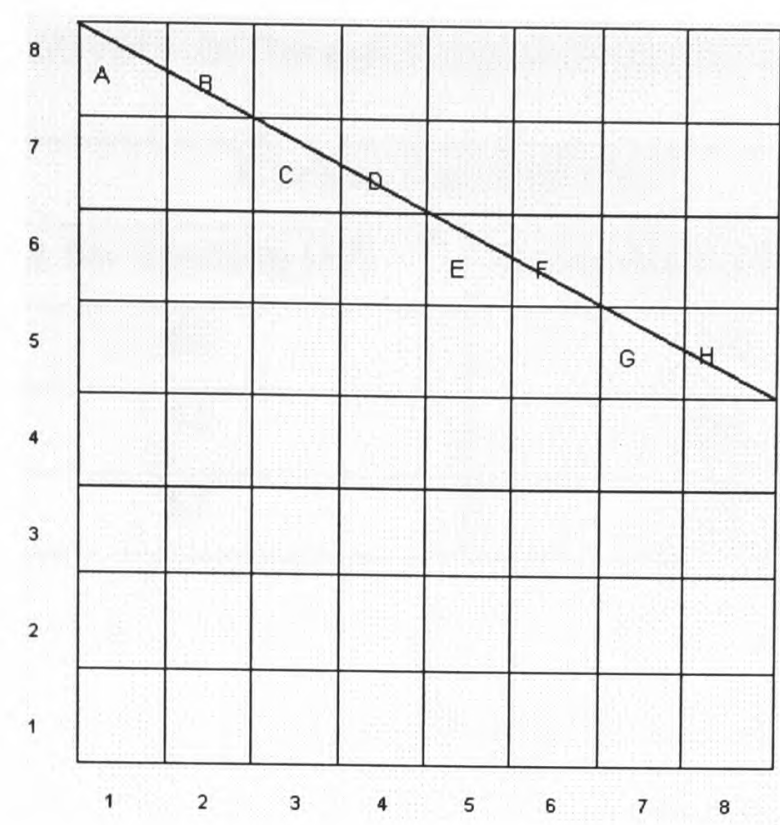

Figure 5.3.5: The computational mesh in the compartment opening (each cell measures $0.1 \mathrm{~m} \times 0.11 \mathrm{~m}$ ) and the gas-sampling probe being placed from the top left corner to the middle of other side of the opening.

\subsubsection{Results and discussions}

The carbon recovery, i.e. the ratio of the total amount of carbon in the different compounds to the total amount of carbon burned, was calculated in report [LBM96]. Table 5.3.2 presents the average carbon recovery in different positions and at different time periods. As seen in Table 5.3.2, the carbon recovery in the opening is very low while being approximately $100 \%$ in the duct. In the experiment, 'the yield of CO in the duct is almost twice as large as in the opening' especially when the GER is low. The reason for this is that 'Fresh air probably diluted the smoke gas' [LBM96]. Therefore, the predicted species concentrations at the opening are multiplied by the carbon recoveries there (see Table 5.3.2) and then are compared with the measured species concentrations at this location.

Because the insulation at the ceiling fell after 22 minutes, the simulation was only run for an equivalent of 22 minutes of real test time. The predictions are depicted in Figures 5.3.6-5.3.11. The ignition time was 1 minute and 25 seconds after the test started; the predictions are only compared with the experimental data after three minutes. As seen in Figure 5.3.5, the measuring probes occupy eight cells in the opening. The average concentration at the eight positions is compared with the measurement. 
Table 5.3.2: Carbon recovery in the opening and the duct (data from [LBM96]).

\begin{tabular}{|c|c|c|c|}
\hline \multirow{2}{*}{$\begin{array}{c}\text { Time period } \\
\text { (Min) }\end{array}$} & \multicolumn{2}{|c|}{ Carbon recovery (\%) } & Ratio of r1 to \\
\cline { 2 - 3 } & In the opening (r1) & In the duct (r2 ) & (\%) \\
\hline $\mathbf{4 - 1 1}$ & 63 & 103 & 61.16 \\
\hline $\mathbf{1 5 - 1 7}$ & 71 & 96 & 73.96 \\
\hline $\mathbf{1 9 - 2 1}$ & 62 & 95 & 65.26 \\
\hline
\end{tabular}

\subsubsection{Temperature}

The predicted and measured temperatures at the opening and the front corner are depicted in Figure 5.3.6. The measured temperatures increase rapidly between 3 minutes and 4 minutes, followed by a gradual increase. The predicted temperatures appear to follow this trend. Generally, the predicted temperatures over-estimate the measured temperatures through time at both locations. After 17 minutes, the temperatures in the front corner and at the opening are over predicted by approximately $9.8 \%$ and $15.9 \%$ respectively.

\subsubsection{Global equivalence ratio}

The predicted GER was calculated using the predicted airflow rate into the room and the measured fuel loss rate and is depicted in Figure 5.3.7. As can be seen from the measured values, the GER is around 0.5 between 4 minutes and 14 minutes. It increases after 14 minutes and reaches to the maximum 1.38 at 18 minutes, and then gradually decreases. The predicted GER follows this measured trend after 4 minutes. However, it is higher than the measured value. The largest measured GER 1.38 at 18 minutes is over-predicted by 0.247 or $17.9 \%$.

\subsubsection{3 $\mathrm{CO}$ and $\mathrm{CO}_{2}$}

The predicted $\mathrm{CO}$ volume concentration as a function of time is depicted in Figure 5.3.8. The measured concentrations in the first 15 minutes are low, since the GER is 
just about 0.5 in this period of time (see Figure 5.3.7). The measured concentration of $\mathrm{CO}$ rapidly increases after 15 minutes and reaches to $1.10 \%$ after 18 minutes. The simulation accurately reproduces this trend, generating the local maxima in the GER at 5 minutes and 18 minutes. The peak concentration at 18 minute is over predicted by $0.22 \%$ or a relative error of $20.0 \%$.

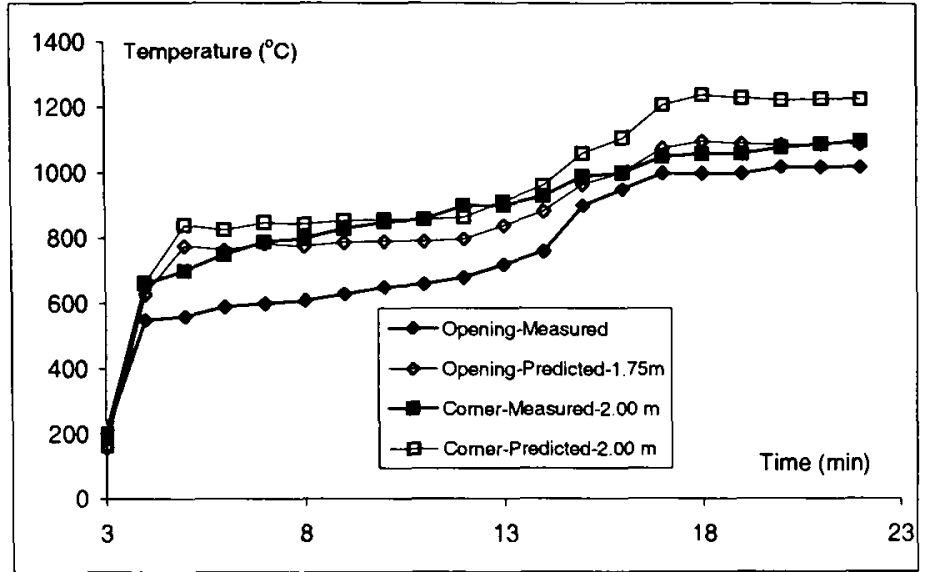

Figure 5.3.6: Predicted (open) and measured (solid) temperatures as functions of time.

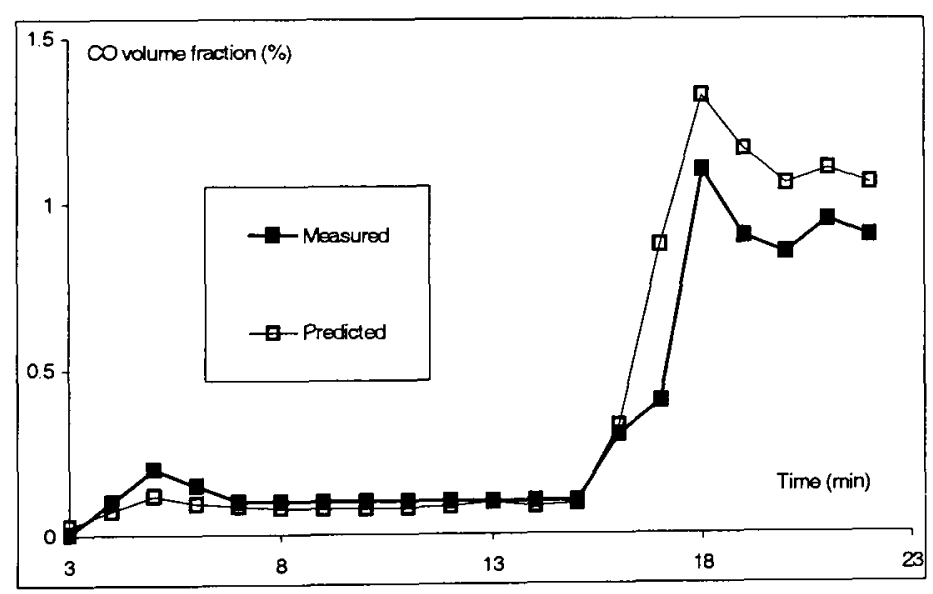

Figure 5.3.8: Predicted (open) (at positions from $\mathrm{A}$ to $\mathrm{H}$ and their average) and measured (solid) $\mathrm{CO}$ as functions of time.

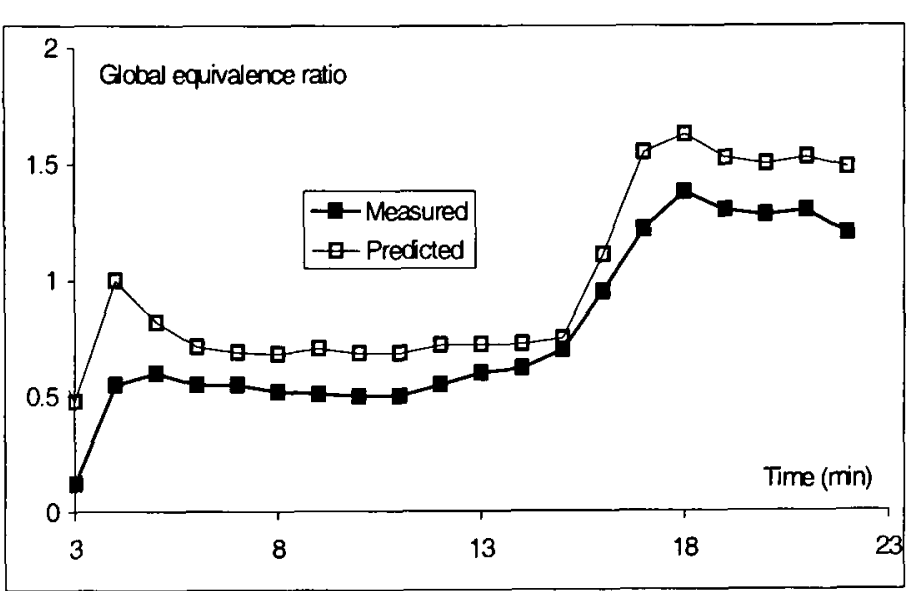

Figure 5.3.7: Predicted (open) and measured (solid) GER as a function of time.

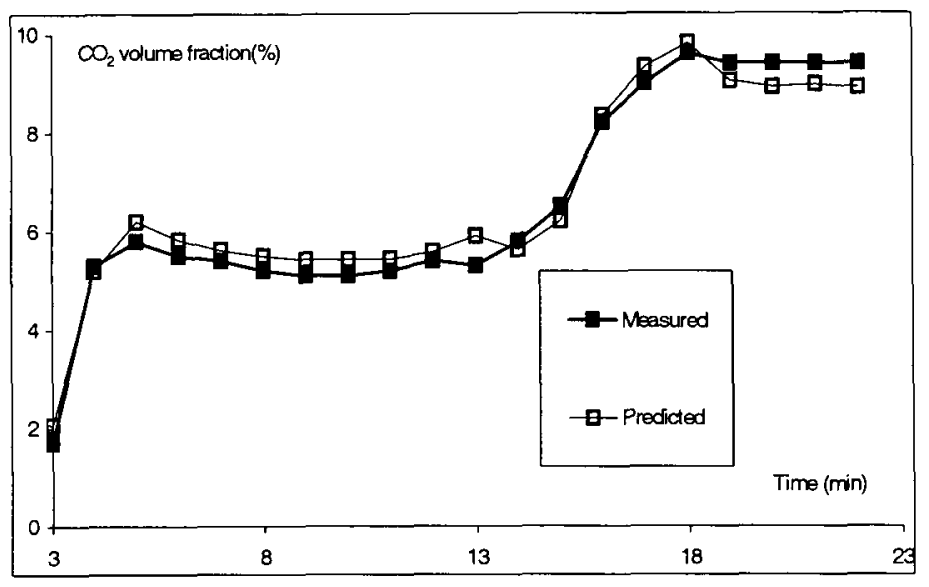

Figure 5.3.9: Predicted (open) and measured (solid) $\mathrm{CO}_{2}$ volume fraction (\%) as a function of time.

The predicted $\mathrm{CO}_{2}$ volume fraction against time is depicted in Figure 5.3.9. The trend in the change of $\mathrm{CO}_{2}$ concentrations is also well predicted. The predictions are in good agreement with the measured values from 3 minutes to 22 minutes. The peak concentration is over-predicted by 0.2 ) or a relative error of $2.1 \%$. At the two quasisteady states (from 5 minutes to 13 minutes and from 18 minutes to 22 minutes), the relative average error of predictions are $6.2 \%$, and $3.3 \%$ respectively. 


\subsubsection{Hydrocarbon and smoke}

The predicted volume fraction of unburned hydrocarbon (propane) is depicted in Figure 5.3.10. After 16 minutes, the predicted values are significantly higher than the measured values. This discrepancy maybe stem from the inconsistency associated with the instrumentation measuring hydrocarbon in experiments. It is known that determining the concentration of unburned hydrocarbon is sensitive to the type of instrumentation used. The type of instrumentation used to measure the concentration of the unburned hydrocarbon in PP3 may be different from that used in the test, which generated correlation (5.3.2). Therefore, the actual correlation of hydrocarbon to the equivalence ratio in test PP3 may be quite different from that estimated by correlation (5.3.2).

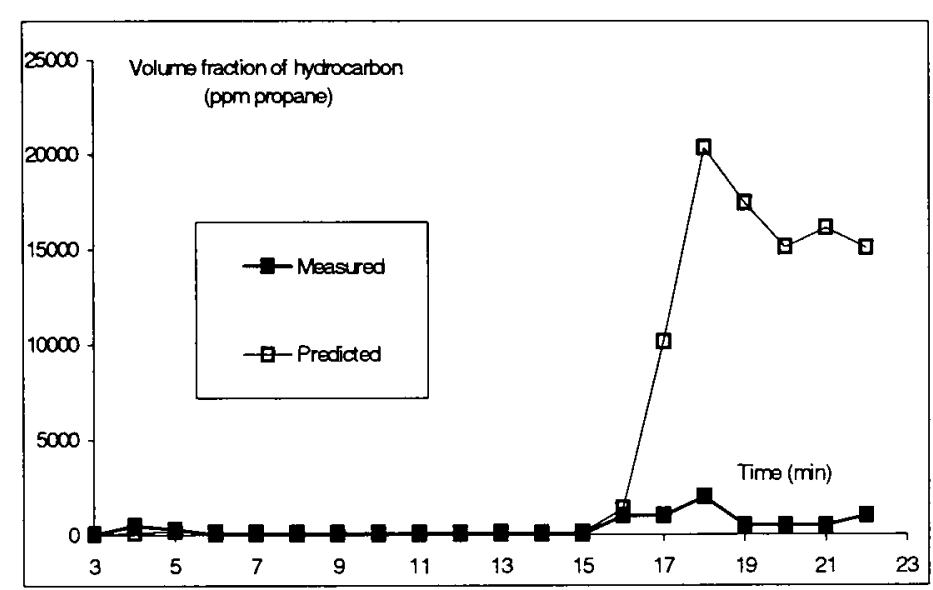

Figure 5.3.10: Predicted (open) and measured (solid) hydrocarbon volume fractions (ppm) in the opening as functions of time.

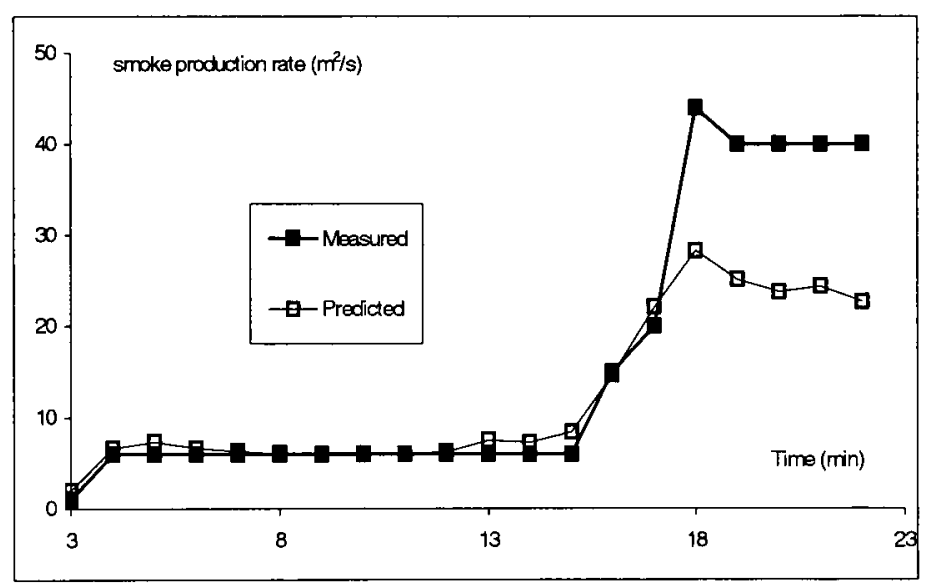

Figure 5.3.11: Predicted (open) and measured (solid) soot rates $\left(\mathrm{m}^{2} / \mathrm{s}\right)$ in the opening as functions of time.

The smoke production rate was calculated using the soot mass fraction at position $\mathrm{D}$ in the opening. As seen from Figure 5.3.11, the predicted smoke rates are in good agreement with the measured values over the first 17 minutes. However, they are lower than the measurements after 18 minutes.

\subsection{Simulation of W14-B3 Fire Test}

\subsubsection{Description of the experiment}

The experiment used here is one of the tests in Scenario B of CIB W14 Round Robin [HK98]. Scenario B consisted of three different fire scenarios, B1, B2, and B3. Case 
B1 has previously been successfully simulated using the SMARTFIRE software in Appendix A (or see [WJ00, WJ01a] for detail). The results presented here are for scenario B3 in which the CO concentrations were measured. Case B3 is an example of a situation where the fire spreads from one source to another in a low space, similar to fire spread from one car to another in a tunnel.

The fire compartment measured $7.4 \mathrm{~m} \times 7.2 \mathrm{~m}$ in plan and $3.6 \mathrm{~m}$ in height. It contained a window of dimensions $3.0 \mathrm{~m} \times 1.24 \mathrm{~m}$ and a ceiling mounted concrete down stand (see Figure 5.4.1). The walls of the compartment were made of aerated concrete blocks (with siporex mortar) with a thickness of $0.3 \mathrm{~m}$ and with the following material properties: specific heat $1.05 \mathrm{~kJ} / \mathrm{kg} . \mathrm{K}$, thermal conductivity 0.12 $\mathrm{W} / \mathrm{m} . \mathrm{K}$ and density $500 \mathrm{~kg} / \mathrm{m}^{3}$. The initial air temperature was measured as $20.0^{\circ} \mathrm{C}$.
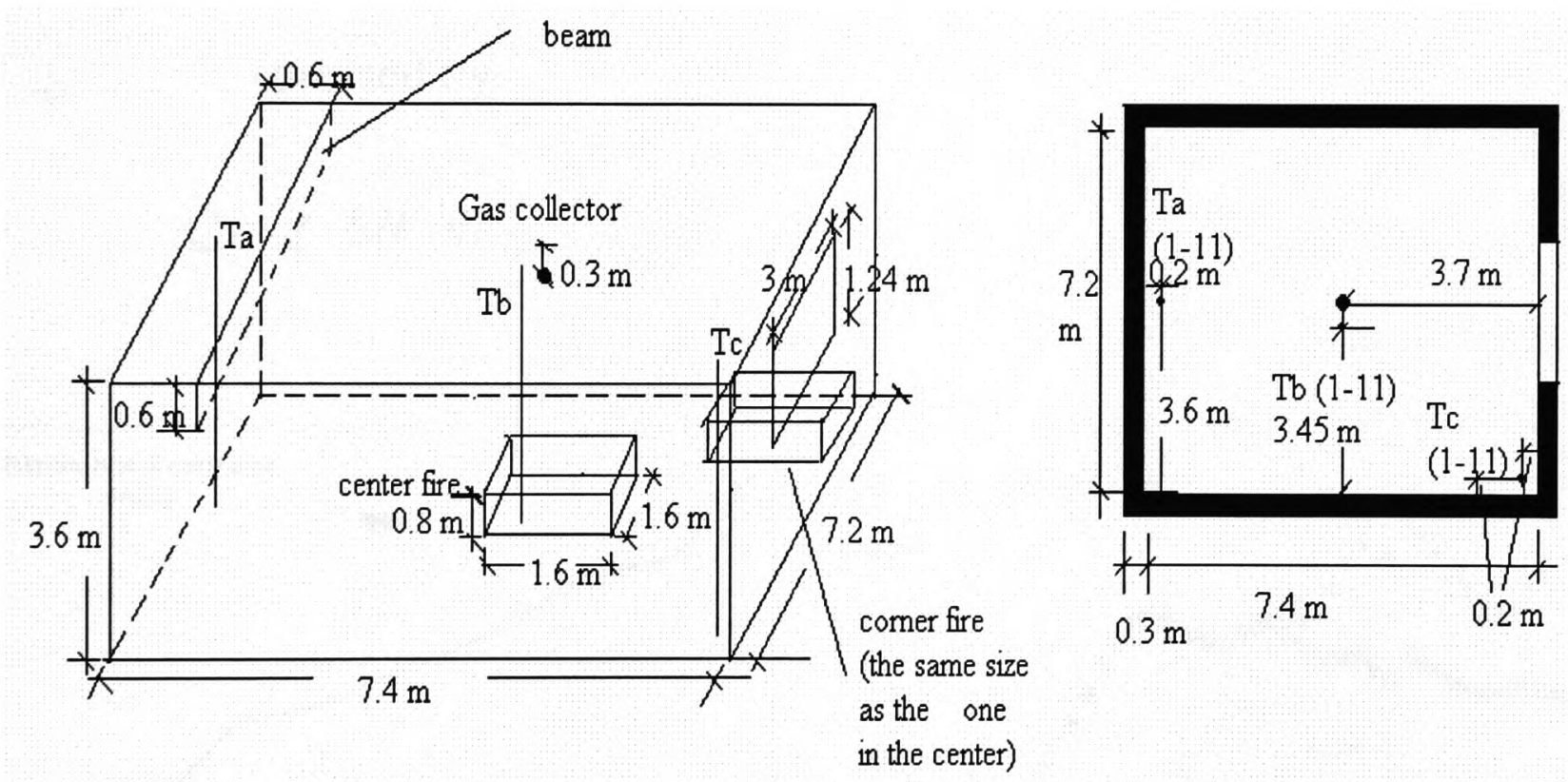

Figure 5.4.1: The geometry of W14-b3 test and the locations of the instrumentation.

One fire was located on the floor in the centre of the room while the other was located in the left front corner (see Figure 5.4.1). The fire fuel consisted of softwood (Pinea ecelsa, $\left.\mathrm{CH}_{1.7} \mathrm{O}_{0.83}\right)$ timber cribs nailed into $40 \mathrm{~mm} \times 40 \mathrm{~mm}$ battens. The two cribs measured $1.6 \mathrm{~m}$ in length, $1.6 \mathrm{~m}$ in width and $0.8 \mathrm{~m}$ in height. The corner fire is ignited first followed by the central fire after 11 minutes. Gas temperatures were measured with three vertical rakes of thermocouples located in the right front corner, centre and near the centre of the rear wall. The concentrations of $\mathrm{O}_{2}, \mathrm{CO}_{2}$ and $\mathrm{CO}$ were all measured at a single location in the centre of the compartment and just $0.3 \mathrm{~m}$ 
under the ceiling. The positions of the thermocouple rakes and the gas collector are illustrated in Figure 5.4.1.

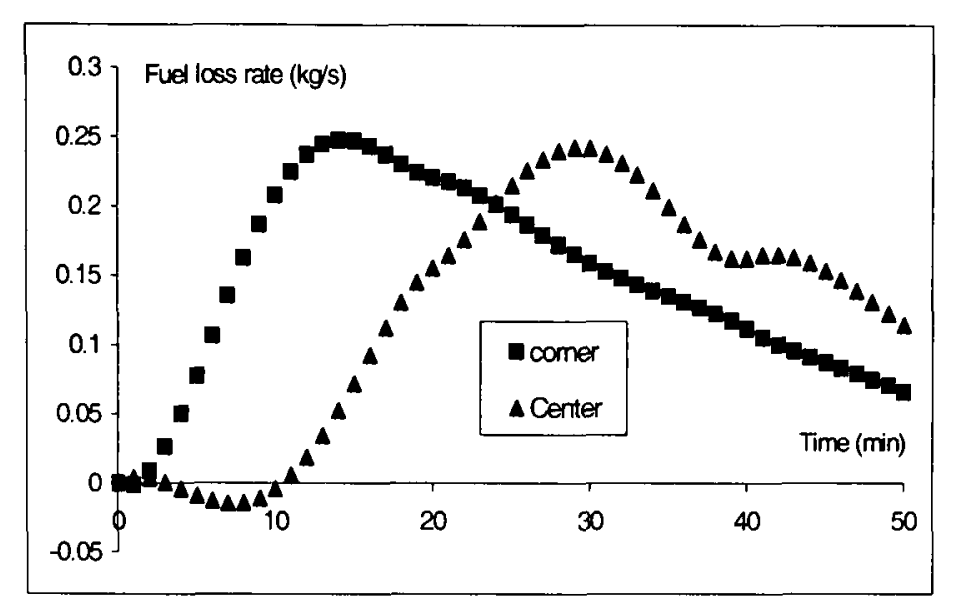

Figure 5.4.2: Fuel loss rates of the two fires.

The fuel loss rates of the two fire sources in scenario B3 were measured using load cells. They are shown in Figure 5.4.2. The correlation of the heat release rate to the burning rate is expressed by

$$
\dot{Q}=\chi \cdot \Delta H \cdot \dot{m}
$$

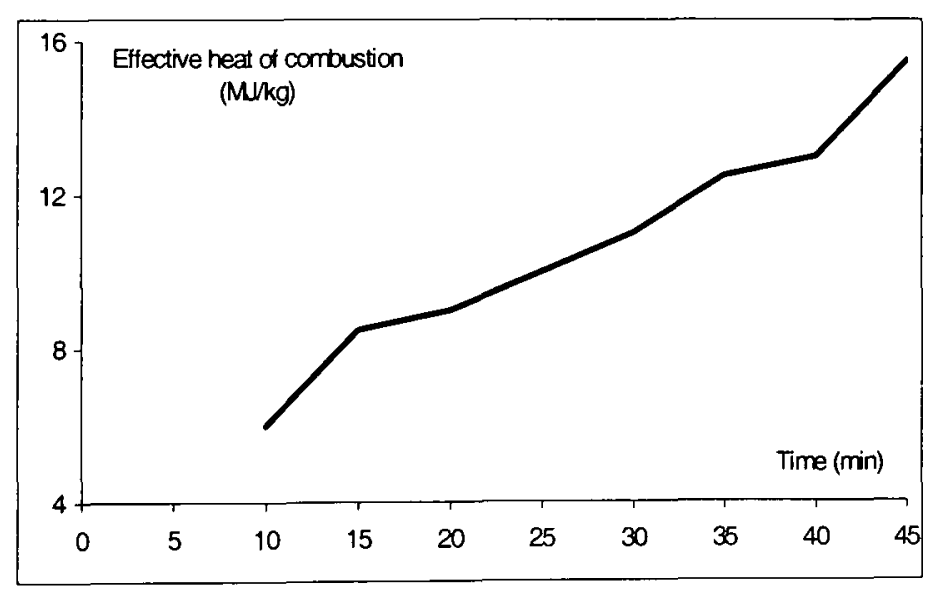

(a)

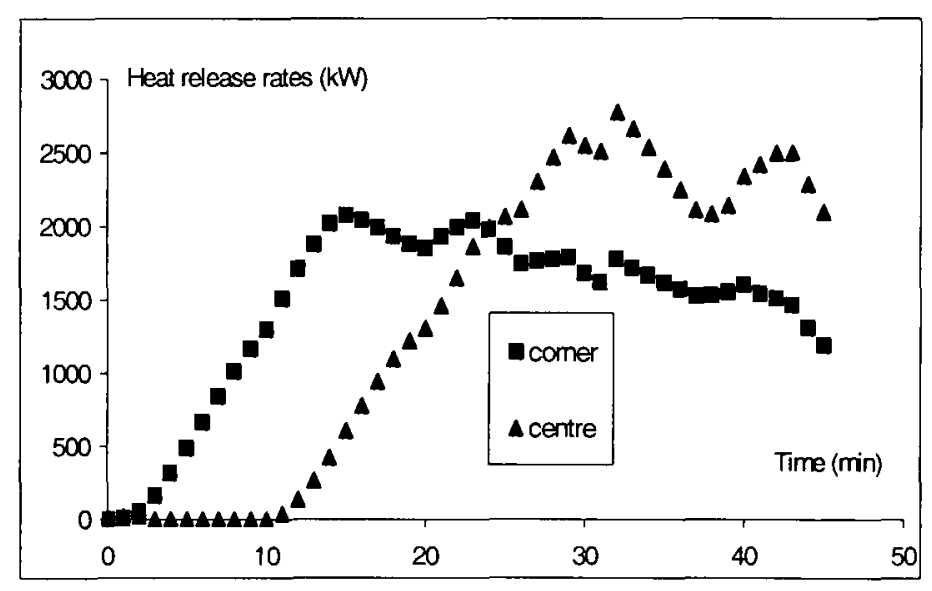

(b)

Figure 5.4.3: (a) The effective heat of combustion (data from Figure 8 in [HK98]); (b) the heat release rates of the two fires.

The efficiency factor and heat of combustion were given in [HK98] as $\chi=0.7$ and $\Delta H$ is $17.8 \mathrm{MJ} / \mathrm{kg}$ for $\mathrm{B} 1$ and $\mathrm{B} 2$ tests. But varied combustion efficiency factors are found for B3 test and used in this study. The effective heat of combustion $(\chi \cdot \Delta H)$ as function of time for B3 test are depicted in Figure 5.4.3(a) [HK98] while the heat release rates calculated using formula (5.4.1) with the corresponding varied efficiency 
factor $\chi$ are depicted in Figure 5.4.3(b). There are errors in the measured fuel loss rates due to the high levels of turbulence generated during the experiment. These errors manifest themselves as situations where the measured fuel loss rate is negative. In these cases, the fuel loss rates are assumed zero.

Unfortunately, the GER was not measured in the experiment [HK98] and so predicted GERs cannot be compared with measured values. The purpose of this simulation is to demonstrate the capability of the model to reproduce the basic trends in combustion product generation for a complex fire scenario.

\subsubsection{Simulation}

The volumetric heat source model is used to represent the two heat sources. The release rates of fuel and heat for the two fire sources are depicted in Figures 5.4.2 and 5.4.3(b). The mesh consisted of 14175 (i.e. $35 \times 15 \times 27$ ) computational cells. Yields of species of wood burning are given by (2.2.3), which is repeated here by

$$
y_{i}(\phi)=y_{i, \infty}\left[1+\frac{\alpha}{\exp (\phi / \beta)^{-\zeta}}\right]
$$

All parameters in correlation (5.4.2) for wood have been provided in Table 5.2.1 for the RI fire scenario.

\subsubsection{Results and discussions}

\subsubsection{Temperature}

The simulation was run for an equivalent of 45 minutes of real time. The temperature at the third thermocouple $(0.9 \mathrm{~m}$ under the ceiling) of each thermocouple tree is shown in Figure 5.4.4. In the first 15 minutes, the highest temperatures are measured at locations $C$ (i.e. front corner of the room) and the lowest temperatures are measured at location A (close to rear wall). After 25 minutes, the order is reversed. The predicted temperatures essentially follow this trend. However, the predictions are somewhat higher than the measurements from 15 to 30 minutes. 


\subsubsection{2 $\mathrm{CO}_{2}$}

The predicted GERs gradually increase from start of the fire and reach a maximum of 1.13 at 32 minutes. The predicted LER at the position for gas sampling reaches a maximum of 1.63 at 29 minutes. The predicted GER suggests that this fire is only slightly under-ventilated at the peak of the fire. As can be seen in Figure 5.4.5, the measured $\mathrm{CO}_{2}$ concentration increases gradually over the first 32 minutes and reaches its peak value $18.19 \%$ after 32 minutes and then slowly decreases. The prediction is in good agreements with this trend. The predicted peak value is $18.01 \%$ at 29 minutes. It is only under-predicted by $0.18 \%$ or a relative error of $1.0 \%$.

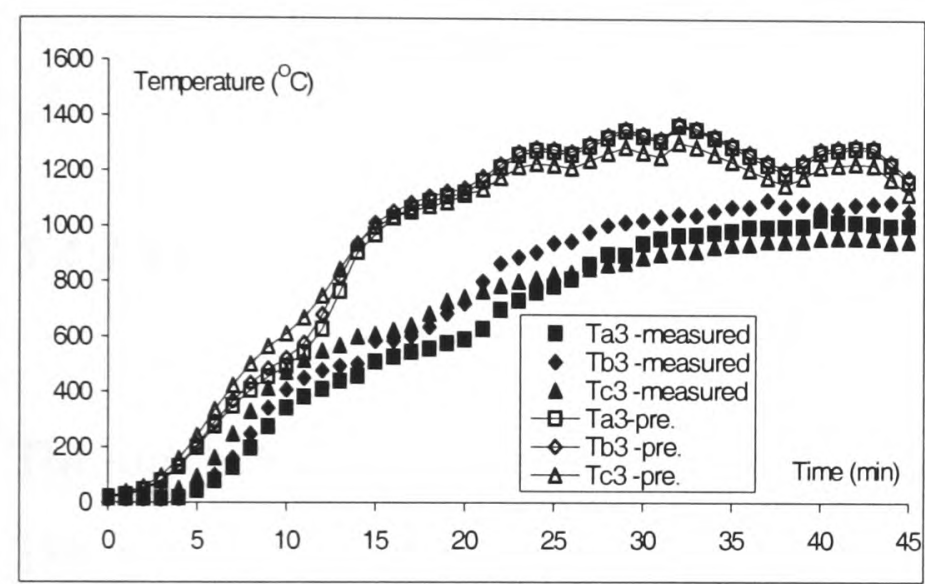

Figure 5.4.4: Predicted (open) and measured (solid) temperatures as functions of time.

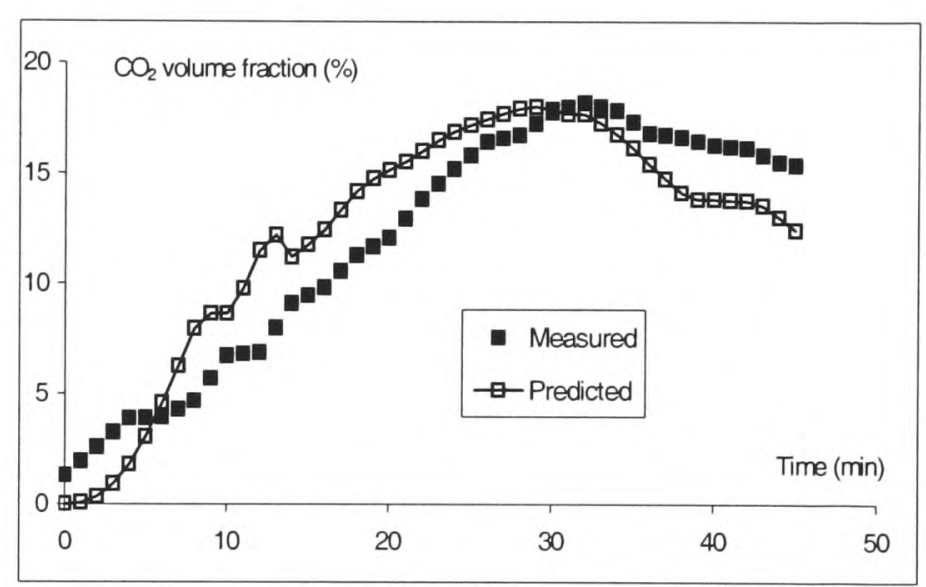

Figure 5.4.5: Predicted (open) and measured (solid) concentrations of $\mathrm{CO}_{2}$ as functions of time.

\subsubsection{3 $\mathrm{O}_{2}$}

The predicted $\mathrm{O}_{2}$ concentrations are depicted in Figure 5.4.6. The measured oxygen concentration is almost a constant value of $19.19 \%$ in the first 6 minutes. It decreases to $5 \%$ at 30 minutes and maintains this level until 45 minutes. Generally, the model predictions are lower than the measurements. The predictions fall to 0 after 28 minutes and remain at this value until 2 minutes have elapsed. A minimum measured value of $5 \%$ after 30 minutes is shown in Figure 5.4.6. It is reported that this value is the result of some kind of saturation in the measurement system and does not correspond to the reality [HK98]. As seen in Figure 5.4.2, the total fuel loss rate suggests that the fire was in its peak phase at around 30 minutes. This would suggest that the $\mathrm{O}_{2}$ concentration would be at its minimum value around this time. Therefore, 
the predicted minimum in $\mathrm{O}_{2}$ concentration is in qualitative agreement with this however, quantitatively it is under predicted.

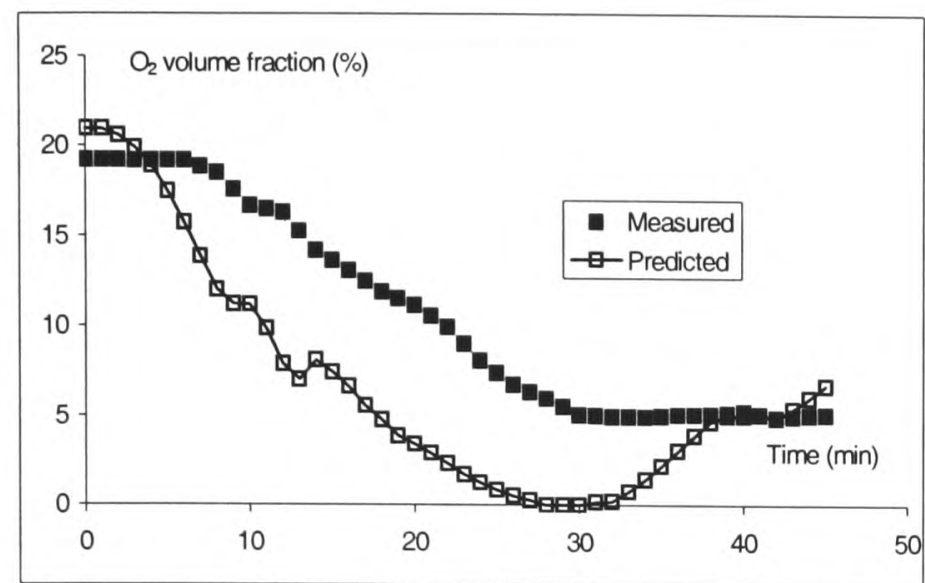

Figure 5.4.6: Predicted (open) and measured (open) concentrations of $\mathrm{O}_{2}$ as functions of time.

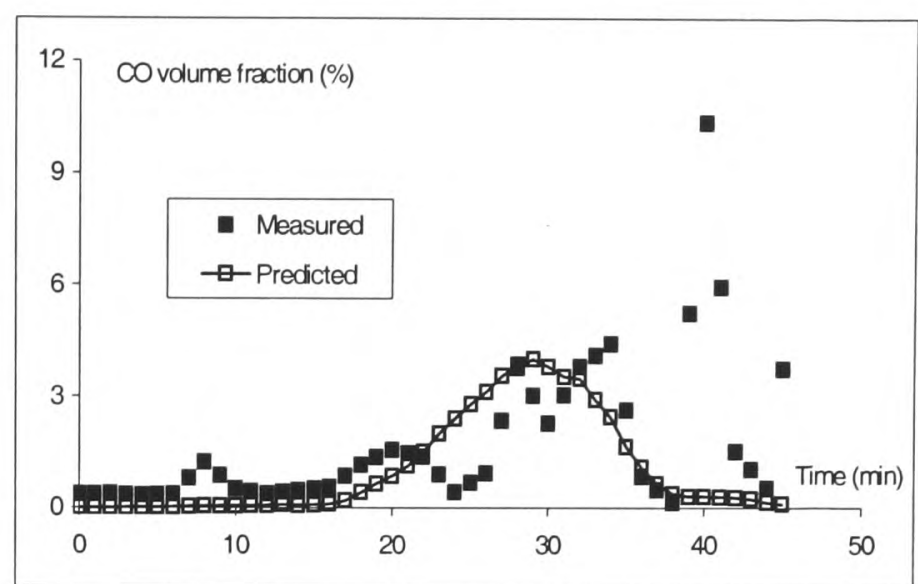

Figure 5.4.7: Predicted (open) and measured (solid) concentrations of $\mathrm{CO}$ as functions of time.

\subsubsection{CO}

The predicted $\mathrm{CO}$ concentrations as functions of time are depicted in Figure 5.4.7. The measured $\mathrm{CO}$ concentration in the first 6 minutes is about $0.35 \%$. The first local maximum is $1.23 \%$ at 8 minutes. It is about $0.5 \%$ between 10 minutes and 16 minutes. After 35 minutes, especially between 38 minutes and 45 minutes, there are fluctuations in the test result. For example, the measured $\mathrm{CO}$ concentration at 38 minutes was only $0.13 \%$. It was $10.35 \%$ at 40 minutes and was only $1.52 \%$ at 42 minutes (see Figure 5.4.7). The measured peak $\mathrm{CO}$ concentration in the first 35 minutes is $4.41 \%$ at 34 minutes.

The predicted CO concentrations are close to zero in the first 15 minutes. The trend in predicted CO concentration in the simulations is reasonable from 15 to 35 minutes. The predicted peak values are $4.01 \%$ at 29 minutes. The peak concentration of $\mathrm{CO}$ is over predicted by $0.40 \%$ or a relative error of $9.07 \%$. No conclusion can be drawn after 38 minutes because of the intense fluctuations in the measurements.

The ratio of $\mathrm{CO}$ to $\mathrm{CO}_{2}$ of wood combustion has been studied in [Ts94]. The combustion of wood includes three different stages. The first stage is pre-flaming pyrolysis, in which, the rate of generation of $\mathrm{CO}$ and $\mathrm{CO}_{2}$ steadily increases with time. 
The ratio of $\mathrm{CO}$ to $\mathrm{CO}_{2}$ is high at this stage. The second stage is flaming pyrolysis. As soon as the wood is ignited, the ratio of $\mathrm{CO}$ to $\mathrm{CO}_{2}$ rapidly falls to its lowest value. The third stage is char combustion or glowing, in which the ratio of generation rate of $\mathrm{CO}$ to $\mathrm{CO}_{2}$ is about 0.2 . The central fire in the test simulated here was ignited some 10 minutes after the first fire (see Figure 5.4.3(b)). Therefore, the wood in the centre of the floor was in the first combustion stage at 8 minutes and much $\mathrm{CO}$ was generated by pyrolysis. After 10 minutes, the combustion of the central wood cribs was in the flaming stage and then the $\mathrm{CO}$ generation rate decreased compared with that in the pyrolysis process. This is probably the reason for the first local $\mathrm{CO}$ maximum at 8 minutes. The present model cannot predict this local $\mathrm{CO}$ maximum. One reason is that the $\mathrm{CO}$ formation by the pyrolysis of wood is not modelled in the present model. Another reason is that only one scalar is used to represent the gas mixture. The mass fractions of fuel originated from the two fire sources cannot be separately expressed. However, the predictions of $\mathrm{CO}$ do reflect the trend during the flaming stage.

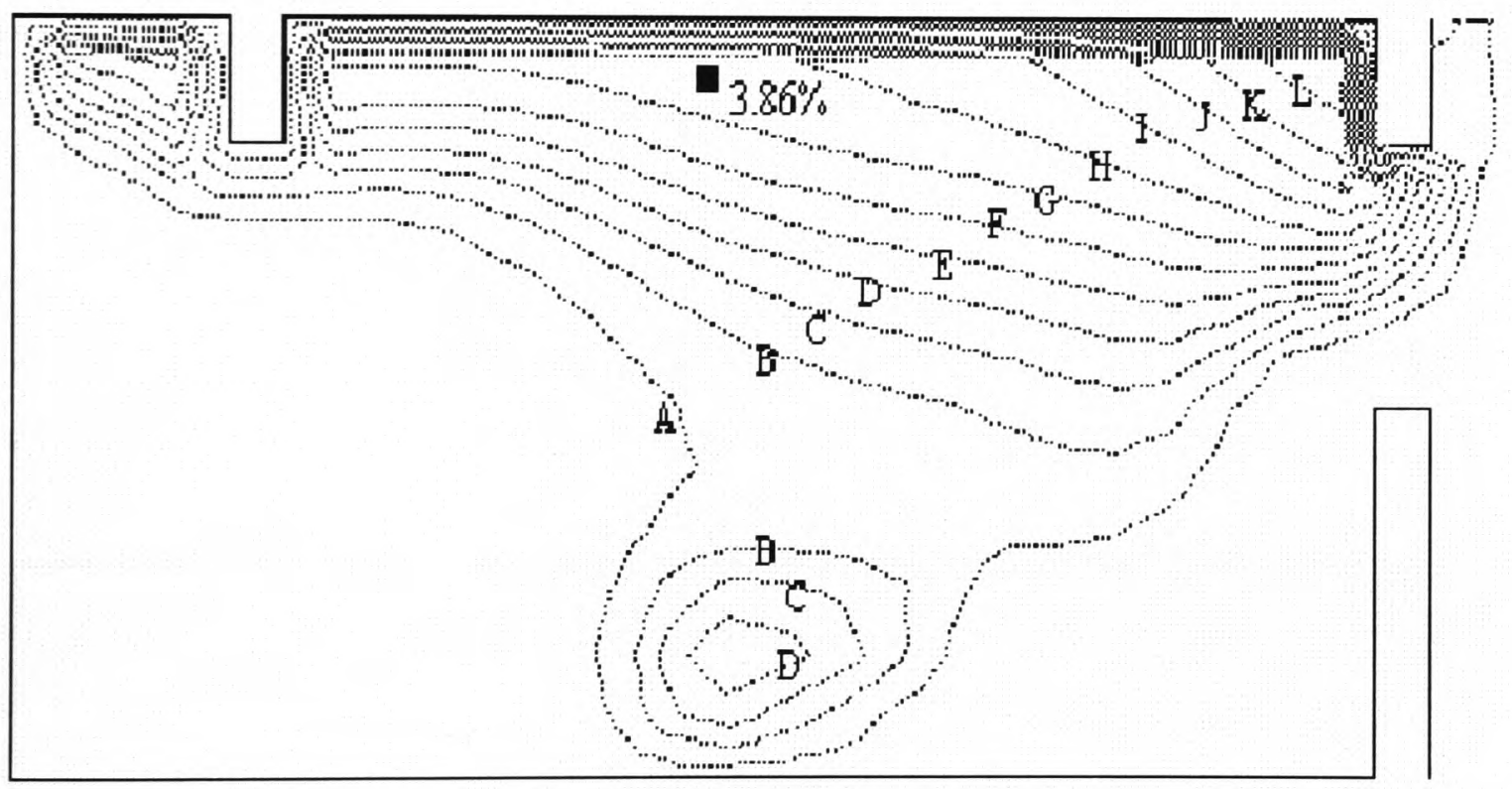

CO Volume Fraction $(\%$, dry)

A $\quad 0.5$ B 1.0

C 1.5 D 1.0

E $2.5 \quad$ F 3.0

$\begin{array}{llll}\mathrm{G} & 3.5 & \mathrm{H} & 4.0\end{array}$

I $\quad 4.5 \quad \mathrm{~J} \quad 5.0$

$\begin{array}{llll}\mathrm{K} & 5.5 & \mathrm{~L} & 6.0\end{array}$

Figure 5.4.8: Predicted CO contours in the central plane through the opening and the gas collector at 28 minutes. Note: the black square depicts the gas collector and the measured $\mathrm{CO}$ volume fraction is $3.86 \%$.

The predicted $\mathrm{CO}$ contours at 28 minutes in three different planes are depicted in Figures 5.4.8 to 5.4.10. As seen in the three Figures, the distributions of $\mathrm{CO}$ are significantly affected by the position of the fire sources. The front corner fire generates the high $\mathrm{CO}$ concentrations near the ceiling in the front (see Figure 5.4.8). The CO concentrations in the right half of the room are higher than that in the left half because the two fires located on the centre and the right side of the floor respectively 
(see Figure 5.4.9). In the opening window, high $\mathrm{CO}$ concentration (as high as 4\%) is observed in the area of the right top corner (see Figure 5.4.10). Locations with relative low $\mathrm{CO}$ concentrations, where the $\mathrm{CO}$ concentrations are below $1 \%$ (see Figure 5.4.10), account for approximately one third of the opening area.

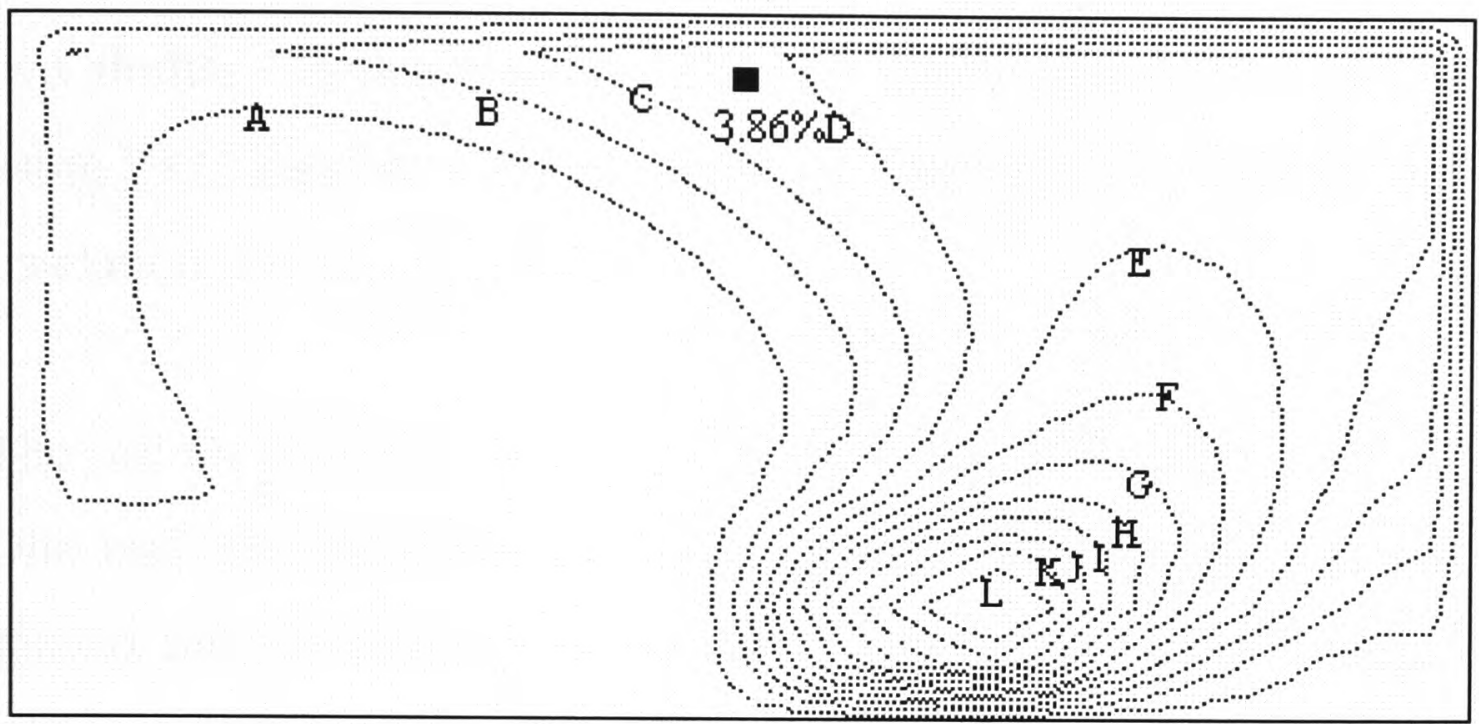

$\mathrm{CO}$ Volume Fraction (\%, dry)

A $1.0 \quad$ B 2.0

C $\quad 3.0 \quad \mathrm{D} \quad 4.0$

E $\quad 5.0 \quad$ F $\quad 6.0$

G $\quad 7.0 \quad \mathrm{H} \quad 8.0$

I $\quad 9.0 \mathrm{~J} \quad 10$.

K 11. L 12 .

Figure 5.4.9: Predicted CO contours in the plane through the gas collector and thermocouple tree $\mathrm{Tb}$ at 28 minutes. Note: the black square depicts the gas collector and the measured $\mathrm{CO}$ volume fraction is $3.86 \%$.

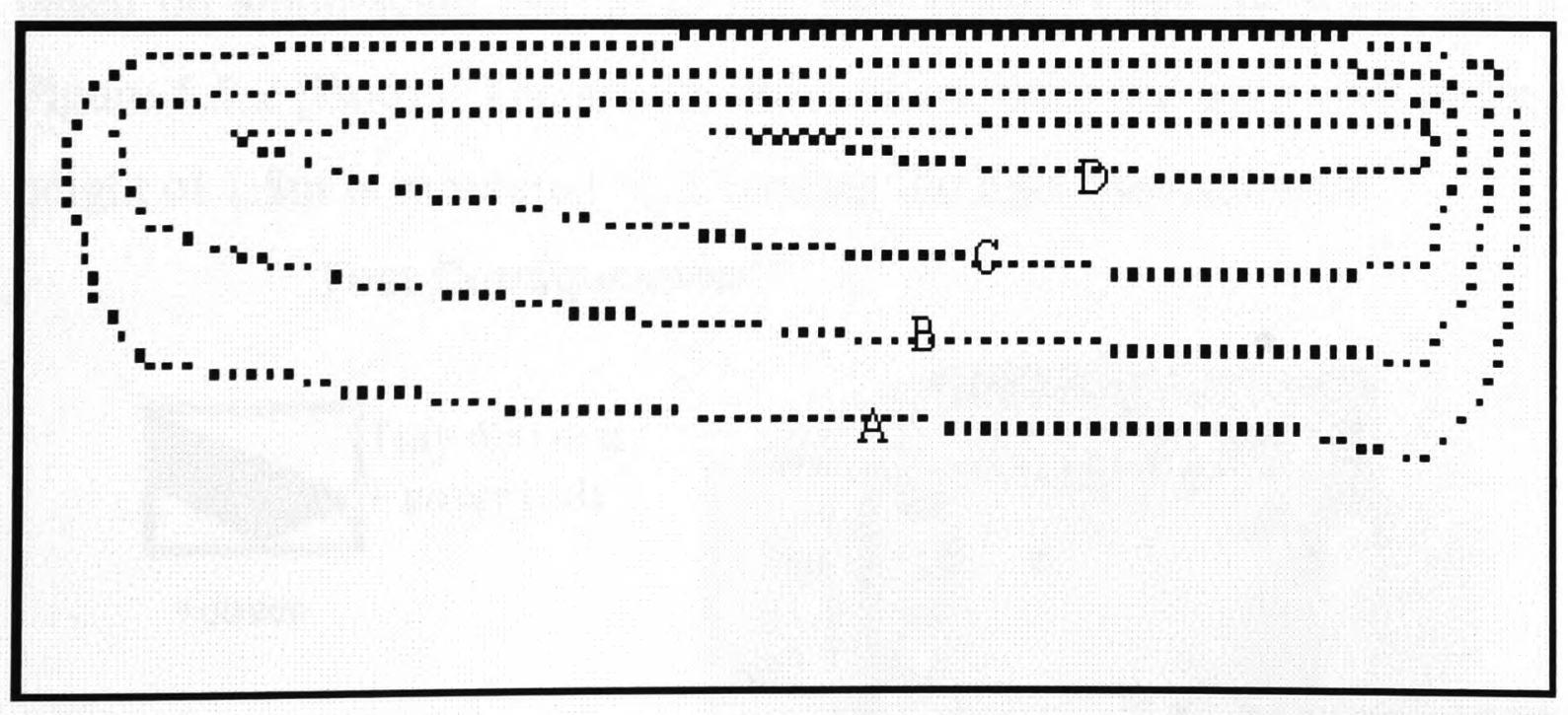

CO Volume

Fraction $(\%$, dry)

A $\quad 1.0$

B $\quad 2.0$

$\mathrm{C} \quad 3.0$

D $\quad 4.0$

Figure 5.4.10: Predicted $\mathrm{CO}$ contours in opening window at 28 minutes. 


\section{5 Simulation of the Borealis Cable Fire Test}

\subsubsection{Description of the experiment}

In 1999 a series of large-scale cable fire tests were undertaken in the Borealis Antwerp fire research laboratory [Bo01, RS02, RS04]. The investigation involved two similar German standard 220 volt mains cables. The NYM cable was produced using PVC insulation sheath while the NHMH cable was produced using polyolefin insulation and sheath [Bo01].

The cell for the cable fire tests with dimensions of $3 \times 3 \times 3 \mathrm{~m}$ is shown in Figure 5.5.1. One tray of cables ran vertically up the wall opposite the cell opening while the second ran horizontally across the ceiling of the cell. The opening door is of $0.8 \mathrm{~m}$ width and $2.0 \mathrm{~m}$ height. The tray was of $400 \mathrm{~mm}$ width and $50 \mathrm{~mm}$ depth. The cables were attached by means of conventional polypropylene cable ties. A protective cover was placed on the vertical part of the installation. The test was carried out with the door of the cell open. An extraction system above the door opening removed the combustion fumes and permitted heat release determination by oxygen depletion.

The heat release rates and the rates of generation of $\mathrm{CO}$ and $\mathrm{CO}_{2}$ were measured in the tests while the generation rates of $\mathrm{HCl}$, Acrolein and Formaldehyde were calculated based on small-scale test data. The heat release rates for NYM fire test are given in Figure 5.5.2 [Bo01]. The smoke obscuration from the front wall to the back wall at the height of $1.8 \mathrm{~m}$ is measured by recording the light transmittance.

Test Configuration

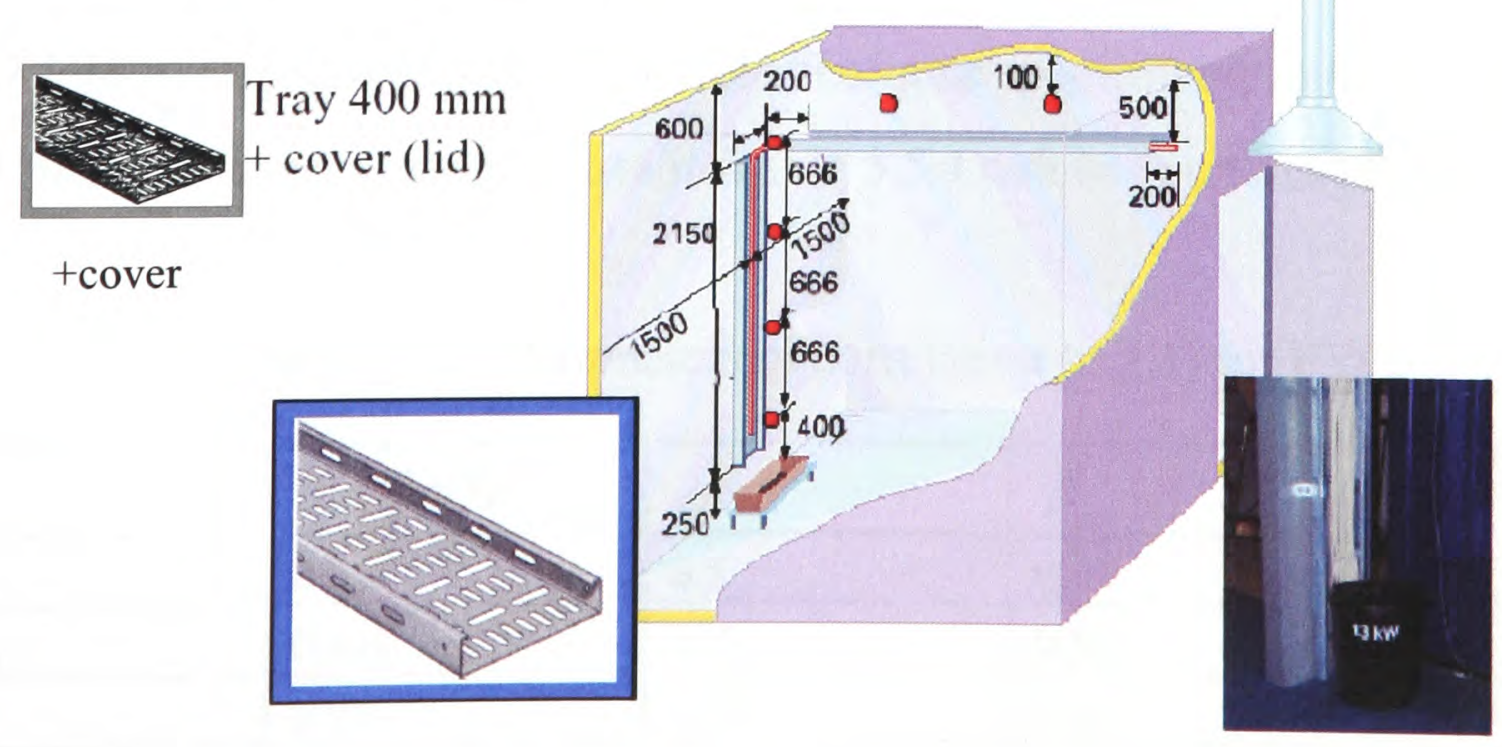

Figure 5.5.1: Schematic of cable fire tests set up [Bo01]. 


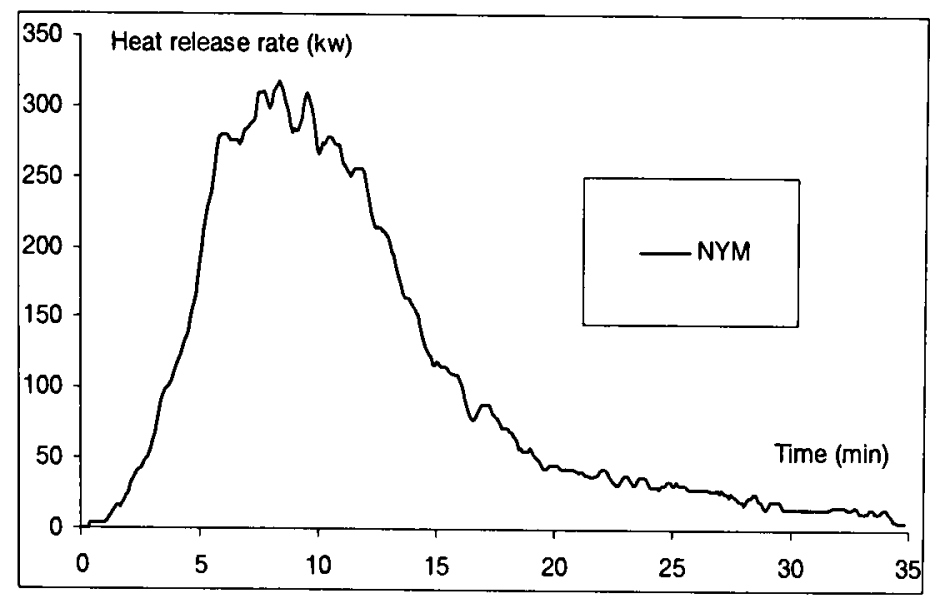

Figure 5.5.2: Heat release rate of NYM room cable fire.

\subsubsection{Simulation}

The SMARTFIRE V3.0 software is used to perform the simulation. In the position of cable trays, two fire sources are set up. The vertical one is $0.2 \mathrm{~m} \times 0.2 \mathrm{~m} \times 2.4 \mathrm{~m}$ height and horizontal one is $0.2 \mathrm{~m} \times 1.5 \mathrm{~m} \times 0.4 \mathrm{~m}$. In the simulations, the EBU model is used to simulate the released heat due to combustion.

The fuel loss rate of NYM cable is converted from the heat release rate (see Figure 5.5.2) with a constant heat of combustion $5.913 \mathrm{MJ} / \mathrm{kg}$, which is calculated from the measured total released heat and total lost fuel in the test [Bo01]. The yields of species for PVC cable fire have the form of equation (2.2.3), which is

$$
y_{i}(\phi)=y_{i, \infty}\left[1+\frac{\alpha}{\exp (\phi / \beta)^{-\zeta}}\right]
$$

where yields $y_{i, \infty}$ for $\mathrm{CO}_{2}$ and $\mathrm{CO}$ at fully ventilation condition are $0.678 \mathrm{~kg} / \mathrm{kg}$ and $0.0177 \mathrm{~kg} / \mathrm{kg}$ respectively (derived from the cone calorimeter data for PVC cable in [Bo01]) and other parameter values in Table 5.5.1 can be found in [Te95].

Table 5.5.1: Parameters of correlation (5.5.1) for PVC.

\begin{tabular}{|l|l|l|l|l|}
\hline & $y_{i, \infty}(\mathbf{k g} / \mathbf{k g})$ & $\alpha$ & $\beta$ & $\zeta$ \\
\hline $\mathbf{C O}$ & 0.0177 & 6.5 & 0.42 & -8.0 \\
\hline $\mathrm{CO}_{2}$ & 0.678 & -0.3 & 0.53 & -1.1 \\
\hline $\mathbf{O}_{2}$ & 0.71 & -0.3 & 0.53 & -1.1 \\
\hline
\end{tabular}


In the fire experiment, the light transmission at the height of $1.8 \mathrm{~m}$ from the front wall to the rear wall in the central plane through the cable tree was measured. A method developed in Chapter 4 is able to predict light extinction coefficient. It uses the mixture fraction in the CFD simulation and the mass optical density of the fuel. For PVC material, the mass optical density is about $0.33 \mathrm{~m}^{2} / \mathrm{g}$ [Mu95]. In order to simulate the smoke property, a large volume to cover the line for the smoke measurement from $1.6 \mathrm{~m}$ to $2.0 \mathrm{~m}$ in height, from $1.3 \mathrm{~m}$ to $1.7 \mathrm{~m}$ in width and from $0.4 \mathrm{~m}$ to $3.0 \mathrm{~m}$ in length is selected here. The average of mixture fractions in such a volume $\bar{\xi}$ is calculated by (5.5.2), i.e.

$$
\bar{\xi}=\frac{\sum_{i=1}^{m} \xi_{i} \rho_{i} \Delta v_{i}}{\sum_{i=1}^{m} \rho_{i} \Delta v_{i}}
$$

where $\xi$ is mixture fraction, $\rho$ is the density, $\Delta v$ is the volume and $m$ is the number of control volumes. The average density in this volume is

$$
\bar{\rho}=\frac{\sum_{i=1}^{m} \rho_{i} \Delta v_{i}}{\sum_{i=1}^{m} \Delta v_{i}}
$$

With the average mixture fraction and the average density, the average light extinction coefficient within this large volume is calculated by (4.4.13), i.e.

$$
\bar{K}=2.3 D_{m} \bar{\rho} \bar{\xi}
$$

The light transmission is then converted from the light extinction coefficient using equation (4.4.5), i.e.

$$
\frac{I_{\lambda}}{I_{\lambda}^{0}}=e^{-\bar{K} L}
$$

where the light path length $L$ is $3.0 \mathrm{~m}$ in this test. The visibility distance is estimated using equation (4.4.28), i.e. 


$$
S=\frac{C}{2.3 D_{m} \bar{\rho} \bar{\xi}}
$$

where $C$ is 3 for light-reflecting sign or 8 for light-emitting sign.

The mass rate of species passing through the opening doorway, $\dot{m}$ is calculated by

$$
\dot{m}=\sum_{i=1}^{l} Y_{i} u_{i} \rho_{i} \Delta S_{i}
$$

where $l$ is the number of cells in the opening, $Y_{i}$ is the mass fraction of the species concerned, $\rho_{i}$ is the density, $u_{i}$ is the normal perpendicular velocity and $\Delta S_{i}$ is the area of the surface with its normal perpendicular to the opening of the control volume $i$. Let $\Delta t_{j}$ stand for the time step (seconds) in the simulation. At the $n$th simulation step (with real fire $t_{n}=\sum_{j=1}^{n} \Delta t_{j}$ seconds), the accumulative mass passing through the opening is calculated with

$$
M \approx \sum_{j=1}^{n} \dot{m}_{j} \Delta t_{j}
$$

where $\dot{m}_{j}$ is the mass rate in (5.5.7) at $j$ th time step.

\subsubsection{Results and discussions}

\subsubsection{Accumulative $\mathrm{CO}_{2}$ and $\mathrm{CO}$}

The predicted total mass of $\mathrm{CO}_{2}$ and $\mathrm{CO}$ passing though the doorway and the measured data for NYM cable fire are compared in Figure 5.5.3 and 5.5.4. As seen in these figures, the predicted accumulative mass passing through the opening are in good agreement with the measured data. The predicted accumulative $\mathrm{CO}_{2}$ is slightly less than that in the test while the predicted accumulative $\mathrm{CO}$ is higher than the measured data. At 35 minutes, the measured accumulative $\mathrm{CO}_{2}$ is under predicted by $1281 \mathrm{~g}$ or $10.0 \%$. The measured $\mathrm{CO}$ is over predicted by $240 \mathrm{~g}$ or $22.9 \%$. The accumulative mass of species are predicted at the doorway while $\mathrm{CO}$ and $\mathrm{CO}_{2}$ were measured in the exhaust 
duct in the test. Since CO may be further oxidise when being transferred from the doorway to the hood, the over predicted $\mathrm{CO}$ or under predicted $\mathrm{CO}_{2}$ is not unexpected.

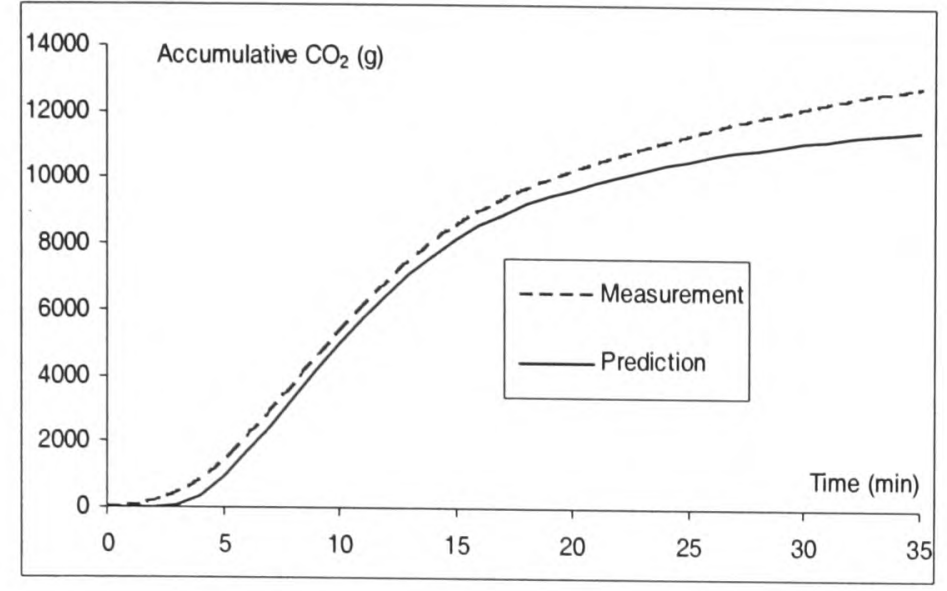

Figure 5.5.3: Accumulative $\mathrm{CO}_{2}$ in $\mathrm{NYM}$ fire.

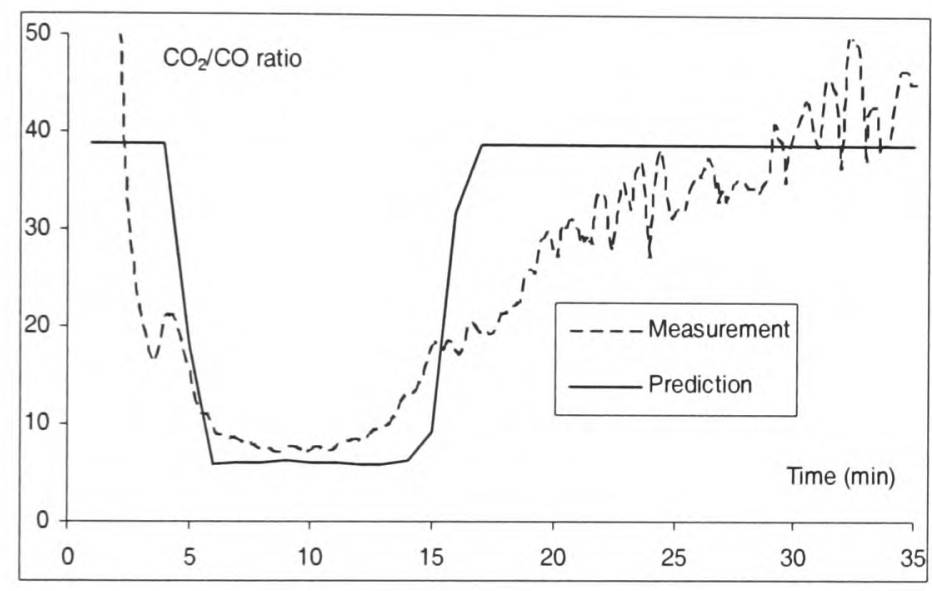

Figure 5.5.5: Ratio between yield rates of $\mathrm{CO}_{2}$ and $\mathrm{CO}$.

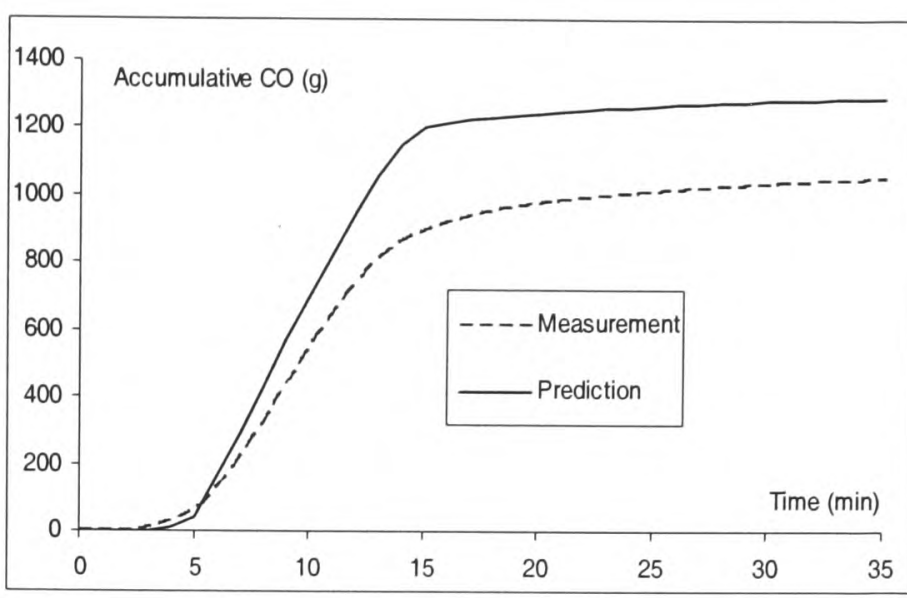

Figure 5.5.4: Accumulative CO in NYM fire.

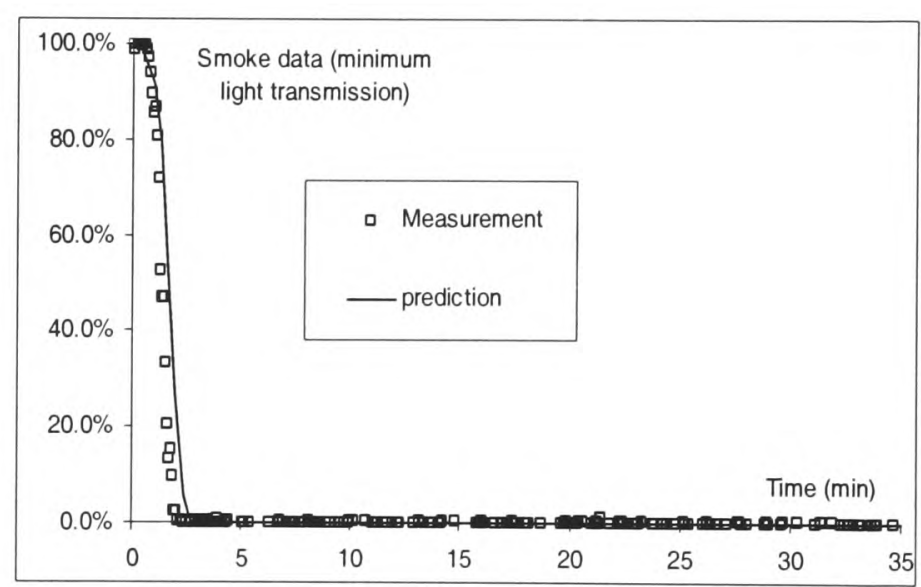

Figure 5.5.6: Measured and predicted light transmission as function of time for NYM cable fire.

The $\mathrm{CO}_{2} / \mathrm{CO}$ mass ratios as function of time are depicted in Figure 5.5.5. The measured $\mathrm{CO}_{2} / \mathrm{CO}$ mass ratio decreases quickly in the first 5 minutes. The agreement between the predictions and the measurements is quantitatively quite good, in particular, in the first 15 minutes.

\subsubsection{Light transmission}

The light transmissions as function of time are depicted in Figure 5.5.6. The measured light transmission decreases from $100 \%$ at 0.5 minutes to values close to $0 \%$ after 2 minutes. The predictions capture the measured trend. The time at which the predicted light transmission becomes zero is 0.6 minutes later than that in the experiment. 
The predicted averaged light extinction coefficients at the position for the measurement of light transmission as function of time are depicted at Figure 5.5.7. The light transmission is close to zero if the extinction coefficient is above $1.8 / \mathrm{m}$. Since both of the measured and predicted light transmission is 0 after 3 minutes, no conclusion can be drawn about the accuracy of the predicted extinction coefficient after 3 minutes.

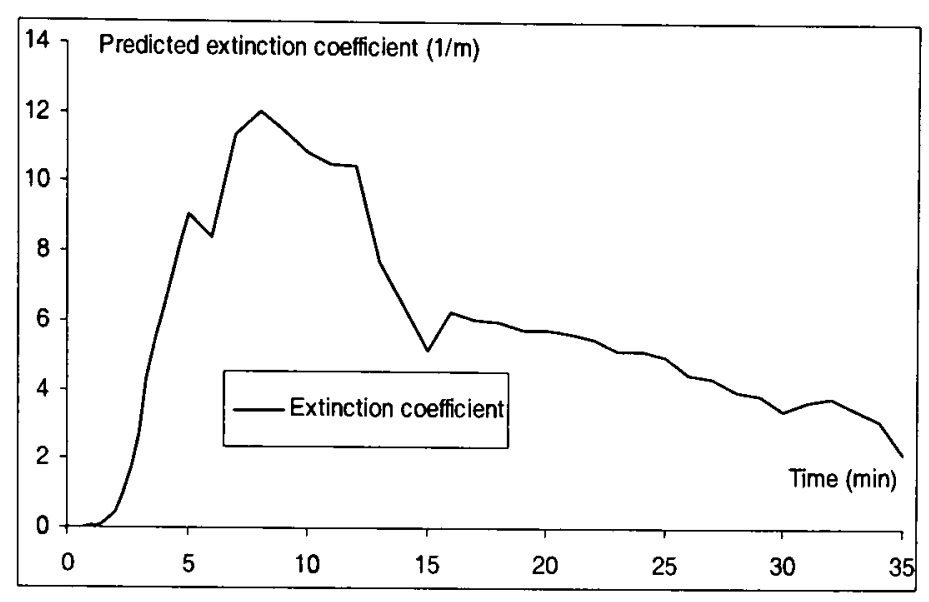

Figure 5.5.7: Predicted light extinction coefficient as function of time for NYM cable fire.

\subsubsection{Visibility distance}

The changes of smoke in the test room within the first 3 minutes of the real fire are captured in Figure 5.5.8. The predicted averages of visibility distances at the height of $1.0 \mathrm{~m}, 1.6 \mathrm{~m}$ and $2.2 \mathrm{~m}$ within the test room are given in Table 5.5.2. At 1 minute, there is a little smoke inside the room; the cable tray and the fire plume are clearly visible through the opening doorway. The predicted visibility distance is still more than $12.3 \mathrm{~m}$ at the worst case (at $2.2 \mathrm{~m}$ and for light-reflecting sign). At 2 minutes, the horizontal cable tray under the ceiling disappeared because of the heavy smoke in the upper layer of the test room. The predicted visibility distance is $2.26 \mathrm{~m}$ for lightemitting sign and $0.8 \mathrm{~m}$ for light-reflecting sign respectively at the height of $2.2 \mathrm{~m}$. At 3 minutes, only the bottom of the cable tray could be seen. The predicted visibility distance at $1.6 \mathrm{~m}$ height is only $1.9 \mathrm{~m}$ (light-reflecting sign). The fire plume in the upper part of the vertical cable tree could not be seen due to the heavy smoke (see Figure 5.5.8(c)). The predicted visibility distance at the height of $2.2 \mathrm{~m}$ for light emitting sign is only $0.6 \mathrm{~m}$. 


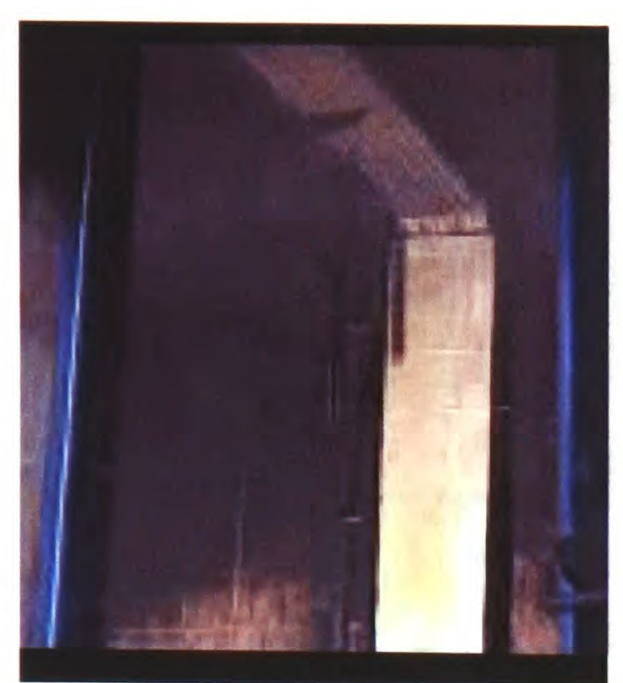

(a)

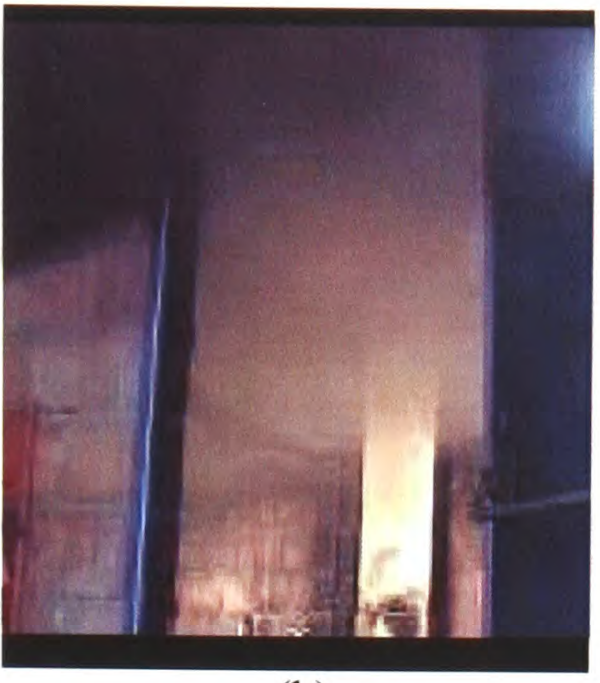

(b)

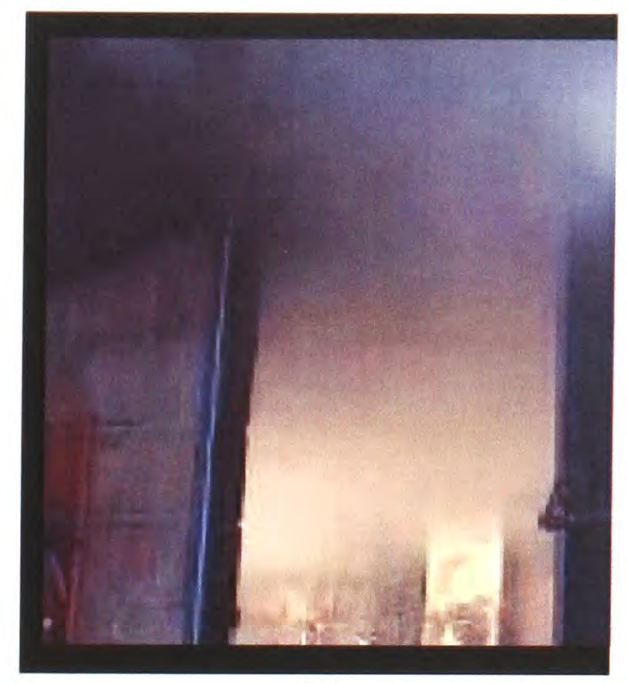

(c)

Figure 5.5.8: Smoke viewed through the doorway at (a): 1 minute; (b) 2 minutes and (c) 3 minutes.

Table 5.5.2: Predicted visibility distances within the test room.

\begin{tabular}{|c|c|c|c|c|c|c|}
\hline & \multicolumn{3}{|c|}{ Visibility distances for light-emitting } & \multicolumn{3}{c|}{$\begin{array}{l}\text { Visibility distances for light-reflecting } \\
\text { sign (m) }\end{array}$} \\
\hline $\begin{array}{c}\text { Time } \\
\text { (min) }\end{array}$ & $\begin{array}{c}\text { At } \mathbf{2 . 2} \mathbf{~ m} \\
\text { height }\end{array}$ & $\begin{array}{c}\text { At 1.6 } \mathbf{~ m} \\
\text { height }\end{array}$ & $\begin{array}{c}\text { At 1.0 } \mathbf{~ m} \\
\text { height }\end{array}$ & $\begin{array}{c}\text { At 2.2 } \mathbf{~ m} \\
\text { height }\end{array}$ & $\begin{array}{c}\text { At 1.6 } \mathbf{~ m} \\
\text { height }\end{array}$ & $\begin{array}{c}\text { At 1.0 } \mathbf{~ m} \\
\text { height }\end{array}$ \\
\hline $\mathbf{1}$ & 32.9 & 343.1 & 486.0 & 12.3 & 128.6 & 182.0 \\
\hline $\mathbf{2}$ & 2.2 & 32.9 & 132.0 & 0.8 & 12.3 & 49.5 \\
\hline $\mathbf{3}$ & 0.6 & 5.0 & 44.3 & 0.2 & 1.9 & 16.6 \\
\hline
\end{tabular}

\section{6 Simulation of a Compartment-hallway Fire Test}

\subsubsection{Description of the experiment}

A series of fire tests [Go92, LE94, LV97, LV98] were conducted in an enclosure designed to mimic the condition in hood experiments. In these experiments, the air is naturally drawn into the plenum through a circulator duct. The air enters the fire compartment through a thermally shield opening. The combustion gases exit the compartment through a window. The GERs for these kinds of experiments can be calculated with a high level of accuracy as the airflow rate into the compartment can be measured accurately. Therefore, these experiments are excellent for validation of the toxic gas model developed in this study. The scenario considered here is one where the fire gasses exit into a hallway [LE94]. The simulation of this experiment is to demonstrate the capability of the present method on predicting the GER of fire compartment and the combustion products. 


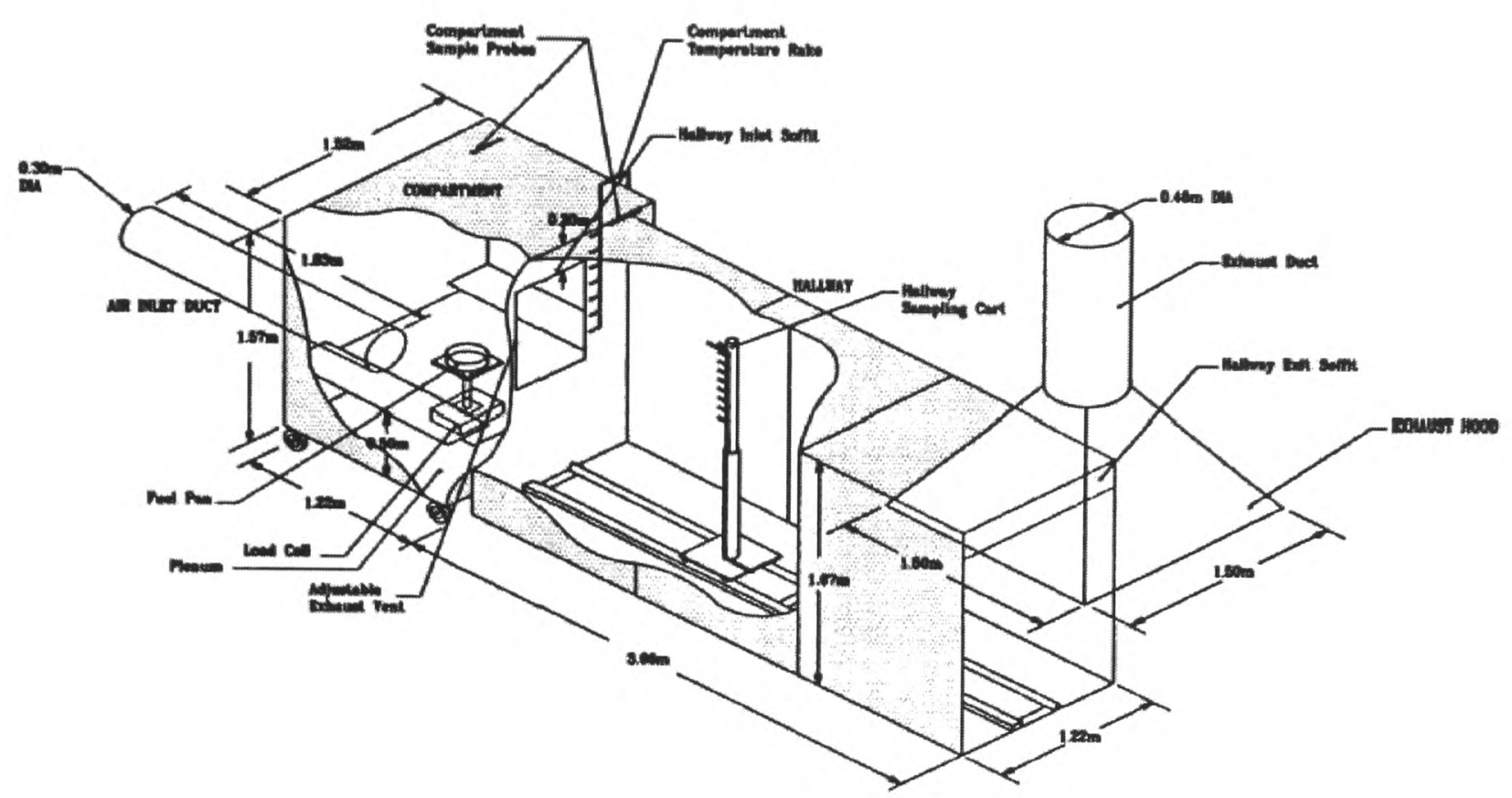

Figure 5.6.1: The compartment-hallway fire facility (reproduced from [LE94]).

The schematic of the experimental apparatus for hexane reduced room-hallway fires is shown in Figure 5.6.1. The $1.52 \mathrm{~m}$ wide, $1.22 \mathrm{~m}$ deep and $1.20 \mathrm{~m}$ high fire apartment is located above a $1.52 \mathrm{~m}$ wide, $1.22 \mathrm{~m}$ deep and $0.37 \mathrm{~m}$ high plenum. Air is naturally drawn from the surroundings into the plenum through a $0.3 \mathrm{~m}$ diameter and $1.83 \mathrm{~m}$ long circulator duct and enters the fire compartment through a thermally shield opening. The combustion gases exit the compartment through a $1200 \mathrm{~cm}^{2}(50 \mathrm{~cm}$ by $24 \mathrm{~cm}$ ) window with a $20 \mathrm{~cm}$ high soffit and enters a $1.22 \mathrm{~m}$ wide, $1.67 \mathrm{~m}$ high and $3.66 \mathrm{~m}$ long hallway. The average fire size and the equivalence ratio was $575 \mathrm{~kW}$ and 2.78 respectively. The species concentrations and temperatures in the hallway were measured at $0.051 \mathrm{~m}$ under the ceiling and $0.46 \mathrm{~m}, 1.83 \mathrm{~m}, 3.2 \mathrm{~m}$ away from the compartment. Details of the experiment can be found in [LE94].

\subsubsection{Simulation}

In the experiments, hexane fires usually increase and reach a maximum burning rate, followed by a short period of quasi-steady combustion. In the simulation presented here, the fire is simply treated as a steady state problem with a fire load of $575 \mathrm{~kW}$. The duct for air entrainment into the plenum is treated as an inlet. Since the rate of air entrained in to the fire compartment is not reported in [LE94], the fuel loss rate and the equivalence ratio in the experiment are used to calculate this value.. By the definition of the GER, the velocity of air at the duct can be calculated from the GER and the fuel loss rate by the following equation 


$$
v=\frac{\dot{m} r}{\phi s \rho Y_{o}}
$$

where $v$ is the air velocity at the duct, $\dot{m}$ is the fuel loss rate, $\phi$ is the GER of the fire, $r$ is the stoichiometric oxygen to fuel ratio, $s$ is the area of the inlet, air density $\rho$ and oxygen mass fraction $Y_{o}$ here are the ambient values. The calculated velocity is then set at the inlet.

The fire is simulated using with the EBU combustion model. The yields correlations (2.2.8-2.2.11) in Chapter 2 are used for the calculation of $\mathrm{CO}_{2}, \mathrm{O}_{2}$. The unburned hydrocarbon is estimated by

$$
\begin{array}{ll}
f_{T H C}=0 & \phi<1 \\
f_{T H C}=1-1 / \phi & \phi \geq 1
\end{array}
$$

The effect of temperature on the $\mathrm{CO}$ production for hexane fire are reflected from correlations (2.2.15) and (2.2.16), which are represented as following

$$
\begin{aligned}
& y_{C O}^{1}=(0.19 / 180) * \tan ^{-1}(10(\phi-0.8))+0.095 \text { for } T<800 K \\
& y_{C O}^{2}=(0.22 / 180) * \tan ^{-1}(10(\phi-1.25))+0.11 \text { for } T>900 K
\end{aligned}
$$

In fully developed compartment fires, for high temperatures and high equivalence ratios, correlation (5.6.5) produces a good approximation to the yield of CO. However, for low equivalence ratio, (5.6.5) represents the lower limit of $\mathrm{CO}$ yield while (5.6.4) represents the upper limit of CO yield (see figure 2-7.7 in [GR95]). As a result, the average of the yields given in (5.6.4) and (5.6.5) is used here to represent an approximation to the generation of $\mathrm{CO}$ for control volumes where the LERs are less than 1.4. Thus, combining this information, we produce the formulation given in (5.6.6) to describe the approximate yield of $\mathrm{CO}$ as 


$$
y_{C O}= \begin{cases}\left(y_{C O}^{1}+y_{C O}^{2}\right) / 2 & \phi<1.4 \\ y_{C O}^{2} & \phi \geq 1.4\end{cases}
$$

The curves described by equations (5.6.4-5.6.6) are depicted in Figure 5.6.2.

Only half of the geometry is simulated due to the symmetry. Because the fire is relatively large compared to the size of the enclosure, a fine mesh of consisting of $37128(84 \times 26 \times 17)$ cells and a time step size of 0.1 seconds are adopted.

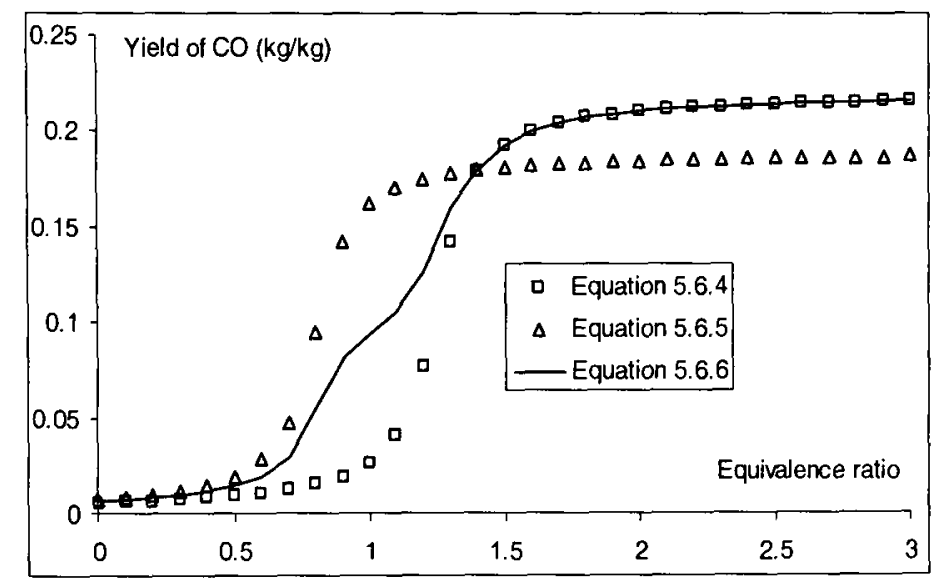

Figure 5.6.2: Yield of CO for hexane fire.

\subsubsection{Results and discussions}

In the experiments, the quasi-steady state burning of hexane fire usually lasts a short period (2-3 minutes) [Go92]. Details about the transient nature of the slow are not reported in [LE94]. The reported temperatures, species concentrations and GER are averages within 20 seconds of burning time, which are around the time corresponding to the maximum value of measured CO concentrations [LE94]. Because cold air entered the room only through the duct (an inlet in the simulation) and the combustion gases exited through the open window, the LERs at the opening should represent well the GER of the apartment. In the present study, the fire is simulated until the LERs at the opening window are close to the measured GER. The time to reach this state in the simulation is approximately 60 seconds. Thus, the predictions are compared with the measurements at the simulated time of 60 seconds at which the predicted GER is very close to the measured values. 


\subsubsection{Equivalence ratio}

In the experiment, the GER at the steady state is 2.78 . The predicted LERs at the window vary between 1.97 and 2.53 at 40 seconds and between 2.73 and 2.93 at 60 seconds (see Figure 5.6.3). At 60 seconds, the predicted rate of total mass flow through the window is $0.0797 \mathrm{~kg} / \mathrm{s}$ and the rate of mass (that from the fuel source) through the window is $0.0127 \mathrm{~kg} / \mathrm{s}$. Therefore, the predicted GER is 2.91, which is higher than the experimental data by around $4.7 \%$.

At 60 seconds, the predicted LERs in the hallway decrease quickly as we move away from the fire compartment (see figure 5.6.4). The LERs at $0.05 \mathrm{~m}$ beneath the ceiling are $>1.0$ almost all the way through the hallway, which means unburned fuel existed just underneath the ceiling. This prediction is supported by the fact that the flame length in the experiment was observed at about $90 \%$ of the length of the hallway for a $550 \mathrm{~kW}$ fire [Le94].

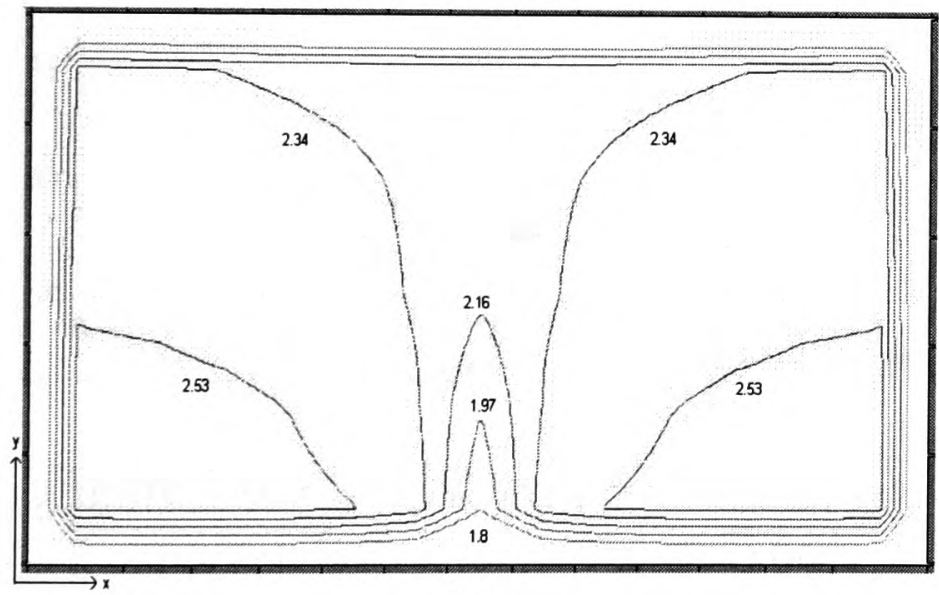

(a)

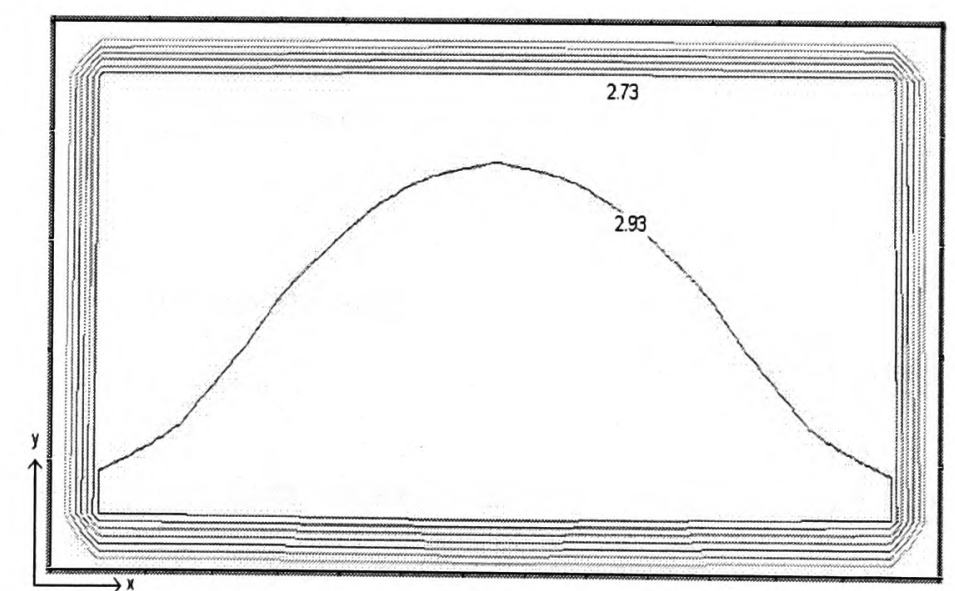

(b)

Figure 5.6.3: Predicted LERs contours at the window at (a) 40 seconds and (b) 60 seconds.

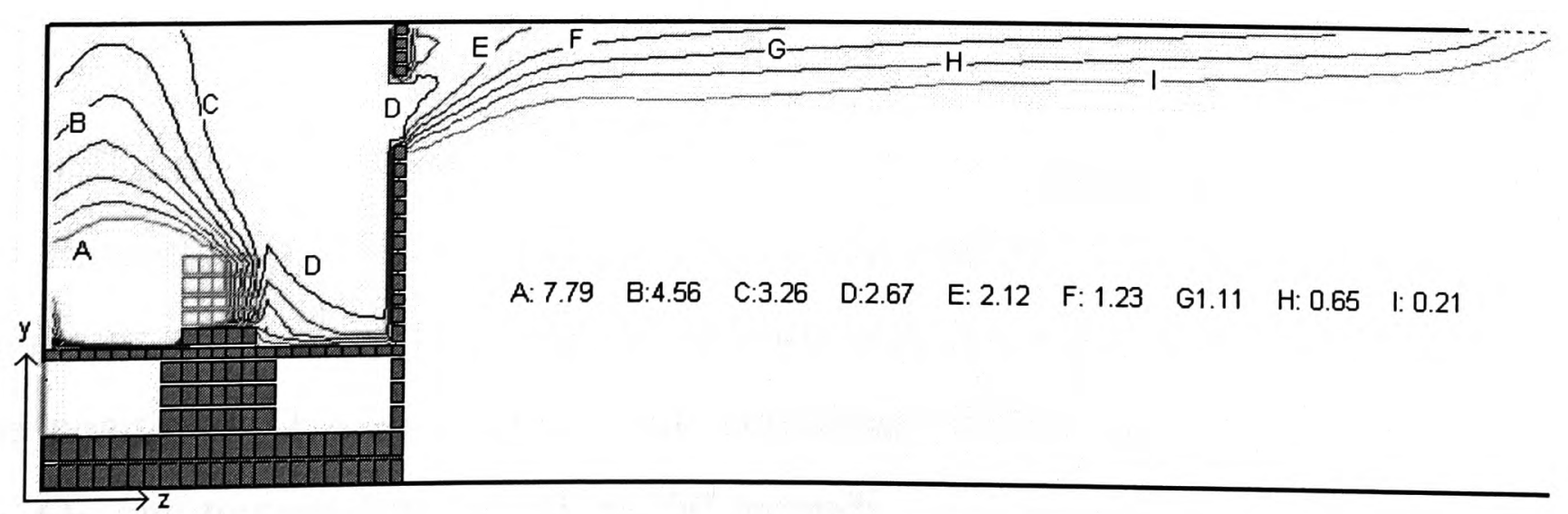

Figure 5.6.4: Predicted LERs contours at the central plane through the window at 60 seconds. 


\subsubsection{Temperature}

The temperatures and species concentrations in the following sections are in a normalized form. The normalized temperature $\left(T / T_{0}\right)$, where $T_{o}=1300 K$ and the normalized concentration $\left(\mathrm{C} / \mathrm{C}_{0}\right)$, where the value for $\mathrm{C}_{\mathrm{o}}$ varies from species to species. The constants used to calculate the normalized values for any species and temperature are given in the figures for temperature and species concentrations. As seen in Figure 5.6.5, the measured temperatures gradually decrease from the compartment to the end of the hallway. The predictions of temperatures follow this trend. The measured temperatures $0.51 \mathrm{~m}$ under the ceiling are $1237 \mathrm{k}, 1090 \mathrm{k}$ and $927 \mathrm{k}$ at positions $0.46 \mathrm{~m}$, $1.83 \mathrm{~m}$ and $3.2 \mathrm{~m}$ away from the compartment respectively. The predicted temperatures at these positions are $1180 \mathrm{k}, 1092 \mathrm{k}$ and $1002 \mathrm{k}$ respectively.

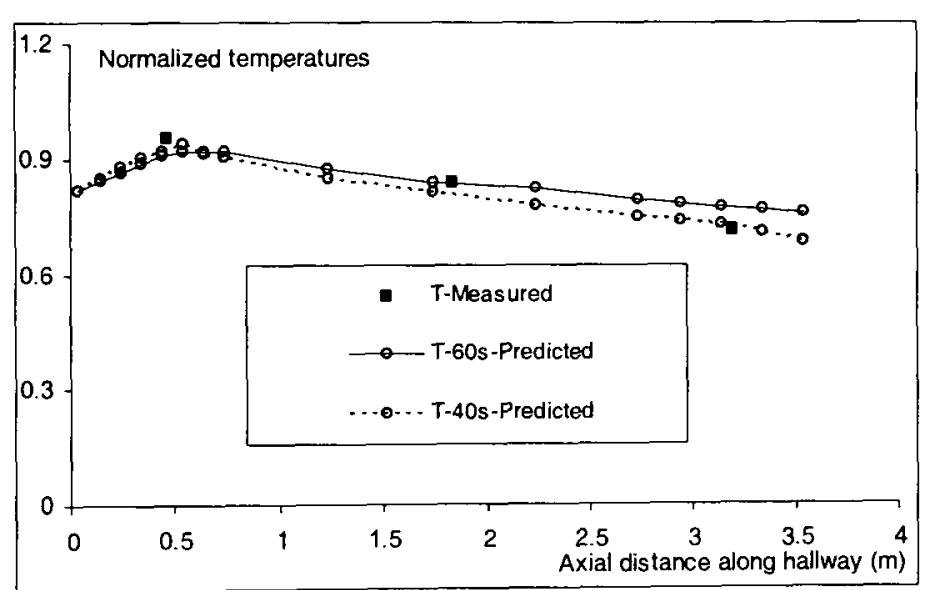

Figure 5.6.5: Axial temperatures in the upper-layer of the hallway. The normalizing value is $1300 \mathrm{~K}$.

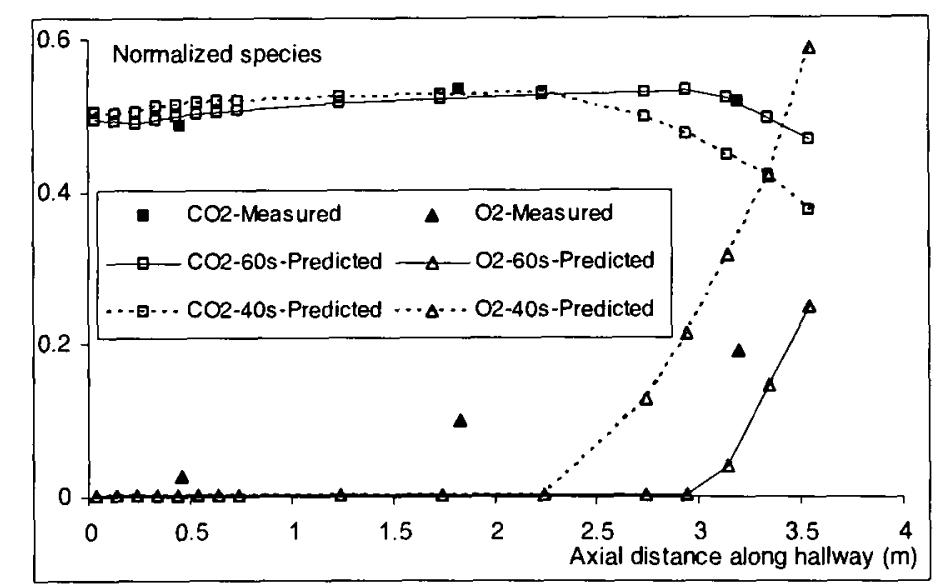

Figure 5.6.6: Axial profile of $\mathrm{CO}_{2}$ and $\mathrm{O}_{2}$ in the upper-layer of the hallway. The normalizing values are $20 \%\left(\mathrm{CO}_{2}\right)$ and $10.5 \%\left(\mathrm{O}_{2}\right)$.

\subsubsection{3 $\mathrm{CO}_{2}$ and $\mathrm{O}_{2}$}

The predicted $\mathrm{CO}_{2}$ concentrations are in good agreements with the measured data (See Figure 5.6.6). The measured $\mathrm{O}_{2}$ concentrations are less than $2 \%$ and the predictions are less than $3.6 \%$ at 40 seconds and less than $0.4 \%$ at 60 seconds at the three positions. Overall, there are no significant differences between the predicted $\mathrm{CO}_{2}, \mathrm{O}_{2}$, and temperatures at 40 and 60 seconds. 


\subsubsection{4 $\mathrm{CO}$ and hydrocarbon}

As seen in Figure 5.6.7, the measured $\mathrm{CO}$ and unburned hydrocarbon (UHC) $\left(\mathrm{C}_{2} \mathrm{H}_{4}\right.$ [LE94]) at positions $0.46 \mathrm{~m}$ away from fire compartment are $2.56 \%$ and $4.36 \%$ respectively. They decrease and reach $1.16 \%$ and $0.98 \%$ at a location of $1.83 \mathrm{~m}$ and then $0.49 \%$ and $0.27 \%$ at a location of $3.2 \mathrm{~m}$. The predicted $\mathrm{CO}$ and UHC concentrations at 40 seconds are lower than the measured data. The predicted $\mathrm{CO}$ concentrations at 60 seconds are in good agreements with the measurements. The predicted UHC concentrations at 60 seconds follow the measured trends.

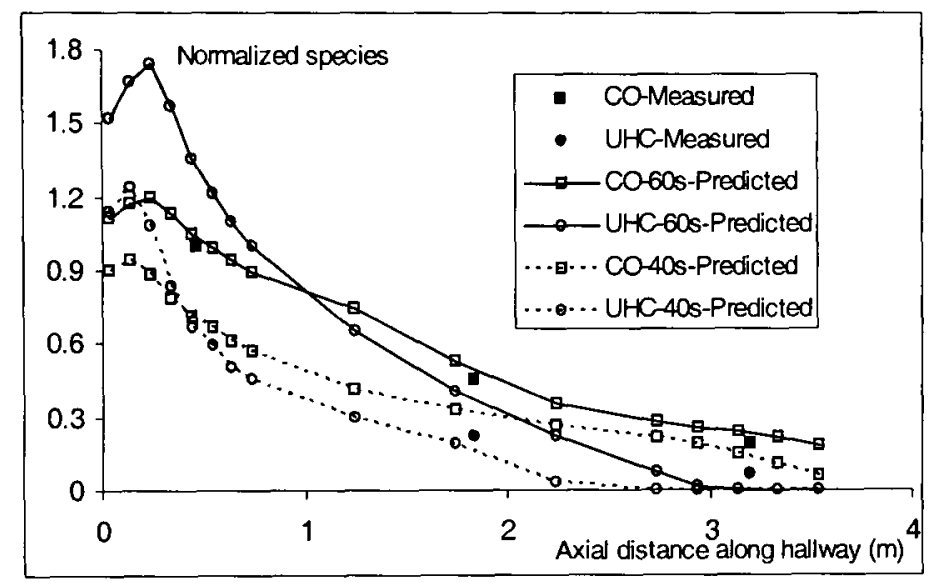

Figure 5.6.7: Axial profile of the $\mathrm{CO}$ and unburned hydrocarbon (UHC) the upper-layer of the hallway. The normalizing values are $2.56 \%(\mathrm{CO})$ and $4.36 \%(\mathrm{UHC})$.

\subsection{Concluding Remarks}

In this chapter, a wide range of enclosure fires with various fuel and geometries have been used to validate the toxicity models and the calculation of smoke properties developed in Chapter 4. These models are used in conjunction with either the volumetric heat source approach or the EBU combustion model in the simulations.

In the simulation of RI fire[RI00], where pinewood is the fuel, three toxic models are applied in the predictions of combustion products. All three toxicity models resulted in qualitatively good predictions of measured mass fractions of $\mathrm{O}_{2}$ and $\mathrm{CO}_{2}$, and reasonable predictions of $\mathrm{CO}$. Compared with the average measured $\mathrm{CO}_{2}$ volume fractions inside the room and at the doorway, the three models produced a maximum error of $15.4 \%$. Compared with the measured $\mathrm{CO}$ concentrations, the predicted 
averages of $\mathrm{CO}$ volume fractions predicted by the three models between 4-9 minutes and 10-15 minutes are reasonable.

Only Model I is used to simulate PP3, W14-b3, Borealis fire and the compartmenthallway fire. The predictions of temperatures, the GER and the volume fractions of $\mathrm{CO}$ and $\mathrm{CO}_{2}$ of the PP3 fire are in good agreements with the experimental data while the predictions of hydrocarbon and smoke production in the opening are relatively poor.

In the simulation of W14-B3 test, the predictions of temperatures are somewhat higher than the measurements but follow the measured trend. The simulation of the burning wood cribs produced reasonably good predictions for species concentrations in the flaming combustion stage. The $\mathrm{CO}$ generated by the pyrolysis of wood is not specifically considered in the present model. In addition, it is difficult to distinguish the mass fractions of fuel which originate from the two fire sources in a given control volume by using only a single mixture fraction. Therefore, the local maximum of CO caused by the pyrolysis of the central fire at 8 minute was not predicted by the present model.

In the simulation of cable fire, the predicted accumulative $\mathrm{CO}_{2}$ and $\mathrm{CO}$ exiting the doorway are in good agreement with the measured accumulative masses. The predicted light transmission follows the measured trend in the test. The predicted visibility distances are reasonable compared with experimental observations.

The compartment-hallway fire simulation was generally in good agreement with experimental data. The accurate measured GER makes this type of experiments is good for model validation. The predicted $\mathrm{CO}_{2}$ and $\mathrm{O}_{2}$ concentrations are steady from 40-60 seconds and are in good agreements with the measured data. The predicted CO concentrations at 60 seconds are reasonable, at which the predicted GER is very close to the measured value.

The simulations with different fire scenarios in this chapter show that the models developed in this study is promising to predict the production and movement of 
smoke and toxic gases in full-scale fire enclosures. The correlations between the yields of combustion products and the GERs used in the simulations are derived from small-scale experiments. Some of the applications of the approaches to smoke properties are reported in [GG03, GL03, GL04].

It should be noted that the toxicity models developed in Chapter 4 with an assumptions that the combustion conditions within a control volume and the associated LER and temperature is assumed approximately equivalent to that of a small-scale fire experiment with the same value of GER and upper layer temperature. This makes these models validate mainly at locations within or close the fire plume. The species measurements locations in the scenarios RI, PP3, W14-B3 and Borealis test were within the room or at the doorway and close to the fire flame. Although the gas sampling locations in the reduced compartment-hallway $(\mathrm{RCH})$ were in the hallway that was adjacent to the fire compartment and furthermore in this case the flame extended from the fire compartment to about $90 \%$ of the length of the hallway as been observed in the experiments.

What about the species concentrations at remote locations from the fire origin, where there are no flames? This question will be answered in the next chapter. 


\section{Chapter 6}

\section{APPROACH TO TOXIC GASES AT REMOTE LOCATIONS}

\subsection{Introduction}

The models developed in Chapter 4 have successfully reproduced the measured species concentrations at locations within or close to the combustion region in a wide range of fire scenarios as seen in Chapter 5. The models implicitly assume that all the gaseous fuel is burnt and combustion can occur remote from the fire origin during the transportation process. This can result in poor model predictions of toxicity levels at remote locations from the fire source in fire scenarios in which not all of the available gaseous fuel is actually completely combusted.

Two-thirds of the fatalities were found at locations distant from the original fire and carbon monoxide (CO) was the main cause for these deaths [GB94]. It is desirable that fire models are equipped with the capability to predict not only the generation but also the transportation of $\mathrm{CO}$ within fire enclosures including locations remote from the fire source. This chapter focuses on predicting the species concentrations at remote locations.

The transportation of high levels of $\mathrm{CO}$ to remote locations has been experimentally studied in [LE94, LV97, LV05]. In these studies, a hallway connected the fire compartment through an opening window. Air enters the fire compartment only through a duct under the compartment. If no further reaction occurs outside the compartment, species levels transported away from the compartment will be equal to those generated inside the compartment; otherwise, if oxidation occurs outside the compartment, the unburned hydrocarbon and $\mathrm{CO}$ levels are expected to decrease. The degree of further oxidation of $\mathrm{CO}$ in a hallway adjacent to the fire compartment has been found to be a function of the amount of oxygen entrained into the gases burning in the hallway [LE94, LV97, LV05]. 
A concept of control volume equivalence ratio (CVER) has been proposed in their study [LV05]. A control volume is defined as the fire compartment and a space extended into the hallway up to the location where the species need to be determined. For remote locations, the control volume needs to extend as far as to the flame tips in the adjacent hallway. The CVER is calculated using the fuel loss rate and the rate of air entering the defined control volume. It was concluded that the CVER can be used to predict the species level at a location downstream of a post-flashover fire [LV05]. The use of CVER and the results in these experiments further demonstrated that the yields of $\mathrm{CO}$ in the combustion region are local conditions dependent. Like the LER at a computational cell of a field fire model, the CVER tries to reflect the mixing state of gases at the gas sampling location. For remote locations outside the combustion region, the control volume contains an area right up to the flame tip in the adjacent space (See Figure 6.1.1) [LV05].

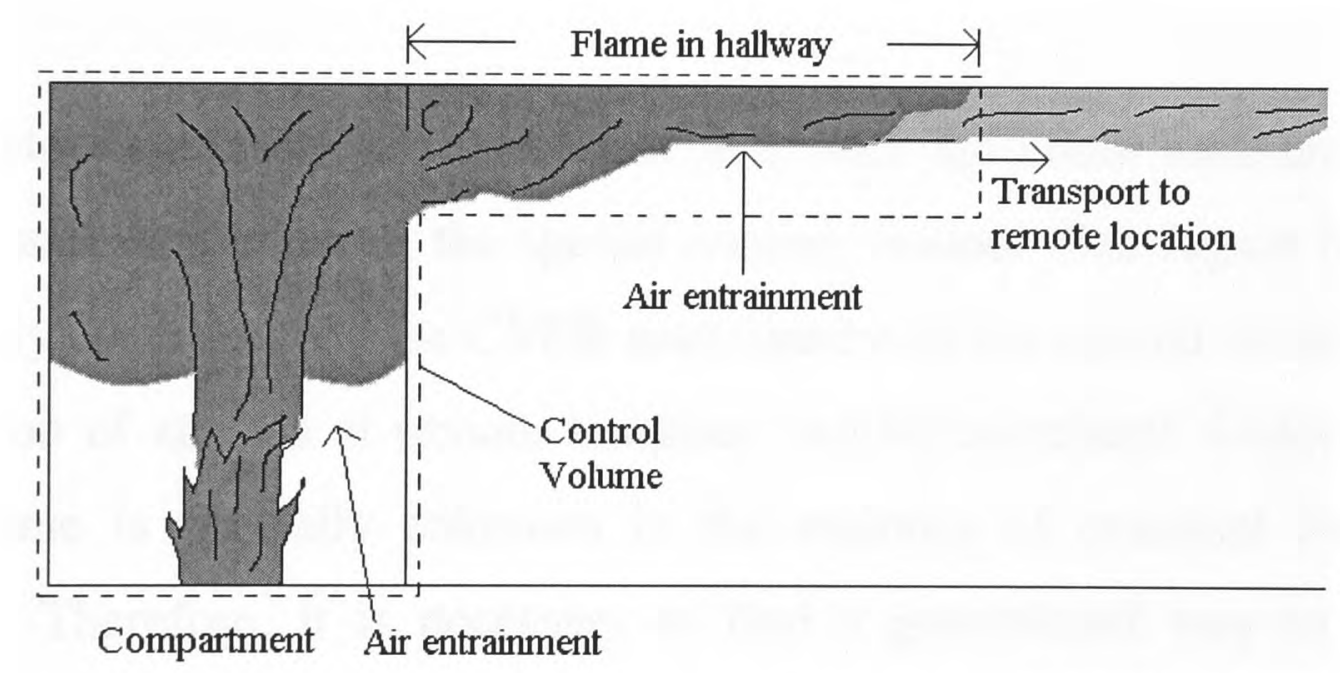

Figure 6.1.1: Control volume used for calculating the CVER by Lattimer et al.

This chapter is a further application and continuing development of the toxicity models developed in Chapter 4. In order to improve the prediction of species concentrations remote from the fire source, the author introduced the concept of a control region (CR), outside which the changes of species are only the result of mixing processes. A methodology to divide the computational domain into the control region and transport region (TR) is proposed in Section 6.2. Different calculation methods for the species concentrations in each region are also given in this section. Two room-corridor fire scenarios have been simulated using the present model, and the model predictions are compared with the experimental results in Section 6.3 and Section 6.4 respectively. Finally, conclusions are drawn in Section 6.5. 


\subsection{Methodology}

\subsubsection{Division of the computational domain}

The method proposed by Lattimer et al [LV05] for predicting toxic gases at a location downstream of a post-flashover fire essentially divides the fire enclosure into two parts for the calculation: Region I and Region II. The calculations of toxic gases within the two regions are different. At locations within Region I, the toxic gases are calculated with a control volume equivalence ratio (CVER), which is location dependent. At any location within Region II (remote locations), the toxic gases are determined by a fixed value of CVER, which is based on a special control volume including the fire compartment and the flaming area in its adjacent hallway (See Figure 6.6.1).

For a laboratory controlled experimental fire, with sufficient measurements of air entrainment and temperatures, the special control volume (See Figure 6.1.1) may be approximately estimated and the CVER associated with the special control volume for the calculation of species at remote locations can be calculated. Unfortunately, this control volume is normally unknown in the majority of practical fire simulation applications. Therefore, it is necessary to find a generalized way to estimate the special control volume and its associated equivalence ratio in practical fire simulations. In this study, the combustion efficiency of the fire will be used to determine the division of the computational domain.

The combustion efficiencies $\lambda$ correlate the equivalence ratios well [Te95, Go92]. equation (6.2.1) represents this correlation for non-halogenated polymers [Te95] while equation (6.2.2) is a general approach for all fuels [Go92]. In these equations, the constants $A$ and $B$ are determined experimentally. For example, the value of $B$ for Hexane is 0.91 based on Gottuk's reduced room experiments [Go92].

$$
\lambda=A\left(1-\frac{0.97}{\exp (\phi / 2.15)^{-1.2}}\right)
$$




$$
\lambda= \begin{cases}B & \phi \leq 1 \\ B / \phi & \phi>1\end{cases}
$$

For a fire scenario with known combustion efficiency (normally it is an input parameter for fire simulations), an equivalence ratio corresponding to this efficiency can be calculated using correlations such as equation (6.2.1) or (6.2.2). This equivalence ratio is denoted as $\phi_{C R}$ in the rest of the chapter. As with the special CVER used by Lattimer et al, $\phi_{C R}$ is used in this study for the calculation of toxic gases at remote locations.

With this critical value of equivalence ratio $\phi_{C R}$, the computational domain is then divided into two parts. A control region (CR) is defined as $\phi \geq \phi_{C R}$, which is consistent with the fact that considerable amount of $\mathrm{CO}$ is normally generated when the equivalence ratio is greater than a certain value for a given fuel. Within this region, the toxic gas levels are determined by local burning conditions such as the local equivalence ratios and temperatures. The transport region is then defined as the region in which $\phi<\phi_{C R}$. In this region, toxic gas levels are determined by $\phi_{C R}$, a fixed value for a given fire. Since the local equivalence ratio is a function of the mixture fraction (See Equation (6.2.3) below), the use of the LER contour $\left(\phi=\phi_{C R}\right)$ to represent the boundary of the $\mathrm{CR}$ in this study is similar to that taken by a flamelet model in [BK94], in which the boundary of the combustion region is approximately calculated by the mixture fraction contour with a fixed value which is in turn derived from the $\mathrm{OH}$ concentrations.

The simple correlation (6.2.2) cannot provide a unique critical equivalence ratio $\phi_{C R}$ if the combustion efficiency happens to be $B$, which represents high values of combustion efficiency under fuel controlled conditions. A more precise correlation such as that described by equation (6.2.1) is necessary for fires of this kind. However, for ventilation controlled fires, which normally have low values of the combustion efficiency, equation (6.2.2) is sufficient for the calculation of species concentrations at remote locations. 


\subsubsection{Toxic gases in the control region}

The yields of toxic gases within the CR are determined by the local combustion conditions. The toxicity model Model I developed in Chapter 4, which considers the effect of LER and local temperature on the yields of species, can be used for the calculation of species levels in this region. The LER $\phi$ is calculated using the mixture fraction $\xi$ by equation (4.2.8), i.e.

$$
\phi=\frac{\xi /(1-\xi)}{r_{s t}}
$$

The mass fraction $Y_{i}$ of species $i\left(\mathrm{CO}\right.$ and $\left.\mathrm{CO}_{2}\right)$ is a function of the mixture fraction $\xi$ and the local equivalence ratio $\phi$ and temperature $T$

$$
Y_{i}=\xi y_{i}(\phi, T)
$$

where $y_{i}(\phi, T)$ is the yield of species $i$ per unit mass of the fuel evaporated. The mass fraction of oxygen is expressed using

$$
Y_{O 2}=0.23(1-\xi)-\xi y_{O 2}(\phi, T)
$$

where 0.23 is the mass fraction of oxygen in the ambient air and $y_{O 2}(\phi)$ is the consumption of oxygen when per unit mass of fuel. The volume fraction $C_{i}$ of species $i$, can be converted from the mass fraction with the following equation

$$
C_{i}=Y_{i} \frac{W}{W_{i}}
$$

where $W$ and $W_{i}$ represent the molecular weights of the gas mixture and species $i$ respectively. Assume that the $\mathrm{CO}_{2} / \mathrm{H}_{2} \mathrm{O}$ mole ratio in the reaction is the same as the stoichometric mole ratio of $\mathrm{CO}_{2}$ to $\mathrm{H}_{2} \mathrm{O}$ in the complete one-step reaction. The dry volume fraction of species $i$ is then calculated with Equation (6.2.7)

$$
C_{i}^{d r y}=\frac{Y_{i}(\phi) W}{\left(1-18 Y_{C O 2} /(44 \alpha)\right) W_{i}}
$$

where $\alpha$ is the $\mathrm{CO}_{2} / \mathrm{H}_{2} \mathrm{O}$ mole ratio. 


\subsubsection{Toxic gases in the transport region}

The generation of combustion products in the $\mathrm{CR}$ is dependent on the local equivalence ratio (LER) and temperature. Although the boundary of the CR has a unique LER value, the species concentrations on the boundary may not be constant under certain circumstances [BK94]. In order to calculate the species transportation in the transport region without solving extra equations, an assumption is made.

\section{Assumption 6.2.1}

The species concentrations at the boundary of the control region are only determined by the LER $\phi_{C R}$.

With this assumption, the species concentrations at remote locations can be calculated with the method adopted in the flamelet model in [BK94]. As discussed in [BK94], $\mathrm{CO}$ concentrations outside the control region obey the diffusion equation without a source term. Therefore, the two equations for $\mathrm{CO}$ and for the mixture fraction respectively are the same within the transport region. If the $\mathrm{CO}$ concentrations on the boundary between the control region and the transport region are known, the $\mathrm{CO}$ concentrations in the transport region can be linearly approximated with the mixture fraction [BK94]. The species mass fractions at the interface with LER $\phi=\phi_{C R}$ are obtained from equations (6.2.4-6.2.5). Therefore, in the transport region where the LER $\phi$ is smaller than $\phi_{C R}$, the mass fractions of species $i\left(\mathrm{CO}\right.$ and $\left.\mathrm{CO}_{2}\right)$ is

$$
Y_{i}(\phi)=Y_{i}\left(\phi_{C R}\right) \cdot \xi / \xi_{C R}
$$

where $\xi_{C R}$ is the mixture fraction corresponding to the equivalence ratio $\phi_{C R}$. The consumed oxygen in a control volume in the transport region is also a linear function of the mixture fraction,

$$
0.23-Y_{O 2}(\phi)=\left(0.23-Y_{O 2}\left(\phi_{C R}\right)\right) \cdot \xi / \xi_{C R}
$$

Then, the concentration of oxygen is derived from equation (6.2.9) 


$$
Y_{O 2}(\phi)=0.23\left(1-\xi / \xi_{C R}\right)+Y_{O 2}\left(\phi_{C R}\right) \cdot \xi / \xi_{C R}
$$

\subsubsection{Model Implementation}

Within the framework of the CFD fire simulation package SMARTFIRE [EJ02], the present approach to the toxic gases has been incorporated with both the volumetric heat source representation and the EBU combustion model. The methodology for the prediction of toxic gases in fire enclosures in this study is summarised as follows

- The mixture fraction, a conserved scalar, is used to calculate the LERs by equation (6.2.3).

- A critical equivalence ratio $\phi_{C R}$ is derived via such correlations as equation (6.2.1) or (6.2.2) utilising the combustion efficiency of the fire scenario.

- In the Control Region ( $\phi \geq \phi_{C R}$ ), with the prescribed correlations between the yields of species and the equivalence ratio, which are derived from small-scale experiments, the species concentrations are calculated from equations (6.2.46.2.7).

- In the Transport Region $\left(\phi<\phi_{C R}\right)$, the species concentrations are calculated with equations (6.2.8) and (6.2.10).

\subsubsection{Applicable Conditions}

Currently, temperature dependent correlations for the yields of species are not sufficiently reliable to be used in determining species concentrations. Therefore, we limit our calculation of species concentrations in the CR on the LERs alone for most fire scenarios. This means that the species on the boundary of the CR is uniform. Therefore, a simple method using the key Assumption 6.2.1 is proposed for the calculation of $\mathrm{CO}$ in the transport region. Use of this assumption may be subject to a certain level of error under particular circumstances. However, simulation results presented in this study demonstrate that this assumption is reasonably accurate. 


\subsection{Simulations of one of the Compartment-hallway Hexane Fires}

\subsubsection{Description of the experiment}

A series of fire tests were conducted with a scaled room connecting a side hallway to measure the effect of oxygen entrainment on the transport of $\mathrm{CO}$ in building fires [LV97]. The schematic of the experimental apparatus for these reduced room-hallway fires is shown in Figure 6.3.1(a). The $1.52 \mathrm{~m}$ wide, $1.22 \mathrm{~m}$ deep, $1.20 \mathrm{~m}$ high fire compartment was located above a $1.52 \mathrm{~m}$ wide, $1.22 \mathrm{~m}$ deep $0.37 \mathrm{~m}$ high plenum. The walls were made from Fibreboard with a thickness of $0.025 \mathrm{~m}$ [La06]. The air was naturally drawn from atmosphere into the plenum through a $0.3 \mathrm{~m}$ diameter, $3.96 \mathrm{~m}$ long circulator duct and entered the fire compartment through a thermally shield opening. The combustion gases exited the compartment through a window and entered a $1.22 \mathrm{~m}$ wide, $1.67 \mathrm{~m}$ high and $3.66 \mathrm{~m}$ long hallway. In these tests, all samples were taken $0.05 \mathrm{~m}$ below the ceiling of the hallway and the compartment except for the sample location at the opening.

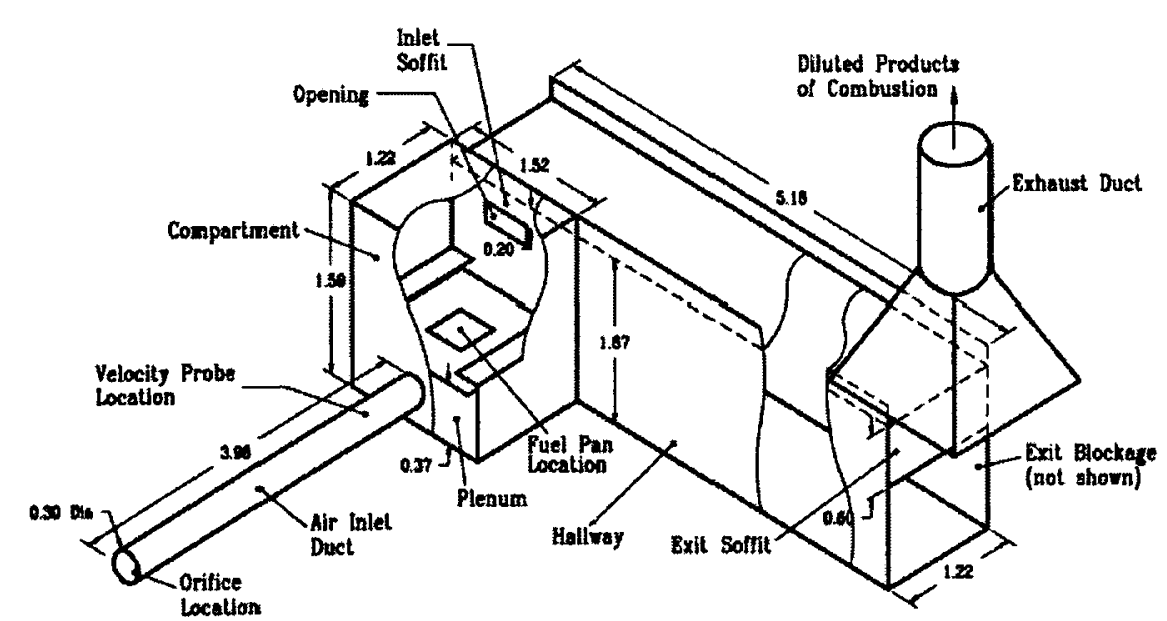

(a)

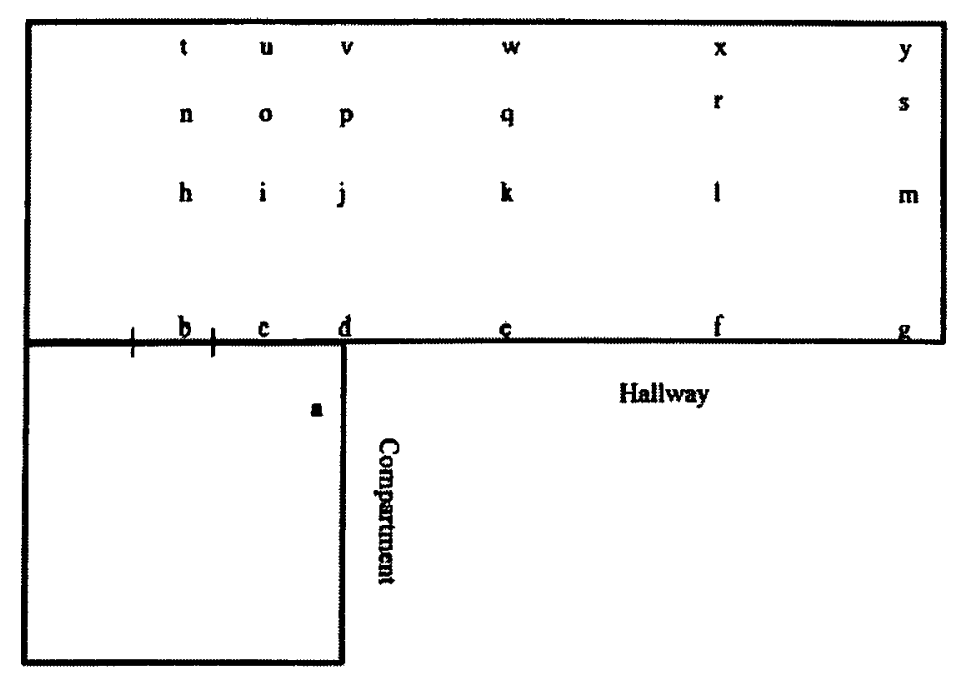

(b)

Figure 6.3.1: (a) Fire test facility and (b) sample locations ([LV97]). 
The test selected for the model validation is an $n$-hexane fire with a $0.2 \mathrm{~m}$ soffit at the inlet and a $0.6 \mathrm{~m}$ soffit at the exit of the hallway respectively. The size of the window was $0.04 \mathrm{~m}^{2}(0.25 \mathrm{~m}$ by $0.16 \mathrm{~m})$. The heat of combustion and the stoichiometric fuel air mass ratio for hexane are $44735 \mathrm{~kJ} / \mathrm{kg}$ and 0.0658 respectively. In order to map the hallway species concentrations, the same fire was repeated 25 times by varying the gas sampling location (see Figure 6.3.1(b) for gas sampling locations). The species concentrations reached a steady state within a short transient time after flashover occurred. The 25 repeated tests have similar curves of the fuel loss rates and air mass flow rates into the compartment. The fuel loss rates and air mass rates as functions of time for the typical experiments provided in [La06] are presented in Figure 6.3.2.

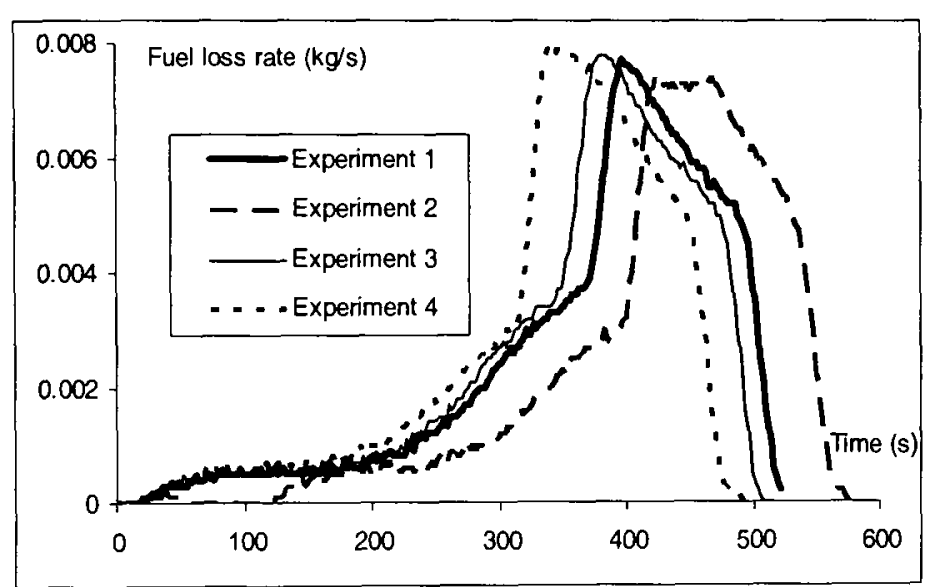

(a)

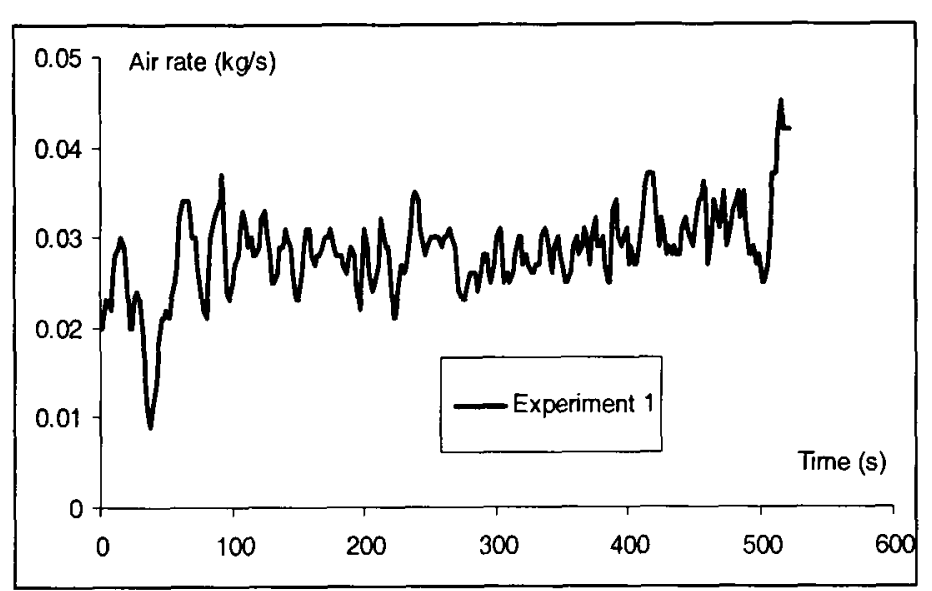

(b)

Figure 6.3.2: (a) Fuel loss rates and (b) air mass flow rates into fire compartment.

\subsubsection{Simulations}

Figure 6.3.3 is the mesh used in the SMARTFIRE fire simulations. A mesh of 40,734 cells for the L-shape geometry and a time step size of 1.0 second are adopted in the simulation. The model calculated heat loss through the walls and ceiling with the walls assuming the material properties of fibreboard, with the default settings in SMARTFIRE for this material being used [EJ02].

The combustion efficiency, which is used to estimate the critical equivalence ratio $\phi_{C R}$, must be specified as part of the proposed toxicity model input requirements. Unfortunately, the combustion efficiency was not reported in [LV97]. However, the GERs based on the fuel loss rates and the air entrainments to the fire compartment were given in [La06]. As the fire only occurred inside the compartment, the critical 
equivalence ratio determining the $\mathrm{CR}$ in this scenario takes the measured compartment GER values (See Figure 6.3.4(a)). Since there are considerable fluctuations in the measured compartment equivalence ratios, a curve of averaged measured compartment equivalence ratios has been structured. The average value at any time is calculated from the measured equivalence ratios in a period of 30 seconds prior to this time. The CR at any time in the simulation is determined by the averaged equivalence ratio curve.

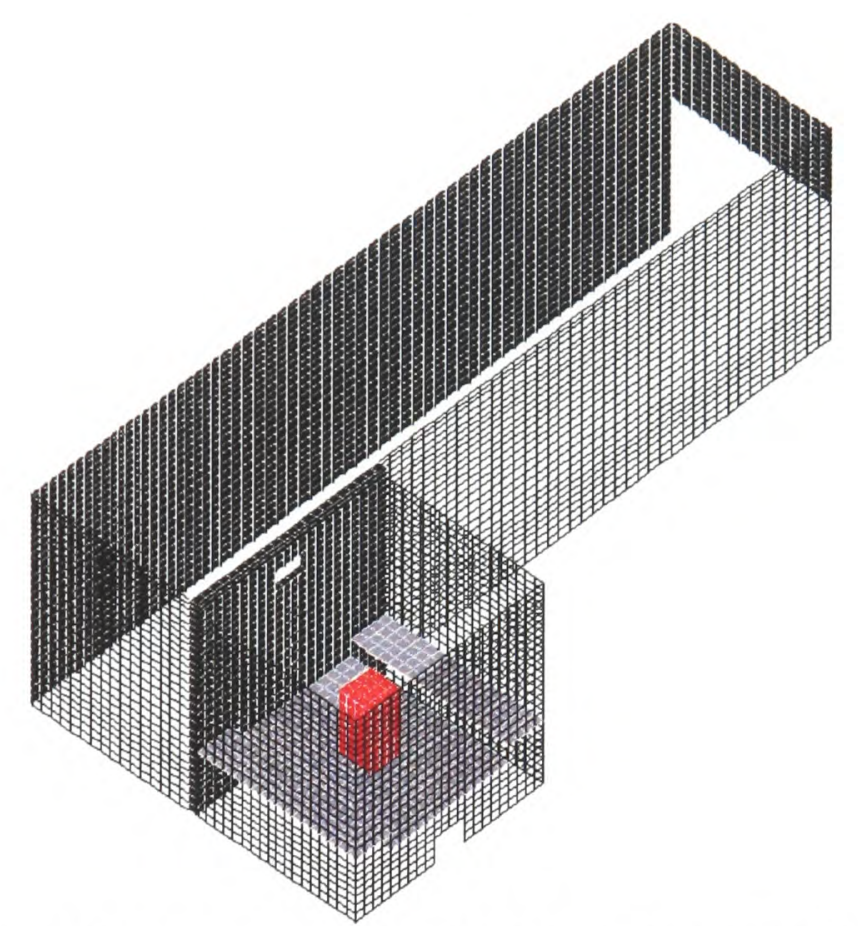

Figure 6.3.3: Set up of the compartment-hallway hexane fire by SMARTFIRE (ceiling and floor are removed to allow visualizations of the inside compartment and hallway).

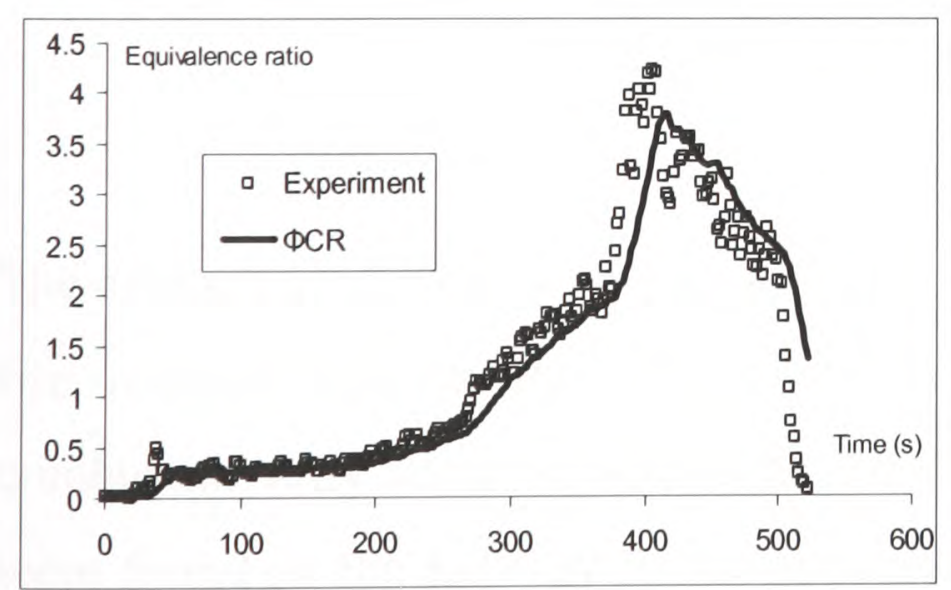

(a)

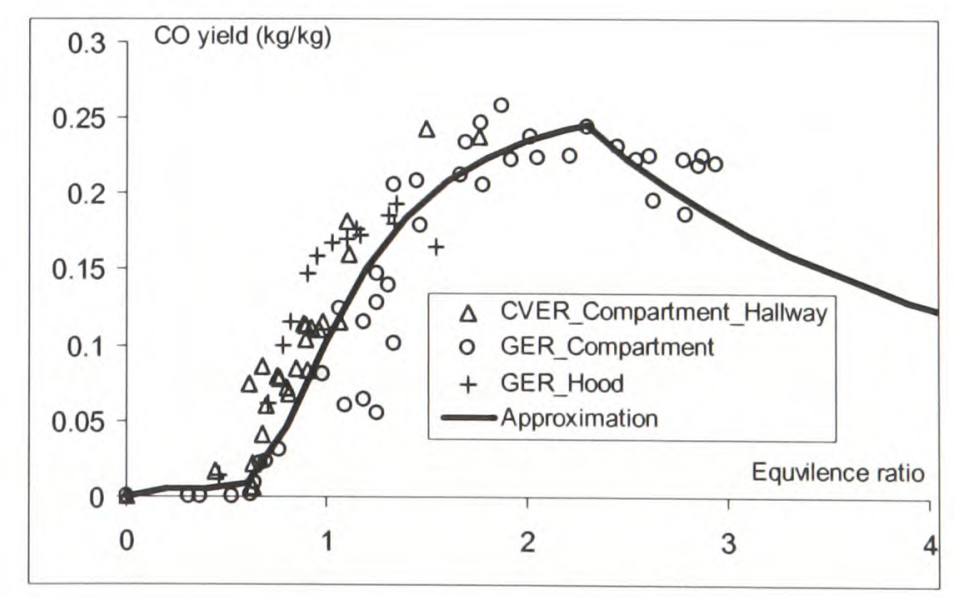

(b)

Figure 6.3.4: (a) Measured compartment equivalence ratios and (b) correlations between the yields of CO and CVER and GER.

The correlation between the yields of combustion products and the equivalence ratio is expressed in the form of equation (2.2.3), i.e. (6.3.1) and the parameters in the equation are summarised in Table 6.3.1. 


$$
y_{i}(\phi)=y_{i_{\infty}}\left[1+\frac{\alpha}{\exp (\phi / \beta)^{-\zeta}}\right]
$$

Table 6.3.1: Parameters for the yields of combustion products of $n$-Hexane.

\begin{tabular}{|c|c|c|c|c|}
\hline & $y_{i, \infty}(\mathrm{kg} / \mathrm{kg})$ & $\alpha$ & $\beta$ & $\zeta$ \\
\hline $\mathrm{CO}$ & 0.005 & 53.0 & 1.0 & -2.8 \\
\hline $\mathrm{CO}_{2}$ & 2.79 & -1.0 & 1.7 & -1.4 \\
\hline $\mathrm{O}_{2}$ & 3.34 & -0.97 & 1.7 & -1.5 \\
\hline
\end{tabular}

These parameters are derived by matching equation (6.3.1) with the experimental data for $n$-hexane by Beyler (as cited in [GR95]), Gottuk et al [GR95] and by Lattimer et al [LV05] (See Figure 6.3.4(b) for CO yields). The correlation (6.3.1) for hexane with parameters in Table 6.3.1 are similar to those expressed with formula (5.6.2), (5.6.3) and (5.6.6) in the earlier simulation to a hexane fire in Chapter 5.6. As discussed in Chapter 4, a maximal equivalence ratio for $\mathrm{CO}$ generation is required for the use of equation (6.3.2) to account for the reduction of $\mathrm{CO}$ generation at large LERs. The inflexion point of equivalence ratio $\phi_{\text {inflexion }}$ in equation (6.3.2) used in this simulation is 2.3 that is determined from the experimental data reported in [GR95, LV05] (see Figure 6.3.4 (b)).

$$
y_{C O}(\phi)= \begin{cases}y_{C O}(\phi) & \phi<\phi_{\text {inflexion }} \\ y_{C O 2}(\phi) \cdot \frac{y_{C O}\left(\phi_{\text {inflexion }}\right)}{y_{C O 2}\left(\phi_{\text {inflexion }}\right)} & \phi \geq \phi_{\text {inflexion }}\end{cases}
$$

The average air flow rate of $0.03 \mathrm{~kg} / \mathrm{s}$ (See Figure 6.3.2(b)) is set up at the duct of the fire compartment in the simulation. For this specially designed fire scenario, combustion only occurred within the compartment while high levels of unburned fuel were found in the hallway in the experiment. The volumetric heat source model is used for the purpose of confining the fire plume within the compartment. The heat is released within a prescribed volume with dimensions of $0.25 \mathrm{~m}$ by $0.25 \mathrm{~m}$ in square and $0.5 \mathrm{~m}$ in height. The heat release rates are calculated from the fuel loss rates labelled as Experiment 1 in Figure 6.3.2(a). Corresponding to the air flow rate of 0.03 $\mathrm{kg} / \mathrm{s}$ and the stoichometric ratio of air to fuel for complete combustion, the maximal 
effective heat release rate within the fire compartment is approximately $81.4 \mathrm{~kW}$ and this value is used to limit the heat release rate in the simulation.

As a sensitivity analysis of the calculated CR to the fire volume, an additional simulation with the same fire input but an extended fire volume with dimensions of $0.25 \mathrm{~m}$ by $0.25 \mathrm{~m}$ in square and $1.0 \mathrm{~m}$ in height has been performed. It has been found from two different fire volume simulations that the distributions of predicted local equivalence ratios and temperatures at the opening of the compartment and the hallway are almost identical over the entire simulation time. Although the distributions of LERs within the fire volumes are somewhat different, the calculated CRs are quite similar and the CR boundaries at the vicinity of the compartment opening become identical after 300 seconds, from which the fire becomes ventilation controlled. Therefore, it is deemed that the size of the fire volume in this scenario does not significantly affect the application of the present toxicity model for the calculation of species concentrations at remote locations. The following results are taken from the simulation with the fire volume with dimensions of $0.25 \mathrm{~m} \times$ $0.25 \mathrm{~m} \times 0.5 \mathrm{~m}$.

This fire scenario is simulated using Model I too for model comparison.

\subsubsection{Results and Discussion}

\subsubsection{Transient compartment temperature}

The predicted temperatures in the upper layer of the compartment (represented by temperatures at $1.54 \mathrm{~m}$ and $1.0 \mathrm{~m}$ high of location $a$ ) as functions of time are depicted in Figure 6.3.5. As seen in Figure 6.3.5, the predicted upper layer temperatures inside the compartment reach a steady state after 300 seconds in the simulation. The experimental temperatures are reported at three different time intervals, twice just before and once right at the onset of the steady state for species concentrations according to the fire growth parameters [LV97, La06]. However, the precise time of the temperature measurement was not reported and so the experimental temperature data points displayed in Figure 6.3.5 for each time interval represent the start and end of the time interval. For Experiment 1, these times are approximately 363-367 
seconds, 395-399 seconds and 419-423 seconds respectively. As seen in Figure 6.3.5, the predicted temperatures at $1.54 \mathrm{~m}$ high of location $a$ in the steady state are very close to the measured temperatures at three points of the steady state stage.

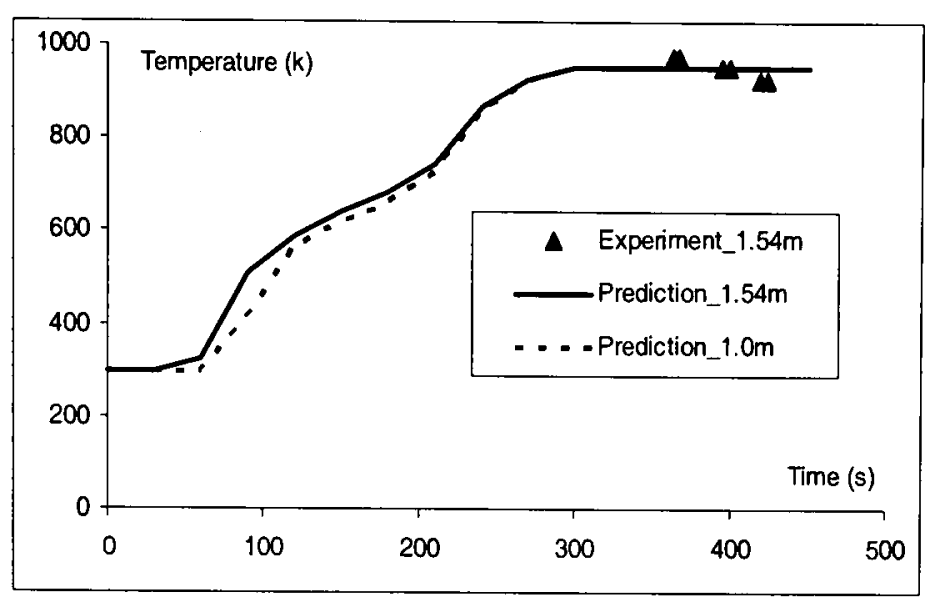

Figure 6.3.5: Measured and predicted temperatures at location $a$ inside the compartment.

\subsubsection{Predicted control region during steady state}

Figure 6.3.6(a) depicts the distributions of the predicted local equivalence ratios in the vertical plane passing through the centre of the window, which connects the fire compartment and the hallway. The hatched area bounded by the equivalence ratio contour 3.5 is the predicted CR at 420 seconds. As seen in Figure 6.3.6(a), the predicted $\mathrm{CR}$ is almost completely confined within the compartment as indicated by the experimental observations, i.e. no external burning occurred in the hallway. The predicted distributions of velocities, temperatures and $\mathrm{O}_{2}$ concentrations are of help to explain why the fire did not spread into the hallway. The predicted temperatures at the window are approximately $886 \mathrm{~K}$ (see Figure 6.3.6(b)), which is within the range of flame tip temperatures (815-940K) observed from a series of hexane fire experiments [LV05]. For fuel rich hexane fire, 'the upper layer of a compartment fire can be considered unreactive at a temperature of $800 \mathrm{~K}$ or less for residence times less than $15 s, 750 K$ for times less than 92s, and 700K for times less than 760s' [GR95]. The predicted gas velocities range from $1.0 \mathrm{~m} / \mathrm{s}$ (towards the wall) to $2.6 \mathrm{~m} / \mathrm{s}$ (just outside the window) in the hallway (see Figure 6.3.6(c)) and the gas temperatures decreases from $886 \mathrm{~K}$ at the window to a value of $522 \mathrm{~K}$ at locations with the same height of the window bottom. With the $1.22 \mathrm{~m}$ wide hallway, the transport time is approximately one second. With such a rapid decrease of temperatures to below $800 \mathrm{~K}$ and such a 
short residence time of less than one second, continuing burning in the hallway is unlikely. On the other hand, as seen in Figure 6.3.6(d), the oxygen concentrations near the window are as low as $0.8 \%$. The low $\mathrm{O}_{2}$ levels would produce little heat even if continuing burning somehow occurs around the window and this inevitably results in the gas temperature decreasing quickly. The temperature falls below the necessary temperature for continuing combustion before more oxygen is mixed into the fuel rich layer in the hallway.

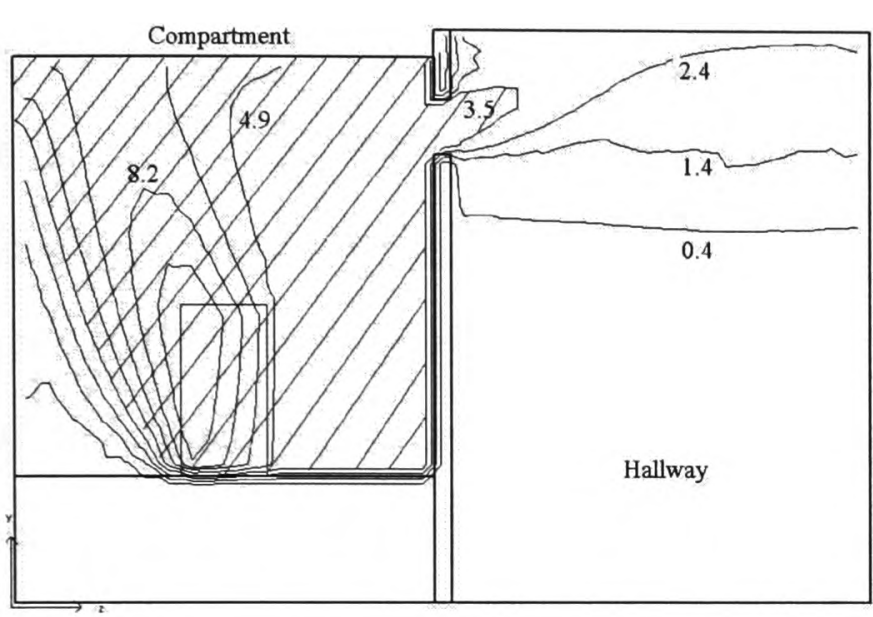

(a) LERs and extent of CR (hatched region)

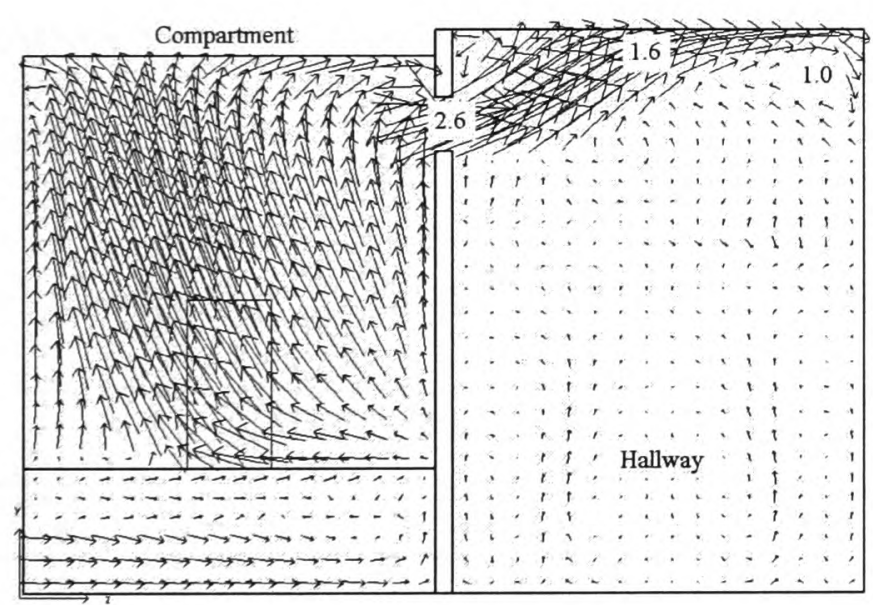

(c) Velocity $(\mathrm{m} / \mathrm{s})$

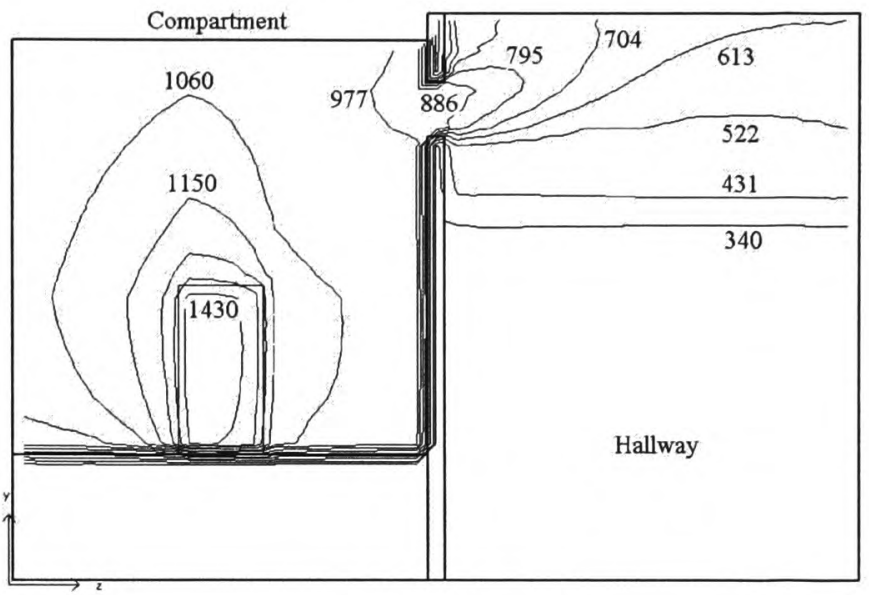

(b) Temperature $(\mathrm{K})$

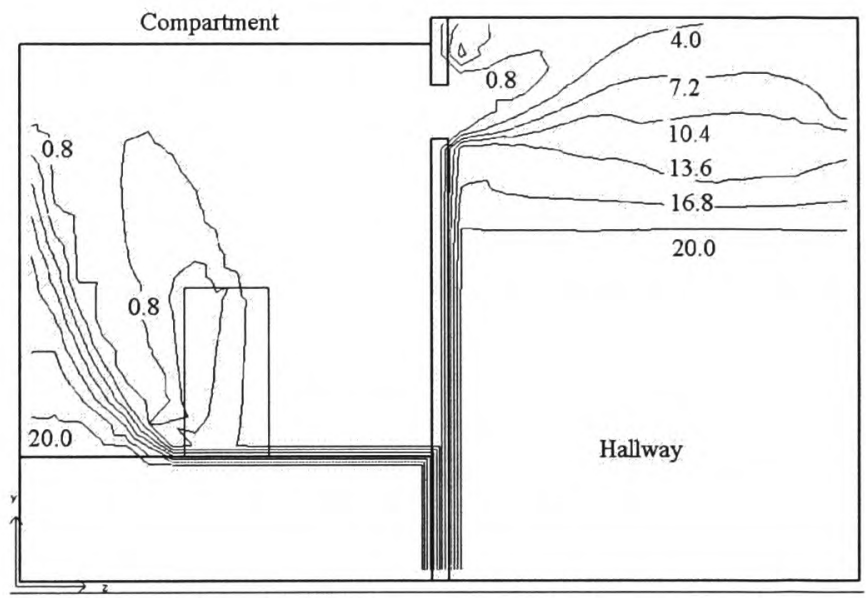

(d) Oxygen $(\%)$

Figure 6.3.6: Predicted distributions of (a) local equivalence ratios and the control region (shadow); (b) temperatures; (c) velocities; and (d) Oxygen in the vertical plane passing through the window.

\subsubsection{Temperatures and species during steady state}

Among the three sampling times in Experiment 1, the first two took place before the onset of the steady state (as measured by the species concentration) and species concentrations were undergoing dramatic changes during these periods of time. Therefore, the predicted species concentrations for Experiment 1 are only compared 
with the experimental data at different locations in the steady state. In Experiment 1 , species concentrations were found to be at steady state after 419-423 seconds according to the fire growth parameters [LV97, La06], while the predicted species concentrations reach a quasi-steady state after 420 seconds in the simulation.

The predicted temperatures and species concentrations (wet) at the onset of the steady state are compared with the measured data at four typical locations $(a, h, k$, and $m$ in Figure 6.3.1(b)) in Table 6.3.2. The measured data in Table 6.3.2 are estimated from the distribution maps in the experimental report [LV97]. The predicted temperatures at location $a(950 \mathrm{~K})$ and location $k(528 \mathrm{~K})$ are very close to the measured temperatures of $925 \mathrm{~K}$ and $530 \mathrm{~K}$ respectively. Location $a$ is in the front corner of the fire compartment, which is within the predicted control region. The temperature level at this location is just within the range of temperatures at which combustion may occur. The measured temperature of $725 \mathrm{~K}$ at location $h$ is under predicted by $55 \mathrm{~K}$ or a relative error of $7.6 \%$. The measured temperature at location $m$ is just below $500 \mathrm{~K}$ [LV97] while the model prediction is $495 \mathrm{~K}$. Generally, the predicted temperatures within the compartment and the hallway are in good agreement with the measured data.

Table 6.3.2: Measured and predicted temperatures and species concentrations.

\begin{tabular}{|c|c|c|c|c|c|c|c|c|}
\hline \multirow{2}{*}{ Location } & \multicolumn{2}{|c|}{ Temperature (K) } & \multicolumn{2}{c|}{ CO (\%) } & \multicolumn{2}{c|}{$\mathbf{C O}_{\mathbf{2}}$ (\%) } & \multicolumn{2}{c|}{$\mathbf{O}_{2}$ (\%) } \\
\cline { 2 - 9 } & Exp. & Pre. & Exp. & Pre. & Exp. & Pre. & Exp. & Pre. \\
\hline $\boldsymbol{a}$ & 925 & 950 & 2.6 & 2.5 & 9.6 & 9.2 & 0.0 & 0.0 \\
\hline $\boldsymbol{h}$ & 725 & 670 & 2.4 & 2.3 & 8.0 & 8.1 & 4.2 & 4.5 \\
\hline $\boldsymbol{k}$ & 530 & 528 & 1.9 & 1.7 & 6.4 & 6.2 & 7.8 & 8.3 \\
\hline $\boldsymbol{m}$ & $<500$ & 495 & 1.8 & 1.7 & 6.6 & 6.1 & 8.4 & 8.3 \\
\hline
\end{tabular}

The measured $\mathrm{CO}$ concentrations decreased from $2.6 \%$ at location $a$ in the compartment to $1.8 \%$ at location $m$ in the far end of the corridor in the steady state. The predicted CO concentrations at those locations varied between $2.5 \%$ and $1.7 \%$. The relative errors between the measured and predicted $\mathrm{CO}$ concentrations are less than $11 \%$.

The measured $\mathrm{CO}_{2}$ concentrations at location $a, h, k$ and $m$ are $9.6 \%, 8.0 \%, 6.4 \%$ and $6.6 \%$ while the model predictions are $9.2 \%, 8.1 \%, 6.2 \%$ and $6.1 \%$ respectively. The relative prediction errors at those locations are less than $7.6 \%$. The predicted $\mathrm{O}_{2}$ 
concentrations at the three locations are quite close to the measured data (see Table 6.3.2).

\subsubsection{Comparison with Model I}

The toxicity model developed in Chapter 4 assumes that combustion will continue in the transportation region until all fuel is burnt. This will lead the $\mathrm{CO}$ concentrations at remote locations to be poorly predicted in scenarios in which not all fuel is burnt. Location $m$ is at the far end of the hallway. The predicted species concentrations at location $m$ using the present model and the Model I (see Chapter 4) are compared in Table 6.6.3. The measured $\mathrm{O}_{2}$ at location $m$ is approximately $8.4 \%$. Model I predicts that there is no oxygen present at location $m$. This results in Model I significantly over predicting the measured $\mathrm{CO}(1.8 \%)$ and $\mathrm{CO}_{2}(6.6 \%)$ (by $50 \%$ and $82 \%$ respectively). Introducing the assumption that combustion occurs only in the $\mathrm{CR}$ results in a considerable improvement in the quality of the predictions for the toxic gas species as shown in Table 6.3.3. Using the present model, the relative errors in predicting $\mathrm{CO}$, $\mathrm{CO}_{2}$ and $\mathrm{O}_{2}$ are within $7.6 \%$.

Table 6.3.3: Experimental and predicted species concentrations at location $m$ generated using Model I and the present model.

\begin{tabular}{|c|c|c|c|}
\hline & Experiment & Model I & Present model \\
\hline $\mathbf{C O}(\boldsymbol{\%})$ & 1.8 & 2.7 & 1.7 \\
\hline $\mathbf{C O}_{\mathbf{2}} \mathbf{( \% )}$ & 6.6 & 12.0 & 6.1 \\
\hline $\mathbf{O}_{\mathbf{2}} \mathbf{( \% )}$ & 8.4 & 0.0 & 8.3 \\
\hline
\end{tabular}

\subsubsection{Sensitivity of species on critical equivalence ratio}

The sensitivity of the predicted gas species concentrations to the critical equivalence ratio $\phi_{C R}$ was examined for the predictive simulation to this scenario.

The predicted species concentrations presented in Table 6.3.2 are at 420 seconds with a $\phi_{C R}$ value of 3.5. The changes in the predicted species concentrations at location $m$ corresponding to a $+/-10 \%$ change in $\phi_{C R}$ are compared with the predictions with $\phi_{C R}$ of 3.5 in Table 6.3.4. As seen in Table 6.3.4, if the critical equivalence ratio decreases 
by $10 \%$, the predicted species concentrations are subject to a maximum relative change of $15.7 \%$ compared with the predictions using $\phi_{C R}$ of 3.5 . If the critical equivalence ratio increases by $10 \%$, the relative changes in the predictions of species concentrations are within $14.5 \%$.

In this scenario, the critical equivalence ratio at 420 seconds is 3.5. As seen in Figure 6.3.4(b), the yields of $\mathrm{CO}$ are almost linearly dependent on the equivalence ratios at around $\phi_{C R}$ value of 3.5. Therefore, the variations in the critical equivalence ratio $\phi_{C R}$ result in proportionate changes of the predicted species concentrations.

Table 6.3.4: Sensitivity of remote species concentrations on the critical equivalence ratio at location $m$ for the compartment-hallway fire.

\begin{tabular}{|c|c|c|c|}
\hline \multirow{2}{*}{$\begin{array}{c}\text { Relative change } \\
\text { in } \phi_{C R}\end{array}$} & \multicolumn{3}{|c|}{ Corresponding change in species concentration compared with those } \\
with $\phi_{C R}$ of 3.5 \\
\cline { 2 - 4 } & $\mathbf{C O}$ & $\mathrm{CO}_{2}$ & $\mathbf{O}_{2}$ \\
\hline$-10 \%$ & $+14.4 \%$ & $+11.5 \%$ & $-15.7 \%$ \\
\hline$+10 \%$ & $-11.5 \%$ & $-11.5 \%$ & $+14.5 \%$ \\
\hline
\end{tabular}

\subsection{Simulations of a Corridor Cable Fire}

\subsubsection{Description of the experiment}

A series of cable fire tests were performed by the Department of Fire Technology at SP Swedish National Testing and Research Institute as part of a joint study conducted by the Borealis $\mathrm{AB}$ and Universities of Greenwich and Bolton [Pe06]. The test geometry consisted of a $U$ shaped corridor with a total length of $44.6 \mathrm{~m}$ (See Figure 6.4.1). The inner height and width of the corridor were $2.4 \mathrm{~m}$ and $2.0 \mathrm{~m}$ respectively. This corridor was divided into five parts by four $0.4 \mathrm{~m}$ high soffits (at location $\mathrm{A}, \mathrm{B}$, $\mathrm{C}$ and $\mathrm{D})$. At the inner corner, between soffit $\mathrm{A}$ and soffit $\mathrm{B}$, a room was connected to the corridor through a $2 \mathrm{~m} \times 0.4 \mathrm{~m}$ window at $2.0 \mathrm{~m}$ high. The room had a floor area of $4 \mathrm{~m} \times 3 \mathrm{~m}$ and the same ceiling height as the corridor. The corridor was constructed of modules made of a frame of wooden joists covered with $10 \mathrm{~mm}$ Promatect ${ }^{\circledR}-\mathrm{H}$, non-combustible boards. Because of the heat exposure to the wall close to the fire source, $1.2 \mathrm{~m}$ of the wall behind the cable tray had one extra layer of a Promatect ${ }^{\circledR}-\mathrm{H}$ board $(2 \times 10 \mathrm{~mm})$. Wall conductivity and specific heat capacity are approximately 
$0.188 \mathrm{~W} / \mathrm{m}^{\mathrm{O}} \mathrm{C}$ and $1.13 \mathrm{~kJ} / \mathrm{kg}^{\mathrm{O}} \mathrm{C}$ respectively. Details on the material properties in the experiment can be found in [Pe06].

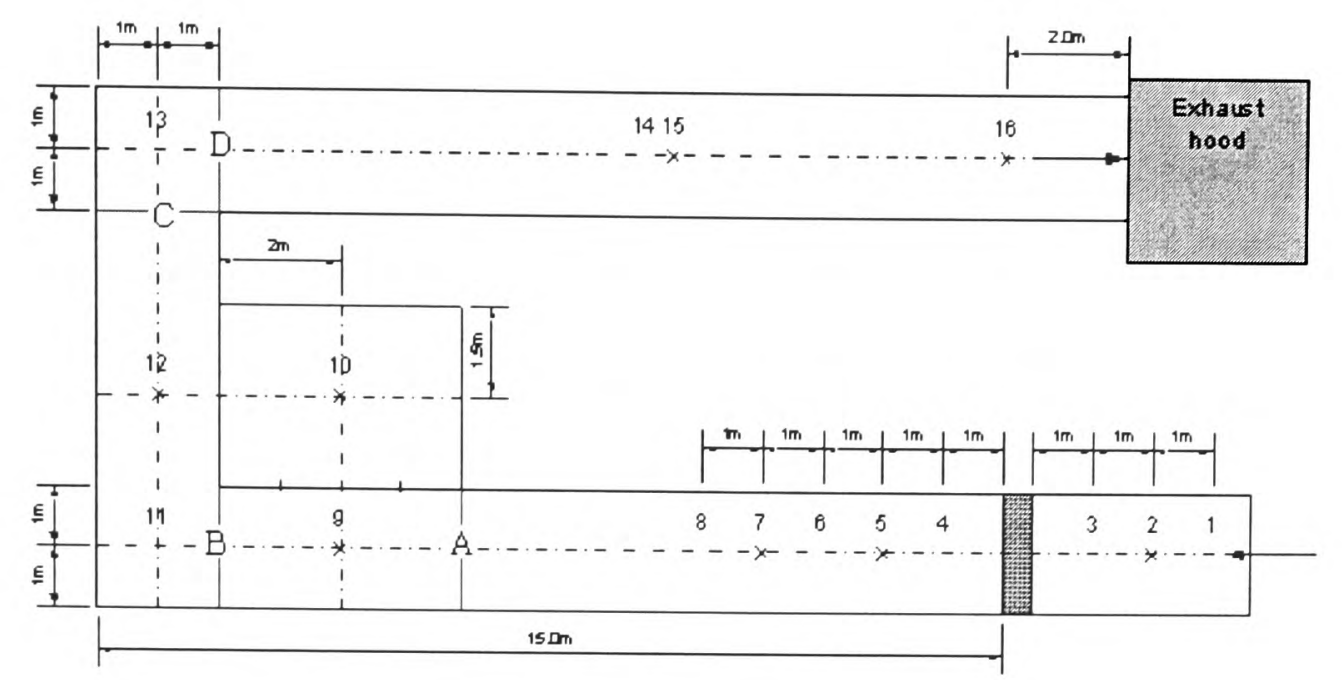

Figure 6.4.1: Top views of test geometry and measurement locations.

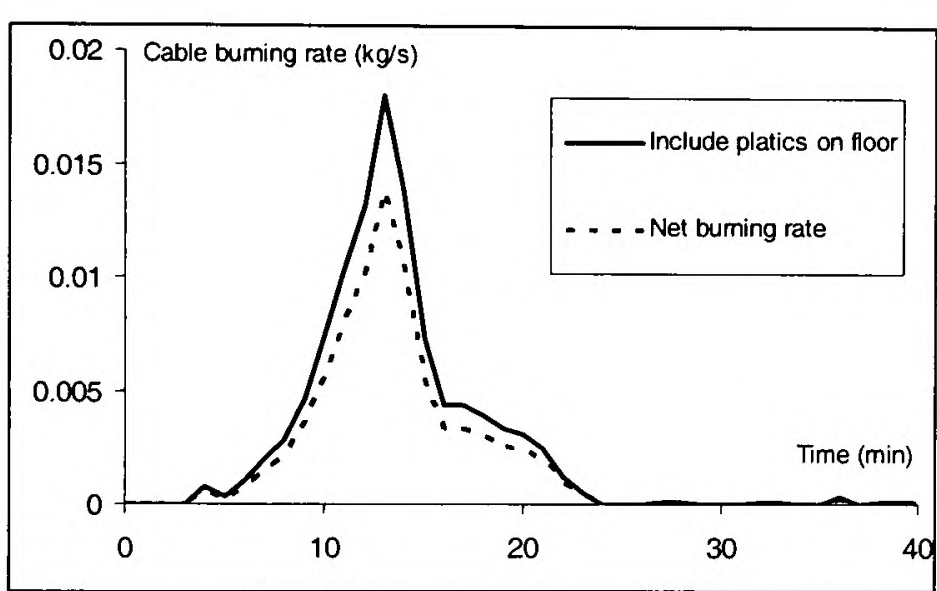

(a)

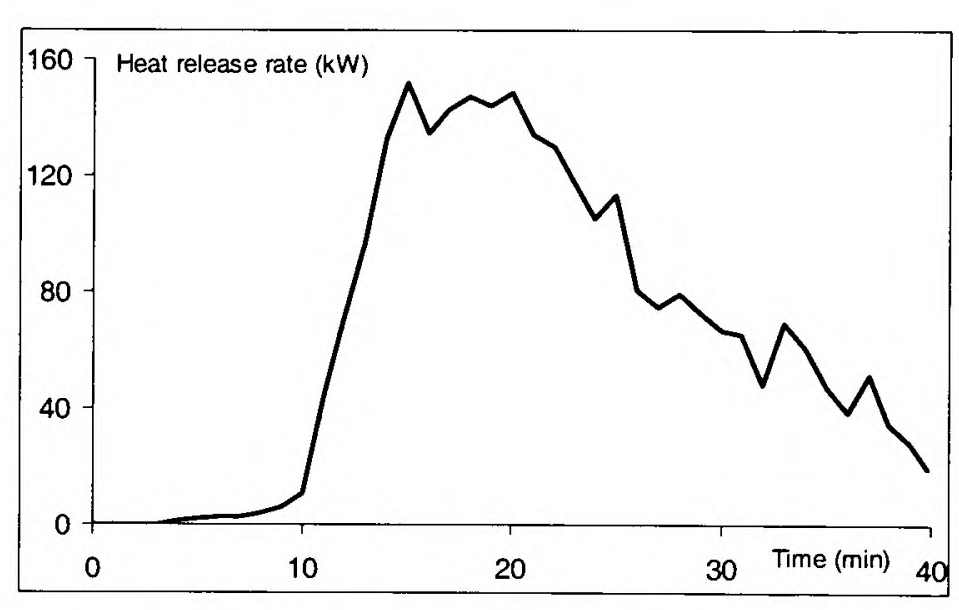

(b)

Figure 6.4.2: Measured (a) cable burning rate and (b) total heat release rate of the NHMH cable fire.

The principal gases of interest for measurements were oxygen, carbon dioxide, carbon monoxide and hydrogen chloride (only for PVC cable). In addition temperatures were also measured. The measurements were taken at some of the locations identified by the numbers 1 to 16 in Figure 6.4.1. The gas sampling locations were $0.2 \mathrm{~m}$ below the ceiling for most of the species except $\mathrm{CO}$ at location $5(0.1 \mathrm{~m}$ below the ceiling). The thermocouples were at heights of $2.2 \mathrm{~m}, 2.0 \mathrm{~m}, 1.5 \mathrm{~m}, 1.0 \mathrm{~m}$ and $0.5 \mathrm{~m}$. The mass loss of the cables was measured by positioning the entire cable tray arrangement on a load cell platform. The fire effluent was collected via a hood to a calorimeter. The test selected here is one with a NHMH cable tray fire. Beside the main fuel - NHMH cables - a propane burner generated $30 \mathrm{~kW}$ heat during the entire test time. In this test, the corridor was blocked from the floor to a height of $1.5 \mathrm{~m}$ at location $\mathrm{B}$. The 
measured cable loss rate and total heat release rate are shown in Figure 6.4.2. The cable mass loss after the test was $6.3 \mathrm{~kg}$, which includes $1.45 \mathrm{~kg}$ of unburned plastics (approximately 23\%) collected from the floor [Pe06]. Therefore, a net burning rate is derived from the measured burning rate including the plastics on the floor by multiplying a factor of 0.77 in Figure 6.4.2(a).

\subsubsection{Simulations}

In this simulation, the EBU combustion model is used to generate the heat due to combustion. The main materials of NHMH cable can be found in [SE98, HH03]. The molecular structure of this cable is set to be $\mathrm{C}_{3} \mathrm{H}_{6}$ (for polypropylene) in the EBU model in the present study as in [MJ06]. As described previously, approximately $23 \%$ of the $6.3 \mathrm{~kg}$ cables were not involved in combustion as they dropped to the floor. Therefore, the net burning rate in Figure 6.4.2(a) is used in the present simulation. The correlations between the yields of combustion products and the equivalence ratio for NHMH cables have been previously studied using the Purser Furnace at the University of Bolton [HL05]. The parameters for these correlations with the form of equation (6.3.1) are derived from these test data and presented in Table 6.4.1. When the equivalence ratio is larger than 3.5 , the combustion will be in non-flaming mode for most solid fuel [Te95]. It is supposed here that the inflexion point of the CO yield curve against the equivalence ratio $\phi_{\text {inflexion }}$ beyond which the yield of $\mathrm{CO}$ decreases (See equation 6.3.2) is 3.5. Figure 6.4.3(a) shows that the derived yields of CO with parameters in Table 6.4.1 are in very good agreement with the experimental data.

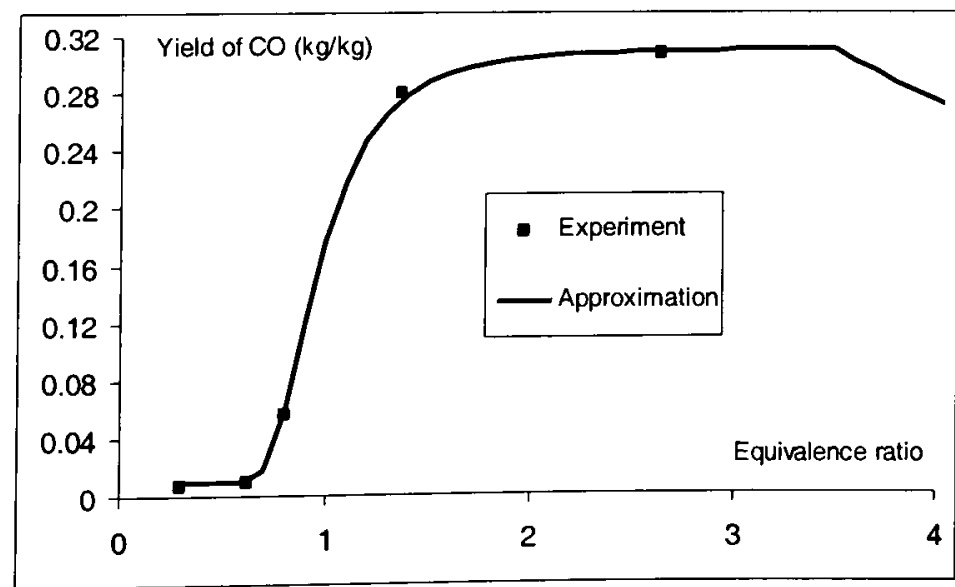

(a)

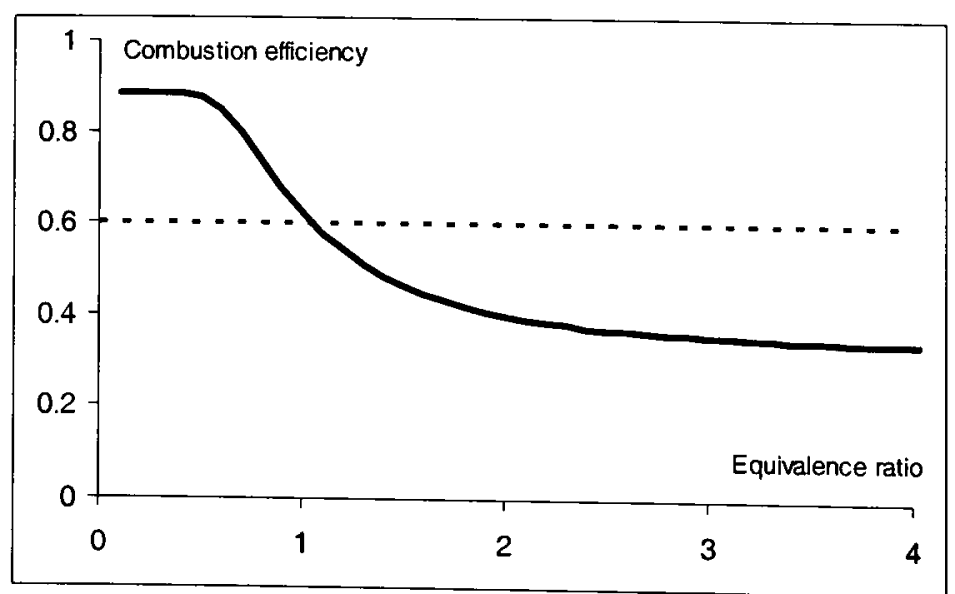

(b)

Figure 6.4.3: Yield of $\mathrm{CO}$ (a) and combustion efficiency as function of equivalence ratio (b) for NHMH cable. 
Table 6.4.1: Parameters for the yields of combustion products of NHMH cable.

\begin{tabular}{|l|l|l|l|l|}
\hline & $y_{i, \infty}(\mathrm{kg} / \mathrm{kg})$ & $\alpha$ & $\beta$ & $\zeta$ \\
\hline $\mathrm{CO}$ & 0.009 & 33.5 & 0.9 & -5.0 \\
\hline $\mathrm{CO}_{2}$ & 3.25 & -0.627 & 0.9 & -2.5 \\
\hline $\mathrm{O}_{2}$ & 3.20 & -0.627 & 0.9 & -2.5 \\
\hline
\end{tabular}

Combustion efficiency $\lambda$ can be defined as [Te95]

$$
\lambda=\Delta H_{c h} / \Delta H
$$

where $\Delta H_{c h}$ and $\Delta H$ are the chemical heat of combustion and the net heat of complete combustion respectively. The chemical heat of combustion in this case can be calculated as a function of the amount of oxygen consumed $y_{O 2}$ by

$$
\Delta H_{c h}=12.63 \cdot y_{O 2}
$$

where $y_{O 2}$ is defined by equation (6.3.1) with parameters listed in Table 6.4.1 and $12.62(\mathrm{MJ} / \mathrm{kg})$ is the heat of combustion per unit oxygen consumed for polypropylene. The net heat of combustion per unit mass of polypropylene is $46.37 \mathrm{MJ} / \mathrm{kg}$. Therefore, the Combustion efficiency for the NHMH cable fire can be calculated by

$$
\lambda=0.87 \cdot\left[1-\frac{0.627}{\exp (\phi / 0.9)^{-2.5}}\right]
$$

Figure 6.4.3(b) depicts the combustion efficiencies of the NHMH cable derived from equation (6.4.3).

The measured average heat of combustion in the present test was $27285 \mathrm{~kJ} / \mathrm{kg}$. By the definition of combustion efficiency (equation (6.4.1)), the average combustion efficiency of this test is approximately 0.60. From equation (6.4.3), the equivalence ratio corresponds to the combustion efficiency of 0.60 (See the broken line in Figure 6.4.3(b)) is approximately 1.0. Therefore, this critical equivalence ratio value will be used to determine the control region for this cable fire scenario.

Figure 6.4.4 is the set up of the geometry produced by the SMARTFIRE fire simulation tool. The computational mesh used in the simulation consisted of 86,080 
cells. The model calculated heat loss through the walls and the ceiling assuming the material properties of Promatect®-H board from [Pe06]. The time step size varied between 1 and 2 seconds. Since the combustion stopped at approximately 24 minutes, only this duration of the fire test is simulated.

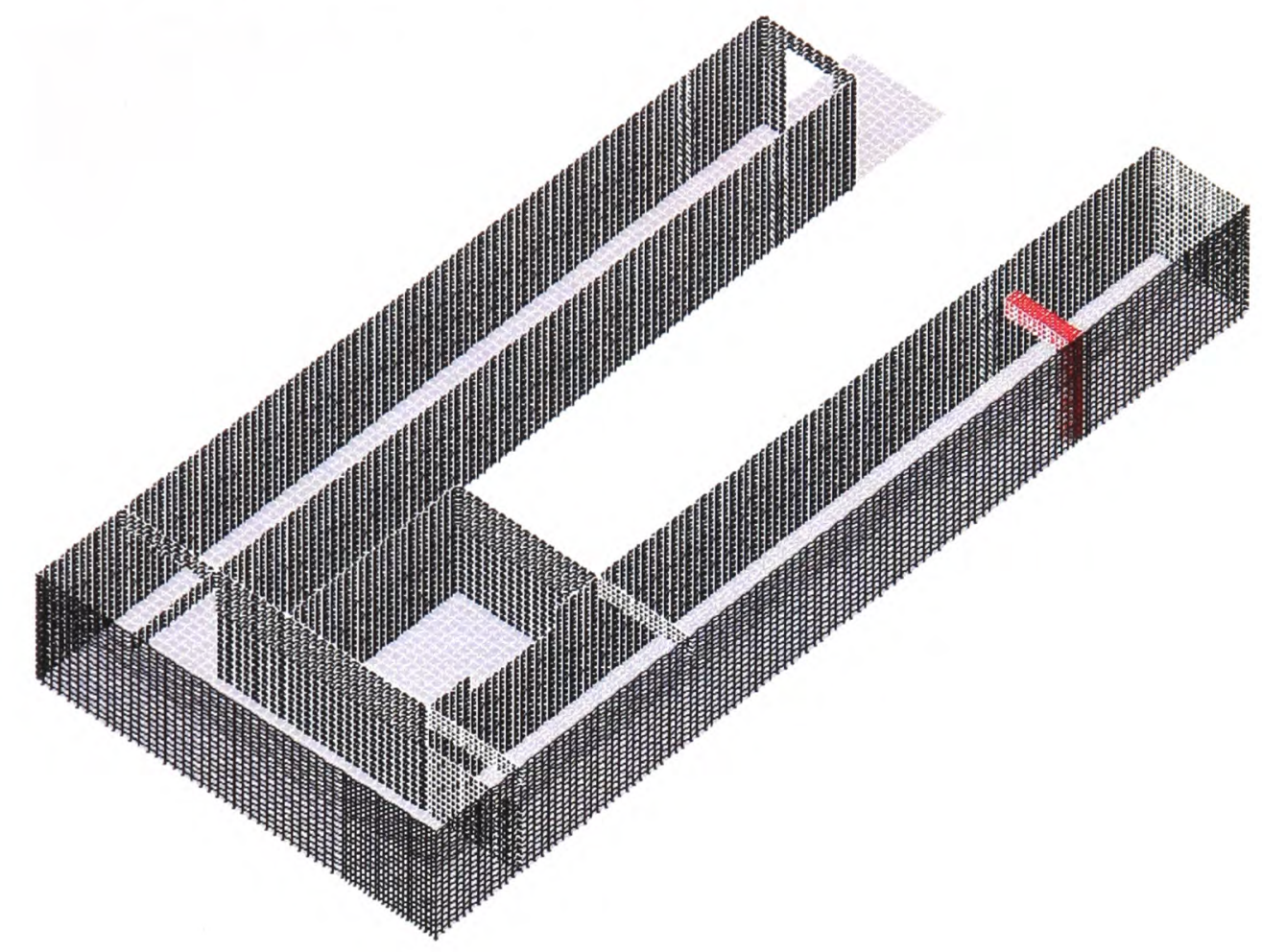

Figure 6.4.4: Set up of the room-corridor cable tray fire by SMARTFIRE (ceilings are removed to allow visualization of the inside corridor and room).

This scenario is simulated using Model I too for model comparison.

\subsubsection{Results and discussion}

The predicted CO concentrations in the horizontal plane $0.1 \mathrm{~m}$ below the ceiling at 13 minutes (corresponding to the peak burning rate) are shown in Figure 6.4.5. As seen in Figure 6.4.5, from the cable tray to the exit of the corridor, the predicted $\mathrm{CO}$ concentrations decrease from values above $1.0 \%$ to approximately $0.28 \%$. The CO concentrations in various sections which are divided by soffits A-D are almost uniform in each section. The CO levels within the room are similar to those in the section between soffit $B$ and $C$.

Therefore, the simulation results will be compared with the experimental data only at locations 5, 9, 12 and 15. These locations are at or close to the middle of each section of the corridor and the temperatures and species concentrations at these locations are representative of the air conditions across the whole enclosure. 


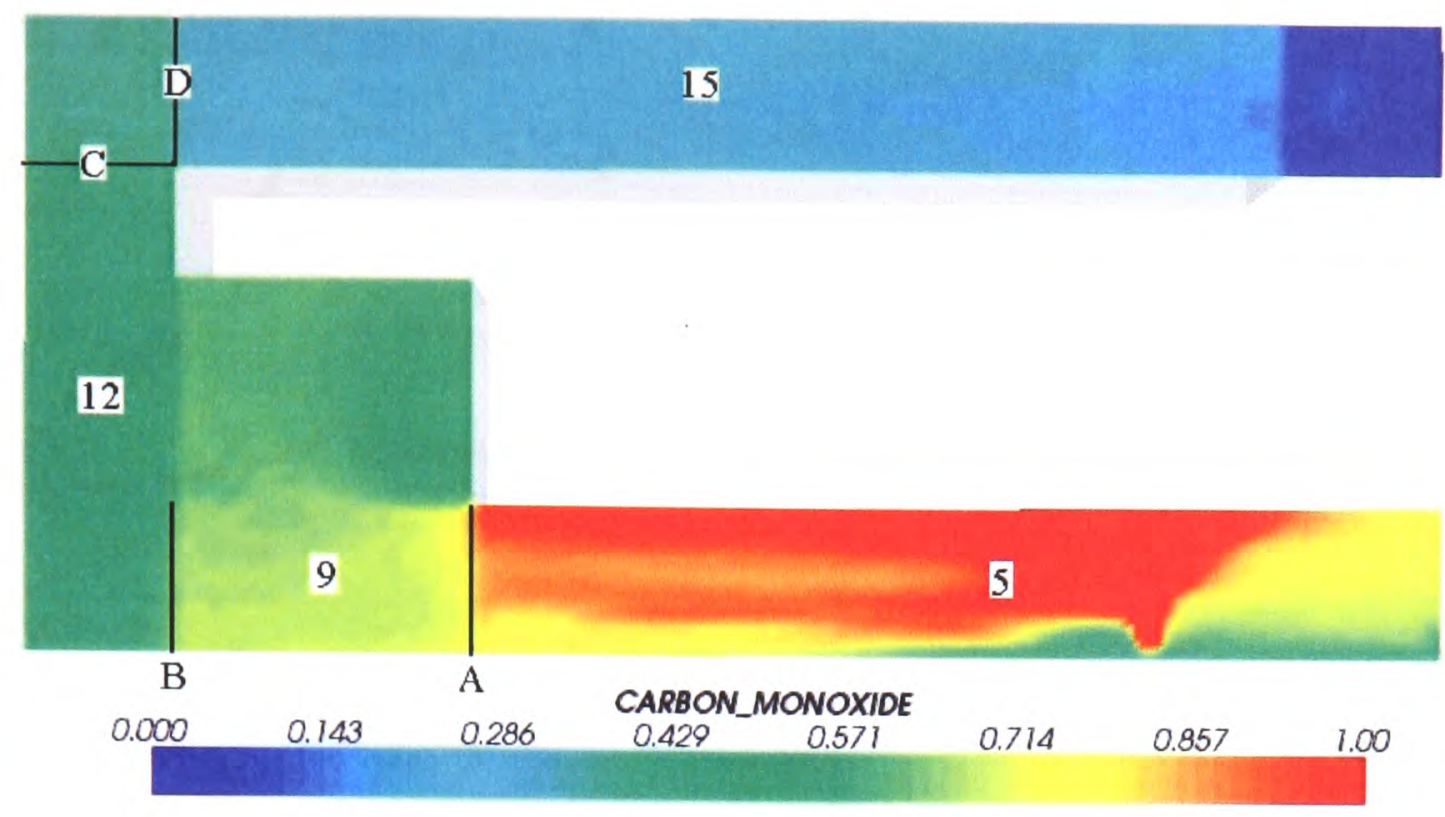

Figure 6.4.5: Predicted CO (\%) contours at the horizontal plane $0.1 \mathrm{~m}$ below the ceiling at 13 minutes.

\subsubsection{Temperatures}

The measured and predicted temperatures at location 5, 9, 12 and 15 are depicted in Figure 6.4.6. Location 5 is $2 \mathrm{~m}$ from the cable tray. After 3 minutes the measured temperatures at location 5 at a height of $2.2 \mathrm{~m}$, gradually increased to a peak value of $360^{\circ} \mathrm{C}$ at 13 minutes. After this peak time, the measured temperatures quickly decreased to $175^{\circ} \mathrm{C}$ at 17 minutes and then slowly decreased to $125^{\circ} \mathrm{C}$ at 24 minutes. Although the measured temperatures at other locations had similar trends to those at location 5 , the decrease rates of temperatures after the peak time become slower with the increase of distance from the fire source. The predicted temperatures at each location follow the measured trends.

The measured and predicted peak temperatures at a height of $2.2 \mathrm{~m}$ in locations 5,9 , 12 and 15 are $360^{\circ} \mathrm{C}, 140^{\circ} \mathrm{C}, 82^{\circ} \mathrm{C}$ and $40^{\circ} \mathrm{C}$ (experiment) and $397.0^{\circ} \mathrm{C}, 172.0^{\circ} \mathrm{C}$, $93.0^{\circ} \mathrm{C}$ and $41.0^{\circ} \mathrm{C}$ (predicted), respectively. The relative errors at these locations were $10.3 \%, 22.9 \%, 13.4 \%$ and $2.5 \%$ respectively. At a height of $1.0 \mathrm{~m}$, the measured peak temperatures were $78^{\circ} \mathrm{C}$ at location 5 and $69^{\circ} \mathrm{C}$ at location 9 , which are over predicted by approximately $7^{\circ} \mathrm{C}$ and $13^{\circ} \mathrm{C}$ or $9.0 \%$ or $18.8 \%$ respectively. At locations 12 and 15, both the measured and predicted temperatures at a height of 1.0 $\mathrm{m}$ are near the ambient values. 


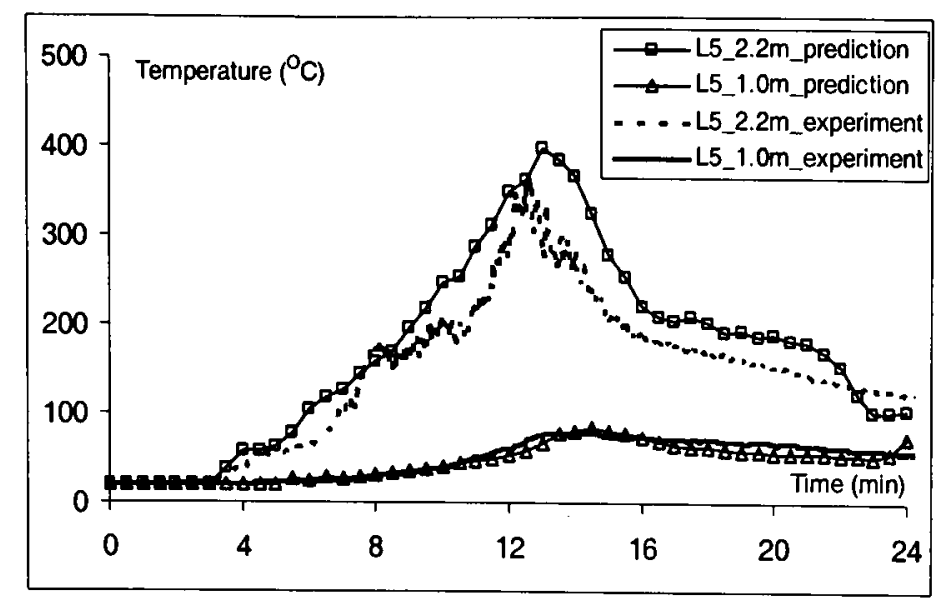

(a)

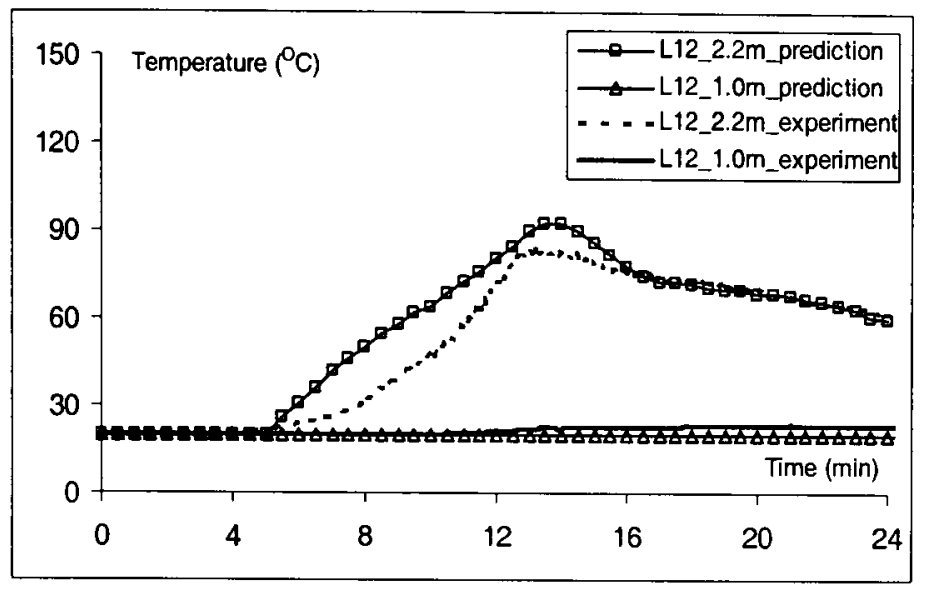

(c)

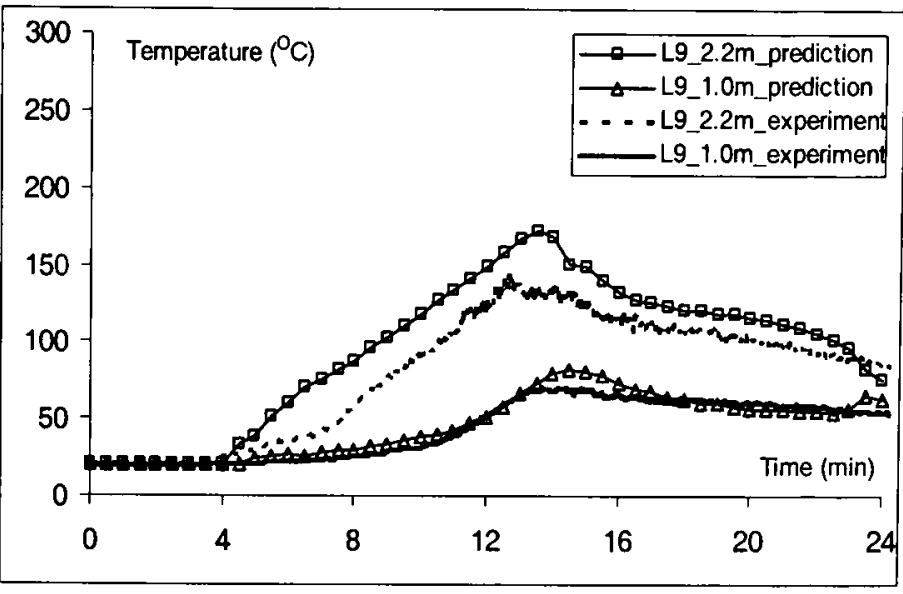

(b)

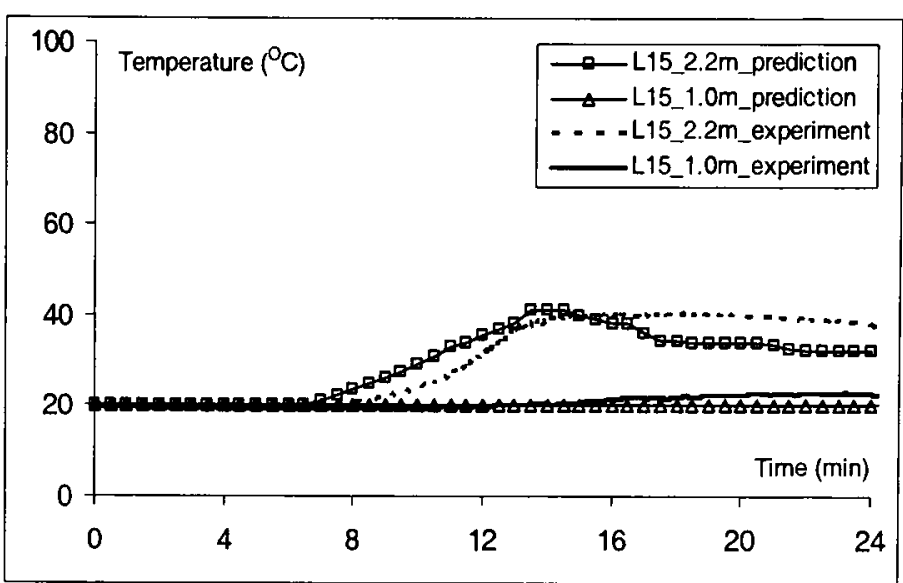

(d)

Figure 6.4.6: Measured and predicted temperatures at (a) location 5; (b) location 9; (c) location 12 and (d) location 15.

\subsubsection{CO concentrations}

The measured and predicted CO concentrations as functions of time are depicted in Figure 6.4.7 and the predicted peak species concentrations are compared with the measured data in Table 6.4.2. As seen in Figure 6.4.7, the measured $\mathrm{CO}$ curves at the

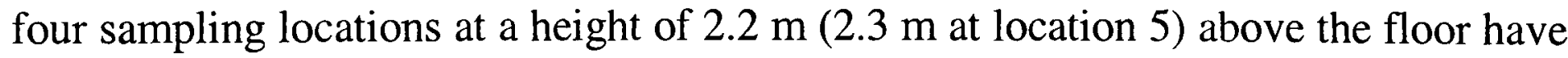
similar shapes to the measured temperatures (see Figure 6.4.6). Generally, the predicted $\mathrm{CO}$ concentrations follow the measured trends at the four gas sampling locations and the predictions of the time at which the concentration reaches its peak value very closely match the measured values.

In the first 15 minutes, $\mathrm{CO}$ predictions at locations 9,12 and 15 are very close to the measured values whereas after 15 minutes, the predicted $\mathrm{CO}$ concentrations are higher than the measured results. The $\mathrm{CO}$ concentrations at location 5 at 20 minutes are over predicted by a relative error of approximately $150 \%$. The over predictions of CO after 
15 minutes may be caused by the use of an averaged combustion efficiency. The actual combustion efficiency at this point in time may be somewhat higher than the average assumed here. The measured peak $\mathrm{CO}$ concentrations at location 5 was $1.62 \%$ (See Table 6.4.2) and the change of $\mathrm{CO}$ concentrations around the peak time was very sharp (See Figure 6.4.7(a)). The predicted peak CO concentration at this location is only $1.00 \%$ (see Table 6.4.2) and the predictions appear to change quite smoothly around the peak time.

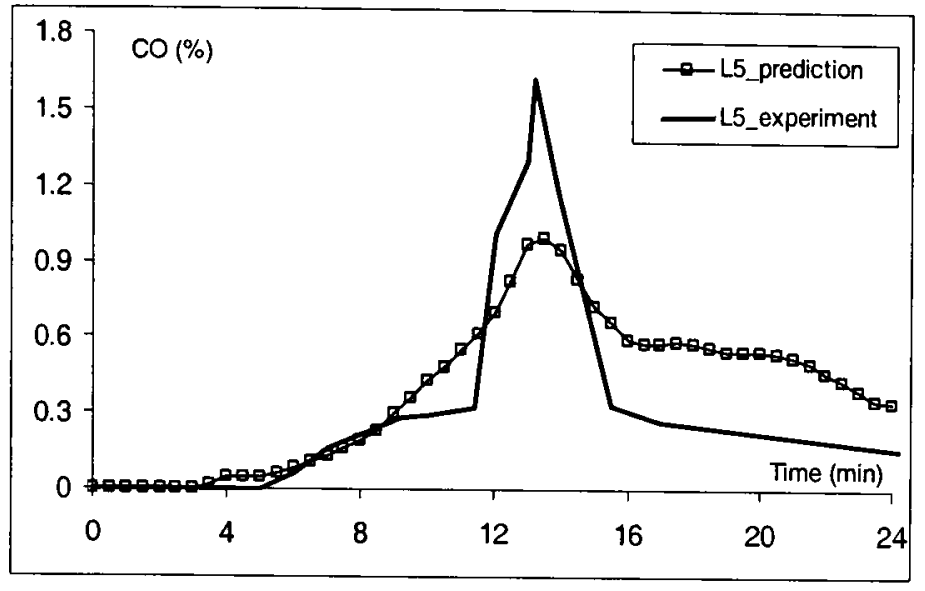

(a)

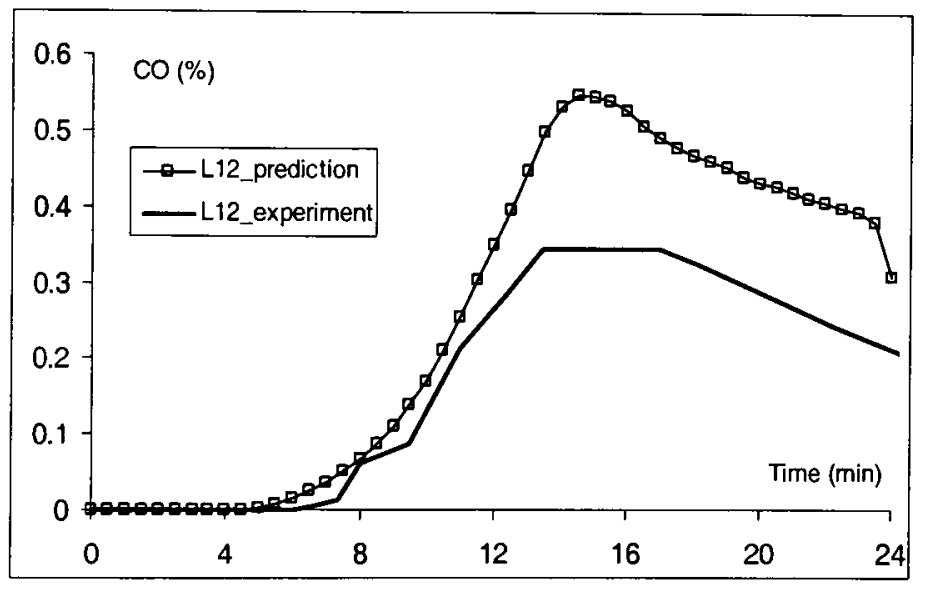

(c)

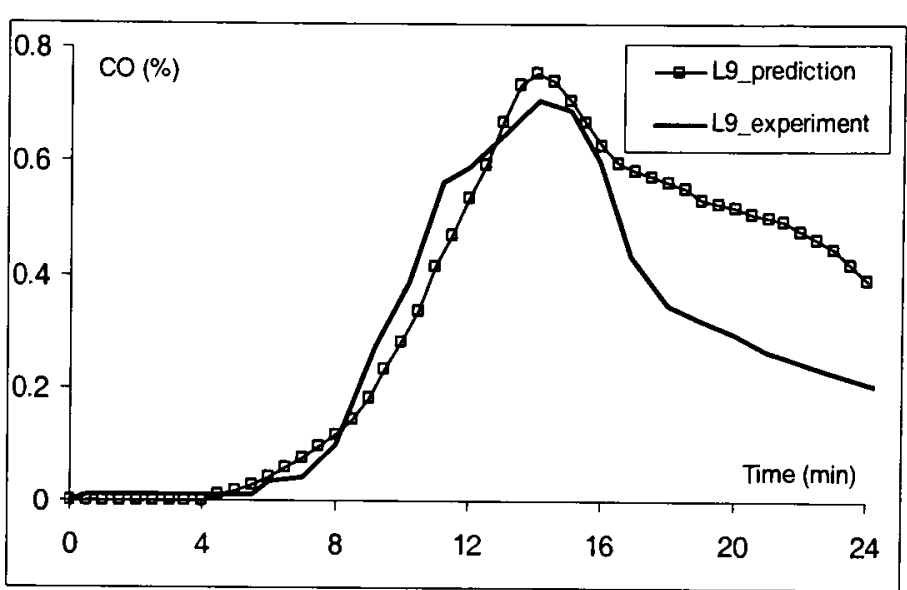

(b)

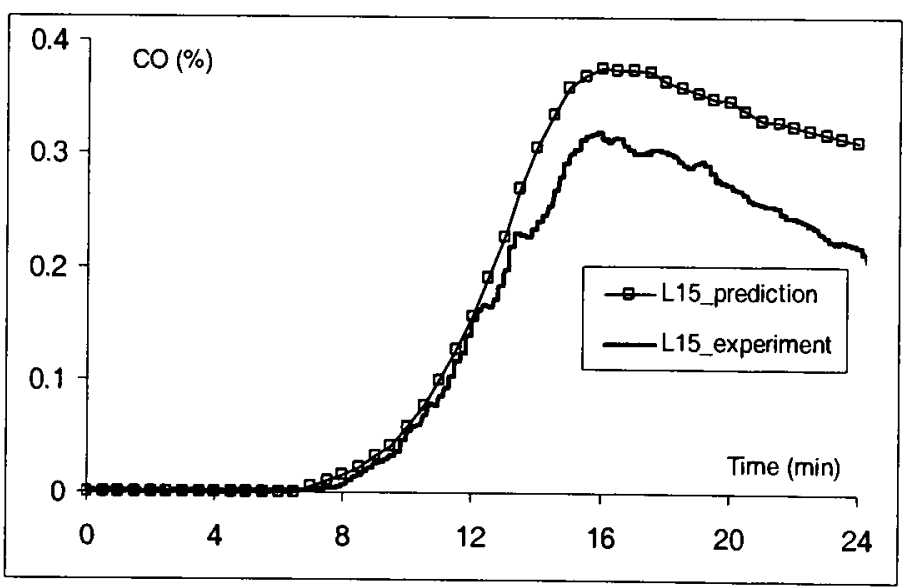

(d)

Figure 6.4.7: Measured and predicted CO concentrations at (a) location 5; (b) location 9; (c) location 12 and (d) location 15.

At location 12, the gas analyser was saturated at around the time of peak concentration. The $\mathrm{CO}$ concentration trace shows a measured $\mathrm{CO}$ concentration of $0.34 \%$ which lasted more than 3 minutes (see Figure 6.4.7(c)). The measured value during this time is therefore unreliable and likely to exceed $0.3 \%$, the upper limit for the gas analyser at this location. The predicted peak CO concentration at location 12 is $0.54 \%$. Finally, the predicted peak CO concentrations at location $9(0.76 \%)$ and location $15(0.37 \%)$ are in good agreement with the measured results $(0.71 \%$ and $0.31 \%$ respectively). 
Table 6.4.2: Measured and predicted peak species concentrations.

\begin{tabular}{|c|c|c|c|c|c|c|}
\hline \multirow{2}{*}{$\begin{array}{c}\text { Sampling } \\
\text { Location }\end{array}$} & \multicolumn{2}{|c|}{$\mathbf{C O}(\%)$} & \multicolumn{2}{c|}{$\mathbf{C O}_{2}(\%)$} & \multicolumn{2}{c|}{$\mathbf{O}_{\mathbf{2}}$ (\%) } \\
\cline { 2 - 7 } & measured & predicted & measured & predicted & measured & predicted \\
\hline $\mathbf{5}$ & 1.62 & 1.00 & 10.51 & 9.00 & 10.40 & 8.14 \\
\hline $\mathbf{9}$ & 0.71 & 0.76 & 5.61 & 6.35 & 12.45 & 12.32 \\
\hline $\mathbf{1 2}$ & $0.34^{*}$ & 0.54 & 4.41 & 4.59 & -- & 14.66 \\
\hline $\mathbf{1 5}$ & 0.31 & 0.37 & 3.31 & 3.15 & 16.41 & 16.61 \\
\hline
\end{tabular}

*gas analyser was saturated; the upper limit of the $\mathrm{CO}$ analyser at location 12 was $0.3 \%$.

\subsubsection{3 $\mathrm{CO}_{2}$ concentrations}

The measured and predicted $\mathrm{CO}_{2}$ concentrations as functions of time are depicted in Figure 6.4.8. The predicted $\mathrm{CO}_{2}$ concentrations are in good agreement with the measured data in the first 15 minutes at locations 5 and 9 . At locations 12 and 15, the predicted $\mathrm{CO}_{2}$ concentrations are in good agreement with the measured data within the entire simulation time of 24 minutes. The measured peak $\mathrm{CO}_{2}$ concentrations were $10.51 \%$ at location $5,5.61 \%$ at location $9,4.41 \%$ at location 12 and $3.31 \%$ at location 15 (See Table 6.4.2), which are under or over predicted by relative errors of $9.5 \%$, $13.2 \%, 4.1 \%$ and $4.8 \%$ respectively.

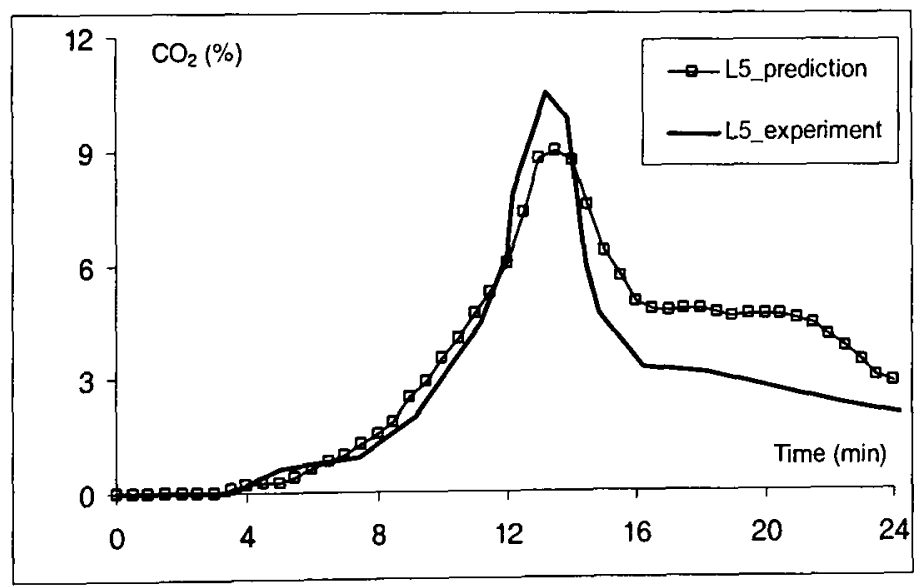

(a)

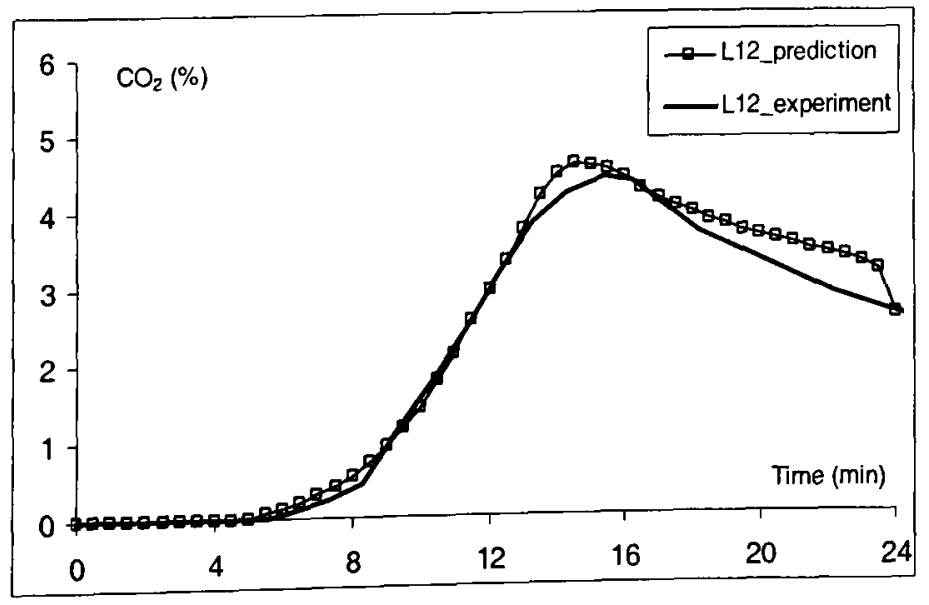

(c)

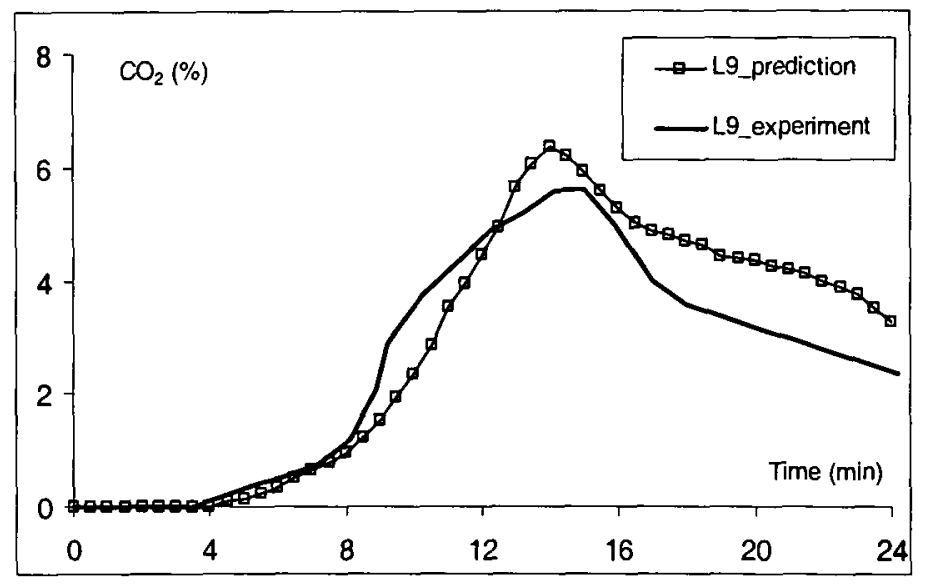

(b)

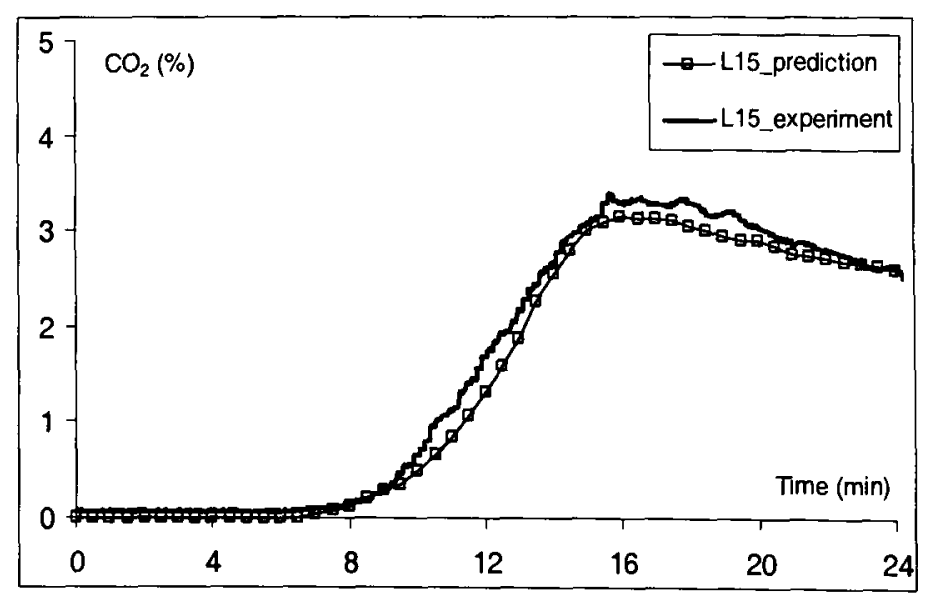

(d)

Figure 6.4.8: Measured and predicted $\mathrm{CO}_{2}$ concentrations at (a) location 5; (b) location 9; (c) location 12 and (d) location 15. 


\subsubsection{4 $\mathrm{O}_{2}$ concentrations}

The measured $\mathrm{O}_{2}$ concentration curves (See Figure 6.4.9) have the reversed curve shapes of $\mathrm{CO}_{2}$. As the absolute changes of $\mathrm{O}_{2}$ concentrations are not significant, the predicted $\mathrm{O}_{2}$ concentrations appear in good agreement with the measured data. The measured peak $\mathrm{O}_{2}$ concentrations of $10.40 \%$ at location $5,12.45 \%$ at location 9 , and $16.41 \%$ at location 15 are under or over predicted by relative errors of $21.7 \%, 1.2 \%$ and $1.2 \%$ respectively.

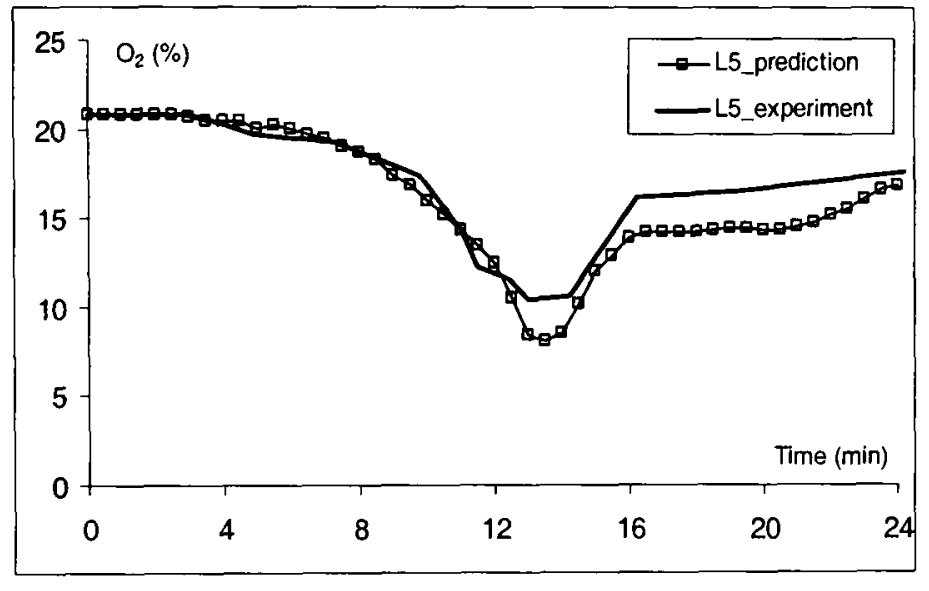

(a)

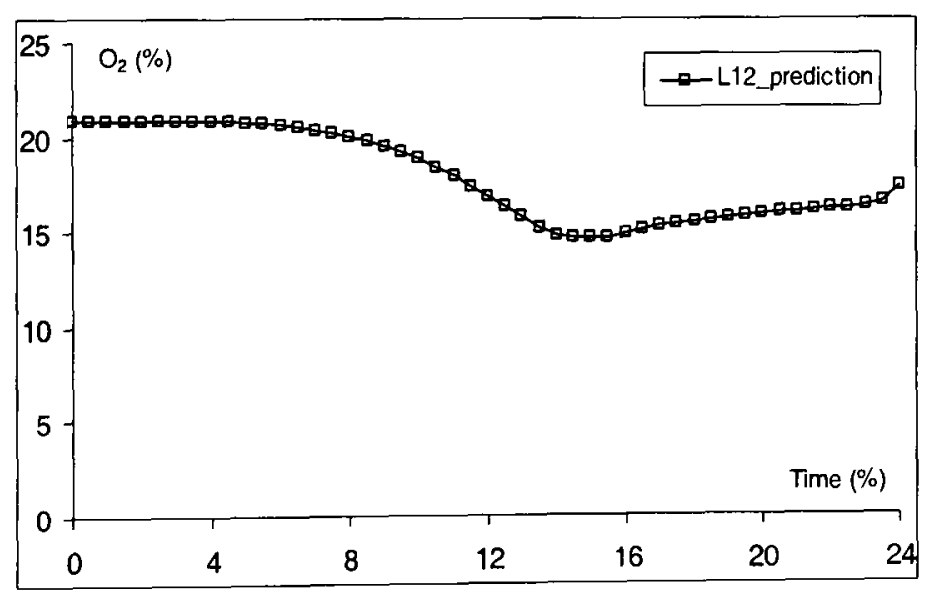

(c)

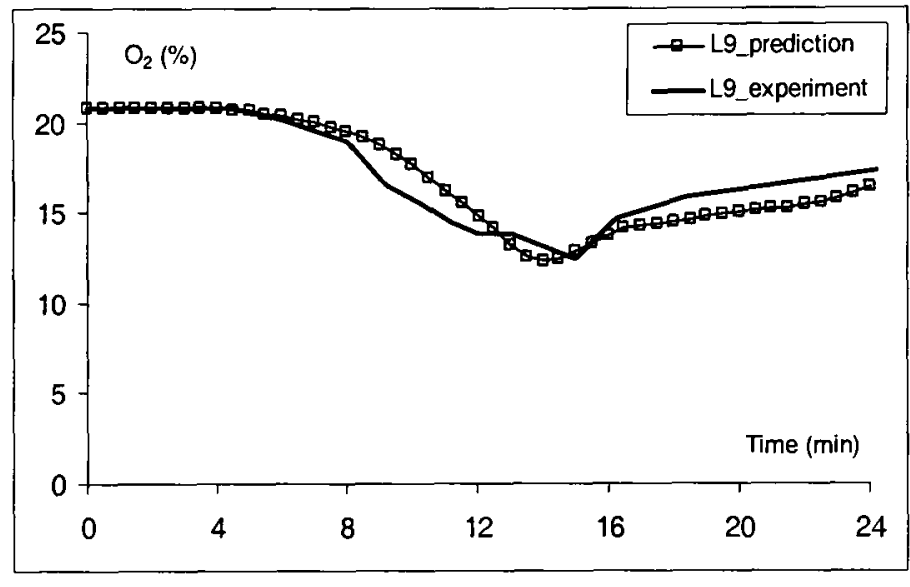

(b)

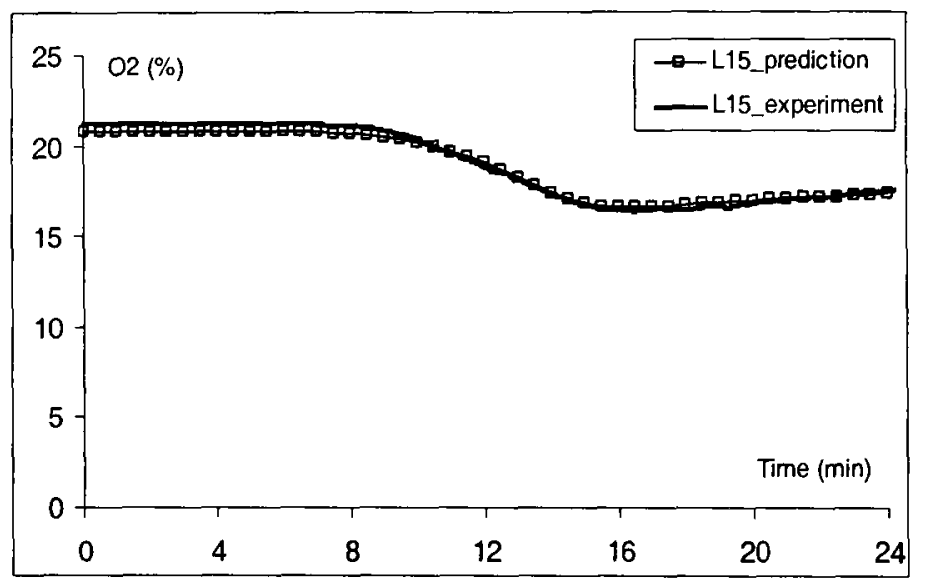

(d)

Figure 6.4.9: Measured and predicted $\mathrm{O}_{2}$ concentrations at (a) location 5; (b) location 9; (c) location 12 and (d) location 15

\subsubsection{Predicted control region at time of peak burning rate}

After the fire had fully developed in the experiment, the fire plume could not be seen due to the poor visibility caused by the thick smoke. From the measured peak $\mathrm{CO}$ and $\mathrm{O}_{2}$ levels in Table 6.4.2, it is estimated that location 5 is outside the control region. The gas probes at location 5 were in the central line of the corridor and $2 \mathrm{~m}$ from the 
cable trays. The $\mathrm{CO}$ probe was $0.1 \mathrm{~m}$ below the ceiling and $\mathrm{CO}_{2}$ and $\mathrm{O}_{2}$ probes were $0.2 \mathrm{~m}$ below the ceiling. At 13 minutes (around the peak burning rate as seen in Figure 6.4.2(a)), the predicted combustion region in the horizontal plane of $2.25 \mathrm{~m}$ high $(0.15 \mathrm{~m}$ below the ceiling) which is passing through the middle of these gas probes is shown in Figure 6.4.10. Figure 6.4.10 shows that location 5 is inside the predicted transport region but close to the predicted control region when the fire reached its peak.

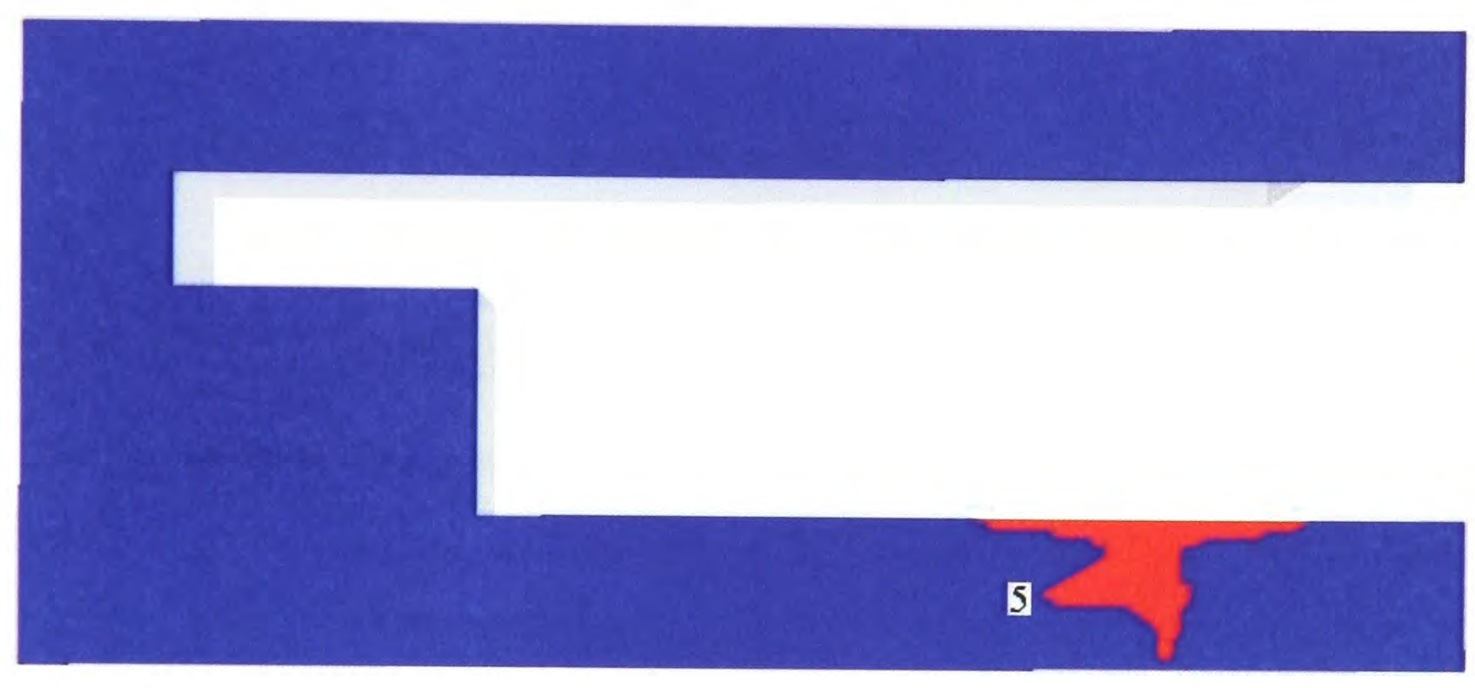

Figure 6.4.10: Predicted control region in the horizontal plane $0.15 \mathrm{~m}$ below the ceiling at 13 minutes.

\subsubsection{Comparison with Model I}

The predicted peak species concentrations at location 5 and location 15 , using the improved model and the Model I in Chapter 4 are compared in Table 6.4.3. Location 5 is $2.0 \mathrm{~m}$ from the cable tray and is just outside the predicted CR in the simulation using the modified model. Whilst the improvement in the predictions of $\mathrm{CO}_{2}$ and $\mathrm{O}_{2}$ using the modified model is marginal, the $\mathrm{CO}$ prediction has improved significantly with $38 \%$ of the relative error by the modified model compared with $75 \%$ for the original model. Location 15 is approximately $29.5 \mathrm{~m}$ away from the cable tray. The predicted peak $\mathrm{CO}$ concentration is as low as $0.02 \%$ with the original model, compared with the measured CO concentration of $0.31 \%$. The modified model has achieved greater improvement in the prediction accuracy with a predicted $\mathrm{CO}$ concentration of $0.37 \%$. The modified model is also able to generate better predictions for $\mathrm{CO}_{2}$ and $\mathrm{O}_{2}$ concentrations at location 15. Comparing the predictions at location 5 and 15 , it appears that the improvement in the predictions of toxic gases by the modified model becomes significant as the distance from the CR increases. 
Table 6.4.3: Predicted peak species concentrations at location 5 and 15 using Model I and the present modified model.

\begin{tabular}{|c|c|c|c|c|}
\hline Location & Species & Experiment & Model I & Present Model \\
\hline \multirow{4}{*}{5} & $\mathbf{C O}(\%)$ & 1.62 & 0.40 & 1.00 \\
\cline { 2 - 5 } & $\mathbf{C O}_{\mathbf{2}}(\boldsymbol{\%})$ & 10.51 & 9.70 & 9.00 \\
\cline { 2 - 5 } & $\mathbf{O}_{\mathbf{2}}(\boldsymbol{\%})$ & 10.40 & 7.30 & 8.14 \\
\hline \multirow{3}{*}{15} & $\mathbf{C O}(\boldsymbol{\%})$ & 0.31 & 0.02 & 0.37 \\
\cline { 2 - 5 } & $\mathbf{C O}_{\mathbf{2}}(\boldsymbol{\%})$ & 3.31 & 4.40 & 3.15 \\
\cline { 2 - 5 } & $\mathbf{O}_{\mathbf{2}}(\boldsymbol{\%})$ & 16.41 & 15.03 & 16.61 \\
\hline
\end{tabular}

\subsubsection{Sensitivity of species on combustion efficiency}

The sensitivity of the predicted gas species concentrations on the combustion efficiency, which determines the critical equivalence ratio $\phi_{C R}$ was examined in this section.

The predicted peak species concentrations for the cable fire simulation presented in Table 6.4.2 are with a $\phi_{C R}$ value of 1.0 determined at a combustion efficiency of 0.6. Presented in Table 6.4.4 are the relative changes in the predicted peak species concentrations at location 15 if the combustion efficiency has an error of $+/-10 \%$. As seen in Table 6.4.4, the predicted change in peak $\mathrm{CO}$ concentration at location 15 can be as much as $+40.5 \%$ if there is an error of $-10 \%$ in estimating the combustion efficiency. The corresponding changes in $\mathrm{O}_{2}$ and $\mathrm{CO}_{2}$ concentrations are $+3.2 \%$ and $-14.9 \%$ respectively. If the error in estimating the combustion efficiency is $+10 \%$, the corresponding change in $\mathrm{CO}, \mathrm{O}_{2}$ and $\mathrm{CO}_{2}$ concentrations are $-32.4 \%,-1.9 \%$ and $+4.8 \%$ respectively.

Table 6.4.4: Sensitivity of remote peak species concentrations on combustion efficiency at location 15 for the U-shaped corridor.

\begin{tabular}{|c|c|c|c|c|}
\hline \multirow{2}{*}{$\begin{array}{c}\text { Relative changes } \\
\text { in Combustion } \\
\text { efficiency }\end{array}$} & \multicolumn{4}{|c|}{$\begin{array}{c}\text { Corresponding change in critical equivalence ratio and predicted species } \\
\text { concentrations compared with combustion efficiency of 0.6 }\end{array}$} \\
\cline { 2 - 5 } & $\phi_{C R}$ & $\mathbf{C O}$ & $\mathbf{C O}_{2}$ & $\mathbf{O}_{2}$ \\
\hline$+10 \%$ & $-10 \%$ & $-32.4 \%$ & $+4.8 \%$ & $-1.9 \%$ \\
\hline$-10 \%$ & $+15 \%$ & $+40.5 \%$ & $-14.9 \%$ & $+3.2 \%$ \\
\hline
\end{tabular}


In this fire scenario, the average combustion efficiency is 0.6 , which corresponds, in this particular case, to a critical equivalence ratio of 1.0. As seen in Figure 6.4.3(a), the yields of $\mathrm{CO}$ varies dramatically when the equivalence ratio has a value of around 1.0. As a result, the predicted $\mathrm{CO}$ concentrations at location 15 are very sensitive to the changes in the combustion efficiency.

If $\phi_{C R}$ falls in the range of equivalence ratio values in which the species yields change rapidly with small changes in equivalence ratio, the species concentrations in the far field will be sensitive to the precise value of $\phi_{C R}$. In this case it is advisable to undertake a sensitive analysis in order to determine the likely degree of variation in species concentrations in the far field.

\subsection{Concluding Remarks}

This chapter is an attempt to address the relatively poor predictions of the species concentrations made by Model I in Chapter 4 at locations remote from the fire source for scenarios in which significant amounts of fuel are not fully combusted. In the approach outlined in this chapter, the computation domain is divided into two zones: a control region and a transport region. The division of the computational domain is based on a critical equivalence ratio that is associated with the combustion efficiency of the fire scenario. Unlike Model I in which one single equation for the prediction of each species was used over the entire computation domain, different calculations for the two regions are employed in the modified model.

Two experimental fire scenarios have been simulated using the modified approach. One fire scenario involved a reduced compartment-hallway $n$-Hexane fire. In this scenario, the fire that originated within the room was extinguished before it could spread to the adjacent hallway. The simulation successfully reproduced the distributions of the measured species concentrations in the fire compartment and the adjacent hallway during the quasi-steady state period. The second fire scenario consisted of a cable fire in a large $U$ shape corridor. The predicted temperatures and species concentrations at different locations within the fire enclosure follow the overall measured experimental trends, although there was considerable difference 
between the predicted and measured peak $\mathrm{CO}$ concentrations near to source of the fire i.e. the cable tray. The observed over predictions of $\mathrm{CO}$ concentrations some 15 minutes after fire initiation could be the result of using an averaged combustion efficiency which is no longer representative of the burning conditions in this phase of the fire development.

In both cases the modified model has lead to considerable improvements in the predictions of $\mathrm{CO}, \mathrm{CO}_{2}$ and $\mathrm{O}_{2}$ at locations remote from the fire source when compared to Model I in Chapter 4.

In this study, the combustion efficiency is assumed to be known and used to determine the control region. The sensitivity of the predicted species concentrations on the combustion efficiency is scenario dependent. Model predictions of species concentrations at remote locations are sensitive to the combustion efficiency if the correspond critical equivalence ratio happens to be a value near which yields of species are subject to rapid changes. Where this is the case, it is advisable to undertake a sensitive analysis in order to determine the likely degree of variation in species concentrations in the far field.

Work is necessary to further refine the model, for example, rather than rely simply on the combustion efficiency to define the CR, other methodologies involving the effect of temperature, residence time and oxygen concentrations need to be explored. In addition, the assumption that the species concentrations on the boundary of the $\mathrm{CR}$ are solely dependent on the LER alone (Assumption 6.2.1) should be further tested for more complex scenarios. 


\section{Chapter 7}

\section{TWO-FUEL TOXICITY MODEL AND APPLICATION}

\subsection{Introduction}

In real enclosure fires, fuels include not only the construction materials but also some furniture. Morikawa et al. [MY93] conducted house fire tests to study the toxicity in upstairs rooms caused by fire within downstairs room. In the tests, the combustible contents in the burning room are a wardrobe and its contents, a chest of drawers and its contents, a table, foam chairs, acrylic and wool carpet, wall, ceiling and floor material (plywood) and curtains (acrylic and polyester). High levels of $\mathrm{CO}$ were measured on the second floor while the fire room was in the ground floor. Therefore, it is important to develop a mathematical model for predicting the generation of toxic gases from enclosure fires with more than one fuel and their transport within the building.

A townhouse fire in Sharon, Pennsylvania, USA resulted in the death of three people [LV97]. As introduced in [LV97], a full-scale test by Levine and Nelson shows that a large load of wood in the upper layer of a kitchen was the source of high CO levels in the bedroom in the second floor, which caused the deaths of the three people. Pitts, et al [PJ94] conducted tests using a reduced-scale compartment with the ceiling and upper portion of the walls lining with wood. In those tests, $\mathrm{CO}$ level were measured to be as high as $12 \%$. Lattimer, et al [LV98] conducted a series of hexane fire tests within a reduced compartment and an adjacent hallway with and without wood under the ceiling of the compartment. This set of tests was to study the effect of wood on the generation of $\mathrm{CO}$ in enclosure fires, and the transport of $\mathrm{CO}$ from the fire room to other locations. The measured peak CO concentration with wood in the compartment reaches to $14 \%$ that is 4 times higher than that measured without wood in the upper layer.

The pyrolysis of wood in the hot upper layer of fire enclosures releases certain amount of $\mathrm{CO}$. To simulate this phenomenon, a mathematical model is developed to predict the toxic gases for two-fuel fires in this chapter. The two-fuel EBU (eddy 
break-up) combustion model [CFX97] is used to calculate the heat released due to combustion. The concentrations of carbon monoxide, carbon dioxide and oxygen are calculated based on the local equivalence ratio (LER) concept, which is obtained from the two mixture fractions. Hexane fires with and without wood in the upper layer of the compartment [LV98] are simulated using the combination of the two-fuel EBU model and the new developed two-fuel toxicity model.

The calculation of the LER based on two fuels is presented in Section 7.2. The toxicity model is discussed in Section 7.3. Model validations are described in Section 7.4. Conclusions are drawn in Section 7.5.

\subsection{Local Equivalence Ratio for Two-fuel Fires}

The LER $\phi$ at a control volume, which corresponds to the mixture fraction, is the ratio of the mass that originates from the fuel source to the mass that originates from the air stream divided by the stoichiometric fuel-to-air ratio. In the one-fuel model, the LER in equation (4.2.8) is a function of the mixture fraction, i.e.

$$
\phi=\frac{\xi /(1-\xi)}{r_{s t}}
$$

where $r_{s t}$ is the stoichiometric fuel-air ratio.

When calculating the LER for multi-fuel fires, the stoichiometric fuel-air ratio changes with both space and time. The stoichiometric ratio is constant only if the ratio of the mass from the first fuel source to the mass from the second fuel source at any control volume remains constant. This is impossible in real enclosure fires. In the fire tests in [LV98], a temporal global stoichiometric fuel-air ratio is calculated. A stoichiometric equation is balanced using appropriate mole contributions from each fuel source. In [LV98] the mole contribution from one fuel is the ratio of its mole loss rate to the total fuel mole loss rate. After obtaining the temporal stoichiometric equation, the stochiometric fuel-air ratio can be calculated. 
In the present study, the LER is derived from the mixture fractions. In the two-fuel EBU model [CFX97], the combustion is supposed to be complete. The oxygen is shared by the two different fuels and the two mixture fractions can be expressed with

$$
\xi^{i}=Y_{f}^{i}+\frac{Y_{p}^{i}}{1+r_{s t}^{i}}
$$

where $i=1,2$ stand for the two different fuels and $r_{s t}^{i}$ stoichiometric air-fuel mass ratio. Mixture fractions, $\xi^{1}$ and $\xi^{2}$ represent the mixture of fuels, air and combustion products. The mass fraction of oxidant and products are calculated from the two fuel mass fractions and the two mixture fractions.

Similar to the study in Chapter 4, it is supposed that fuel, $\mathrm{CO}_{2}, \mathrm{CO}$, hydrocarbon and soot include the carbon element in the mixture of gases. As assumed in the one-fuel toxicity model, all chemical elements have the same diffusion coefficient in the twofuel fires. Therefore, the ratios among elements, which come from the same fuel source, will not change in the processes of chemical reactions and transport. If the combustion is incomplete, the mixture fraction based on the conservation of carbon, which come from fuel $i$, can be expressed by the following equation

$$
\xi^{i}=\frac{\left(\mu_{f, c}^{i} Y_{f}^{i}+\frac{12}{44} Y_{c o 2}^{i}+\frac{12}{28} Y_{c o}^{i}+Y_{s o o t}^{i}+\mu_{H C, c} Y_{H C}^{i}\right)-0}{\mu_{f, c}^{i}-0}
$$

where $Y$ represents the mass fractions of fuel and combustion products containing carbon, $\mu_{k, j}$ represents the mass fraction of element $j$ in species $k$ and $i=1,2$ stand for the two different fuels, and the mass fraction of carbon in the air stream is 0 .

In the two-fuel scenario, the LER depends on the mass ratio of the two fuels. According to the definition of the two mixture fractions (7.2.3), in each control volume, the mass that comes from the two different fire sources are $\xi^{1} \rho V$ and $\xi^{2} \rho V$ respectively, here $\rho$ is the average fluid density in the control volume and $V$ is the volume. The total mass that originates from the two fuel sources is $\left(\xi^{1}+\xi^{2}\right) \cdot \rho \cdot V$ and the mass that originates from the air stream is $\left(1-\xi^{1}-\xi^{2}\right) \cdot \rho \cdot V$. Let $r_{s t}^{i}$ be the stoichiometric fuel-air ratio for fuel $i, i=1$ or 2 . The stoichiometric air for the 
combustion of $\xi^{1} \rho V$, the first fuel, and $\xi^{2} \rho V$, the second fuel, are $\xi^{1} \rho V / r_{s t}^{1}$ and $\xi^{1} \rho V / r_{s t}^{2}$ respectively. Therefore, the stoichiometric air for the combustion of $\xi^{1} \rho V+\xi^{2} \rho V$ fuels is $\xi^{1} \rho V / r_{s t}^{1}+\xi^{1} \rho V / r_{s t}^{2}$. The fuel to air ratio at this control volume is $\left(\xi^{1}+\xi^{2}\right) \cdot \rho \cdot V /\left(\left(1-\xi^{1}-\xi^{2}\right) \cdot \rho \cdot v\right)$. Therefore, the LER (7.2.1) for one-fuel fires is now extended to

$$
\phi=\frac{\left(\xi^{1}+\xi^{2}\right) \cdot \rho \cdot V /\left(\left(1-\xi^{1}-\xi^{2}\right) \cdot \rho \cdot V\right)}{\left(\xi^{1}+\xi^{2}\right) \cdot \rho \cdot V /\left(\xi^{1} \rho V / r_{s t}^{1}+\xi^{2} \rho V / r_{s t}^{2}\right)}=\frac{\xi^{1} / r_{s t}^{1}+\xi^{2} / r_{s t}^{2}}{1-\xi^{1}-\xi^{2}}
$$

for two-fuel cases. In a control volume, if the mass only comes from one of the two fuel sources and the air stream, the value of one of the mixture fractions should be zero. Let $\xi_{2}$ be zero, the LER at this control volume given by (7.2.4) becomes

$$
\phi=\frac{\xi^{1} / r_{s t}^{1}}{1-\xi^{1}}=\frac{\xi^{1} /\left(1-\xi^{1}\right)}{r_{s t}^{1}}
$$

Equation (7.2.5) means that the LER given by (7.2.4) at locations, where the mass comes from the air stream and only one fuel source, is the same as the LER (7.2.1) for one-fuel fire.

\subsection{Two-fuel Toxicity Model}

In Chapter 4, the mass fraction of product $i$ and the oxygen mass fraction are calculated using the species yields and the mixture fraction by Model I with the following equations

$$
\begin{aligned}
& Y_{i}=y_{i}(\phi, T) \xi \\
& Y_{O 2}=\mu_{a i r, o}(1-\xi)-y_{O}(\phi, T) \xi
\end{aligned}
$$

where correlation $y_{i}(\phi, T)$ is the yield of product $i(\mathrm{~kg} / \mathrm{kg}), y_{O}(\phi, T)$ is the oxygen consumption and $\mu_{\text {air,o }}$ is the oxygen mass fraction in the air stream. 
The mass fraction of a combustion product in two-fuel fires consists of two parts. One part comes from the oxidation of the first fuel and the other comes from the oxidation of the second fuel.

An equivalent of the equation (7.2.3) is

$$
\rho V\left(\xi^{i}-Y_{f}^{i}\right) \mu_{f, c}^{i}=\rho V\left(\frac{12}{44} Y_{c o 2}^{i}+\frac{12}{28} Y_{c o}^{i}+Y_{\text {sooT }}^{i}+\mu_{H C, C} Y_{H C}^{i}\right)
$$

Equation (7.3.3) means that the mass of carbon in each control volume from fuel $i$ is conserved in the process of the chemical reactions and species transport.

Expressing that the yields per $\mathrm{kg}$ fuel $i$ with the condition of LER $\phi$ and temperature $T$ as $y_{f}^{i}(\phi, T) \mathrm{kg}$ of fuel, $y_{c o 2}^{i}(\phi, T) \mathrm{kg}$ of $\mathrm{CO}_{2}, y_{c o}^{i}(\phi, T) \mathrm{kg}$ of $\mathrm{CO}, y_{\text {soot }}^{i}(\phi, T) \mathrm{kg}$ of soot, $y_{h c}^{i}(\phi, T) \mathrm{kg}$ of hydrocarbon, then the conservation of the carbon element is expressed by

$$
\begin{aligned}
\mu_{f, c}^{i} & =\mu_{f, c}^{i} y_{f}^{i}(\phi, T)+\frac{12}{44} y_{c o 2}^{i}(\phi, T) \\
& +\frac{12}{28} y_{c o}^{i}(\phi, T)+y_{s o o T}^{i}(\phi, T)+\mu_{H C, c} y_{H C}^{i}(\phi, T)
\end{aligned}
$$

The conservation equation (7.3.4) is equivalent to the following by multiplying $\rho V \xi^{i}$

$$
\begin{gathered}
\rho V \xi^{i}\left(1-y_{f}^{i}(\phi, T)\right) \mu_{f, c}^{i}=\rho V \xi^{i}\left(\frac{12}{44} y_{c o 2}^{i}(\phi, T)+\frac{12}{28} y_{c o}^{i}(\phi, T)\right. \\
\left.+y_{\text {SoOT }}^{i}(\phi, T)+\mu_{H C, c} y_{H C}^{i}(\phi, T)\right)
\end{gathered}
$$

By comparing equation (7.3.3) and equation (7.3.5), mass fractions of combustion products from fuel $i$ can be expressed with the mixture fraction $\xi^{i}$ and the LER $\phi$ by the following equations

$$
\begin{aligned}
& Y_{c o 2}^{i}=y_{c o 2}^{i}(\phi, T) \xi^{i} \\
& Y_{c o}^{i}=y_{c o}^{i}(\phi, T) \xi^{i}
\end{aligned}
$$




$$
\begin{aligned}
& Y_{\text {soor }}^{i}=y_{\text {soor }}^{i}(\phi, T) \xi^{i} \\
& Y_{H C}^{i}=y_{H C}^{i}(\phi, T) \xi^{i} \\
& Y_{f}^{i}=y_{f}^{i}(\phi, T) \xi^{i}
\end{aligned}
$$

From the balance equation (7.3.4) the fraction of the unburned fuel $i$ to the total mass, which comes from the fire source $i$, is expressed by

$$
\begin{aligned}
y_{f}^{i} & =1-\left(\frac{12}{44} y_{\text {co2 }}^{i}(\phi, T)+\frac{12}{28} y_{c o}^{i}(\phi, T)+y_{\text {soor }}^{i}(\phi, T)\right) / \mu_{f, c}^{i} \\
& +\mu_{H C, c} y_{H C}^{i}(\phi, T) / \mu_{f, c}^{i}
\end{aligned}
$$

The total mass fractions of the combustion products in two-fuel fires have the following forms

$$
\begin{aligned}
& Y_{C O 2}=y_{C O 2}^{1}(\phi, T) \xi^{1}+y_{C O 2}^{2}(\phi, T) \xi^{2} \\
& Y_{C O}=y_{C O}^{1}(\phi, T) \xi^{1}+y_{C O}^{2}(\phi, T) \xi^{2} \\
& Y_{S O O T}=y_{S O O T}^{1}(\phi, T) \xi^{1}+y_{S O O T}^{2}(\phi, T) \xi^{2} \\
& Y_{H C}=y_{H C}^{1}(\phi, T) \xi^{1}+y_{H C}^{2}(\phi, T) \xi^{2}
\end{aligned}
$$

For two-fuel fires, it is difficult to express the mixture fractions with $O$ (oxygen) element because the two different fuels share the oxygen. Treating the right side of equation (7.3.2) as the difference between the total mass fraction of oxygen and the consumed mass fraction of oxygen, the calculation of oxygen mass fraction in onefuel toxic gas can be extended to two-fuel cases

$$
Y_{O 2}=\mu_{a i r, o}\left(1-\xi^{1}-\xi^{2}\right)-y_{o}^{1}(\phi, T) \xi^{1}-y_{o}^{2}(\phi, T) \xi^{2}
$$

where the superscripts correspond to the first and the second fuels. 
The volume fraction of species $j, C_{j}$, can be converted from the mass fraction with the following equation

$$
C_{j}=Y_{j} \frac{W}{W_{j}}
$$

where $W$ and $W_{j}$ represent the molecular weights of the gas mixture and species $j$ respectively. Just as assumed in the one-fuel toxic gas model, the mole ratio of $\mathrm{CO}_{2}$ to $\mathrm{H}_{2} \mathrm{O}$ for each fuel with any value of LER is the same as the stoichometric $\mathrm{CO}_{2}$ to $\mathrm{H}_{2} \mathrm{O}$ mole ratio in the complete one-step reaction. Therefore, the dry volume fraction of species $j$ is then calculated with equation (7.3.18)

$$
C_{j}^{d r y}=\frac{Y_{j} W}{\left(1-18\left(Y_{\mathrm{CO} 2}^{1} / r_{\mathrm{CO} / \mathrm{H} 2 \mathrm{O}}^{1}+Y_{\mathrm{CO} 2}^{2} / r_{\mathrm{CO} / \mathrm{H} 2 \mathrm{O}}^{1}\right) / 44\right) W_{i}}
$$

where $r_{\mathrm{CO} / \mathrm{H} 2 \mathrm{O}}^{i}$ is the stoichiometic $\mathrm{CO}_{2} / \mathrm{H}_{2} \mathrm{O}$ mole ratio in the one-step completely reaction of fuel $i$.

\subsection{Application of Two-fuel Model}

\subsubsection{Description of fire experiments}

Ten experiments of hexane fires were reported in [LV98] (five with wood and five without wood) to examine the effect of wood in the upper layer of the fire compartment on the $\mathrm{CO}$ levels and to study the transport of $\mathrm{CO}$ from the fire room to remote locations. The fire facility, shown in Figure 7.4.1(a), consisting of a compartment, a hallway, and an exhaust system. The compartment was $1.52 \mathrm{~m}$ wide, $1.22 \mathrm{~m}$ high and $1.22 \mathrm{~m}$ deep. The adjacent hallway was $1.22 \mathrm{~m}$ wide, $1.67 \mathrm{~m}$ high and $3.66 \mathrm{~m}$ long. There is an opening window with $0.12 \mathrm{~m}^{2}(0.5 \mathrm{~m}$ wide, $0.24 \mathrm{~m}$ long) between the compartment and the hall. In the tests, the air entered into the fire compartment from a plenum below the compartment and the hot fire gases were exhausted through the opening. 
The fire source inside the compartment was an $n$-hexane pool located on the floor and in the middle of the compartment. In the test with wood in the upper layer, a truss system on top of the compartment was used to hang Douglas fir plywood approximately $0.05 \mathrm{~m}$ below the ceiling. The plywood was $6.35 \mathrm{~mm}$ thick and had overall dimensions of $1.22 \mathrm{~m}$ long and $0.90 \mathrm{~m}$ wide. The position of the wood is shown in Figure 7.4.1(b). The volatile compositions of the fuels are $\mathrm{C}_{6} \mathrm{H}_{14}$ for $n$ hexane and $\mathrm{CH}_{2.056} \mathrm{O}_{1.071}$ for Douglas wood. The corresponding heats of combustion are $44735 \mathrm{~kJ} / \mathrm{kg}$ for hexane and $10400 \mathrm{KJ} / \mathrm{kg}$ for wood respectively.

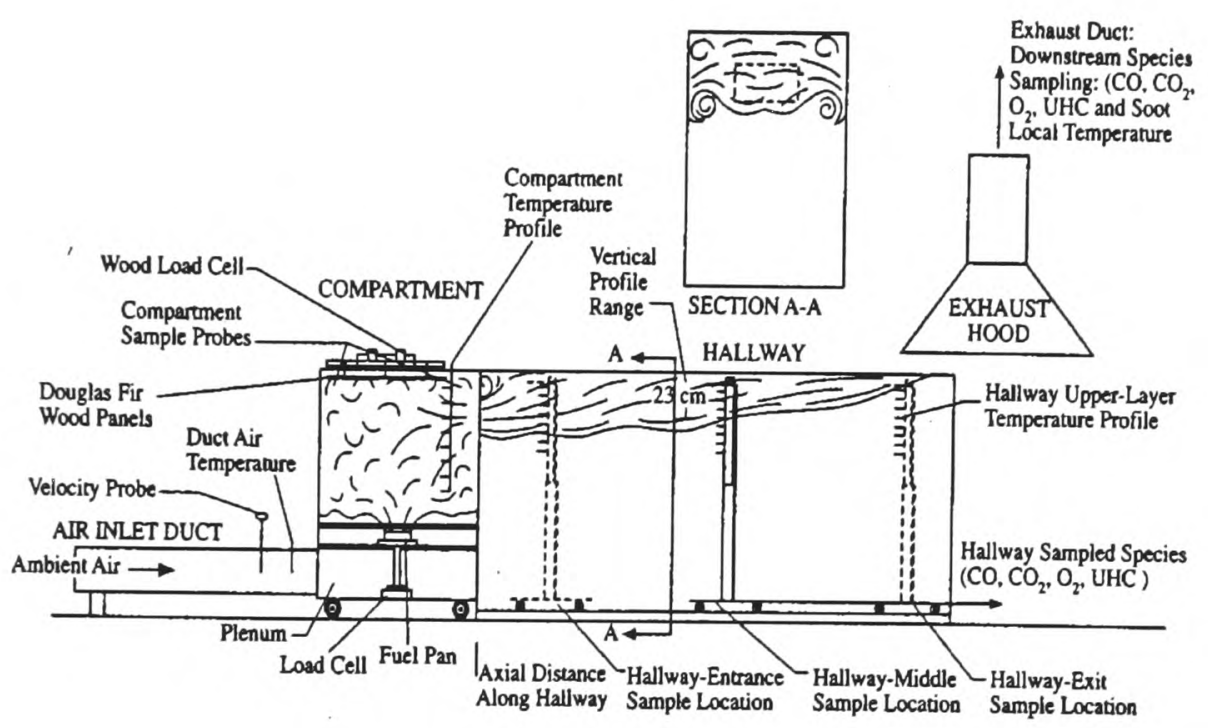

(a)

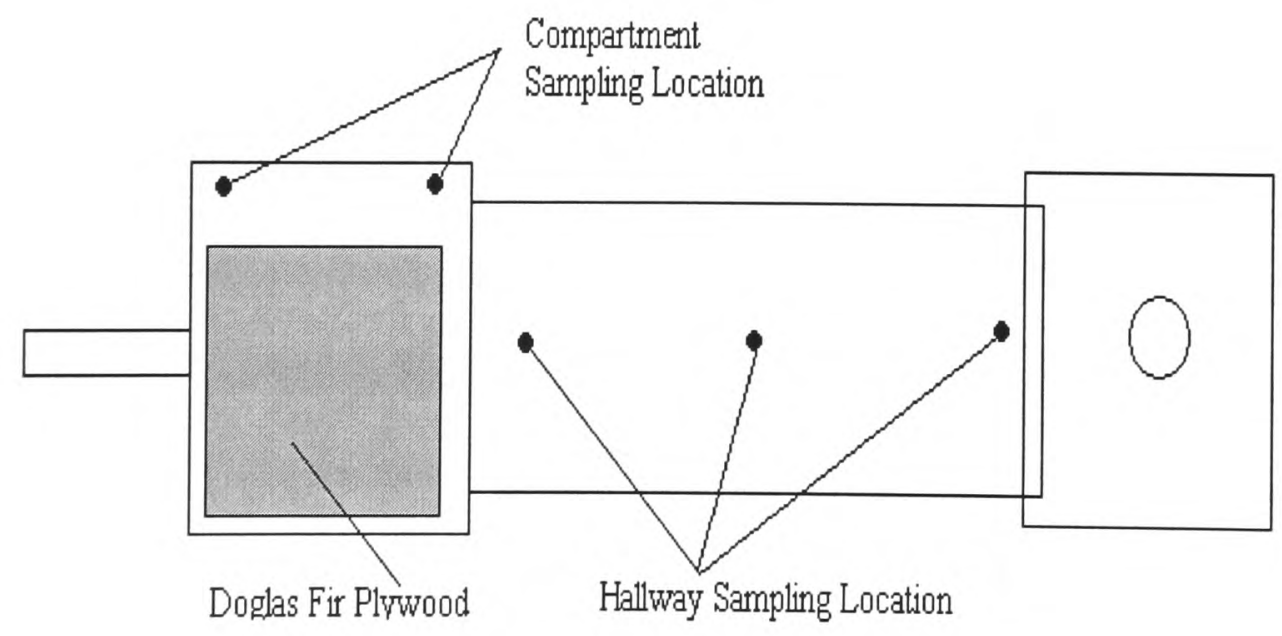

(b)

Figure 7.4.1: Schematic of hexane-wood fires: (a) Side view of the facility used in the test (reproduce from [LV98]) and (b) plan view of the facility showing the wood in the upper layer of the compartment and the gas sampling positions.

The concentrations of $\mathrm{O}_{2}, \mathrm{CO}_{2}, \mathrm{CO}$ and unburned hydrocarbon (UHC) are measured in the tests. Two gas positions for the measurement in the fire room were $0.1 \mathrm{~m}$ under the ceiling, $0.23 \mathrm{~m}$ to the side wall and $0.1 \mathrm{~m}$ to the front wall or the rear wall respectively. The gas positions in the hallway were along the central line of the 
corridor and $0.05 \mathrm{~m}$ below the ceiling. They were $0.46 \mathrm{~m}, 1.83 \mathrm{~m}$, and $3.2 \mathrm{~m}$ from the compartment respectively. As seen in Figure 7.4.1(a), the vertical temperature profiles were measured in the front corner of the compartment and the centre plane of the hallway.

In the five tests without wood in the upper layer, the average of fire sizes was $535 \mathrm{~kW}$. A typical mass-loss rate of the five tests with wood in the upper layer of the fire compartment is given in Figure 7.4.2.

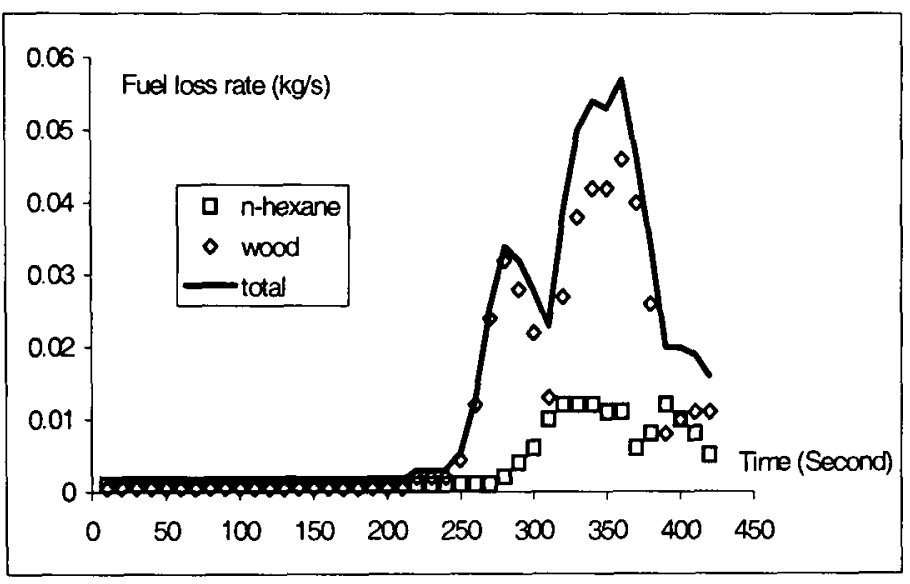

Figure 7.4.2: Fuel loss rates of the liquid $n$-hexane and the wood (data from [LV98]).

\subsubsection{Simulations}

The toxicity model in section 7.3 is implemented within the CFD environment of software CFX-4.2. The two-fuel EBU model is used to calculate the heat released due to the combustion. The six-flux radiation model represents the transfer of heat via thermal radiation. The species volume fractions are calculated using the present twofuel toxicity model.

Two fire scenarios are simulated in this study. The first one, called 'Hexane' fire, is the experiment without wood in the upper layer of the fire compartment as described previously. The averaged heat release rate in the five repeated hexane fire tests without wood was $535 \mathrm{~kW}$ [LV98]. Since there is no detailed fuel loss rate for this type of experiments, a constant fuel loss rate $0.016 \mathrm{~kg} / \mathrm{s}$, which is supposed to be the maximum of the fuel loss rates, is used in the present simulation. In the simulation of the 'Hexane' fire, the one-fuel toxic gas model-Model I developed in Chapter 4 (Note: if the loss rate of the second fuel is zero, the two-fuel model is the same as the one-fuel model) is applied. 
The second fire scenario, called 'Wood-hexane' fire, is the experiment of hexane fire with wood in the upper layer of the fire compartment. In the simulation of the test with wood in the upper layer, the fire source of wood is simply set in the centre of the compartment, which is slight different from the position in the experiment (see Figure 7.4.1(b)). The fuel loss rates used in the simulation are the typical fuel loss rates of the five repeated experiments (see Figure 7.4.2).

The two simulations use the same mesh, which consisted of 31464 (i.e. $72 \times 23 \times 19$ ) computational cells (see Figure 7.4.3).

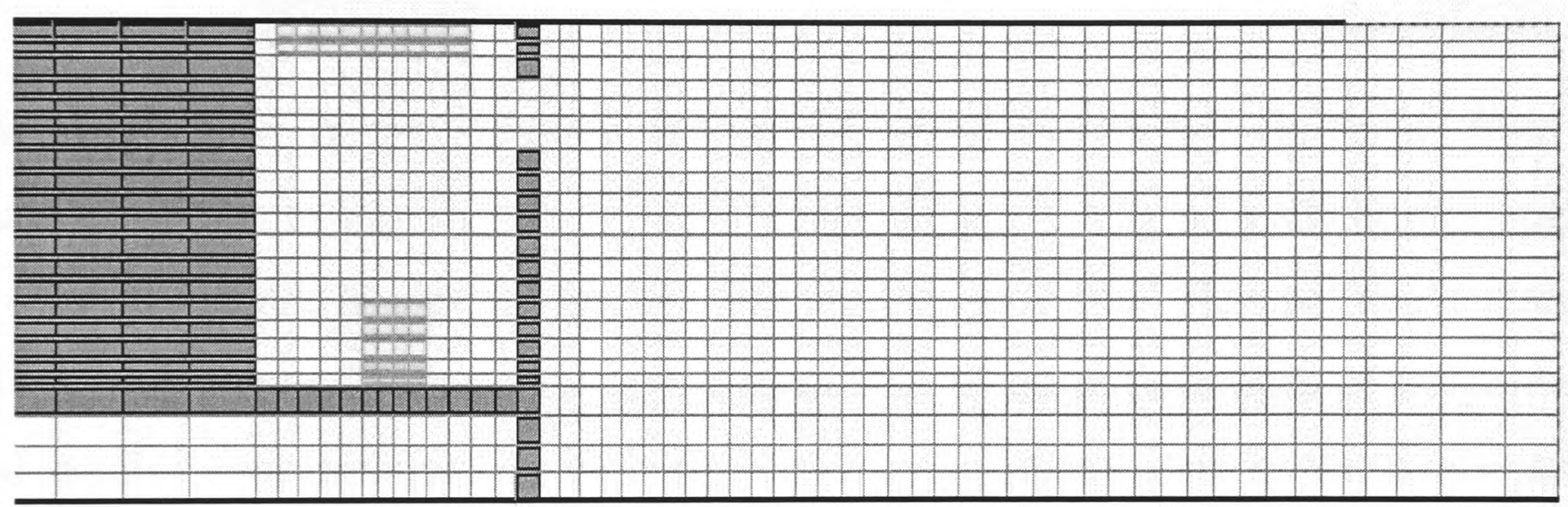

Figure 7.4.3: Vertical plane through the middle of the fire sources depicting the computational mesh.

The species yields required in equations (7.3.12) to (7.3.16) for hexane and wood are given as following. As applied in the simulation of a compartment-corridor hexane fire in Chapter 5, the combination of yield given by (2.2.15) and (2.2.16), which is expressed by equation (7.4.1), approximately represent the generation of $\mathrm{CO}$ for hexane fires

$$
y_{C O}=\left\{\begin{array}{cc}
\left(0.19 \times \tan ^{-1}(10 \times(\phi-0.8))\right. & \\
\left.+0.22 \times \tan ^{-1}(10 \times(\phi-1.25))\right) / 360+0.1025 & \phi<1.4 \\
0.22 \times \tan ^{-1}(10 \times(\phi-1.25)) / 180+0.11 & \phi \geq 1.4
\end{array}\right.
$$

The yields $(\mathrm{kg} / \mathrm{kg})$ of species of Douglas wood are [Te95]

$$
y_{\mathrm{CO} 2}(\phi)=1.31\left[1-\frac{1.0}{\exp (\phi / 2.15)^{-1.2}}\right]
$$




$$
y_{\mathrm{CO}}(\phi)=0.004\left[1+\frac{44}{\exp (\phi / 1.3)^{-3.5}}\right]
$$

The oxygen consumption is

$$
y_{0}=1.12\left[1-\frac{0.97}{\exp (\phi / 2.14)^{-1.2}}\right]
$$

\subsubsection{Results and Discussions}

\subsubsection{Hexane fire}

The steady time of the 'Hexane' fire is not given in [PV98]. However, from the hexane loss rate in the 'Hexane-wood' fire (see Figure 7.4.2), a steady burning period of over 70 seconds is seen for hexane. The simulation for the 'Hexane' fire is then run for an equivalent of 70 seconds of real time. The measured averages of temperatures and species concentrations in the period of a quasi-steady state in the five repeated tests are compared with the predictions of a steady fire with a burning rate of $537 \mathrm{~kW}$, which is the average heat release rate of the five tests. In some of the following figures, the coordinate origination of the horizontal axis is at the opening between the compartment and the corridor. As seen in Figure 7.4.4, the predicted temperature in the compartment is higher than that in the hallway while the measured one inside the compartment is relatively low. In the hallway, the predicted temperatures are reasonable compared with the measurements.

The predicted normalised species concentrations are compared with the measured data in Figure 7.4.5. The normalised species concentration here is the ratio of the species concentration to a fixed concentration $\left(\mathrm{C} / \mathrm{C}_{\mathrm{o}}\right)$. The fixed values $\left(C_{o}\right)$ for $\mathrm{CO}, \mathrm{CO}_{2}$ and $\mathrm{O}_{2}$ are $15 \%, 20 \%$ and $10.5 \%$ respectively. The measured $\mathrm{CO}$ concentration in front of the burning room is higher than that in the rear of the room. From the room to the hallway, the measured $\mathrm{CO}$ concentration decreases quickly because of its further oxidation. The simulation has reproduced the previously mentioned trends. The predicted $\mathrm{CO}$ in the hallway is very close to the measurements while the accuracy of 
the $\mathrm{CO}$ predictions in the rear of the compartment is relatively poor. From the compartment to the position with a distance of $0.46 \mathrm{~m}$ in the hallway, the measured $\mathrm{CO}$ concentrations go down about $30 \%$ and the prediction of this decrease is about $40 \%$.

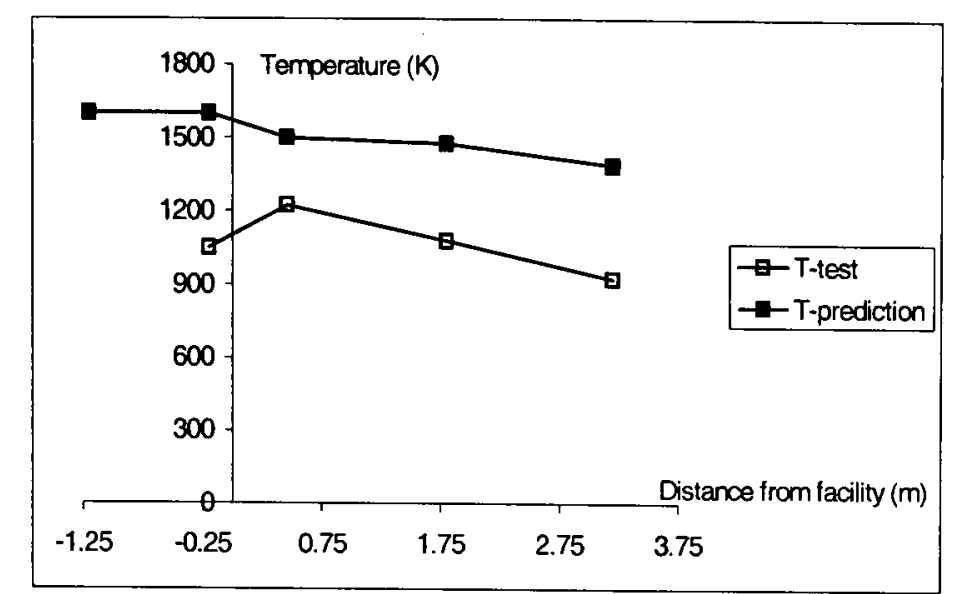

Figure 7.4.4: Measured and predicted temperatures for the 'Hexane' fire.

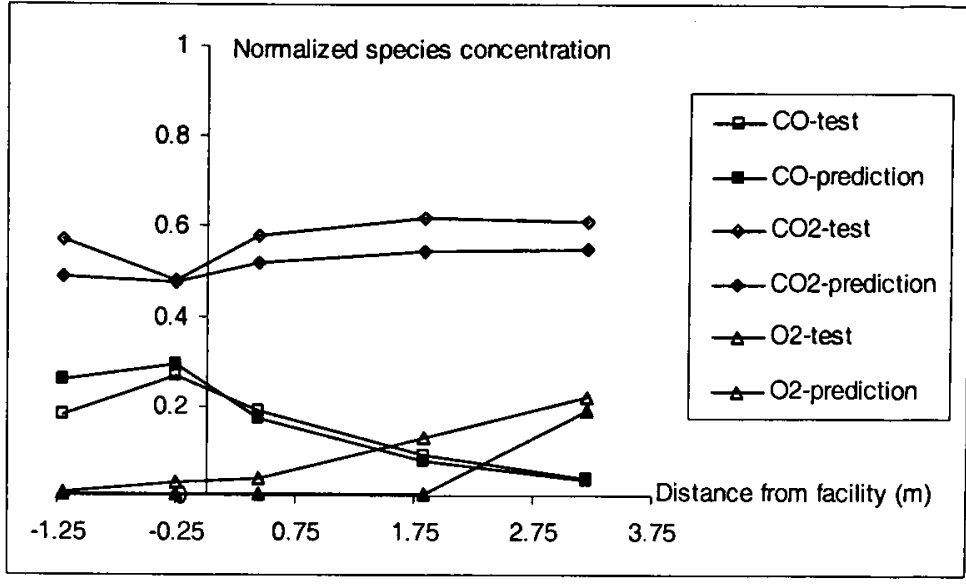

Figure 7.4.5: Measured and predicted normalised species concentrations in the 'Hexane' fire. Symbols and normalised values: square-CO (15\%), diamond- $\mathrm{CO}_{2}$ $(20 \%)$, and triangle- $\mathrm{O}_{2}(10.5 \%)$.

The trend of the predicted $\mathrm{CO}_{2}$ concentrations is similar to the measured data, i.e. the concentration increases from the room to the end of the hallway. The predicted $\mathrm{O}_{2}$ concentrations look reasonable.

\subsubsection{Wood-hexane fire}

Since the burning rate of 'Wood-hexane' fire is very low in the first 220 seconds (see Figure 7.4.2), the simulation is undertaken for an equivalent of 200 seconds (from 220 seconds to 420 seconds) of real time only. The predicted temperatures at the positions for gas sampling as functions of time are depicted in Figure 7.4.6. As seen in this figure, the predicted temperatures are above $1400 \mathrm{~K}$ inside the fire compartment and still $1200 \mathrm{~K}$ in the hallway.

From 340 seconds to 360 seconds, the predicted temperatures are nearly steady. The averages of predicted temperatures during this period are compared with the averages of the measurements within a steady period from the five repeated tests in Figure 7.4.7. As seen in this figure, the predicted temperatures in the hallway are reasonable compared with the measured data. 


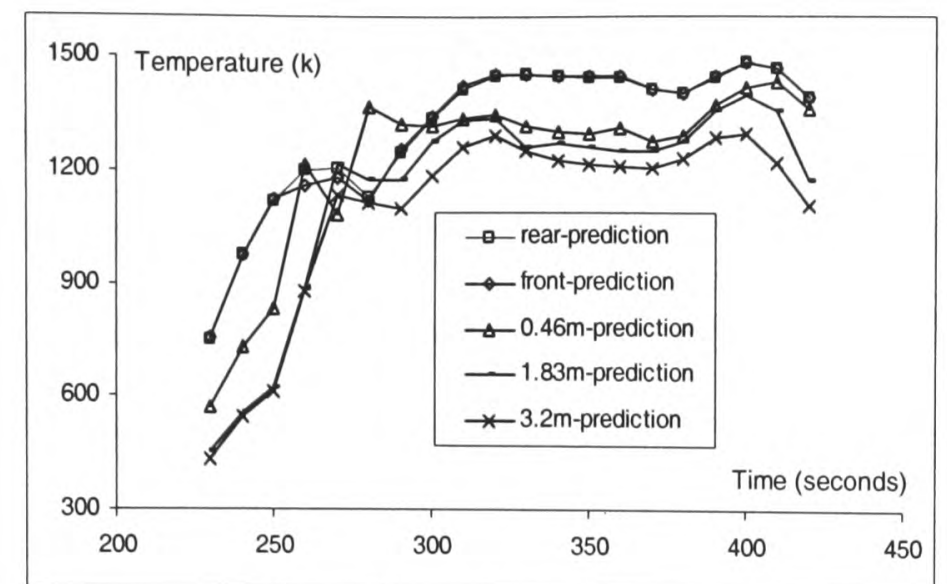

Figure 7.4.6: Predicted temperatures at the five positions for gas sampling in the 'Wood-hexane' fire.

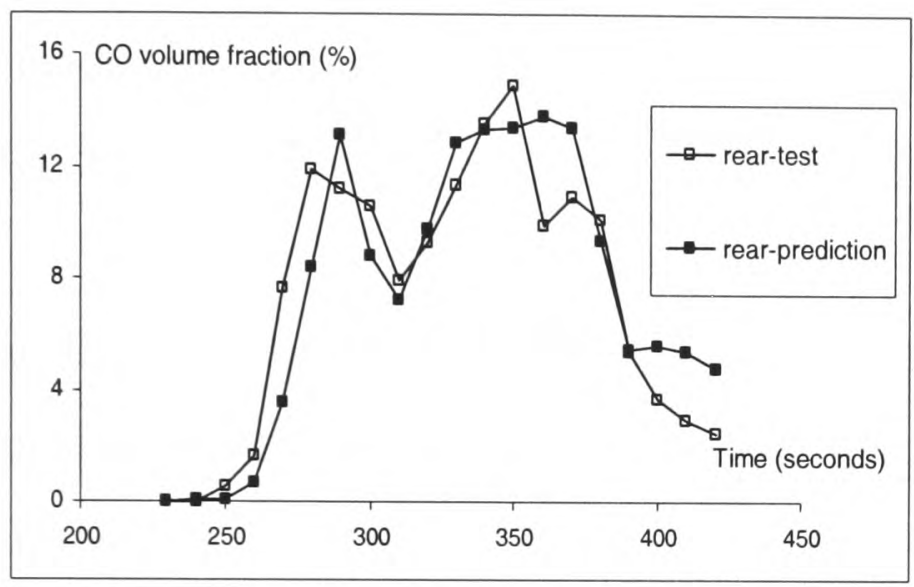

(a)

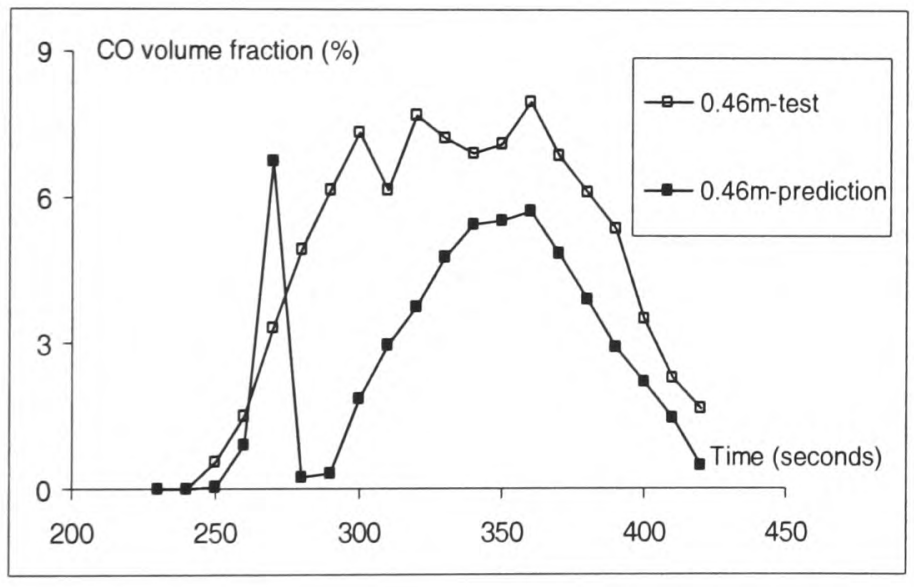

(c)

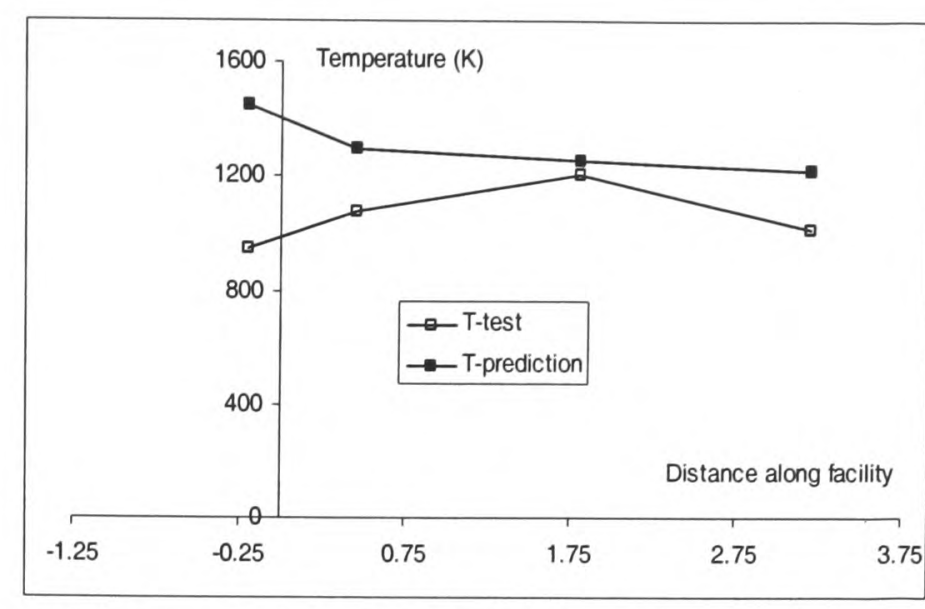

Figure 7.4.7: Measured and predicted averages of temperatures in the 'Woodhexane' fire.

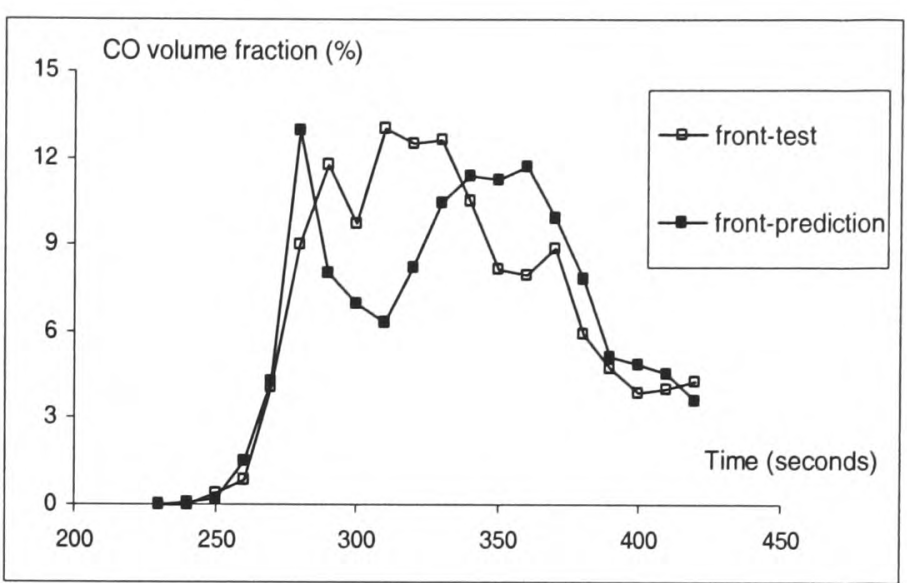

(b)

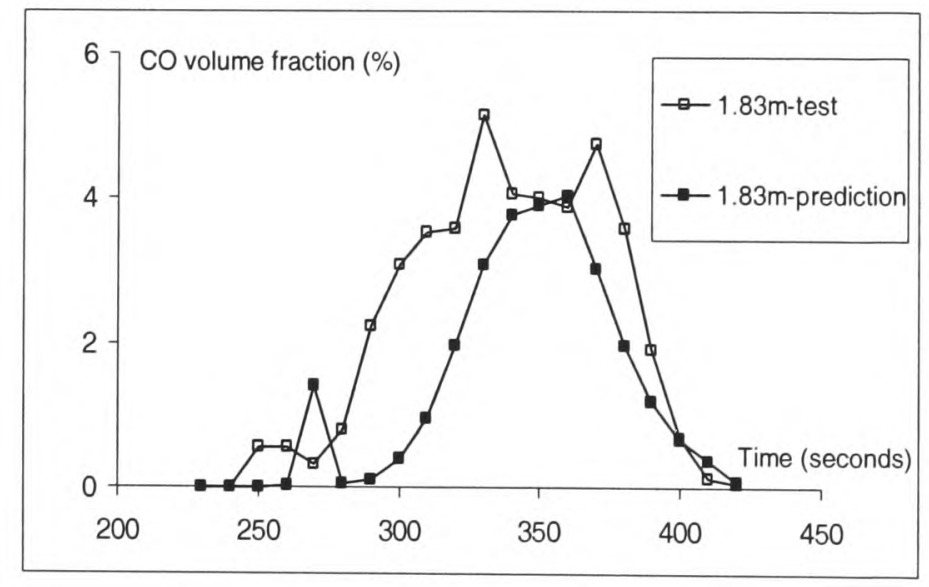

(d)

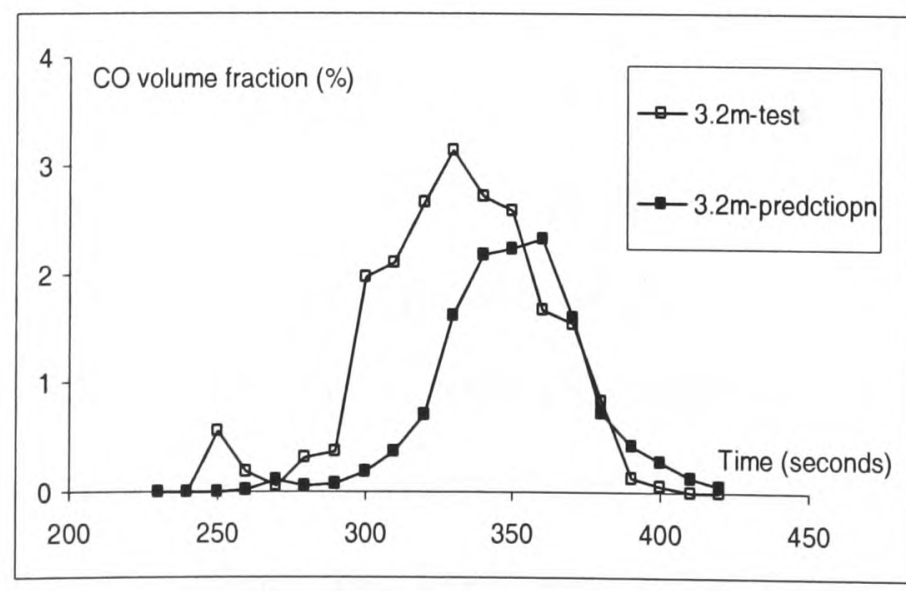

(e)

Figure 7.4.8: Measured and predicted $\mathrm{CO}$ volume fractions at (a) the rear of the compartment; (b) the front of the compartment and at locations in the hallway with (c) $0.36 \mathrm{~m}$, (d) $1.83 \mathrm{~m}$ and (e) $3.2 \mathrm{~m}$ to the compartment. 
The measured and predicted $\mathrm{CO}$ volume concentrations of the 'Wood-hexane' fire at the five positions as functions of time are depicted in Figure 7.4.8. In Figure 7.4.8, the measured $\mathrm{CO}$ concentrations are from one of the five repeated tests and the predictions are based on the test with the fuel loss rate given in Figure 7.4.2. The measured $\mathrm{CO}$ concentrations inside the compartment are approximately $11 \%$ on average (see Figure 7.4.8(a-b)). The maximum of the measured $\mathrm{CO}$ concentrations is as high as $14.9 \%$ in the rear of the compartment. The peak predicted CO concentrations inside the room are around $13.8 \%$. The two peaks in the wood loss rates in the 'Wood-hexane' fire leads to the two peaks of measured CO concentrations inside the compartment. The simulation reproduced the two measured peaks of the CO concentrations.

In the hallway, the $\mathrm{CO}$ concentrations decrease as the distances of the gas sampling positions increase (i.e. moving away from the window) (see Figure 7.4.8(c-e)). In the hallway, the maxima of the measured CO concentrations are around $7.96 \%, 5.15 \%$ and $3.15 \%$ at positions $0.46 \mathrm{~m}, 1.83 \mathrm{~m}$ and $3.2 \mathrm{~m}$ outside the room, respectively. These maxima are under-predicted by absolute values of around $1.23 \%, 1.13 \%$ and $0.83 \%$ at these positions, respectively. The relative errors, corresponding to the above absolute errors, range from $15.4 \%$ to $26.3 \%$ at the three positions. The predictions of $\mathrm{CO}$ in the hallway are considerably lower than the experimental data between 300 and 350 seconds. The measured peak of $\mathrm{CO}$ concentrations, at position $0.46 \mathrm{~m}$ to the fire room, appeared around 350 seconds while the predicted peak occurred at 270 seconds.

The averages of predicted normalised species concentrations of the five repeated tests, with wood in the upper layer of the compartment, are reported in [LV98]. The predicted averages of species concentrations from 340 seconds to 360 seconds are compared with the averages of those measured in the five repeated tests and are presented in Figure 7.4.9. As the measured $\mathrm{CO}$ concentrations for the typical fire simulated in this study are known, the normalized CO concentrations in Figure 7.4.9 are averages of the measured values and averages of the predictions from 340 seconds to 360 seconds, which are depicted in Figure 7.4.8. The predicted averages of CO concentrations are close to the measured values. 


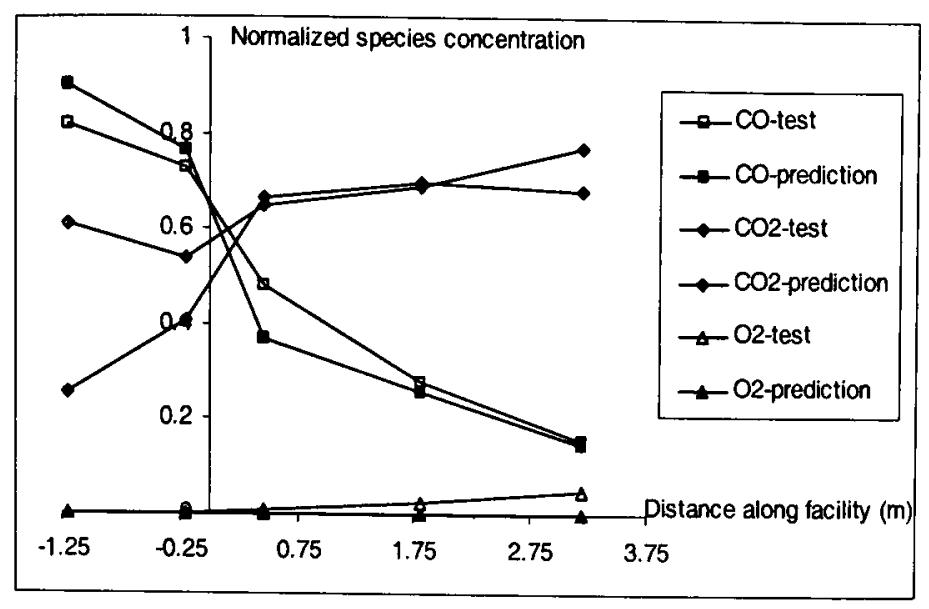

Figure 7.4.9: The measured and predicted normalised species concentrations of the

'Wood-hexane' fire (Symbols and normalised values see Figure 7.4.5).

Unlike the averages of the $\mathrm{CO}$ concentrations, the $\mathrm{O}_{2}$ and $\mathrm{CO}_{2}$ concentrations are averages of the measurements of the five repeated experiments during the steady combustion state. The maximum of measured $\mathrm{O}_{2}$ concentrations at the five positions for gas measurements are $0.3 \%$ while the predictions are 0 . The measured $\mathrm{CO}_{2}$ concentrations inside the room are much under-predicted while good agreements are obtained between the measured and predicted $\mathrm{CO}_{2}$ concentrations in the corridor (see Figure 7.4.9).

\subsubsection{Effect of wood on combustion products}

Compared with the pure hexane fire case (see Figure 7.4.5), wood in the 'Woodhexane' fire (see Figure 7.4.9) causes a moderate increase of the average measured $\mathrm{CO}_{2}$ concentrations within the fire compartment. The average measured $\mathrm{CO}_{2}$ concentration increases from $10.4 \%$ (Hexane) to $11.6 \%$ (Wood-hexane). The average predicted $\mathrm{CO}_{2}$ concentrations within the fire compartment decrease from $9.6 \%$ (Hexane) to $6.7 \%$ (Wood-hexane).

Significant increases appear for the CO concentrations. The average measured CO concentrations within the compartment increases from $3.2 \%$ (Hexane) to $11.7 \%$ (Wood-hexane). The average predicted $\mathrm{CO}$ concentrations increases from $4.1 \%$ to $12.5 \%$ for the two fire scenarios. The simulations, using the two-fuel toxicity model, have captured the effect of wood on the CO concentrations, the impact of which is tabulated in table 7.4.1. 
Table 7.4.1: Effect of wood on the averaged productions of combustion products within the fire compartment and the model predictions.

\begin{tabular}{|c|c|c|c|c|}
\hline \multirow{2}{*}{ Tests } & \multicolumn{2}{|c|}{$\mathrm{CO}_{2}$ concentration (\%-dry) } & \multicolumn{2}{c|}{ CO concentration (\%-dry) } \\
\cline { 2 - 5 } & $\begin{array}{c}\text { Experimental } \\
\text { data }\end{array}$ & Prediction & $\begin{array}{c}\text { Experimental } \\
\text { data }\end{array}$ & Prediction \\
\hline 'Hexane' fire & 10.4 & 9.6 & 3.2 & 4.1 \\
\hline $\begin{array}{c}\text { 'Wood-hexane' } \\
\text { fire }\end{array}$ & 11.6 & 6.7 & 11.7 & 12.5 \\
\hline
\end{tabular}

The measured and predicted $\mathrm{O}_{2}$ concentrations at the end of the hallway and the $\mathrm{CO}$ reduction percentages from the fire compartment to the end of the hallway are represented in Table 7.4.2. The average measured $\mathrm{O}_{2}$ concentrations for both the hexane fire and the 'Wood-hexane' fire are less than $2.1 \%$. The predictions of $\mathrm{O}_{2}$ concentrations compared reasonably well with the measured results. For the hexane fire, the ratio of the average measured $\mathrm{CO}$ concentration in the hallway $(3.2 \mathrm{~m}$ to the compartment) is $2.3 \%$. The percentage reduction of the measured $\mathrm{CO}$ average from the fire compartment to the end of the hallway is around $86 \%$, which compares well with the predicted value of $89 \%$. Similarly, for the 'Wood-hexane' fire, measured percentage reduction of $\mathrm{CO}$ was $79 \%$ compared to that $84 \%$ predicted.

Table 7.4.2: $\mathrm{O}_{2}$ concentration at the end of the hallway and the $\mathrm{CO}$ reduction percentage from the fire compartment level to that at the end of the hallway.

\begin{tabular}{|c|c|c|c|c|}
\hline \multirow{2}{*}{ Tests } & \multicolumn{2}{|c|}{$\mathbf{O}_{2}$ concentration (\%-dry) } & \multicolumn{2}{c|}{ Percent reduction of CO } \\
\cline { 2 - 5 } & $\begin{array}{c}\text { Experimental } \\
\text { data }\end{array}$ & Prediction & $\begin{array}{c}\text { Experimental } \\
\text { data }\end{array}$ & Prediction \\
\hline 'Hexane' fire & 2.1 & 2.0 & 86 & 89 \\
\hline $\begin{array}{c}\text { 'Wood-hexane' } \\
\text { fire }\end{array}$ & 0.3 & 0.0 & 79 & 84 \\
\hline
\end{tabular}

\subsection{Concluding Remarks}

By extending the concept of LER from one-fuel fire to two-fuel fire, a two-fuel toxicity model based on the concept of LER has been developed, in this study, to predict the generation and movement of combustion products in enclosure fires. The heat released due to combustion is modelled with the two-fuel EBU model while the 
concentrations of combustion products are calculated using the newly developed toxicity model.

The developed toxicity model can be used to study the effect of wood on the CO concentration in enclosure fires. Simulations of $n$-hexane fire tests with or without wood in the upper layer (called 'Hexane' fire and 'Wood-hexane' fire respectively) have been performed. The present toxicity has demonstrated that it can predict "good" results taking into consideration the effect of wood in the upper layer, in terms of the generation of $\mathrm{CO}$ and its transport from fire room to an the adjacent hallway. The average $\mathrm{CO}$ concentration within the fire compartment increases from $3.2 \%$ in the 'Hexane' fire to $11.7 \%$ in the 'Wood-hexane' fire whereas the predicted values increase from $4.1 \%$ to $12.5 \%$ for the two fire scenarios. The predicted $\mathrm{CO}$ concentrations in the five sampling positions of the 'Wood-hexane' fire test follow the measured trends. The prediction of the maximum $\mathrm{CO}$ concentration inside the room is in good agreement with the experimental data, while the maximum (under-predicted) error for all locations in the hallway ranged from $15.4 \%$ to $26.3 \%$ (peak concentration). 


\section{Chapter 8}

\section{DEPOSITION OF HCL ON SOLID SURFACES}

\subsection{Introduction}

Fires involving fuels containing the element chlorine such as PVC, will produce hydrogen chloride $(\mathrm{HCl})$. $\mathrm{HCl}$ is a sensory and pulmonary irritant and as such posses a threat to humans subjected to prolonged exposures during fires[Ha96]. Recently, coupled evacuation [GG01], FED [Pu02] and fire [EG99b] simulations of a hypothetical PVC cable fire within a single story building [GG04] suggested that $\mathrm{HCl}$ may have been a significant contributor to the predicted fatalities in fire scenarios. Unlike other toxic fire gases produced within enclosure fires, such as $\mathrm{CO}$, the concentration of $\mathrm{HCl}$ in the fire effluent decreases rapidly due to absorption by solid surfaces. However, the fire model used in the earlier study[GG04] did not predict the decay of $\mathrm{HCl}$ resulting from absorption by wall surfaces but rather assumed a range of predetermined decay fraction. Evacuation simulations were then performed for each assumed decay factor.

As already reviewed in Chapter 2, many experimental studies have been performed to study the absorption of $\mathrm{HCl}$ within fire enclosure [MP79, LBM96, LBM96b, GH89, GH90, GH91a, GH91b, GH92, CV98].

As $\mathrm{HCl}$ levels in fire atmospheres may have an important effect on evacuees exposed to fire effluent, it is essential for fire models to be equipped with the capability of simulating the $\mathrm{HCl}$ decay processes including for absorption. A mathematical model to predict the decay of $\mathrm{HCl}$ in fire gases due to absorption has been developed by Galloway and Hirschler [GH89]. The model was particularly suited for use in fire zone models [JF00]. The input parameters for the $\mathrm{HCl}$ decay model are the volumes of the fire compartment, gas/surface temperatures, gas humidity and mass exchange rates between compartments. The model has been used for predicting $\mathrm{HCl}$ decay in several small-scale and full-scale tests [GH89, GH90, GH91a, GH91b, GH92], and has been incorporated within the CFAST [JF00] fire zone model. 
In this chapter, the deposition model reported in [GH89], linked to zone models, is modified and applied in a field fire modelling framework, to predict the decay of $\mathrm{HCl}$ in fire enclosures due to the wall effect. Simulation results using the modified $\mathrm{HCl}$ deposition model are compared with data from HVAC (heating, ventilation and air conditioning) tests [GH91], small-scale chamber PVC fires [CV98] and a roomcorridor cable fires [GH92]. An early simplified form of an $\mathrm{HCl}$ deposition model and the improved model used in a fire field model have been reported in [WJ04] and [WJ06b] respectively.

The symbols and terms used in this chapter should be specially addressed. The species concentrations usually refer to the mass fraction or volume fraction in this thesis. In the HCL decay model [GH89, JF00], the $\mathrm{HCl}$ concentration is the mass of $\mathrm{HCl}$ per unit volume (gas mixture). In order to distinguish the concept of concentration in the $\mathrm{HCl}$ decay model from the concept of concentration in the common fire simulations, symbol $\psi\left(\mathrm{kg} / \mathrm{m}^{3}\right)$ is used to represent the mass of a species per unit volume of gases mixture while $Y$ (dimensionless) is still for the mass fraction. Furthermore, the $\mathrm{HCl}$ concentration on wall furnaces refers to the $\mathrm{HCl}$ mass per unit area and is expressed as $D\left(\mathrm{~kg} / \mathrm{m}^{2}\right)$.

\subsection{The Original Model}

\subsubsection{Movement of HCL to Boundary Layer}

The description of the original $\mathrm{HCl}$ decay model developed by Galloway and Hirschler can be found in [GH89, GH90, GH91a, GH91b, GH92, JF00]. The mechanisms of $\mathrm{HCl}$ deposition on walls include three processes, which are shown in Figure 8.2.1. A gas boundary layer is supposed to connect the gas phase and the solid walls.

The first process involves the movement of $\mathrm{HCl}$ from the gas phase into the boundary layer. The gas transport equation for $\mathrm{HCl}$ is

$$
\frac{d}{d t} m_{H C l}=S O U R C E-k_{c} \times\left(\psi_{H C l}-\psi_{B, H C l}\right) \times A_{W}
$$


where $m_{H C l}$ is the $\mathrm{HCl}$ mass $(\mathrm{kg})$ in the gas phase of a considered zone. SOURCE is the sum of the production rate of $\mathrm{HCl}$ due to the combustion and the exchange of $\mathrm{HCl}$ with other zones. $\psi_{\mathrm{HCl}}$ and $\psi_{B, \mathrm{HCl}}$ are the $\mathrm{HCl}$ concentration (as indicated in the introduction of this chapter, its unit is $\mathrm{kg} / \mathrm{m}^{3}$ ) in the gas phase and in the boundary layer. $A_{W}\left(\mathrm{~m}^{2}\right)$ is the area of the wall surfaces in the zone in question. $k_{c}(\mathrm{~m} / \mathrm{s})$ is a mass transfer coefficient. Equation (8.2.1) represents the total rate of change $\mathrm{HCl}$ within the zone. The second term on the right hand side of this conservation equation is the $\mathrm{HCl}$ deposition rate, an unknown quantity that needs further modelling. A second process is consequently considered.

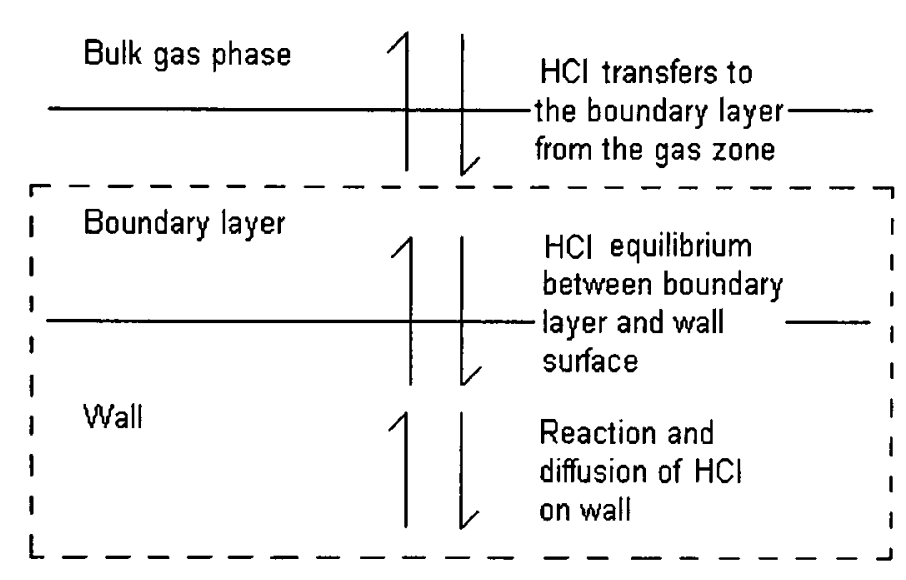

Figure 8.2. 1: Mechanisms of $\mathrm{HCl}$ deposition on wall.

\subsubsection{Equilibrium between Boundary Layer and Wall Surfaces}

In the second process, some of the $\mathrm{HCl}$ gas in the boundary layer sticks to the wall surface and some moves within the boundary layer due to convection and diffusion processes. It is assumed that there is a dynamic equilibrium between the $\mathrm{HCl}$ in the boundary layer and the $\mathrm{HCl}$ absorbed on the surfaces of walls $D_{W, H C l}$ (as indicated in the introduction of this chapter, its unit is $\mathrm{kg} / \mathrm{m}^{2}$ ). This equilibrium is expressed with equation (8.2.2).

$$
\psi_{B, H C l}=D_{W, H C l} / k_{e}
$$

where the equilibrium coefficient $k_{e}(\mathrm{~m})$ is calculated by equation (8.2.3). As seen in equation (8.2.3), the equilibrium coefficient depends on the wall temperature $T_{W}$, the $\mathrm{HCl}$ concentration and the gas humidity in the boundary layer. In equation (8.2.3), 
$\psi_{\mathrm{H} 2 \mathrm{O}}$ is the boundary layer water concentration, $\psi_{\mathrm{H} 2 \mathrm{O}, \mathrm{sat}}$ is the saturation concentration of water (as indicated in the introduction of this chapter, the unit is $\mathrm{kg} / \mathrm{m}^{3}$ ) corresponding to the boundary layer temperature and the boundary layer $\mathrm{HCl}$ concentration, and $b_{1}, b_{2}, b_{5}, b_{6}$ and $b_{7}$ are model parameters dependent on wall materials.

$$
k_{e}=\frac{b_{1} e^{1500 / T_{W}}}{1+b_{2} e^{1500 / T_{W}} \psi_{B, H C l}}\left(1+\frac{b_{5}\left(\psi_{H 2 O}\right)^{b_{6}}}{\left(\psi_{H 2 O, s a t}-\psi_{H 2 O}\right)^{b_{7}}}\right)
$$

\subsubsection{Diffusion and Reaction with Wall}

The change of $D_{W, H C l}$ is modelled in a third process. In the third process, the $\mathrm{HCl}$ on the wall surfaces can diffuse into and react with the wall material or covering. For wall surface $\mathrm{HCl}$ concentrations, the change rate is

$$
\frac{d}{d t} D_{W, H C l}=k_{c} \times\left(\psi_{H C l}-\psi_{B, H C l}\right)-k_{r} D_{W, H C l}
$$

where $k_{r}$ (dimensionless) is a factor to the reaction between $\mathrm{HCl}$ on the wall surface and the surface material. This factor is wall temperature and wall material dependent and is calculated by

$$
k_{r}=b_{3} e^{-\left(\frac{b_{4}}{R T_{w}}\right)}
$$

where $b_{3}$ and $b_{4}$ are parameters, $R$ is the universal gas constant, $T_{W}$ is wall temperature. Equation (8.2.4) represents the $\mathrm{HCl}$ mass conservation on the wall surface. The first term on the right hand side of this equation is the $\mathrm{HCl}$ deposition rate per unit surface area, standing for the amount of $\mathrm{HCl}$ available to the wall surface and the second term on the right hand side represents the amount of $\mathrm{HCl}$ disappearing at this surface due to $\mathrm{HCl}$ reaction with the wall material.

\subsubsection{Solution Approach}

Equations (8.2.1), (8.2.2) and (8.2.4) represent the three processes of the $\mathrm{HCl}$ decay model. In these equations, the $\mathrm{HCl}$ mass $(\mathrm{kg})$ in the gas phase $m_{H C L}$, the $\mathrm{HCl}$ 
concentration in the boundary layer $\psi_{B, H C l}$ and $\mathrm{HCl}$ absorbed on the surfaces of walls $D_{W, H C l}$ are the variables that need to be solved. These three equations are coupled and iterative methods are required to solve them. In order to save computational time, instead of the $\mathrm{HCl}$ concentration in the boundary layer $\left(\psi_{B, H C l}\right)$, the gas phase value $\left(\psi_{\mathrm{HCl}}\right)$ was used to calculate the equilibrium coefficient $k_{e}$ in equation (8.2.3) in the implementation of this model in [GH89]. With this simplification, the equilibrium coefficient $k_{e}$ does not contain any solved variable. Thus, the $\mathrm{HCl}$ concentration in the boundary layer $\left(\psi_{B, H C l}\right)$ is a linear function of the $\mathrm{HCl}$ absorbed on the surfaces of walls $D_{W, H C l}$. Substituting $\psi_{B, H C l}$ by equation (8.2.2), equation (8.2.4) becomes a firstorder differential equation of $D_{W, H C l}$, which has an analytical solution.

\subsection{The Modified Model}

The previous $\mathrm{HCl}$ decay model [GH89] was developed for application in zone models. The purpose of the model is to predict $\mathrm{HCl}$ concentrations remaining in the fire atmosphere following the decay of atmospheric $\mathrm{HCl}$ due to deposition onto wall surfaces as given by equation (8.2.1). In this study, an attempt is made to extend the application of Galloway and Hirschler's model to fire field models. The original model needs to be modified to allow incorporation within the concept and framework of fire field models.

\subsubsection{Transport Equation for $\mathrm{HCl}$}

Like any other solved variables in fire field modelling, an equation is introduced for $\mathrm{HCl}$ that represents the total effect of accumulation, convection, diffusion, production and destruction of $\mathrm{HCl}$ during its course of transportation. This equation is called the $\mathrm{HCl}$ governing equation that is expressed in the following form,

$$
\frac{\partial}{\partial t}\left(\rho Y_{H C l}\right)+\frac{\partial}{\partial x_{j}}\left(\rho u_{j} Y_{H C l}\right)=\frac{\partial}{\partial x_{j}}\left(\rho D \frac{\partial Y_{H C l}}{\partial x_{j}}\right)+S_{H C l}
$$

where $Y_{H C l}$ is the mass fraction of $\mathrm{HCl}$ and $S_{H C l}\left(\mathrm{~kg} /\left(\mathrm{m}^{3} \mathrm{~s}\right)\right)$ is its source term. 


\subsubsection{HCl deposition Model}

\subsubsection{On the movement of $\mathrm{HCl}$ to boundary layer}

In the zone modelling approach, in order to determine the amount of $\mathrm{HCl}$ being transported from the core of a zone to its boundary layer, an estimated convective coefficient $k_{c}$ must be provided. With this convective coefficient, the differential equation (8.2.1) is used to calculate the $\mathrm{HCl}$ exchange rate between the gas zone and the wall boundary layer. However, in field modelling, the movement of species to the boundary layer is actually solved by its governing partial differential equation (8.3.1) and the boundary layer model employed. Therefore, the first process of the three processes in the original model and its representation using equation (8.2.1), are not required in fire field modelling.

The three $\mathrm{HCl}$ deposition mechanisms shown in Figure 8.2.1 can hence be reduced to two processes governing $\mathrm{HCl}$ equilibrium between the gas boundary layer and the wall surfaces, and the reaction of $\mathrm{HCl}$ with walls. These two processes are highlighted via a broken lined rectangle in Figure 8.2.1. Corresponding to this simplification, the differential equation (8.2.1) can be removed from the decay model. However, the second term on the right hand side of equation (8.2.1) forms part of the source term of the $\mathrm{HCl}$ governing equation (8.3.1). Its calculation in fire field modelling will be addressed later in this section.

\subsubsection{On the equilibrium equation}

Equation (8.2.2) must also be re-examined to fit into the fire field modelling framework. It is assumed here that the cell just adjacent to the wall surface in the computational domain is in the boundary layer. This assumption is consistent with the practice in the original model of using the gas phase value $\left(\psi_{H C l}\right)$ in the calculation of the equilibrium coefficient $k_{e}$, instead of the $\mathrm{HCl}$ concentration in the boundary layer $\left(\psi_{B, H C l}\right)$. With this assumption, the $\mathrm{HCl}$ concentration at this cell can be used to calculate the equilibrium coefficient $k_{e}$ with equation (8.2.3). Then by re-arranging equation (8.2.2), the equilibrium wall $\mathrm{HCl}$ concentration is obtained as follows, 


$$
D_{W, H C l}=k_{e} \times \psi_{B, H C l}
$$

Due to the absorption of wall surfaces, the $\mathrm{HCl}$ concentration in the boundary layer should be less than that in the core of the gas flow. Therefore, this assumption may cause the $\mathrm{HCl}$ concentration on the wall surface to be over-predicted if this cell is not in the boundary layer. This issue will be further discussed later in this section. Equation (8.3.2) does not change the equilibrium correlation expressed within equation (8.2.2), only highlighting the use of available boundary layer $\mathrm{HCl}$ concentrations in field modelling. The expression of equilibrium equation (8.3.2) makes the calculation of $\mathrm{HCl}$ concentrations on wall surfaces at the equilibrium state more convenient.

\subsubsection{Source term due to absorption}

Finally, an overhaul of equation (8.2.4), representing the third process of the original $\mathrm{HCl}$ decay model, is required to adapt it for calculation in fire field models. An approach different from the original $\mathrm{HCl}$ decay model is proposed in this study to calculate the first term on the right hand side of equation (8.2.4) that forms part of the source term of the $\mathrm{HCl}$ governing equation. This term represents the $\mathrm{HCl}$ deposition rate on the wall surface. By re-arranging equation (8.2.4) and multiplying $A_{w}$ on each side of the equation we have,

$$
k_{c} \times\left(\psi_{H C l}-\psi_{B, H C l}\right) \times A_{W}=\left(\frac{d}{d t} D_{W, H C l}+k_{r} D_{W, H C l}\right) \times A_{W}
$$

Here $A_{w}$ is the area of the cell face that is next to the wall (this corresponds to the surface area of the zone of interest in equation (8.2.1)). The left hand side of equation (8.3.3) is the $\mathrm{HCl}$ deposition rate. As the $\mathrm{HCl}$ concentration on wall surfaces can easily be obtained from equation (8.3.2), the first term on the right hand side of equation (8.3.3), the rate of change of $\mathrm{HCl}$ concentrations on wall surfaces, can be computed as follows,

$$
\frac{d}{d t} D_{W, H C l}=\left(D_{W, H C l}-D_{W, H C l}^{\text {old }}\right) / \Delta t
$$


where $D_{W, H C l}^{\text {old }}$ is the $\mathrm{HCl}$ concentration on wall surfaces at the last time step and $\Delta t$ is the time step size. With equation (8.3.4) and re-naming the left hand side of equation (8.3.3), equation (8.3.3) becomes

$$
\begin{aligned}
S_{B, H C l} & =-k_{c} \times\left(\psi_{H C L}-\psi_{B, H C l}\right) \times A_{W} \\
& =-A_{W}\left(\left(D_{W, H C l}-D_{W, H C l}^{\text {old }}\right) / \Delta t+k_{r} D_{W, H C l}\right)
\end{aligned}
$$

This deposition rate $S_{B, H C l}(\mathrm{~kg} / \mathrm{s})$ is a source term for $\mathrm{HCl}$ in the boundary layer cell adjacent to the solid surface.

Returning to the use of equation (8.3.2) in the derivation of equation (8.3.5), it is assumed that an equilibrium exists between the $\mathrm{HCl}$ in the boundary layer and the wall surfaces. If the wall material has a weak absorption capability (for example PMMA [GH89]) and the $\mathrm{HCl}$ mass flux to the wall surface by convection and diffusion is strong, a state of equilibrium between the $\mathrm{HCl}$ in the boundary layer and wall surfaces would be attained very quickly. In these circumstances, the above formulations are considered reasonable.

However, if the wall material has a large absorption capability (for example cement [GH89]) and the $\mathrm{HCl}$ mass flux to the wall surface is relatively weak, it may take a long time for the wall surface $\mathrm{HCl}$ concentration to rise and reach equilibrium levels. Preliminary numerical results [WJ04] have demonstrated that in these situations the amount of $\mathrm{HCl}$ absorbed by a cement block target can be over predicted by as much as $80 \%$ when using equation (8.3.5) due to the delay in reaching $\mathrm{HCl}$ equilibrium.

To address this issue consider the actual $\mathrm{HCl}$ mass flux to the wall surface (representing the available amount of $\mathrm{HCl}$ actually being deposited onto the wall surface) given by $\dot{f}_{H C l}\left(\mathrm{~kg} /\left(\mathrm{m}^{2} \mathrm{~s}\right)\right)$. We now assume that before the wall surface reaches $\mathrm{HCl}$ equilibrium, the wall surface absorbs a proportion of the $\mathrm{HCl}$ available to it. Thus the left hand side of equation (8.3.3) (i.e. the $\mathrm{HCl}$ deposition rate), can be expressed as,

$$
k_{c} \times\left(\psi_{H C l}-\psi_{B, H C l}\right) \times A_{W}=\alpha \times \dot{f}_{H C l} \times A_{W}
$$


where $\alpha$ is the fraction of $\mathrm{HCl}$ absorbed by the wall surface. Equation (8.3.3) hence becomes

$$
\alpha \times \dot{f}_{H C l} \times A_{W}=\left(\frac{d}{d t} D_{W, H C l}+k_{r} D_{W, H C l}\right) \times A_{W}
$$

and

$$
S_{B, H C l}=-k_{c} \times\left(\psi_{H C l}-\psi_{B, H C l}\right) \times A_{W}=-\alpha \times \dot{f}_{H C l} \times A_{W}
$$

Combining Equation (8.3.5) and (8.3.8), the deposition rate is hence corrected as

$$
\begin{aligned}
S_{B, H C L} & =-k_{c} \times\left(\psi_{H C l}-\psi_{B, H C L}\right) \times A_{W} \\
& =-A_{W} \min \left\{\left(\left(D_{W, H C l}-D_{W, H C l}^{\text {old }}\right) / \Delta t+k_{r} D_{W, H C l}\right), \quad \alpha \times \dot{f}_{H C l}\right\}
\end{aligned}
$$

As there is no experimental quantification of this fraction, in the simulations presented in this thesis it is assumed that the parameter takes the value used in the earlier study, i.e. 1.0 [WJ04].

In the earlier study [WJ04], the $\mathrm{HCl}$ concentration on the wall surface $D_{W, H C l}^{\text {old }}$ was simply treated as the equilibrium concentration calculated from equation (8.3.2) at the last time step. However, when the deposition rate is controlled by $\dot{f}_{\mathrm{HCl}}$, the corresponding $\mathrm{HCl}$ concentration on the wall surface does not reach the equilibrium value. In this case, from equation (8.3.7), we have

$$
\frac{d}{d t} D_{W, H C l}=\alpha \times \dot{f}_{H C l}-k_{r} D_{W, H C l}
$$

In this study, the $\mathrm{HCl}$ concentration on the wall surface $D_{W, H C l}^{\text {old }}$ takes the minimum of the solutions of equation (8.3.10) (non-equilibrium) and equation (8.3.2) (equilibrium) at the previous time step.

As mentioned previously, the average room temperature in the fire compartment was used in [WJ04] for the calculation of the saturation concentration of water. The 
saturation concentration of water in air is dependent on both the temperature and the $\mathrm{HCl}$ concentration [GH89,GH90]. The formulation used in [GH89,GH90] for the calculation of the saturation concentration of water in the atmosphere, which is a complicated polynomial expression of temperature and $\mathrm{HCl}$ concentration, while used in the present study is not reproduced here. However, in the present study, local temperatures are used instead of averaged room temperatures.

\subsubsection{Implementation of the $\mathrm{HCl}$ decay model}

The $\mathrm{HCl}$ decay model described above is implemented in the framework of the CFD fire model SMARTFIRE [EJ02] to create an integrated field fire model capable of predicting the transport and decay process of $\mathrm{HCl}$ generated in a fire.

The modified $\mathrm{HCl}$ decay model is implemented as follows:

- Calculate the equilibrium coefficient using Equation (8.2.3);

- Calculate equilibrium $\mathrm{HCl}$ concentration on wall surface using Equation (8.3.2);

- Calculate the reaction factor using equation (8.2.5);

- Calculate the $\mathrm{HCl}$ deposition rate in the cell adjacent to the solid surface via equation (8.3.9);

- In situations where the $\mathrm{HCl}$ mass flux to the wall surface, $\dot{f}_{H C l}$, is the controller of the deposition rate, calculate the $\mathrm{HCl}$ concentration on the wall surface using equation (8.3.10).

\subsection{Model Verification}

In this section, three tests specially designed to study $\mathrm{HCl}$ transport within enclosures are used to verify the modified $\mathrm{HCl}$ decay model. Two of the three tests have been used to demonstrate the capabilities of the earlier model [WJ04]. The SMARTFIRE software is used to simulate the three tests and the simulation results are compared with corresponding experimental data. The material dependent parameters (see equation (8.2.3) and (8.2.5)) of the decay model, $b_{i}, \mathrm{i}=1,2, \ldots, 7$, for painted gypsum, PMMA and cement block have been reported in [JF00]. Unpainted gypsum and 
concrete have similar model parameters [GH91]. All the parameters used in the present simulations are listed in Table 8.4.1. Since it has been found in experiments that gypsum has similar absorption capability to cement blocks, the values of $b_{2}-b_{7}$ for gypsum in Table 8.4.1 are taken the same values of cement blocks from [JF00].

Table 8.4.1: Model parameters for various wall materials [GH91, JF00].

\begin{tabular}{|l|l|l|l|l|l|l|l|}
\hline Materials & $\boldsymbol{b}_{\mathbf{1}}(\mathbf{m})$ & $\boldsymbol{b}_{\mathbf{2}}\left(\mathbf{m}^{\mathbf{3}} / \mathbf{k g}\right)$ & $\boldsymbol{b}_{\mathbf{3}}(\mathbf{1} / \mathbf{s})$ & $\boldsymbol{b}_{\mathbf{4}}(\mathbf{J} / \mathbf{k} \cdot \mathbf{m o l e})$ & $\boldsymbol{b}_{\mathbf{5}}(\mathrm{a})$ & $\boldsymbol{b}_{\mathbf{6}}(\mathrm{b})$ & $\boldsymbol{b}_{\mathbf{7}}(\mathrm{b})$ \\
\hline Painted gypsum & 0.0063 & 191.8 & 0.0587 & 7476.0 & 193 & 1.021 & 0.431 \\
\hline PMMA & 0.000096 & 0.0137 & 0.0205 & 7476.0 & 29 & 1.0 & 0.431 \\
\hline Cement block & 0.018 & 5.48 & 0.497 & 7476.0 & 30 & 1.0 & 0.431 \\
\hline Gypsum & 0.015 & 5.48 & 0.497 & 7476.0 & 30 & 1.0 & 0.431 \\
\hline
\end{tabular}

a: unit of $b_{5}$ is $\left(\mathrm{kg} / \mathrm{m}^{3}\right)^{\wedge}\left(b_{7}-b_{6}\right)$

b: non-dimensional

\subsubsection{HCl in HVAC system}

\subsubsection{Description of experiments}

Transport of $\mathrm{HCl}$ in air has been experimentally and numerically studied in a simulated HVAC system in [GH91a]. The test system was a tube of square cross section, $0.305 \mathrm{~m}$ on each side, with a total length of $122.0 \mathrm{~m}$ (see Figure 8.4.1). A fan provided the gas velocity at the entrance of the HVAC system. After a steady airflow was established, the $\mathrm{HCl}$ was injected into the first straight section at $0.61 \mathrm{~m}$ from the air inlet.

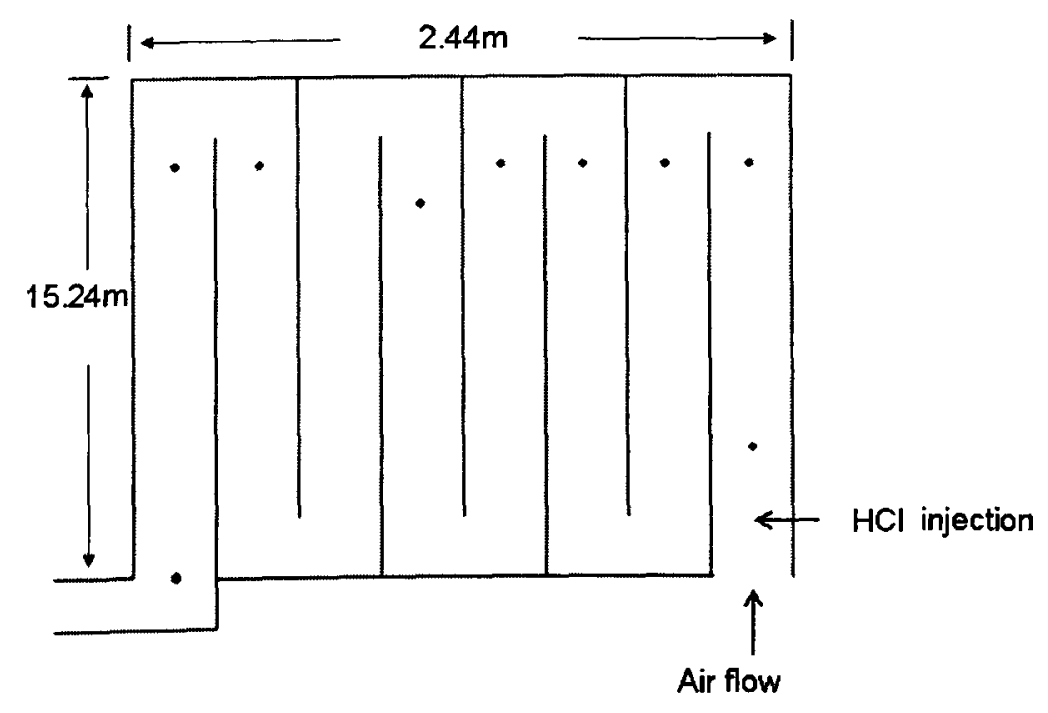

Figure 8.4.1: Schematic layout of HVAC experimental system (dots are positions for the measurements of $\mathrm{HCl}$ ). 
Two of the four cases in [GH91a], here called Case 1 and Case 2, are selected for comparison purposes. In Case 1 , the surfaces of the first $76.3 \mathrm{~m}$ of tube were painted gypsum and the rest of the tube was unpainted gypsum. In Case 2, three sides of the first 76.3m were PMMA and the fourth side was painted gypsum, and the rest was unpainted gypsum. The ambient temperature, gas velocity, absolute humidity and the average $\mathrm{HCl}$ injection rate were $297 \mathrm{~K}, 1.05 \mathrm{~m} / \mathrm{s}, 1.8 \%$, and $0.000386 \mathrm{~kg} / \mathrm{s}$ for Case 1 , and $295 \mathrm{~K}, 1.01 \mathrm{~m} / \mathrm{s}, 1.9 \%$ and $0.0005 \mathrm{~kg} / \mathrm{s}$ for Case 2 , respectively.

\subsubsection{Simulation and results}

The computational mesh for the HVAC system consisted of $30,800(40 \times 154 \times 5)$ cells. The time step size used in the simulation was 1 second. Figure 8.4.2 gives a graphical comparison of the predicted and measured $\mathrm{HCl}$ concentrations of Case 1 at 2, 10 and 28 minutes respectively. In this case, the $\mathrm{HCl}$ concentration at the injection location is greater than $2400 \mathrm{ppm}$, which corresponds to high equilibrium $\mathrm{HCl}$ concentrations on the wall surface. Because the area of the HVAC cross section is small, the measured $\mathrm{HCl}$ concentrations decay quickly with distance from the inlet. The measured $\mathrm{HCl}$ concentrations decay from about $2400 \mathrm{ppm}$ at $3 \mathrm{~m}$ from the inlet to a value less than $200 \mathrm{ppm}$ at approximately $50 \mathrm{~m}$ in 2 minutes, at $70 \mathrm{~m}$ in 10 minutes and $100 \mathrm{~m}$ in 28 minutes respectively. The measured $\mathrm{HCl}$ concentrations gradually increases from 2 minutes to 10 minutes and reaches a steady value at around 28 minutes.

The results shown in Figure 8.4.2 labelled 'old' are the predictions made using the previous version of the model from the earlier study [WJ04]. First consider the results using the old model. The predicted $\mathrm{HCl}$ concentrations at 2 minutes from $5 \mathrm{~m}$ to $80 \mathrm{~m}$ are much higher than the measured data (see Figure 8.4.2(a)). We also note that there is little change in the predicted $\mathrm{HCl}$ concentrations from 2 minutes to 28 minutes. Clearly, the predicted $\mathrm{HCl}$ concentrations using the old model have reached a quasi-steady state much sooner than is indicated by the experimental results. Indeed, the old model suggests that quasi-steady conditions are attained after only approximately 2 minutes. Now consider the results produced by the modified model, see Figure 8.4.2, the predicted $\mathrm{HCl}$ concentrations follow the measured trends. The prediction of $\mathrm{HCl}$ concentrations are in good agreement with the measured data in the 
first $20 \mathrm{~m}$ at 2 minutes and in the first $50 \mathrm{~m}$ at 10 and 28 minutes. The mass flow in this case was $1.01 \mathrm{~m} / \mathrm{s}$ and the total length of the tube was $122 \mathrm{~m}$. Had the walls of the tube not absorbed $\mathrm{HCl}$, the $\mathrm{HCl}$ concentrations within the tube would be uniform after approximately 2 minutes. As can be seen from the $\mathrm{HCl}$ concentrations at 28 minutes, a considerable amount of $\mathrm{HCl}$ has been absorbed by the painted gypsum part of the tubing (i.e. the first $76.3 \mathrm{~m}$ ) in both the experiment and model predictions (see Figure 8.4.2(c)).

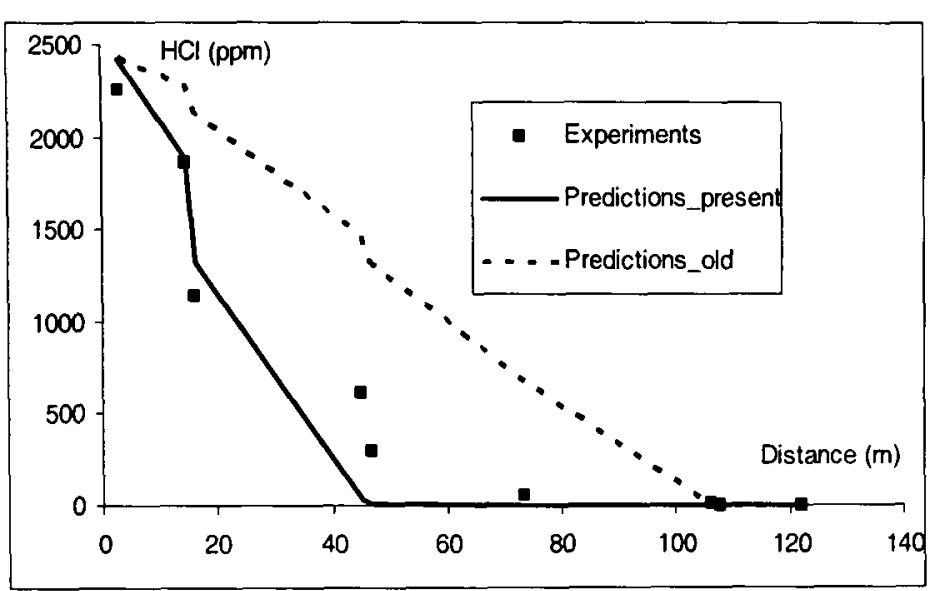

(a)

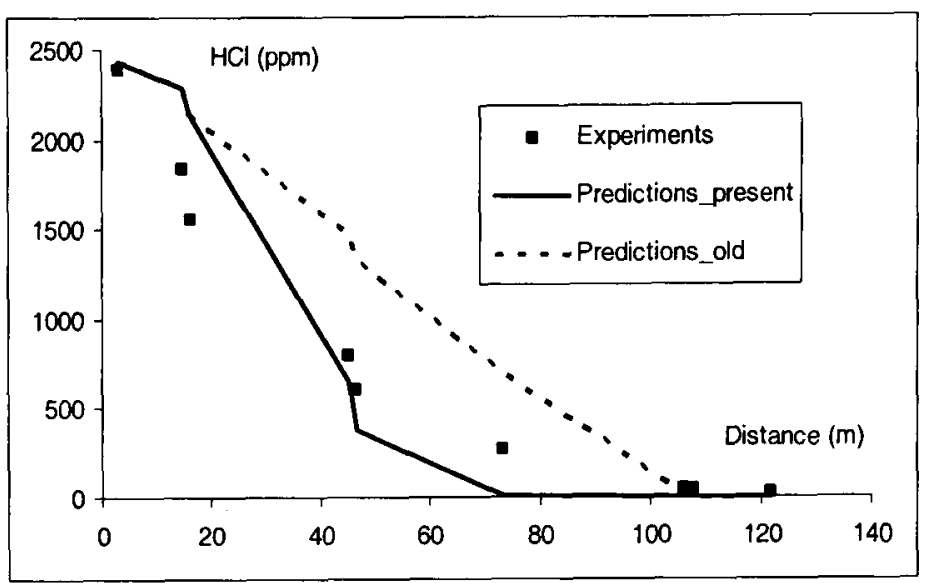

(b)

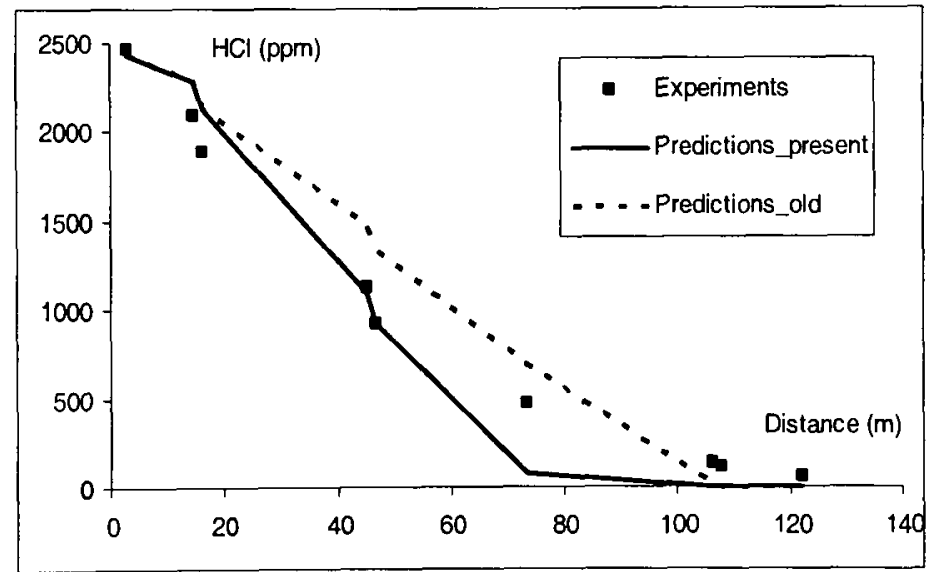

(c)

Figure 8.4.2: Measured and predicted $\mathrm{HCl}$ concentrations of Case 1 at (a) 2 minutes;

(b) 10 minutes and (c) 28 minutes using the modified model (present) and earlier model (old).

In the course of $\mathrm{HCl}$ transportation within the $\mathrm{HVAC}$ system, the main $\mathrm{HCl}$ flow follows the tube with the $\mathrm{HCl}$ mass fluxes to the side walls being weak in comparison. This suggests that it will take a considerable time for equilibrium between the $\mathrm{HCl}$ concentration in the boundary layer and wall surfaces to be attained. In a nonequilibrium situation, the walls will continually absorb $\mathrm{HCl}$ from the gas phase. However, in the old model [WJ04], the wall $\mathrm{HCl}$ concentrations were simply assumed 
to be the equilibrium value. This assumption would therefore result in rapidly (and incorrect) establishing an equilibrium state. Thus less $\mathrm{HCl}$ would be absorbed by the walls in the non-equilibrium situations. The approach adopted in the revised model to predict wall $\mathrm{HCl}$ concentrations has greatly improved the predictions in this scenario.

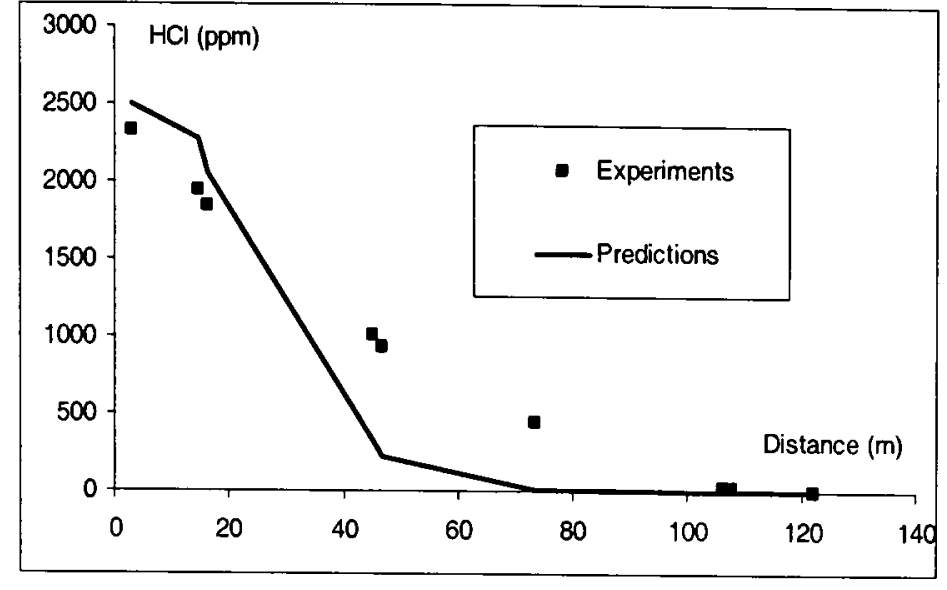

(a)

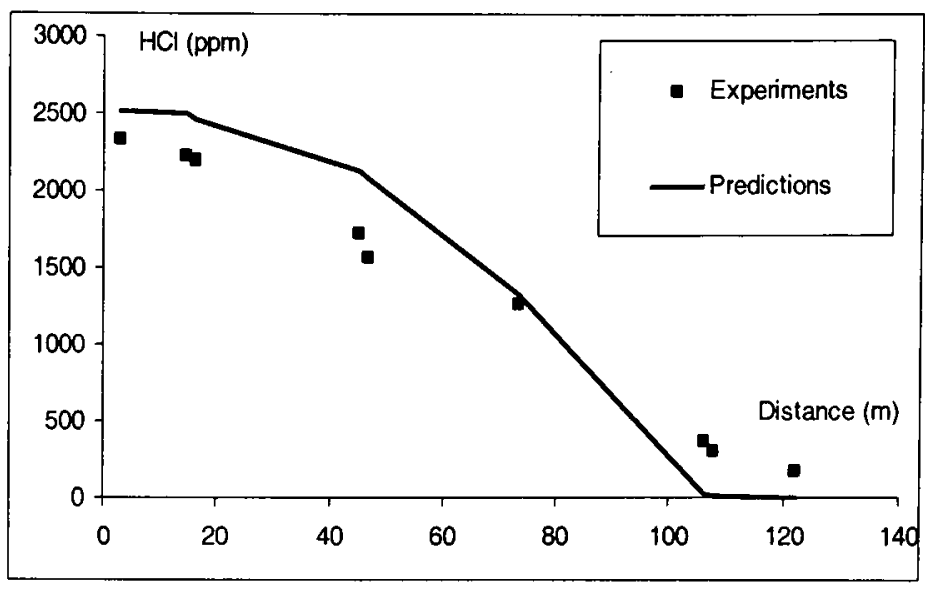

(b)

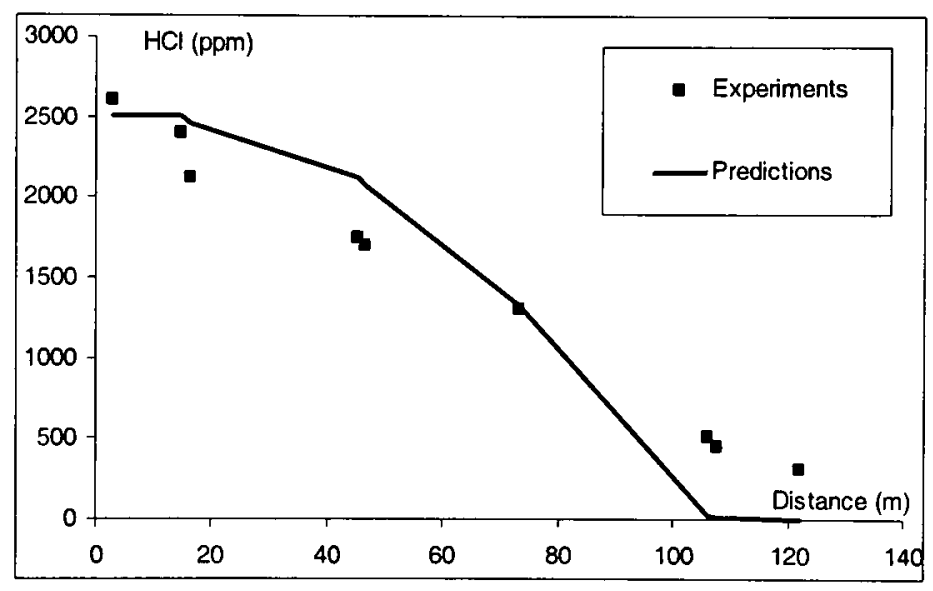

(c)

Figure 8.4.3: Measured and predicted $\mathrm{HCl}$ concentrations of Case 2 at (a) 2 minutes; (b) 10 minutes and (c) 28 minutes.

The measured and predicted $\mathrm{HCl}$ concentrations for Case 2 at 2, 10 and 28 minutes are compared in Figure 8.4.3. It should be recalled that in Case 1 all the walls in the first $76.3 \mathrm{~m}$ were painted gypsum, while in Case 2, one quarter of the tube surfaces in the first $76.3 \mathrm{~m}$ are PMMA, a material with poorer $\mathrm{HCl}$ absorption properties. Together with a higher $\mathrm{HCl}$ injection rate $(0.000386 \mathrm{~kg} / \mathrm{s}$ and $0.0005 \mathrm{~kg} / \mathrm{s}$ for the two cases respectively), this results in the $\mathrm{HCl}$ equilibrium in Case 2 being attained sooner than that in Case 1. This can be seen from the relative changes of the measured $\mathrm{HCl}$ concentrations at $73.2 \mathrm{~m}$ between 10 minutes and 28 minutes (74\% in Case 1 and 5\% in Case 2). As a result, we expect that the differences between the old and current model to be less pronounced in this case and so only therrolts from the current 
model are presented here. As a whole, the predicted distribution trends of $\mathrm{HCl}$ within the duct at the three different points in time match those of the measured $\mathrm{HCl}$ concentrations. Furthermore, the predictions are in good agreement with the experimental $\mathrm{HCl}$ data.

It can be seen from Figure 8.4.2 and Figure 8.4.3 that the predicted $\mathrm{HCl}$ concentrations reduce to zero faster compared with the experimental data. As indicated by equation (8.3.9), when the $\mathrm{HCl}$ flow is the controlling factor for the $\mathrm{HCl}$ deposition rate, the absorption rate is a proportion to the $\mathrm{HCl}$ flow toward the surface. This fact behaviour is represented with the parameter $\alpha$ in equation (8.3.9). As indicated in Section 8.3.2.3, $\alpha$ is assumed to equal 1.0 as there are not experimental data to derive appropriate values. The actual value of $\alpha$ may in fact be less than 1.0. Thus the predicted $\mathrm{HCl}$ concentrations reduce faster than they should due to the inappropriate value of $\alpha$ used. Further studies are required for the derivation of representative values of $\alpha$.

\subsubsection{Small-scale chamber PVC fires}

\subsubsection{Description of experiments}

Climent-Llorca et al [CV98] have studied the absorption and diffusion of chloride with concrete in small-scale PVC resin fires. The test chamber had dimensions of $40 \mathrm{~cm}$ wide, $40 \mathrm{~cm}$ high and $80 \mathrm{~cm}$ long and was constructed of $14 \mathrm{~mm}$ thick PMMA sheet (see Figure 8.4.4). Concrete specimens of different exposed surface areas were located in the test chamber in order to study the chloride contamination of concrete by interaction with PVC combustion gases (see [CV98] for details). A vitrified ceramic hot plate, with dimensions of $22 \times 22 \mathrm{~cm}$, was used for the thermal decomposition of PVC resin samples. PVC samples were located on a Pyrex Petri dish over the hot plate. Several calibration tests were performed to determine the maximum weight of PVC resin that could be completely decomposed within 30 minutes, which was approximately $15 \mathrm{~g}$; and the heating power and times needed for a total sample decomposition without damaging the PMMA test chamber. Three fire scenarios with different ratios of PVC mass to the area of concrete surface (Called Case 1, Case 2 and Case 3 respectively) were investigated. The amount of PVC decomposed was 
$2.075,12.926$ and $12.9 \mathrm{~g}$ respectively. The relative atmospheric humidity in the chamber before the test was maintained between 50 and $65 \%$. The chloride content in the PVC was $56.7 \%$. These tests lasted 30 minutes. Climent-Llorca et al studied the chloride concentrations in concrete while the main concern of the present study is how much $\mathrm{HCl}$ is absorbed by solid surfaces.

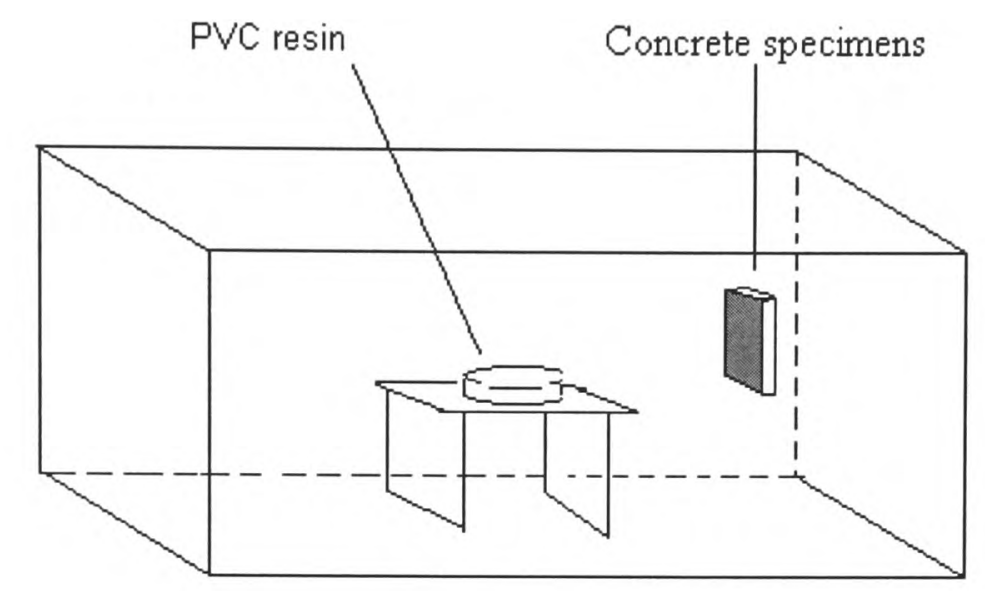

Figure 8.4.4: Schematic of small-scale chamber PVC fire tests.

\subsubsection{Simulation and results}

The plate surface temperature was not reported in [CV98]. In the experiments, the atmospheric temperatures inside the chamber were between 30 and $35^{\circ} \mathrm{C}$. Several preliminary numerical simulations, with the 24-ray radiation model for the exchange of energy by thermal radiation, were carried out to decide on a suitable plate surface temperature required to represent chamber temperatures between 30 and $35^{\circ} \mathrm{C}$. These simulations revealed that a constant temperature of $200^{\circ} \mathrm{C}$ for the plate surface could make the atmosphere temperatures similar to those in the experiments. In addition, $200^{\circ} \mathrm{C}$ is a critical temperature for PVC decomposition since $\mathrm{HCl}$ can be generated from pyrolysis above this temperature [MP79]. Since the maximum possible weight of PVC decomposed within 30 minutes was approximately $15 \mathrm{~g}$, it is assumed here that the $12.926 \mathrm{~g}$ PVC in Case 2 and $12.9 \mathrm{~g}$ PVC in Case 3 was evenly decomposed in the entire experimental time of 30 minutes. It is also assumed that the $2.075 \mathrm{~g} \mathrm{PVC}$ in Case 1 was decomposed with the same decomposition rate as Case 2 and Case 3, i.e. the $2.075 \mathrm{~g}$ PVC was decomposed within approximately 5 minutes. The computational mesh used in these simulations consisted of $6,528(24 \times 16 \times 17)$ computational cells and the time step size was one second. 
The measured and predicted absorption percentages of chloride by the PMMA walls and the concrete specimens at 30 minutes are given in Table 8.4.2. Concrete is much more efficient at absorbing $\mathrm{HCl}$ than PMMA. The area of the PMMA walls is 40 times greater than the exposed concrete specimen area in Case 1, while the measured absorption percentage of chloride by concrete surfaces is above half of the total $\mathrm{HCl}$. The area of the PMMA walls is 66.8 times bigger than the exposed concrete specimen area while the measured absorption percentage of chloride by concrete surfaces is as high as $15 \%$ in Case 2 . The area of exposed concrete surfaces is only $0.3 \%$ of the total solid surfaces of the test chamber in Case 3. The measured absorption percentage of chloride by concrete surfaces in this case is between 2.3 and $6.2 \%$.

Table 8.4.2: Absorption percentages of chloride by PMMA walls and concrete specimens.

\begin{tabular}{|l|c|c|c|c|}
\hline $\begin{array}{l}\text { Tests (area ratio of } \\
\text { PMMA } \\
\text { concrete) }\end{array}$ & \multicolumn{2}{|c|}{ Concrete (\%) } & PMMA (\%) & $\begin{array}{c}\text { Predicted HCI } \\
\text { mass ratio per unit } \\
\text { area of PMMA to } \\
\text { concrete }\end{array}$ \\
\cline { 2 - 5 } & & Predictions & Predictions & 0.0228 \\
\hline Case 3.2-1 (40.0) & 54 & 43.0 & 39.2 & 0.0224 \\
\hline Case 3.2-2 (66.8) & 15 & 22.5 & 33.7 & 0.0229 \\
\hline Case 3.2-3 (334.7) & $2.3-6.2$ & 5.15 & 39.5 & \\
\hline
\end{tabular}

The predicted absorption percentages by concrete surfaces are in good agreement with the measured values in Case 1 and Case 3 while the prediction in Case 2 is relatively poor compared with the measurements. These agreements for cases 1 and 3 are within $20 \%$. In Case 1 , the predicted absorption percentage of chloride by PMMA walls is only $39.2 \%$, which is less than that by concrete (43.0\%). Because the area of PMMA walls is 40 times bigger than the area of exposed concrete surfaces, this prediction demonstrates that PMMA is much weaker at absorbing $\mathrm{HCl}$ compared to concrete. Although the burnt PVC mass and the exposed concrete surfaces are different in the three scenarios, the predicted $\mathrm{HCl}$ mass ratio per unit area of PMMA to that of concrete are almost the same (See Table 8.4.2). Taking the equilibrium value as the wall surface $\mathrm{HCl}$ concentration and the average temperature inside the chamber for the calculation to the saturation water concentration in the earlier study [WJ04], the predicted absorption percentages by concrete were $59.5 \%, 25.5 \%$ and $4.42 \%$ for the three cases respectively. The use of the corrected $\mathrm{HCl}$ concentrations on wall surfaces 
and the local temperatures in the current study slightly improves the prediction accuracy in Case 2 and makes the predicted results to Case 1 slight worse.

The relatively strong absorption capability of concrete for $\mathrm{HCl}$ compared with PMMA can also be seen from the predicted $\mathrm{HCl}$ deposition rates on solid surfaces in Figure 8.4.5 and the last column of Table 8.4.2 which lists the predicted $\mathrm{HCl}$ mass ratio per unit area of PMMA to that on concrete. Because the combustion gases go upwards to the ceiling, then descend and reach the concrete specimens, the deposition rate on concrete surfaces is initially very low at the beginning of the simulation while that on PMMA walls is high with an approximate value of $1.5 \mathrm{e}-6 \mathrm{~kg} / \mathrm{s}$. In Case 1 , the total PVC is supposed to completely burn within 5 minutes as discussed previously. After 5 minutes, the predicted $\mathrm{HCl}$ deposition rate on concrete surfaces has surpassed that on the PMMA wall surfaces (see Figure 8.4.5(a)). Although the total deposition rates on the PMMA walls are higher than those on the concrete surfaces for Case 2 and especially for Case 3 (see Figure 8.4.5(b-c)), the deposition rates per unit area of PMMA are much smaller than that per unit area of concrete.

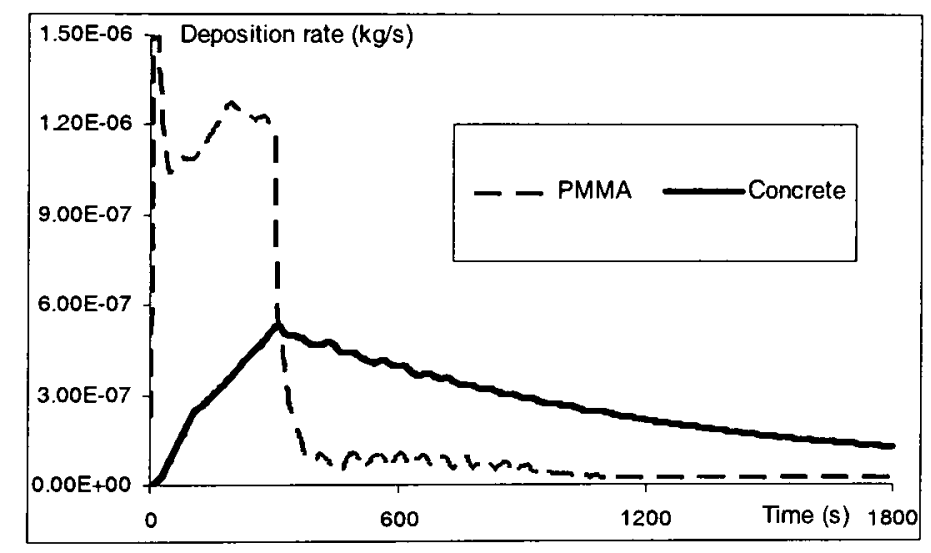

(a)

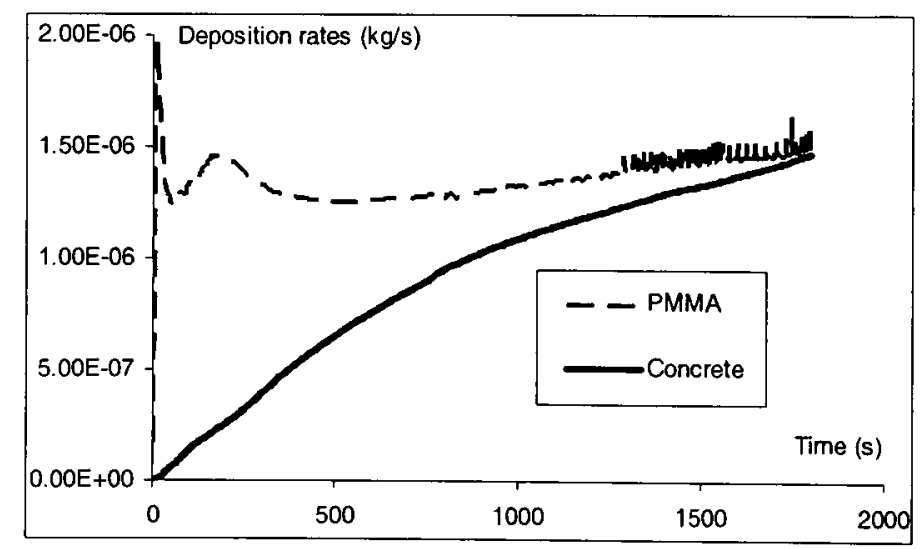

(b)

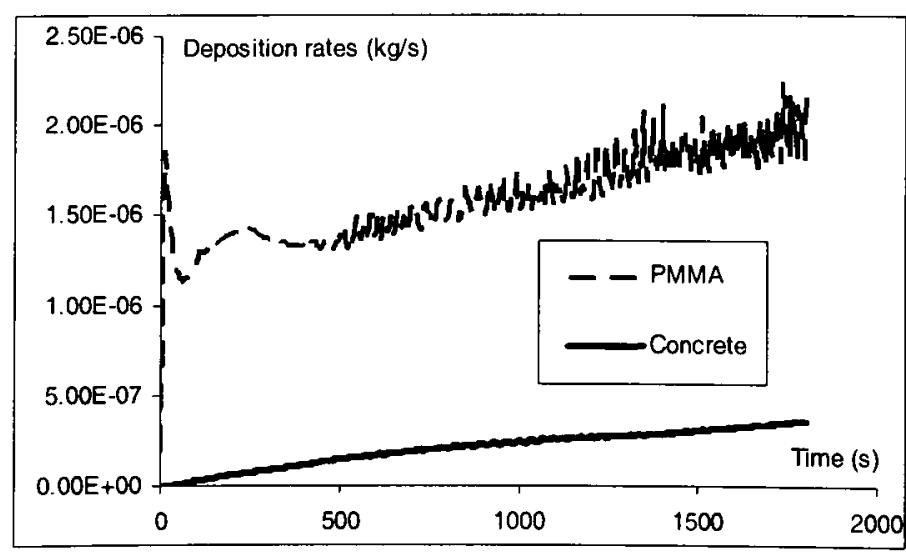

(c)

Figure 8.4.5: Predicted deposition rates of $\mathrm{HCl}$ on $\mathrm{PMMA}$ wall surfaces and concrete species surfaces (a) Case 1; (b) Case 2 and (c) Case 3. 


\subsubsection{Room-corridor PVC Fire}

\subsubsection{Description of experiment}

One of a series of PVC room-corridor fires [GH92], has been selected to verify the modified $\mathrm{HCl}$ decay model. Only a single test fire is selected from this series as all the fires display similar $\mathrm{HCl}$ decay trends. The test scenario involved a burn room of dimensions of $3.6 \times 2.4 \mathrm{~m}$ with a height of $2.4 \mathrm{~m}$, and a corridor of dimensions of $19.0 \mathrm{~m} \times 1.65 \mathrm{~m}$ with a height of $2.44 \mathrm{~m}$ (see Figure 8.4 .6 ). The test room and corridor were constructed with painted gypsum walls. The test selected here for comparison purposes involved a fire fuelled by $401 \mathrm{~g}$ of PVC and a $6.474 \mathrm{~kW}$ burner. The $\mathrm{HCl}$ analysis samplers were located at the doorway, in the middle of the corridor and $1.24 \mathrm{~m}, 6.15 \mathrm{~m}, 11.02 \mathrm{~m}$ and $15.91 \mathrm{~m}$ from the closed end of the corridor. $\mathrm{HCl}$ sampling was taken at a height of $2.3 \mathrm{~m}$.

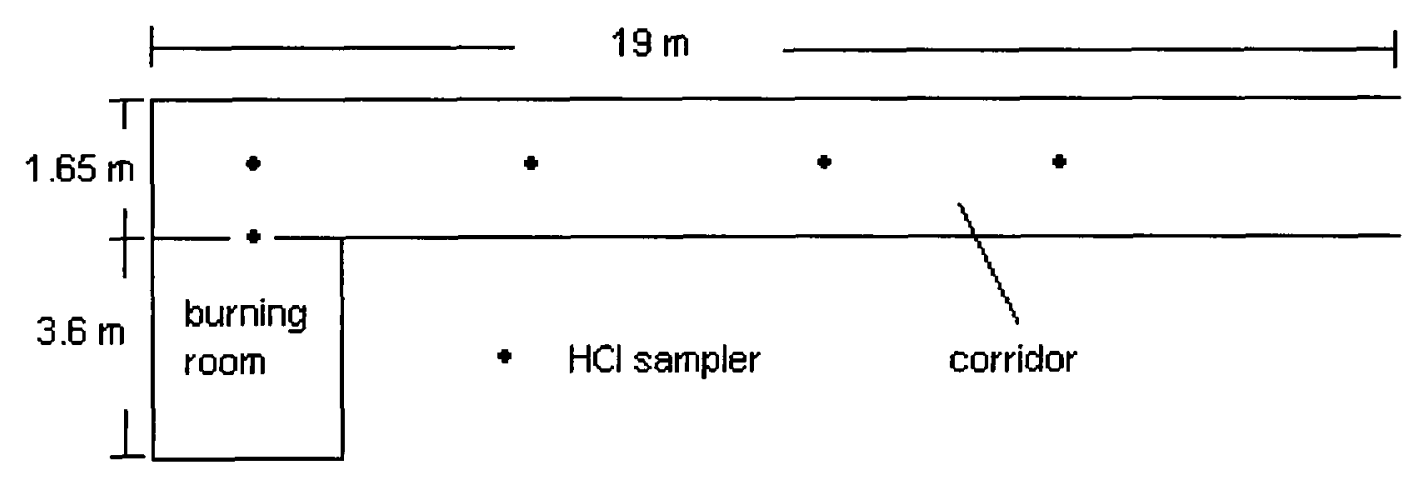

Figure 8.4.6: Plan view of Schematic of PVC room-corridor fire.

\subsubsection{Simulation and results}

The burning rate of PVC was not given in [GH92]. Instead, the measured $\mathrm{HCl}$ concentrations at the doorway as a function of time were reported. This curve against time is the basis on which the burning rate of $\mathrm{HCl}$ is derived for this study. It is assumed that the shape of the PVC burning rate curve is proportional to the one of the measured $\mathrm{HCl}$ concentrations at the doorway. The burning rate of $\mathrm{PVC}$ is then determined such that the total calculated mass of burned PVC in half an hour is equal to 401g, which is the total burned PVC in the half hour test. The estimated PVC burning rates are shown in Figure 8.4.7. The yield of $\mathrm{HCl}$ for rigid PVC used in the simulation is $0.55 \mathrm{~kg} / \mathrm{kg}$ [Te95]. According to the description of this experiment in an 
earlier paper [GH89], the ambient absolute humidity was $2.0 \%$ in mole fraction. In this simulation, the volumetric heat source model is used to represent the fire while the six-flux radiation model is used for the exchange of energy by thermal radiation. The computational mesh consisted of 31,350 computational cells and the time step size was two seconds.

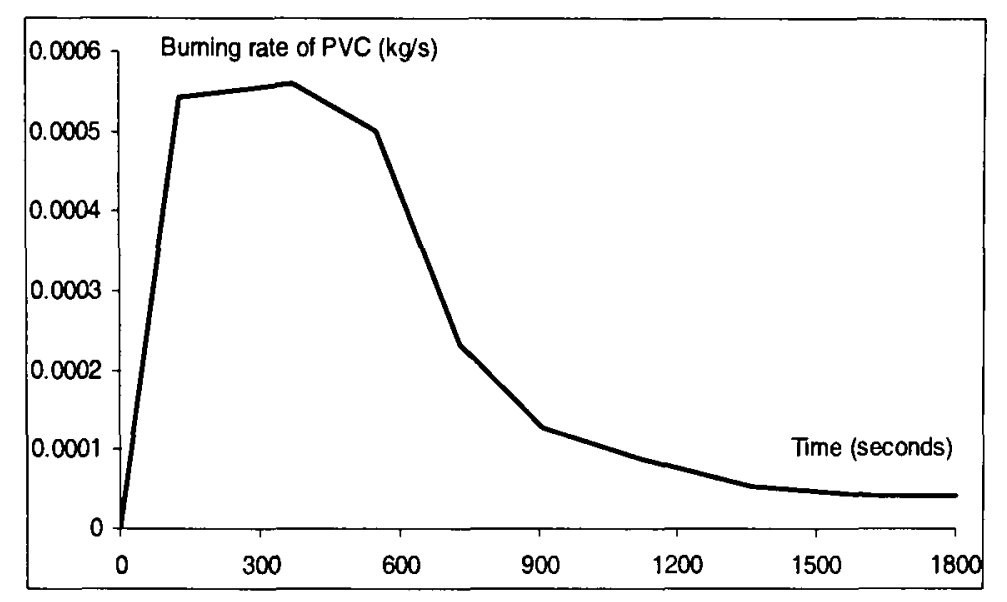

Figure 8.4.7: Burning rate of PVC.

Two types of simulation were undertaken, one involving the $\mathrm{HCl}$ decay model and one without using the $\mathrm{HCl}$ decay model. The predicted and measured $\mathrm{HCl}$ concentrations, as functions of time, at different locations are depicted in Figures 8.4.8 (a-e). The predicted $\mathrm{HCl}$ concentrations at all of the measurement locations captured the basic measured trends. However, predictions using the decay model agree more closely with the measured $\mathrm{HCl}$ concentrations than the predicted results derived from the simulations without the decay model. The differences between the two sets of predicted results are only relatively small as the painted gypsum walls have a weak $\mathrm{HCl}$ absorption capability. However, we note that the differences between the two sets of predicted results increase with distance from the fire room. At the doorway, there is only an approximately $30 \mathrm{ppm}$ difference between the two predicted peak $\mathrm{HCl}$ concentrations. However, at the position $15.91 \mathrm{~m}$ from the closed end of the corridor, the difference between the two predicted peak values is over 90 ppm or approximately $20 \%$ of the predicted peak values (see Figure 8.4.8(e)).

In this scenario, we note that there are no significant differences in the predicted $\mathrm{HCl}$ concentrations between the present study and the earlier study [WJ04]. This can be seen from Figure 8.4.9 which depicts predicted $\mathrm{HCl}$ concentrations at the location $6.15 \mathrm{~m}$ from the closed end of the corridor for both models. A possible explanation 
for the lack of difference between the two sets of predictions could be due to the small amounts of $\mathrm{HCl}$ involved and the relatively large compartment volume found in this experiment.

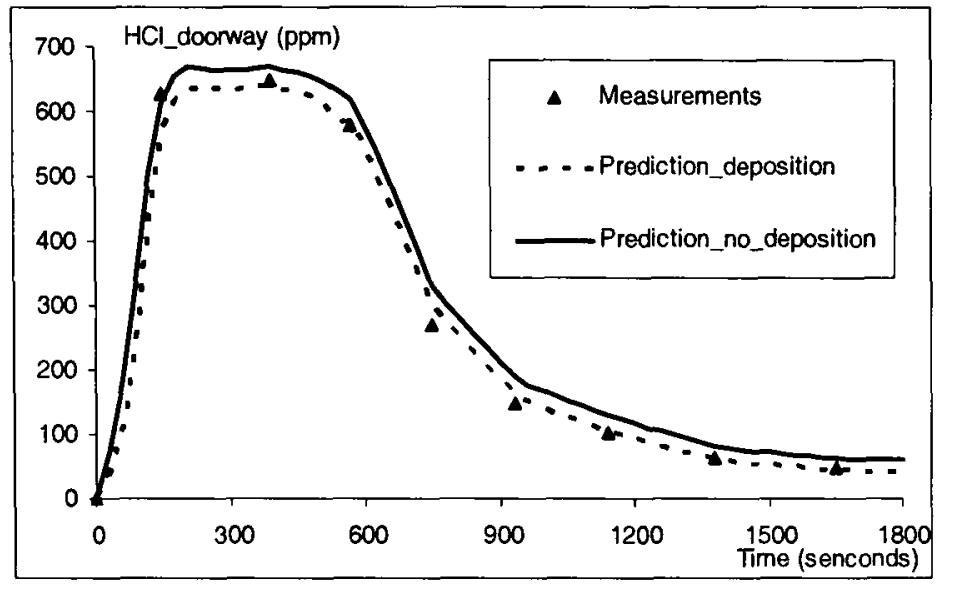

(a)

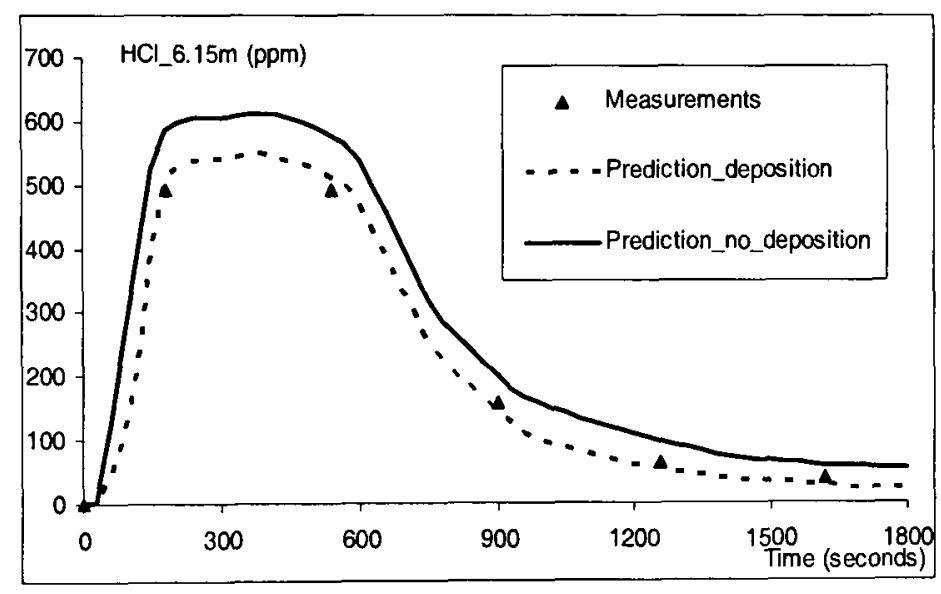

(c)

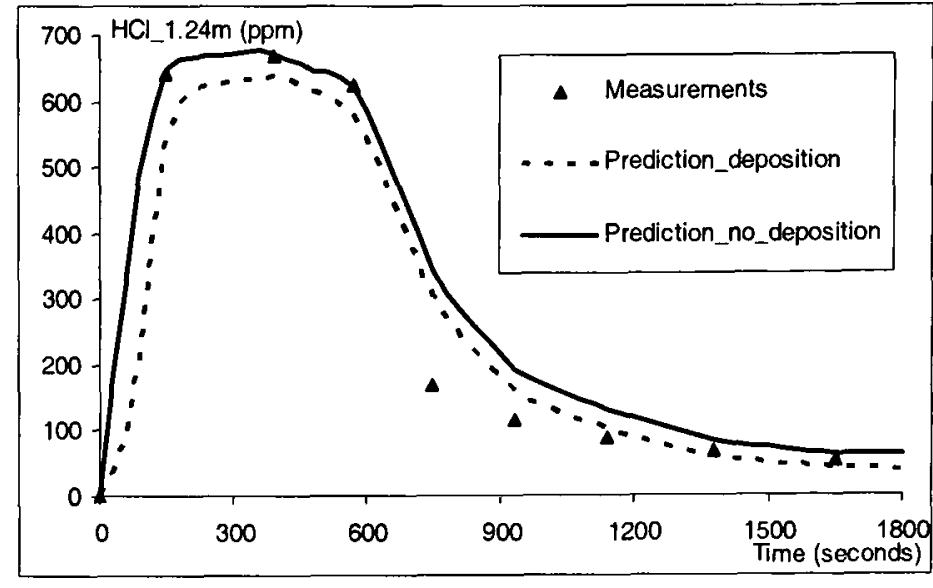

(b)

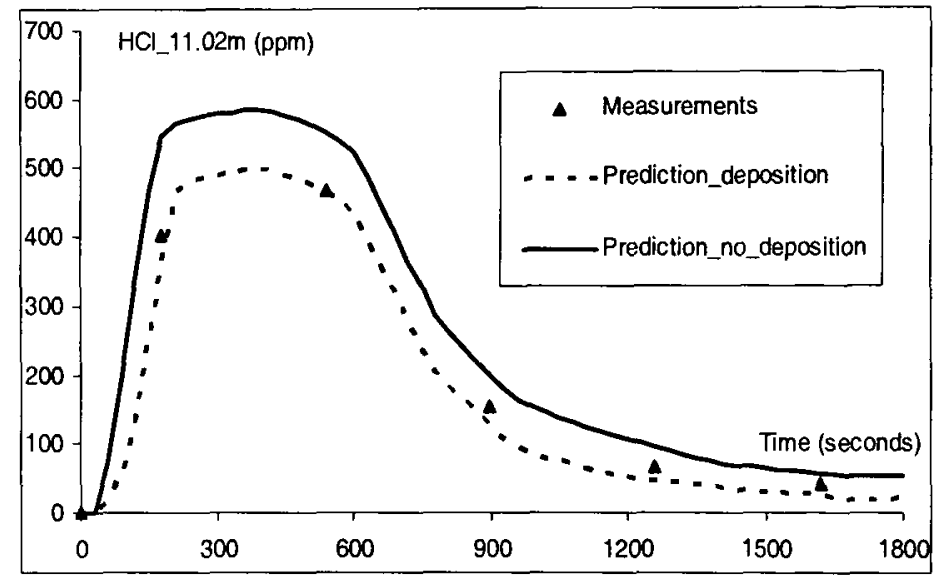

(d)

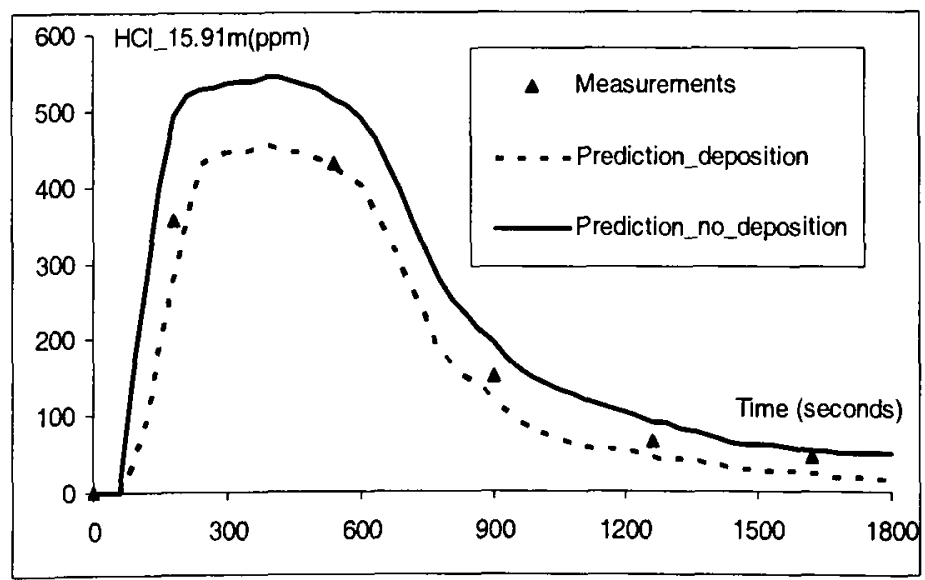

(e)

Figure 8.4.8: Measured and predicted $\mathrm{HCl}$ concentrations at (a) doorway and locations in the corridor with distances of (b) $1.24 \mathrm{~m}$, (c) $6.15 \mathrm{~m}$, (d) $11.02 \mathrm{~m}$ and (e) $15.91 \mathrm{~m}$. 


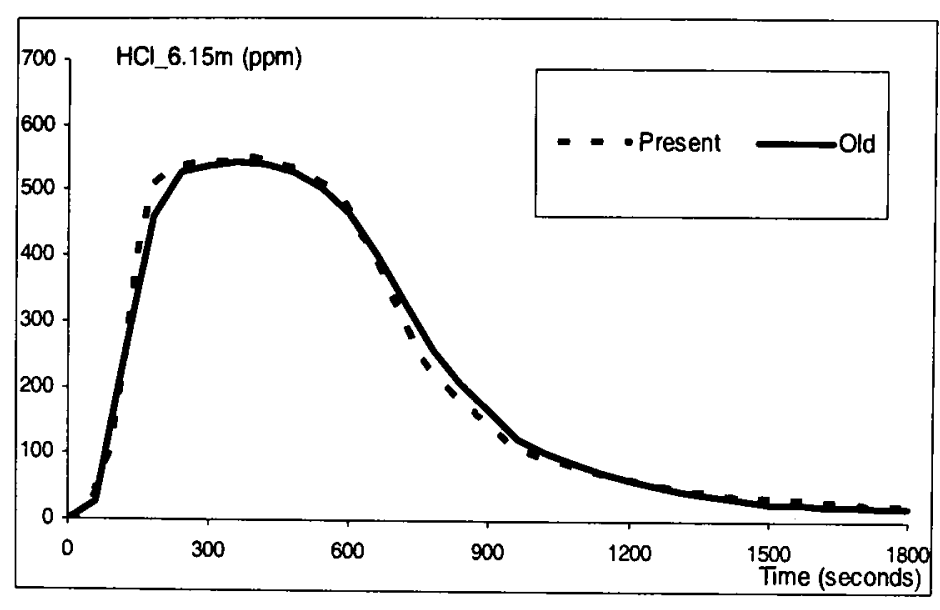

Figure 8.4.9: Predicted $\mathrm{HCl}$ concentrations at $6.15 \mathrm{~m}$ from the closed end of the corridor produced by the current model (present) and the earlier model (old).

\subsection{Concluding Remarks}

A previously defined $\mathrm{HCl}$ decay model used in zone models has been adapted for use in CFD based fire field models. The model is used to predict the decay of $\mathrm{HCl}$ in fire enclosures due to the wall absorption effect. By taking advantage of the capabilities of the CFD framework several simplifying assumptions inherent in the original model have been removed. These model enhancements mainly concern:

- The process involving the movement of $\mathrm{HCl}$ from the gas phase into the boundary layer is realistically represented within the CFD model and does not need to be represented via a first-order conservation equation.

- The $\mathrm{HCl}$ mass fluxes to wall surfaces are introduced as a controller of the deposition rate.

- Actual $\mathrm{HCl}$ concentrations on wall surfaces are calculated rather than using equilibrium values.

Model predictions were then compared with experimental data derived from three different experiments involving $\mathrm{HCl}$ transport. One experiment involved a $\mathrm{HVAC}$ system operating at ambient temperature conditions. In this case the model was found to be capable of capturing the observed trends in $\mathrm{HCl}$ deposition. Simulations of PVC fires in a small-scale test chamber also produced reasonable predictions of $\mathrm{HCl}$ absorption by concrete specimen targets. Finally, a fire test using a room-corridor system with painted gypsum walls demonstrated that the $\mathrm{HCl}$ model was capable of producing reasonable agreement with measured $\mathrm{HCl}$ gas concentrations at locations distant from the fire compartment. Of particular note, the simplification to the wall 
surface $\mathrm{HCl}$ concentrations in earlier studies failed to predicted the $\mathrm{HCl}$ concentrations in the HVAC system. The new methodology developed in this study greatly improved the model predictions to this test.

The key model parameters used in these simulations are based on data derived from experiments with a limited range of temperatures. Furthermore, the verification work presented in this study, and that of other researchers, has only addressed scenarios in which the room temperatures are relatively low. In real enclosure fires, temperatures in the fire compartment may be significantly higher than those used in the verification experiments and the experiments used to define the model parameters. These higher temperatures may speed up both $\mathrm{HCl}$ deposition onto and evaporation from wall surfaces. Thus, further verification is necessary to test the robustness of these model parameters in more severe fire scenarios.

It is also interesting to derive an appropriate value of $\alpha$ in Equation (8.3.9) in the future study. 


\section{Chapter 9}

\section{CONCLUSIONS}

Uncontrolled fires within buildings release heat and a range of combustion products. If the combustion is incomplete, fuels involved in the combustion such as furniture and building materials may produce a large mount of smoke and toxic gases. Toxic gases and smoke in enclosure fires pose a major threat to human lives. Carbon monoxide $(\mathrm{CO})$ is the main toxic gas, which is responsible for deaths at remote locations from the fire source.

Fire field modelling provides a powerful tool for the simulation of enclosure fires. Some advanced combustion models, such as the laminar flamelet model, provide information of incomplete combustion products. However, these models are difficult to use in most applications due to a lack of chemical kinetic data for common building materials. The main objective of this study was to develop relatively simple and practical engineering fire models within a CFD framework to simulate the production and movement of toxic gases.

The main achievements on these objectives are presented here by briefly answering the questions raised in Chapter 1. A practical toxicity model (Model I) based on the concept of local equivalence ratio (LER) has been developed in Chapter 4 for predicting the generation and transport of toxic gases within fire enclosures. In addition, similar toxicity approaches (Model II and Model III) using $\mathrm{CO} / \mathrm{CO}_{2}$ and $\mathrm{H}_{2} / \mathrm{H}_{2} \mathrm{O}$ mole ratios have been developed and presented in Chapter 4. In order to study the toxic gas levels at remote locations (non-combustion region), a methodology has been proposed in Chapter 6, which divides the computational domain into two parts, a control and transport region. In the control region, the toxic gases are dependent on the LER and temperature and are calculated using Model I. In the transport region, the toxic gas levels are the result of the mixing of hot gases with fresh air and are proportionate to the mixture fraction. All these models have been validated with fire tests and simulation results to these fire tests have demonstrated that these models are capable of producing reasonably accurate predictions to toxicity levels within fire enclosures. 
While the associated questions to the fundamental question have been answered in the relevant chapters, the achievements of this study are summarised below.

A review of the research background of this study has been presented in Chapter 1. The aims and objectives of this study have been discussed and outlined. A number of questions related to this study have been raised. Developing practical engineering toxicity models in a CFD fire modelling framework has been identified as the main goal of the study.

Chapter 2 has reviewed the background of the experimental studies and mathematical modelling of toxic gases in enclosure fires. It has been found, in various fire experiments, that ventilation condition is the main factor that determines the yield of toxic gases in flaming fires. Some fire researchers have introduced GER to represent the combustion condition of a fire scenario and reported that the yields of combustion products can be correlated with GER. However, it is problematic to apply GER data directly in large-scale fire simulations, particularly zone fire models. This is largely due to the direct air entrainment into a fuel rich upper layer.

Chapter 3 has focused on the CFD techniques used in field fire modelling. The governing equations for turbulent flames have been described in this chapter. The sub-models, which are available to describe the physical and chemical process of fires, are discussed. They are the volumetric heat source approach, the Eddy Break-up model, the laminar flamelet model, the mixture fraction model and radiation models. Simulation to a complicated warehouse fire showed that the simple volumetric heat source approach together with the six-flux radiation model and the $k$ - $\varepsilon$ turbulence model are capable of providing good temperature predictions if a good mesh is used. Combustion has been ignored in the simple heat source model.

The concept of LER has been introduced in Chapter 4 to predict combustion products in fire enclosures. Firstly, the reason for the failure of GER in certain circumstances to predict correctly the levels of toxic gases within full-scale fire enclosures has been discussed. The failure of GER in most fire scenarios is due mainly to the variation of the local conditions (the mixing of fuel with air, local temperatures and residence time) 
with space within the fire enclosure and the fact that one single value of GER is not capable of representing the varying local burning conditions. This has been demonstrated in the analyses conducted in Chapter 4.1 and 4.2. A CFD simulation to a fire test has shown that LERs within the upper layer are quite different from the GER. The analyses have highlighted that it is possible to make use of the LER values instead of GER to obtain better predictions of the $\mathrm{CO}$ concentrations at different locations.

Secondly, an assumption has been proposed in Chapter 4.3 for the application of the concept of LER in predicting toxic gases within fire enclosures, i.e. the combustion conditions within a control volume and the associated LER and temperature are assumed approximately equivalent to those of a small-scale fire experiment with the same value of GER and temperature.

Thirdly, with this assumption, three mathematical models based on the species yields (Model I) or the $\mathrm{CO} / \mathrm{CO}_{2}$ mole ratio (Model II) or the mole ratios of $\mathrm{CO} / \mathrm{CO}_{2}$ and $\mathrm{H}_{2} / \mathrm{H}_{2} \mathrm{O}$ (Model III) have been developed to predict combustion products in this chapter. In these models, the LER is calculated from the mixture fraction. Therefore, only one scalar variable is adopted if the heat released due to combustion is modelled with the volumetric heat source approach and no additional equation is needed if the EBU model is applied. All three models produced similar predictions of combustion products except at locations with extremely high LERs.

A method to calculate the smoke optical density and light extinction coefficient based on local equivalence ratios (or mixture fractions) has also been developed in Chapter 4.4 .

In Chapter 5, a range of full-scale fire tests, RI (Rockwool International) fire, PP3 fire, W14-b3 fire, Borealis cable fire and one reduced compartment-corridor fire have been simulated by using the toxicity models and the smoke calculation method developed in Chapter 4. The fuels of these experiments included wood, Polypropylene, PVC or PVC cable and hexane. Models I, II and III have produced similar predictions of species concentrations to the RI fire test. Later on only Model I has been applied in the simulations to other fire scenarios. Good agreements have been obtained between 
the predictions of temperatures and combustion products and experimental data of each of these fire tests. Simulations have shown that the toxicity models developed in this study are promising to use in conjunction with the volumetric heat source approach or the EBU model for predicting combustion products in enclosure fires. It should also be noted that the gas sampling locations in the experiments for model validation in Chapter 5 are all within or close to the fire flaming region.

The method of calculating the smoke properties has been validated with a cable fire in Chapter 5.5. Good agreements between the measured and predicted light transmissions have been obtained. The predicted visibility distances at various times are in reasonably good agreement with observations in the experiment.

In Chapter 6, a methodology to calculate the species concentrations at remote locations has been proposed. In this method, the computational domain in a CFD fire simulation is divided into a control and transport regions. A critical value $\phi_{C R}$ of LER is required to determine the two regions. The combustion coefficient $\lambda$ (normally it is an input parameter for fire simulations) can be used to derive the critical equivalence ratio $\phi_{C R}$. The species concentrations in the control region are LER and temperature dependent and are calculated with the toxicity model developed in Chapter 4 . With the assumption that species concentrations at the boundary of the control region are only determined by the LER $\phi_{C R}$, the species concentrations in the transport region are proportionate to the mixture fractions with a proportional factor that links to $\phi_{C}$.

Two room-corridor fire scenarios have been simulated using this approach to predict the toxic gases within the two fire enclosures. Significant improvement on the predictions of $\mathrm{CO}, \mathrm{CO}_{2}$ and $\mathrm{O}_{2}$ at remote locations has been achieved by the method compared with Model I developed in Chapter 4. If $\phi_{C R}$ is determined by combustion efficiency, the model predictions of species concentrations at remote locations may be sensitive to the change of the combustion efficiency if the corresponding critical equivalence ratio happens to be a value near which yields of species are subject to rapid changes. For fire scenarios of this kind, it is advisable to undertake a sensitive analysis in order to determine the degree of variation in species concentrations in the far field. 
By extending the concept of the equivalence ratio to two-fuel fires, a two-fuel toxicity model has been developed in Chapter 7 to predict the toxic gases in enclosure fires in which two different types of fuel are involved. The mixing-limited reaction rate is modelled with the two-fuel EBU model while the concentrations of the products are calculated with the concept of LER. The correlations between the species yields and equivalence ratios in this model are from small-scale experiments with a single fuel. Simulations to a fire scenario with two fuels involved have been performed. Compared with the experimental results, the present two-fuel toxicity model has predicted the effect of wood on the generation and transport of $\mathrm{CO}$ and $\mathrm{CO}_{2}$.

A mathematical model has been developed to study the deposition of $\mathrm{HCl}$ on solid surfaces in Chapter 8, which is a modification of an $\mathrm{HCl}$ decay model in zone modelling. As the process involving the movement of $\mathrm{HCl}$ from the gas phase into the boundary layer is represented within the CFD model, there is no need to model this process via a first-order conservation equation as the original model did. Therefore, the modified model cuts down the three processes of the original model to two process of the absorption of HCL by walls and the reaction between walls and HCL. An assumption that a cell adjacent to a wall surface in the computational domain is in the boundary layer has been made. With this assumption, the equilibrium $\mathrm{HCl}$ concentration on the solid surfaces can be calculated from the $\mathrm{HCl}$ in the adjacent air cell (boundary layer). The $\mathrm{HCl}$ mass fluxes to the wall surfaces are applied to limit the deposition rate, which is obtained from the equilibrium equation. Reasonably accurate predictions have been obtained using this deposition model to simulate the absorption of $\mathrm{HCl}$ by gypsum walls in a room-corridor PVC fire, and by PMMA walls and concrete specimens in small-scale chamber PVC fires.

The study has addressed the concerns and questions raised in Chapter 1 as the main aim and motivation of this study.

Can the correlations derived from small-scale experiments be applied to predict the generation and transport of toxic gases within fire enclosures, and if so, how can they be used? 
This study has positively demonstrated that the correlations with GER derived from small-scale experiments can be applied in field fire modelling to predict the generation and transport of toxic gases within fire enclosures which is the main aim of this study.

- Why does the GER approach fail in certain circumstances to predict correctly the levels of toxic gases within full-scale fire enclosures? How can a field model address this problem?

Chapter 4 has given a detailed discussion to the two questions and has been summarised earlier in this chapter. Here only the work related the second question is highlighted. This study takes advantage of field modelling being able to provide local information at a control volume. The local combustion conditions at the control volume can be approximately represented by the LER and local temperature in field modelling, unlike applying a single value of GER to represent the varying burning conditions over the entire enclosure of interest (Chapter 4.3). The local mixing state of fuel, air and combustion products at the control volume can be reflected in the LER, which links to fire field modelling via the mixture fraction.

- How are species concentrations calculated using the concept of local equivalence ratio (LER)? In what region is the approach valid?

Formulas of species concentrations, which can be easily slotted into the framework of field fire modelling, have been derived based on the correlation between yields of the species and the GER (Chapter 4 and 6). Application of these formulas in field fire modelling is achived by replacing GER with LER which is calculated locally at each control volume through the mixture fraction. The yield data used in the formula can be obtained through small scale fire tests. The models proposed in Chapter 4 are valid only within or close to the fire flaming region while the revised model proposed in Chapter 6 can be applied both in the near and far field.

- How is the region, in which the changes of species concentrations are only due to mixing of hot gases with fresh air, determined? Furthermore, how are the species concentrations calculated in this region? 
To accurately predict the species concentrations at locations far from the fire origin of enclosure fires, dividing the whole computational domain into two non overlapping regions with different formulas of calculation of species in each region is proposed in Chapter 6. This is a generic concept and there can be a number of different realizations of the concept. As a preliminary test of this idea, a methodology based on critical equivalence ratio for the division of the computational domain has been put forward. The use of a critical equivalence ratio to determine the two regions is generic in its own right. It is materialized in this study through the use of the combustion efficiency of a considered fire scenario. In the transport region, the species concentrations are only the result of the mixing of hot gases and fresh air and can be linearly approximated with the local mixture fraction.

- Can smoke properties, such as optical density or visibility distance, be calculated using the LERs via simple relationships?

As seen in Chapter 4.3.2, the soot mass fractions which is LER dependent are available from the toxicity Model I. The light extinction coefficient, smoke optical density and visibility distance can be calculated from the soot concentrations and the specific extinction coefficient (Chapter 4.4).

- How are the species concentrations in two-fuel fires calculated using correlations from the experiments with a single burning fuel?

A model for predicting toxic gas concentrations of fire scenarios involving two fuels has been developed in Chapter 7. Two mixture fractions are introduced to represent the two different fuels. The key technique in this model is the calculation of LER using the two mixture fractions. The yields of species can then be calculated from correlations for a single fuel derived from small-scale experiments.

Finally, the features of the toxicity models developed in this study are summarised here:

(1) The species yields or the $\mathrm{CO} / \mathrm{CO}_{2}$ mole ratio used in the toxicity model can be obtained from literatures or derived from small-scale experiments for most 
furniture and building materials. The empirical parameters in the $\mathrm{HCl}$ deposition model are available for common building materials too.

(2) The models can easily be integrated within a CFD fire modelling framework.

(3) The simulation overheads incurred for the toxicity models is minimal when compared to typical CFD runtime requirements because only one variable (mixture fraction) is introduced when the volumetric heat source approach is applied and no extra equation is involved when the EBU model is applied. For the $\mathrm{HCl}$ deposition model, tiny extra calculations are involved because only the boundary conditions need to be addressed. 


\section{Chapter 10}

\section{FURTHER WORK}

There is room for the improvement for the models developed in this study. In this chapter, further work to the toxicity models are discussed.

\subsection{Effect of High Temperatures on $\mathrm{CO}$}

For most polymers, the yields of toxic gases depend mainly on the LERs [Te95, Pu03]. Simulations presented in this study using the correlation between the yields of combustion products and the GERs have demonstrated this point. However, besides the LERs, the upper layer temperature also plays an important role in the production of toxic gases for some fuels. Although the toxicity models developed in this study have involved the effect of temperature, only the concept of LER has been validated in this study because of the lack of reliable correlations with temperature. Some specially designed fire scenarios with gas fuels [GR95] have demonstrated that the effect of temperature on $\mathrm{CO}$ level can be significant. Therefore, the present toxicity models need further validation with proper correlations between the yields of $\mathrm{CO}$ and combustion conditions (equivalence ratio and temperature here). This work depends on the availability of these correlations. At least for solid fuels, it is hopeful to obtain this type of correlations with experimental apparatus like the Purser Furnace [BS03, PF94, HP99, HQ02].

An alternative method to address the effect of temperature is to apply simplified chemical reaction mechanisms in the EBU model (this is usually called multi-step EBU model). Bilger [Bi94] has mentioned an idea of using the EBU model based on a 4-step reduced mechanism for the prediction of CO. The multi-step EBU model, in which $\mathrm{CO}$ is one of the intermediate products, was sufficient to account for the effect of inefficient oxygen, slow fuel evaporation and slow reaction rate on the extinction processes [BK99, BM00, HC97], produced good flame base height and other flame characteristics of cylindrical tube methane flames [KC99]. Hyde et al [HM99] simulated axi-symmetric flame using a two-step EBU model, in which the reaction 
rates of the two steps are controlled by the mixing rate but with different coefficient for each step. The predicted CO level was modest in Hyde's simple model.

The multi-step EBU could be used in conjunction with the toxicity model using the LER for some gas-fuel fires in which the toxic gases are strongly temperature dependent [Pi94b, PB95, GRB95] and for which the multi-step chemical reaction rates are available from literatures [HD81,WD81,JL88].

\subsection{Effect of Pyrolysis of Solid Fuel on CO}

The concept of equivalence ratio usually reflects the ventilation conditions of flaming fires. As reviewed in Chapter 2, many factors affect the $\mathrm{CO}$ level in fire enclosures. Pyrolysis of solid fuel is another important $\mathrm{CO}$ formation mechanism. The presence of wood in an oxygen-depleted upper layer of a fire enclosure can generate a significantly high CO level [Pi95, LV98]. Limited data on the yields of toxic gases in the pyrolysis stage of solid fuels are available [Oh95, Pu95, Ts94]. The effect of pyrolysis of solid fuels on the $\mathrm{CO}$ level should be specially addressed in the future model development.

\subsection{Criterion for Determining the Control Region}

In Chapter 6, a simple method has been proposed to determine the boundary of the control region. The boundary is based on a critical equivalence ratio, which is calculated from the combustion efficiency of a fire scenario considered and the correlation between the combustion efficiency and equivalence ratio derived from small-scale experiments. Because the combustion efficiency (obtaining from the actual heat release rate and fuel loss rate) is not known in general fire applications, it is necessary to develop an alternative method solely based on the fuel loss rate to determine the control region.

Preliminary simulations have demonstrated that the combination of the traditional Eddy Break-up (EBU) combustion model [MH77] and flammability limit [Dr85] has the potential to simulate the extinction or continuing burning of the post flashover fire in the hallway that is adjacent to the original fire compartment. This method may be 
useful to determine a dynamical control region without the information of actual heat release rates.

The approach to the control region and transport region will be extended to the twofuel toxicity model in the future study.

\subsection{Toxicity Models for $\mathrm{HCN}$ and Soot}

Besides $\mathrm{CO}, \mathrm{HCN}$ is another main toxic gas responsible for fire deaths [Pu95]. Using the laminar flamelet concept, a kinetics model for the generation of $\mathrm{HCN}$ has been developed [TH04]. However, with the same intent of this study for the generation of $\mathrm{CO}$, a practical method with easily available model inputs for the simulation of $\mathrm{HCN}$ could be developed.

The ratio of the converting efficiency of fuel carbon to $\mathrm{CO}$ and the fuel nitrogen to $\mathrm{HCN}$ is almost close to one in full-scale enclosure fires [Pu00]. Therefore, the yield of $\mathrm{HCN}$ can be estimated from the yield of $\mathrm{CO}$ and hence, the $\mathrm{HCN}$ concentration could possibly be calculated from the prediction of $\mathrm{CO}$ concentration with the models developed in this study.

Soot in fire effluent is an important component in determining the hazardous impact of fire. The presence of soot in fire products will influence the temperature and thermal radiation produced by the fire as well as the light transmittance of the fire, all of which will in turn influence the survivability of a particular fire environment. Correlation between the generation of soot and equivalence ratio has been derived by Tewarson [Te95] and has been modelled in the present toxicity model in Chapter 4. However, this has been validated only with one scenario in Chapter 5.3. Further work on soot models and the model validation is necessary.

\subsection{Species Deposition Model}

The deposition rate of $\mathrm{HCl}$ in the model in Chapter 8 is expressed by equation (8.3.9), i.e. 


$$
\begin{aligned}
S_{B, H C l} & =-k_{c} \times\left(\psi_{H C L}-\psi_{B, H C l}\right) \times A_{W} \\
& =-A_{W} \min \left\{\left(\left(D_{W, H C l}-D_{W, H C l}^{\text {old }}\right) / \Delta t+k_{r} D_{W, H C l}\right), \quad \alpha \times \dot{f}_{H C l}\right\}
\end{aligned}
$$

In this equation, a parameter $\alpha$ is introduced when the mass flux $\dot{f}_{H C l}$ is the controller of the deposition rate. This parameter reflects the time for the $\mathrm{HCl}$ to reach equilibrium between the gas phase and the solid surface. As there is no experimental quantification of this parameter, in the simulations presented in this study it is assumed that the parameter takes a value of 1.0. It is possible to obtain an optimal value of $\alpha$ for a wall material via performing a series of simulations with varied $\alpha$ to reliable experiments.

Since $\mathrm{HCl}$ gas is highly water-soluble, it could attach to small water droplets [BM04]. Another future work is to extend the present $\mathrm{HCl}$ deposition model involving the absorption by water mist [Gr03].

Finally, it appears that the approach to the decay of $\mathrm{HCl}$ for zone models used by Galloway and Hirschler [GH89] and the modified version for field models in this study could be applied for other fire gases such as $\mathrm{HBr}, \mathrm{HF}, \mathrm{HCN}$ etc. It is believed that the transport of $\mathrm{HBr}$ should behave like $\mathrm{HCl}$ [BM04]. A similar model for $\mathrm{HBr}$ or $\mathrm{HCN}$ can be developed if the necessary experimental data are available. For species such as HCN with weak absorption dependence on humidity [BM04], the calculation of the partition (gas/wall equilibrium) coefficient in equation (8.2.3) might be much simple. 


\section{REFERENCES}

[AF00] Alvares A. and Fernandez_Pello A.C., Fire initiation and spread in overload communication system cable trays, Experimental thermal and fluid science, 21, (2000), 51-57.

[AM03] Andersson B., Markert F. And Holmsteds G., Combustion products generated by hetero-organic fuels on four different fire test scales, in 'Combustion products from fires, influence from ventilation conditions', Report 1029 by Andersson B., Lund University, 2003.

[Ba92] Babrauskas V., From Bunsen burner to heat release rate colorimeter, in 'Heat release in fires', Edited by Babrauskas V. and Grayson S.J., Published by E \& FN Spon, 1992.

[Ba95] Barbauskas V., The generation of $\mathrm{CO}$ in Bench-scale fire tests and the prediction for real-scale fires, Fire and Materials, Vol. 19, 1995, pp. 205213.

[BB96] Brizuela E. A. and Bilger R. W., On the eddy break-up coefficient, Combustion and Flame, Vol. 104, 1996, pp. 208-212.

[Be86a] Beyler C. L., Major species production by diffusion flames in a twolayer compartment fore environment. Fire Safety J. 10(1):47-56, 1986.

[Be86b] Beyler C. L., Major species production by solid fuels in a two-layer compartment fore environment, fire safety science-proceedings of first international symposium, (Eds, Grant, C. E. and Pagni, P. J.), Hemisphere, Washington, D.C. 431-440, 1986.

[BG98] Babrauskas V., Gann R. G., Levin B. C., Paabo M., Harris R. H., Peacock R. G. and Yusa S., A methodology for obtaining and using toxic potency data for fire hazard analysis, Fire Safety Journal, Vol. 31, 1998, pp. 345-358.

[Bi76] Bilger R. W., The structure of diffusion flames, Combustion Science and Technology, Vol. 13, 1976, pp. 155-170.

[Bi80] Bilger R. W., Turbulent flow with non-premixed reactants. In: Libby PA, Williams FA, editors. Turbulent reacting flow, Topics in Applied Physics. New York: Springer-Verlag, 1980. pp. 65-114.

[Bi89] Bilger R. W., Turbulent diffusion flames, Ann. Rev. Fluid Mech., 1989, 21: 101-135.

[Bi94] Bilger R. W., Computational field models in fire research and engineering, Fire Safety Science: Proceedings of the fourth international symposium/Editor, Takashi, Kashiwagi, 1994, pp. 95-109. 
[BJ94] Bryner N. P., Jonsson E. L. and Pitts W. M., Carbon monoxide production in compartment fires-reduced scale enclosure test facility, NIST internal report, NISTIR 5568, December 1994.

[BK94] Buriko Y.Y., Kuznetsov V.R., Volkov D.V., Zaitsev S.A. and Uryvsky A.F., A test of a flamelet model for turbulent nonpremixed combustion, Combustion and Flame, Vol. 96, pp104-120, 1994.

[BK99] Brink A., Kilpinen P., Hupa M. and Kjaldman L., Study of alternative descriptions of methane oxidation for CFD modelling of turbulent combustors, Combust. Sci. and Tech., Vol. 141, 1999, PP. 59-81.

[BM00] Brink A., Mueller C., Kilpinen P. and Hupa M., Possibilities and limitation of the Eddy Break-up model, Combustion and Flame, Vol. 123, 2000, PP. 275-279.

[BM04] Butler K. M. and Mulholland G. W., Generation and transport of smoke components, Fire Technology, Vol. 40, 2004, pp. 149-176.

[Bo01] Experimental data, provided by Borealis, 2001.

[Bo77] Boussinesq J., Theorie de L'Ecoulement Toubillant, Mem. Acad. Sci., $23(46), 1877$.

[Br97] Brushlinsky N., Formulating statistics for a safer planet, Fire International, October/November, 1997, p.26.

[Br04] Brink A., Private communication, 2004.

[BS03] Tuber furnace method for the determination of toxic products yield in fire effluent, British Standard, BS7900, 2003.

[BW87] Burn A. D. and Wilkes N. S., "A finite-difference method for the computation of fluid flow in complex three dimensional geometry", U.K. Atomic Energy Authrity Harwell Report, AERE-R 12342, 1987.

[CFX97] CFX-4.1/4.2 flow solver use guide, AEA Technology, October, 1997.

[CH98] Chow W. K., On safety systems for under ground car parks, Tunnelling and Underground Space Technology, Vol. 13, pp. 281-287.

[Co94] Cooper L. Y., The generation rate and distribution of products of combustion in two-layer fire environments: a model and application, Fire Safety Journal, Vol. 23, 1994, pp. 245-270.

[Co95] Cox G., Compartment Fire Modelling, in: Combustion of Fundamentals of Fire. Edited by Cox G., Acdemic Press, 1995.

[Co95b] Cox G., Basic consideration, in: Combustion of Fundamentals of Fire. 
Edited by Cox G., Acdemic Press, 1995.

[Co00] Conachy J., Christmas night fire kills 311 in central China, World Socialist Web Site, www.wsws.org.

[CR93] Chui E.H. and Raithby G.D., Computation of radiant heat transfer on a nonorthogonal mesh using the finite-volume method, Numerical Heat Transfer, Part B, Vol. 23, pp. 269-288, 1993.

[CV98] Climent-Llorca M. A., Viqueira-Perez E., Ver-Almenar G. and LopezAtalaya M. M., Chloride contamination of concrete by interaction with PVC combustion gases, Cement and Concrete Research, Vol. 28, No. 2, pp. 209-219, 1998.

[Do94] Dobbins R. A., Comparison of a fractal smoke optics model with light extinction measurements, Atmospheric environment, Vol. 28, No. 5, 1994, pp. 889-897.

[Dr85] Drysdale D., An introduction to fire dynamics, John Wiley \& Sons Ltd., 1985.

[EJ02] Ewer J., Jia F., Grandison A., Galea E. R., Knight B. and Patel M., User guide and technical manual, SMARTFIRE V3.0, 2002.

[EG99a] Ewer J., Galea E. R., Patel M., and Knight B., The Development and Application of Group Solvers in the SMARTFIRE Fire Field Model. Proceedings Interflam 99, Edinburgh, UK, pp 939 - 950, 1999.

[EG99b] Ewer J., Galea E. R., Patel M., Taylor, S., Knight B., Petridis M., SMARTFIRE: An Intelligent CFD Based Fire Model, Journal of Fire Protection Engineering, 10 (1), pp 13-27, 1999.

[FB01] Floyd J. E., Baum H. R. and McGrattan K. B., A mixture fraction combustion model for fire simulation using CFD, Reprinted from the International Conference on Engineered Fire Protection Design Proceedings, 2001, Building and Fire Research Laboratory, National Institute of Standard and Technology, USA.

[FP04] Fardell P. J., Purser D. A., Purser J. A. Marshall N. R. and Clark P., Fires reduced oxygen conditions, $10^{\text {th }}$ International Interflame, 2004, volume 1 , pp. 129-141.

[FR94] Fardell P.J., Rowley J.A., Vollam S.J. and Bridgeman B.B., Oxygen staved (vitiated) fires: a full scale test rig, Fire research Station, BRE, Report, CR 14/94, 1994. (With permission D. Purser BRE ).

[FSEG] www: http://fseg.gre.ac.uk/

[Ga89] Galea E. R., On the field modelling approach to the simulation of enclosure fires, J. Fire Protection Engng 1, 11-22, 1989. 
[Ga97] Galea E. R., The use of mathematical modelling in fire safety engineering, paper No. 97/IM/25, CMS Press, University of Greenwich.

[Ga98] Galea E. R. "A General Approach to Validating Evacuation models with an application to EXODUS", J of Applied Fire Science, 1998, vol. 16, Nov/Dec, pp. 414-436.

[GB94] Gann R. J., Babrauskas, V. And Peacok, R.D., Fire conditions for smoke toxicity measurements, Fire and Materials, Vol. 18, 1994, pp. 193-199.

[GB96] Galea E.R, Berhance D. and ., Hoffmann N., CFD analysis of fire plumes emerging from windows with external protrusions in high-rise buildings, Proceedings Interflames'96, Compiled by C. Franks and S. Grayson, ISBN 09516320 94, pp. 835-839.

[GG00] Galea E. R., Gwynne S., Lawrence P. J., Filippdis L. and Blacksnields D, Building Exodus V3.0, User Guide and Technical Manual, the University of Greenwich, 2000.

[GG01] Gwynne S., Galea E.,R., Lawrence P.,J. and Filippidis L., Modelling Occupant Interaction with Fire conditions Using The buildingEXODUS Evacuation Model, Fire Safety Journal, 36, pp327-357, Elsevier, 2001.

[GG03] Galea E. R., Gwynne S., Lawrence P., Blackshields D., Ewer J., Wang Z., Hurst N. and Mawhinney N., Fire Safety Engineering and Ship Design Using Advanced Fire and Evacuation Simulation.. $1^{\text {st }}$ International Conference - Fire on Ships: Prevention, Detection and Control on Ships and Offshore Structures, 11-12/3/03.

[GG03b] Galea E. R., Gwynne S., Lawrence P., Blackshields D., Ewer J., Wang Z., Hurst N. and Mawhinney N, The Application of Fire and Evacuation Simulation in Ship Design. COMPIT 2003, 2nd International EuroConference on Computer and IT Applications in the Maritime Industries, Hamburg, pp55-69, 2003.

[GG04] Galea E.R., Gwynne S., Lawrence P., and Wang Z., Introducing occupant sensitivity to irritant fire gases into evacuation analysis, Proceedings Interflames'2004, Volume 1, pp. 353-364.

[GH89] Galloway F. M. and Hirschler M.M., A model for the spontaneous removal of airborn hydrogen chloride by common surface, Fire Safety Journal, Vol. 14, 1989, pp. 251-268.

[GH90] Galloway F. M. and Hirschler M. M., Transport and decay of hydrogen chloride: use of a model to predict hydrogen chloride concentrations in fires involving a room-corridor-room arrangement, Fire Safety Journal, Vol. 16, 1990, pp. 33-52.

[GH91a] Galloway F. M. and Hirschler M. M., Experiments for hydrogen chloride 
transport and decay in a simulated heating, ventilating and air conditioning system and comparison of the results with predictions from a theoretical model, Journal of Fire Sciences, Vol. 9, 1991, pp. 259-275.

[GH91b] Galloway F. M., Hirschler M. M and Smith G. F., Surface parameters from small-scale experiments used for measuring $\mathrm{HCl}$ transport and decay in fire atmosphere, Fire and Materials, Vol. 15, 1991, pp. 181189.

[GH92] Galloway F.M. and Hirschler M.M., The use of a model for hydrogen chloride transport and decay to predict airborne hydrogen chloride concentrations in a full-scale room-corridor scenario, Fire Safety Journal, 1992, pp. 73-101.

[GK99] Galea E.R., Knight B., Patel M., Ewer J., Petridis M., and Taylor S.[1999], "SMARTFIRE V2.01 build 365, User Guide and Technical Manual”, SMARTFIRE CD.

[GL03] Galea E. R., Lawrence P, Gwynne S., Filippidis L., Blackshields D., Sharp G., Hurst N., Wang Z., and Ewer J. Simulating Ship evacuation under fire conditions. Proc $2^{\text {nd }}$ Int Pedestrian and Evacuation Dynamics Conference, Ed: E. R. Galea, CMS Press, Greenwich, UK, ISBN 1904521088, pp. 159-172, 2003.

[GL04] Galea E. R., Lawrence P, Gwynne S., Filippidis L., Blackshields D., Sharp G., Hurst N., Wang Z., and Ewer J. the Simulation of Ship evacuation under fire conditions, Proc Fire and Safety at Sea, Rocarm Pty Ltd, Melbourne Australia 17-19 March 2004, day2, session 1, pp $1 / 14-14 / 14,2004$.

[GM91] Galea E. R. and Markatos N.C., The mathematical modelling and computer simulation of fire development in aircraft, Int. J. Heat Mass Transfer, Vol. 34, pp. 181-197.

[Go92] Gottuk D. T., Generation of carbon monoxide in compartment fires, NIST-GCR-92-619, 1992.

[GP97] Galea E. R. and Patel M. K., Principles and practice of fire modelling, Paper No. 97/IM/18, Volume 1, CMS Press, University of Greenwich.

[GR95] Gottuk D. T. and Roby R. J., Effect of combustion conditions on species production, in 'the SFPE handbook of fire protection engineering', $2^{\text {nd }}$ edition, published by the National fire protection, 2.64-2.84, 1995.

[Gr03] Grandison A.J., Improving the redulatory acceptance and numerical performance and numerical performance of CFD based fire-modelling software, $\mathrm{PhD}$ thesis, University of Greenwich, 2003.

[GRB95] Gottuk D. T., Roby R.J. and Beyler C. L., The role of temperature on carbon monoxide production in compartment fires, Fire Safety Journal, 
Vol. 23, 1995, pp. 315-331.

[HA82] Harland W. A. and Anderson R. A., Cause of fire death, proceedings smoke and toxic gases from burning plastics, pp. 15/1-15/19, London, 1982.

[Ha96] Hartzell G. E., Overview of combustion toxicology, Toxicology, Vol. 115, 1996, pp. 7-23.

[HC97] Hadjisophocleous G. and Cao S., Numerical simulations of aircraft cabin fire suppression, AGARD conference proceedings, AGARD-CP-587, 1997, pp. 5.1-5.12.

[HD81] Hautman D. J., Dryer F. L., Schug K. P. and Glassman I., A multi-step overall kinetic mechanism for the oxidation, Combustion Science and Technology, Vol. 25, 1981, pp. 219-235.

[HG88] Hoffman N., Galea E. R. and Markatos N. C., "A computer simulation of fire sprinkler inaction: a two-phase phenomena", $12^{\text {th }}$ MACS World Congress.

[HH89] Harwood B. and Hall J. R., What kills in fires: smoke inhalation or burns, Fire Journal, Vol. 83, 1989, pp. 29-34.

[HH99] Hayashi Y., Hasemi Y. and Ptchelintsev A., Smoke layer formation by fires in forced-ventilation enclosure, Sixth fire safety science-proceeding of the international symposium, 1999, 805-816.

[HH03] Hermansson A., Hjertberg T. and Sultan B-A, The flame retardant mechanism of polyolefins modified with chalk and silicone elastomer, Fire and Materials, Vol. 27, pp 51-70, 2003.

[HK85] Hagen E. and Haksever A. Contribution for the investigation of natural fires in large compartments. IAFSS $1^{\text {st }}$ Int Symp, Oct 1985, Gaithersburg USA, Hemisphere Pub Corp NY. pp 149-158.

[HK98] Hostikka S and Keski-Rahkonen O., Results of CIB W14 Round Robin for Code Assessment Scenario B. Draft 31/08/98, VTT Technical Research Centre of Finland.

[HL05] Hull R. and Lebek K., Summary of data obtained by burning 3 Power cables at $800^{\circ} \mathrm{C}$ under different phi ratios, unpublished data, the University of Bolton, 2005.

[HM88] Hoffman N. and Markatos N.C., Thermal radiation effects on fire enclosures, Appl. Math. Modelling, Vol. 12, pp129-140, 1988.

[HM99] Hyde S. M. and Moss J. B., Field modelling of carbon monoxide production in fires,_Proceedings Interflam 99, Edinburgh, UK, pp 951962. 
[HP99] Hull T. R., Price D., Carman J. M. and Purser D., Studies of carbon/oxygen chemistry under different fire conditions, Proceedings Interflam 99, Edinburgh, UK, pp 189-199.

[HP04] Fardell P. J., Purser D. A., Purser J. A. Marshall N. R. and Clark P., Fires reduced oxygen conditions, $10^{\text {th }}$ International Interflame, 2004, volume 1, pp. 129-141.

[HQ02] Hull T. R., Quinn R. E., Areri I. G. and Purser D., Combustion toxicity of fire retarded EVA, Polymer Degradation and Stability, 77 (2002), 235-242.

[HT78] Hubbard G. L. and Tien C. L., Infrared mean absorption coefficients of luminous flames and smoke, J. Heat Transfer, 1978, vol. 100, pp. 235239.

[Hy00] Hyde S.M., Field modelling of carbon monoxide production in vitiated compartment fires, $\mathrm{PhD}$ thesis, Cranfield University, 2000.

[IP99] Isaksson S., Persson B. and Yuovinen H., Simulation of smoke detection on a room with a perforated suspended ceiling, $8^{\text {th }}$ International Interflam, 1999, volume 1, pp. 441-452.

[JF00] Jones W. W., Forney G. P., Peacock R.D. and Reneke P. A., A Technical Reference for CFAST: An Engineering Tool for Estimating Fire and Smoke Transport, National Institute of Standards and Technology, Technical Note 1431, 2000.

[JG95] Jia F., Galea E. R., Patel M. and N. Hoffmann, Combustion models of turbulent diffusion flames, Paper No. 95/IM/09, CMS Press, University of Greenwich, 1995.

[JG96] Jia F., Galea E. R. and Patel M. K., The prediction of fire propagation in enclosure fires, Paper No. 96/IM/10, CMS press, University of Greenwich, 1996.

[JG97] Jia F., Galea E. R. and Patel M. K., The prediction of fire propagation in enclosure fires, Fire Safety Science- Proc $5^{\text {th }}$ Int Symp, 1997, pp.345354.

[JG99a] Jia F., Galea E. R. and Patel M. K Simulating "FLASHOVER" and "BACKDRAFT" type events using fire field models - a fist approximation, Journal of Fire Protection Egineering, Vol. 9, 1999, No. 4, pp.1-17.

[JG99b] Jia F., Galea E. R. and Patel M. K., The numerical simulation of the noncharring pyrolisis process and fire development within a compartment, Applied Mathematical Modelling, Vol. 23, 1999, pp.587607. 
[JG99c] Jia F., Galea E. R. and Patel M. K., The Numerical Simulation of Fire Spread within a Compartment using an Integrated Gas and Solid Phase Combustion Model, J. of Applied Fire Sciences, vol. 8, pp. 291-316, 1999.

[Ji99] Jia F., The simulation of fire growth and spread within enclosures using an integrated CFD fire spread model, PhD thesis, 1999, University of Greenwich.

[JL88] Jones W. P. and Lindstedt R. P., Global reaction schemes for hydrocarbon combustion, Combustion and Flame, Vol. 73, 233-249.

[JP06a] Jia F., Patel M. K., Galea E. R., Grandison, A. and Ewer, J., CFD fire simulation of the Swissair flight 111 in-flight fire Part I: Prediction of the pre-fire air flow within the cockpit and surrounding areas, The Aeronautical Journal of the Royal Aeronautical Society, pp.44, January 2006.

[JP06b] Jia F., Patel M.K., Galea E.R., Grandison A. and Ewer J., CFD Fire Simulation of the Swissair Flight 111 In-flight Fire - Part II: Fire Spread within the Simulated Area, The Aeronautical Journal of the Royal Aeronautical Society, pp. 303, May 2006.

[JR94] Jackson M. A. and Robin I., Gas sensing for fire detection: measurements of $\mathrm{CO}, \mathrm{CO}_{2}, \mathrm{H}_{2}, \mathrm{O}_{2}$ and smoke density in European standard fire tests, Fire Safety Journal, Vol. 22, PP. 181-205.

[JT92] Janssens M. and Tran H. C., Data reduction of rooms tests for zone model validation, Journal of Fire Science, Vol. 10, 528-555, 1992.

[Ka92] Karlsson B., "Modelling fire growth on combustible lining materials in enclosures", 992, Report TVBB-1009, Lund University, Department of Fire Safety Engineering, Lund, Sweden, 1992.

[KB97] Keramida E. and Boudouvis A., Radiation model implementation, Project draft, National Technical University of Athens, 1997.

[KB99] Klimenko A. Y. and Bilger R. W., Conditional moment closure for turbulent combustion, Progress in Energy and Combustion Science, Vol. 25,1999 , pp. 595-687.

[KC85] Kumar S. and Cox G., "Mathematical modelling of fire in tunnels", $5^{\text {th }}$ Int. Conf. On Aerodynamics and Ventilation of Vehicle Tunnels, 1985, p. 61 .

[KC99] Kheireddine A. S., Chaturvedi S. K. and Mohieldin T. O., Computing of flame base height of a turbulent diffusion flame resulting from a methane jet, JSME International Journal, Vol. 42, No. 1, pp. 78-90. 
[KG91] Kumar S., Gupta A .K. and Cox G., "Effects of Thermal Radiation on the Fluid Dynamics of Compartment Fires", Fire Safety Science - Proc. of the Third Intl. Symp., 1991, pp 345-354.

[KG94] Kerrison L., Galea E. R., Hoffmann N. and Patel M. K., "A comparison of FLOW3D based fire field model with experimental room fire data", Fire Safety J., Vol. 23, 1994, pp. 387-411.

[KH97] Keski-Rahkonen O. and Hostikka S, Design and simulation reports of CIB W14 Round Robin for code assessment Scenario B. VTT Building Technology Internal Report: RTE119-IR-2/1997.4 + 272p.

[KK91] Kennedy IM, Kollmann W. Predictions of soot in laminar diffusion flames. AIAA J 1991, 29:1452-7.

[KM94] Kerrison L., Mawhinney N., Galea E. R., Hoffmann, N. and Patel M. K., "A comparison of two fire models with experimental room fire data", Fire Safety Science-Proc $4^{\text {th }}$ Int Symp, 1994,pp. 161-172.

[KQ00] Karlsson B. and Quintiere J. G., Enclosure fire dynamics, CRC Press, 2000.

[Ku83] Kumar S., Mathematical modelling of natural convection in fire- a state of art review of the field modelling of variable density turbulent flow, Fire and Materials, Vol. 7(1), pp 1-24, 1983.

[Ku86] Kuo, K. K., Principles of combustion, John Wiley \& Sons, 1986.

[La06] Lattimer B. Y., Experimental data files, private communication, 2006.

[LB94] Luo M. and Beck V., The fire environment in a multi-room buildingcomparison of predicted and experimental results, Fire safety Journal, Vol. 23, 1994, pp. 413-438.

[LB96a] Luo M. and Beck V., Stoiometric combustion model with oxygen threshold improved predictions for fire simulation using CFD model, Fire Safety Science-proceedings of the fifth international symposium, 1996, pp. 559-570.

[LB96b] Luo M. and Beck V., A study of non-flashover and flashover fires in a full-scale multi-room building, Fire safety Journal, Vol. 26, 1996, pp. 191-219.

[LBM96] Lonnermark A., Blomqvist P., Mansson M. and Persson H., TOXFIREfire characteristic and smoke gas analysis in under-ventilation largescale combustion experiments, SP report, 1996:45, ISBN 91-7848-6505.

[LBM96b] Lonnermark A., Blomqvist P., Mansson M. and Persson H., TOXFIRE- 
fire characteristic and smoke gas analysis in under-ventilation largescale combustion experiments: Storage configuration tests, SP report, 1996:46, ISBN 91-7848-651-3.

[LE94] Lattimer Brain Y., Ewens David S., Vandsburger Uri and Roby Richard J., Transport and oxidation of compartment fire exhaust gases in an adjacent corridor, J. of Fire Prot. Engr., 6(4), 1994, pp. 163-181.

[Le98] Lewis M. J., "Field modelling of flame spread for enclosure fires", $\mathrm{PhD}$ thesis, Cranfield University, 1998.

[Li83] Liew K. S., Flamelet model of turbulent non-premixed combustion, PhD thesis, 1983, the University of Highfield.

[LM97] Lewis M. J., Moss MB and Rubini PA, CFD modelling of combustion and heat transfer in compartment fires, Fire Safety Science, Proc. of the $5^{\text {th }}$ Int. Symp, 1997, pp. 463-474.

[LS74] Launder B.E. and Spalding D.B., "The numerical computation of turbulent flows", Comp. Meth. in Applied Mech. and Engineering, 3, 269-289, (1974).

[LS81] Lockwood F. C. and Shah, N. G., a new radiation solution method for incorporation in general combustion prediction procedures, $18^{\text {th }} \mathrm{Sym}$. (Int.) on Combustion, The Combustion Institute, 1981, pp. 1405-1414.

[LV97] Lattimer B. Y. and Vandsburger U., The transport of high concentrations of carbon monoxide to locations remote from the burning compartment, NIST-GCR-97-713.

[LV98] Lattimer B. Y., Vandsburger U. and Roby R. J., Carbon monoxide levels in structure fires: effect of wood in the upper layer of a post-flashover compartment fire, Fire Technology, Vol. 34, No. 4, 1998, 325-355.

[LV05] Lattimer B.Y., Vandsburger U. and Roby Richard, Species transport from post-flashover fires, Fire Technology, Vol. 41, 2005, pp. 235-254.

[LW93] Liñán A. and Williams F. A., Fundamental aspects of combustion, Oxford University Press, 1993.

[MC01] Masaík I., Charvátová V. and Dvoák O., Influence of variable temperature and air flow on the release of main toxicants during burning of plastics, $9^{\text {th }}$ International Interflam, 2001, PP. 1183-1188.

[Mc05] McGrattan K., FDS/Smokeview examples. 1 March 2005. (FDS_SV_Survey_2005_7.pdf, http://fire.nist.gov/fds).

[MH77] Magnussen B. F. and Hjertager B. H., On mathematical modelling of turbulent combustion with special emphasis on soot formation and combustion, $16^{\text {th }}$ Symp. (Int.) on Combustion, the Combustion Institute, 
1977, pp. 719-729.

[MH78] Magnussen B. F., Hjertager B. H., Olsen, J. G. and Bhaduri, D., Effect of turbulent structure and local concentration on soot formation and combustion in $\mathrm{C}_{2} \mathrm{H}_{2}$ diffusion flames, $17^{\text {th }}$ Symp. (Int.) on Combustion, the Combustion Institute, 1978, pp. 1383-1393.

[MJ06] Mahalingam A., Jia, F., Patel M.K. and Galea, E.R., Modelling generation and transport of toxic combustion products during fire using Bench-scale test data, Workshop on cable fires on building evacuation, 17, October, 2006, BSI, UK.

[MJ91] Mulholland G., Janssens M., Yusa S., Twilley W. and Babrauskas, The effect of oxygen concentration on $\mathrm{CO}$ and smoke produced by flames, Fire Safety Science - Proc. of the Third Intl. Symp., 1991, pp 585-594.

[MK99] Miles S. D., Kumar S. and Cox G., Comparison of 'blind predictions' of a CFD model with experimental data, Fire Safety Science, Proc. of the $6^{\text {th }}$ Int. Symp, 1999, pp. 543-554.

[MP79] Martin K. G. and Powell D. A., Toxic gas and smoke assessment studies on Vinyl floor covering with fire propagation test, Fire and materials, Vol. 3, No. 3, 1979, pp. 132-139.

[Mu95] Mulholland G. W., Smoke production and properties, in 'the SFPE handbook of fire protection engineering', $2^{\text {nd }}$ edition, published by the National fire protection, 2.217-2.227, 1995.

[MT94] Milov D. and Tewarson A., Comment on chemical efficiency of combustion as reported by Tewarson et al. Author's reply. Combustion and Flame, Vol. 98, pp. 175-178, 1994.

[MY93] Morikawa T., Yanai E., Okada T. and Sato K., Toxicity of the atmosphere in an upstairs room caused by inflow of fire effluent gases rising from a burn room, J. of Fire Sciences, Vol. 11, 1993, pp. 195-209.

[Na00] Nam S., Numerical simulation of smoke movement in clean room environments, Fire Safety J., Vol. 34, 2000, pp.169-189.

[NM96] Novozhilov V., Moghtaderi B., Fletcher D. F. and Kent J. H., Computational fluid dynamics modelling of wood combustion, Fire Safety Journal, Vol. 27, 1996, pp. 69-84.

[No01] Novozhilov V., Computational fluid dynamics modelling of compartment fires, Progress in Energy and Combustion Science, Vol. 27, 2001, pp. 611-666.

[Oh95] Ohlemiller T. J., Smoldering combustion, SFPE Handbook of Fire Protection Engineering, 2nd Edition, Chapter 11, Section 2, pp. 2/171179. 
[Op95] Opstad, K. "Modelling of thermal flame spread on solid surfaces in a large-scale fires", MTF-Report, 1995:114(D), Department of Applied Mechanics, Thermo- and Fluid Dynamics, The Norwegian Institute of Technology, University of Trondheim.

[Pa80] Pantakar S. V., Numerical Heat Transfer and Fluid Flow, Intertext Books, McGraw Hill, New York, 1980.

[PB95] Pitts W. M., Bryner N. P. and Jonsson E. L., Combustion product formation in under and over ventilated full-scale enclosure fires, Proceeding combustion fundamentals and applications, Combustion Institute/Central and Western state, Mexican National Section and American Flame Research Committee, 1995.

[Pe06] Persson H., LABYRINTH project-Full scale tests in a corridor arrangement, Brs 6119, SP Swedish National Testing and Research Institute, 2006.

http://www.sp.se/fire/Sv/PDF_reports/Final_report_BRs6119.pdf.

[Pe84] Peters N., Lamina diffusion flamelet models in non-premixed turbulent combustion, Prog. Energy Combust. Sci., 1984, Vol.10, 319-339.

[Pe86] Peters N., Lamina flamelet concept in turbulent combustion, Twenty-fist Symposium (International) on combustion, the Combustion Institute, 1986, pp. 1231-1250.

[PF94] Purser D. A., Fardell P. J., Rowley J., Vollam S., Bridgeman B. and Ness E. M., An improved tube furnace method for the generation and measurements of toxic combustion products under a wide range of fire conditions, Flame Retardants '94 Conference, London, 1994, Proceedings pp. 263-274.

[Pi94a] Pitts W. M., The global equivalence ratio concept and the prediction of carbon monoxide formation in enclosure fires, National institute of standards and technology, Gaithersburg, MD 20899, 1994.

[Pi94b] Pitts W. M., Application of thermodynamic and detailed chemical kinetic modelling to understanding combustion products generation in enclosure fires, Fire safety Journal, Volume 23, 271-303, 1994.

[Pi95] Pitts W. M., The global equivalence ratio concept and the formation mechnisms of carbon monoxide formation in enclosure fires", Prog. Energ. Combust. Sci,, 21, 1995, pp. 197-237.

[Pi97] Pitts W. M., An algorithm for estimating carbon monoxide formation in enclosure fires, Fire safety science--proceeding of the fifth international symposium, 535-546, 1997.

[Pi01] Pitts W. M., Toxic yield, National institute of standards and technology, 
Gaithersburg, MD 20899, 2001.

[PJ94] Pitts W. M., Johnsson E. L. and Bryner N. P., Carbon monoxide formation in fires by high-temperature anaerobic wood pyrolysis, Twenty-fifth symposium (international) on combustion, 1455-1462, The combustion Institute, Pittsburgh, PA, 1994.

[PR99] Purser D. A., Rowley J. A., Fardell P. J. and Bensilum M., Fully enclosed design fires hazard assessment in relation to yields of carbon monoxide and hydrogen cyanide, Interflam'99, pp. 1163-1169.

[PS72] Patank S. V. and Spalding, D. B., A calculation procedure for heat, mass and momentum transfer in three-dimensional parabolic flows, International Journal of Heat and Mass transfer, 15, 1787-1806, 1972.

[PS99] Pope C. J., Shandross R. A. and Howard J. B., Variation of equivalence ratio and element ratio with distance from burner in premixed onedimensional flames, Combustion and flame, Vol. 116, 605-614.

[Pu95] Purser D. A., Toxicity assessment of combustion products, in 'the SFPE handbook of fire protection engineering', $2^{\text {nd }}$ edition, published by the National fire protection, 2.85-2.146, 1995.

[Pu00] Purser D. A., Toxic product yields and hazard assessment for fully enclosed design fires, Polymer International, Vol. 49, 2000, pp. 12321255 .

[Pu02] Purser D.A., Toxicity Assessment Of Combustion Products, The SFPE, Handbook Of Fire Protection Engineering ( $3^{\text {rd }}$ Edition), Ed: Dilenno, P.J., Drysdale, D., Beyler, C.L., Walton, W.D., Custer, R.L.P., Hall, J.R., and Watts, J.M.W., National Fire Protection Association,Quincy,Ma, pp (2-83)-(2-171), 2002.

[Pu03] Purser, D. A., Private communication, 2003.

[Qu95] Quintiere J. G., Compartment fire modelling, in the SFPE handbook of Fire Safety Protection Engineering, $2^{\text {nd }}$ edition, 1995, pp. 3.125-3.133.

[RI00] Experimental data of wood cribs fires, Rockwool International, Danmark, 2000.

[RS02] Robinson J. E. \& Samson F., In search of Flashover, IWMA, Proc of Conf "Fire Performance of Cables", Dusseldorf, p 32-35, Oct 2002.

[RS04] Robinson J. E., Samson F. and Sultan B., The development of toxic gas yield in a format suitable for evacuation modelling, Interflam'2004, pp. 781-786.

[Sa86] Sarofim A. F., Radiative heat transfer in combustion: friend or foe, $21^{\text {st }}$ Sym. (Int.) on Combustion, The Combustion Institute, 1986, pp. 1-23. 
[SE98] Sultan B., Ericsson K., Hirvensalo M., Hjertberg T. and Hänninen M., Novel halogen free flame retardant polyolefin intended for internal wiring - properties and flame retardant mechanism, $47^{\text {th }}$ International Wire \& Cable Symposium, Philadelpha, USA, Nov. 1998.

[SF90] Sivathanu Y. R. and Faeth G. M, generalized state relationship for scalar properties in nonpremixed hydrocarbon/air flames, Combustion and flame, Vol. 82, 1990, 211-230.

[SFPE95] The SFPE Handbook of Fire Protection Engineering, $2^{\text {nd }}$ Edition, 1995.

[Si95] Simmons R.F., Fire chemistry, in: Combustion Fundamentals of Fire, Edited by Cox G., Acdemic Press, 1995.

[Sm63] Smagorinsky, J., Mon. Weath. Rev., Vol. 91, Part 3, pp99.

[Smar99] Smartfire Verification and Validation Report. Software Version 2.01, Report Version 1.01 Fire Safety Engineering Group, University of Greenwich. Revision Date 25/05/99

[Sp71] Spalding D. B., Mixing and chemical reaction in steady confined turbulent flames, $13^{\text {th }}$ Symp. (Int.) on Combustion, the Combustion Institute, 1971.

[Sp81] Spalding D. B., A general purpose computer program for multidimensional one- and two-phase flow, in Mathematics and Computers in Simulations, North Holland (IMACS), Vol. XXIII, 1981, p. 267.

[SQ82] Steckler K. D., Quintiere J. G. and Rinkinen W. J., "Flow Induced By Fire in a Compartment", NBSIR 82-2520, National Bureau of Standards, Washington, 1982.

[St95] Stroup D. W., Using field modelling to simulate enclosure fires, in The SFPE Handbook of Fire Protection Engineering, $2^{\text {nd }}$ edition, 1995, pp. 3152-3-159.

[ST96] Sekizawa, A., Takemoto, A., Kozeki, D., Yanai E. and Suzuki K., Experimental study on fire hazard of residential fires before and after sprinkler activation, Thirteenth meeting of the UJNR panel on fire research and safety, March 13-20, 1996, pp. 145-157.

[SW92] Simcox S., Wilkes, N. S. and Jones, I. P., Computer simulation of the flows of hot gases from the fire at King's Cross underground station, Fire Safety Journal, Vol. 18, 1992, pp. 49-73.

[TB04] Tuovinena H., Blomqvista P. and Saricb F., Modelling of hydrogen cyanide formation in room fires, Fire Safety Journal, Vol. 39, 2004, pp. 735-755. 
[TC94] Tewarson A., Chu F. and Jiang F. H., Combustion of halogenated polymers, Proceedings of the fourth international symposium on fire safety science, Ottawa, 1994, 563-574.

[Te84] Tewarson A., Fully enveloped enclosure fires of wood cribs, Twentieth symposium (international) on combustion, the Combustion Institute, Pittsburgh, PA, 1555 (1984)

[Te95] Tewarson A., generation of heat and chemical compounds in fires, in 'the SFPE handbook of fire protection engineering', $2^{\text {nd }}$ edition, published by the National fire protection, 3.53-3.124, 1995.

[Te96] Tewarson A., Ventilation effect on combustion products, Toxicology, 115(1996), 145-146.

[TH04] Tuovinen H., Blomqvist P. and Saric F., Modelling of hydrogen cyanide formation in room fires, Fire Safty Journal, Vol. 39, pp.737-755, 2004.

[TL95] Tien C. L., Lee K. Y. and Stretton A. J., Radiation heat transfer, in the SFPE handbook of Fire Safety Protection Engineering, $2^{\text {nd }}$ edition, 1995, pp. 1.65-1.79.

[TP97] Taylor S., Petridis M., Knight B., Ewer J., Galea E. R. and Patel M., SMARTFIRE: An Integrated Computational Fluid Dynamics code and Expert System for Fire Field Modelling. Fire Safety Science Proceedings of the Fifth International Symposium, 1997, pp 1285-1296.

[Ts94] Tsuchiya $\mathrm{Y}$., $\mathrm{CO} / \mathrm{CO}_{2}$ ratios in fires, Fire Safety Science - Proceedings of the 4th International Symposium, 1994, pp 515-526.

[Ts95] Tsuchiya Y., Chemical modelling of fire gases, Journal of Fire Sciences, Vol. 13, 1995, pp. 214-223.

[Tu95] Tuovine H., Simulation of combustion and fire-induced flows in enclosures, Report Lutvdg(TVBB-1010), 1995, Department of Fire Safety Engineering, Lund University, Sweden.

[TZ87] Toner S. J., Zukoski, E. E. and Kubota, T., Entrainment, chemistry and structure of fire plumes, National Bureau of standards government contractors report, GCR-87-528 (April, 1987)

[Wa95] Walton D.W., Zone computer fire models for enclosures, in the SFPE handbook of Fire Safety Protection Engineering, $2^{\text {nd }}$ edition, 1995, pp. 3.148-3.151.

[WB00] Wu Y. and Bakar M. Z. A., Control of smoke flow in tunnel fires using longitudinal ventilation systems-a study of the critical velocity, Fire Safety Journal, Vol. 35, 2000, PP. 363-390.

[WD81] Westbrook C. K. and Dryer F. L., Simplified reaction mechanisms for 
the oxidation of hydrocarbon, Combustion science and Technology, 1981, Vol. 27, pp. 31-43.

[WF82] Wooley W. D. and Fardell P. J., Basic aspects of combustion toxicology, Fire Safety Journal, Vol. 5, 1982, pp. 29-48.

[WG00] Wetson L., Gamble J. and Schofield R., Fire statistics, United Kingdom 1999, ISSUE 20/00, 2000, London: Home Office.

[WH00] Wen J. X. and Huang L. Y., CFD modelling of confined jet fires under ventilation-controlled conditions, Fire Safety Journal, Vol. 34, 2000, pp. $1-24$.

[WJ00] Wang Z., Jia, F., Galea, E. R., Patel, M. K. and Ewer, J., "Simulating one of the CIB W14 round robin test cases using the SMARTFIRE fire field models", Paper No. 00/IM/53, CMS Press, University of Greenwich, 2000.

[WJ01a] Wang Z., Jia F., Galea E. R., Patel M. K. and Ewer, J., "Simulating one of the CIB W14 round robin test cases using the SMARTFIRE fire field models", Fire Safety Journal, 36, 2001, pp. 661-677.

[WJ01b] Wang Z., Jia F., Galea E. R., Applying the local equivalence ratio concept to fire field models, $9^{\text {th }}$ International Interflam, 2001, volume 2, pp. 1409-1414.

[WJ02] Wang Z., Jia F., Galea E.R., Predicting toxic gas concentrations resulting from enclosure fires using the local equivalence ratio concept linked to fire field models, Paper No. 02/IM/101, CMS Press, the university of Greenwich, 2002.

[WJ04] Wang Z., Jia F., Galea E.R. and Ewer J., Predicting hydrogen chloride concentrations in fire enclosures using a deposition model linked to fire field models, $10^{\text {th }}$ International Interflam, 2004, volume 1, pp. 665-670.

[WJ06] Wang Z., Jia F., and Galea E.R., Predicting toxic gas concentrations resulting from enclosure fires using local equivalence ratio concept linked to fire field models, Fire and Materials, Vol. 31, No. 1, 2007, pp $27-51$.

[WJ06b] Wang Z., Jia F., Galea E.R., Patel M.K. and Ewer J, Predicting HCl concentrations in fire enclosures using an $\mathrm{HCl}$ decay model coupled to a CFD-based fire field model, Accepted by Fire and Materials, November, DOI: 10.1002/fam.942, 2006.

[WJ06c] Wang Z., Jia F., and Galea E.R., The use of local equivalence ratio in field fire modelling for the prediction of carbon monoxide within fire enclosures, FIRENET workshop on fire in enclosures, Ulster University, 30-31 May, 2006. 
[WM99] Warnatz J., Maas U. and Dbble R.W., Combustion, $2^{\text {nd }}$ Edition, Springer, 1999.

[WV99] Wieczorek C. J., Vandsburger U., Lattimer B. and McKay C., The applicability of correlations between the species formation and the global equivalence ratio in a $1 / 2$-scaled ISO compartment with nongaseous fuel, Proceedings of the sixth international symposium on fire safety science, 1999, pp. 965-976.

[WV04a] Wieczorek C. J., Vandsburger U. and Floyd J., An evaluation of the global equivalence ratio concept for compartment fires: data analysis methods, J. of Fire Protection Engineering, Vol. 14, 2004, pp. 9-31

[WV04b] Wieczorek C. J., Vandsburger U. and Floyd J., Evaluating the global equivalence ratio concept for compartment fires: Part II-Limitation for correlating species yields, J. of Fire Protection Engineering, Vol. 14, 2004, pp. 175-197.

[XH01] Xue H., Ho J.C. and Cheng Y. M., Comparison of different combustion models in enclosure fires simulation, Fire Safety Journal, Vol. 36, 2001, PP. 37-54.

[YH96] Yan,Z. and Holmstedt G, "CFD and experimental studies of room fire growth on wall lining materials", Fire Safety J., Vol. 27, 1996, pp. 201238.

[YC95] Yeoh G. H., Chandrasekaran V. and Leonardi E, Numerical prediction of fire and smoke, AIRAH Journal, Vol. 49, pp. 13-18.

[YT94] Yamada S. and Tanaka T., A model for predicting concentration of CO in building fire, Proceeding of the $4^{\text {th }}$ international symposium on fire safety science, Ottawa, 1994, 539-530

[Yu93] Yu H., A united fire property data correlation scheme for combustibles burning different ventilation/oxygen concentration conditions, Fire Safety Journal, Vol. 20, 1993, pp. 175-181.

[ZT89] Zukoski E. E., Toner S. J., Morehart J. H. and Kubota T., Combustion process in two-layer configurations, in Fire Safety science- proceedings of second International Symposium, (Ed. Wakamastsu, T.), Hemisphere, Washington, D.C. 295-304 (1989). 


\title{
APPENDIX A
}

\section{SIMULATING ONE OF THE CIB W14 ROUND ROBIN TEST CASES USING THE SMARTFIRE FIRE FIELD MODEL}

\author{
Z. Wang, F. Jia, E.R. Galea, M.K. Patel and J. Ewer. \\ Fire Safety Engineering Group. \\ University of Greenwich, \\ Wellington St \\ London SE18 6PF. \\ UK \\ $\underline{\text { http://fseg.gre.ac.uk }}$
}

\begin{abstract}
Numerical predictions produced by the SMARTFIRE fire field model are compared with experimental data. The predictions consist of gas temperatures at several locations within the compartment over a 60 minute period. The test fire, produced by a burning wood crib attained a maximum heat release rate of approximately $11 \mathrm{MW}$. The fire is intended to represent a non-spreading fire in a moderately sized ventilated room. The experimental data formed part of the CIB Round Robin test series. Two simulations are produced, one involving a relatively course mesh and the other with a finer mesh. While the SMARTFIRE simulations made use of a simple volumetric heat release rate model, both simulations were found capable of reproducing the overall qualitative results. Both simulations tended to overpredict the measured temperatures. However, the finer mesh simulation was better able to reproduce the qualitative features of the experimental data. The maximum recorded temperature $\left(1214^{\circ} \mathrm{C}\right.$ after 39 minutes) was overpredicted in the fine mesh simulations by $12 \%$.
\end{abstract}

\section{INTRODUCTION}

This paper is concerned with producing further validation/verification for the SMARTFIRE fire field model [1-4]. Here, the term validation is taken as the systematic comparison of model predictions with reliable experimental data (see reference [5]). Validation is an essential step in the continual development and acceptance of computer models used in fire safety engineering. While no degree of successful validation will prove a computer model correct, confidence in the technique is established the more frequently it is shown to be successful in as wide a range of applications as possible. Validation should be viewed as an on-going activity and an integral part of the life cycle of the software.

The SMARTFIRE model is under continual development and evaluation. As part of the testing and validation procedure, model predictions are compared with other commercial CFD codes and against data generated through physical experimentation $[1,6]$. Test cases fall into one of two categories, standard CFD benchmark cases such as the backward facing step and fire test cases such as the Steckler room fire experiments [7]. Results produced by SMARTFIRE for all these cases compare favourably with experimental data and/or those produced using commercial CFD 
codes such as CFX and PHOENICS [1,6]. In this paper an additional fire test case is introduced into the SMARTFIRE validation suite. The test case involves a naturally ventilated moderately large room with a large non-spreading fire represented by a burning wood crib.

In 1997, CIB W14 Subgroup 2 undertook a Round Robin Testing exercise designed to investigate the accuracy of computer based fire models and their users in simulating a range of test fire scenarios [8]. The exercise included a range of zone and field models $[9,10]$ and a range of modellers, from consultant fire safety engineers to the actual software developers. The tasks involved simulating a range of fire experiments however, the modellers were blind to the actual results of the fire experiments. While the modellers did not have access to the results of the fire experiments that they were intended to simulate, they were provided with necessary and sufficient information to set up their models. Participants were allowed to specify the problem free parameters in whatever manner they chose thus for example, while the mass loss rate of the fire, heat of combustion, and burning efficiency was specified, users of field models were free to chose the number of cells and cell distribution. In this way the Round Robin was intended to test both the fire modelling software and the skill of the users.

In this paper we use one of the test cases as part of the validation process for the SMARTFIRE fire field model. In this case however, the authors are not blind to the experimental results but are fully aware.

\section{OVERVIEW OF THE SMARTFIRE FIRE FIELD MODEL}

SMARTFIRE is an open architecture CFD environment with an integrated knowledge based system that attempts to make fire field modelling accessible to non-experts in CFD. There are three major components to the software: CFD code, User Interfaces, and Expert System. By embedding expert knowledge into the CFD software, it is hoped that fire field modelling is made more accessible to the fire engineer with limited CFD expertise. The expertise currently embedded within the code is used to support the critical task of mesh specification of fire field simulation scenarios.

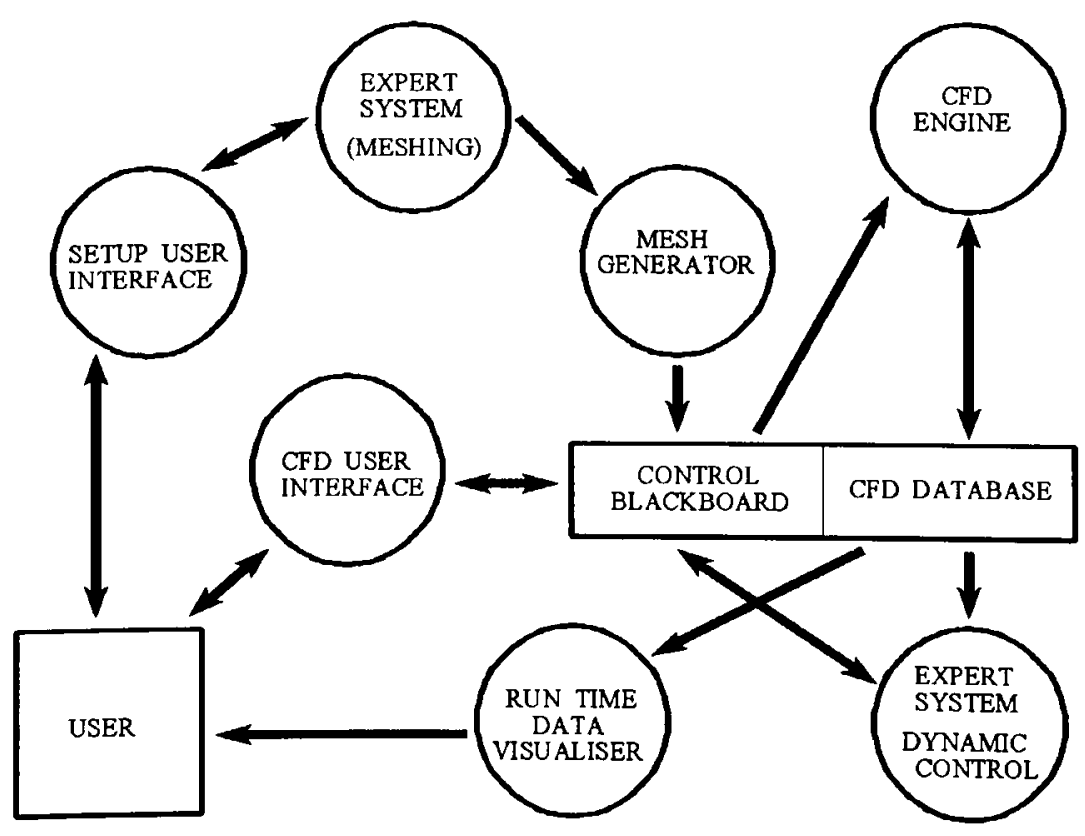

FIGURE 1: The modular architecture of SMARTFIRE. 
The software is fully developed by the University of Greenwich using a combination of in-house and proprietary software building blocks. The software is designed to run on PC's under the WIN95/98 or NT operating systems. The minimum computer platform required is a Pentium PC with 32 Mbytes of memory. The functionality and construction of the SMARTFIRE system have been described in previous publications [1-4], and so only a brief outline is presented here. Depicted in figure 1 is the conceptual architecture of the software and its modules.

The CFD engine of SMARTFIRE, is written in C++ and has been developed in-house from an existing FORTRAN code. It uses validated numerical methods from the legacy FORTRAN code enhanced by object oriented developments in $\mathrm{C}++$ and additional physics features that are required for fire field modelling [1]. These include the addition of a six-flux radiation model, provision for heat transfer through walls and buoyancy modification to the turbulence equations. It uses threedimensional unstructured meshes, enabling complex irregular geometries to be meshed without the need for body fitted co-ordinate grids. Unlike conventional CFD technology such as PHOENICS [11], JASMINE [12] and SOFIE [13], this allows extremely complex geometries to be meshed efficiently.

The current release of the software is V2.01 [1]. In this version, the software represents fires as a transient volumetric heat and mass source. Standard models for gaseous combustion (i.e. diffusion and eddy-dissipation models $[9,10]$, while not enabled in the general release software, are implemented within the in-house beta version and undergoing testing. The code uses the SIMPLE [14] algorithm and can solve turbulent (two equation K-Epsilon closure with buoyancy modification $[9,10]$ ) or laminar flow problems under transient or steady state conditions. Radiation is represented through the use of an enhanced six-flux radiation model [1].

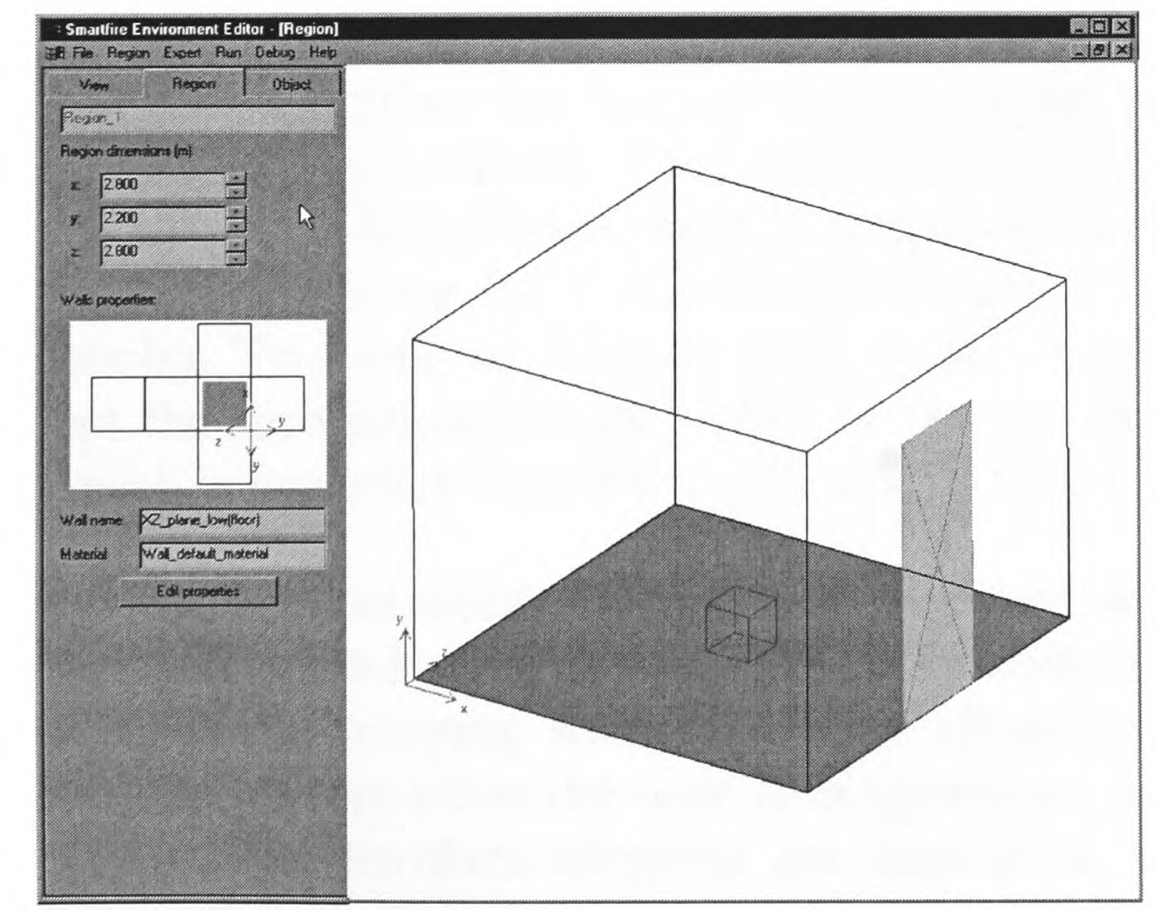

FIGURE 2: Main geometry Setup User Interface

The main user interface (UI) is used to specify the problem. Through the UI the user sets the geometry, specifying the location of walls, wall materials, internal compartments, obstacles, the location of vents, specifies the fire, activates the radiation model, specifies gaseous properties such as absorption coefficients, meshes 
the geometry and starts the CFD engine (see figure 2). In specifying the geometry, the user specifies the compartment(s) dimensions. On each wall of the compartment it is possible to position vents. Here, the generic term vent is used to represent any opening to the outside such as a door or window. The software does not currently include the provision of forced vents. Each vent's size and location is also specified. The user must also specify the wall material. The material type is specified using user familiar terms such as 'brick' or 'insulator', and the conversion to material properties and boundary conditions is performed internally. The fire is specified as a volumetric heat source. The user must specify the physical size of the fire, its location and heat output. The fire can be specified either as a constant heat release rate or as a transient heat release rate. If the transient option is selected, the user is presented with a menu which allows the specification of a range functional forms (see figure 3 ). The heat release rate can also be specified through an input table.

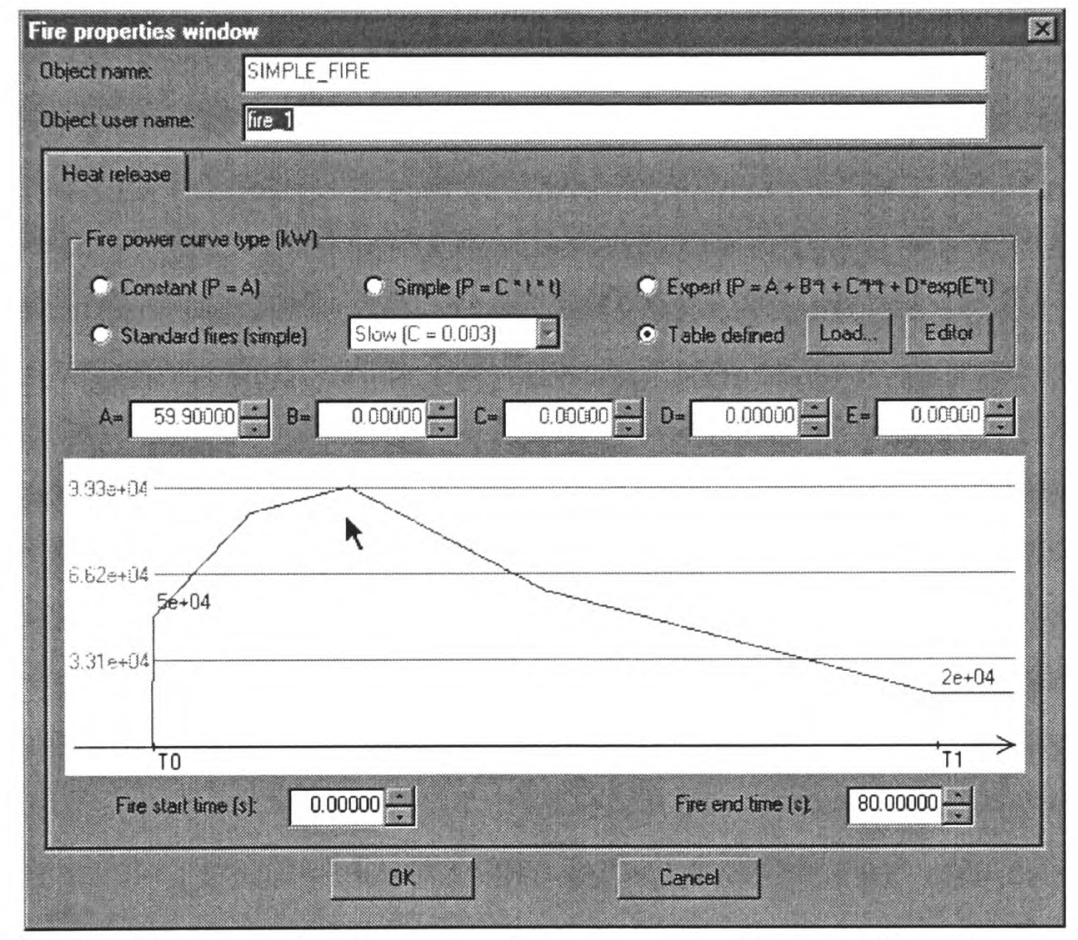

FIGURE 3: Volumetric heat release rate (transient) specification.

Once the problem has been specified, the mesh generation expert system is launched from the main user interface to generate the CFD grid for the problem. It is important here to note that the Knowledge Base System (KBS) component of SMARTFIRE will determine a cell budget appropriate for a reasonable solution to the problem (see $[1,2,4]$ for more details). This will be reported back to the user who is given the opportunity to accept the recommended cell budget or modify the cell budget (see figure 4). Once the mesh is generated the CFD engine can be launched.

The CFD code has its own unique windows based user interface depicted in figure 5. Unlike traditional fire field models, this allows the user to interact with the solution through observation of the developing solution and by allowing the user to make adjustments to control parameters while the code is in operation. Adjustment such as this in traditional CFD codes involves stopping the simulation, editing input files (possibly searching through large files containing nothing but floating point numbers), and restarting the simulation. With SMARTFIRE, this form of dynamic user control is considerably easier. All that is required is point and click of various buttons on the user interface.

Further information concerning SMARTFIRE including a demonstration may be found on the World Wide Web [15]. 
APPENDIX A SIMULATING ONE OF THE CIB W14 ROUND ROBIN TEST CASES USING THE SMARTFIRE FIRE FIELD MODEL

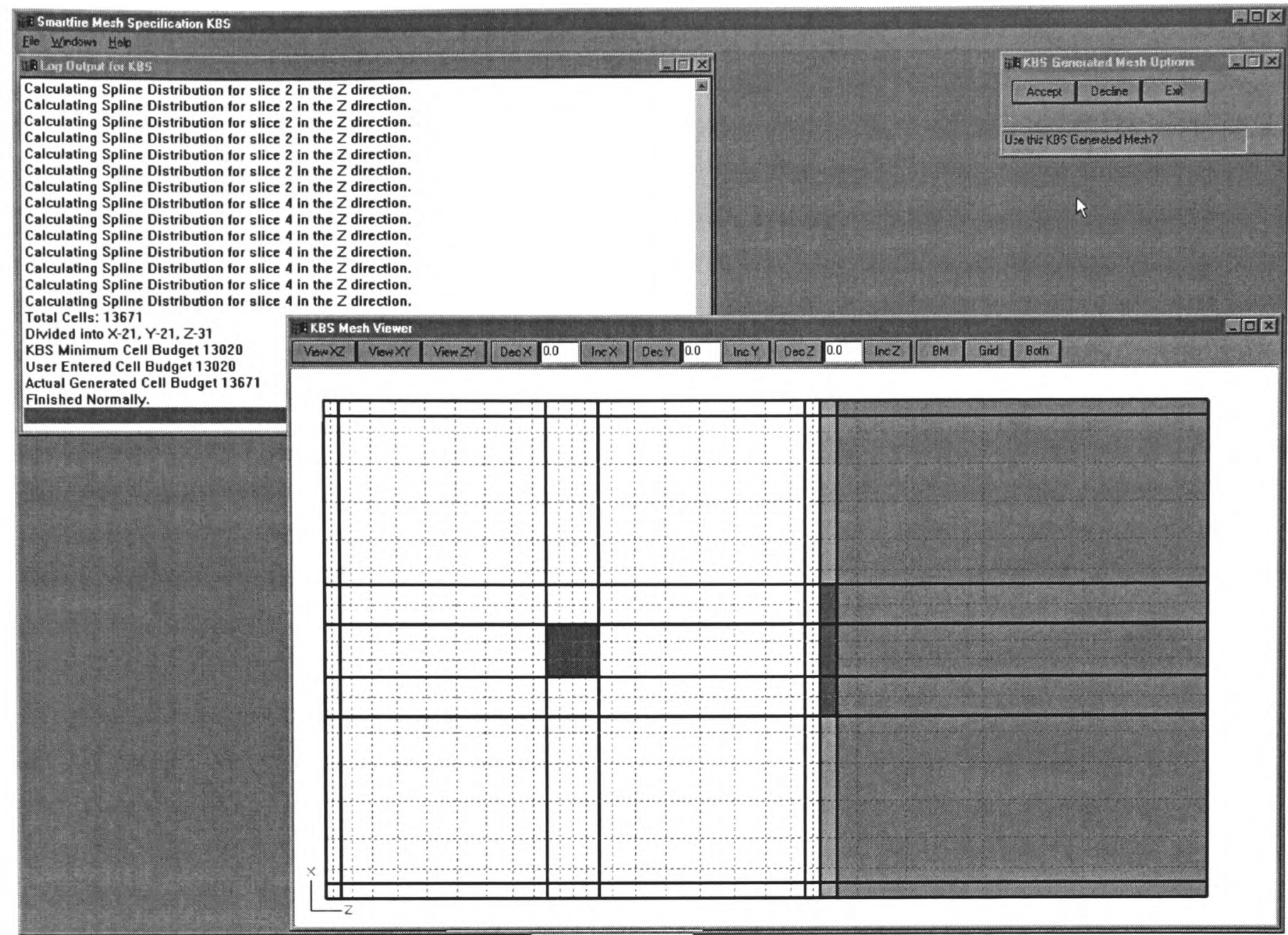

Figure 4: Mesh generation environment.

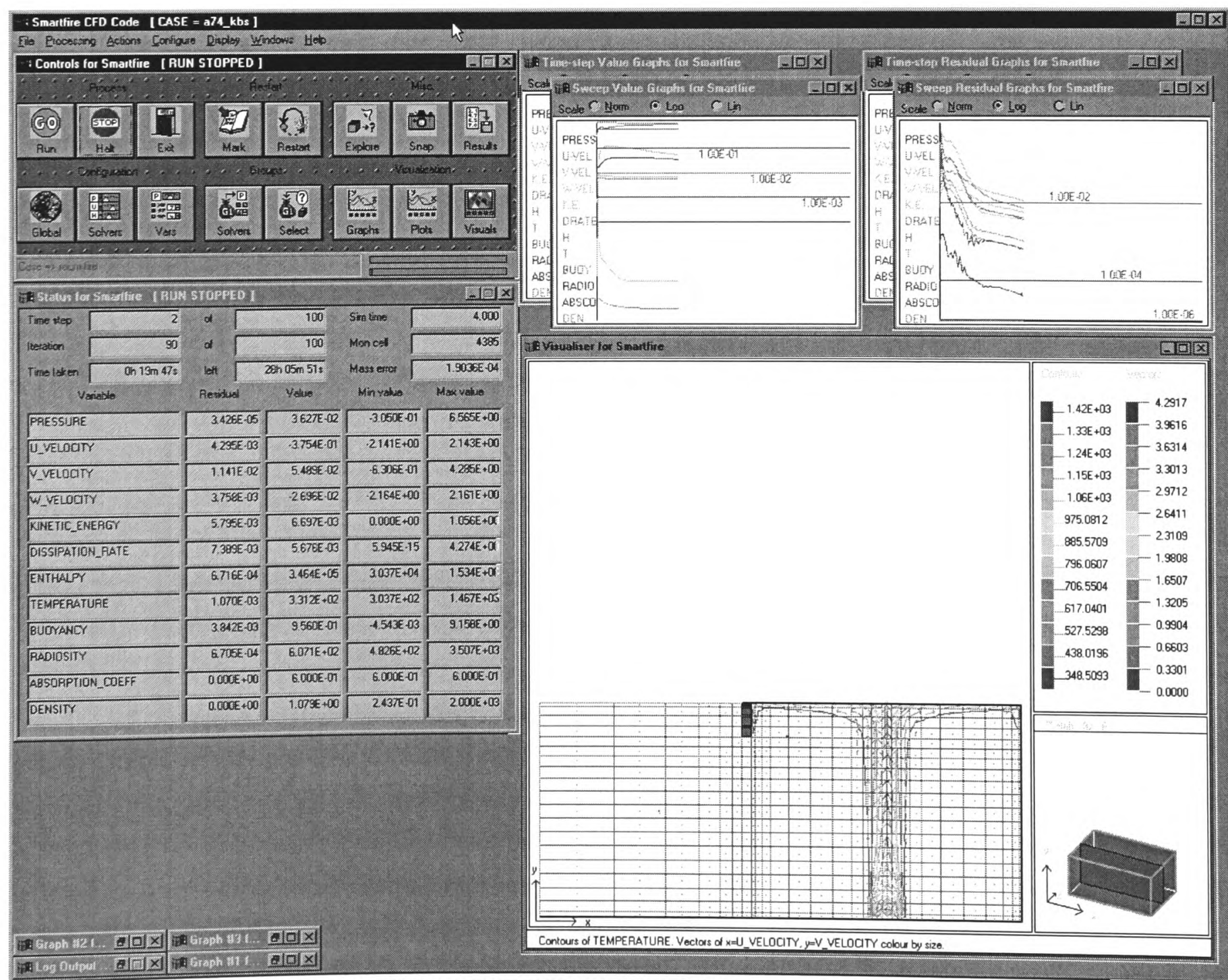

FIGURE 5: SMARTFIRE CFD Engine User Interface 


\subsection{DESCRIPTION OF EXPERIMENT}

The experiment used in this analysis corresponds to Scenario B of the CIB W14 Round Robin [8]. Scenario B consisted of three separate test cases, B1, B2 and B3. The specific results reported here are for scenario B1. This test was conducted at the VTT laboratory by VTT and Technische Universitat Braunschweig in $1983[8,16,17]$. The test series was devised to study compartment fire development. Test B1 was intended to represent an industrial building in which the fire source was concentrated in a single location.

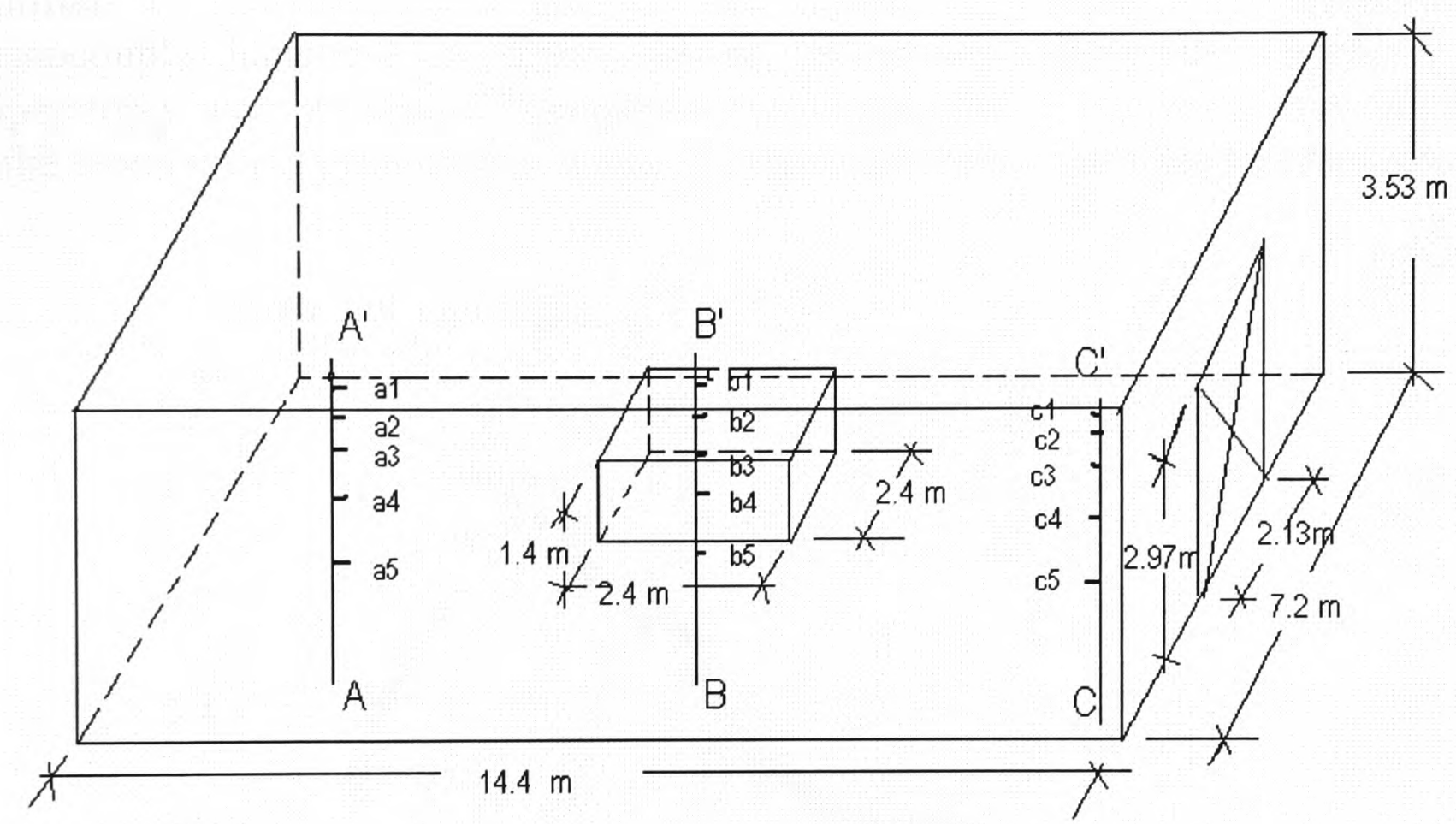

Figure 6a

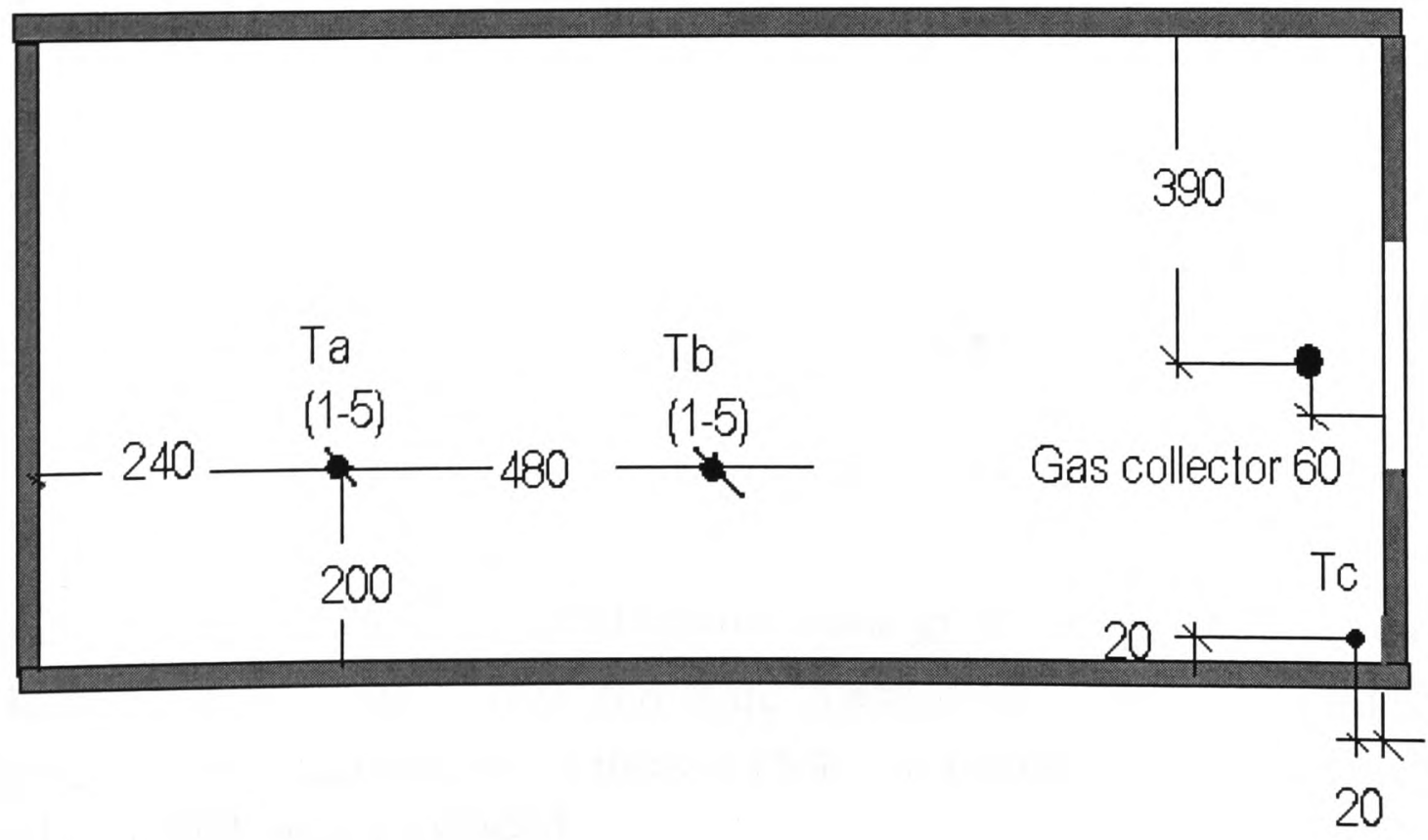

Figure 6b.

Figure 6: Depiction of fire compartment geometry showing location of fire source (figure 6a) and the location of the three thermocouple trees (figure 6b). 
The fire compartment measured $14.4 \mathrm{~m} \times 7.2 \mathrm{~m}$ in plan and $3.53 \mathrm{~m}$ in height and contained a door of dimensions $2.97 \mathrm{~m} \times 2.13 \mathrm{~m}$ (see figure 6). The walls of the compartment were made of aerated concrete blocks (with siporex mortar) with thickness $0.3 \mathrm{~m}$ and the following material properties: specific heat $1.05 \mathrm{~kJ} / \mathrm{kg} . \mathrm{K}$, thermal conductivity $0.12 \mathrm{~W} / \mathrm{m} . \mathrm{K}$ and density $500 \mathrm{~kg} / \mathrm{m}^{3}$. The initial air temperature was measured as $20.0^{\circ} \mathrm{C}$.

The fire was located on the floor in the centre of the room. The fire fuel consisted of softwood (Pinea ecelsa) timber cribs nailed into $40 \mathrm{~mm} \times 40 \mathrm{~mm}$ battens. The crib measured $2.4 \mathrm{~m}$ in length, $2.4 \mathrm{~m}$ in width and $1.4 \mathrm{~m}$ in height.

Gas temperatures were measured using three vertical rakes of thermocouples identified as location $\mathrm{A}, \mathrm{B}$ and $\mathrm{C}$ (see figure 6). Each rake contained five thermocouples located $20 \mathrm{~cm}, 50 \mathrm{~cm}, 100 \mathrm{~cm}, 180 \mathrm{~cm}$ and $250 \mathrm{~cm}$ from the ceiling. Gas temperatures were measured throughout the experiment. The mass loss rate $(\mathrm{kg} / \mathrm{s})$ for the wood crib is presented in figure 7. The wood cribs burnt for 100 minutes.

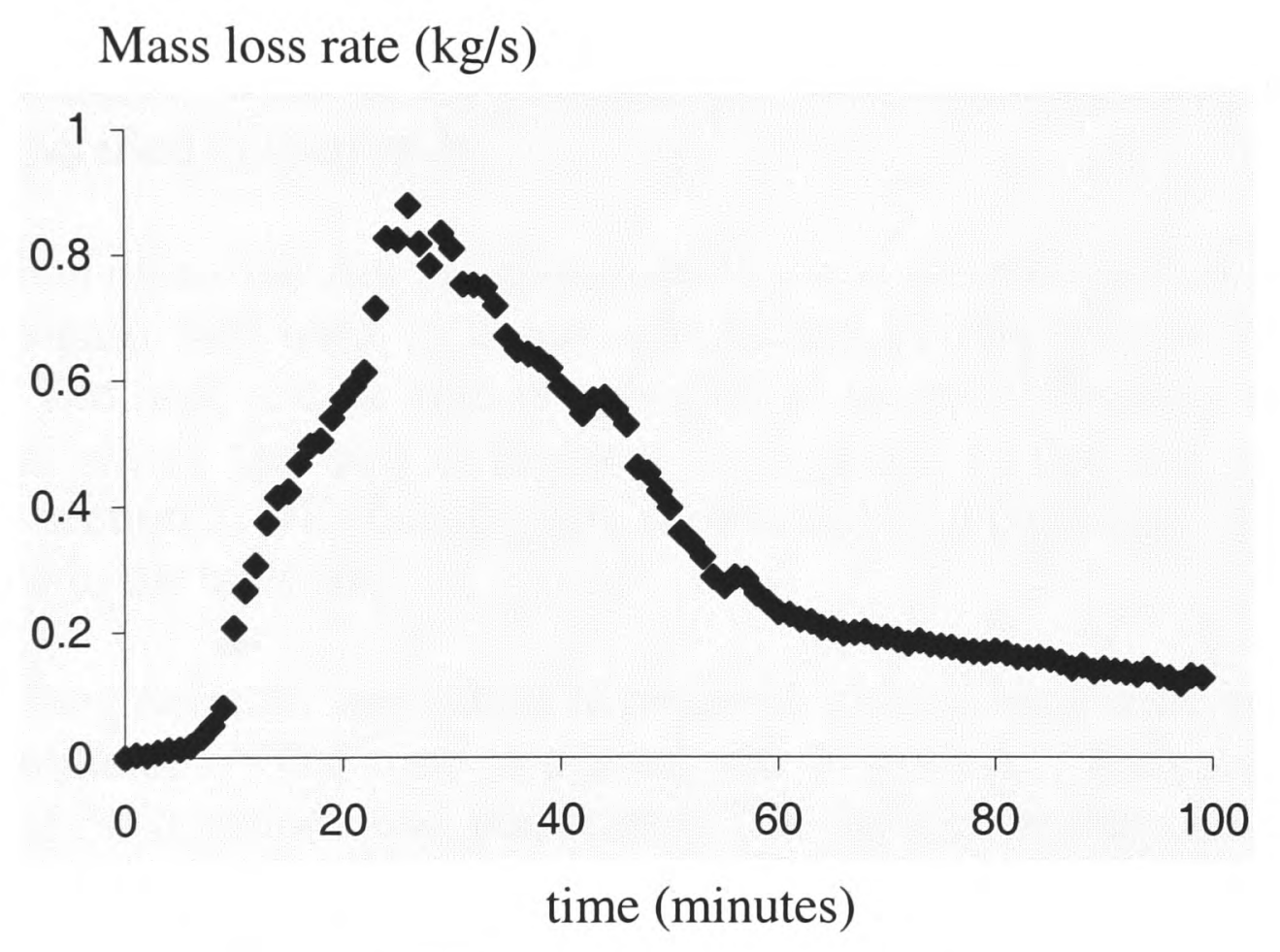

Figure 7: Mass loss rate (kg/s) for burning crib in Scenario B1.

The heat release rate of the fire is calculated by multiplying the mass loss rate with the effective heat of combustion and an efficiency factor (see equation 1).

$$
\dot{Q}=\chi \cdot \Delta H_{c} \cdot \dot{m}
$$

The efficiency factor and heat of combustion were given as $\chi=0.7$ and $\Delta H_{c}$ is 17.8 $\mathrm{MJ} / \mathrm{kg}$ for burning wood with a $10 \%$ moisture content [8]. The derived heat release rate is reported to be accurate to within $\pm 15 \%$. A maximum heat release rate of approximately $11 \mathrm{MW}$ was produced.

This completes the information passed onto the participants of the blind round robin test. The experimental results for this scenario are reported in [18]. 


\section{DESCRIPTION OF MODEL SETUP.}

The SMARTFIRE V2.01 [1] software was used to perform the above simulations. Thermal radiation is represented using the six-flux radiation model (see section 2) with absorption coefficient specified in equation (2).

$$
\begin{aligned}
& a=0.01, \text { if } T<323 \mathrm{~K} ; \\
& a=0.01+0.305 / 377(T-323), \text { if } 323<=T<700 ; \\
& a=0.315+0.315 / 700(T-700), \text { if } T>700 .
\end{aligned}
$$

where $T$ is the gas temperature.

This functional representation of the absorption coefficient (i.e. equation 2) is a modification of the curves found in figure 1 of reference 19 . The wall emissivity was assumed to be 0.8 . The initial temperature was set to that specified in the experiments while the insulating walls of the compartment were modelled using the wall thermal properties specified in section 3.

In these calculations the fire is represented by a volumetric heat release rate model. The heat release rate used in these calculations corresponds to that described in section 3. The heat release rate is specified in watts $/ \mathrm{m}^{3}$ through a table file. The volume over which the heat is released corresponds to the size of the fire source specified in section 3 . The burning rate is obtained by a piecewise linear interpolation with the data in the table file.

As a fairly long duration simulation is required, quite a large time step size was used in these simulations. The time step used was 5 seconds. Within each time step, a maximum of 50 iterations was performed. The default convergence tolerance 0.001 was adopted. This means that the expected maximum reference norm of the errors for every variable in a simulation time step is 0.001 .

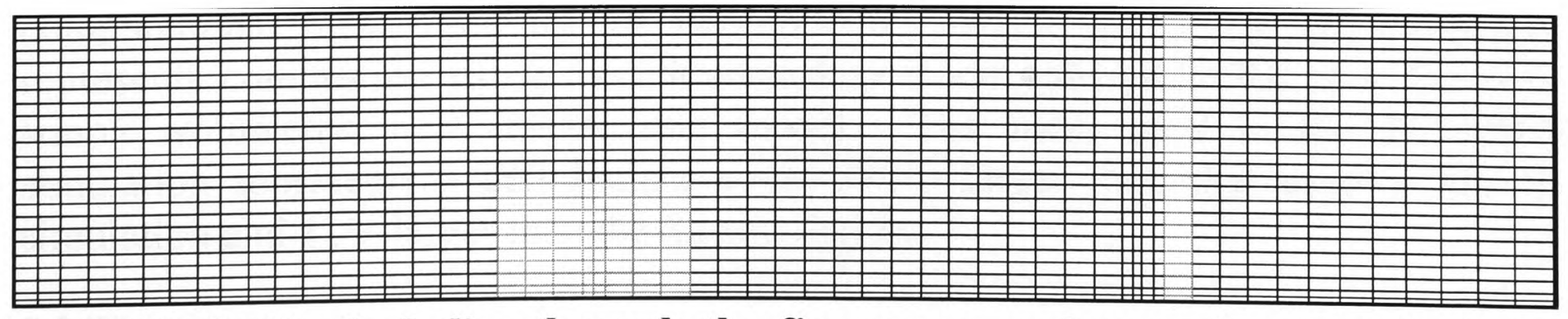

FIGURE 8: Vertical slice through the fire source and open doorway depicting the fine computational mesh.

Two meshes were used in the simulations. The first corresponding to a course mesh consisted of 12,480 (i.e. $39 \times 16 \times 20$ ) computational cells while the second finer mesh consisted of 41,325 (i.e. $57 \times 25 \times 29$ ) computational cells. The computational mesh distribution was determined and generated by the SMARTFIRE KBS. The KBS recommended a minimum cell distribution of 11,552 cells. Note that the KBS has generated a non-uniform mesh distribution and that an extended region was 
automatically included beyond the open door of the compartment (see figure 8). Free surface boundary conditions were automatically applied on all open surfaces of the extended region. All air entrained into the solution field brought in air at ambient conditions.

Results for the course mesh are denoted as $\mathrm{S} 1$ while results for the fine mesh are denoted as S2. S1 was computed using a $200 \mathrm{MHz}$ Pentium PC with $64 \mathrm{MB}$ while S2 was computed using a $400 \mathrm{MHz}$ Pentium III PC with $256 \mathrm{MB}$ of memory.

\section{RESULTS AND DISCUSSION.}

\subsection{The Round Robin Models.}

Throughout this discussion, results from the SMARTFIRE predictions will primarily be compared with the temperatures directly measured during the experimental trial. In addition, the SMARTFIRE predictions will be compared with values produced by the fire field models participating in the Round Robin, namely the JASMINE model (calculations produced by the Fire Research Station U.K.) [12] and SOFIE (calculations produced by SP in Sweden) [13].

The JASMINE model made use of the k-eps turbulence model with buoyancy modification, eddy-breakup combustion model, six-flux radiation model, a mesh comprising of 46,208 (i.e. $19 \times 38 \times 64$ ) cells and a time step of 2 seconds. The SOFIE model made use of the k-eps turbulence model with buoyancy modification, eddy-breakup combustion model, discrete transfer radiation model, a mesh comprising of 65,076 (i.e. $29 \times 34$ x 66) cells and a time step of 5 minutes [18]. The results for these two models are reported in reference [18] however, these results are only reported for locations A-A' and B-B'. In addition, the results are only presented in graphical form and so all results quoted in this paper for these models should be considered as only approximate. Furthermore, a programming error occurred with the SOFIE code resulting in the numerical predictions being unreliable after 20 minutes.

Of the two simulations, the JASMINE results are likely to be more similar to those produced by SMARTFIRE as the physics of the component submodels for these two models are similar. The main differences lie in the treatment of combustion and in the computational mesh used by the two models. While the JASMINE simulation represents combustion using the eddy-breakup model, SMARTFIRE uses the more simple volumetric heat release rate model. The mesh used in the JASMINE simulation represents a cell budget some $370 \%$ larger than that used in the S1 simulation and $11 \%$ larger than the $\mathrm{S} 2$ simulation.

\subsection{The S1 Simulation.}

The S1 simulation was run for an equivalent of 30 minutes of real time. These predictions required 67 hours of computational time. The predictions are depicted in figures 9-11, while table 1 shows a comparison of measured and predicted temperatures at certain times through the simulation.

Figure 7 shows the burning rate (and hence the heat release rate) within the compartment as a function of time. Over the first 10 minutes there is a very slow 
increase in burning rate, this increases rapidly from 10 to approximately 20 minutes and even more rapidly from 20 to approximately 25 minutes. From 25 to 60 minutes there is a rapid decline in burning rate which decreases significantly in the latter stages of the experiment from 60 to 100 minutes. These trends in heat release rate are reflected in the measured and predicted temperature distributions at the various locations.

TABLE 1: Comparison of measured and predicted $(\mathrm{S} 1)$ temperatures $\left({ }^{\circ} \mathrm{C}\right)$ at the three thermocouple tree locations as a function of time.

\begin{tabular}{|c|c|c|c|c|c|c|c|}
\hline \multirow{2}{*}{ Time } & \multirow{2}{*}{ Position } & \multicolumn{2}{|c|}{$\mathbf{A}-\mathbf{A}$} & \multicolumn{2}{|c|}{ B-B } & \multicolumn{2}{|c|}{ C-C } \\
\hline & & $\operatorname{Exp}\left({ }^{\circ} \mathrm{C}\right)$ & Pred $\left({ }^{\circ} \mathrm{C}\right)$ & $\operatorname{Exp}\left({ }^{\circ} \mathrm{C}\right)$ & Pred $\left({ }^{\circ} \mathrm{C}\right)$ & $\operatorname{Exp}\left({ }^{\circ} \mathrm{C}\right)$ & Pred $\left({ }^{\circ} \mathrm{C}\right)$ \\
\hline \multirow{5}{*}{$5 \min$} & 1 & 32.2 & 68.1 & 38.6 & 74.9 & 31.0 & 66.0 \\
\hline & 2 & 28.2 & 66.3 & 35.9 & 71.5 & 31.3 & 66.3 \\
\hline & 3 & 25.5 & 66.5 & 25.8 & 66.0 & 27.6 & 66.6 \\
\hline & 4 & 24.6 & 64.7 & 24.9 & 63.4 & 23.4 & 65.6 \\
\hline & 5 & 25.2 & 61.2 & 23.7 & 58.7 & 23.7 & 61.7 \\
\hline \multirow{5}{*}{$10 \mathrm{~min}$} & 1 & 75.3 & 380 & 149 & 453 & 81.6 & 344 \\
\hline & 2 & 69.1 & 358 & 86.0 & 424 & 81.9 & 341 \\
\hline & 3 & 64.9 & 344 & 61.0 & 375 & 56.9 & 338 \\
\hline & 4 & 29.5 & 317 & 31.9 & 311 & 27.0 & 327 \\
\hline & 5 & 29.2 & 286 & 26.3 & 263 & 25.5 & 311 \\
\hline \multirow{5}{*}{$15 \mathrm{~min}$} & 1 & 526 & 717 & 924 & 938 & 510 & 651 \\
\hline & 2 & 498 & 693 & 884 & 918 & 521 & 642 \\
\hline & 3 & 478 & 659 & 670 & 868 & 511 & 633 \\
\hline & 4 & 314 & 586 & 465 & 789 & 443 & 616 \\
\hline & 5 & 204 & 524 & 324 & 756 & 194 & 599 \\
\hline \multirow{5}{*}{$20 \mathrm{~min}$} & 1 & 616 & 856 & 998 & 1110 & 656 & 739 \\
\hline & 2 & 604 & 833 & 942 & 1100 & 670 & 731 \\
\hline & 3 & 587 & 798 & 819 & 1090 & 665 & 721 \\
\hline & 4 & 517 & 750 & 713 & 1070 & 630 & 701 \\
\hline & 5 & 406 & 716 & 574 & 1070 & 484 & 682 \\
\hline \multirow{5}{*}{$25 \mathrm{~min}$} & 1 & 839 & 1050 & 1081 & 1310 & 909 & 825 \\
\hline & 2 & 847 & 1040 & 1089 & 1320 & 931 & 815 \\
\hline & 3 & 844 & 1020 & 1087 & 1330 & 930 & 803 \\
\hline & 4 & 819 & 999 & 1081 & 1380 & 908 & 779 \\
\hline & 5 & 794 & 988 & 1057 & 1430 & 872 & 757 \\
\hline \multirow{5}{*}{$30 \mathrm{~min}$} & 1 & 919 & 1240 & 1128 & 1390 & 945 & 859 \\
\hline & 2 & 921 & 1240 & 1134 & 1390 & 971 & 853 \\
\hline & 3 & 918 & 1240 & 1130 & 1370 & 979 & 833 \\
\hline & 4 & 905 & 1240 & 1110 & 1270 & 970 & 786 \\
\hline & 5 & 889 & 1240 & 1078 & 1130 & 950 & 746 \\
\hline
\end{tabular}

As can be seen from figure 9 , the measured temperatures at location $A-A^{\prime}$ show a very gradual increase over the first 10 minutes. From 10 to 25 minutes, the lower thermocouples record a rapid and almost uniform temperature increase. The upper thermocouples show a very rapid increase in temperature from 10 to approximately 12.5 minutes and then a more gradual increase from 12.5 to 25 minutes. From 10 to 25 minutes there is a clear stratification within the compartment with the lower regions being considerably cooler than the upper region. After 25 minutes the temperature distribution measured at this location appears to very rapidly become uniform, gradually increasing to reach a maximum value at approximately 30 minutes. 
The predicted temperatures appear to follow this trend throughout the first 30 minutes. However, the predicted temperature increase in the last five minutes is still very rapid, unlike that seen in the experiment. While the trends are similar between the predicted and measured temperatures, there is a significant difference in the actual values (see table 1). Furthermore, the average predicted temperature after 30 minutes at location A-A ${ }^{\prime}$ is approximately $1240^{\circ} \mathrm{C}$ while the measured average temperature is $910^{\circ} \mathrm{C}$. Thus, the predicted average value exceed the measured average value by $330^{\circ} \mathrm{C}$ or $36 \%$ after 30 minutes (see tables 1 and 3 ).

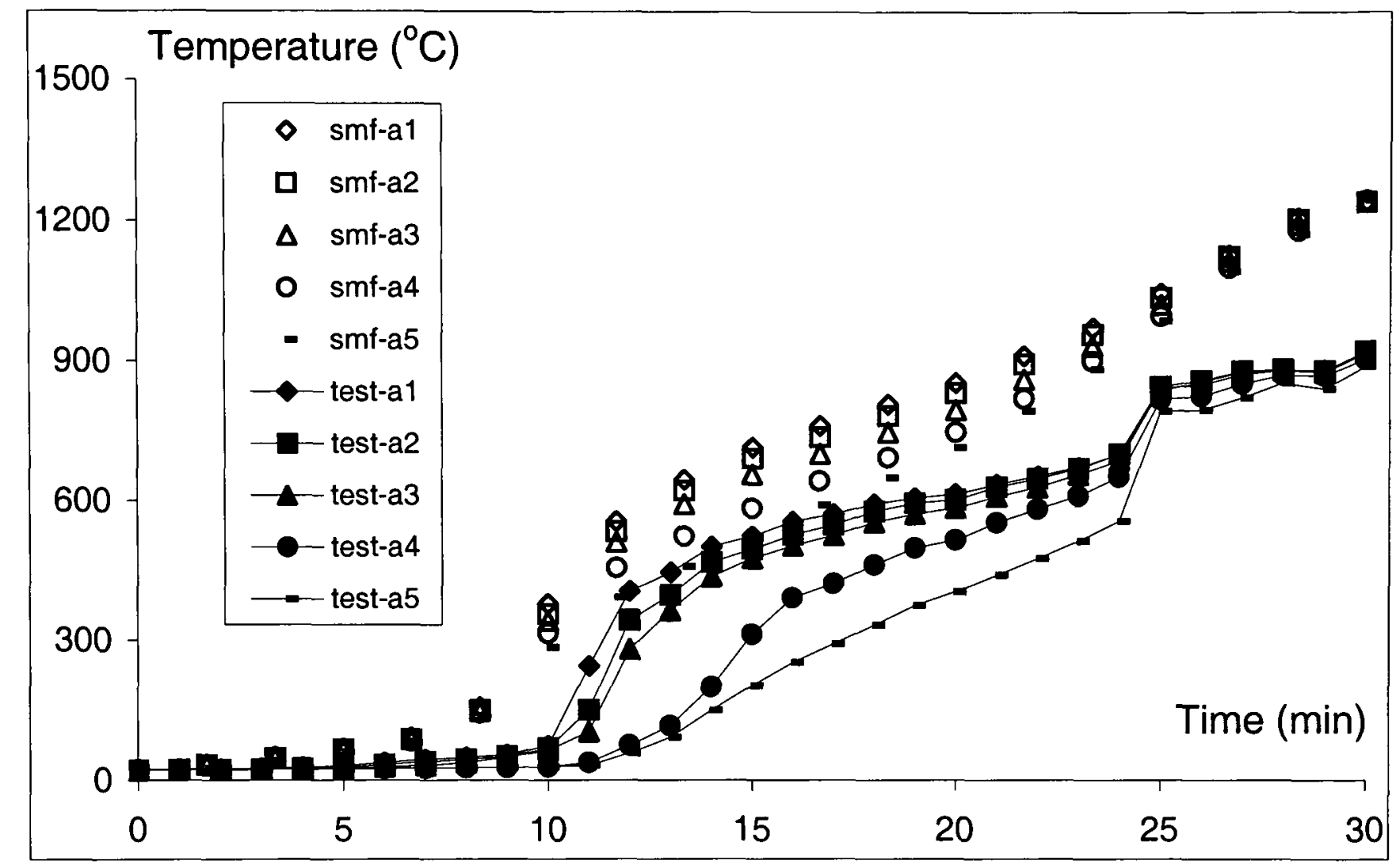

Figure 9: Predicted (symbols) and measured (solid lines) temperatures $\left({ }^{\circ} \mathrm{C}\right.$ ) on line $\mathbf{A}-\mathbf{A}^{\prime}$ as a function of time (min) for simulation $\mathrm{S} 1$.

Over the first 25 minutes, the difference between the predicted and measured temperatures is most apparent for the lower thermocouple values. The measured values show a more significant temperature difference between the upper and lower temperatures than is observed in the numerical predictions. At 15 minutes, the measured difference between the upper and lower temperatures is $322^{\circ} \mathrm{C}$ or $158 \%$ while the predicted temperature difference is $193^{\circ} \mathrm{C}$ or $37 \%$ and at 20 minutes, the measured difference is $210^{\circ} \mathrm{C}$ or $52 \%$ while the predicted temperature difference is $140^{\circ} \mathrm{C}$ or $20 \%$.

It is worth comparing these values with those produced by the fire field models participating in the Round Robin, namely the JASMINE model [12] and SOFIE [13]. The trends in the JASMINE results follow that of SMARTFIRE and generally overpredict the experimental values. However, the JASMINE predicted temperatures corresponding to the lower thermocouple appear to be significantly closer to the measured values. The JASMINE peak predicted temperature after 30 minutes is approximately $1180^{\circ} \mathrm{C}$ thus, the predicted temperature exceeds the measured value by $261{ }^{\circ} \mathrm{C}$ or $28 \%$. The SMARTFIRE value at this location exceeds that predicted by JASMINE by some $60^{\circ} \mathrm{C}$. Over the first 20 minutes, the SOFIE predictions appear to most closely match the observed qualitative and quantitative results. After 20 minutes, the measured maximum and minimum temperatures are $616^{\circ} \mathrm{C}$ and $406^{\circ} \mathrm{C}$ respectively while the predictions by SOFIE, JASMINE and SMARTFIRE are approximately 
$645^{\circ} \mathrm{C}$ and $570^{\circ} \mathrm{C}$, approximately $755^{\circ} \mathrm{C}$ and $630^{\circ} \mathrm{C}$, and $856^{\circ} \mathrm{C}$ and $716^{\circ} \mathrm{C}$ respectively.

The differences between the various models should come as no surprise as the SMARTFIRE predictions do not make use of a combustion model and the mesh used by SMARTFIRE in the S1 simulation is very course compared to that used by the other models.

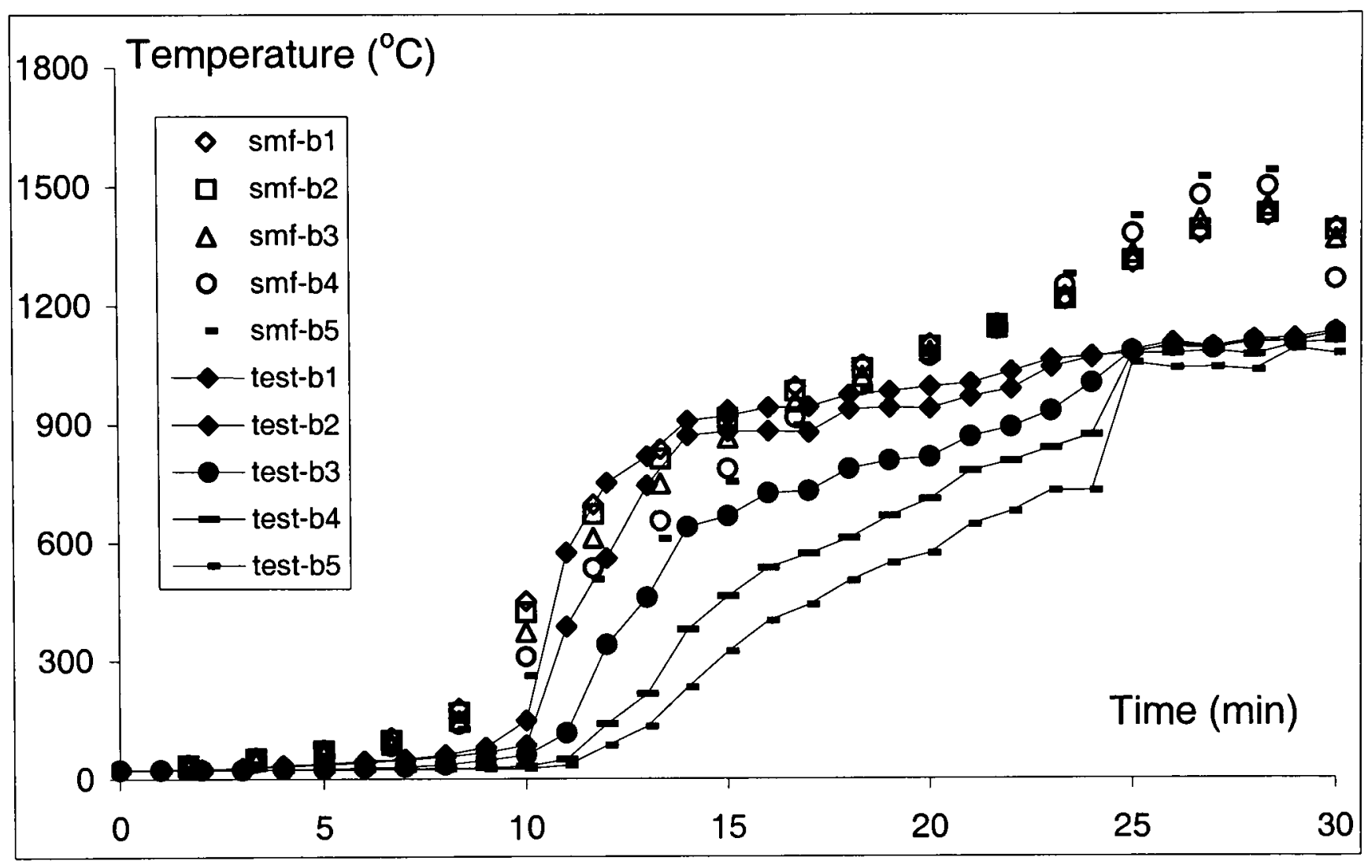

Figure 10: Predicted (symbols) and measured (solid lines) temperatures $\left({ }^{\circ} \mathrm{C}\right)$ on line

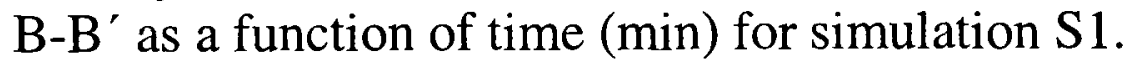

The trend in measured temperatures at location $\mathrm{B}^{-\mathrm{B}^{\prime}}$ follows that for location $\mathrm{A}-\mathrm{A}^{\prime}$ (see figure 10). However, temperatures at location $\mathrm{B}^{-\mathrm{B}^{\prime}}$ are considerably hotter than those at location $\mathrm{A}-\mathrm{A}^{\prime}$. The average temperature at location $\mathrm{B}^{-\mathrm{B}^{\prime}}$ after 30 minutes being $1116^{\circ} \mathrm{C}$ compared with $910^{\circ} \mathrm{C}$ at location $\mathrm{A}-\mathrm{A}^{\prime}$.

As with the SMARTFIRE predictions at location A-A', the numerical predictions at location B-B' tend to follow the trends in the measured results. However, there are two notable exceptions, firstly the predicted temperatures are closer to the measured values than at location A-A' (see table 1). At 15 minutes, the predicted temperature at location 1 (i.e. near ceiling) is $938^{\circ} \mathrm{C}$ compared with a measured value of $924^{\circ} \mathrm{C}$, a difference of only $14^{\circ} \mathrm{C}$ or $2 \%$. The average predicted temperature after 30 minutes at location $\mathrm{B}^{-\mathrm{B}^{\prime}}$ is approximately $1310^{\circ} \mathrm{C}$ while the measured average temperature is $1116^{\circ} \mathrm{C}$. Thus after 30 minutes, the predicted average values exceed the measured average values by $194^{\circ} \mathrm{C}$ or $17 \%$ (see table 1). However, as noted at location A-A', the numerical predictions do not show as marked a difference between upper and lower layers as is observed in the experiments.

The second difference between locations $A-A^{\prime}$ and $B-B^{\prime}$ concerns the surprising result that the temperatures in the lower layer are hotter than those in the upper layer. This observation occurs after approximately 23 minutes and continues up to approximately 27 minutes (see figure 10 and table 1). This phenomena does not 
correspond to behaviour observed in the measured temperatures and is thought to be due to the lack of resolution in the computational mesh.

As with location A-A', the trends in the SMARTFIRE results follow that of JASMINE. However, the unusual behaviour concerning the heating of the lower layer to a temperature above that of the upper layer is not observed. This lends support to the conjecture that this behaviour is a result of insufficient mesh resolution in the S1 simulation. The JASMINE maximum predicted temperature after 30 minutes (at location B-B') is approximately $1360^{\circ} \mathrm{C}$ thus, the predicted temperature exceeds the measured value by $226^{\circ} \mathrm{C}$ or $20 \%$. The SMARTFIRE value at this location exceeds that predicted by JASMINE by some $30^{\circ} \mathrm{C}$. Once again, over the first 20 minutes, the SOFIE predictions appear to most closely match the observed qualitative and quantitative results. At 20 minutes, the measured maximum and minimum temperatures are $998^{\circ} \mathrm{C}$ and $574^{\circ} \mathrm{C}$ respectively while the predictions by SOFIE, JASMINE and SMARTFIRE are approximately $990^{\circ} \mathrm{C}$ and $565^{\circ} \mathrm{C}$, approximately $1000^{\circ} \mathrm{C}$ and $740^{\circ} \mathrm{C}$ and $1110^{\circ} \mathrm{C}$ and $1070^{\circ} \mathrm{C}$ respectively.

Thermocouple tree C-C' is located in the front corner of the compartment. However, the overall trend in measured temperatures follows that for the other locations (see figure 11). Temperatures at location $\mathrm{C}^{-\mathrm{C}^{\prime}}$ are considerably cooler than those at location $\mathrm{B}-\mathrm{B}^{\prime}$ and are similar to those at location $\mathrm{A}-\mathrm{A}^{\prime}$. The average temperature at

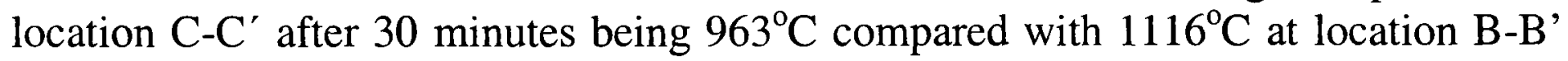
and $910^{\circ} \mathrm{C}$ at location $\mathrm{A}-\mathrm{A}^{\prime}$.

The SMARTFIRE predictions at location C-C', tend to follow the trends in the measured results. Compared with the numerical predications at the other locations, a notable difference with the predictions at location C-C' is that here we find that in the last five minutes, the numerical results tend to underpredict the measured values (see table 1). At 15 minutes, the average predicted temperature is $628^{\circ} \mathrm{C}$ compared with a measured average value of $436^{\circ} \mathrm{C}$, a difference of $192^{\circ} \mathrm{C}$ or $44 \%$. The average predicted temperature after 30 minutes at location $\mathrm{C}-\mathrm{C}^{\prime}$ is approximately $815^{\circ} \mathrm{C}$. Thus after 30 minutes, the predicted average values underpredict the measured average values by $148^{\circ} \mathrm{C}$ or $15 \%$ (see table 1 ). 


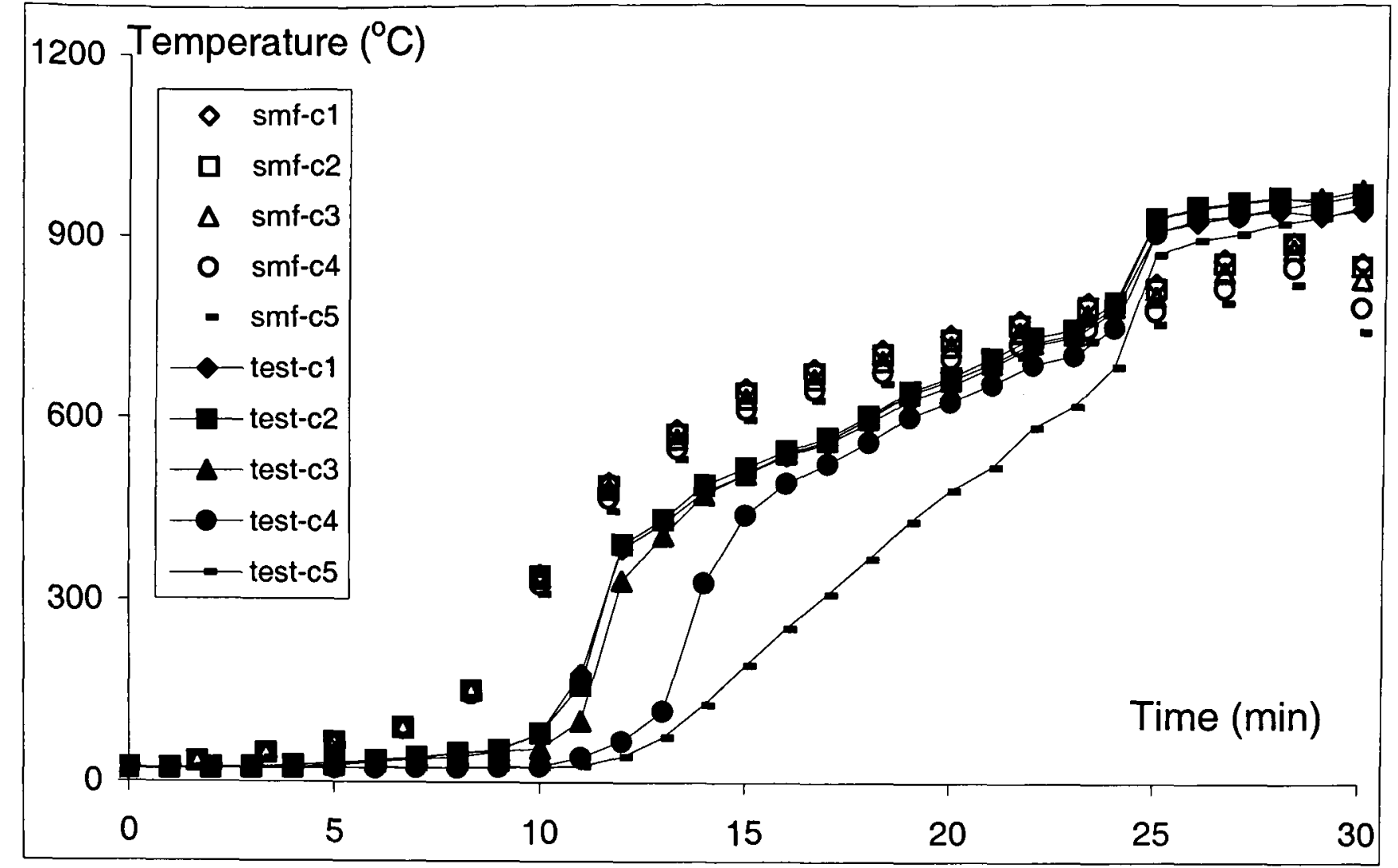

Figure 11: Predicted (symbols) and measured (solid lines) temperatures $\left({ }^{\circ} \mathrm{C}\right)$ on line $\mathrm{C}-\mathrm{C}^{\prime}$ as a function of time (min) for simulation $\mathrm{S} 1$.

It is also interesting to note that a temperature inversion exists in the experimental data at location C-C' (see tables 1 and 2). This occurs at the higher thermocouple locations. Initially (after 5 minutes), the temperature at thermocouple 2 is slightly higher than that at location 1 . This differences progresses until it reaches a maximum difference at 30 minutes after which time the third thermocouple is some $34^{\circ} \mathrm{C}$ hotter than the first thermocouple. After this time the temperature difference decreases until at 60 minutes, there is only a $3^{\circ} \mathrm{C}$ difference between the first and second thermocouple.

Unlike the temperature inversion observed in the numerical predictions at location BB', this is thought to be the result of a real fluid behaviour. The temperature inversion may occur due to the physical location of the thermocouple tree being in the region where the spreading ceiling jet impacts the corner walls and is forced down towards the floor. The lower thermocouples therefore are exposed to the hotter downward moving jet while the upper thermocouples are exposed to the resulting cooler recirculation region. The S1 simulation, due to the coarseness of the mesh, does not resolve this behaviour.

Finally, over the first 30 minutes, a maximum temperature of $1134^{\circ} \mathrm{C}$ was recorded in the experiment at location B-B' after 30 minutes. This corresponded to a predicted temperature of $1390^{\circ} \mathrm{C}$ at the same location, producing an error of $23 \%$.

\subsection{The S2 Simulation.}

The $\mathrm{S} 2$ simulation was a repeat of the $\mathrm{S} 1$ simulation with a finer mesh consisting of 41,325 computational cells. In addition to improving the accuracy of the SMARTFIRE simulations, the mesh used in the $\$ 2$ simulation more closely resembles that used in the JASMINE simulation (which used some 46,208 computational cells). The S2 simulation was run for an equivalent of 60 minutes of real time and required approximately 174 hours of computational time. The predictions are depicted in 
figures 12-15, while tables 2 and 3 show a comparison of measured and predicted temperatures at certain times through the simulation.

Figure 12 depicts the measured temperature distribution at location A-A' from 0 to 60 minutes, a further 30 minutes than was depicted in figure 9. As can be seen from figure 12 , the measured temperatures at location $\mathrm{A}-\mathrm{A}^{\prime}$ reach a maximum value of some $944^{\circ} \mathrm{C}$ after approximately 40 minutes and then show a gradual decline for the next 20 minutes. From 40 minutes through to 60 minutes, the level of stratification increases with the lower layer becoming progressively cooler.

The predicted temperatures appear to follow this trend throughout the first 60 minutes. Over the first 30 minutes, the $\mathrm{S} 2$ predictions produce a better representation of the measured temperatures than the $\mathrm{S} 1$ predictions. In particular, the overprediction in temperatures has decreased and there is a greater level of stratification predicted. At 20 minutes, the measured difference between the upper and lower temperatures has increased from $140^{\circ} \mathrm{C}$ or $20 \%$ in the $\mathrm{S} 1$ simulation to $262^{\circ} \mathrm{C}$ or $50 \%$ in the $\mathrm{S} 2$ simulation (compared with $210^{\circ} \mathrm{C}$ or $52 \%$ in the experiment). In addition, the temperature predicted at the lower level has decreased significantly - inline with observation. The measured temperature at the lower thermocouple is $406^{\circ} \mathrm{C}$ while the $\mathrm{S} 1$ prediction is $716^{\circ} \mathrm{C}$ (an error of $76 \%$ ) and the $\mathrm{S} 2$ prediction is $527^{\circ} \mathrm{C}$ (an error of $30 \%$ ). At 20 minutes, the measured maximum and minimum temperatures at thermocouple location 3 are $616^{\circ} \mathrm{C}$ and $406^{\circ} \mathrm{C}$ respectively while the predictions by SOFIE, JASMINE and SMARTFIRE are approximately $645^{\circ} \mathrm{C}$ and $570^{\circ} \mathrm{C}$, approximately $755^{\circ} \mathrm{C}$ and $630^{\circ} \mathrm{C}$ and $789^{\circ} \mathrm{C}$ and $527^{\circ} \mathrm{C}$ respectively.

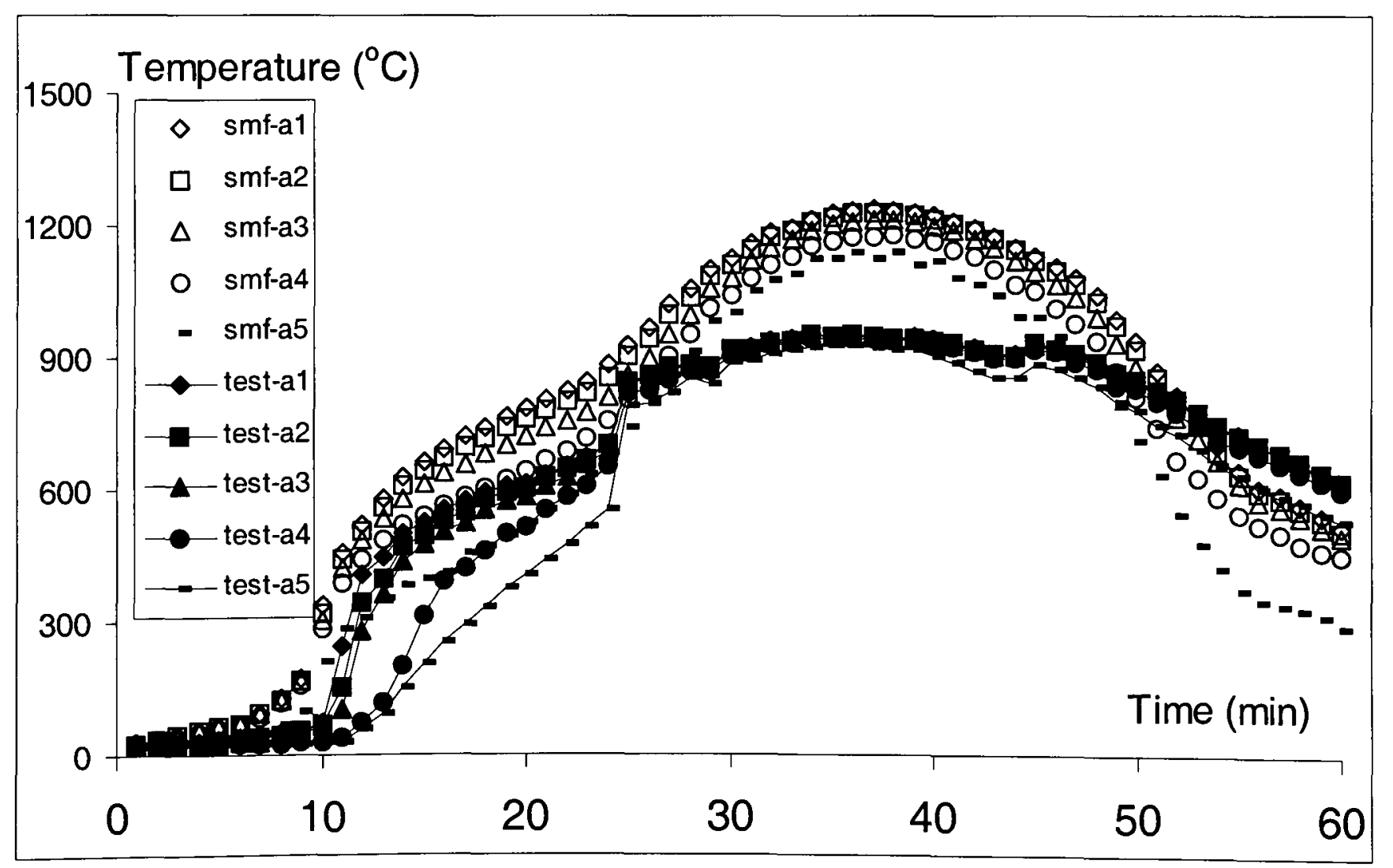

Figure 12: Predicted (symbols) and measured (solid lines) temperatures $\left({ }^{\circ} \mathrm{C}\right.$ ) on line $\mathbf{A}-\mathrm{A}^{\prime}$ as a function of time (min) for simulation $\mathrm{S} 2$.

The maximum measured temperature at the A-A' location over the first 30 minutes is $921^{\circ} \mathrm{C}$, while the maximum predicted temperature for the $\mathrm{S} 1$ simulation is $1240^{\circ} \mathrm{C}$ (an error of $35 \%$ ) and that for the $\mathrm{S} 2$ simulation is $1130^{\circ} \mathrm{C}$ (an error of $23 \%$ ). Furthermore, the average temperature after 30 minutes at location $A-A^{\prime}$ predicted by the $S 2$ simulation is approximately $1072^{\circ} \mathrm{C}$ while the measured average temperature is $910^{\circ} \mathrm{C}$. 
While the S2 simulation produced an error of $162^{\circ} \mathrm{C}$ or $18 \%$, this represents a reduction of $168^{\circ} \mathrm{C}$ compared to the $\mathrm{S} 1$ predictions (see tables 1-3). The $\mathrm{S} 2$ prediction of the peak temperature after 30 minutes is comparable to that predicted by JASMINE (i.e. approximately $1120^{\circ} \mathrm{C}$ ).

After 30 minutes, the $\mathrm{S} 2$ predicted temperatures show a very gradual increase, reaching a maximum at approximately 40 minutes. The average predicted

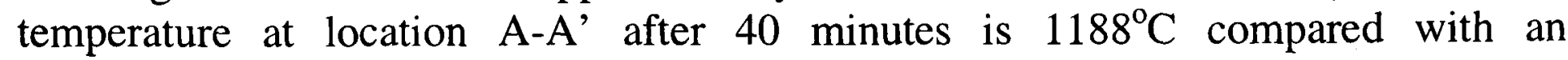
approximate average temperature of $932^{\circ} \mathrm{C}$, producing an overprediction of $27 \%$ (see table 3). From 40 to 60 minutes, the predicted temperatures fall and the level of stratification is predicted to increase in line with observations. However, the degree of stratification in the numerical predictions is greater than that observed in the experiment (see table 2) and the numerical predictions tend to underpredict the measured temperatures. At 60 minutes the average measured temperature at location $\mathrm{A}-\mathrm{A}^{\prime}$ is $596^{\circ} \mathrm{C}$ while that predicted is $452^{\circ} \mathrm{C}$, an underprediction of $24 \%$.

Figure 13 depicts the measured temperature distribution at location B-B' from 0 to 60 minutes, a further 30 minutes than was depicted in figure 10. As can be seen from figure 13 , the measured temperatures at location $\mathrm{B}-\mathrm{B}^{\prime}$ reach a maximum value of some $1199^{\circ} \mathrm{C}$ after approximately 40 minutes and then show a gradual decline for the next 20 minutes. As with the measured temperatures at A-A', the level of stratification increases with the lower layer becoming progressively cooler from 40 minutes through to 60 minutes.

TABLE 2: Comparison of measured and predicted (S2) temperatures $\left({ }^{\circ} \mathrm{C}\right)$ at the three thermocouple tree locations as a function of time.

\begin{tabular}{|c|c|c|c|c|c|c|c|}
\hline \multirow{2}{*}{ Time } & \multirow{2}{*}{ Position } & \multicolumn{2}{|c|}{$\mathbf{A}-\mathbf{A}$} & \multicolumn{2}{|c|}{ B-B } & \multicolumn{2}{|c|}{ C-C } \\
\hline & & $\operatorname{Exp}\left({ }^{\circ} \mathrm{C}\right)$ & $\begin{array}{c}\text { Pred } \\
\left({ }^{\circ} \mathrm{C}\right)\end{array}$ & $\operatorname{Exp}\left({ }^{\circ} \mathrm{C}\right)$ & $\begin{array}{l}\text { Pred } \\
\left({ }^{\circ} \mathrm{C}\right)\end{array}$ & $\operatorname{Exp}\left({ }^{\circ} \mathrm{C}\right)$ & $\begin{array}{c}\text { Pred } \\
\left({ }^{\circ} \mathrm{C}\right)\end{array}$ \\
\hline \multirow{5}{*}{$5 \mathrm{~min}$} & 1 & 32.2 & 62.5 & 38.6 & 69.2 & 31.0 & 60.4 \\
\hline & 2 & 28.2 & 62.3 & 35.9 & 63.1 & 31.3 & 60.4 \\
\hline & 3 & 25.5 & 62.5 & 25.8 & 60.9 & 27.6 & 60.3 \\
\hline & 4 & 24.6 & 59.6 & 24.9 & 58.0 & 23.4 & 59.8 \\
\hline & 5 & 25.2 & 38.5 & 23.7 & 46.8 & 23.7 & 54.1 \\
\hline \multirow{5}{*}{$10 \mathrm{~min}$} & 1 & 75.3 & 338 & 149 & 402 & 81.6 & 308 \\
\hline & 2 & 69.1 & 321 & 86.0 & 357 & 81.9 & 307 \\
\hline & 3 & 64.9 & 307 & 61.0 & 311 & 56.9 & 307 \\
\hline & 4 & 29.5 & 287 & 31.9 & 270 & 27.0 & 298 \\
\hline & 5 & 29.2 & 212 & 26.3 & 202 & 25.5 & 268 \\
\hline \multirow{5}{*}{$20 \mathrm{~min}$} & 1 & 616 & 789 & 998 & 1050 & 656 & 691 \\
\hline & 2 & 604 & 761 & 942 & 1040 & 670 & 688 \\
\hline & 3 & 587 & 722 & 819 & 995 & 665 & 681 \\
\hline & 4 & 517 & 646 & 713 & 891 & 630 & 663 \\
\hline & 5 & 406 & 527 & 574 & 737 & 484 & 632 \\
\hline \multirow{6}{*}{$25 \mathrm{~min}$} & 1 & 839 & 928 & 1081 & 1260 & 909 & 788 \\
\hline & 2 & 847 & 904 & 1089 & 1260 & 931 & 785 \\
\hline & 3 & 844 & 859 & 1087 & 1250 & 930 & 776 \\
\hline & 4 & 819 & 805 & 1081 & 1220 & 908 & 754 \\
\hline & 5 & 794 & 742 & 1057 & 1110 & 872 & 717 \\
\hline & 1 & 919 & 1130 & 1128 & 1360 & 945 & 829 \\
\hline
\end{tabular}


APPENDIX A SIMULATING ONE OF THE CIB W14 ROUND ROBIN TEST CASES USING THE SMARTFIRE FIRE FIELD MODEL

\begin{tabular}{|c|c|c|c|c|c|c|c|}
\hline \multirow{4}{*}{$30 \mathrm{~min}$} & 2 & 921 & 1110 & 1134 & 1350 & 971 & 826 \\
\hline & 3 & 918 & 1080 & 1130 & 1340 & 979 & 813 \\
\hline & 4 & 905 & 1040 & 1110 & 1300 & 970 & 779 \\
\hline & $\overline{5}$ & 889 & 1000 & 1078 & 1200 & 950 & 731 \\
\hline \multirow{5}{*}{$40 \mathrm{~min}$} & 1 & 944 & 1220 & 1199 & 1340 & 954 & 821 \\
\hline & 2 & 939 & 1220 & 1197 & 1330 & 965 & 821 \\
\hline & $\overline{3}$ & 937 & 1200 & 1156 & 1310 & 957 & 813 \\
\hline & 4 & 932 & 1160 & 1078 & 1260 & 951 & 791 \\
\hline & 5 & 907 & 1120 & 996 & 1150 & 929 & 756 \\
\hline \multirow{5}{*}{$50 \mathrm{~min}$} & $\overline{1}$ & 852 & 937 & 999 & 1130 & 846 & 765 \\
\hline & 2 & 849 & 918 & 975 & 1110 & 853 & 761 \\
\hline & 3 & 846 & 879 & 973 & 1080 & 846 & 750 \\
\hline & 4 & 831 & 810 & 987 & 1000 & 840 & 727 \\
\hline & 5 & 781 & 713 & 921 & 844 & 828 & 694 \\
\hline \multirow{5}{*}{$60 \mathrm{~min}$} & 1 & 619 & 515 & 703 & 630 & 634 & 480 \\
\hline & 2 & 617 & 508 & 693 & 598 & 637 & 476 \\
\hline & 3 & 617 & 497 & 675 & 545 & 625 & 469 \\
\hline & 4 & 598 & 453 & 643 & 486 & 602 & 451 \\
\hline & 5 & 529 & 288 & 629 & 387 & 576 & 404 \\
\hline
\end{tabular}

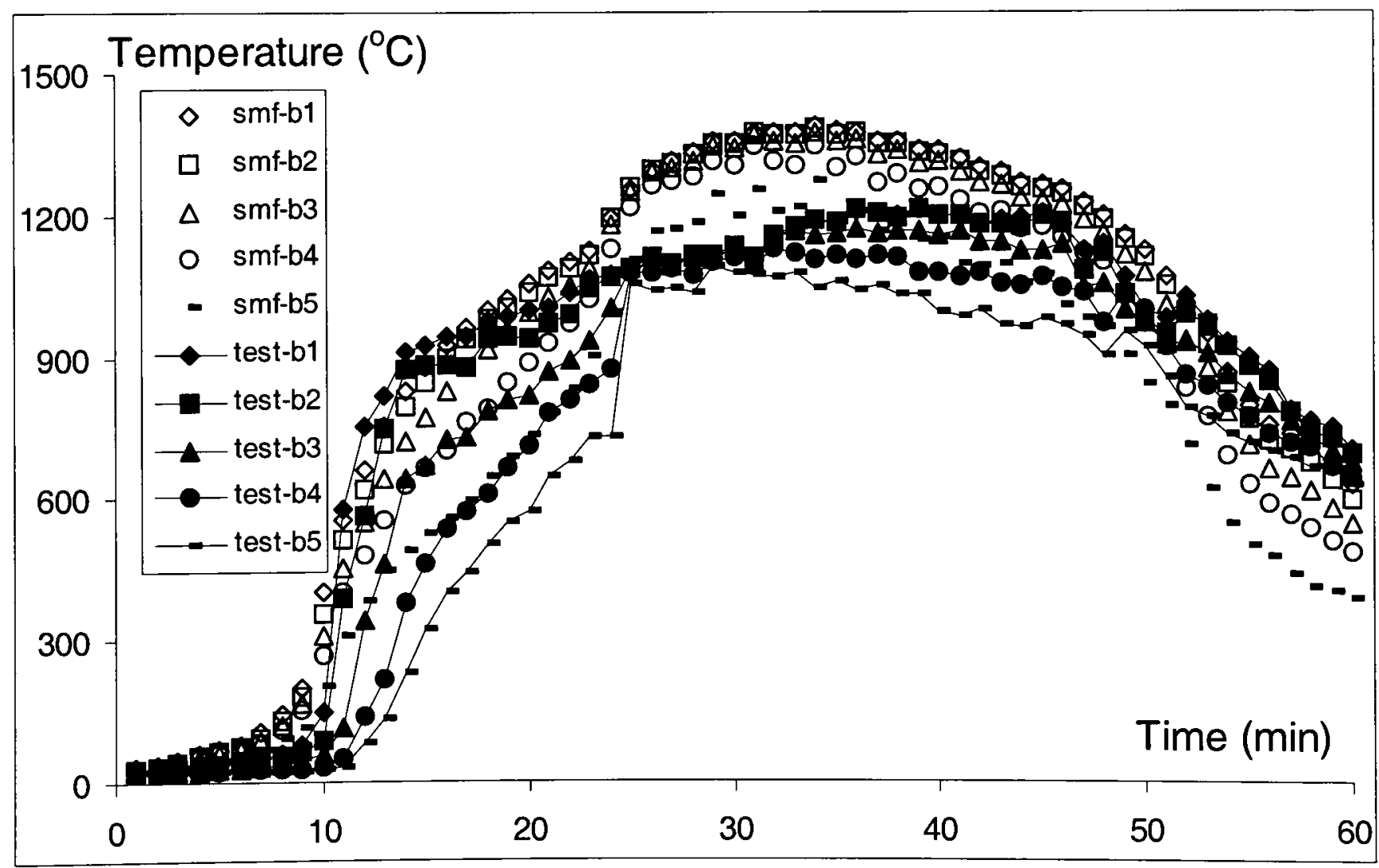

Figure 13: Predicted (symbols) and measured (solid lines) temperatures $\left({ }^{\circ} \mathrm{C}\right)$ on line $\mathrm{B}-\mathrm{B}^{\prime}$ as a function of time (min) for simulation $\mathrm{S} 2$.

As with the previous case, the predicted temperatures appear to follow this trend throughout the first 60 minutes. Over the first 30 minutes, the $\mathrm{S} 2$ predictions produce a better representation of the measured temperatures than the $S 1$ predictions (see tables 2 and 3). In particular, the overprediction in temperatures has decreased and there is a greater level of stratification predicted. Furthermore, the mesh refinement in the $\mathrm{S} 2$ simulation has eliminated the unusual temperature inversion at location $\mathrm{B}$ $B$ ' previously observed in the S1 simulations (see table 2). This shows the 
importance of performing mesh refinement studies, especially when unusual numerical results are predicted.

The peak temperature observed in the experiment at location $\mathrm{B}-\mathrm{B}^{\prime}$ is $1214^{\circ} \mathrm{C}$ and occurs after 39 minutes. The peak in the numerical predictions is $1360^{\circ} \mathrm{C}$ (an error of $12 \%$ ) and occurs after 30 minutes. However, the predicted temperature shows very little variation over the next 10 minutes, decreasing by only $20^{\circ} \mathrm{C}$ or $1.5 \%$ measured at the same thermocouple. The average predicted temperature after 30 minutes at location $\mathrm{B}-\mathrm{B}^{\prime}$ is approximately $1310^{\circ} \mathrm{C}$ while the measured average temperature is $1116^{\circ} \mathrm{C}$. Thus after 30 minutes, the predicted average values exceed the measured average values by $194^{\circ} \mathrm{C}$ or $17 \%$ (see table 3). Between 25 minutes and 50 minutes, the predicted average temperature at location B-B' differ from the measured values by between 6 and $17 \%$.

As with location A-A', the trends in the JASMINE results follow that of SMARTFIRE. JASMINE appears to produce a peak temperature of approximately $1360^{\circ} \mathrm{C}$ after 30 minutes which decreases to approximately $1280^{\circ} \mathrm{C}$ after 40 minutes. At 20 minutes, the measured maximum and minimum temperatures are $998^{\circ} \mathrm{C}$ and $574^{\circ} \mathrm{C}$ while the predictions by SOFIE, JASMINE and SMARTFIRE are approximately $990^{\circ} \mathrm{C}$ and $565^{\circ} \mathrm{C}$, approximately $1000^{\circ} \mathrm{C}$ and $740^{\circ} \mathrm{C}$ and $1050^{\circ} \mathrm{C}$ and $737^{\circ} \mathrm{C}$ respectively. Here again, the mesh refinement has improved the SMARTFIRE predictions, bringing them closer to the experimental results and the JASMINE predictions.

TABLE 3: Comparison of average measured and predicted (S1 and S2) temperatures $\left({ }^{\circ} \mathrm{C}\right)$ at the three thermocouple tree locations and associated errors.

\begin{tabular}{|c|c|c|c|c|c|c|}
\hline \multirow{2}{*}{$\begin{array}{l}\text { Time } \\
\text { (min) }\end{array}$} & \multirow{2}{*}{ Location } & \multirow{2}{*}{$\begin{array}{l}\text { Exp } \\
\text { Average } \\
\text { Temp }{ }^{\circ} \mathrm{C}\end{array}$} & \multicolumn{2}{|c|}{ S1 Average } & \multicolumn{2}{|c|}{ S2 Average } \\
\hline & & & $\operatorname{Temp}^{\circ} \mathrm{C}$ & $\%$ error & $\operatorname{Temp}^{\circ} \mathrm{C}$ & \% error \\
\hline \multirow{3}{*}{5} & A-A & 27 & 65 & 140 & 58 & 115 \\
\hline & B-B & 30 & 67 & 123 & 60 & 100 \\
\hline & C-C & 27 & 65 & 141 & 59 & 119 \\
\hline \multirow{3}{*}{10} & A-A & 54 & 337 & 524 & 293 & 443 \\
\hline & B-B & 71 & 365 & 414 & 308 & 334 \\
\hline & $\mathrm{C}-\mathrm{C}$ & 55 & 332 & 504 & 298 & 442 \\
\hline \multirow{3}{*}{20} & A-A & 546 & 791 & 45 & 689 & 26 \\
\hline & B-B & 629 & 1088 & 73 & 942 & 50 \\
\hline & $\mathrm{C}-\mathrm{C}$ & 621 & 715 & 15 & 671 & 8 \\
\hline \multirow{3}{*}{25} & A-A & 829 & 1019 & 23 & 848 & 2 \\
\hline & B-B & 1079 & 1354 & 25 & 1220 & 13 \\
\hline & $\mathrm{C}-\mathrm{C}$ & 910 & 796 & 13 & 764 & 16 \\
\hline \multirow{3}{*}{30} & $\mathrm{~A}-\mathrm{A}$ & 910 & 1240 & 36 & 1072 & 18 \\
\hline & B-B & 1116 & 1310 & 17 & 1310 & 17 \\
\hline & $\mathrm{C}-\mathrm{C}$ & 963 & 815 & 15 & 796 & 17 \\
\hline \multirow{3}{*}{40} & $\mathrm{~A}-\mathrm{A}$ & 932 & - & - & 1184 & 27 \\
\hline & B-B & 1125 & - & - & 1278 & 14 \\
\hline & $\mathrm{C}-\mathrm{C}$ & 951 & - & - & 800 & 16 \\
\hline \multirow{3}{*}{50} & A-A & 831 & - & - & 851 & 2 \\
\hline & B-B & 971 & - & - & 1032 & 6 \\
\hline & $\mathrm{C}-\mathrm{C}$ & 843 & - & - & 739 & 12 \\
\hline
\end{tabular}




\begin{tabular}{|l|l|l|l|l|l|l|}
\hline \multirow{3}{*}{60} & A-A & 596 & - & - & 452 & 24 \\
\cline { 2 - 7 } & B-B & 669 & - & - & 529 & 21 \\
\cline { 2 - 7 } & C-C & 615 & - & - & 456 & 26 \\
\hline
\end{tabular}

Figure 14 depicts the measured temperature distribution at location C-C' from 0 to 60 minutes, a further 30 minutes than was depicted in figure 12. As can be seen from figure 14 , the measured temperatures at location $\mathrm{C}-\mathrm{C}^{\prime}$ reach a maximum value of some $1120^{\circ} \mathrm{C}$ after approximately 30 minutes, remain approximately constant for the next 10 minutes and then show a gradual decline for the next 20 minutes. Unlike the situation noted at location A-A' and B-B', after 30 minutes the level of stratification as measured at location C-C' does not increase significantly. As with the observations at the other locations, the predicted temperatures appear to follow this trend throughout the first 60 minutes. Over the first 30 minutes, the S2 predictions produce a better representation of the measured temperatures than the $\mathrm{S} 1$ predictions (see tables 1-3).

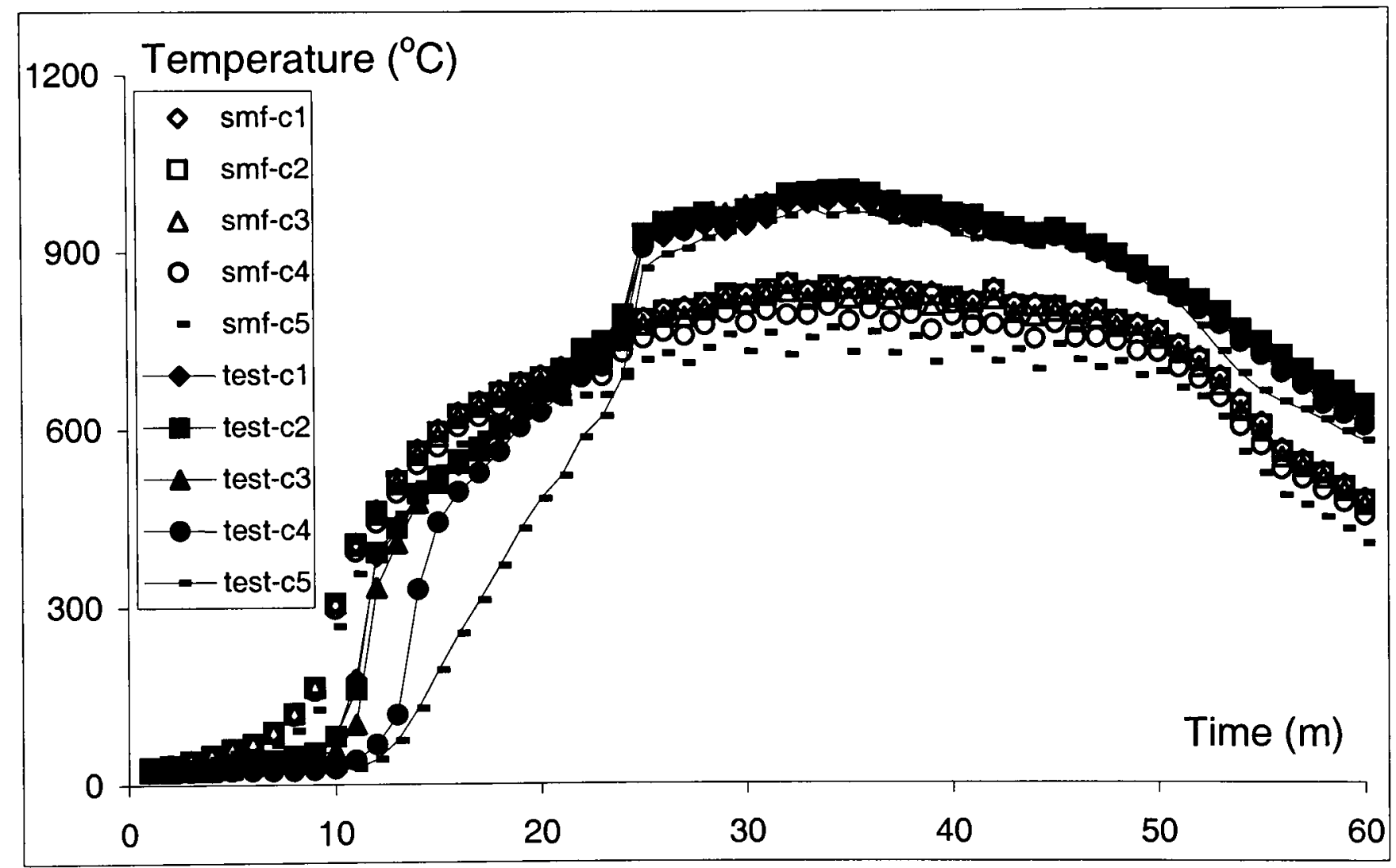

Figure 14: Predicted (symbols) and measured (solid lines) temperatures $\left({ }^{\circ} \mathrm{C}\right.$ ) on line $\mathbf{C}-\mathrm{C}^{\prime}$ as a function of time (min) for simulation $\mathrm{S} 2$.

The underprediction trend noted previously in the $S 1$ simulation from 25 to 30 minutes is also apparent in the S2 simulation and continues through to 60 minutes (see table 2). At 20 minutes, the average $\mathrm{S} 2$ predicted temperature is $671^{\circ} \mathrm{C}$ compared with a measured average value of $621^{\circ} \mathrm{C}$, a difference of $50^{\circ} \mathrm{C}$ or $8 \%$. The average predicted temperature after 40 minutes is approximately $800^{\circ} \mathrm{C}$ while the measured average temperature is $951^{\circ} \mathrm{C}$. Thus after 40 minutes, the predicted average values underpredict the measured average values by $151^{\circ} \mathrm{C}$ or $16 \%$ (see table 3 ). While the temperature predictions for the S2 simulations underpredict the measured values, there is good agreement with the level of stratification observed, primarily in the final 10 minutes. 
The peak temperature observed in the experiment at location $\mathrm{C}-\mathrm{C}$ ' is $1004^{\circ} \mathrm{C}$ and occurs after 35 minutes. The peak in the numerical predictions is $842^{\circ} \mathrm{C}$ (an error of $16 \%$ ) and occurs after 34 minutes. However, the measured peak temperature occurs at thermocouple three while the predicted peak temperature occurs at thermocouple 1 . At first glance, the temperature inversion noted in the experimental results at location C-C' (see table 2) is not apparent in the S2 numerical predictions. However, a closer examination of the data generated by the $\mathrm{S} 2$ simulation does in fact reveal that a slight temperature inversion does begin at around 20 minutes after the start of the simulation. While the temperature inversion is not as pronounced as that observed in the experimental results and it occurs at a higher location, (between the ceiling and thermocouple two), it does nevertheless occur. As suggested earlier, the location of the temperature inversion region corresponds to the downturn region of the ceiling jet (located in the upper corner of the domain) (see figure 15). A more refined computational mesh is required to better resolve this behaviour.

Finally, it should be noted that all the numerical predictions produced in this paper are strongly dependent on the value imposed on the burning efficiency factor $\chi$ (see equation 1). The value used in these calculations was the value recommended for the Round Robin. If this value is too large, this could explain the observed overprediction of the temperatures. Indeed, while the burning efficiency factor $\chi$ is treated as a constant, it is known to vary - in some cases by a large amount - throughout the development of the fire.

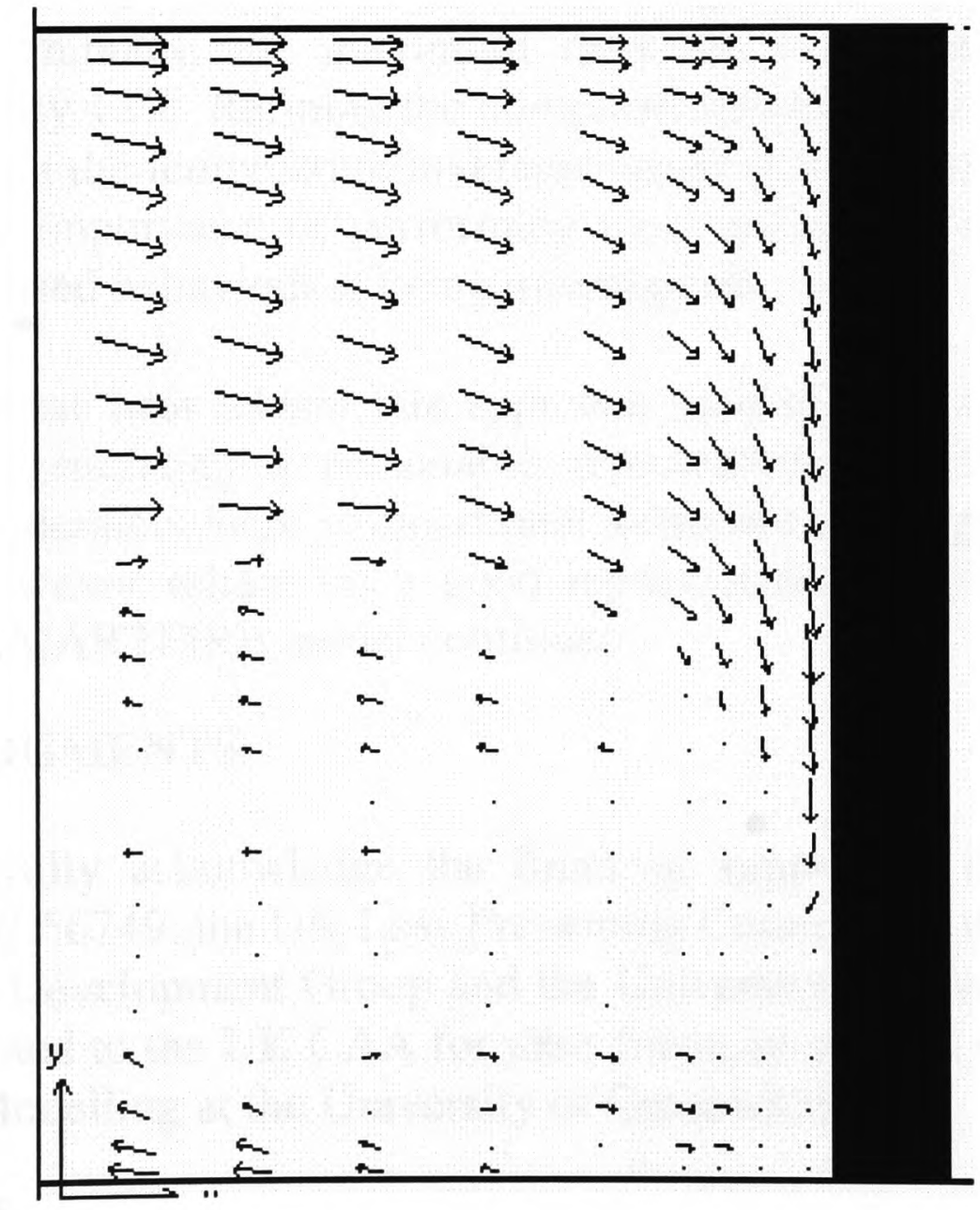

FIGURE 15: Section of flow field generated by $S 2$ simulation showing impact of ceiling jet with front wall of the test compartment.

\section{CONCLUSIONS}


SMARTFIRE was capable of producing reasonable agreement with experimental temperature measurements derived for a non-spreading fire in a moderately large ventilated fire compartment. The test fire, produced by a burning wood crib attained a maximum heat release rate of approximately $11 \mathrm{MW}$.

The course mesh simulation, while performed on a relatively crude computational mesh was able to represent the broad features of qualitative trends in the observed temperature distributions at the three measuring locations. Quantitatively, temperatures were generally overpredicted and the level of stratification within the compartment was not well represented. After an initial 20 minute "burn in", the average predicted temperature at each of the three measuring sites varied from the experimentally derived average temperatures by between 15 and $73 \%$. In addition, a temperature inversion was noted in the numerical predictions at one measuring location which was not seen in the experimental results. Over the first 30 minutes, a maximum temperature of $1134^{\circ} \mathrm{C}$ was recorded in the experiment at location B-B', thermocouple 2 at 30 minutes. This corresponded to a predicted temperature of $1390^{\circ} \mathrm{C}$ at the same location, producing an error of $23 \%$.

The finer mesh simulation was better able to reproduce the qualitative features of the experimental data. In addition, temperatures were quantitatively better represented as was the level of stratification within the compartment. After the initial 20 minute "burn in", the average predicted temperature at each of the three measuring sites varied from the experimentally derived average temperatures by between 2 and $50 \%$. Over the first 60 minutes, the maximum recorded temperature of $1214{ }^{\circ} \mathrm{C}$ was overpredicted by only $12 \%$. Refining the computational mesh eliminated the spurious behaviour generating the temperature inversion noted in the course mesh simulations. This highlights the importance of performing mesh refinement exercises, especially when detailed localised behaviour is being investigated.

Finally, the volumetric heat release rate approach used in these simulations is shown to be capable of generating a reasonable representation of the gas temperature distribution in a moderately large compartment subjected to a large non-spreading fire. The results are however reliant on a good representation of the heat release rate. Validation of the SMARTFIRE model continues.

\section{ACKNOWLEDGMENTS}

The authors gratefully acknowledge the financial support of the EPSRC through research grant GR/L56749, the UK Loss Prevention Council and the UK Home Office Fire Research and Development Group and the University of Greenwich. Professor E Galea is also indebted to the UK CAA for their financial support of his personal chair in Mathematical Modelling at the University of Greenwich.

\section{REFERENCES}

1. Galea E.R., Knight B., Patel M., Ewer J., Petridis M., and Taylor S., "SMARTFIRE V2.01 build 365, User Guide and Technical Manual", SMARTFIRE CD, 1999.

2. Taylor S., Petridis M., Knight B., Ewer J., Galea E.R. and Patel M., SMARTFIRE: An Integrated Computational Fluid Dynamics code and Expert System for Fire 
Field Modelling. Fire Safety Science - Proceedings of the Fifth International Symposium, 1997, pp 1285-1296.

3. Ewer, J., Galea E.R., Patel M., Taylor, S., Knight B., Petridis M., SMARTFIRE: An Intelligent CFD Based Fire Model, Journal of Fire Protection Engineering, 10 (1), pp 13-27, 1999.

4. Ewer J., Galea E.R., Patel M., and Knight B. The Development and Application of Group Solvers in the SMARTFIRE Fire Field Model. Proceedings Interflam 99, Edinburgh, UK, pp 939 - 950, 1999

5. Galea E.R. "A General Approach to Validating Evacuation models with an application to EXODUS", J of Applied Fire Science, 1998, vol. 16, Nov/Dec, pp. 414-436.

6. Smartfire Verification and Validation Report. Software Version 2.01, Report Version 1.01 Fire Safety Engineering Group, University of Greenwich. Revision Date 25/05/99

7. Steckler K.D., Quintiere J.G. and Rinkinen W.J., "Flow Induced By Fire in a Compartment", NBSIR 82-2520, National Bureau of Standards, Washington, 1982.

8. Hostikka S and Keski-Rahkonen O., Results of CIB W14 Round Robin for Code Assessment Scenario B. Draft 31/08/98, VTT Technical Research Centre of Finland.

9. Galea E.R., On the field modelling approach to the simulation of enclosure fires, Journal of Fire Protection Engineering, vol 1 (1), 1989, pp 11-22.

10. Combustion Fundamentals of Fire, Editor: Cox G., Academic Press 1995.

11. Spalding D.B., "A General Purpose computer Program For Multi-Dimensional One- and Two- Phase Flow", Mathematics and Computers in Simulations, North Holland (IMACS), Vol. XXIII, 1981, 267.

12. Kumar S., Gupta A.K. and Cox G., "Effects of Thermal Radiation on the Fluid Dynamics of Compartment Fires", Fire Safety Science - Proc. of the Third Intl. Symp., 1991, pp 345-354.

13. Lewis M.J., Moss M.B. and Rubini P.A., "CFD Modelling of Combustion and Heat Transfer in Compartment Fires", Fire Safety Science, Proc. of the $5^{\text {th }}$ Int. Symp., Ed: Hasemi Y., 1997, pp 463-474.

14. Pantakar S.V., "Numerical Heat Transfer and Fluid Flow", Intertext Books, McGraw Hill, New York, 1980.

15. www: http://fseg.gre.ac.ukJ

16. Hostikka S and Keski-Rahkonen O., Design and simulation reports of CIB W14 Round Robin for code assessment Scenario B. VTT Building Technology Internal Report: RTE119-IR-2/1998.4 + 272p.

17. Hagen, $\mathrm{E}$ and Haksever, A. Contribution for the investigation of natural fires in large compartments. IAFSS $1^{\text {st }}$ Int Symp, Oct 1985, Gaithersburg USA, Hemisphere Pub Corp NY. pp 149-158.

18. Results of CIB W14 Round Robin for Code Assessment Scenario B. Draft 31/08/99, VTT Technical Research Centre of Finland.

19. Hubbard, G. L. and Tien, C. L., "Infrared mean absorption coefficients of luminous flames and smoke", J. Heat Transfer, 1978, vol. 100, pp. 235-239. 


\section{APPENDIX B}

\section{PUBLICATIONS}

\section{PAPERS:}

Wang Z., Jia F., Galea E.R., Patel M.K. and Ewer J, Predicting $\mathrm{HCl}$ concentrations in fire enclosures using a $\mathrm{HCl}$ decay model coupled to a CFD based fire field model, Accepted by Fire and Materials, Nov., DOI: 10.1002/fam.942, 2006.

Wang Z., Jia F., and Galea E.R., Predicting toxic gas concentrations resulting from enclosure fires using local equivalence ratio concept linked to fire field models, Fire and Materials, Vol. 31, No. 1, 2007, pp 27-51.

Wang Z., Jia F., Galea E.R., Predicting toxic gas concentrations resulting from enclosure fires using the local equivalence ratio concept linked to fire field models, Paper No. 02/IM/101, CMS Press, the university of Greenwich, 2002.

Wang Z., Jia F., Galea E. R., Patel M. K. and Ewer J., Simulating one of the CIB W14 round robin test cases using the SMARTFIRE fire field models, Fire Safety Journal, Vol. 36, 2001, pp 661-677.

Wang Z., Jia, F., Galea, E. R., Patel, M. K. and Ewer, J., Simulating one of the CIB W14 round robin test cases using the SMARTFIRE fire field models, Paper No. 00/IM/53, CMS Press, University of Greenwich, 2000.

\section{CONFERENCE PUBLICATIONS:}

Wang Z., Jia F., Galea E.R. and Patel M. K., Predicting toxic gases in a full-scale vitiated fire, accepted by $11^{\text {th }}$ International Interflam, 2007, London.

Wang Z., Jia F. and Galea E.R., The use of local equivalence ratio in field fire modelling for the prediction of carbon monoxide within fire enclosures, FIRENET workshop on fire in enclosures, Ulster University, 30-31 May, 2006.

Wang Z., Jia F., Galea E.R. and Ewer J., Predicting hydrogen chloride concentrations in fire enclosures using a deposition model linked to fire field models, $\underline{10^{\text {th }}}$ International Interflam, 2004, volume 1, pp. 665-670.

Wang Z., Jia F., Galea E. R., Applying the local equivalence ratio concept to fire field models, $9^{\text {th }}$ International Interflam, 2001, volume 2, pp. 1409-1414.

Galea E. R., Lawrence P, Gwynne S., Filippidis L., Blackshields D., Sharp G., Hurst N., Wang Z., and Ewer J. the Simulation of Ship evacuation under fire conditions, Proc Fire and Safety at Sea, Rocarm Pty Ltd, Melbourne Australia 17-19 March 2004, day2, session 1, pp 1/14 -14/14, 2004. 
Galea E. R., Lawrence P, Gwynne S., Filippidis L., Blackshields D., Sharp G., Hurst N., Wang Z., and Ewer J., Simulating Ship evacuation under fire conditions, Proc $2^{\text {nd }}$ Int Pedestrian and Evacuation Dynamics Conference, Ed: E. R. Galea, CMS Press, Greenwich, UK, ISBN 1904521088, pp. 159-172, 2003.

Galea E. R., Gwynne S., Lawrence P., Blackshields D., Ewer, J. Wang Z., Hurst N. and Mawhinney N., Fire Safety Engineering and Ship Design Using Advanced Fire and Evacuation Simulation.. $1^{\text {st }}$ International Conference - Fire on Ships: Prevention, Detection and Control on Ships and Offshore Structures, 11-12/3/03.

Galea E R, Gwynne S., Lawrence P., Blackshields D., Ewer, J., Wang Z., Hurst N. and Mawhinney $\mathrm{N}$, The Application of Fire and Evacuation Simulation in Ship Design, COMPIT 2003, 2nd International EuroConference on Computer and IT Applications in the Maritime Industries, Hamburg, pp55-69, 2003.

\section{SUBMITTED PAPERS:}

Wang, Z., Jia F., Galea E.R., Patel M.K., Predicting toxic gas concentrations at locations remote from the fire source, submitted to Fire and Materials in November 2006. 